A UNTED STATES XEPARTMENT OF COMMERCE PUBLICATION

watlonal Bureau of Standards

Library, E-01 Admin. Bldg.

\section{OCi 1970 \\ NBS TECHNICAL NOTE 554}

\title{
Annotated Accession \\ List of Data Compilations of the NBS Office of Standard Reference Data
}

U.S. DEPARTMENT OF COMMERCE 2C National 100 Bureau of 5753 tandards 554 


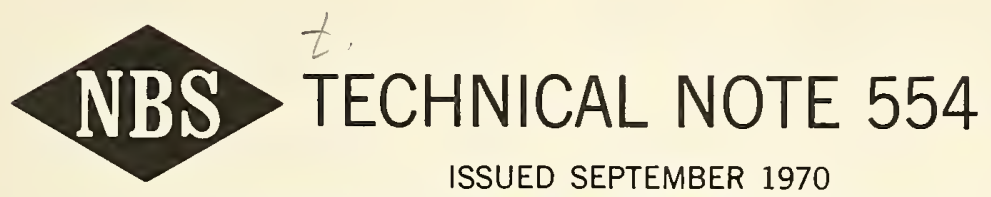

Nat. Bur. Stand. (U.S.), Tech. Note 554, 196 pages (Sept. 1970) CODEN: NBTNA

\section{Annotated Accession List of Data Compilations of the Office of Standard Reference Data}

Herman M. Weisman and Gertrude B. Sherwood

Office of Standard Reference Data

National Bureau of Standards

Washington, D.C. 20234

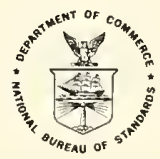

NBS Technical Notes are designed to supplement the Bureau's regular publications program. They provide a means for making available scientific data that are of transient or limited interest. Technical Notes may be listed or referred to in the open literature.

For sale by the Superintendent of Documents, U.S. Government Printing Office, Washington, D.C., 20402.

(Order by SD Catalog No. C 13.46:554), Price $\$ 1.50$. 
The National Bureau of Standards, Office of Standard Reference Data has attempted to acquire all significant reference data compilations on a worldwide basis. This publication lists the collection of documents so acquired, together with their abstracts. The documents are organized in the following categories: General Collections, Nuclear Properties (including Fundamental Particles Properties), Atomic and Molecular Properties, Solid State Properties, Chenical Kinetics, Colloid and Surface Properties, Mechanical Properties, and Thermodynamic and Transport Properties. Sources of availability for the listed publications are also provided.

Key words: Accession List, Atomic and Molecular Properties, Chemical Kinetics, Colloid and Surface Properties, Fundamental Particles Properties, General Collections, Mechanical Properties, Nuclear Properties, Solid State Properties, Thermodynamic and Transport Properties 
Table of Contents

Introduction 1

Page

Genera1 Collections 4

Nuclear Properties (Including

$\begin{array}{ll}\text { Fundamental Particles Properties) } & 18\end{array}$

Atomic and Molecular Properties 57

Solid State Properties 94

$\begin{array}{ll}\text { Chemical Kinetics } & 121\end{array}$

Colloid and Surface Properties 132

$\begin{array}{lll}\text { Mechanica1 Properties } & 135\end{array}$

Thermodynamic and Transport Properties 141 

The scientist or engineer seeking specific data is often confronted with a number of difficulties. Where can the data be found? Are they complete? Are they compatible with other sources? Most important, how reliable are they? Each year the problem for the scientist and engineer becomes greater. As the volume of literature expands, he finds even more difficulty in ${ }^{2} r v i n g$ to keep abreast of literature relevant to his field. This is why the National Standard Reference Data System (NSRDS) was created. The NSRDS is a nationwide program to help provide the U.S. technical community optimum access to the quantitative data of the physical sciences, critically evaluated and compiled in convenient formats. It was established in 1963 by action of the President's Office of Science and Technology with responsibility for administering the effort given to the National Bureau of Standards. The Office of Standard Reference Data was set up within NBS to manage and coordinate the program. The National Standard Reference Data System operates, for the most part, as a decentralized cooperative network of data centers and data compilation projects for which the office of Standard Reference Data (OSRD) is the program management vehicle. The data centers are composed of individuals or groups working on the collection and evaluation of all the data within specific technical areas. These centers, many of which are located in the experimental divisions of the NBS, some in other government laboratories, at universities or at private companies, are the source of the critically evaluated compilations, reviews, and bibliographies published by the NSRDS program.

The scope of the program has been limited to the physical sciences. For management purposes, the Office of Standard Reference Data has divided its activity into specific technical areas: nuclear data, atomic and molecular data, solid state data, thermodynamic and transport data, chemical kinetics, mechanical properties, and colloid and surface properties. Fach of the technical areas is organized in a similar way, having a program manager who monitors data compiling groups who may be working at the NBS or may be under contract, or who might be a group sponsored by the other government agencies. Each area program has an advisory panel of expert consultants, who aid in appraising activities, assessing priorities and recommending what the NSRDS should do in a given area.

Among the primary duties of the Office of Standard Reference Data, according to the directive from the office of Science and Technology which established the program is the operation of an information services center for the NSRDS. Because the major available resources of the NSRDS program have been applied thus far to the production of compilations and critical reviews, the development of a vigorous information service activity has been delayed. Nevertheless, some basic and significant services have been implemented. One of the more active is the operation of a Data File. The mission of the OSRD Data File (lihrary) is to acquire selectively, on a world-wide basis, reference data compilations and to organize the collection systematically. The Data File houses a collection of about 1300 reference data compilations, critical reviews, bibliographies and other ancillary reference works. It has been judged as one of the most extensive collections of its kind in the world.

Systematic compilations of data contribute in a fundamental way to the progress of science and technology. Data Compilations have been among the 
basic tools of scientists and engineers throughout the history of technology. Each worker owns at least one handbook containing, among other useful information, table after table of data on the properties of the substances and systems that he deals with daily. Recognizing that knowledge of the items within its collection could be of value to many scientists and engineers, the Office of Standard Reference Data has organized the documents and prepared an annotated accession list to be made available to the technical community. Individuals as well as organizations might be interested in single items within the collection of groupings of them.

The Annotated Accession List has been organized in accordance with the present shelving arrangements of the documents in the office of Standard Reference Data library. We consider this a first approach to classification and indexing of the holdings of the program, while searching for a classification and indexing system that can withstand the test of filegrowth, time, and complexity. A modest examination has shown that no existing classification scheme is satisfactory for NSRDS needs. The present size of the Data File and its highly specialized nature and use were important factors in the evolvement of the scheme presently being used. Classifying publications for shelf location and for convenient browsing is a somewhat different problem than that of indexing contents to permit rapid location of individual data points.

A classification scheme for shelving does not need to have a large number of partitions of a subject matter. Factors in the development of the system included the needs of the user, his familiarity with or capability to become acquainted with the shelving system, and his subjective reactions while browsing. For these reasons, the approach to shelving the holdings of the Office of Standard Reference Data File has emphasized the needs of those who use the file for browsing -- that is, the professional staff of the office.

Nine classification categories based on the NSRDS technical areas are used. The categories are further partitioned by a decimal type of breakdown. The scheme is as follows:

General Collections

Fundamental Particles Properties

Nuclear Properties

Atomic and Molecular Properties

Solid State Properties

Chemical Kinetics

Colloid and Surface Properties

Mechanical Properties

The rmodynamic and Transport Properties
$0.1,0.2, \ldots$

$1.0,1.1,1.2$

$2.0,2.1,2.1, \ldots$

$3.0,3.1,3.2,3.3$

$4.0,4.1,4.2,4.3$

$5.0,5.1,5.2,5.3$

$6.0,6.1,6.2,6.3$

$7.0,7.1,7.2,7.3$

$8.0,8.1,8.2,8.3$

Associated with the classification numbers are two additional digits. These last two digits are shelving control codes. With the exception of the General Collections, Fundamental Particles Properties and Nuclear Properties categories, the number after the decimal point indicates the type of compounds discussed in the publication: .1 indicates only inorganic compounds, .2 both inorganic and organic, and .3 only organic compounds. For example, the classification 3.1 indicates that the publication deals with atomic and molecular properties of only inorganic compounds, 8.2 indicates the publication contains information on thermodynamic and transport properties of both inorganic and organic compounds.

The Fundamental Particles Properties and Nuclear Properties are subdivided by the categories: stationary states (1.1 and 2.1) and processes $(1.2$ and 2.2). Further, nuclear properties, stationary states, and processes are subdivided by a particular state (i.e., quantum numbers, 2.15) or a particular reaction (i.e., interactions with neutrons, 2.25 ). 
The General Collections classification contains publications appropriate to several categories, housing material like data collections (0.11), handbooks $(0.21)$, and bibliographies $(0.31)$.

The classification number is preceded by the document's accession number and appears on the first line of the item's listing. The items in the Annotated Accession List are arranged by the nine subject cateogories in accordance with the indicated scheme. For the convenience of the reader, items shelved in one technical area but applicable to still another or several have been repeated in the separate area listings.

This Annotated Accession List does not include an index. Its classification scheme is intended essentially as an aid to shelf retrieval of documents. However, the decimal breakdown used provides limited capability for substance-property retrieval. The office of Standard Reference Data is, at present, heginning exploratory work on substanceproperty retrieval.

We have attempted to provide information on the sources of availability for the listed publications. To conserve space, the Clearinghouse for Federal Scientific and Technical Information (CFSTI), the Defense Documentation Center (DDC), the Superintendent of Documents, Government Printing Office (GPO), and the Division of Technical Information, Atomic Energy Commission, (DTI, AEC) have been abbreviated as indicated.

We wish to emphasize that the Office of Standard Reference Data does not necessarily vouch for the validity, criticality, or currency of the data within these documents. They have been selected on the basis of the judgment by the technical staff of the office as providing some information or data of a useful and reference nature. Most of the annotations are based on author abstracts in the original documents.

The Office of Standard Reference Data has attempted to acquire all significant reference data compilations in existence on a world-wide basis. Availability and financial considerations may have precluded acquisition of some documents which properly might have been included. The office of Standard Reference Data invites readers to inform it of any additions which might enhance the collection.

The compilers of this Annotated Listing want to acknowledge their indebtedness to a number of individuals without whose help this Listing would not have been possible. These individuals include: Edmonia Kearney, Lyle D. Joyce, Allen M. We inberger, Alfred Weissberg, David R. Lide, Jr., Edward L. Brady, Howard J. White, Jr., L. H. Gevantman, David T. Goldman, and Joseph Hilsenrath. We want to express our appreciation to Robert Thompson, Carla Messina, and Robert McClennon for the help in mechanizing the Annotated Listing. A special note of thanks is due to Mrs. Darlene Graham for keyboarding the items in this List and to Mrs. Roberta Kadesch, Mrs. Barbara Sommervill, Leonard Loewentritt and Elizabeth Wilson for the painstaking and diligent proofreading. 
General Collections 
$\$ 6500568$

$\star 0.2113$

Abramowitz, M.; Stegun, I. A. (Editors)

HANDBOOK OF MATHEMATICAZ FUNCTIONS WITH FORMULAS, GRAPHS, AND MATHEMATICAL TABLES

NBS Applied Mathematics Series 55 (2nd printing with corrections), National Bureau of Standards, Washington, D. C.

November 1964

$1046 \mathrm{p}$

Available from Superintendent of Documents, GPO

This Handbook contains tabulations of mathematical methods, mathematical functions, and tabulations of their values. The explanatory sections were written by various authors; the tables were calculated by means of a digital computer. The following functions have been tabulated: logarithmic, exponential, trigonometric, hyperbolic, exponential integral, gamma function, Legendre functions, Bessel functions of integer and fractional order, integrals of Bessel functions, Struve functions, confluent hypergeonetric functions, Coulomb wave functions, hypergeometric functions Jacobian elliptic functions, theta functions, elliptic integrals, Weierstrass elliptic and related functions, parabolic cylinder functions, Mathieu functions, spheroidal wave functions, orthogonal polynomials, Bernoulii and Euler polynomials, Riemann zeta function, combinatorial analysis, numerical interpolation, differentiation and integration, probability functions, miscellaneous functịons, scales of notation and Laplace transforms.

$\# 6508014$

$\star 0.2113$

Allen, C. W.

ASTROPHYSICAL QUANTITIES, SECOND EDITION

London: Athlone Press (University of London)

1955

$291 \mathrm{p}$

This book presents recommended values for astronomical and astrophysical quantities. These valuss were arrived at from an examination of literature published through 1955. The following types of data have been included: general constants and units, atoms, spectra, radiation, the earth, planets and satelites, interplanetary matter, the sun, normal stars, special stars, stellar populations, interstellar space, star clusters, galaxies, and groups of galaxies.

$\$ 6811014$

$\star 0.5411$

Alt, Franz i.

INFORMATION HANDLING IN THE NATIONAL STANDARD

NBS Technical Note 290, National Bureau of Standards, Washington, D. C.

July 1,1966

$24 \mathrm{p}$

Available from Superintendent of Documents, GPO

This is a preliminary and academic exploration of approaches to information handing by the office of Standard Reference Data. It speculates on means for the selection, acquisition, intellectual organization, and storage of the information which might underlie the Information Services Operation of the National Standard Reference Data System, as well as for methods of locating desired information items in storage, retrieving, and displaying or communicating ther. Questions of the use of computers for these purposes are discussed, including selection of equipment, arrangement of digital storage, input format, remote access, and the economics of choosing certain functions of the system for mechanization. Also, an interim system, based on conventional and, in the main, manually operated files, is described.
$6902012,6902051,6902052$

$\star 0.5413-8.213$

American Institute of Chemical Engineers

AIChE PHYSICAL PROPERTY ESTIMATION SYSTEM, VOLUME I: CHAPTER 8: SURFACE TENSION (ROUGH), CHAPTER 9: VISCOSITY; VOLUME II: USER'S MANUAL; VOLUME III: SYSTEMS MANUAL

New York: Arthur D. Little, Inc.

September 1965

$600 \mathrm{p}$

These loose-leaf documents, the AIChE Physical Property Estimation System, are manuals directed primarily at computer oriented staff who are familiar with the operation of the computer system on which programs are compiled and executed. Volume II instructs the user on how he can communicate with the estimation system. The input requirements, alternatives, and options are described together with the various outputs that the system can generate. Volume II further describes how the system programs are delivered and how they can be compiled, assembled into a system, and executed on a computer. Volume II discusses the process of updating the system, system table requirements, the subject of transfer function link construction, and the passing of information from program to program through a pass tape system. This volume contains a functional description and flow charts for the main system programs. The layout of common storage for each main program is also shown. Chapter 8 discusses the reliable techniques to estimate surface tensions of pure liquids, the variation of surface tension with temperature and pressure, and surface tension estimation methods for non-aqueous mixtures and aqueous mixtures. Interfacial tensions are briefly considered. Chapter 9 deals with the viscosity of gases and liquids. Methods are recommended for correlating low-pressure viscosity data with temperature estimating viscosities when no experimental data are available, estimating the effect of pressure on viscosity, and estimating the viscosities of mixtures. The molecular theory of viscosity is also considered.

\#6500560 $* 0.2113$

Atack, F. W. (Editor)

HANDBOOK OF CHEMICAL DATA

Altrincham, Great Britain: John Shenatt and Son

1957

$629 \mathrm{p}$

This handbook contains tables of atomic weights, densities, melting points, specific and latent heats, thermal expansion coefficients, viscosities, vapor pressures, solubilities, refractive indices, dissociation constants, properties of minerals, inorganic compounds, and organic compounds, methods of qualitative and quantitative analysis, conversion tables, logarithms, powers of numbers, sines and tangents.

$\$ 6500569$ *0.2113

Baumeister, T. (Editor)

MECHANICAL ENGINEERS' HANDBOOK, SIXTH EDITION

New York: McGraw-Hill Book Company, Inc.

1958

$2277 p$

This Handbook contains tables and text material useful in the field of mechanical engineering. The following topics have been included: mathematical tables, mathematical methods, solid mechanics, fluid mechanics, thermodynamics, mechanics of materials, general properties of materials, fuels and furnaces, machine elements, bearings, pipe and pipe fittings, steam boilers, engines and turbines, internal-combustion engines, hoisting and conveying, transportation engineering, construction of buildings, heating, ventilation and air conditioning, machine shop practice, pumps, electrical engineering, instruments and controls industrial engineering, refrigeration and surveying. 
16811024

${ }^{*} 0.8113$

Brady, E. L. (Editor)

STATUS REPORT NATIONAL STANDARD REFERENCE DATA SYSTEM, APRIL 1968

NBS Technical Note 448, National Bureau of Standards, Washington, D. C.

June 1968

$129 \mathrm{p}$

Available from Superintendent of Documents, GPO

This report summarizes the status of activities of the NBS Office of Standard Reference Data as of April 1968. It provides a detailed review of the data compilation activities within the seven broad categories of the technical scope of the program: (1) nuclear data, (2) atomic and molecular data, (3) solid state data, (4) thermodynamic and transport data, (5) chemical kinetics, (6) colloid and surface properties, and (7) mechanical properties, Progress in data systems design and development and in information services are reviewed. Certain problem areas of the program are identified. The appendix includes: (1) a listing of information and data centers associated with the Office of Standard Reference Data, (2) publications of the National Standard Reference Data System, and (3) a listing of organizations, groups, or individuals compiling or evaluating data.

$46809022 * 0.8113$

Brady, E. L.; Wallenstein, M. B.

NATIONAL STANDARD REFERENCE DATA SYSTEM PLAN OF OPERATION

NSRDS-NBS-1, National Bureau of Standards, Washington, D. C.

December 30,1964

$12 \mathrm{p}$

Available from Superintendent of Documents, GPO

In June 1963, the National Bureau of Standards was assigned the responsibility, by the President's Office of Science and Technology, of administering a government-wide program to promote and coordinate systematic data compilation and evaluation activities in all fields of the physical sciences. This government-wide program has been called the National Standard Reference Data System. The present report describes the background of this program and the mode of approach adopted by the management of the National Bureau of Standards in fulfilling its responsibility.

$\$ 6603020$

$* 0.2113$

Brady, G. S

MATERIALS HANDBOOK AN ENCYCLOPEDIA FOR PURCHASING AGENTS, ENGINEERS, EXECUTIVES, AND FOREMEN, NINTH EDITION

New York: McGraw-Hill Book Company, Inc.

1963

$968 \mathrm{p}$

This Handbook contains data on the properties and uses of materials, the elements of material economics, and miscellaneous topics. The materials properties and uses section makes up the largest section of the book, including physical, chemical, and mechanical properties, as well as uses of the materials. A materials index is provided.

$\# 6500591$

* 0.3113

Brown, L. M.; Friedman, A. S.; Beckett, C. W.

BIBLIOGRAPHY' OF RESEARCH ON DEUTERIUM AND TRITIUM COMPOUNDS 1945 TO 1952

NBS Circular 562, National Bureau of Standards, Washington, D. C.

January 27,1956

$85 p$

Available from Superintendent of Documents, GPO

A bibliography of 2,482 references to published research on the properties of deuterium and tritium compounds is given. The subject matter of each entry in the bibliography is indicated by letter codes related to a list of broad subject headings shown in the Introduction. An index of deuterium and tritium compounds and a subject index are included.

$\$ 6803001$

* 0.2123

Condon, E. U.; Odishaw, H. (Editors)

HANDBOOK OF PHYSICS SECOND EDITION

New York: McGraw-Hill Book Company, Inc.

1967

$1684 \mathrm{p}$

The Handbook of Physics is a compendium of articles and tabulations of data, written by about 90 contributors, on many topics of physics. Among the topics considered are: mathematics, mechanics of particles and rigid bodies, mechanics of deformable bodies, electricity and magnetism, heat and thermodynamics, optics, atomic physics, solid-state physics, and nuclear physics. Tables and graphs of data are included.

\section{$\# 6500671$}

$\star 8.222-0.3322$

CONSOLIDATED INDEX OF SELECTED PROPERTY VALUES, PHYSICAL CHEMISTRY AND THERMODYNAMICS

NAS-NRC Publication 976, National Academy of Sciences-National Research Council, office of Critical Tables, Washington, D. C.

1962

$275 p$

Available from Superintendent of Documents, GPO

The Consolidated Index is a key to the contents of publications that present critically evaluated numerical property values. This initial volume contains in codified form the contents of the following six compilations of physicochemical and thermodynamic data: Selected Values of properties of Hydrocarbons and Related Conpounds (American Petroleum Institute Research Project 44), Selected Values of Properties of Chemical Compounds (Manufacturing Chemists Association Research Project), Selected Values of Chemical Thermodynamic Properties (NBS Circular 500), Thermodynamic Properties of the Elements (D.R. Stull and G.C. Sinke), Contributions to the Data on Theoretical Metallurgy (Bureau of Mines Bulletins 383,384 $393,406,407,477,542,584)$, and Selected values for the Thermodynamic properties of Metals and Alloys (Minerals Research Laboratory, University of California). Each element, compound, or system of pure substances appears as a separate index entry. For a given substance, all properties that appear in one or more of the compilations are listed separately under each. A given property, if appearing in several, is repeated under each publication.

6902029 $\star 0.1123$

DuMond, J. W. ; Cohen, E. R.

A LEAST SQUARE ADJUSTMENT OF THE ATOMIC CONSTANTS AS OF DECEMBER 1950, A REPORT TO THE NATIONAL RESEARCH COUNCIL COMMITTEE ON CONSTANTS AND CONVERSION FACTORS OF PHYSICS

California Institute of Technology and North American Aviation, Inc., Pasadena and Downey, California

1951

$202 p$

This is a preliminary report to the National Research Council consisting of a reevaluation of the atomic constants and conversion factors of physics. As a result of the precision microwave and atomic beam techniques, a great advance has been accomplished in the accuracy of such constants as $e$ and $m$, the charge and mass of the electron, $h$, planck's constant, Avogadro's number, the velocity of light, the conversion factor from the Siegbahn nominal scale of $x$-units to milliangstroms, and a list of some sixty or more useful derived constants and conversion factors which can be computed from the preceding with the use of accurately known other constants, such as the Rydberg, the gas constant, etc. In this report the least-squares method was employed to adjust the data to arrive at 
a set of consistent values for the constants in question.

16906009

*3.123-8.123-0.2523

Eisenberg, D.: Kauzmann, W.

THE STRUCTURE AND PROPERTIES OF WATER

New York and Oxford: Oxford University press 1969

$296 \mathrm{p}$

In this book the authors summarize the many experimental observations on water and present theoretical discussions which effectively correlate the data. The topics covered include the water molecule and forces between water molecules; the thermodynamic properties of steam; the structures of the ices; the thermodynamic, electrical, spectroscopic and transport properties of the ices and of liquid water; hydrogen bonding in ice and water; and models for liquid water. The authors emphasize the relation of the properties of ice and water to their structures, and discuss properties such as infrared and Raman spectra because they reveal a great deal about these structures. The inclusion of some background material in physical chemistry makes the book accessible to the biologist, biochemist, and geologist as well as the chemist and physicist. An addendum listing a number of recent articles on the structures and properties of water completes the volume.

\#6500575

0.2133

Forsy the, W. E.

SMITHSONIAN PHYSICAL TABLES, NINTH REVISED EDITION Smithsonian Miscellaneous Collections Vol. 120, (Publication 4169), Smithsonian Institution, Washington, D. C .

1959

$827 \mathrm{p}$

The Smithsonian Physical Tables consist of 901 tables giving data of general interest to scientists and engineers. The topics covered in the table include general physical constants, units of measurement, photometry, characteristics of light sources, exissivity, densities, boiling, and freezing points of materials, specific and latent heats, mechanical properties of materials, viscosity, electrical properties of materials, dielectric properties of materials, magnetic properties of materials, optical properties of materials, air and the atmosphere, kinetic theory of gases, atomic and $10^{-9}$ molecular physics, radioactivity, $X$-rays, fission, cosmic rays, gravitation, solar radiation, astronony, astrophysics, and oceanography.

$\$ 6500564$

*0.2133

Frankel, M.; Patai, S. (Compilers)

TABLES FOR IDENTIFICATION OF ORGANIC COMPOUNDS SUPPLEMENT TO HANDBOOK OF CHEMISTRY AND PHYSICS, SECOND EDITION

Cleveland. Ohio: Chemical Rubber Company

1964

$301 \mathrm{p}$

These tables list the melting and boiling points and indices of refraction of organic compounds. The tables are arranged by class of compound, and by melting and boiling point within each class. The classes of compounds considered are: alkanes and cycloalkanes, alkenes, cycloalkenes and dienes, alkynes, aromatic hydrocarbons, halides, alcohols, phonols, ethers, alkehydes, ketones, quinones carboxylic acids, carboxylic acid halides, anhydrides, amides, imides, esters, amino acids, amines, carbohydrates, nitro compounds, nitriles. and sulfonic acids.
1 6509004

0.2142

THE INDUSTRIAL GRAPHITE ENGINEERING HANDBOOK

New York: Union Carbide Corporation.

Carbon Products Division

1969

$150 \mathrm{p}$

In this handbook of engineering data, the Carbon Products Division of Union Carbide Corporation brings together a comprehensive collection of data on the properties and applications of manufactured graphite. The data, test results and recommentations are offered as a guide in the use of these materials. The handbook is kept current by the release of revised data with the objective of providing supplementary information on new developments and additional data. As important restricted military data are released, they also are added.

1) 6500594

0.3143

Johnson, V.R.; Brown, L. M.; Friedman, A. S.

BIBLIOGRAPHY OF RESEARCH ON DEUTERIUM AND TRITIUM COMPOUNDS 1953 AND 1954

NBS Circular 562, Supplement 1, National Bureau of Standards, Washington, D. C

July 15,1957

$31 p$

Available from Superintendent of Documents, GPO

A bibliography of 720 references to published research on the properties of deuterium and tritium compounds is given. The subject matter of each entry in the bibliography is indicated by letter codes related to a list of broad subject headings as shown in the Introduction. An index of deuterium and tritium compounds and a subject index are included.

\#6500562

$* 0.2143$

Kaufman, H. C.

HANDBOOK OF ORGANOMETALLIC COMPOUNDS

New York: D. Van Nostrand Company, Inc.

1961

$1546 p$

This book contains data on the properties of organometallic compounds. Data were taken from literature published on the subject. The properties tabulated include color, state at room temperature, solubility, refractive index, specific gravity, melting point, boiling point, vapor pressure, viscosity, thermodynamic values, surface tension, dipole moment, and references to original literature.

$\# 6500549$

Kaye, G. W. C.; Laby, T. H.

TABLES OF PHYSICAL AND CHEMICAL CONSTANTS AND SOME MATHEMATICAL FUNCTIONS

New York: John Hiley \& Sons, Inc.

1959

$231 \mathrm{p}$

This book contains tables of data and constants from general physics, chemistry, atomic physics and some mathematical functions. The general physics data compiled are: definitions of units and conversion factors, gravity, the physical and astronomical constants of the earth, moon and solar system, densities of common materials and of water and mercury as a function of tomperature, elasticities and tensile strengths of metals and alloys, viscosities, nolecular diameters, surface tension, temperature scales, thermometry, thermal conductivities, specific heats, thermal expansion, velocity of sound, acoustics, photometry, refractive indices, light transmission, electric resistances, dielectric constants, and magnetic properties of materials. The chemical data includes: properties and abundances of the elements, physical properties of inorganic and certain organic compounds, vapor pressures, boiling points as a function of pressure of organic compounds, density of gases, solubility of gases, 
density of aqueous solutions, dipole moments, heat capacity of solids, latent heats and thermodynamic functions. The atomic physics data include: ion and electron mobilities, recombinations and diffusion. coefficient, electron configurations of elements, ionization potentials, X-ray spectra, atomic constants, units of radioactivity, absorption of radiation, fundamental particles, and properties of known isotopes. The mathematical functions tabulated include: logarithms, antilogarithms and exponential functions.

\#6004006

$\star 0.2143$

Kit, B.; Evered, D. S

ROCKET PROPELLANT HANDBOOK

New York: The Macmillan Company

1960

$354 \mathrm{p}$

This book contains data on the chemical propellants used in rocket engines. It also includes a general discussion of rocket propulsion and of liquid and solid propellants. The following inorganic propellants are discussed: aluminum, ammonia, boron compounds, fluorine and related compounds, hydrazine, hydrogen, hydrogen peroxide, lithium, lithium hydride, nitrates, nitric acid, nitrogen oxides, oxygen and ozone, perchlorates and water. The following organic compounds are discussed: alcohols, ethers, amines, benzene, metal-organic compounds, nitroparaffins, nitrates, petroleum fractions, polymers, saturated hydrocarbons, and unsaturated hydrocarbons. The properties tabulated for each compound include some or all of the following: molecular weight, color, odor, melting and boiling point, critical point, triple point, coefficient of thermal expansion, vapor pressure surface tension, heats of fusion and vaporization, heat capacity, heats of formation and combustion, heat of solution in water, free energy of formation, entropy, enthalpy, free energy, viscosity, thermal conductivity, velocity of sound, magnetic susceptibility, electrical conductivity, dielectric constant, dipole moment and index of refraction.

Knowlton, A. E. (Editor) ${ }^{*} 0.2143$

STANDARD HANDBOOK FOR ELECTRICAL ENGINEERS, NINTH EDITION

New York: McGraw-Hill Book Company, Inc.

1957

$2230 \mathrm{p}$

This handbook contains data useful in the field of electrical engineering. The topics covered by the handbook are: units and conversion factors, electric and magnetic circuits, measurements, properties of materials, circuit elements, transformers, regulators, reactors, a1ternating current generators and motors, direct-current generators and motors, rectifiers and converters, power-plants, power economics, power system electrical equipment, power transmission, power distribution, wiring design for commercial and industrial buildings, illumination industrial and commercial power, electric heating and welding, electricity in transportation, electrochemistry and electrometallurgy, batteries, wire telephone and telegraph, electronics and electron tubes, radio, radar and carrier communication, codes and standard practices, and electro-physics.

$\$ 6809005$

$*^{*} 0.3241$

Kuchowicz, B.

NUCLEAR ASTROPHYSICS A BIBLIOGRAPHICAL SURVEY

New York: Gordon and Breach, Science Publishers 1967

$450 \mathrm{p}$

A survey is given on the abundance of chemical elements, nuclear reactions in stars and stellar energy sources, stellar structure and evolution, solar system, novae and supernovae, superdense stellar matter, nucleosynthesis, neutrino emission by stars, origin of cosmic radiation, radio and gamma astronomy, cosmology and cosmogony problems and related nuclear physics. Twenty-one hundred and one papers are quoted from 1335 to the present.

\# 6500525

*0.1151-3.151

LANDOLT-BORNSTEIN, NUMERICAI, DATA AND FUNCTIONAL RELAT IONSHIPS IN PIYYSICS, CHEMISTRY, ASTRONOMY GEOPIIYSICS AND TECHNOLOGY, SIXTH EDITION,

VOLUME I: ATOMIC AND MOLÉCULAR PHYSICS, PART 1: ATOMS NND IONS

Eucken, A.; Hellwege, K.-H. (Editors)

Berlin, New York: Springer-Verlag

1950

$441 p$

In German

This section of the Landolt-Bornstein tables contains data on the fundamental physical constants and the properties of atoms and ions. Information for the use of the tables, including data on systems of measurement and units, has also been included. A discussion of atomic spectra, including wavelength standards, electron terms, important spectral line wavelengths, ionization potentials, electron affinities, $X$-ray spectra, Zeeman and Stark effect data, pressure broadening and pressure displacement of spectral lines, and oscillator strengths and lifetimes of excited states is presented. Other properties of atoms and ions compiled include electron distribution, X-ray scattering and absorption, cross sections, magnetic moments, magnetic and electric polarizability, and Faraday effect data.

\#6500526

*0.1151-3.151-5.151-8.151

LANDOLT-BORNSTEIN, NUMERICAL DATA AND FUNCTIONAL RELATIONSHIPS IN PHYSICS, CHEMISTRY, ASTRONOMY, GEOPHYSICS AND TECHNOLOGY, SIXTH EDITION,

VOLUME I: ATOMIC AND MOLECULAR PHYSICS,

PART 2: MOLECULES I (NUCLEAR STRUCTURE)

Eucken, A.; Hellwege, K.-H. (Edi tors)

Berlin, New York: Springer-Verlag

1951

$571 p$

In German

This section of the Landolt-Bornstein tables contains atomic physical data of molecules which describes the structure and dynamics of their nuclear framework. The following properties have been tabulated: atomic distances and structures, valence energies of chemical bonds, dissociation energies of chemical bonds, and molecular vibrations and rotations.

\#6500527

*0, 1151-2, 2051-3,151

LANDOLT-BORNSTEIN, NUMERICAL DATA AND FUNCTIONAL RELATIONSHIPS IN PHYSICS, CHEMISTRY, ASTRONOMY, GEOPHYSICS AND TECHNOLOGY, SIXTH EDITION

VOLUME I: ATOMIC AND MOLECULAR PHYSICS,

PART 3: MOLECULES II (ELECTRON SHELL) WITH AN APPENDIX TO VOLUME I, PARTS 1,2 , AND 3

Eucken, A.; Hellwege, K.-H. (Editors)

Berlin, New York: Springer-Verlag

1951

$724 \mathrm{p}$

In German

This section of the Landolt-Bornstein tables contains data on the electron shells of molecules. The volume includes data published through September 1951. The following properties have been tabulated: band spectra of diatomic molecules electron band spectra of multiatomic molecules, light absorption of solutions in the infrared and visible region, ionization energies of molecules. optical rotary power of molecules, electrical and optical polarizability of molecules, magnetic moments of molecules, diamagnetic polarizability of molecules, and quanta output of photochemical reactions. 
\$ 6500528

*0,1151-2,2051-3,151-4,151

LANDOLT-BORNSTEIN, NUMERICAL DATA AND FUNCTIONAL RELATIONSHIPS IN PHYSICS, CHENISTRY, ASTRONOMY, GEOPHYSICS, AND TECHNOLOGY, SIXTH EDITION,

VOLUME I: ATOMIC AND MOLECULAR PHYSICS,

PART 4: CRYSTALS

Hellwe ge, K. -H. (Editor)

Berlin, New York: Springer-Verlag

1955

$1007 p$

In German

This section contains data on the atomic physical interpretation of crystal physics as of November 1954. The following properties have been tabulated: the characteristics of symmetry, e'ements, the 32 crystal classes, space groups; lattice types, structures, crystal dimensions of inorganic and organic crystals, atomic and ionic radii, lattice energies, internal vibrations of crysta1s, electron emission, energy bonds, X-ray spectra and bonding states, electron spectra of crystals, and high frequency spectra of crystals.

\#6500529

*0.1151-2.2051-3.151

LANDOLT-BORNSTEIN, NUMERICAL DATA AND FUNCTIONAL RELATIONSHIPS IN PHYSICS, CHEMISTRY, ASTRONOMY, GEOPHYSICS AND TECHNOLOGY, SIXTH EDITION,

VOLUME I: ATOMIC AND MOLECULAR PHYSICS,

PART 5: ATOMIC NUCLEI AND ELEMENTARY PÁRTICLES

He 1lwege, K.-H. (Edi tor)

Berlin, New York: Springer-Verlag

1952

$470 p$

In German

This section of the Landolt-Bornstein tables contains tables on atomic nuclei and elementary particles. These tables were compiled by the authors from literature on these subjects published through November 1951. The properties tabulated are: the hyperfine structure of atomic terms and atomic lines, a table of all atomic nuclei and their properties, mass spectrographic measurements, nuclear reaction energies, electron line spectra of artificially radioactive atoms, the radioactive families, nuclear level schemes of radioactive transformations, energy schemes of the light atomic nuclei, beta-spectra, common naturally radioactive atoms and their alpha- and beta-spectra, scattering of charged particles by atomic nuclei, nuclear transition products, passage of alpha-particles, electrons, protons and gamma-rays through matter and cosmic radiation.

\section{$\# 6602004 \quad * 0.1151-8.151$}

LANDOLT-BORNSTEIN, NUMERICAL DATA AND FUNCTIONAL RELATIONSHIPS IN' PHYSICS, CHEMISTRY, ASTRONOMY, GEOPHYSICS AND TECHNOLOGY, SIXTH EDITIÓN,

VOLUME II: PROPERT IES OF MATTER IN ITS AGGREGATED STATES,

PART 2a: VAPOR-LIQUID EQUILIBRIUM AND OSMOTIC

PHENOMENA

Schaefer, K. ; Lax, E. (Editors)

Berlin, New York: Springer-Verlag

1960

$974 \mathrm{p}$

In German

This section contains 22 mechanical-thermal constants for the equilibrium of heterogeneous systems. Vapor pressure under various conditions, literature references, density, fusion and allotropic changes, temperature changes of crystalline liquids, and other data are given for one component systems. The same type of data as well as heterogeneous equilibrium, freezing point lowering, Lewis-Randall functions, boiling point elevation, osmotic pressure diagrams and references are listed for 222 multi-component systems.
\# 6500530

$* 0.1151-4.151-8.151$

LANDOLT-BORNSTEIN, NUMERICAL DATA AND FUNCTIONAL RELATIONSIIIPS IN PHYSICS, CHEMISTRY, ASTRONOMY, GEOPHYSICS AND TECHNOLOGY, SIXTH EDITION,

VOLUME II: PROPERTIES OF MATTER IN ITS AGGREGATED STATES,

PART 2b: EQUILIBRIA EXCEPT FUSION EQUILIBRIA,

EQUILIBRIA OF SOLUTIONS I

Schaefer, K.; Lax, E. (Editors)

Berlin, New York: Springer-Verlag

1962

$982 \mathrm{p}$

In German

This section of the Landolt-Bornstein tables contains information on solution equilibria of gaseous substances in liquids, solid and liquid metals and alloys, as well as the solubilities of solid and liquid substances in liquid solution media. Except for the section "gases in metals," on $1 y$ solution equilibria in the temperature region of about $-200^{\circ} \mathrm{C}$ to $300^{\circ} \mathrm{C}$ are presented. The dat were taken from literature published through 1962 . Solution equilibria are presented for gases with water, aqueous solutions, non-aqueous inorganic liquids, liquids and mixtures of organic and inorganic liquids. Also, solution equilibria of hydrogen, oxygen and nitrogen in pure metals are presented. Solution equilibria are presented for inorganic materials in water and other inorganic liquids, organic liquids, organic materials in water, and solutions with organic solvents.

$\# 6510028 \quad *_{0} .1151-8.151$

LANDOLT-BORNSTEIN, NUMERICAL DATA AND FUNCTIONAL RELATIONSHIPS IN PHYSICS, CHEMISTRY, ASTRONOMY, GEOPHYSICS AND TECHNOLOGY, SIXTH EDITION,

VOLUME II: PROPERTIES OF MATTER IN ITS AGGREGATED STATES,

PART 2C: EQUILIBRIA EXCEPT FUSION EQUILIBRIA, EQUILIBRIA OF SOLUTIONS II

Sch aefer, K,; Lax, E. (Editors)

Berlin, New York: Springer-Verlag

1964

$731 \mathrm{p}$

In Ge rman

This section of the Landolt-Bornstein tables includes data on solution equilibria of multicomponent systems for solutions of solid and liquid organic materials in organic liquids and equilibria in systems with several non-miscible phases.

H 6500531

${ }^{*} 0.1151-4.151-6.151-8.151$

LANDOLT-BORNSTEIN, NUMERICAL DATA AND FUNCTIONAL RELATIONSHIPS IN PHYSICS, CHEMISTRY, ASTRONOMY, GEOPHYSICS AND TECHNOLOGY, SIXTH EDITION,

VOLUME II: PROPERTIES OF MATTER IN ITS "AGGREGATED STATES,

PART 3: FUSION EQUILIBRIA AND INTERFACIAL PHENOMENA

Schaefer, K. ; Lax, E. (Editors)

Berlin, New York: Springer-Verlag

1956

$535 \mathrm{p}$

In German

This section of the Landolt-Bornstein tables contains data on fusion equilibria and interfacial tensions. The section includes data from literature published through September 1955. Where several values for one measurement were found, the average was presented. Data on fusion equilibria are presented for binary and ternary systems of inorganic compounds, silicate systems, organic systems, and organic-inorganic systems. Interfacial tension data are presented for pure liquids towards their vapors or air, solutions towards air, two non-miscible systems, and interfacial filns on water. Data are also presented on absorption from the gas phase and the liquid phase, and on paper chromatography. 
LANDOLT-BORNSTEIN, NUMERICAL DATA AND FUNCTIONAL RELATIONSHIPS IN PHYSICS, CHEMISTRY, ASTRONOMY, GEOPIYSICS AND TECHNOLOGY, SIXTH ENITION

VOLUME II: PROPERTIES OF'MATTER IN ITS AGGREGATES STATES,

PART 4: CALORIC QUANTITIES OF STATE

Schaefer, K.; Lax, E. (Edi tors)

Berlin, New York: Springer-Verlag

1961

$863 p$

In German

This section of the Landolt-Bornstein tables contains data on the thermodynamic state functions of alloys, compounds and elements, as well as reaction enthalpies for the formation of compounds from the elements and heats of reaction for several important reactions, such as acid-base neutralization and moistening of solid surfaces with liquids. The numerical values are given in joules, except that certain tables for the theoretical calculations of functions present values in joules and calories. Because of the large number of substances and systems listed, numerical data could be given only for a selection of compounds; preference was given to substances which had been accurately investigated or which could claim a greater interest on the part of the user. The following quantities are tabulated: experimental and theoretical basic quantities of thermochenistry, standard value of molar heats, entropies, formation enthalpies and free formation enthalpies; values of thermodynamic properties as a function of temperature for selected materials; heat capacity as a function of temperature; tables for calculating thermodynamic functions in the presence of internal molecular vibrations (including Planck-Einstein and Debye functions); Joule-Thoms on effect; magnetocaloric effect of lowest temperatures on paramagnetic salts; thermodynamic data of metal solutions; adsorption heats and heats of moistening; and heats of neutralization.

\section{\#6909060 *0.1151-5.151-8.151}

LANDOLT-BORNSTEIN, NUMERICAL DATA AND FUNCTIONAL RELATIONSHIPS IN PHYSICS, CHEMISTRY, ASTRONOMY, GEOPHYSICS AND TECHNOLOGY, SIXTH EDITION,

VOLUME II : PROPERTIES OF MATTER IN ITS AGGREGATED STATES,

PART '5b: TRANSPORT PHENOMENA II, KINETIC HOMOGENOUS GAS EQUILIBRIA

Schaefer, K. (Editor)

Berlin, New York: Springer-Verlag

1968

$397 \mathrm{p}$

In German

part 5b of Volume II contains some kinetic properties of the elements and selected compounds. The first series of tables lists diffusion constants for elements and ions in metals, salts, and alloys, both liquid and solid. A short tablé of values for diffusion constants of some gases in selected metals is included. The second series of tables deals with thermal conductivity data for gases, liquids and solids. The constants are reported as a function of temperature, pressure and composition. The next series of tabies deals with thermal diffusion constants for binary and ternary gas mixtures. Constants are also reported in liquid mixtures and solutions (Ludwig-Soret Effect). The next section lists a series of kinetic rate constants for reaction in gas phase and includes: dissociation, atom-radical and ionmolecule reactions. Another series of tables lists rate constants and equilibrium constants for reactions in solid and mixed phase. Several addenda to the main sections are appended.
\#6500533

$* 0.1151-4.151-8.151$

LANDOLT-BORNSTEIN, NUMERICAL DATA AND FUNCTIONAL RELATIONSHIPS IN PHYSICS, CHEMISTRY, ASTRONOMY, GEOPHYSICS, AND TECHNOLOGY, SIXTH EDITION,

VOLUME II: PROPERTIES OF MATTER IN ITS ÁGGREGATED STATES,

PART 6: ELECTRICAL PROPERTIES

Hellwege, K. $-H_{0}$; Hellwege, A.M. (Editors)

Berlin, New York: Springer-Verlag

1959

$1018 \mathrm{p}$

In German

This section of the Landolt-Bornstein tables contains data on the electrical properties of matter (except electrochemical systems) published through February 1959. The properties tabulated include the conductivities of pure metals and certain alloys at $0{ }^{\circ} \mathrm{C}$ and their temperature variations, resistance ratios, effect of magnetic fields on resistance, super-conducting metals and alloys and their transition temperature, magnetic field effects on super conductivity, galvanomagnetic and thermomagnetic effects, ionic conductivity in crystals, transfer numbers in solids. Also tabulated are the constants of homogeneous semiconductors; photoelectric conduction; piezoelectric equations; and the elastic, piezoelectric and dielectric constants of piezoelectric crystals; dielectric properties of inorganic and organic crystals; crystalline fluids and pure fluids; dielectric properties at high frequencies; temperature and pressure dependence of dielectric constants; and the dielectric properties of gases. Thermionic emission and work functions, thermopotentials, peltier and Thoms on heats, and photoemission data are also listed.

\#6500534

$* 0.1151-8.151$

LANDOLT-BORNSTEIN, NUMERICAL DATA AND FUNCTIONAL RELATIONSHIPS IN PHYSICS, CHEMISTRY, ASTRONOMY, GEOPHYSICS AND TECHNOLOGY, SIXTH EDITION,

VOLUME II: PROPERTIES OF MATTER IN ITS AGGRFGATED STATES,

PART 7: ELECTRICAL PROPERTIES II (ELECTROCHEMICAL SYSTEMS)

Hellwe ge, K. $-H_{0}$; Hellwege, A.M. (Editors)

Berlin, New York: Springer-Verlag

1960

$959 \mathrm{p}$

In German

This section of the Landolt-Bornstein tables contains data on the electrical properties of electrochenical systems. The data were taken from the literature published through 0ctober 1960. The data compiled include the electrical conductivity of molten salts, pure liquid, and aqueous electrolyte and field solutions; frequency and field strength dependence of conductivity of aqueous solutions; conductivity of ions in aqueous solutions; electrical conductivity of non-aqueous solutions; electrophoretic potentials and electrokinetic potentials, standard electrode potentials, dissociation constants of inorganic and organic compounds and acid-base indicators.

\#6500535

*0.1151-3.151-4.151-8.151

LANDOLT-BORNSTEIN, NUMERICAL DATA AND FUNCTIONAL RELATIONSHIPS IN PHYSICS, CHEMISTRY, ASTRONOMY, GEOPHYSICS AND TECHNOLOGY, SIXTH EDITION,

VOLUME II: PROPERT IES OF MATTER IN ITS 'AGGREGATED STATES,

PART 8: OPTICAL CONSTANTS

Hellwege, K.-H.; Hellwege, A.M. (Editors)

Berlin, New York: Springer-Verlag

1962

$90 \mathrm{Ip}$

In German

This section of the Landolt-Bornstein tables contains tables and figures for the optical properties of substances. Data are compiled from literature published through June 1962. Data are compiled for: the optical constants and reflection of metals, alloys, and thin metal layers; 
refractive indices of inorganic and organic materials as a function of wavelengths; optical constants of selected solid materials in broad wavelength regions; rotation of the polarization plane in crystals; double refraction; piezooptical and electrooptical constants of piezoelectric crystals; light refraction by glasses and transparent plastic; light permeability, double refraction, Faraday effect and Kerr effect on glasses: refractive index of crystalline fluids; refractive index and optical rotatory power of pure liquids and solutions; Cotton-Mouton effect of liquids; double refraction due to current in liquids; Kerr constants of liquids; refractive indixes of gaseous elements and compounds; refraction and dispersion of air and of hydrogen compounds; Cotton-Mouton effect of gases and Kerr constants for gases.

\#6500536 *0.1151-4.151

LANDOLT-BORNSTE1N, NUMERICAL DATA AND FUNCTIONAL RELATIONSH1PS IN PHYSICS, CHEMISTRY, ASTRONOMY, GEOPHYS1CS AND TECHNOLOGY, SIXTH EDITION,

VOLUME 11: PROPERT IES OF MATTER IN ITS AGGREGATED STATES,

PART 9: MAGNETIC PROPERTIES

He llwe ge, K.-H.; Hellwege, A.M. (Editors)

Berlin, New York: Springer-Verlag

1962

$900 \mathrm{p}$

In German

This section of the Landolt-Bornstein tables contains tables and curves of magnetic data for paramagnetic, ferromagnetic, ferrimagnetic and antiferromagnetic substances, taken from the literature published through september 1962. The following quantities have been tabulated: fundamental magnetic properties (dia- and paramagnetic susceptibility, spontaneous magnetization, and Curie temperatures) for metaliic elements and alloys, exchange energy, crystal energy, magnetostriction, magnetocaloric effects, Faraday effect, magnetooptical effect, Kerr effect, gyromagnetic effects and resonance in ferromagnetic metals and alloys, properties of ferrospinels, magnetic garnets, perovskites and hexagonal ferrites, magnetic properties of transition element compounds, lanthanide series compounds and actinide series compounds paramagnetic centers in crystals, paramagnetic ions as impurities, magnetic susceptibility, cyclotron resonance, de Haas-schubnikow effect and Faraday effect in semi-conductors paramagnetic relaxation and nuclear magnetic relaxation.

\section{\#6802003 *0.1151-4.151}

LANDOLT - BORNSTEIN, NUMERICAL DATA AND FUNCTIONAL RELATIONSHIPS IN PHYSICS, CHEMISTRY, ASTRONOMY, GEOPHYSICS AND TECHNOLOGY, SIXTH EDITION,

VOLUME 1I: PROPERTIES OF MATTER IN ITS AGGREGATED STATES,

PART 10: MAGNETIC PROPERTIES 11

Hellwege, K. -H.; Hellwege, A.M. (Editors)

Berlin, New York: Springer-Verlag

1967

$173 \mathrm{p}$

in German

This section of the Landolt-Bornstein tables gives the paramagnetic and diamagnetic properties of substances. The compiler has listed one or more values for each substance, and marked with an asterisk those considered acceptable and reliable. These, and other selected values considered worthy of inclusion, are listed under "Used References" and values far outside the others under "Further References." The values were taken from literature up to April 1966. Molar magnetic susceptibilities are listed for compounds, and magnetic mass susceptibilities for elements. Diamagnetic susceptibilities are listed for isotropic organic and inorganic substances, and principal susceptibilities for anisotropic substances. Paramagnetic susceptibilities are listed for organic compounds only.
\# 6500537 $* 0.1151-2.2051-3.151$

LANDOLT-BORNSTEIN, NUMERICAL DATA AND FUNCTIONAL RELATIONSHIPS $1 \mathrm{~N}$ PHYS1CS, CHEMISTRY, ASTRONO? $\mathrm{K}$, GEOPHYSICS AND TECHNOLOGY, SIXTH EDITION, VOLUME III: ASTRONOMY AND GEOPHYSICS

Bartels, J.; Bruggencate, P. T. (Editors)

Berlin, New York: Springer-Verlag

1952

795 p

In German

This volume of the Landolt-Bornstein series contains data on astronomy and geophysics. The material has been taken from literature published through 1951. The astronomical section includes data on astronomical instruments, position and time determination, abundance of the elements in the universe, the sun, mechanics and physics of the planets, moons, comets and meteors, stellar radiation, stellar spectra, stellar positions and movements, double stars, variable stars, novae, the galaxy, star clusters and extra-galactic nebulae. The section on geophysics contains data on the earth's gravity and tidal forces, radioactivity density; elastic, electrical, magnetic and thermal properties of minerals and rocks; seismicity and earthquake waves; properties of the earth's interior, surface and magnetism; physical and chemical properties of sea water; sea tides, hydrography, meteorology and climatology; and upper atmosphere physics.

\#6500538

*0.1151-4.151-7.151-8.151

LANDOLT - BORNSTEIN, NUMERICAL DATA AND FUNCTIONAL RELATIONSHIPS IN PHYSICS, CHEMISTRY, ASTRONOMY, GEOPHYSICS AND TECHNOLOGY, SIXTH EDITION,

VOLUME IV: TECHNOLOGY

PART 1: MATER1AL VALUES AND MECHANICAL PROPERTIES OF NON-METALS

Schmidt, E. (Edi tor)

Berlin, New York: Springer-Verlag

1955

$881 \mathrm{p}$

In Ge rman

This section of the Landolt-Bornstein tables contains material values and mechanical behavior of non-metallic substances, and their technical applications. Data are compiled from literature published through autumn 1964. The following general information is included: measuring systems, units and conversion tables, atomic weights, reduction to standard states, densities of water and mercury. Physical and mechanical properties of non-metalic solids are given for natural and synthetic building materials, organic natural materials (wood, paper, cellulose, wood pulp), fibrous materials, ceramics, glasses, synthetic materials, and natural synthetic materials. Also presented are data on friction and rolling resistance of non-metallic and metallic substances, viscosity, flow through tubes circuitous flow of bodies during two dimensional flow and rough flow, flotation, speed of sound, sound radiation and absorption, the human ear, sound spectra, vibrational numbers, spatial and building acoustics, sound recording and biological effects of ultrasonics.

$\# 6500539$

$\star 0.1151-3.151-4.151-7.151-8.151$

LANDOLT-BORNSTEIN, NUNERICAL DATA AND FUNCTIONAL RELAT1ONSHIPS IN PIIYSICS, CIIEMISTRY, ASTRONOMY, GEOPHYS1CS AND TECHNOLOGY, SIXTH EDITIÓN,

VOLUME IV: TECINOLOGY,

PART 2a: MATERIAL VALUES AND PROPERT1ES OF INDUSTRIAL METALS, BAS1C PR1NC1PLES, TEST1NG PROCESSES, INDUSTRIAL STEELS

Borchers, If.; Schmidt, E. (Editors)

Berlin, New York: Springer-Verlag

1963

$888 \mathrm{p}$

In German

This section of the Landolt-Bornstein tables

includes data on metallographic principles and concepts, testing properties, and the properties of 
iron and its alloys. Data are given on diagrams of state, strength testing and destruction free materials. The physical properties of iron tahulated are lattice constants, density, thermal expansi-on, diffusion, atomic heats, specific heats, enthalpies, entropies and free enthalpies, phase diagrams and transition points, thermal conductivity, electrical properties, magnetic properties, optical constants and excitation crosssections. Composition, treatment, strength values and special chemical properties of pure iron, unalloyed steels, highly alloyed steels, special steels, and cast iron are presented.

\#6510029

*0.1151-4.151-7.151

LANDOLT-BORNSTEIN, NUMERICAL DATA AND FUNCTIONAL RELATIONSIIIPS IN" PIYYICS, CHEMISTRY, ASTRONOMY, GEOPIYSICS AND TECHNOLOGY, SIXTH EDITIÓN,

VOLUME IV: TECIINOLOGY,

PART 2b: MATERIAL V́ALUES AND PROPERTIES OF INDUSTRIAL METALS, SINTERED INDUSTRIAL MIATERIALS, IIEAVY METALS (WITHOUT SPECIAL INDUSTRIAL MATERIALS) Borchers, H.; Schmidt, E. (Editors)

Berlin, New York: Springer-Verlag

1964

$1000 \mathrm{p}$

In German

This section of the Landolt-Bornstein series deals with numerical data and behavior of both metallic and powdered industrial materials. The materials covered include the metals tungsten, tantalum, niobium, vanadium, chromium, cobalt, nickel, manganese, gold, silver, copper, antimony, zinc, cadmium, lead, bismuth, tin, and their alloys. Data are provided for the physical and mechanical properties of these materials, their corrosion behavior and some of their applications to technology.

$\$ 6605008$

*0.1151-4.151-7.151

LANDOLT-BORNSTEIN, NUMERICAL DATA AND FUNCTIONAL RELATIONSHIPS IN PHYSICS, CIIEMISTRY, ASTRONOMY, GEOPIYSICS AND TECHNOLOGY, SIXTH EDITIÓN, VOLUME IV: TECHNOLOGY

PART 2C: MATERIAL V́LUES AND PROPERTIES OF INDUSTRIAL METALS, ALKALINE EARTHS, SPECIAL INDUSTRIAL MATERIALS, SEMICONDUCTORS, CORROSION

Borchers, H.; Schmidt, E. (Editors)

Berlin, New York: Springer-Verlag

1965

$976 \mathrm{p}$

In German

This section is the third part in a series of Landolt-Bornstein tables presenting the physical properties and behavior cliaracteristics of most of the 75 elements in the periodic system exhibiting metallic characteristics as well as data concerning their alloys. Special emphasis is directed toward light metals, special work metals, binding materials, enamel properties, corrosive behaviors, reactor metals, metals of the rare earths, semiconductors, and current developnents in binary alloy systems. The volume is divided into sections entitled: titanium, beryllium, aluminum, magnesium, lithium, rubidium, cesium, liquid metals, general survey of reactor metals, uranium, plutorium, zirconium, hafnium, thorium, rare earths, semiconductors, bearing metals, enamels, solder, welding and cutting metals, metal adhesives, and corrosive behavior of materials.

\# 6500540

*0.1151-3.151-4.151

LANDOLT-BORNSTEIN, NUMERICAL DATA AND FUNCTIONAL RELATIONSHIPS IN PHYSICS, CIIEMISTRY, ASTRONOMY, GEOPIIYSICS AND TECHNOLOGY, SIXTH EDITIÓN, VOLUME IV: TECHNOLOGY

PART 3: ELECTROTECHNOLOGY, OPTICS, X-RAY TECHNOLOGY Schmidt, E. (Editor)

Berlin, New York: Springer-Verlag

1957

$1076 \mathrm{p}$

In German
This section of the Landolt-Bornstein tables deals with the technological aspects of electricity, light and $X$-rays. It is compiled from the literature published through 1956. Wherever possible, the compilers have drawn, from several values available for a given quantity, the value they feel is most accurate. The properties tabulated in the electrical section are electrical conductivity, resistance and temperature coefficient of resistance; conductivity of molten metals; effect of pressure, tension, and cold machining on electrical resistance; resistance as functions of frequencies; resistance of thin layer semiconductors; thermoelectric emf of metal pairs and thermocouples in use; effects of pressure and cold treatment on thermocouples; conduction of electricity in gases, breakdown potentials of gases; insulating liquids, filling and impregnating media, fibrous material insulators, insulating foils, mica insulators, rubber insulators; resin, plastics, quartz, glass, ceramic materials and rocks as insulators; and magnetic materials. The quantities tabulated in the light section are: sensitivity of the human eye, illuminating materials, light sources, light filters and photographic films. The $X-r a y$ section includes data on measurements and units of X-ray technology.

\#6804008

*0.1151-4.151-8.151

LANDOLT-BORNSTEIN, NUMERICAL DATA AND FUNCTIONAL RELATIONSHIPS IN PHYSICS, CHEMISTRY, ASTRONOMY, GEOPHYSICS AND TECHNOLOGY, SIXTH EDITION,

VOLUME IV: TECHNOLOGY,

PART 4a: HEAT TECHNOLOGY, HEAT TECHNOLOGY ENGINEERING MEASURING METHODS, THLRMODYNAMIC PROPERTIES OF HOMOGENOUS MATERIALS

Hausen, H. (Editor)

Berlin, New York: Springer-Verlag

1967

$944 p$

In German

This section of Landolt-Bornstein is a compilation of data dealing with the technology of thermal measurements and the thermodynamic properties of gases, vapors, liquids, and solid materials. Data are included for such areas as the basis of temperature measurement; the temperature measurement with contact thermometers; optical temperature measurement (Pyrometry); hygrometry; thermodynamic properties of homogeneous materials, gases, water and steam, refrigerating agents: thermal expansion of solids and liquids; and the specific thermal properties of technically important materials.

\#6500524

*0.1151-2.2651-3.151

LANDOLT-BORNSTEIN, NUMERICAL DATA AND FUNCTIONAL RELATIONSHIPS IN SCIENCE AND TECHNOLOGY, NEW SERIES,

GROUP I: NUCLEAR PHYSICS AND TECHNOLOGY

VOLUME 1 : ENERGY LEVELS OF NUCLEI: $\mathrm{A}=5^{\circ}$ TO $\mathrm{A}=257$ Hellwege, A.M.; Hellwege, K.-H. (Editors)

Berlin, New York: Springer-Verlag

1961

$813 \mathrm{p}$

In German and English

This first volume of the New Series of Landolt. Bornstein contains the known energy levels and transitions for all nuclei from $A=5$ to $A=257$. The essential parts of the material are the level and decay schemes. These are supplemented by tables and special data. The tables list the following parameters for each level: excitation energy above ground state; the total angular momentum quantum number and the parity; the isobaric spin quantum number; the half 1 ife or mean life of the state, its half-width; the known modes of decay of the states; binding energies for various nucleon combinations, and the $Q$-values for the relevant nuclear reactions. Much of the same information is incorporated in diagrams in which the energy levels of the various nuclei are exhibited schematically, together with the reactions by which they are formed. Recent 
references to papers dealing in a more general way with the properties of the nucleus in question are included. This compilation includes the data available through May 1960 with a few references to work done since that time.

$\# 6802002$

*0.1151-2.1351-3.151

LANDOLT-BORNSTEIN, NUMERICAL DATA AND FUNCTIONAL RELATIONSHIPS IN SCIENCE AND TECHNOLOGY, NEW SERIES,

GROUP I: NUCLEAR PHYSICS AND TECHNOLOGY,

VOLUME 2: NUCLEAR RADII

Schopper, H. (Editor)

Berlin, New York: Springer-Verlag

1967

$54 \mathrm{p}$

In German and English

The volume contains nuclear radii and other characteristic parameters of atomic nuclei which are deduced from electron scattering experiments and the observation of mesic X-rays. The first section contains data on nuclear charge distributions derived from sources other than scattering, primarily mu-mesic atoms for spherical nuclei. Data are given on energy levels and radius parameters for mu-mesic X-ray transitions; also for neighboring nuclides, deformed nuclei, and radii of isomers. The second section deals with nuclear radii determined by electron scattering. Both electric charge and magnetic radii are given. Because a variety of nuclear models are available, each nucleus is discussed separately, in addition to listing the size parameters.

\section{\#6902044}

$\star 0.1151-2.2051-3,151$

LANDOLT-BORNSTEIN, NUMERICAL DATA AND FUNCTIONAL RELATIONSHIPS IN SCIENCE AND TECHNOLOGY, NEW SERIES,

GROUP I: NUCLEAR PHYSICS AND TECHNOLOGY,

VOLUME 3: NUMERICAL TABLES FOR ANGULAR CORRELATION COMPUTATIONS IN alpha-, beta-, AND gammaSPECTROSCOPY: $\quad 3 j-, 6 j-, 9 j-S Y M B O L S, F-A N D$ GAMMACOEFFICIENTS

Appe l, $\mathrm{H}$.

Schopper, H. (Editor)

Berlin, New York: Springer-Verlag

1968

$1206 \mathrm{p}$

In German and English

The various types of angular correlation coefficients have been tabulated in the literature. These tables provide the coefficients for a com: plete calculation of the basic angular correlations in alpha-, beta-, and gamma-spectroscopy. In chapter 2, the angular correlation formalism is given in a - necessarily incomplete-collection of relations involving the tabulated coefficients. i.e., 3j-symbols, 6j-symbols, $9 \mathrm{j}$-symbols, F-coef: ficients, and GAMMA-coefficients. In the computer tables these symbols are given in upright capital letters: $J=j, J_{1}=j_{1}$ etc. and GAMMA $=\gamma$. These quantities are given solely by angular momentum conservation for the total system in consideration. When answering the question of how the angular momenta of the various subsystems of particles may combine to fulfill the conservation condition, one ends up with Clebsch-Gordan-coefficients for two angular momenta and with Racah-coefficients for more than two. To achieve a higher degree of symmetry, Wigner has rewritten both types of coefficients as the $3 j-$ and $6 j-s y m b o l s$ respectively. In more compilicated correlations (e.g. triple correlations, where the intermediate transition also is observed) a new combination of $6 j-s y m b o l s$ appears which is usually called the 9 j-symbol. For alpha-, beta-, and gama-spectroscopy a combination of one $3 j-$ and one $6 j-s y m b o l$ with statistical factors is frequently used and is called the F-coefficient. The GAMMA-coefficient is a slightly modified form of this F-coefficient useful for beta-spectroscopy. The coefficients mentioned above are extensively tabulated in this volume. For the $9 j$-symbol, however, only selected ones are listed. In addition, there is a chapter dealing with angle functions. Since angular correlations are generally developed in a series of Legendre polynomials, these functions should be very useful.

$\# 6912054$

*0.1151-2.2651

LANDOLT-BORNSTEIN, NUMERICAL DATA AND FUNCTIONAL RELATIONSHIPS IN SCIENCE AND TECHNOLOGY, NEW SERIES,

GROUP I: NUCLEAR PHYSICS AND TECHNOLOGY,

VOLUME 4: NUMERICAL TABLES FOR BETÁ-DECAY AND ELECTRON CAPTURE

Behrens, H.; Jaenecke, J. (Authors)

Schopper, H. (Editor)

Berlin, New York: Springer-Verlag

1969

$322 \mathrm{p}$

In German and English

These beta-decay tables present certain combinations of electron radial wave function and phase shifts for both screened and unscreened betadecay functions. The tables for electron capture give the amplitudes and values of the electron radial wave functions at the nuclear radius for the $K, L$ and $M$ shell for all values of $Z$ (the $L$ and $M$ shells are excluded for very light nuclei). A brief compilation of all the relevant formulae used in the treatment of beta-decay and electron capture is given. An explanation of the details of the computations and a description of the tables is included; in addition, 56 references are given.

$\# 6605007$

$* 0.1151-3,151$

LANDOLT-BORNSTEIN, NUMERICAL DATA AND FUNCTIONAL RELATIONSHIPS IN SCIENCE AND TECHNOLOGY, NEW SERIES,

GROUP II: ATOMIC AND MOLECULAR PHYSICS,

VOLUME 1: MAGNETIC PROPERTIES OF FREE RADICALS

Fischer, H.

Hellwege, K.-H.; Hellwege, A.M. (Editors)

Berlin, New York: Springer-Verlag

1965

$154 \mathrm{p}$

In German and English

This book contains data on the magnetic properties of free radicals, including inorganic and organic uncharged free radicals and radical ions. These data were taken from published results of ESR and atomic beam resonance experiments. The data compiled include: name and structural formula of a free radical compound, method of generation, crystal matrix or solvent used for the radical, temperature of measurement, frequency of measurement, g-factor, hyperfine splitting parameter, primary references for the values quoted, and further references on the same subject.

$\# 6706020$

*0.1151-3.151-4.151

LANDOLT-BORNSTEIN, NUMERICAL DATA AND FUNCTIONAL RELATIONSHIPS IN SCIENCE AND TECHNOLOGY, NEW SERIES,

GROUP II: ATOMIC AND MOLECULAR PHYSICS, VOLUME 2: MAGNETIC PROPERTIES OF COORDINATION AND ORGANO-METALLIC TRANSITION METAL COMPOUNDS

Koenig, E.

Hellwege, K.-H.; Hellwege, A.M. (Editors)

Berlin, New York: Springer-Verlag

1966

$578 \mathrm{p}$

In German and English

Magnetic properties are 1 isted for coordination and organo-metallic transition metal compounds. The magnetic susceptibility section lists for each compound gram susceptibility and molar susceptibility at a particular temperature; temperatures, and the experimental method used to obtain data. The electron paramagnetic resonance section lists for each compound the spectroscopic splitting g-factor, fine structure and hyperfine structure parameters, half-width of the EPR line, and temperature and frequency of measurement. 
$\$ 6706036$

*0.1151-3.151-5.151

LANDOLT-BORNSTEIN, NUMERICAL DATA AND FUNCTIONAL RELATIONSHIPS IN SCIENCE AND TECHNOLOGY, NEW SERIES,

GROUP II: ATOMIC AND MOLECULAR PHYSICS

VOLUME 3: LUMINESCENCE OF ORGANIC SUBSTANCES

Schmillen, A.; Legler, R.

Hellwege, K.-H.; Hellwege, A.M. (Editors)

Berlin, New York: Springer-Verlag

1967

$416 \mathrm{p}$

In German and English

The most important data on the luminescence of over 1000 luminescent organic substances investigated have been assembled into a main table. Most substances are shown note only in a pure state (liquid or solid) but diluted in several solvents as wel1. On compiling the literature, it was noticed that not all data were of equal reliability, e.g. owing to insufficient purity of the substances investigated or to the application of theories which had become out of date in the meantime. For these reasons the main table only contains the physical magnitudes which are known with sufficient reliability for the majority of substances investigated. Data appearing in the main table for each compound include: its state, solvent used, if any, temperature, wavelength used for stimulation, absorption and emission wavenumbers for fluorescent and phosphorescent enission, quantum efficiency, decay time, and literature reference. Following the main table, a selection of typical luminescence spectra are given. Tables of the wavenumbers of very sharp single bands are given for some important spectra. Data are given concerning applications of luminescence by radiation damage. The tables were taken from literature dating from 1949 to 1964.

\#6708004

$* 0.1151-3,151$

LANDOLT - BORNSTEIN, NUNERICAL DATA AND FUNCTIONAL RELATIONSHIDS IN SCIENCE AND TECHNOLOGY, NEN SERIES,

GROUP II : ATOMIC AND MOLECULAR PHYSICS

VOLUME 4: MOLECULAR CONSTANTS FROM' MICROWAVE SPECTROSCOPY

Stark, B.

Hellwege, K. -H.; Hellwege, A.M. (Editors)

Berlin, New York: Springer-Verlag

1967

$813 \mathrm{p}$

In German and English

This collection provides data on the rotation of molecules in the gaseous state obtained by microwave spectroscopy. The following molecular constants are presented: rotational constants, centrifugal distortion constants, rotationvibration interaction constants, beta-type doubling constants, and isotopic masses or mass ratios. These constants are separately tabulated for diatomic molecules, linear molecules, symmetric top molecules and asymmetric top molecules. Also presented are electric dipole moments, nuclear quadrupole constants, and hindered rotation constants. There are also diagrams of the structural arrangement, including the position of the electric dipole moment, for those molecules whose structure could be deduced from the microwave measurements with sufficient reliability. References are tabulated with a special table for magnetic constants determined by Zeeman effect.

46802001

$\star 0.1151-4.151-8.151$

LANDOLT - BORNSTEIN, NUMERICAL DATA AND FUNCTIONAL RELATIONSHIPS IN SCIENCE AND TECHNOLOGY, NEW SERIES,

GROUP II. ATOMIC AND MOLECULAR PHYSICS, VOLUME 5: MOLECULAR ACOUSTICS

Schaaffs, W.

Hellwege, K.-H.; Hellwege, A.M. (Editors)

Berlin, New York: Springer-Verlag

1967

$286 \mathrm{p}$

In German and English
This volume lists data on acoustics based essentially on the molecular properties of matter, and values of sound velocity, absorption coefficients, and dispersion data. The measurements were made at frequencies ranging from $10 \mathrm{Kc}$ to $200 \mathrm{Mc}$. preference was generally given in compiling data to measurements for which statements on density, chenical purity, and the temperature coefficient were added. Often, however, several statements can be found for a measured value. Generally, the limit of accuracy is $1 \%$ to $2 \%$. Data are given on sound velocity in gases and liquids; for pure gases and liquids as a function of temperature, pressure, and structure; also for mixtures of gases, mixtures of liquids, and solution. Data are compiled on sound absorption and dispersion in gases; sound absorption in homogenecus liquids, in solution, and on sound propagation in polymers, also on sound propagation in isotropic or quasi-isotropic solids (metals, inorganic non-metals, organic solids). There are special sections on sound propagation in liquid helium and shock wave velocity.

\#6608030

$* 0.1151-4.151$

LANDOLT-BORNSTEIN, NUMERICAL DATA AND FUNCTIONAL RELATIONSHIPS IN SCIENCE AND TECHNOLOGY, NEW SERIES,

GROUP III: CRYSTAL AND SOLID STATE PHYSICS

VOLUME 1: ELASTIC, PIEZOELECTRIC, PIEZOOPTIC AND ELECTROOPTIC CONSTANTS OF CRYSTALS

Bechmann, R.; Hearmon, R.F.S.

Hellwege, K.-H.; Hellwege, A.M. (Editors)

Berlin, New York: Springer-Verlag

1966

$160 \mathrm{p}$

In German and English

This volume of the new Landolt-Bornstein series gives the elastic, piezoelectric, piezooptic, and electrooptic constants of crystals. The data included were taken from data published in the literature through 1965. For materials where one or two sets of measurements of a given constant were found, they are quoted as published; for more than two sets of measurements, the mean and coefficient of variation are given. For nonpiezoelectric constants, the elastic stiffness and compliance constants, together with their temperature and pressure variations, are given. For piezoelectric constants, the elastic, piezoelectric, dielectric, piezooptic, and electrooptic constants, plus the electromechanical coupling factors are given. The temperature coefficients of the elastic stiffness, compliance, piezoelectric stress and strain constants are also given.

\#7001036

* $0.1151-4.151-8.151$

LANDOLT-BORNSTEIN, NUMERICAL DATA AND FUNC'TIONAL RELATIONSHIPS IN SCIENCE AND TECHNOLOGY, NEW SERIES,

GROUP III: CRYSTAL AND SOLID STATE PHYSICS, VOLUME 2: ELASTIC, PIEZOELECTRIC, PIEZOOPTIC, ELECTROOPTIC CONSTANTS, AND NONLINEAR DIELECTRIC SUSCEPTIBILITIES OF CRYSTALS

Bechmann, R.; Hearmon, R.F.S.; Kurtz, S.K. Heliwege, K.-H.; Hellwege, A.M. (Editors)

Berlin, New York: Springer-Verlag

1969

$242 p$

In German and English

Volume III/2 of the New Series of Landolt-Bornstein continues Volume III/l only three years after publication of the latter. All the tables contained in III/l are brought up to date by supplements in III/2. In addition, the elastic and electrooptic constants of higher order and the nonlinear optical susceptibilities have now been included. Readers should note that the Index of Substances at the end of III/2 refers to both volumes. 
\#6605010

$* 0.1151-3.151$

LANDOLT-BORNSTEIN, NUMERICAL DATA AND FUNCTIONAL RELATIONSHIPS IN SCIENCE AND TECHNOLOGY, NEW SERIES,

GROUP VI: ASTRONOMY, ASTROPHYSICS AND SPACE

RESEARCH,

VOLUME 1: ASTRONOMY AND ASTROPHYSICS

Voigt, H.H. (Editor)

Berlin, New York: Springer-Verlag

1965

7110

In German and English

This volume of the Landolt-Bornstein New Series contains data useful in the fields of astronomy and astrophysics. The following topics are covered: optical instruments, radio-astronomical devices, performance of telescopes, photoelectric photometry, position and time determination, astronomical constants, abundances of the elements in the universe; physical and mechanical properties of the sun, planets, satellites, comets, meteors, artificial earth satelites, interplanetary space, stellar positions and motions, stellar spectra, star colors and magnitudes, physics of stellar atmospheres, stellar structure and evolution, double stars, variable stars, novae and supernovae, planetary nebulae, star clusters and association, properties of the nearest stars, galactic structure, kinematic and hynamic properties of the galaxy, interstellar space, galaxies, and clusters of galaxies.

$\# 6500570$

$\star 0.2153$

Lange, N. A. (Editor)

HANDBOOK OF CHEMISTRY, TENTH EDITION

New York: McGraw-Hill Book Company, Inc.

1961

$1969 p$

The Handbook of Chemistry is a reference volume containing chemical and physical data for the use of chemists. It contains data on life and fire hazards of chemicals, chemical and physical properties of elements, minerals, inorganic compounds, organic compounds, industrial materials, chemical analysis, and numerical tables. Quantities tabulated in the tables of miscellaneous specific properties include solubilities, densities, electrical properties, refractive indices, X-ray table, hygrometry, vapor, pressure, and thermodynamic properties.

$\# 6908006$

$* 2.063-0.3163$

Penny, S. K.; Trubey, D. K.; Gurney, J.

BIBLIOGRAPHY, SUBJECT INDEX, AND AÚTHOR INDEX OF THE LITERATURE EXAMINED BY THE RADIATION SHIELDING INFORMATION CENTER

ORNL-RSIC-5 (Rev. 1), Radiation Shielding Information Center, Oak Ridge National Laboratory, Oak Ridge, Tennessee

May 1966

$603 \mathrm{p}$

This bibliography is the fourth cumulative edition of the literature concerned with nuclear reactors, nuclear weapons, and radioisotopes which has been selected by the Radiation.- Shielding Information Center. It supersedes ORNL-RSIC-2 and ORNL-RSIC-5 (Dec. 1964). The Key Word Index, which should serve as an aid in locating bibliographic references to a given subject, has also been revised. The pages associated with a word in the index will contain a list of documents that might be relevant to the word. This bibliography is synchronized with abstracts published as ORNL-RSIC- 6 in a looseleaf binder. New abstracts, corresponding to accessions since the original issue, are now available. The two major sections in this bibliography are a 1 ist of documents by subject category and an author index. The document list covers nine major subjects with a total of 147 categories. If a document applies to more than one category, it is listed under each. The author index includes cross references to the categories under which the author's publications appear.
Preceding the bibliography is a list of the subject categories, their corresponding category numbers, and the pages on which they appear in the bibliography.

$\$ 6500561 \quad * 0.2163$

Perry, R. H.; Chilton, C. H.; Kirkpatrick, S. D. (Editors)

CHEMICAL ENGINEERS' HANDBOOK FOURTH EDITION

New York: McGraw-Hill Book Company, Inc.

1963

$1900 \mathrm{p}$

This handbook consists of mathematical tables and methods, physical and chemical data, reaction kinetics, reactor design, thermodynamics, fluid and particle mechanics, transport and storage of fluids and solids, size reduction and enlargement, heat generation, transport and storage, heat transmission, heat-transfer equipment, refrigeration, distillation, gas absorption and solvent extraction, humidification and drying, absorption and ion exchange, other diffusion operations, multiphase contacting and separations, process control, materials of construction, mechanical plant and project engineering, and cost of profitability estimation.

\#6500634

*2.373-0.2173

RADIATION QUANTITIES AND UNITS, ICRU REPORT 10A

NBS Handbook 84, National Bureau of Standards, Washington, D. C.

October 1964

$17 \mathrm{p}$

Available from Superintendent of Documents, GPO

This handbook presents recommendations agreed upon at the meeting of the International Commission on Radiological Units and Measurements held in Montreux, Switzerland, in April 1962. Quantities, units, and the names of radiation are given.

\#6809001

$* 8.273-3.273-0.2573$

Reid, R. C. Sherwood, T. K.

THE PROPERTIES OF GASES AND LIQUIDS ESTIMATION AND CORRELATION, SECOND EDITION

New York: McGraw-Hil1 Book Company, Inc.

1966

$646 \mathrm{p}$

This book presents a critical review of the various estimation procedures for a limited number of properties of gases and liquids. These include critical properties, P-V-T and thermodynamic properties, vapor pressures, latent heats, heats of formation, free energies of formation, heat capacities, surface tensions, viscosities, thermal conductivities, diffusion coefficients, and equilibrium properties of mixtures. Comparisons of experimental and estimated values are shown in the form of tables for indication of the degree of reliability of the methods discussed. The book is designed to be used as a text for molecular physics or a reference source for engineers. A set of Appendixes summarizes material referred to in the chapters and contains a large volume of tabulated data as well as references to the literature.

\#6803002 $\star 0.2173-8.173$

Samsonov, G. V. (Editor)

HANDBOOK OF THE PHYSIOCHEMICAL PROPERTIES OF THE ELEMENTS

New York, Washington: IFI/Plenum

1968

$941 p$

Translated from Russian

This compendium was compiled from literature data available to the authors ( 30 Russian experts). An attempt was made to present the most reliable numerical values; the criteria for reliability were the experimental techniques used to obtain these values, the purity and state of the samples used, and statistical criteria. One recomended 
numerical value is given for each property, but additional literature references are listed. The properties included in the handbook are: 1) general information on properties of the elements and atomic structure; relative abundances, atomic weights and atomic volumes, atomic and ionic radii, density, crystal structure, optical and $x$-ray spectra; 2) nuclear properties of the elements; isotopes, nuclear energy levels, neutron interaction cross sections, range and scattering of electrons; 3) thermodynamic and heat properties: entropy, heat capacity, melting and boiling points, heats of fusion and sublimation, vapor pressures, critical parameters, thermal conductivity, crystal lattice energy, surface tensions, viscosity diffusion parameters, ion mobilities; 4) electrical and magnetic properties; electrical conductivity superconductivity, thermoelectric properties, magnetic properties, enission properties, dielectric properties; 5) optical properties color, emissivity, reflectivity, refractive index; 6) mechanical properties; elastic and shear moduli, Poisson ratio, compressibility, velocity of sound, tensile, yield and compressive strength, fatigue limit, creep, and hardness; 7) electrochemical properties; electrode potentials, oxidationreduction potentials; 8) chemical properties: interaction of the elements with various media and other elements.

\section{\#6809006}

$* 0.2173$

Sober, H. A. (Editor)

HANDBOOK OF BIOCHEMISTRY, SELECTED DATA FOR MOLECULAR BIOLOGY

Cleveland, Ohio: The Chemical Rubber Company

1968

$980 p$

This handbook gives evaluated data for selected areas of biochemistry including the principal research frontiers in medicine, modern biology, genetics, biophysics, and molecular biology. The material is divided into the major sections of abbreviations and nomenclature, amino acids, peptides and proteins, carbohydrates, 1ipids, steroids, purines, pyrimidines, nucleotides and oligonucleotides, nucleic acids, genetics and biology, physical and chemical data, and a miscellaneous section.

\$ 6810020

$* 0.2173$

Stecher, P. G. (Editor)

THE MERCK INDEX, AN ENCYCLOPEDIA OF CHEMICALS AND DRUGS EIGHTH EDITION

Rahway, New Jersey: Merck \& Co., Inc.

1968

$1214 \mathrm{p}$

This eighth edition of the Merck Index includes nearly 10,000 descriptions of individual substances, more than 4,500 structural formulas, and about 42,000 names of chemicals and drugs. References to the cited original papers are given. The Merck Index has a strong medicinal element, but it is essentially an organic chemical work with the inclusion of useful inorganic substances and a separate section on organic name reactions. The Cross Index of Names and Formula Index provide a key to the monographs included. The handbook includes some data and conversion factors in tabular form; however, a majority of the information is given in paragraph form under each alphabetical entry.

\$6512008

${ }^{*} 0.2172-3.172$

TABLES OF COULOMB WAVE FUNCTIONS, VOLUME I

NBS Applied Mathematics Series No. 17, National

Bureau of Standards, Washington, D. C.

April 1952

$141 p$

Available from Superintendent of Documents, GPO

This report presents numerical values of the Coulomb wave functions in somewhat peculiar notation. However, an introduction by $M$.
Abramowitz defines all quantities and it is possible to reconstruct the actual Coulomb wave functions from the tables presented herein. In addition, there is a discussion of the significance of these wave functions in a preface by G. Breit.

\#6708016

$\star 0.2173$

TABLES RELATING TO MATHIEU FUNCTIONS

NBS Applied Mathematics Series 59, National Bureau of Standards, Washington, D. C.

August 1,1967

$311 p$

Available from Superintendent of Documents, GPO

This second edition of "Tables Relating to Mathieu Functions" (Columbia University Press, New York, 1951) incorporates the article "Table of Characteristic Values of Mathieu's Equation for Large Values of the Parameter" by Gertrude Blanch and Ida Rhodes, Journal of the Washington Academy of Sciences, $45(6), 166-196$, June 1955, but makes

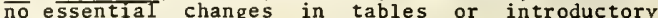
material. However, a number of misprints in the first edition have been corrected and the bibliography updated.

\#6500541-6500548, inclusive

$* 0.1083$

Washburn, E. W. (Editor)

INTERNATIONAL CRITICAL TABLES OF NUMERICAL DATA, PHYSICS, CHEMISTRY AND TECHNOLOGY VOLUMES 1 TO 7 AND INDEX

New York: McGraw-Hill Book Company, Inc.

1926-1933

$1-415 p ; 2-616 p ; 3-444 p ; 4-481 p ; 5-465 p ; 6-471 p$; 7. $507 p ;$ Index- $321 p$

The International Critical Tables, covering the literature from 1910-1923, provide evaluated data on physical, chemical, and technological materials. The seven volumes of data are organized into more than 300 sections. Among the sections and properties included are national and local systens of weights and measures, symbols, basic constants, conversion data, dimensions, definitions, chemical elements and atoms, thermometry laboratory techniques, laboratory methods for producing and maintaining constant temperatures, constant humidity, pressure, volume of liquid menisci, weights and weighing, calibration of volumetric vessels, buffer solutions and indicators, high vacuum technique, building material properties, abrasives, glass, fuels and petroleums, paints and varnishes, $x$-ray diffraction data for industrial materials, metallurgy, liquid and gas kinetics, spectroscopy, photometry, photography, properties of soaps, electrical conductivity and resistivity, magnetism, acoustics, refractivity, optical properties, explosives, PVT relations for one-phase systems, phase equilibrium data, osmotic pressures, surface properties, and a great volume of other related areas and properties. The last volume is a comprehensive index of all materials and properties listed in the International Critical Tables.

\# 6711003

Weast, R. C. (Editor)

$* 0.2183$

HANDBOOK OF CHEMISTRY AND PHYSICS, 49 th EDITION, 1968-1969

Cleveland, Ohio: Chemical Rubber Company

1968

$2450 p$

This Handbook is a compendium of data from all fields of physical science which have been experientally determined and published by scientists over the years, plus a collection of tables of important mathematical functions. The mathematical tables include logarithnic and trigonometric functions, numerical tables of squares, cubes, powers, roots and reciprocals, statistical tables, derivatives and integrals, algebraic functions and other formulas. Data on elements and inorganic compounds includes atomic weights, electron configurations, neutron cross sections, and physical constants. Data on organic compounds include naming rules, 
physical constants, organometallic physical constants, properties of steriod hormones, amino acids, carbohydrates, and waxes. General chemical data includes thermodynamic constants, dissociation and ionization constants, conductances, electrolytes, melting and boiling points, vapor pressure, and properties of aqueous solutions. General physical constants data includes thermal conductivity, dielectric properties, superconductivity, magnetic properties of matter, X-ray and optical spectral index of refraction.

\section{$\$ 6809004$}

$* 0.2183$

Wick, 0. J. (Editor)

PLUTONIUM HANDBOOK, A GUIDE TO THE TECHNOLOGY, VOLUME 1

New York: Gordon and Breach, Science Publishers

1967

$520 \mathrm{p}$

The Plutonius Handbook provides information on the range of topics concerning plutonium technology. The book is divided into seven separate sections: physics; metallurgy and ceramics; chemistry; chemical separations; fabrication and utilization; analys is and inspection; and health and safety. References are included and data are given both graphically and in tabular form.

6603015

$\star 0.2183$

Wilhoit, R. C.; Ha thaway, W.

TABLES OF CONVERSION FÁCTORS BASED ON ACCEPTED CONSTANTS AS OF 1965

College Station, Texas: Texas Agricultural and
Mechanical University, Chemical Thermodynamic Properties Research Center, College Station, Texas November 1965

$70 \mathrm{p}$

These tables contain values of the defined, basic and derived physical constants, and tables of conversion factors for physical quantities measured in different units, presented in matrix form.

\section{Wilson, B. S. (Editor)}

THE RADIOCHEMICAL MANUAL, SECOND EDITION

Amersham, England: Radiochemical Centre

1966

$327 p$

The manual is intended as a guide for all who use radioactive substances professionally; its aim is to helo the individual user choose the right material for his purposes and to make effective use of it. The text portion of the book includes information on preparation of radioisotopes; radiation sources; problems of using radioactive materials; radioactive chemicals as pharmaceuticals; quantification of radioactive substances; radiologica1 safety and waste disposa1; packaging and carrying radioactive materials; ownership and use regulations and a guide to literature. The tabulated data includes: physical data on the more common radioisotopes, including physical characteristics, availability, beta and gamma decay energies and half-lives; radiation sources, synthetic routes to labelled compounds, decomposition rates of labelled compound, measurement and safety. 
Nuclear Properties

(Including Fundamental Poarticles Properties) 
$\$ 6509015$

$* 2.1013$

Abadie-taumert, F. A

EPPIRICAL DETERYINATION OF TIIE RELATIVE ATOMIC IVEIGIITS OF TIIE ELEIENTS AND OF TIIE ?HSS OF TIIEIR ISOTOPES

Energie Nucléaire, 5:526-532

November 1963

In French

The decinal variations (d) of relative atomic weights (m) in the form d = $1-A+1$, are graphically represented as a function of the mass number $A$ by 5 linear segments.

\#6500683

*2. 1413

Ajzenberg-Selove, F.; Lauritsen, T.

ENERGY LEVELS OF' LIGIT NUCLEI. VI

Nuclear Physics, 11:1-340

Tay 1959

In this 6 th article of a series of compilations sumarizing experimental information on the energy levels of the nuclei from He-5 to Ne-24 each nucleus is represented by a diagram and a table in which the known energy levels are indicated, together with the nuclear reactions in which they are involved. The listings are supported by a brief text for each reaction, intended as an annotated bibliography, hut abstracting as far as possible the most relevant information.

6604066

Akiyana, $Y$.

TABLES OF TIIE SU, FRACTIONAL-PARTNTAGE AND CLEBSCI1-GORDAN COEFFICIENTS IN TIIE $2 S-1 \mathrm{~d}$ S!IELL (I) Nuclear Data, A2:403-428

November 1966

The $\mathrm{SU}_{3}$ fractional-parentage and Clebsch-Gordan coefficients in the $2 \mathrm{~s}-1 \mathrm{~d}$ she11 have been calculated nunerically for systers of up to six nucleons. The classification scheme of the states is characterized by the group chain $\mathrm{SU}_{5} \supset \mathrm{SU}_{3}>\mathrm{R}_{3}$, and the basis functions are represented in $a$ completely orthogonal system. A new reciprocity relation (phase convention) for the Clebsch-Gordan coefficients is proposed and adopted in the tabulation. A table of the coefficients of fractional parentage is presented here. A sample of the table of Clebsch-Gordan coefficients is given. The complete table is available from the American Documentation Institute Auxiliary Publications Project.

\section{$\# 6909065$}

2.2413

Alexander, G.; Benary, 0.: Manor, $\mathrm{U}$.

DATA COIPILATION ON BARYON-BARYON INTERACTIONS. I. PROTON-PROTON COLLISIONS BETIVEN 1 AND $32 \mathrm{GeV} / \mathrm{C}$ Nuclear Physics, B5:1-28

Apri1 1968

A data compilation on pp interactions is reported as the beginning of a systematic review of the strong-interaction properties of elenentary particles. The list of references is followed by curves showing the total and elastic pp cross sections as a function of the incident momentum. Following these are differential cross sections and polarization curves for various proton incident momenta. The curves are presented in the same manner as they appear in print. Finally, tables are presented of some partial-reactions cross sections. This report covers the data published during the period January 1, 1954 to October 31, 1967.
66509010

*2.2413

Altamirano, C. P.; Perkins, S. T. ANGULAR DISTRIBUTION COEFFICIENTS

SCATTERING OF NEUTRONS

FOR

ELAST IC

AN-1321, Aerojet-General Nucleonics,

San Ramon, California

December 1964

$60 \mathrm{p}$

Available from CFSTI, TID 21629

The results of an analysis which utilized the experimental differential cross section for elastic scattering of neutrons are presented. The calculated quantities for all materials were the elastic scattering cross section, the average logarithmic energy decrement per collision, and the Legendre expansion coefficients. The neutron energy range was approximately from 0.1 to $14 \mathrm{MeV}$. These parameters were presented graphically as a function of neutron energy when sufficient data were available. For materials in which this was not the case, the results are presented in tabular form.

\#6908007 *2.511

ARGONNE CODE CENTER: BENCHMARK PROBLEM BOOK, NUMERICAL DETERMINATION OF THE SPACE, TIME, ANGLE, OR ENERGY DISTRIBUTION OF PARTICLES IN AN' ASSEMBLY ANL-7416, Argonne National Laboratory,

Argonne, Illinois

February 1968

$73 p$

Available from CFSTI

This initial collection contains benchmark problems relating to numerical determination of space, time, angle, or energy distribution of particles in an assembly. The primary objective is to provide reliable solutions to diverse problems of varying. complexity. This book should serve as a source book of solutions to mathematically well-defined problems for which either analytical or very accurate approximate solutions are known.

\section{\#6500154 *2.2413-3.113}

Aron, W. A.; Hoffman, B. G.; Willians, F. C.

RANGE ENERGY CURVES, SECOND REVISION, 1949

UCRL-121 (AECU-663), California University,

Lawrence Radiation Laboratory.

Berkeley, California

1949

$99 \mathrm{p}$

Two tables are presented, one for protons and one for electrons, which enable the computation of the rate of energy loss, given the value of the mean excitation potential. While the value of this latter constant has been taken, in this paper, as being given by

\section{$I=\alpha z=11.5 \mathrm{Z} \mathrm{eV}$,}

the validity of such a simplistic formulation has been increasingly questioned, several recent experiments yielding results considerably different from those expected. The range-energy curves therefore are tentative, pending a better understanding of the mean excitation potential. The energy-loss considered in this paper derives only from collision effects. The losses due to bremsstrahlung and meson and pair production result from an essentially different process. The calculation of the Fermi effect is ill-advised pending a better determination of the constants.

$\# 6500058$

Ashby, V. J.; Catron, H. C.

TABLES OF NUCLEAR REACTION Q VALUES

UCRL-5419, California University, Lawrence Radiation Laboratory, Livermore, California February 10 , 1959

$337 \mathrm{p}$

Available from CFSTI

Tables of nuclear reaction $Q$ values have been calculated from nuclide masses, when possible, for those 42 reactions involving gamma, $n, p, d, t$, 
$\mathrm{He}-3$, or $\mathrm{He}-4$ as either incident or product particle for about 650 target nuclides. Approximately $8000 \mathrm{Q}$ values are tabulated.

\#6509013

$\star 1.2413$

Barashenkov, V. S.

INTERACTIONS OF MESONS

Fortschritte der Physik, 12:347-387

1964

A review is given of the experimental and theoretical data on $\pi-\pi, K-\pi$ and $K-K$ interactions.

\section{$\sharp 6907017$}

Barash-Schmidt, N.; et al.

REVIEW OF PARTICLE PROPERTIES

Reviews of Modern Physics, 41:109-192

January 1969

This review of the properties of leptons, mesons, and baryons is an updating of Rosenfeld et al. [Rev. Mod. Phys. 40,77 (1968)]. Data are evaluated, 1 isted, averaged, and summarized in tables and wallet sheets. A data booklet is also available.

$\sharp 6500053$

$\star 2.2413$

Bartholomew, G. A.; Higgs, L. A.

COMPILATION OF THERMAL NEUTRON CAPTURE GAMMA RAYS

CRGP-784, Atomic Energy of Canada Limited, Cha1k River, Ontario

July 1958

$146 \mathrm{p}$

This is a compendium of energies, absolute intensities, and spectral distributions, together with a complete bibliography, of neutron capture gamma-rays compiled from information available as of June 1, 1958.

\section{\#6604064}

$* 2.083-2.2583$

Bartholomew, G. A.; et al.

COMPEND IUM OF THERMAL-NEUTRON-CAPTURE GAMMA-RAY MEASUREMENTS PART $1 . Z \leqq 46$

Nuclear Data, A3:367-650

December $\frac{1967}{1967}$

A compilation is presented of thermal-neutroncapture gamma-ray energies and intensities with decay schemes, examples of original data, and fully corrected spectral distributions. A table of stable isotopes with abundances, thermal-neutron radiative-capture cross sections, spins, parities, and other information of value to neutron-capture gamma-ray spectroscopy is included.

\author{
$\# 6509028$ \\ $\star 2.013$ \\ Beckurts, K. H.; Wirtz, K. \\ Dresner, L. (Translator) \\ NEUTRON'PHYSICS \\ Berlin and New York: Springer-Verlag \\ 1964 \\ $454 p$ \\ In German
}

This text presents a survey of: the physical properties of the neutron, neutron sources and detectors, and cross-section measurements; the theory of neutron fields; the theory of diffusion, slowing-down, and thermalization; the determination of neutron fluxes and distributions; and the determination of neutron transport properties.

$\# 6908049$

$\star 2.2213$

Berger, M. J.

ENERGY DEPOSITION IN WATER BY PHOTONS FROM POINT ISOTROPIC SOURCES

Journal of Nuclear Medicine, Supplement 1 , No.

2:17-25

February 1968
Buildup factors and related data which describe the deposition of energy by photons in water medium are presented. Nineteen photon source energies between $15 \mathrm{keV}$ and $3 \mathrm{MeV}$ are included. Data are given on energy-absorption buildup factor for point isotropic sources in water, coefficients of polynomial representation of energy absorption buildup factors for water, specific absorbed fractions and absorbed dose rates in water and muscle, and radii of spheres for specific absorbed fractions.

$\# 6910030$

$\star 2.2513$

Bertin, M. C.; et al.

NEUTRON CROSS SECTIONS OF U-238, U-235, U-239, U$234, \mathrm{U}-236, \mathrm{Pu}-239$, Pu-240, W, Pb, Ni, Cr, C, Li-6, Li-7, AND T

UNC-5099, United Nuclear Corporation, Development Division, White Plains, New York

December 31,1964

$303 p$

Available from CFSTI, AD 616629

Neutron cross-section sets have been prepared for 16 elements or isotopes for neutron energies from $0.037 \mathrm{eV}$ to $18 \mathrm{MeV}$. The cross sections tabulated include the total, elastic, inelastic, $(n, 2 n)$, and fission cross sections, as well as cross sections for charged particle emission. Information is also given on the angular distribution of elastically scattered neutrons and on the energy distribution of neutrons and gamma-rays following nonelastic scattering.

$\# 6508024$

$* 2,1113-3,113$

Bhanot, V. B.; Gupta, S. C.

MASS-SPECTROSCOPIC ATOMIC MASS DIFFERENCES

Nuovo Cimento, 10:1011-1023

June 1963

New mass-spectroscopic data on atomic mass differences in the medium and heavy mass regions were compiled and tabulated. Corresponding values in the new scale based on $\mathrm{C}-12$ as the standard are also given. Unadjusted experimental values for nuclidic masses in the new scale were listed. For a large number of stable nuclides in the region from strontium to antimony the newer values obtained from the data of Demirkhanov et al., are more accurate than the corresponding values 1 isted in the recent exhaustive mass table of Koenig et al. Attention is drawn to the advantages of the isotopic doublets over the published conventional mass doublets in the heavy mass region.

$\sharp 6604066 \quad$ \#2.083-2.2483

Bichse 1, H.; Tschalaer, C.

A RANGE-ENERGY TABLE FOR HEAVY PARTICLES IN SILICON Nuclear Data, A3:343-360

october 1967

Stopping-power $S$ and range $R$ tables for protons, deuterons, tritons, He-3, He-4, and Li-7 ions of energies between $1-$ and $200-\mathrm{MeV}$ penetrating silicon absorbers are presented. Auxiliary data are included in tables for the coefficients $C$ and alpha needed for the approximate expression $S=C \cdot E$ alphand in figures giving the energy dependence for the coefficients of $R=C C_{B} \cdot E$ beta. Included is a short review of principles to be considered in detector applications.

\#6508027

22.2413

Bormann, $M$.

NEUTRON SHELL EFFECTS IN THE $(n, 2 n)$ CROSS-SECTIONS AT $14 \mathrm{MeV}$

Nuclear Physics, 65:257-274

May 1965

The systematics of the available total (n,2n) reaction cross sections for $14 \mathrm{MeV}$ neutrons were investigated for even-neutron target nuclei. The existence of neutron shell effects is verified and discussed on the basis of the statistical theory. 
Minima in the $(n, 2 \pi)$ cross sections at the magic neutron numbers $N=28$ and 50 for even- and $N=20$ for odd-proton target nuclei can be accounted for by similar trends in the $Q$ values. Cross-section maxima at the closed neutron shells $N=82$ and 126 for even- and $N=28,50,82$, and 126 for oddproton target nuclei can be understood as an effect of shell closure on the nuclear level density, the relative decrease of which on passing across a closed shell is larger for higher excitation energies. The ( $n, n^{\prime}$ gamma) reaction is the principle one competing with the $(n, 2 n)$ process at $14 \mathrm{MeV}$ in the medium and heavy mass regions. Since for the (n,n'gamma) reaction the residual excitation energy 1 ies in the region up to $12 \mathrm{MeV}$, whereas for the $(n, 2 n)$ reaction it does not exceed $4 \mathrm{MeV}$, and since the relative change in the level density is greater at the higher excitation energies, the competition between ( $n, n^{\prime}$ gamma) and $(\pi, 2 \pi)$ reactions favors the latter in the region of closed neutron shells and thus accounts for the observed maxima in the $(n, 2 \pi)$ cross-section systematics. Ninety-six references are included.

$\# 6911012$

$\star 2.1413$

Braglia, G. L.; de'Munari, G. M.; Mambriani, G.

ELECTRON ENERGY DISTRIBUTIONS IN RARE GASES IN A dC ELECTRIC F1ELD AT LOW E/N VALUES

$\mathrm{RT} / \mathrm{FI}=(66) 35$, Parma University, 1stituto di Fisica, Parma, Italy

September 1966

$66 \mathrm{p}$

Available from AEC Dep. Libraries

The steady-state distributions of electron energies in helium, neon, argon, krypton, and xenon are given at values of $E / N$ (ratio of dc electric field to gas atom number density) up to $E / N$ approximately equal to $10-17 \mathrm{~V} / \mathrm{sq} . \mathrm{cm}$

\#6509032

$\approx 2.1313$

Brahmavar, S. M.; Ramaswamy, M. K.

CLASSIFICATION OF EXCITED STATES OF EVEN-EVEN NUCLE I

Proceedings of the lndian Academy of Science, Section $A, 59: 102-114$

February 1964

The possible usefulness of the product $\mathrm{AE}$ (mass number times excitation energy) in classifying excited states of even-even nuclei is discussed. A table of $E$ and $A E$ for even-even nuclei having $0^{+}$, $2^{+}, 0^{+1}, 2^{+1}, 3^{-}$and $5^{-}$levels is given. It is shown that this criterion may be useful in understanding the excitation mechanism.

\section{$\# 6908048 \quad * 2.2213$}

Browne 11, G. L.; E1lett, W. H.; Reddy, A. R.

ABSORBED FRACTIONS FOR PHOTON DOSIMETRY

Journal of Nuclear Medicine Supplement 1, No. 3:2939

\section{February 1968}

The photon absorbed fraction, which is the fraction of the emitted photon energy that is absorbed by a volume, depends on the source and absorber geometry, the photon energy, and the intervening material. Data from Monte Carlo calculations of absorbed fractions for a number of geometries and photon energies are presented.

$\# 6500057$

$\star 2.2313$

Bruninx, E.

HIGH-ENERGY NUCLEAR REACTION CROSS-SECTIONS

CERN 61-1, European Organization for Nuclear Research, Geneva, Switzerland

January 1961

$40 \mathrm{p}$

A compilation is presented of formation cross sections in the region beryllium to copper for nuclear reactions above $50 \mathrm{MeV}$.
Bruninx, E.

HIGH-ENERGY NUCLEAR REACTION CROSS-SECTIONS II

CERN 62-9, European Organization for Nuclear Research, Geneva, Switzerland

February 15,1962

$200 \mathrm{p}$

Data on formation cross sections for target elements in the $2 \mathrm{n}$ to $\mathrm{U}$ region are presented. The cross section values were recalculated on the basis of the monitor reaction, Al-27(p,3pn) Na-24, where necessary. These values appear in CERN 61-1.

\#6509037

*2.2313

Bruninx, E.

HIGH-ENERGY NUCLEAR REACTION CROSS-SECTIONS II CERN 64-17, European Organization for Nuclear Research, Geneva, Switzerland

March 18,1964

$65 \mathrm{p}$

Cross sections based on new values (given) for the cross sections of the monitor reactions A1-27 $(\mathrm{p}, 3 \mathrm{pn}) \mathrm{Na}-24$ and $\mathrm{C}-12(\mathrm{pn}, \mathrm{pn}) \mathrm{C}-11$ are tabulated for the production of specific nuclei in proton reactions on nuclei from Be through $U$. Cross sections for production by gamma reactions on $B$ and $\mathrm{Ni}$; deuteron reactions on $\mathrm{Cu}$, Th, $\mathrm{U}, \mathrm{Ar}, \mathrm{Ne}, \mathrm{Mg}, \mathrm{N}$, $C$, and $A 1$; alpha reactions on $U, T h, A l$, and $C$ $C-12$ ions on $A u$ and $A 1$; and by $L i-6, N-14$, and $0-16$ ions on $\mathrm{A} 1$ are also included.

\#6908023 $\approx 2.2213$

Busza, W.; Diebold, R.; Ecklund, S.

PHOTOPRODUCTION KINEMATICS TABLES FROM 0.5 TO 45 $\mathrm{GeV}$

SLAC Report No, 77, Stanford Linear Accelerator Center, Stanford, California

October 1967

$205 p$

Available from CFST

The tables list kinematic quantities for two-body and quasi two-body photoproduction reactions. A1though the laboratory angle and momentum are given for both outgoing particles, differential quantities such as the solid angle transformation, Jacobians, etc., are given only for the long-1ived charged particles $\left(\pi^{*}, K^{+}, p\right)$; to this extent the tables are oriented toward spectrometer experiments. Each table is characterized by the laboratory photon energy shown in the upper righthand corner. Tables are not given for photon energies below the kinematic threshold for the reaction. Each table lists the various kinematic quantities every $5^{\circ}$ in the center of mass from $0^{\circ}$ to $180^{\circ}$.

\#6508067 *2.513

Buyst, L.

TABLES OF $12-\mathbf{j}$ COEFFICIENTS

BLG 142, Centre d'Etude de L'Energie Nucleaire, Brusse1s, Be1gium

October 1962

$42 \mathrm{p}$

The 12-j coefficients which are used in nuclear physics are defined by: $(-)^{c-d} \sum_{0}(2 x+1)$ W(ajbj; $\left.x c\right)$ $W(b j f j ; x d) W(f j j ' e ; x j ;) W(j ' e a j \cdot x j)$ with $x=1 / 2$, $3 / 2,5 / 2$... where the W's stand for Racahcoefficients. The tables give the following values of the couple $\left(j^{\prime}, j^{\prime}\right)$ : $(3 / 2,3 / 2) ; \quad(3 / 2,5 / 2)$; $(3 / 2,7 / 2) ; \quad(3 / 2,9 / 2) ; \quad(5 / 2,5 / 2) ; \quad(5 / 2,7 / 2)$; $(5 / 2,9 / 2)$ and for all possible values of $a, b, c$, $d$, e, f with the restrictions that a, c, d, f are even or zero and that $b$ and $e$ are integers or zero. Computations were programed for Ferranti-MERCURY computer, and the program can be used to calculate the coefficients for any other values of $j$ and $j$ '. 
Caswe 11, R. S.

A FORTRAN CODE FOR CALCULATION OF EIGENVALUES AND EIGENFUNCTIONS IN REAL POTENTIAL WELLS

NBS Technical Note 159, National Bureau of Standards, Washington, D. C.

August 1962

$29 \mathrm{p}$

Available from Superintendent of Documents, GPO

A Fortran code has been developed for the calculation of eigenvalues and eigenfunctions for neutrons in real potential wells. A systematic procedure is given for approximate location of the eigenvalue and an automatic search procedure to determine the exact location. The code may be used for either bound or scattering states. In the case of scattering states, the criterion for maximum scattering ( $90^{\circ}$ phase shift) is used to deternine the energy of the state. The eigenvalues are determined by matching the numerically calculated logarithmic derivative $(f$, ) inside the nucleus to the appropriate analytical logarithmic derivative for the region outside the nucleus. In an alternate mode of operation, the outside value of $f_{1}$ may be set arbitrarily, and a match made to this value. Sample results for a Woods-Saxon we 11 with spin-orbit coupling for the case of oxygen-16 are shown. The code is in Fortran and was written for an IBM 7090 computer.

\#6609034

$\star 2.423$

Chalmers, J. H.; Walker, G.; Pugh, J. (compilers)

HANDBOOK OF CRITICALITY DATA VOLUME I, REVISED EDITION

United Kingdom Atomic Energy Authority, Authority Health and Safety Branch, Risley, England 1965

$122 p$

Available from AEC Dep. Library

This revised edition of the Handbook of Criticality Data, AHSB(S) Handbook 1, contains the best estimates on the critical parameters of the most reactive systems likely to be encountered in process plants. It presents data on compounds of plutonium and uranium, and some account is taken of the neutron poisoning effects of the nitrate ion and of Pu-240. Metal-water data and unreflected data are included in this edition. No theoretical justification of the data is given, nor is correlation with experiment shown on those graphs where the information is firmly established. In cases where sufficient experimental evidence or data check points are not available, graphs are overprinted as provisional. The basis of calculation is given and quantitative comparison with other known calculations is quoted as appropriate.

\#509023

2.2423

Chatterjee, A.

ALPHA REACTION CROSS SECTIONS FOR 14-MeV NEUTRONS Nucleonics, 22:108-109 August 1964

A comprehensive compilation of the data available through December 1963 , on total cross sections for the ( $\mathrm{n}$, alpha) reaction on nuclei $(6 \leq \mathrm{A} \leq 238)$ at 14 $\mathrm{MeV}$ is presented.

\section{2 *2.022-2.2522}

CINDA 68 AN INDEX TO THE LITERATURE ON MICROSCOPIC NEUTRON DATA

CINDA 68, Atomic Energy Commission, Division of Technical Information Extension, Oak Ridge, Tennessee

1968

$1017 p$

Available from AEC Div, of Tech. Information, TID 24489

CINDA 68 (Computer Index of Neutron Data) is a cumulative bibliography of the literature on microscopic neutron cross sections and allied data for any given target nucleus, arranged first by element and mass number, then by cross section or other quantity. It is intended to indicate where in the journal and report literature information can be found on specific microscopic neutron data and is not a listing of the data themselves. CINDA is limited to reactions induced by neutrons of energy less than $20 \mathrm{MeV}$ for specific elements and isotopes and includes information on (gamma, $n$ ) and (gamma,f) reactions with the restriction that the gamma-ray energy must be less than approximately 15 $\mathrm{MeV}$ and the (gamma, $n$ ) cross section greater than $0.1 \mathrm{mb}$. CINDA 68 contains the full computer file as of June 15, 1968.

\#9910003

$\star 2.022-2.2522$

CINDA 68 SUPPLEMENT AN INDEX TO THE LITERATURE ON MICROSCOPIC NEUTRON DATA

CINDA 68 Supplement, Atomic Energy Commission, Division of Technical Information Extension, Oak Ridge, Tennessee

January 15,1969

$182 \mathrm{p}$

Available from the AEC Div. of Tech. Information

This CINDA Supplement, unlike previous ones contains only new information added to the CINDA library since CINDA 1968 was published. Changes and corrections to existing CINDA entries will not appear until the next cumulation.

\#6910020

*2.022-2.2522

CINDA 69 AN INDEX TO THE LITERATURE ON MICROSCOPIC NEUTRON DATA

EANDC 80'U'; CCDN-C1/24; CINDA 69; Atomic Energy Commission, Division of Technical Information Extension, Oak Ridge, Tennessee

May 1969

$1157 \mathrm{p}$

Available from AEC Div, of Tech. Information

The CINDA (Computer Index of Neutron Data) bibliography allows its users to find the references to specific types of cross section information or other microscopic data from neutroninduced reactions, for any given target nucleus. CINDA entries are ordered in this publication first by element and mass number, then by cross section or other quantity. Within these isotopes and quantity groups, the references are ordered by date of publication. Users should note that experimental, theoretical and evaluation articles are nixed within the listing.

\#6803005

*2. 3521

CINDU CATALOGUE OF NUMERICAL NEUTRON DATA AVAILABLE FROM THE IAEA NUCLEAR DATA UNIT

CINDU-6, International Atomic Energy Agency, Nuclear Data Unit, Vienna, Austria November 1967

$116 \mathrm{p}$

Available from the IAEA Nuclear Data Unit

This issue of CINDU (Catalog of the IAEA Nuclear Data Unit) contains a bibliography of the current holdings of the data unit as of 1 November 1967. Only those references stored in the data storage and retrieval system (DASTAR) are listed in CINDU. (CINDU primarily lists papers on neutron induced and neutron scattering reactions). For each reference, there is listed the name, atomic and mass number of the target nucleus, the quantity measured or calculated, the energy range considered, the publishing laboratory, the type of article (experimental, theoretical, étc.), journal reference, volume, page and date, the author's name, and an accession number to the DASTAR data from the IAEA Nuclear Unit. 
16500079

$\star 2.2423$

Cojocaru, V.

GAMMA RAÝS ARRANGED ACCORDING TO THE ENERGY EMITTED BY RADIOISOTOPES FORMED BY THERMAL NEUTRON ACTIVATION OF NATURAL ISOTOPES

IFA-SN-17, Academia $R$ P $R$ Institutul de Fizica Atomica, Bucharest, Hungary

1962

$36 \mathrm{p}$

Available from CFSTI.

The rays emitted by radioisotopes produced in the thermal neutron activation of natural isotopes are tabulated according to energy. The natural abundance of the isotope, its half life, thermal neutron cross section, and absolute intensity of the radiation are given. The source of each energy tabulation is given. Ninety-one references are included.

$6608018 \quad \star 2.323$

Crocker, G. R.; Connors, M. A.

GAMMA-EMISSION DATA FOR' THE CALCULATION OF EXPOSURE RATES FROM NUCLEAR DEBRIS. VOLUME 1 . FISSION PRODUCTS

USNRDL-TR=876, Naval Radiological Defense Laboratory, San Francisco, California

June 10,1966

$79 \mathrm{p}$

Available from CFSTI.

Photon energies and abundances were compiled and sumarized for some fission-product and other radio-nuclides, using data reported in the literature up to June 1963. The data are presented in tabular form, listing photon energies and abundances for gamma rays, beta rays, and X-rays emitted. A list of multipliers is also presented for converting activities of the radionuclides to infinite-plane exposure rates.

*6911003

Dalton, P.; Turner, J. E.

$$
\star 2.1423
$$

NEW EVALUATION OF MEAN EXCITATION ENERGIES FOR USE IN RADIATION DOSIMETRY

Health Physics, 15:257-262

1968

Atomic mean excitation energies (I values) have been obtained from stopping-power and range data in twelve experiments. The analys is incorporates new values of shell corrections that include contributions made by electrons in all shel1s. Except for one set of data, the values of I obtained from different experiments are consistent with one another. Empirically, it is found that the following formulas fit the I values obtained here:

$$
\begin{aligned}
& I(\mathrm{eV})=11.2+11.7 \mathrm{z}, 2 \leq 13, \\
& I(\mathrm{eV})=52.8+8.71 \mathrm{z}, 2>13 .
\end{aligned}
$$

The numerical values found from the experiments analyzed are in agreement with those suggested in the National Academy of Sciences-National Research Council Report 1133. It appears that a few of the I values given in the National Bureau of Standards Handbook 79 (1961) are now out of date. A table of I values for some thirty-six materials of particular importance in radiation dosimetry is given in addition to the elemental I values found from the new data analysis.

$16508028 \quad 2.2623$

Daniel, H.; Schmitt, H.

FERMI MATRIX ELEMENTS IN ALLOWED BETA DECAY

Nuclear Physics, $65: 481=489$

Apri1 1965

A11 measured Fermi matrix elements of normal allowed beta decay are compiled. For each case an adopted value is given, which is the mean overall consistent results. The Fermi matrix elements turn out to be small or vanishing, in fair agreement with the isospin selection rule. The question of charge dependence of the nuclear forces is $\mathrm{d}$ is cussed.

$\$ 6904001 \quad * 2.021$

Davidson, J. P. (Editor)

THIRD SYMPOSIUM ON THE STRUCTURE OF LOW-MEDIUM MASS NUCLEI

Lawrence, Kansas; University Press of Kansas

1968

$294 \mathrm{p}$

This volume of the proceedings includes all of the invitational papers, formal comments and discussion of the papers presented in the third Symposium on the structure of low-medium mas nuclei held at the University of Kansas on April 18-20, 1968. The papers discuss such topics as the studies in the $2 s-1 d$ shell using triton induced reactions; effective interactions in nuclei and two-nucleon scattering; nuclear forces and the structure of light nuclei; gamma decay of analogue states in the $2 \mathrm{~s}-1 \mathrm{~d}$ she11; $\mathrm{T}=2$ levels in $\mathrm{Ne}-20, \mathrm{Mg}-24$, and $\mathrm{S}-32$ as compound nucleus resonances; the mirror nuclei, $\mathrm{Mg}-25$ and A1-25; gamma decay of states in Al-25; shell-model calculations in the $2 \mathrm{~s}-1 \mathrm{~d}$ she11; theory and experiment in the $2 s-1 d$ shell nuclei; SU 3 symetry and realistic interactions; gamma-ray spectroscopy in light nuclei: Na-22; recent developments on the dynamitron accelerator; and heavy ion acceleration.
$\# 6509039$
$\star 2.023$

Dazai, T.

NUMERICAL TABLES OF THE RACAH COEFFICIENT CONTAINING FOUR HALF-INTEGERS

Bulletin of the Tokyo Institute of Technology, $49: 35$
1962

Numerical values of the Racah coefficient containing four half-integers are shown in fractional form for parameters up to $9 / 2$.

\section{\#6508025}

$\star 2.2623$

Dewdney, J. W.

ISOBARIC ANALYSIS OF TOTAL BETA DECAY ENERGIES Nuclear Physics, 43:303-315

1963

An analysis was made of the total beta decay energies that follow from the nuclidic masses determined by Everling, et a1, and revised by Koenig, Mattauch and Wapstra. At all mass numbers for which there are known at least 3 beta decay energies, a weighted least squares procedure was used to determine, with standard errors, the following isobaric properties of the mass surface: the separation of the parabolas, the location of the common minimum of the two parabolas, and the common breadth of the parabolas. The dependence on mass number of these properties is discussed in terms of nuclear shell structure.

\section{2}

2.2423

Doherty, G.

NEUTRON'CROSS-SECTIONS OF Be-9 IN THE ENERGY RANGE $1 \mathrm{MeV}$ TO $15 \mathrm{MeV}$

AEEW-M 513, Atomic Energy.Establishment, Winfrith, England

1965

$10 \mathrm{p}$

Available from AEC Dep. Libraries

Self consistent neutron cross-sections for the energy range $1 \mathrm{MeV}$ to $15 \mathrm{MeV}$ are tabulated. The cross-section for the $n, 2 n$ reaction in the important energy region $2-4 \mathrm{MeV}$ is still not well determined but the shape has been adjusted to give agreement with a recent integral measurement. The angular distribution of elastically scattered neutrons, and the energy distribution of neutrons from the $n, 2 n$ reaction are not reviewed. The tabular data listed in Table 6 have been used to 
prepare a revised data file DFN 50 for inclusion in the U.K.A.E.A. nuclear data 1 ibrary.

16604066

$2.083-2.2783$

Dragoun, O.; Pauli, H. C.; Schmutzler, F.

TABLES OF INTERNAL CONVERSION COEFFICIENTS FOR

N-SUBSHELL ELECTRONS

Nuclear Data Tables, A6:235-351

\section{September 1969}

Internal conversion coefficients are presented for the first five N-subshells for every value of the atomic number 2 from $2=60$ to $2=100$. The coefficients are given for nuclear transition multipoles $E 1, \ldots, E 4, M 1, \ldots, M 4$ and for transition energies from about $1 \mathrm{keV}$ above threshold to usually $500 \mathrm{keV}$. The finite nuclear size is taken into account as well as atomic screening according to the nonrelativistic, self-consistent field method.

\# 6508047

Dummer, J. E., Jr.

$\star 2.323$

LOS ALAMOS HANDBOOK OF RADIATION MONITOR1NG, THIRD EDI T ION

LA-1835 Los Alamos Scientific Laboratory, Los Alamos, New Mexico

November 1958

$180 \mathrm{p}$

Available from the Superintendent of Documents, GPO

This third edition of the Handbook of Radiation Monitoring contains data for permissible exposures and permissible body burdens, as we 11 as numerous tables, graphs, and tabulated information on the basic data needed frequently in a nuclear laboratory. This manual contains a monitor's check list, radio code, emergency monitoring, protective methods, LASL radiation safety policies. permissible dose and measurement units, permissible contamination levels, decontamination, maximum permissible exposures, table of isotopes. monitoring instruments, alpha monitoring, betagamma monitoring, neutron monitoring, tritium monitoring, personnel dosimetry, urinalysis, air sampling, and respiratory protective equipment information.

\# 6902029 *1.1023

DuMond, J. W. ; Coher, E. R.

A LEAST-SQUARE ADJUSTMENT OF THE ATOMIC CONSTANTS AS OF DECEMBER 1950

California Institute of Technology, Pasadena, California

1951

$202 \mathrm{p}$

This is a preliminary report to the National Research Council consisting of a reevaluation of the atomic constants and conversion factors of physics. It is of historical interest only. As a result of the precision microwave and atomic beam techniques, it reported advances in the accuracy of such constants as $e$ and $m$, the charge and mass of the electron, h, Planck's constant, Avogadro's number, the velocity of light, the conversion factor from the Siegbahn nominal scale of $x$-units to milliangstroms, and a list of some sixty or more useful derived constants and conversion factors which can be computed from the preceding with the use of accurately known other constants, such as the Rydberg, the gas constant, etc. In this report the least-squares method was employed to adjust the data to arrive at a set of consistent values for the constants in question.

\#6509034

2.1623

Dutta, A. K.; et al.

ON NUCLEAR BINDING ENERG1ES

Indian Journal of Phys ics, $38: 57 \div 60$

January $\frac{\text { low } 1964}{10}$

Relations for the binding energies of nuclei of a given mass number were derived, Experimental and calculated binding energies for all the isobars of some mass numbers are tabulated.

$\# 6608019$

$* 2.2423$

Edwards, W. E.

GAMMA RAY PRODUCTION CROSS SECTIONS SUPPLEMENT NO. 1 GEMP-360 (informal), General Electric Company, Atomic Products Division, Cincinnati, Ohio August 1965

$49 \mathrm{p}$

Available from CFST 1

Gamma ray production cross sections are presented as a function of 15 neutron lethargy groups and 11 photon energy groups for 15 elements and isotopes. They were computed by Program GAMMA-P and account for the production of gamma rays by neutron radiative capture, neutron inelastic scattering, neutron induced fissioning, and (n,alpha) reactions.

$\# 6907025$

$\star 2.2552$

ENDF NEWSLETTER

3:MAT1001-1 to MAT1072-3, Brookhaven National Laboratory, Upton, New York

October 1967

$100 \mathrm{p}$

This newsletter contains a summary of neutron cross section data, information and references for $H, D$, $\mathrm{Li}-6$, Li-7, C-12, 0-16, Mg, Ti, V, Mn-55, Mo, $\mathrm{Xe}-135, \mathrm{Sm}-149, \mathrm{Eu}-151, \mathrm{Eu}-153, \mathrm{Gd}, \mathrm{Dy}+164, \mathrm{Lu}-175$ Lu-176, Au-197; U-235, Pu-238, Pu-239, Pu-240, $\mathrm{Pu}-242, \mathrm{Cm}-244, \mathrm{Na}-23, \mathrm{Hf}-176, \mathrm{Hf}-177, \mathrm{Hf}-178$, Hf-179, Hf-180, Hf-174.

$\$ 6500044$

*2.1423

Endt, P. M.; Van der Leun, C.

ENERGY LEVELS OF LIGHT NUCLEI III, $2=11$ to $2=20$ Nuclear Physics, $34: 1-324$

1962

The present compilation of information is for the energy levels of nuclei with $2=11$ to $2=20$. The nuclei are presented in order of increasing $A$, and nuclei of the same $A$, in order of increasing $Z$. Generally, each nuclear reaction is treated under the heading of the final nucleus. Exceptions are reactions where resonances have been observed, and the beta decay of unstable nuclei. in all other cases, the order of the reactions is determined by the initial nucleus, starting with the lowest-2 element. Following the last reaction discussed, for each nucleus a list is given of reactions "Not Reported." Discussions on the energy levels of a particular nucleus which do not fit naturally under the headings of a specific reaction leading to that nucleus have been given as "Remarks" at the end of the survey of that nucleus. References are given for all the information provided in the text, tables or figures. The excitation and resonance energies given in the level diagrams are the weighted mean values of all determinations available. The nuclear mass excesses used throughout this compilation are listed in table 1 . The natural abundances of the isotopes in the mass range discussed in this compilation are listed in table 2, together with nuclear moments.

$\# 6807002$

Endt, P. M.; Van der Leun, C.

ENERGY LEVELS OF $2=11-21$ NUCLEI (1V)

Nuclear Physics, A105:1-488

December $\frac{1967}{1967}$

A compilation is given of experimentally determine properties of energy levels of $2=11$ to 21 nuclei with special emphasis on nuclear spectroscopy. Data are presented such as excitation energies, half-lives, and thermal-n capture cross sections. All available elementary particle resonances are reported and updated values given. over 3200 references are provided. 
\#6908040

$* 2.2723$

Eskola, P.

COMPILATION OF DATA ON ALPHA ACTIVITIES WITH $Z$

GREATE $\dot{R}$ THAN $50, N$ LESS THLAN 126

In International Symposium on Why and How Should We Investigate Nuclides Far Of The Stability Line: Lyseki1, Sweden, August 21-27, 1966, pages 447-49 Uppsala, Sweden: Almquist \& Wiksells Boktryckeri $A B$ September 1967

$48 \mathrm{p}$

The experimental data on alpha energies, half-lives and branching ratios are presented in tables. An attempt has been made to make the calibration energies of various measurements compatible. The experimental alpha-decay energies and those given by the 1964 atomic mass table and mass formulae are presented graphically as a function of the neutron number of the parent nucleus.

$\# 6508040$

$* 2.023$

Everling, F.; et al.

1960 NUCLEAR' DATA TABLES PART 1 , CONSISTENT SET OF ENERGIES LIBERATED IN NUCLEAR REACTIONS I. TARGETS IN THE MASS REGION A $₫ 66$

National Academy of Sciences-National Research Council, Nuclear Data project, Washington, D. C.

February 1961

$230 \mathrm{p}$

Available from the Superintendent of Documents, GPO

These Q-values have been computed from masses of nuclides participating in the reaction, using a consistent set of nuclidic masses derived from all the relevant experimental data. This set, reaching from $n-1$ to $F m-254$, has just been obtained by a series of least-squares adjustments. The results of the first two adjustments, covering the mass range up to $A=70$, have been used here to calculate the Q-values'in the accompanying table.

\section{$\# 6509002$}

*2.1033

Faessler, A.; Greiner, W.; Sheline, R, K,

ROTATION VIBRATION INTERACTION IN DEFORMED NUCLEI

Nuclear Physics, 70:33-88

Augus $t 1965$

The rotation vibration model for deformed nuclei is summarized, various matrix elements are defined, and multipole operators are evaluated. The rotation vibration Hamiltonian is diagonalized up to spin 20 and presented in a manner which is independent of the individual nucleus. Energies and wave functions for the various states in the ground state, beta and gamma rotational bands of even deformed nuclei are given in an extensive table. The use of the table is explained in detail. An extensive comparison of the rotation vibration model and the asymmetric rotator model with available experimental data indicates an approximate equivalence of the two theories in explaining the three lowest rotational bands. However, the rotation vibration model appears to have a decided advantage in explaining the few twophonon vibrations experimentally observed. Small systematic deviations between the rotation vibration mode 1 and experiment for the energies of the vibrational bands are interpreted in terms of the Coriolis anti-pairing and blocking effects. These effects lead to lower limits on the pairing correlation energy and the critical spin above which all pairing ceases.

$\# 6500050$

$$
\star 2.2133
$$

ON THE SCATTERING OF GAMMA RAYS BY NUCLEI

NBS Technical Note 83, National Bureau of Standards, Washington, D. C.

November 1960

$25 p$

Available from CFSTI, PB 161584

The theory of scattering by electric dipole interaction is developed by tensorial techniques, which permit an early separation of geometric and dynamic factors. The geometric relationships are formulated in terms of variables that represent arbitrary partial polarization of the incident and scattered gamma rays. The relevant dynamic properties of a nucleus are represented by a scalar, a vector, and a quadrupole polarizability. These polarizabilities correspond respectively to the values 0,1 , and 2 of the quantum number $j$ which indicates the angular momentum transfer in the scattering process. The analysis of scattering according to angular momentum transfer is compared to the ordinary theory of angular distributions. The nucleat polarizability is discussed from the standpoint of different models. The magnitudes of the three polarizabilities can be determined by experiments with unpolarized nuclei but with some degree of circular polarization of the gamma rays; linear polarization contributes no additional information. Nuclear polarization is required to determine the phases of the polarizabilities.

$\# 6911031$

$* 2.2333$

Farchi, G.

FURTHER RESULTS OF THE MONTE CARLO CALCULATION OF THE $\left(e, e^{\prime} p\right)$ REACTION FOR INCIDENT ENERGIES BETWEEN 300 AND $600 \mathrm{MeV}$

ISS $67 / 9$, Istituto Superiore di Sanita, Laboratori di Fisica, Rome, Italy

March 22, 1967

$51 p$

Available from CFSTI

This paper supplements the results reported in ISS 66/37, "Some Results of the Monte Carlo Calculation of the (e,e'p) Reaction of Incident Energies between 300 and $600 \mathrm{MeV}$," adding the values valid for a s-state with $70 \mathrm{MeV}$ proton binding energy. Electron scattering angles and incident energies are kept the same as ISS 66/37. Ejected proton kinetic energies are still between 40 and $100 \mathrm{MeV}$.

$\# 6604066$

$$
\text { *2.083-2.2183 }
$$

Ford, G. P.; Hoffman, D. C.

FERMI - FUNCT ION INTEGRALS FOR FINDING RELATIVE BETAGROUP INTENSITIES

Nuclear Data, Al:411-433

July 1966

Values of Fermi-function integrals to aid in finding relative beta-intensities are tabulated for allowed beta-transitions with end-point energies, $T$ (rax)' in the range $0.10(0.05) 0.40,0.5(0.1)$ $5.2 \mathrm{for}$ values of $z$ in the range 10 (1) 109.

\section{\$6509031}

$$
\star 2.2633
$$

Ford, G. P.; Hoffman, D. C.

INTEGRALS OF BETA.SPECTRA

LA-3066, Los Alamos Scientific Laboratory, Los Alamos, New Mexico

May 1964

$31 \mathrm{p}$

Available from CFSTI

Integrals of beta-spectra for allowed transitions were calculated for $Z=10$ to 109 and for maximum kinetic energies from 0.1 to $5.5 \mathrm{MeV}$ at $0.1 \mathrm{MeV}$ intervals. The results are tabulated and can be used for a nongraphical determination of relative abundances of beta-spectrum components from their Fermi-Kurie plots.

Ford, K.W.; et al.

Way, K. (Editor)

1960 NUCLEAR DATA TABLES, PART 4, SHORT TABLES

National Academy of Sciences - National Research Council, Nuclear Data Project, Washington, D. C. December 1961

$254 \mathrm{p}$

Available from the Superintendent of Documents, GPO

Tabulations are included on elastic and inelastic scattering of charged particles, distribution of 
charge in the nucleus, nuclear moments, total beta disintegration energies, and gamma rays emitted by radioactive nuclei. A supplement to Directory to Nuclear Data Tabulations, 1958-61, is also included.

\#6508020 *2.1333

Fossan, D. B.; Herskind, B

ROTATIONAL-STATE LIFETIMES

Physics Letters, 2:155-157

\section{September 1,1962}

The half lives of the $2^{+}$first excited states of $W-180$ and $H f-180$ have been measured as $(1.33 \pm 0.05) \times 10^{-9}$ and $(1.50 \pm 0.04) \times 10^{-9}$ sec. respectively. These results $\bar{r}$ re included in summary of a complete investigation of the enhancement of the $2^{+} \rightarrow 0^{+}$internal-conversion process for deformed nuclei, in which half lives of $2^{*}$ first rotational levels were measured for series of even-even nuclei (150 less than A less than 190). The summary includes calculated experimental total conversion coefficients and the corresponding theoretical total conversion coefficients for comparison.

\section{$\# 6910041$}

$\star 1.2033$

Fuj imura, $K$.

NN INELASTIC PROCESSES

Progress of Theoretical physics (Kyoto) Supplement, $41-42: 282-315$

1967

Experimental data obtained from accelerators are summarized for pp inelastic scattering, and their characteristic features are shown. The data are separately compiled for the two final channels; the quasi-elastic scattering (isobar production) and the multiple production. Some cosmic ray data are also included. Twenty-five references are tabulated together with incident momentum, observed quantities and experimental methods.

\#6508036

*2.1533

Fuller, G. H.; Cohen, V. W.

NUCLEAR MOMENTS, A COMPILATION OF VALUES MEASURED BY SPECTROSCOPIC AND RESONANCES METHODS WHICH APPEARED IN THE LITERATURE BEFORE MAY 1964

Appendix 1 to Nuclear Data Sheets, National Academy of Sciences - National Research Council, Nuclear Data Project, and Brookhaven National Laboratory, Oak Ridge, Tennessee and Brookhaven, New York May 1965 $140 \mathrm{p}$

Measured values of nuclear moments and of some of the auxiliary quantities from which moment values are derived are listed in this compilation. There are nine tables. The first contains the measured values for the neutron, proton and anti-proton moments. The other eight contain information obtained by paramagnetic resonance, microwave spectroscopy, quadrupole resonance, nuclear magnetic resonance, atomic and molecular beans, optical spectroscopy, optical double resonance and pumping techniques, and Mbssbauer spectroscopy. Information obtained by angular correlation measurements has not been included in this compilation. Since detailed descriptions of methods of measuring nuclear moments have been presented by others only brief summaries are given with each table. In all tables the values of the magnetic moments tabulated have been adjusted to a standard value of the magnetic moment of the proton by the compilers using the experimentally determined quantities and their adopted standard values of mu and frequency ratios. In the preparation of the compilation all data available up to May 1964 were reviewed. Unless important for the determination of the sign of a moment, older values have been omitted when superseded by more accurate results. The abbreviations used and policies adopted concerning signs, uncertainties, standard values, and corrections are explained in tabular style in the first part of the report. section called Table of Rounded-Off Values and Index is included.

$\# 6604066$

Fuller, G. H.; Cohen, V. W.

NUCLEAR SPINS AND MOMENTS

Nuclear Data Tables, A5:433-612

March 1969

A summarizing table of rounded-off nuclear-moment values, arranged by $Z$ and $A$, is presented. This summary is based on experimentally determined values of the nuclear moments which have been listed in tables according to the specific spectroscopic or resonance technique used. References are given for all values quoted. Each table is preceded by a short introduction describing the experimental technique involved and the method of calculating the moments from the measured quantities. The cutoff date for the information included is August 1968. This tabulation supersedes Nuclear Moments, Appendix to Nuclear Data Sheets, issued May 1965.

\#6904009

Furnish, J .

ALPHA-ALPHA ELASTIC SCATTERING: A BIBLIOGRAPHY

LA-4011-MS, Los Alamos Scientific Laboratory, Los Alamos, New Mexico

October 1968

$16 \mathrm{p}$

Nuclear Science Abstracts was searched from Volume 7 (1955) through Volume 22, Number 15 (August 15, 1968) for material on the elastic scattering of alpha particles on alpha particles. The references are arranged numerically within each volume with the abstracts from the most recent volumes presented first.

\# 6909066

Gajewski, W.; et al.

$* 2.1733$

COMPILATION OF BINDING ENERGY VALUES OF LIGHT HYPERNUCLEI

Nuclear Physics, B 5: 105-113

February 1967

The results of a compilation of the binding energy values of light hypernuclei $(A \leq 16)$ obtained by the European $\mathrm{K}^{-}$Collaboration and the EFINSNorthwestern Collaboration are presented. The events were analyzed by the same computer program and uniform stringent selection criteria were applied.

\#6910025

*2.2533

Galloway, L, A, III; Shrader, E. F.

NEUTRON TOTAL CROSS SECTIONS MEASUREMENTS USING A "WHITE" NEUTRON SOURCE

C00-1573-6, Case Institute of Technology, Nuclear Physics Laboratory, Cleveland, Ohio

September 1966

$131 p$

Available from CFSTI

A method for measuring neutron total cross sections using a neutron intensity spectrum continuous in energy (a "white" spectrum) and a pulsed beam timeof-flight technique is used to measure neutron total cross sections in the 2 to $10 \mathrm{MeV}$ region. Total cross sections for the elements $\mathrm{Mg}, \mathrm{Al}, \mathrm{Ca}$, $\mathrm{V}, \mathrm{Fe}, \mathrm{Pd}, \mathrm{Ag}$ and $\mathrm{Pb}$ were measured to $1 \%$ average uncertainty in steps of $0.08 \mathrm{~ns} / \mathrm{m}$. Energy resolution varied from about $1.5 \%$ at $2 \mathrm{MeV}$ to $3 \%$ at $10 \mathrm{MeV}$. Results of these measurements are compared with measurements on the same samples with neutrons of known energy and with measurements of other workers. 
16911027

*2.2633

Gareev, F. A.; et al.

TABLES OF WAVE FUNCTIONS OF ONE-PARTICLE STATES OF DEFORMED NUCLEI OF THE RARE EARTH REGION FOR A FINITE DIFFUSED POTENTIAL

JINR-P4-3607, Joint lnstitute for Nuclear Research, Laboratory of Theoretical Physics, Dubna, USSR

1967

$50 \mathrm{p}$

Available from AEC Dep. Libraries

in Russian

In addition to the previous paper, tables are presented of wave functions of one-particle states of deformed nuclei of the rare-earth region in the approximation of a finite diffused potential.

\section{\#6500685}

\section{$* 2.033$}

Gibbs, R. C. ; Way, K.

A DIRÉCTORY TO NUĆLEAR DATA TABULATIONS

Vational Academy of Sciences-National Research Council, Washington, D. C.

1953

197 7

Available from Superintendent of Documents, GPO

This is a list of the comprehensive compilations and abstracts of experimental and theoretical information in the fields of basic nuclear physics, published in the years just prior to 1953. The book is divided into sections entitled: abundances, alpha activity, angular momentur coefficients, atonic constants, beta activity, beta decay process, capture gamma rays, Coulomb functions, cross sections of charged particles, neutron cross sections, emulsions, energy levels of nuclei, fission, ganma activity, gamma-ray conversion coefficients, half-lives, masses and mass differences, masses calculated from formulae, mass difference systeratics, mesons and hyperons, miscellaneous, moments, natural radioactivity, neutron producing reactions, nuclear models, comprehensive tables of nuclear properties, charts of nuclear properties, charts, nuclear reactions, penetration through matter of electrons, penetration through matter of gamma rays, penetration through matter of heavy particles, radiochenistry, and x-rays. Title, author, publication data, information on the availability of the tabulation, and a description of the tables are included.

\section{\#6910017}

2.2533

Gibson, G.

A SINGLE LEVEL ANALYSIS OF $U-235$ BASED ON RECENT $\sigma_{t}$ of AND of MEASUREMENTS; $\mathrm{A} \mathrm{n}+\mathrm{U}-235$ MULTIGROUP CROSS SECTION LIBRARY

WANL-TME-1586, Westinghouse Electric Corporation, Astronuclear Laboratory, Pittsburgh, Pennsylvania March 1967

$143 \mathrm{p}$

Total, capture, and fission neutron cross section data for U-235 have been simultaneously fit with a single-level Breit-Wigner least squares analysis. Two sets of resonance parameters for $E$ less than 63 eV are presented. One set was obtained when unit weighting was used in the least squares analysis, and another set was obtained when weighting proportional to the inverse of the cross section was used. The resonance capture and fission integrals calculated from the parameters agree in each case to within three percent with corresponding direct numerical integration of the data. The effects of simultaneously fitting two cross sections at a time: total and fission, and capture and fission have also been studied. Two $\mathrm{n}+\mathrm{U}-235$ multigroup cross section libraries, that differ only in the resonance range where the two sets of resonance parameters described above are used, have been generated for use in reactor calculations. The ratio of the capture resonance integral to the fission resonance integral that is calculated (सात्र $=0.51$ ) with either library agrees with the reactor integral measurements $($ alpha $=0.50 \pm 0.02)$. These libraries are presented.
\# 6509007

$\approx 2.2433$

Glasgow, D. W. ; Foster, D. G.

FAST-NEUTRON TÓTAL CROŚS SECTIONS OF 44 ELEMENTS

HW-SA-2875, General Electric Company, Hanford Atomic Products Operation, Richland, Washington April 1963

$10 \mathrm{p}$

Available from CFSTI

Fast neutrons from the $\operatorname{Li}-7(d, n) B e-8$ reaction were used to measure the neutron total cross sections of 20 elements that have principal isotopes with magic neutron numbers and to remeasure the cross sections of 25 other elements. Neutron energies from 3 to $15 \mathrm{MeV}$ were determined by the pulsed-beam-time-of flight technique. A Fortran program for the Hanford IBM 7090 was used to calculate the cross sections. Ramsauer structure in the twenty cross sections shows a reasonable continuity with those for the remeasured cross sections. Closed neutron shells, particularly for magic numbers greater than 28 , introduce small but distinct anomalies; but closed proton shells produce anomalies only if they are combined with a closed neutron shell. Unresolved anomalies appeared in $\mathrm{As}$ and $\mathrm{Sr}$.

\#6500081, $6500082 \quad * 2.2433$

Goldberg, M. D.; May, V. M.; Stehn, J.R.

ANGULAR DISTRIBUTIONS IN NEUTRON-1NDUCED REACTIONS, VOLUME $1.2=1$ TO 22 , VOLUME II. $2=23$ TO 94 .

BNL-400, Second Edition, Volumes 1 and 2; Brookhaven National Laboratory, Upton, New York October 1962

$300 \mathrm{p}$ each volume

Available from CFSTI

These volumes are issued separately, but are cataloged as a unit. Angular distributions of neutrons and of other products of fast neutron reactions with nuclei are included, Distributions for neutron elastic scattering predominate, but distributions for inelastic scattering and products associated with it are also included. Information for the various elements is arranged in order of their atomic number. The compilation encompasses data available in october 1962 and forms two volumes: Volume $I, 2=1$ to 22 and Volume $11,2=$ 23 to 94 .

\#6908016,6909013,6907027

$\star 2.2533$

Goldberg, M. D.; et al.

NEUTRON CROSS SÉCTIONS, VOLUME II A, $2=21$ to 40 , VOLUME II $B, 2=41$ to 60 , VOLUME $11 \mathrm{C}, 2=61$ to 87 BNL 325, Second Edition, Supplement No. 2, Brookhaven National Laboratory, Upton, New York February 1966, May 1966, August 1966

$350 \mathrm{p}, 350 \mathrm{p}, 350 \mathrm{p}$

Available from CFSTI

The neutron cross section data for the elements 2 = 21 to $40,2=41$ to $60,2=61$ to 87 are presented. The volumes are arranged strictly by chemical elements and by their isotopes. Thermal cross sections, resonance parameters, and cross section curves for one element or isotope are together on successive pages, separate from the next element or isotope. The format is fluid, varying from one page to the next to suit the information to be presented. The reference sources are listed as close to the data as is feasible.

\#6902059

$\star 2.21333$

Goldman, D. T

THE CALCULATION OF NUCLEAR CROSS-SECTIONS BY THE OPTICAL MODEL

Nuclear Data For Reactors, I: $339-364$ 1967

This paper presents a systematic derivation of nuclear cross-sections beginning with the interaction between an incident particle and the target nucleons. The replacement of the exact potential by an effective potential results, to first order, in the ordinary time-independent Schroedinger equation. This equation, including a complex and spin-orbit potential is solved for the 
resultant wave function. By writing the wave function in its scattering solution form, it is possible to compute the shape elastic cross-section and the polarization directly in Legendre moment expansions. The compound nucleus cross-section, as given originally by the statistical model of Hauser and Feshbach, arises naturally by examining the total wave function. By using the entire solution, including the complete interaction potential, it is possible to generalize these calculations to include the effect of non-spherical potentials and to derive the distorted wave Born approximation. The equations derived in this manner have been used in writing ABACUS-1 and OPTIC, two widely utilized optical model programs developed by colleagues and the author at Knolls Atomic Power Laboratory. The results obtained from calculations with suitably adjusted potential parameters, have provided confidence for further use of these optical model techniques in providing cross-section information.

4t 6810017,6810018

$* 2.2533$

Goldman, D. T. (Editor)

NEUTRON ' CROSS SECTIONS AND TECHNOLOGY, PROCEEDINGS OF A CONFERENCE, WASHINGTON, D. C. MARCH 4-7, 1968 NBS Special publication 299, Volumes 1 and 2 , National Bureau of Standards, Washington, D. C. September 1968

$1341 \mathrm{p}$

Available from the Superintendent of Documents, GPO

The Second Conference on Neutron Cross Sections and Technology was held in Washington, D. C. on March 4.7, 1968. Papers from this Conference have been published in two volumes, as follows: Volume 1 , Sessions A-D, pages 1-640; Volume II, Sessions E-H, pages 641-1337. These volumes contain the texts of the invited and contributed papers of the Conference. Topics covered include: the need for neutron data in fields of science and technology; standard data and flux measurements; the determination of neutron cross sections by theoretical and experimental techniques; a presentation of recently measured data and their utilization in a variety of applications.

\#6902068

$\star 2.2533$

Goldman, D. T.; et al.

TWENTY-TWO HUNDRED METER PER SECOND NEUTRON ABSORPTION CROSS SECTIONS

Unpublished manuscript, $63 \mathrm{p}$

As a part of the evaluated nuclear data file sponsored by the Atomic Energy Commission and administered by the Brookhaven National Neutron Cross Section Center, this report is a complete reexamination of the $2200 \mathrm{~m} / \mathrm{s}$ neutron cross sections absorption data. The tables supercede and contain data published since the Supplements to BNL-325 which were published from 1964-1966. Each element is listed separately together with all the isotopes for which the cross sections have been measured.

\#6604064

*2:083-2,2783

Goldstein, G.; Reynolds, S. A.

SPECIFIC ACTIVITIES AND HALF-LIVES OF COMMON RADIONUCLIDES

Nuclear Data, Al:435-452

July 1966

A compilation is presented of the half-1ives, decay constants, and specific activities of 354 wellknown radionuclides with half-lives greater than one day.

" 6508030

Goryachev, B. I.

CROSS-SECTIONS OF PHOTONUCLEAR REACTIONS TABULATED EXPERIMENTAL DATA

Atomic Energy Review, 2:71-148
A summary of experimental results relating to the thresholds, cross-sections and yields of photonuclear reactions is presented. Work published in periodicals up to the middle of 1963 is tabulated and is based on a breakdown of the experimental material by elements (from sodium to the transuranium elements), the papers relating to each element being arranged in alphabetical order. Except for a few old papers, the data contained in a11 published works were used, provided they could be adapted to the tabular arrangement employed. Two-hundred fourteen references are included.

औ 6608024

*2.2633

Gove, N. B

BETA AND GAMMA TRANSITION PROBABILITIES

ORNL-p-1786, Oak Ridge National Laboratory, Oak Ridge, Tennessee

1965

$28 \mathrm{p}$

Available from CFSTI, Conf-65118-3

A survey is presented of systematics of $\log \mathrm{ft}$ values, half lives for gamma transitions, and hindrance and enhancement factors for E1, E2, E3, $M 1, M 2, M 3$, and $M 4$ gamma transitions. Fifteen references are provided.

$\# 6604066$

$* 2.083-2.1283$

Gove, N. B.; Yamada, M. SHELL-INDEPENDENT SÝSTEMATICS $S_{n}$ (ISOTONIC) AND $S_{p}$

Nuclear Data, A4: 237-263

May 1968

Graphs are presented of recent values for the separation energy of a given neutron versus mass number, $S_{n}$ (isotonic), and for a given proton versus neutron number, $S$ (isotopic). The graphs confirm the fact, pointed out by Yamada and Matumoto in 1961, that the separation energy of the nth proton increases monotonically (except for oddeven effects) as the neutron number is increased, showing little, if any, discontinuity at magic neutron numbers. An analogous statement can be made for the separation energy of the $n$th neutron as the proton number is increased. Extrapolation by means of the graphs should, therefore, be particularly reliable since it is essentially magic-number independent. Data available in the summer of 1967 have been considered.

$\# 6908058$

*2.2533

Greenwood, R. C.: Reed, J, H.

PROMPT GAMMA RAYS FROM RADIATIVE CAPTURE OF THERMAL, NEUTRONS VOLUMES 1 AND 2

IITRI-1193-53 (Volumes 1 and 2), IIT Research Institute, Technology Center, Chicago, Illinois October 1965

$898 \mathrm{p}$

Available from CFSTI

An atlas of thermal neutron capture gamma ray spectra measured under standard conditions using NaI(T1) scintilation detectors, and a comprehensive compilation of the neutron capture gamma ray data which was available prior to May 1965, are contained in this report. Included are prompt gamma ray spectra, and data from 74 naturally occurring elements, together with a number of separated isotopes, unstable elements and fissionable materials. The objective of this report is to provide sufficient data concerning neutron capture gamma ray spectra to allow evaluation of practical uses for an analytical technique based upon the detection of neutron capture gamma rays in measurement, analysis and control technology and thereby to facilitate the use of the method in these areas. 
Gregoire, R.

SELECTED CONSTANTS NUCLEAR PHYSICS CONSTANTES

SELECTIONNIES PHYSIQUE NUCLEAIRE

Tables of Constants and Numerical Data, 2:1-147

1948

In French

This is a French version of the Table of Isotopes published under the direction of two Nobel Prize winners, F. Jolit and I. Curie, which is now primarily of historical interest.

$\because 6500065$

Groshev, L. V.; et al.

Sykes, J. B. (Trans1ator)

ATLAS OF GAMMA-RAY SPECTRA FROM RADIATIVE CAPTURE OF THERMAL NEUTRONS

New York: Pergamon Press

1959

$198 \mathrm{p}$

Translated from the Russian

The Atlas contains a table of stable isotopes, their thermal neutron capture cross-sections, and the binding energies of the neutron in the resulting nuclei. This table also includes other data needed in the analysis of gamma-spectra and the construction of gamma-transition diagrams. The main part of the book consists of gamma-spectra. Tables of gamma-ray energies and intensities taken from various sources, and gamm-transition diagrams of nuclei formed by neutron capture, are also given. There are two appendices, which contain data on conversion electrons emitted in thermal neutron capture and on gamma-rays from radioactivity which appear in gamma-spectra from the ( $n, g a m m a)$ reaction. All information concerning gama-rays from radiative capture of thermal neutrons published before January 1958 has been collected and systematically presented.

\#6604066

Groshev, L. V.; et al.

$$
\text { * Z. } 083-2.2583
$$

COMPENDIUM OF THERMAL-NEUTRON-CAPTURE GAMMA-RAY MEASUREMENTS PART II, $Z=47$ TO $2=67$ (Ag to Ho)

Nuclear Data Tables, A5:1-242

November 1968

A compilation is presented of thermal-neutroncapture gamma-ray energy and intensity values with decay schemes, examples of original data, and fully corrected spectral distributions. An auxiliary table of data on stable isotopes with abundances, thermal-neutron radiative-capture cross sections, spins, parities, and other information of value to neutron-capture gama-ray spectroscopy is included.

$\# 6604066$

Groshev, L. V.; et al.

$$
* 2.083-2.2583
$$

COMPENDIUM OF THERMAL-NEUTRON-CAPTURE GAMMA-RAY MEASUREMENTS PART III, $2=68$ TO $Z=94$ (ET TO Pu) Nuclear Data Tables, A5:243-431

February 1969

A compilation is presented of thermal-neutroncapture gamma-ray energy and intensity values with decay schemes, examples of original data, and fully corrected spectral distributions. An auxiliary table of data on stable isotopes with abundances, thermal-neutron radiative-capture cross sections, spins, parities, and other information of value to neutron-capture gamma-ray spectroscopy is included.

$\# 6500116$

Guthrie, J.W. (Editor)

TABLE OF ATOMIC MASSES

Sandia Corp. Monograph SCR-245A, Sandia Corporation, Albuquerque, New Mexico

April 1963

$302 \mathrm{p}$

Available from CFSTI
This table is a compilation of the atomic masses, symbols, atomic numbers, nominal weights, square roots of masses, relative abundance and absolute abundance of the isotopes of nuclides. It contains over 2400 entries.

$\# 6908043$

Hager, R. S.; Seltzer, E. C.

INTERNAL CONVERSION TABLES. PART I, K, L, M-SHELL CONVERSION COEFFICIENTS

CALT-63.60, California Institute of Technology, Pasadena, California

June 1967

$160 \mathrm{p}$

Available from CFSTI

Calculations of $K-, L-$, and $M-s h e 11$ conversion coefficients were performed for nuclei with 2 from 30 to 103 taking into account the four lowest electric and magnetic multipoles. The energies of the continuum electron ranged from 1 to $1500 \mathrm{keV}$ for the M-shel1. The coefficients are presented as a function of the continuum electron energy, the continuum electron momentum, and the gamma-ray transition energy.

\#6604066

$$
\text { *2.083-2.2783 }
$$

Hager, R. S.; Seltzer, E. C.

INTERNAL CONVERSION TÁBLES PART I: K-, L-, M-SHELL CONVERSION COEFFICIENTS FOR $Z=30$ TO $Z=103$

Nuclear Data, A4:1-235 February 1968

Values of the internal conversion coefficient, number of electrons per photon emitted in a nuclear transition, are presented from a new relativistic self-consistent-field calculation which takes into account finite nuclear size, hole and exchange effects, experimental electron binding energies, and vacuum polarization. Coefficients are given for each value of 2 ; for $K, L$, and $M$ electron shells and L- and M-sub-shelis for nucleartransition multipolarities E1...E4,M1...M4; and for various nuclear-transition energies up to $1500 \mathrm{keV}$. A program for finding values for other energies by spline interpolation is appended.

\section{$\$ 6604066$}

Hager, R. S.; Seltzer, E. C

$$
\star 2.083-2.2783
$$

INTERNAL CONVERSION TABLES PART II : DIRECTIONAL AND POLARIZATION PARTICLE - PARAMETERS FOR $Z=30 \mathrm{TO}$ $z=103$

Nuclear Data, A4:397-641

October $\frac{\text { No68 }}{1968}$

Values of particle parameters used for the analysis of angular correlations involving internal. conversion electrons are presented. Directional particle parameters are given for the $\mathrm{K}-$, $\mathrm{L}-$, and M-subshells and for the total L- and M-shells. Polarization particle parameters are given for the $\mathrm{K}$-shell. The calculations have been performed for the four lowest electric and magnetic multipoles for all $Z$-values from 30 to 103 and for electron energies varying from 6 to $1500 \mathrm{keV}$ above threshold. Only the particle parameters of lower rank are given. Those of higher rank can be found from the recurrence relations presented, A table of F-coefficients is included.

\section{$\# 6604066$}

Hager, R. S.; Seltzer, E. C

$$
\text { *2.083-2.2783 }
$$

INTERNAL CONVERSION TÁBLES PART III: COEFFICIENTS FOR THE ANALYSIS OF PENETRATION EFFECTS IN INTERNAL CONVERSION AND EO INTERNAL CONVERSION

Nuclear Data Tables, A6:1-127 May 1969

Coefficients for the analysis of penetration effects (nuclear-structure effects) are presented for internal-conversion coefficients and directional particle parameters. The coefficients are given for the four lowest electric and magnetic multipolarities in the $K-$ shell and for the two 
lowest electric and magnetic multipolarities in the $L$ - and $M-s h e l 1 s$. The coefficients are given for every fourth 2 -value from 30 to 102 and for electron energies varying from 6 to $1500 \mathrm{keV}$ above threshold. Some higher-order coefficients are given for El conversion. Coefficients for E0 internal conversion are included for the $K$ - and $L$ shells, and values of the EO-E2 interference particle parameter are included for the K-she11.

\section{$\$ 6500080 \quad \star 2.433$}

Halperin, J.; Stoughton, R. W.

A TABULATION OF INTEGRATED NEUTRON AND FLUX INTENSITIES FOR FISSION SPECTRA FROM 0.01 TO $25 \mathrm{MeV}$ ORNL-TM-795, Oak Ridge National Laboratory, Oak

Ridge, Tennessee

February 7,1964

$70 \mathrm{p}$

The fission neutron spectrum was tabulated as a function of energy for several analytical expressions that were fitted to experimental data for thermal fission in U-235, U-233, pu-239, and spontaneous fission in $\mathrm{Cf}-252$. The integrated neutron density and flux were calculated for energies from 0.01 to $25 \mathrm{MeV}$ in intervals of 0.05 $\mathrm{MeV}$.

\section{6}

Hamilton, J. H.; et al.

EXPERIMENTAL VALUES OF INTERNAL-CONVERSION COEFFICIENTS OF NUCLEAR TRANSITIONS: TOTAL AND KSHELL COEFFICIENTS AND L-SUBSHELL COEFFICIENT RAT IOS

Nuclear Data, A1:521-602 August $1 \frac{196}{966}$

A tabulation is presented of experimentally measured values of internal-conversion coefficients for the K-she11, alphak, L-subshells (most1y ratios only, $\left.L_{1}: L_{2}: L_{3}\right)$, and for all shells, alpha Results reported prior to November 1965, whose uncertainties are $<25 \%$, are 1 isted and compared with theoretical values. Transition energies and spins and parities of initial and final levels, when known, are included. The theoretical values were obtained from computer interpolation, and in some cases extrapolation, of the tables of Sliv and Band. A brief discussion of the methods of measuring conversion coefficients is given.

if 6909032

Hammond, J. p.

PHYSICAL MECHANICAL, AND IRRADIATION PROPERTIES OF THORIUM AND THORIUM ÁLLOYS

ORNL-4218, Oak Ridge National Laboratory, Oak Ridge, Tennessee

April 1968

$71 \mathrm{p}$

Available from CFSTI

Physical and mechanical property data on thorium and thorium alloys were reviewed, and information pertinent to nuclear reactor application is compiled.

$\$ 6909070$

Hardy, E. p. Jr.; Rivera, J.

HEALTH AND SAFETY LABORATORY FALLOUT PROGRAM QUARTERLY SUMMARY REPORT, MARCH 1, 1967 - JUNE 1, 1967

HASL-182, New York Operations Office, Atomic Energy Commission, New York, New York

July 1967

$167 \mathrm{p}$

Available from CFSTI

This report presents current data from the HASL Fallout Program, Isotopes, Inc, Euratom Joint Nuclear Research Center, Argonne National Laboratory, and the National Radiation Laboratory in New 2ealand. Radionuclide levels in stratospheric air, surface air, fallout, milk, other diet components, and tap water, are given in tabular form. The initial section consists of interpretive reports and notes covering the following topics: fallout of SNAP-9A debris; the ratio of $\mathrm{Cs}-137$ to $\mathrm{Sr}-90$ in monthly fallout; and strontium-90 in human vertebrae. A bibliography of recent publications related to radionuclide studies is also presented.

$\# 6500163,6500164$

Heath, R. L.

SCINTILLATION SPECTROMETRY, GAMMA-RAY SPECTRUM CATALOGUE, SECOND EDITION, VÓLUMES 1 AND 2

IDO-16880-1 and IDO-16880-2, phillips petroleum Company, Idaho Operations Office, Idaho Falls, Idaho

August 1964

Available from CFSTI

This second edition is a complete revision of the original data compilation which was issued as an $A E C R$ and $D$ Report (IDO-16408) in 1958. As in the original catalogue, this edition contains a collection of spectra representing the response of a scintillation spectrometer to individual radioactive nuclides. In addition to the graphs representing the response of a 3 " $x$ 3" NaI detector in a standard geometrical arrangement, the data are presented in digital form for the preparation of punched-card, perforated tape, or magnetic tape libraries for data analysis. An important addition to the catalogue is data for neutron-deficient isotopes. The new edition is prepared in two loose-leaf volumes and contains data for almost 300 isotopes. All spectra are normalized to a standard set of gain scales and a text is presented which describes the fundamentals of gamma-ray spectrometry. This includes a discussion of spectrometer design, electronics, instrumental calibration, and data processing. To facilitate the use of these data, tables of detector efficiency, photopeak efficiency, and other information useful for quantitative data analysis have been included. An extensive index has also been added with separate tables of data listed according to gamma-ray energy, half-life, method of source production, and other specialized categories.

\#6911011 * 2.2232

Hemmings, p. J.; Offord, S. M

PHOTON IŃTERACTION IN THE U.K.A.E.A. NUCLEAR DATA LIBRARY

AHSB(S)-R-109, United Kingdom Atomic Energy Authority, Authority Health and Safety Branch, London, England

1966

$43 \mathrm{p}$

Available from AEC Dep. Libraries

Photon-interaction data for 25 elements and water were collected for use in the UKAEA Nuclear Data Library. The methods of photon interaction are discussed with reference to transmission calculations. Tabulations are given of cross sections for each element, and the angular distribution for Compton scattering is given at 23 energies. The energy range considered is 0.01 to $20 \mathrm{MeV}$.

* 6510012

Hiliman, M

$* 2.1133$

EXTENDED TABLE OF NUCLIDIC MASSES

BNL-846(T-333), Brookhaven National Laboratory, Upton, New York

March 1964

$143 p$

Available from CFSTI

About 7000 nuclidic masses for $A=1$ to 255 were calculated based on parabolic systematics. The parabolic parameters, the masses, and energies available for emission of negatrons, positrons, neutrons, protons, and alpha particles are tabulated. The known nuclidic masses are compared with the calculated masses. 
\# 6509009

$$
* 2.2633
$$

Hogan, O. H.; Zigman, P. E.; Mackin, J. L.

BETA SPECTRA II, SPECTRA OF INDIVIDUAL NEGATRON EMITTERS

USNRL-TR-802, Nava1 Radiological Defense Laboratory, San Francisco, California

December 1964

$60 \mathrm{p}$

Available from CFSTI

Beta spectra for 397 beta $^{-}$-emitters were computed at 200 points from the Fermi theory of beta decay using a correction factor for unique-forbidden transitions. These spectra, after normalization and plotting versus energy, are presented, along with experimental end-point energies, forbiddenness assignments, and half lives.

$\# 6910034$

$\star 2.2733$

Hogan, 0. L.

BETA SPECTRA V. SPECTRA OF INDIVIDUAL POSITRON EMITTERS

USNRDL-TR-1101, Naval Radiological Defense Laboratory, San Francisco, California

November 14,1966

$134 \mathrm{p}$

Available from CFSTI, AD646228

Spectra for 219 positron emitters were computed, with energy intervals of .01 MeV (Nb-92 and Ta-177 at shorter intervals). The computation was based on the Fermi theory of beta decay, with a correction factor for unique-forbidden transitions. These energy spectra, after normalization and plotting, are presented, along with the data used in their computation.

\#6511004

$\star 8,143-2.743$

Holley, C. (Editor)

THERMODYNAMIC AND TRANSPORT PROPERTIES OF URANIUM DIOXIDE AND RELATED PHASES, REPORT OF THE PANEL ON THERMODYNAMIC AND TRANSPORT PROPERTIES OF URANIUM DIOXIDE AND RELATED PHASES HELD IN VIENNA, 16.20 MARCH 1964

STI/DOC/10/39, International Atomic Energy Agency,

Vienna, Austria

January 1965

$105 p$

Available from AEA, Vienna, Austria

This report deals with the physical, thermodynamic and transport properties of uranium dioxide and related phases of the uranium-oxygen system. It includes data and a summary of research work done in the field through 1964. The following topics are included: crystal structure, heat capacity, free energy, enthalpy, and entropy measurements, vaporization processes, surface and oxidation properties, thermal conductivity, electrical properties, optical properties, magnetic properties, and practical implications of thermodynamic and transport properties.

$\# 6907028$

*2.2533

Honeck, H. C.

Pear1stein, S. (Revisor)

ENDF/B SPECIFICATIONS FOR AN EVALUATED NUCLEAR DATA FILE FOR REACTOR APPLICATIONS

BNL 50066 (T-467), ENDF 102, Cross Section Evaluation Center, Brookhaven National Laboratory. Upton, New York

May 1966 (Revised Ju1y 1967)

$114 \mathrm{p}$

Available from CFSTI

This report illustrates and describes the several steps required to process measured nuclear data into a form suitable for input to a reactor design code. It also describes a second part of the ENDF system (a magnetic tape store of data), referred to as ENDF/B, and contains the specifications for a magnetic tape (or punched card) storage for evaluated nuclear data to be used for reactor design calculations. The extension of these formats to other than reactor applications has not been included in this report.

\#6903009 *2.2531

Horsley, A.; Stewart, L.

EVALUATED NEUTRON CROSS SECTIONS FOR DEUTERIUM

LA-3271, Los Alamos Scientific Laboratory, Los Alamos, New Mexico

January 1968

$139 \mathrm{p}$

Available from CFSTI

This collection of tables and graphs evaluates experimental data on neutron cross sections for deuterium. Most of the experimental information has been obtained from the literature. Often these data could not be obtained in numerical form and had to be read from graphical displays. Values are recommended for neutron-deuteron cross sections in the energy range $0.0001 \mathrm{eV}$ to $20 \mathrm{MeV}$ to facilitate neutronics calculations.

\#6604066

Horsley, A.

$2.083-2.2583$

NEUTRON CROSS SECTIONS OF DEUTERIUM IN THE ENERGY RANGE $0.0001 \mathrm{eV}$ TO $20 \mathrm{MeV}$

Nuclear Data, A4:321-357

July 1968

Experimental and theoretical data for the neutron cross sections of D are surveyed and values adopted for total and partial cross sections. To facilitate neutronics calculations, angular distribution functions based on $n+d$ and the conjugate reaction $p+d$ are given. The experimental data used, with some comments, and a brief account of the evaluation procedure are presented.

$\# 6604066$

Horsley, A.

NEUTRON' CROSS SECTIONS OF HYDROGEN IN THE ENERGY RANGE $0.0001 \mathrm{eV}=20 \mathrm{MeV}$

Nuclear Data, A2:243-262

September 1966

Theoretical and experimental data for the neutron cross sections of $\mathrm{H}-1$ are surveyed and values recommended for total and partial cross sections in the energy range $0.0001 \mathrm{eV}$ to $20 \mathrm{MeV}$. Available experimental data are supplemented where necessary by estimates based on nuclear theory. Details of energy and angular dependence are given so that the data are complete for the purposes of neutronics calculations.

$\# 6609014$

*2.2433

Horsley, A.

NEUTRON CROSS SECTIONS OF THE PROTON IN THE ENERGY RANGE $0.0001 \mathrm{eV}-20 \mathrm{MeV}$

AWRE $0-23 / 65$, Atomic Weapons Research Establishment, Aldermaston, England

July 1965

$27 \mathrm{p}$

Available from AEC Dep. Libraries

Recommended values are given for neutron total and partial cross sections of $\mathrm{H}-1$ in the energy range $0.0001 \mathrm{eV}$ to $20 \mathrm{MeV}$. The available experimental data are supplemented where necessary by estimates based on nuclear theory. Details of energy and angular distributions are given so that the data are complete for the purposes of neutronics calculations. The recommended data in this report have been written on punched cards ( $3 \mathrm{~A} / \mathrm{H}$ data, data file number DFN 211, for the cross sections of free hydrogen atoms, and $3 \mathrm{~B} / \mathrm{H}$ data, data file number DFN-212, for the cross sections per hydrogen atom of a water molecule) as part of the UKAEA Nuclear Data Library. 
Howerton, R. J.

SEMI-EMPIRICAL NEUTRON CROSS SECTIONS, PART II, $0.5-15 \mathrm{MeV}$

UCRL-5351, Lawrence Radiation Laboratory,

Livermore, California

November igs8

$274 \mathrm{p}$

Available from CFSTI

Several cross section curves are plotted for most of the elements and isotopes considered. For most of the elements and isotopes, the total cross section has been measured in the energy range under consideration. For elements with $A>40$, optical model calculations have been made for the total cross section in the energy range from 7 to $14 \mathrm{MeV}$. The calculations of Fernbauch and Bjorklund were used to supplement the experimental data where there was a shortage of measured values or where an ambiguity existed between several measured values. These data are of value for historical interest.

$\# 6500061,6500062$

$\star 2.2433$

Howerton, R. J.

TABULATED NEUTRON CROSS SECTIONS, PART 1, VOLUMES 1 AND 2, $0.001-14.5 \mathrm{MeV}$

UCRL-5226 (Rev.) (Part 1, Volumes 1 and 2), Lawrence Radiation Laboratory, Livermore, California

October 1959

$431 \mathrm{p}, 413 \mathrm{p}$

Available from CFSTI

Tables of neutron total, elastic, inelastic scattering, and absorption cross sections are presented for the elements hydrogen through titanium, and vanadium through tin at 0.001 to 14.5 $\mathrm{MeV}$. These data are of value for historical interest.

\section{\#6500063}

Howerton, R, J .

TABULATED NEUTRON CROSS SECTIONS, PART I, VOLUME 3 , $0.001-14.5 \mathrm{MeV}$

UCRL-5226 (Rev.) (Part 1, Volume 3), Lawrence Radiation Laboratory, Livermore, Calfiornia

October 1959

241p

Available from CFSTI

Tables of neutron cross sections for various elements at energies of 0.001 to $14.5 \mathrm{MeV}$ are presented. These data are of historical importance.

\section{$甘 6500064$}

Howerton, R. J.

TABULATED DIFFERENTIAL NEUTRON CROSS SECTIONS, PART 3. VOLUME $1,0-15 \mathrm{MeV}, \mathrm{H}-1$ TO $\mathrm{Pu}=94$

UCRL-5573, Lawrence Radiation Laboratory,

Livermore, California

January 1961

$200 \mathrm{p}$

Available from CFSTI

Part 1, Volumes 1,2 , and 3 was issued as report UCRL-5226, revised October 1959. Part 2 was issued as report UCRL -5351 , November 1958. Tab1es are presented of experimental differential neutron cross sections for the elastic scattering of neutrons by nuclei in the energy range of 0 to 15 MeV. Nuclear reactions induced by neutrons are also included, particularly those that are significant for reactor-type calculations. The tables include nuclei from $H$ to $P u$. These data are of historical value.
46903008

Howerton, R. J.; et a1.

ECSIL, A SYSTEM FOR STORAGE, RETRIEVAL, AND DISPLAY OF EXPERIMENTAL NEUTRON DATA

CURL-50400 Vol. 1, Lawrence Radiation Laboratory, Livermore, California

January 1968

$94 \mathrm{p}$

This is a description of the Experimental Cross Section Information Library (ECSIL), a system, written primarily in basic Fortran II, for the storage, retrieval, and tabular or graphical display of experimental constants associated with neutron-induced nuclear reactions.

\#508009

Howerton, R. J.; et a1

$$
\text { *2.2433,2.2333,2.2533,2.2133 }
$$

THRESHOLDS FOR NEUTRON-INDUCED REACTIONS

UCRL-14000 through UCRL-14007, Lawrence Radiation

Laboratory, Livermore, Californía

1964

$342 p$

Available from CFSTI, TID-21627

Thresholds are tabulated for nuclear reactions initiated by neutrons, protons, deuterons, tritons, He-3 nuclei, He-4 nuclei, and gammas (14000-14006). Gamma-production cross sections, both integrated and differential, are tabulated. The data are categorized according to whether the gammas produced are continuum, discrete, or total over-all gamma energies ( 14007$)$.
$\$ 6904007$
$* 2,2233,3,133$

Hubbell, J. H.; Berger, M. J.

PHOTON ATTENUATION

Engineering Compendium on Radiation Shielding, Vol. 1 , Chapter 4 , pages 167-202 Berlin: Springer-Verlag

1968

"Photon Attenuation" is a section of the Engineering Compendium on Radiation Shielding which discusses and tabulates narrow-beam attenuation coefficients and energy-absorption coefficients. The mass energy-absorption coefficient is only one of many quantities that can be used to compute the transfer of energy to the medium. The mass energytransfer coefficient and the mass absorption coefficient are also tabulated. The principal results for attenuation coefficients and for the energy-absorption and related coefficients are listed in tables. The present compilation is based on a new analysis of all available information. Discussions of the atomic cross sections underlying the interaction coefficients are included.

$\# 6500067 \quad * 2.2543$

Hughes, D. J.; Schwartz, R. B.

NEUTRON CROSS SECTIONS

BNL 325 , Second Edition,

Brookhaven Nationa 1

Laboratory, Upton, New York

July 1,1958

$376 \mathrm{p}$

Available from CFSTI

The material in the first edition of BNL-325 and its addendum, the supplement, and new data received up to May 1958 are included. Thermal cross sections, resonance parameters, and cross section curves are given.

\#6500068 *2. 2543

Hughes, D. J.: Magurno, B. A.; Brusse 1, M. K. NEUTRON CROSS SECTIONS SUPPLEMENT NUMBER 1 BNL 325, Second Edition, Supplement No. Brookhaven Nationa1 Laboratory, Upton, New York January 1,1960

$132 \mathrm{p}$

Available from CFSTI

A compilation of data on neutron cross sections and resonance parameters, published between July 1958 and November 1, 1959, is presented. 
$\# 6500052$

*2.2133

Hunt, D.0.; Brynjolfsson, A.; Cooper, R.D.

HANDBOOK OF PHOTONUCLEAR REACTIONS

Technical Report No. FD-1, U, S. Army Natick

Laboratory, Natick, Massachusetts

December 1963

$231 p$

Available from DDC, AD428 207

A compilation was made of the data that would be most useful in calculating the activities produced in food by high-energy electrons or gamma rays. The known isotopes of each element are listed with their atomic masses, half-life, and methods of decay. Photoneutron and photoproton cross sections are shown and both theoretical and experimental threshold values are listed, References are included for all data. The appendixes include a short description of the Bremsstrahlung cross sections and a list of the common radioactive isotopes found in food irradiated by high-energy electrons. One hundred and five references are provided.

$\# 6500084$

$\star 2.2633$

Hut chins on, J. M. R.

CAL1BRATION OF FIVE GAMMA-EMITTING NUCLIDES FOR EMISSION RATE

NBS Technical Note 71 , National Bureau of Standards, Washington, D. C.

August 1960

$23 p$

Available from CFSTI, PB 161572

$\mathrm{Hg}-203$ and $\mathrm{Nb}-95$ were calibrated by a 4 pi betagamma coincidence method for gamma-emission rate, $2 \mathrm{n}-65$ by comparison with the $1.12 \mathrm{MeV}$ peak of $\mathrm{Sc}-46$, $\mathrm{Na}-22$ by a gamma-annihilation-quanta coincidence method and by a triple coincidence method, and Sr-85 by $x$-gamma coincidence counting. The accuracy of the calibration in all cases was plus or minus $2 \%$. The half life of the isomeric state of $\mathrm{Rb}-85$ was measured and found to be 0.98 microseconds.

$\# 6910042$

Iwaki, A.

ANT INUCLEON-NUCLEON INELASTIC PROCESSES

Progress of Theoretical Physics (Kyoto) Supplement, $41-42: 316-373$

1967

Experimental data on antinucleon-nucleon inelastic interactions published from 1963 to 1966 are summarized. Cross sections are first given of typical antiproton,proton processes at various energies. Experimental results are then summarized for the four types of reactions: annihilation into pions; annihilation yielding $K$ mesons; pion production without annihilation; and hyperon production. Annihilation data include cross sections for various annihilation channels, production angular distributions and mean multiplicity for produced particles, the rates of resonance productions, and distribution of the production and decay angles for resonance particles. Pion production without annihilation and hyperon production data include cross sections, angular distribution of final antibaryons (baryons), and resonance production. Thirty-seven references are arranged according to the reaction types, and include the primary momentum, observed quantities, and method used. Seventeen other references are given.

\# 6500059

Jarmie, N.; Seagrave, J. D. (Editors)

CHARGED PARTICLE CROŚS SECTIONS

LA-2014, Los Alamos Scientific Laboratory, Los Alamos, New Mexico

February 1, 1957

2340

Available from CFSTI
The compilation attempts to include the "best" cross sections of all nuclear processes involving charged projectiles with energies up to the region of about $30 \mathrm{MeV}$. No reactions with beams of neutrons, photons, or subnuclear particles are considered. Only results that are absolute or that can be normalized easily to existing absolute data are included. The first edition is limited to light-element targets up to and including fluorine, and the incident particles with a mass of one through four. It should be noted that the compilation is a graphical representation of experimental data. Tables of results that duplicate information on the curves are not given, nor has any consistent attempt been made to include theoretical information.

\#6508026

Je ronymo, J. M. F. : et al.

ABSOLUTE CROSS-SECTIONS FOR SOME (n,p), (n,alpha)

AND $(\mathrm{n}, 2 \mathrm{n})$ REACT IONS

Nuclear Physics, 47:157-176

August $1 \overline{963}$

The reactions $\mathrm{A} 1-27$ (n,alpha) Na-24, $\mathrm{Mg}-24(\mathrm{n}, \mathrm{p}) \mathrm{Na}-24$, $\mathrm{Si}-28(\mathrm{n}, \mathrm{p}) \mathrm{Al}-28$, Co- 59 (n,p) Fe-59, Co-59(n,2n) Co-58, $\mathrm{Co}-59(\mathrm{n}, \mathrm{alph}$ a) $\mathrm{Mn}-56, \mathrm{Ni}-58(\mathrm{n}, \mathrm{p}) \mathrm{Co}-58$ and $\mathrm{Ni}-$ $58(n, 2 n) \mathrm{Ni}-57$ were studied by an activation method from 12.5 to $21.0 \mathrm{MeV}$ neutron energy. A method of calibrating well-type crystals to obtain absolute efficiency is described. The results for absolute cross sections obtained by this method for the above reactions are compared with statistical model calculations in which the nuclear transmission coefficients were derived from an average optical potential extracted from the scattering data.

$\# 6604066$

$* 2.083-2.2583$

Jessen, P.; et al.

EXPERIMENTAL EXCITATION FUNCTIONS FOR $(n, p),(n, t)$, $(n, a 1 p h a), \quad(n, 2 n), \quad(n, n p), \quad A N D \quad(n, n$ alpha) REACTIONS

Nuclear Data, Al:103-202

February 1966

Fast neutron cross sections for $(n, p)$, $(n, t)$, $(n, a 1 p h a),(n, 2 n), \quad(n, n p)$, and (n, n alpha) reactions, leading to all possible final states, are presented in graphic form. Neutron energies range up to $20 \mathrm{MeV}$. Targets include nuclei from H-2 to U-238. The compilation covers data available in June 1965 .

$\# 6509027$

Johnson, W. H., Jr. (Editor)

NUCLIDIC MASSES PROCEEDINGS OF THE SECOND INTERNAT IONAL CONFERENCE ON NUCLIDIC MASSES, VIENNA, AUSTRIA, JULY 15-19, 1963

New York: Springer-Verlag

1964

$480 \mathrm{p}$

Separate abstracts were prepared for 30 of the 37 papers included. Six other papers were previously abstracted for NSA 17:31406, 32998, 33950, 36639; $18: 1057$; and 19:14607. The remaining paper discusses the interest of the International Union of Pure and Applied Chemistry in the values of the fundamental constants.

$\# 6500070 \quad$ *2.2443

Kalos, M. H. : Ray, J. H.

NEUTRON CROSS-SECTIONS OF NATURAL CHLORINE

UNC-5067 Topical Report, United Nuclear Corporation, White Plains, New York

September 30,1963

$26 \mathrm{p}$

Available from AEC Div, of Tech. Information, AD41981

Complete sets of cross sections for natural chlorine are tabulated for incident neutron energies from $0.02 \mathrm{eV}$ to $18 \mathrm{MeV}$. Cross sections for gamma and neutron production in neutron inelastic 
scattering are included, and Legendre polynomial coefficients to describe the angular distribution of elastically scattered neutrons are given.

\# 6910040

* 1.2043

Kanada, H.; Matsuoka, T.; Suzuki, T.

$K=N$ INELASTIC PROCESSES

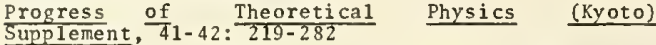
$\frac{5}{1967}$

A review is given of experimental results on the inelastic $K-N$ scattering at various primary momenta above $1.4 \mathrm{GeV} / \mathrm{c}$. Reactions of the following two types are considered: quasi-two-body reactions in which there are more than two particles in the final state, and many-body reactions in which there are more than two particles in the intermediate and/or final state. The cross section for each inelastic channel in the $K-N$ scattering and its momentum dependence are presented. Angular distributions of particles or resonances produced in the $\mathrm{K}-\mathrm{N}$ inelastic processes and decay angular distributions of the produced resonances are shown. Polarizations of the XI particle in the $K^{-} p \rightarrow X I K$ reaction are given. The dominant characteristics of all angular distribution in the inelastic reactions and the characteristics of all resonances produced in the $\mathrm{K}-\mathrm{N}$ scattering are summarized. The 57 references are tabulated in the appendix together with the incident momentum, observed quantities, and experimental methods.

$\# 6604066$

Kantele, J.; Tannila, 0.

*2.083-2.2783

ISOMERIC STÁTES $\left(\mathrm{T}, 2 \geq 10^{-10} \mathrm{~S}\right)$ LISTED ACCORDING TO

INCREASING HALF-L I FE

Nuclear Data, A4:359-395

Ju1y 1968

Values of some 700 half-lives of isomeric states, ranging from $10^{-10}$ seconds to $10^{5}$ years, are presented in order of increasing half-life.

$\# 6609031$

$* 2.2143$

Kerr, W. M. M.; et al.

A 12 GROUP SET OF PHOTON CROSS SECTIONS FOR USE IN AN ISOTROPIC $S_{n}$ CALCULATIONS OF PHOTON TRANSPORT

AWRE $0-79 / 65$, Atomic Weapons Research Establishment, A1dermaston, England

October 1965

$37 \mathrm{p}$

Available from AEC Dep. Libraries

The 12 group set of photon cross sections calculated for use in STRAINT and other programs in which an isotropic scattering can be taken into account is described. All the numbers required are given in the form of SC4020 prints so that the cross sections can be used in other programs.

\#6910008

*2. 1343

Kienle, P.; Kalvius, G. M.; Ruby, S, L.

TABLE ÓF CHANGES IN NUCLEAR RADIÚS

Appendix A from Hyperfine Structure and Nuclear Radiation, International Conference on Hyperfine Interactions Detected by Nuclear Radiations, Pacific Grove, California, August 25-30, 1967, edited by E. Mat thias and D. A. Shirely, pp 971-977 New York: John Wiley \& Sons, Inc.

1968

$8 \mathrm{p}$

This table is an extension of the Table of Nuclear Spins and Moments published in 1965, and contains the nuclear magnetic and quadrupole moments known as of August 1967. Major changes made in the present revision are the omission of spin values (except for identification purposes), the addition of 1 ifetime- or field-dependent quantities or both. the omission of magnetic octupole moments, and the addition of a tabulation of nuclear moment ratios. The table of nuclear moment ratios (Part 3) lists magnetic moment, quadrupole moment, and g-factor ratios determined for two isotopes of the same element and for two levels of the same isotope. The experimental methods used in measuring the nuclear moments are identified by brief symbols which are defined in Part 1 of this table. part 2 is the table of magnetic and quadrupole moments; Part 3, the table of nuclear moment ratios, and Part 4 contains the list of magnetic shielding constants used for each element. References for all parts of the table are given at the end.

$\# 6604066$

*2.083-2.2483

Kim, H. J.; Milner, W. T.; McGowan, F. K.

NUCLEAR CROSS SECTIONS FOR CHARGED-PARTI CLE-INDUCED REACT IONS C

Nuclear Dat $\frac{C}{a}, A 2: 1-241$

Augus t 1966

Nuclear cross sections for charged-particle-induced reactions of isotopes of $C$ are presented in tabular and graphical form. This compilation includes experimental cross-section data available in the literature before January 1,1964 , for nuclear reactions of the type $A(a, b) B$ at all energies, where $\mathrm{M} \geq$ one nucleon mass. Nuclear reactions involving mesons in the exit channel are not included. A large amount of the cross-section data is not presented either in tabular or graphical form in order to limit the size of the present compilation to a manageable one. A number of annotated hihliographies are included in the appendixes to provide a brief summary of these data.

\#6604066

*2.083-2.2483

Kim, H. J.; Milner, W. T.; McGowan, F. K.

NUCLEAR CROSS SECTIONS FOR CHARGED-PARTICLE-INDUCED

REACTIONS Li, Be, B

$\frac{\text { Nuclear Data, A1:203-389 }}{\text { April } 1966}$

Nuclear cross sections for charged-particle-induced reactions of isotopes of $\mathrm{Li}, \mathrm{Be}$, and $\mathrm{B}$ are presented in tabular and graphical form. This compilation includes experimental cross-section data available in the literature before January 1 , 1964, for nuclear reactions of the type $A(a, b) B$ at all energies, where $M>$ one nucleon mass, Nuclear reactions involving mesons in the exit channel are not included. A large amourt of the cross-section data is not presented either in tabular or graphical form in order to limit the size of the present compilation to a manageable one. A number of annotated bibliographies are included in the appendixes to provide a brief summary of these data.

\#6604066

*2.083-2.2483

Kim, H. J. ; Milner, W. T.; McGowan, F. K.

NUCLEAR CROSS SECTIONS FOR CHARGED-PARTICLE-INDUCEN REACTIONS $N$ AND 0

Nuclear Data, A3:123-285

September 1967

Nuclear cross sections for charged-particle-induced reactions of isotopes of $\mathrm{N}$ and 0 are presented in tabular and graphical form. This compilation includes experimental cross-section data available in the literature before January 1 , 1965, for nuclear reactions of the type $A(a, b) B$ at al1 energies, where $\mathrm{M}_{\mathrm{a}} \geqq$ one nucleon mass. Nuclear reactions involving mesons in the exit channel are not included. A large amount of the cross-section data is not presented either in tahular or graphical form in order to limit the size of the present compilation to a manageable one. A numher of annotated bibliographies are included in the appendixes to provide a brief summary of these data. 
\#6511011

$* 2.043$

Koenig, L. A. ; Mattauch, J. H. E.; Wapstra, A. H. 1 1S60 NÚCLEAR DATA TABLES PART 2, CONSISTENT SET OF ENERGIES LIBERATED IN NUCLEAR REACTIONS I. TARGETS IN THE MASS REGION $67 \leq \mathrm{A} \leq 199$

Nuclear Data Project, National Academy of SciencesNational Research Council, Washington, D. C. February 1961

$468 \mathrm{p}$

Available from the Superintendent of Documents, GPO

These tables are the second installment of a list of adjusted 0 -values of which the first installment for targets in the mass range $1 \leqq A \leqq 66$ has been published recently. The least squares adjusted mass values used for the computation of the adjusted $0-v a l u e s$ of the present table are those of a recent publication. As seen from table 2 of this paper the computation for the mass range $71 \leq \mathrm{A} \leq 199$ has been divided into eight separate adjustments.

\#6912028

*2. 2543

Koppe1, J. U. ; Hous ton, D. H.

REFERENCE MANUAL FOR ENDF THERMAL NEUTRON GCATTERING DATA General Atomic, Inc., San Diego, California

December 16, 1968

$112 p$

A study has been made of the neutron cross sections and associated data for U-233. A set of data has beer prepared in the ENDF/B format and includes: resolved and unresolved resonance parameters; pointwise cross sections for total and partial neutron cross sections in the energy region $100 \mathrm{keV}$ to $15 \mathrm{MeV}$; angular distributions for elastically scattered neutrons: energy distributions for secondary neutrons from $\left(n, n^{i}\right),(n, 2 n),(n, 3 n)$ and fission reactions; nu, the number of neutrons produced per fission reaction; fission product yields for thermal neutron fission. A survey was made of the available experimental results for the U-233 neutron cross sections up to June 1968 .

\section{$\# 6500043$}

$* 2.1143-2.1643$

Kravtsov, V. A.

RELATIVE NUCLEIDIC MASSES AND BINDING ENERGIES FOR $84 \leqq \mathrm{~A} \leq 104$ AND $111 \leqq \mathrm{~A} \leqq 125$

Nuclear physics, $41: 330-3 \overline{4} 2$

1963

The tables contain the nucleidic masses for $84 \leq \mathrm{A} \leq 104$ and $111 \leq \mathrm{A} \leq 125$ as well as nuclear total binding energies, nucleon separation energies and pairing energies. The tables are intended to provide, on the strength of new experimental material, more accurate data for the tables of Everling, et al.

$\# 6604066$

$* 2.083-2.1283$

Kravtsov, V. A. ; Skachkov, N. N.

INFLUENCE OF NUCLEAR STRUCTURE ON BINDING ENERGY Nuclear Data, AI:491-519

Augus $t 1966$

Graphs are presented of the intersections of nuclear binding energy surfaces by planes $\cdot \mathrm{I}=\mathrm{N}-\mathrm{Z}=$ constant. Ridges and valleys on the energy surface are shown; the valleys are related to shells while the ridges act mostly as boundaries of regions of deformed nuclei. The sharpness, $D(Z, N)$, of discontinuities, valleys and ridges, is considered and proposed as a new energy characteristic of nuclear shells. Graphs are presented showing the dependence of sharpness on the number of nucleons. In order of decreasing sharpness the shells identified are: $8 \mathrm{p}, 8 \mathrm{n}, 126 \mathrm{n}, 82 \mathrm{p}, 50 \mathrm{n}, 82 \mathrm{n}, 50 \mathrm{p}$, $28 \mathrm{p}, 28 \mathrm{n}, 14 \mathrm{p}, 14 \mathrm{n}, 40 \mathrm{p}, 20 \mathrm{n}, 70 \mathrm{p}, 20 \mathrm{p}, 15 \mathrm{n}$, and $66 \mathrm{p}$. Weak ridges are associated with $4 \mathrm{p}, 10 \mathrm{p}$ and with $4 n, 10 n, 22 n, 88 n$, and $114 n$. The dependence of the separation energy of neutron pairs, $S-2 n$, on the number of protons and of the separation energy of proton pairs, $S-2 p$, on the number of neutrons is presented on other graphs which are analyzed from the viewpoint of nuclear structure. AlI the graphs were prepared on the basis of the 1964 nuclidic masses.

$\# 6804003$

Kunz, W. ; Schintlmeister, J.

NUCLEAR TABLES, PART II, NUCLEAR REACTIONS, VOLUME 1. THE ELFMENTS FROM NEUTRON TO MAGNESIUM

Oxford, New York: Pergamom Press

1968

$699 \mathrm{p}$

These tables contain data on elements and isotopes from $Z=0$ to 12 . Data on elements includes neutron and proton cross sections, parameters for neutron resonances, and nuclear reactions where the resulting mass is unknown. For the individual isotopes, data includes the abundance or half-life and decay mode; its energy levels; all nuclear reactions resulting in that isotope; reaction cross-sections and energies.

$\# 6806003$ *2. 2443

Kunz, W.; Schintlmeister, $J$.

NUCLEAR TABLES, PART II. NUCLEAR REACTIONS, VOLUME 2. THE FLEMENTS FROM ALUMINUM TO SULPHER

Oxford, New York: Pergamon Press

1967

271p

A compilation of the available information on nuclear reactions of $\mathrm{Al}, \mathrm{Si}, \mathrm{P}$, and $\mathrm{S}$ is presented.

\#6500527

*0.1151-2.2051-3.151

LANDOLT-BORNSTEIN, NUMERICAL DATA AND FUNCTIONAL RELATIONSHIPS IN PHYSICS, CHEMISTRY, ASTRONOMY, GEOPHYSICS AND TECHNOLOGY, SIXTH EDITION,

VOLUME I: ATOMIC AND MOLECULAR PHYSICS,

PART 3: MOLECULES II (ELECTRON SHELL) WITH AN

APPENDIX TO VOLUME I, PARTS 1,2 , AND 3

Eucken, A.; Hellwege, $K_{0}-H_{\text {. }}$ (Éditors)

Berlin, New York: Springer-Verlag

1951

$724 \mathrm{p}$

In German

This section of the Landolt-Bornstein tables contains data on the electron shells of molecules. The volume includes data published through September 1951. The following properties have been tabulated: band spectra of diatomic molecules, electron band spectra of multiatomic molecules, light absorption of solutions in the infrared and visible region, ionization energies of molecules, optical rotary power of molecules, electrical and optical polarizability of molecules, magnetic moments of molecules, diamagnetic polarizability of molecules, and quanta output of photochemical reactions.

$\# 650052$

$*_{0.1151-2.2051-3.151}$

LANDOLT-BORNSTEIN, NUMERICAL DATA AND FUNCTIONAL RELATIONSIIIPS IN PHYSICS, CHEMISTRY, ASTRONOMY, GEOPHYSICS, AND TECHNOLOGY, SIXTH EDITION,

VOLUME I: ATOMIC AND MOLECULAR PHYSICS,

PART 4: CRYSTALS

Hellwege, K. H. (Edi tor)

Berlin, New York: Springer-Verlag

1955

$1007 \mathrm{p}$

In German

This volume contains data on the atomic physical interpretation of crystal physics as of November 1954. The following properties have been tabulated: the characteristics of symmetry, elements, the 32 crystal classes, space groups, lattice types, structures, crystal dimensions of inorganic and organic crystals, atomic and ionic radii, lattice energies, internal vibrations of crystals, electron emission, energy bonds, X-ray spectra and bonding states, electron spectra of crystals, and high frequency spectra of crystals. 
LANDOLT-BORNSTEIN, NUMERICAL DATA AND FUNCTIONAL RELATIONSHIPS IN PHYSICS, CHEMISTRY, ASTRONOMY GEOPHYSICS AND TECHNOLOGY, SIXTH EDITION,

VOLUME I: ATOMIC AND MOLECULAR PHYSICS,

PART 5: ATOMIC NUCLEI AND ELEMENTARY PARTICLES

Hellwege, K. $-\mathrm{H}$. (Editor)

Berlin, New York: Springer-Verlag

1952

$470 \mathrm{p}$

In German

This section of the Landolt-Bornstein tables contains tables on atomic nuclei and elementary particles. These tables were compiled by the authors from literature on these subjects published through November 1951. The properties tabulated are: the hyperfine structure of atomic terms and atomic lines, a table of all atomic nuclei and their properties, mass spectrographic measurements, nuclear reaction energies, electron line spectra of artificially radioactive atoms, the radioactive families, nuclear level schemes of radioactive transfornations, energy schemes of the light atomic nuclei, beta-spectra, common naturally radioactive atoms and their alpha- and beta-spectra, scattering of charged particles by atomic nuclei, nuclear transition products, passage of alpha-particles, electrons, protons and gamma-rays through matter and cosmic radiation.

\#6500537

*0.1151-2.2051-3.151

LANDOLT - BORNSTEIN, NUMERICAL DATA AND FUNCTIONAL RELAT IONSHIPS IN PHYSICS, CHEMISTRY, ASTRONOMY, GEOPHYSICS AND TECHNOLOGY, SIXTH EDITION VOLUME III: ASTRONOMY AND'GEOPHYS ICS

Bartels, J.; Bruggencate, P. T. (Editors)

Berlin, New York: Springer-Verlag 1952

$795 \mathrm{p}$

In German

This volume of the Landolt-Bornstein series contains data on astronomy and geophysics. The material has been taken from literature published through 1951. The astronomical section includes data on astronomical instruments, position and time determination, abundance of the elements in the universe and the sun, mechanics and physics of the planets, moons comets and meteors stellar radiation, stellar spectra, stellar positions and movements, double stars, varíable stars, and novae, the galaxy, star clusters, and extra-galactic nebulae. The section on geophysics contains data on the earth's gravity and tidal forces, radioactivity density, and elastic, electrical, magnetic and thermal properties of minerals and rocks, seismicity and earthquake waves, properties of the earth's interior, surface and magnetism, physical and chemical properties of sea water, sea tides, hydrography, meteorology and climatology and upper atmosphere physics.

$\# 6500524$

*0.1151-2.2651-3.151

LANDOLT-BORNSTEIN, NUMERICAL DATA AND FUNCTIONAL RELAT IONSHIPS IN SCIENCE AND TECHNOLOGY, NEW SERIES,

GROUP I: NUCLEAR PHYSICS AND TECHNOLOGY,

VOLUME I: ENERGY LEVELS OF NUCLEI: $\mathrm{A}=5$ TO $\mathrm{A}=257$

Hellwege, A.M.; Hellwege, K.-H. (Editors)

Berlin, New York: Springer-Verlag

1961

$813 p$

In German and English

This first volume of the New Series of LandoltBornstein contains the known energy levels and transitions for all nuclei from $A=5$ to $A=257$. The essential parts of the material are the level and decay schemes. These are supplemented by tables and special data. The tables list the following parameters for each level: excitation energy above ground state; the total angular momentum quantum number and the parity; the is obaric spin quantum number; the half life or mean life of the state, its half-width; the known modes of decay of the states; binding energies for various nucleon combinations, and the $Q-v a l u e s$ for the relevant nuclear reactions. Much of the same information is incorporated in diagrams in which the energy levels of the various nuclei are exhibited schematically, together with the reactions by which they are formed. Recent references to papers dealing in a more general way with the properties of the nucleus in question are included. This compilation includes the data available through May 1960 with a few references to work done since that time.

\# 6802002

*0.1151-2.1351-3.151

LANDOLT-BORNSTEIN, NUMERICAL DATA AND FUNCT IONAL RELATIONSHIPS IN SCIENCE AND TECHNOLOGY, NEW SERIES, GROUP I: NUCLEAR PHYSICS AND TECHNOLOGY, VOLUME 2: NUCLEAR RADI I

Schopper, H. (Editor)

Berlin, New York: Springer-Verlag

1967

$54 \mathrm{p}$

In German and English

The volume contains nuclear radii and other characteristic parameters of atomic nuclei which are deduced from electron scattering experiments and the observation of mesic X-rays. The first section contains data on nuclear charge distributions derived from sources other than scattering, primarily mu-mesic atoms for spherical nuclei. Data is given on energy levels and radius parameters for mu-mesic X-ray transitions; also for neighboring nuclides, deformed nuclei, and radii of isomers. The second section deals with nuclear radii determined by electron scattering. Both electric charge and magnetic radii are given. Because a variety of nuclear models are available, each nucleus is discussed separately, in addition to listing the size parameters.

\# 6902044 * $0.1151-2.2051-3.151$

LANDOLT-BORNSTEIN, NUMERICAL DATA AND FUNCTIONAL RELAT IONSHIPS IN SCIENCE AND TECHNOLOGY, NEW SERIES,

GROUP I: NUCLEAR PHYSICS AND TECHNOLOGY,

VOLUME 3: NUMERICAL TABLES FOR ANGULAR CORRELATION COMPUTATIONS IN alpha-, beta-, AND gammaSPECTROSCOPY: $3 j-, 6 j-, 9 j-S Y M B O L S, F-$ AND GAMMACOEFFICIENTS

Appe 1, H.

Schopper, H. (Editor)

Berlin, New York: Springer-Verlag

1968

$1206 \mathrm{p}$

In German and English

The various types of angular correlation coefficients have been tabulated in the literature. These tables provide the coefficients for a complete calculation of the basic angular correlations in alpha-, beta-, and gamma-spectroscopy. In chapter 2, the angular correlation formalism is given in a - necessarily incomplete- collection of relations involving the tabulated coefficients, i.e., 3j-symbols, 6j-symbols, 9j-symbols, F-coefficients, and GAMMA-coefficients. In the computer tables these symbols are given in upright capital letters: $J_{1}=j J_{1}=j$ etc. and GAMMA $=\gamma$. These quantities are glven solely by angular momentum conservation for the total system in consideration. When answering the question of how the angular momenta of the various subsystems of particles may combine to fulfill the conservation condition, one ends up with Clebsch-Gordan-coefficients for two angular momenta and with Racah-coefficients for more than two. To achieve a higher degree of symmetry, Wigner has rewritten both types of coefficients as the $3 j-$ and $6 j-s y m b o l s$, respectively. In more compilicated correlations (e.g. triple correlations, where the intermediate transition also is observed) a new combination of $6 \mathrm{j}$-symbols appears which is usually called the $9 \mathrm{j}$-symbol. For alpha-, beta-, and gamma-spectroscopy a combination of one $3 j-$ and one $6 j$-symbol with statistical factors is frequently used and is 
called the F-coefficient. The GAMMA-coefficient is a slightly modified form of this F-coefficient useful for beta-spectroscopy. The coefficients mentioned above are extensively tabulated in this volume. For the $9 \mathrm{j}$-symbol, however, only selected ones are listed. In addition, the re is a chapter dealing with angle functions. Since angular correlations are generally developed in a series of Legendre polynomials, these functions should be very useful.

$\# 6912054$

$* 0.1151-2.2651$

LANDOLT - BORNSTEIN, NUMERICAL VALUES AND FUNCT IONAL RELATIONSHIPS IN SCIENCE AND TECHNOLOGY, NEW SERIES,

GROUP I : NUCLEAR PHYSICS AND TECHNOLOGY

VOLUME 4: NUMERICAL TABLES FOR BETÁ-DECAY AND ELECTRON CAPTURE

Behrens, H.; Jaenecke, J. (Authors)

Schopper, H. (Edi tor)

Berlin, New York: Springer-Verlag

1969

$322 p$

In German and English

These beta-decay tables present certain combinations of electron radial wave function and phase shifts for both screened and unscreened betadecay functions. The tables for electron capture give the amplitudes and values of the electron radial wave functions at the nuclear radius for the $K, L$ and $M$ shell for all values of $Z$ (the $L$ and $M$ shells are excluded for very light nuclei). A brief compilation of all the relevant formulae used in the treatment of beta-decay and electron capture is given. An explanation of the details of the computations and a description of the tables is included; in addition, 56 references are given.

$\# 6500682$

Lane, A. M.; Thomas, R. G.

R-MATRIX THEORY OF NUCLEAR REACTIONS

Review of Modern Physics, 30:257-353

Apri1 1958

This is a very detailed exposition of the theory. Among the material presented are: (1) a thorough discussion of the elastic scattering by a potential and of the mathematical properties of Wigner Rfunctions (causality condition); (2) three different derivations of the R-matrix which are collected together. The treatment of the collision matrix is related (although in a very "indirect" way) to the Kapurs-Peierls expansion, and to recent work in this direction. The last chapters give a full account of all the applications of the Rmatrix theory made up to now. A great care is taken to see how the R-matrix theory can be made to describe the direct interaction mechanism.

\section{$\# 6902035 \quad$ *2.2553}

Langner, I.; Schmidt, J. J.; Woll, D.

TABLES ÓF EVALUATED NEUTRON CROSS'SECTIONS FOR FAST REACTOR MATERIALS

KFK750, EUR3715e, EANDC (E) - 88"U", Kernforschungszentrum, Institut fuer Neutronenphysik und Reaktortechnik, Karlsruhe, West Germany January 1968

$063 \mathrm{p}$

This set of tables contains evaluated data as functions of the neutron incident energy $E$ for the following types of neutron cross-sections and nuclear parameters: neutron energy dependent statistical resonance parameters for the fissionable nuclei U-235 and $\mathrm{Pu}-239$ (50 eV $\leqq \mathrm{E} \leftrightharpoons$ $250 \mathrm{keV}$ ), neutron cross-sections and related neutron nuclear data $(0.001 \mathrm{eV}$ less than or equal $\mathrm{E}$ less than or equal $10 \mathrm{MeV}$ ), neutron inelastic excitation cross-sections (lowest threshold $\subseteq \mathrm{E} \subseteq$ a few $\mathrm{MeV})$, and elastic scattering angular distributions (most1y $10 \mathrm{keV} \leq \mathrm{E} \leq 15 \mathrm{MeV}$ ). The materials covered are those of particular interest in fast and intermediate reactor design such as
$\mathrm{H}$ (bound in $\mathrm{H}_{2}$ ), $\mathrm{H}$ (bound in $\left.\mathrm{H}_{2} \mathrm{O}\right), \mathrm{He}-3\left(\sigma_{0}\right)$, He, $\mathrm{C}$, $\mathrm{O}, \mathrm{Na}, \mathrm{Cr}, \mathrm{Fe}, \mathrm{Ni}, \mathrm{Mo}, \mathrm{U}-235, \mathrm{U}-238, \mathrm{Pu}-239$. The data printed in this volume are part of the Karlsruhe microscopic neutron nuclear data file (KFDAK) held in the IBM 7074 computer.

\#6906027 * 2.1453

Lauritsen, T.; Ajzenberg-Selove, F. ENERGY LEVELS OF LIGHT NUCLEI (V'II). $A=5-10$ Nuclear Physics, 78:1-176

March 1966

This paper is a summary of existing experimental information on the structure and properties of the nuclei with mass numbers five through ten. Each nucleus is represented by a diagram and a table exhibiting the known properties of the energy levels and the nuclear reactions from which the information derives. The accompanying text contains an abbreviated discussion and a selected bibliography for each relevant reaction. Fach reaction is listed under both the compound and the residual nuclei. At the beginning of the text material on each nucleus, a general bibliography is listed. Skeletonized level diagrams of isobars for each mass number are included as well as reaction energy and separation energies.

$\# 6510033$

Lawson, J. D.

*2. 2253

DIFFERENTIAL BREMSSTRAHLUNG CROSS SECTIONS FOR LOWENERGY ELECTRONS

ORNL-TM-790, Oak Ridge National Laboratory, Oak Ridge, Tennessee

Feb ruary 1964

$486 \mathrm{p}$

Available from CFSTI

Bremsstrahlung cross sections were calculated for $s$ creened and nonscreened targets for electrons with energies from 0.05 to $5 \mathrm{MeV}$. The cross sections were obtained by numerical integration of the Bethe-Heitler equation for Bremsstrahlung crosssections which are differential in photon energy and in proton and electron emission angles. Values of the Bremsstrahlung cross-sections which are differential in photon energy and doubly differential in photon energy and photon emission angle are tabulated.

\#6910026 *2.1373-2.2673

Lederer, C. M.; Hollander, J. M.; Perlman, I.

TABLE OF I SOTOPES, SIXTH EDITION

New York: John Wiley \& Sons, Inc.

1968

$608 \mathrm{p}$

A table is given which displays data for all radioactive and $s$ table nuclei arranged according to atomic number with increasing mass number for each element. The criterion for the selection of data on each radioactive isotope was that of identifying it in terms of its rate and mode of decay, principal radiations, and how it is prepared. Detailed information on nuclear energy levels and transitions between these levels is given. The general policy adopted for these entries was that they be based on direct experimental information. Spin and parity assignments based wholly, or in large part, on the expectations from nuclear models were avoided. Unobserved transitions that should be present were omitted, with few exceptions. A complete bibliography of the sources of information is included.

$\# 6511009$

$* 2.2453$

Leimdoerfer, M.: Bock, E.; Arkeryd, L.

CROSS SECTIONS FOR NEUTRON INELASTIC SCATTERING AND $(n, 2 n)$ PROCESSES, A REVIEW OF AVAILABLE EXPERIMENTAL, AND THEORETICAL DATA

$\mathrm{AE}-88$, Aktiebolaget Atomenergi, Stockholm, Sweden October 1962

$188 \mathrm{p}$ 
A survey of the present state of knowledge on $\left(n, n^{\prime}\right)$ and $(n, 2 \pi)$ reactions has heen performed. The result is presented in the form of a main index to references on all elements and several special indices to different theoretical ways of approach, such as the direct-interaction concept, the continuum model (including level density theories), the discrete-level statistical model (HauserFeshbach), with a subsection on the optical model. There are 486 references accompanied with abstracts giving essential information pertaining to the field.

\#6500074,6500073,6500072

$\star 2.2453$

Li ikala, R. C

UPDATED RBU BASIC LIBRARY

VOLUME I: RECORES: $1-309$, ISOTOPES: H- 1 TO NO-96: VOLUME 2: RECORDS: $310-664$, ISOTOPES: Mo-97 TO Au200

VOLUME 3: RECORDS: 665-904, ISOTOPES: Hg-196 TO

$\mathrm{Pu}-240$

HW-75716 (Volumes 1, 2, and 3), General Electric Company, Hanford Atomic Products Operation, Richland, Washington

May 20 , 1963

$239 p$

Available from CFSTI

The revised Hanford library of cross sections, resonance parameters, inelastic spectra, and nuclear descendents (product nuclei) for neutron reactions and scattering with isotopes $\mathrm{H}-1$ through Mo-96, Mo-97 through Au-200, and $\mathrm{Hg}-196$ through Pu-240, is presented.

\#6608020

$* 2.453$

Liikala, R. C.

WESTCOTT PARAMETERS DERIVED FROM THE RBU BASIC LIBRARY FOR A DELTA- 4 CUTOFF

HW-77389, General Electric Company, Hanford Atomic Products Operation, Richland, Washington

April 25, 1963

$16 \mathrm{p}$

Available from CFSTI

Westcott parameters were derived for the following is otopes: $\mathrm{U}-235, \mathrm{U}-236, \mathrm{U}-238, \mathrm{Pu}-239, \mathrm{pu}-240$, $\mathrm{Pu}-241, \mathrm{Pu}-242, \mathrm{Am}-241, \mathrm{Am}-242, \mathrm{Am}-243, \mathrm{Cm}-242$, $\mathrm{Cm}-243$, and $\mathrm{Cm}-244$. Results are presented in tabular form.

\#6500069

Liskien, H.; Paulsen, A.

COMPILATION OF CROSS SECTIONS FOR SOME NEUTRON INDUCED THRESHOLD REACTIONS: $\mathrm{Mg}-24(\mathrm{n}, \mathrm{p}) \mathrm{Na}-24$, Al$27(\mathrm{n}, \mathrm{alpha}) \mathrm{Na}-24, \mathrm{Al}-27(\mathrm{n}, \mathrm{p}) \mathrm{Mg}-27, \mathrm{P}-31(\mathrm{n}, \mathrm{p}) \mathrm{Si}_{31}$ $S-32(n, p) P-32, \quad F e-54(n, p) M n-54, F e-56(n, p) M n-56$, $\mathrm{Ni}-58(\mathrm{n}, \mathrm{p}) \mathrm{Co}-58$

EUR-10.e, European Atomic Energy Community, Central Nuclear Measurements Bureau, Gee1, Belgium

November 1961

$50 \mathrm{p}$

Cross sections for neutron threshold reactions $(\mathrm{n}, \mathrm{P})$ with $\mathrm{Mg}-24, \mathrm{Al}-27, \mathrm{P}-31, \mathrm{Fe}-54, \mathrm{Fe}-56$, and $\mathrm{Ni}-58$ and for the (n,alpha) reaction with $\mathrm{Al}-27$ are presented. The cross section values are printed on several sheets of transparent paper enabling a survey of a given series of measurements separately as we 11 as permitting the intercomparison of different measuring series. Uncertainties of cross section values and neutron energies are indicated. The figures of merit for the reactions are also included.

\#6908011

$$
\star 2.2553
$$

Liskien, H.; Paulsen, A.

COMPILATION' OF CROSS-SECTIONS FOR SOME NEUTRON INDUCED THRESHOLD REACTIONS, VOLUME $1(A=55)$, VOLUME $2(A=56)$

EUR-119.e, European Atomic Energy Community, Central Nuclear Measurements Bureau, Geel, Belgium April 1966

$100 \mathrm{p}$
Cross sections are presented for neutron induced reactions in the energy range 0 to 10 or $20 \mathrm{MeV}$. The reactions are generally $(n, a) p h a),(n, 2 n)$, or $(n, p)$. Nuclei considered are: $\mathrm{Na}-23, \mathrm{Mg}-24, \mathrm{Al}-27$, $\mathrm{Si}-26, \mathrm{P}-31, \mathrm{~S}-32, \mathrm{Ti}-46, \mathrm{Ti}-47, \mathrm{Fe}-54, \mathrm{Fe}-56, \mathrm{Ni}=$ 58 , Co-59, Ni-60, Cu-63, Cu-65, and Zñ-64. Comments on the measuring techniques and accuracies, Q-values for these and other threshold reactions, and information on the data of the various contributors are included.

\#6912029, I \& I I

*2. 2052

LRL EXPERIMENTAL CROSS SEC'IION BIBLIOGRAPHY, VOLUME I . BIBLIOGRAPHY, VOLUME II. CROSS SECTION'DATA California University, Lawrence Radiation Laboratory, Livermore, California

May 21,1968

$424 \mathrm{p}, 370 \mathrm{p}$

Tables of isotopes are given showing cross sections with the energy ranges given as E-min and E-max. The number of points included in each experiment is noted as well as the type of reaction. The tables are arranged in three ways: 1) according to increasing A (and 2) numbers; 2) according to the reference numbers; and 3) according to the type of reaction. A separate volume includes a list of bibliographic sources, alphabetic reference list, alphabetic author list, and a numeric reference list. In al1, 1548 references are given.

\#6510011 *2.1073

McCullen, J. D.; Bayman, B. F.; Zamick, L.

WAVE FUNCTIONS IN THE $1 \mathrm{f}$, SHELL

NYO-9891, Princeton Univefsity, Palmer Physical Laboratory, Princeton, New Jersey

June 1964

$73 p$

Available from CFSTI

Eigenvalues and eigenvectors of matrices of a twoparticle interaction, calculated with respect to states of several neutrons and protons in the $f$ shell, are tabulated. The interaction ${ }^{7}$ characterized by eight numbers giving the interaction energies in the eight levels availahle to two $f$ particles. Calculational methods and computer-priftout results are giver for Sc-43, 44 , $45,46,47$ and $\mathrm{Ti}-43,44,45,46,47,48,49$.

\#6500086

$* 2.2353$

McGowan, F. K.; Milner, W. T.; Kim, H. J

NUCLEAR CROSS SECTIONS FOR CHARGED-PART ICLE INDUCED REACTIONS, Mn, Fe, Co

ORNL-CPX-1, Oák Ridge National Laboratory, ChargedParticle Cross-Section Data Center, Oak Ridge, Tennessee

July 1964

$443 p$

Available from the AEC Div. of Tech. Information

This volume is a compilation of nuclear cross section data given for charged-particle-induced reactions of $\mathrm{Mn}=55, \mathrm{Fe}=54,56,57,58$, and Co-59, taken from literature before January 1, 1964. Both graphical and tabular data are included.

\#6500087 *2. 2353

McGowan, F. K.; Milner, W. T.; Kim, H. J

NUCLEAR CROSS SECTIONS FOR CHARGED-PART ICLE INDUCED REACTIONS, Ni, Cu

ORNL-CPX-2, Oak Ridge National Lahoratory, ChargedParticle Cross-Section Data Center, Oak Ridge, Tennessee

September 1964

$511 \mathrm{p}$

Available from the AEC Div. of Tech. Information

A compilation of nuclear cross sections for charged-particle-induced reactions of the nuclei $\mathrm{Ni}$ and $\mathrm{Cu}$ is presented. The compilation includes experimental cross-section data available in the literature before January 1, 1964. A number of bibliographies are included in the appendices to 
provide coverage to important information which is not included in the graphical presentation.

$\# 6604066$

*2.083-2.2683

McGowan, F. K. ; et al.

REACT ION LIST FOR CHARGED-PARTICLE-INDUCED NUCLEAR

REACTIONS PART A: $2=3$ TO $\mathrm{Z}=27$ ( $\mathrm{Li}$ to $\mathrm{Co}$ )

Nuclear Data Tab1es, A6:353-644

October 1969

This reaction list for charged-particle-induced nuclear reactions has been prepared from the journal literature for the period from 1948 through April 1969. Each published experimental paper is listed under the target nucleus in the nuclear reaction with a brief statement of the type of data in the paper. The nuclear reaction is denoted by $A(a, b) B$, where $M \geq$ (one-nucleon mass). There is no restriction on energy. Nuclear reactions involving mesons in the outgoing channel are not included.

\section{\# 6908035}

$* 2.2453$

McMaster, W. H.

COMPILATION OF $X$-RAY CROSS SECTIONS

UCRL-70525 (preprint), California University, Lawrence Radiation Laboratory, Livermore,

California

June 29,1967

$15 \mathrm{p}$

Available from CFSTI

A compilation of $x$-ray cross sections has been made in the range $1 \mathrm{keV}$ to $1 \mathrm{MeV}$ from a survey of the experimental $x$-ray total cross section data existing in the data files being maintained at the Lawrence Radiation Laboratory and at the X-Ray Attenuation Coefficient Information Center, National Bureau of Standards.

\section{$\# 6706033$}

McMaster, W. H.: et al.

COMPILATION OF $X$-RAY CROSS SECTIONS SECTION II

UCRL -50174 , California University, Lawrence Radiation Laboratory, Livermore, California

January 1967

$96 \mathrm{p}$

Preliminary graphs and tables of $x-r a y$ cross sections in the range of $1 \mathrm{keV}$ to $1 \mathrm{MeV}$ are given. The total cross sections presented are the sum of computed scattering cross sections and the fit to derived experimental photoelectric cross sections. Form factors available in the literature were used to compute the scattering cross sections. Where solutions to the Hartree-Foch equation were unavailable, Bewilogua's values for the ThomasFermi model were used. $L$ and $M$ she11 jump ratios were calculated from the theoretical values of Rakavy and Ron. The graphs for each element show the experimental data points used to obtain the fit.

\#6908034 *2.2753

Maples, C.; Goth, G. W.; Cerny, J.

NUCLEAR REACT ION' $\mathrm{Q}$-VALUES

UCRL-16964, California University, Lawrence Radiation Laboratory, Berkeley, California

July 1966

$366 \mathrm{p}$

Available from CFSTI

Tables of Q-values for nuclear reactions have been calculated from the currently available mass data on the nuclides. The calculations were carried out on all stable and naturally occurring radioactive isotopes from a 2 of 2 to 92 , as well as on a few common long-lived isotopes.
\#6508048

$\star 2.383$

A MANUAL OF RADIOACTIVITY PROCEDURES

NBS Handbook 80, National Bureau of Standards, Washington, D. C.

November 20,1961

$159 \mathrm{p}$

Available from Superintendent of Documents, GPO

This handbook prepared by the subcommittee on St andards and Measurements of Radioactivity for Radiological Use presents a survey of the practice of radioactivity measurement with special reference to clinical and biological applications. The manual is divided into three parts. The first part deals with basic standardization procedures, and the second and third parts deal with specialized clinical and biological applications. Tables of nuclear data and references to the literature are included.

$\$ 6604066$ *2. $083-2.2083$

Maples, C.; Goth, G. W. ; Cerny, J.

NUCLEAR REÁCTION'Q-VALUES

Nuclear Data, A2:429-612

December 196

Tables of Q-values for nuclear reactions have been calculated from currently available mass data. The calculations were performed for reactions whose targets include al1 the stable or naturally occurring radioactive isotopes with $2=2$ to 92 and long-lived isotopes of $2 \leq 20$. The incoming particles considered for $2=2$ to 92 are gamma, $n$, $\mathrm{P}, \mathrm{d}, \mathrm{t}, \mathrm{He}-3, \mathrm{He}-4, \mathrm{Li}-6, \mathrm{Li}-7, \mathrm{C}-12$, and $0-16$. The outgoing particles considered for $2=2$ to 92 are gamma, $\mathrm{n}, 2 \mathrm{n}, \mathrm{p}, \mathrm{d}, \mathrm{t}, \mathrm{He}-3, \mathrm{He}-4,(\mathrm{He}-4, \mathrm{n})$. In addition, the following outgoing particles are also considered for $5 \leq 2 \leq 22$ : He-6, Li-6, Li-7, $\mathrm{Li}-8, \mathrm{Be}-7, \mathrm{Be}-9, \mathrm{Be}-10, \mathrm{~B}-8, \mathrm{~B}-10$, and $\mathrm{C}-12$.

\# 6910011 *2. 1453

Marelius, A.; Sparrman, P.; Sundstroem, T.

TABLE OF' NUCLFAR LIFFT IMES

Appendix $D$ in Hyperfine Structure and Nuclear Radiation, International conference on Hyperfine Interactions Detected by Nuclear Radiation, Pacific

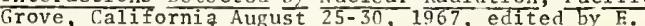
Matthis and D.A. Shi riey, page 10 '43-1087. New York: John Wiley \& Sons, Inc. 1968

$45 p$

This table contains results of direct lifetime measurements of excited nuclear states, as we 11 as results of excitation measurements vielding reduced transition probabilities and level widths that have been reported previous to August 1967. The on $1 y$ restrictions on the range of measured quantities have been that lifetimes shorter than about $10^{-20}$ sec., corresponding to level widths of the order of $10^{4} \mathrm{eV}$, have been omitted, and that the large number of closely spaced highly excited levels (less than $7 \mathrm{MeV})$, especially in neutron capture reactions, have been excluded. Entries are listed for ali nuclear levels that have so far been subjected to the above-mentioned measurements and the entries are arranged by increasing (i) mass number, (ii) atomic number of the nuclide, and (iii) energy of the excited state.

\# 6509036 *2. 2653

Margulies, S.; Engler, C.

EVALUATION OF' THE LAMBDA-4 AND LAMBDA-6 COULOMB CORRECTION FACTORS IN FIRST-FORBIDDEN BETA-DECAY Nuclear Instruments and Methods, 24:149-151

In the equations given by Kotani for the energy and angular dependence of the various observables in first-forbidden beta-decay, the effects of the Coulomb field of the daughter nucleus on the emitted electron are contained in Coulomb correction factors denoted by lambda-i. Tabulations of 1 ambda- 1 and 1 ambda- 2 are given by Kotani and Ross. As an aid for the evaluation of 
experimental data, tables of lambda- 4 and lambda- 6 are presented. The first factor appears in both the beta-circularly polarized gamma correlation and in the longitudinally polarized beta-gamma correlation; the second in the transversely polarized beta-gama correlation. With the appearance of these tables, all of the Coulomb corrections found in kotani's equations are now either given explicitly, or else can he determined by simple multiplication.

$\# 6604066$

Marion, J. B.

$\star 2.083-2.2283$

GAMMA-RAY CAL1BRATION ENERGIES

Nuclear Data, A4:301-319

May 1968

A tabulation is given of gamma-ray energies which are suitable for calibrating high-resolution gammaray spectrometers. Data from studies of both radioactive sources and nuclear reactions are included, allowing precision calibration in the range $25 \mathrm{keV}<\mathrm{E}$ amma $<11 \mathrm{MeV}$.

$\# 6500015,6500014 \quad * 2.1053-2.2053$

Marion, J. B.; Nakasima, R. (Editors)

NUCLEAR THEORY REFERENCE BOOK FOR 1957 AND 1958 , AND FOR 1959 AND 1960. COMPILATION OF NUCLEAR THEORY INDEX CARDS

National Academy of Sciences-National Research Council, Nuclear Data Project, Washington, D. C. April 1963

$136 \mathrm{p}, 152 \mathrm{p}$

Available from Superintendent of Documents, GPO

Guides to theoretical papers on nuclear-structure physics, formerly issued on cards, are presented for the years 1957-1958 and 1959-1960. The articles covered appeared in journals or reports during the given years. The bibliographies are categorized according to nuclear structure, nuclear models, nuclear properties, nuclear reactions, electromagnetic radiation, beta decay and parity, alpha decay, and special topics. Tables and computational aids as well as an author index are included.

\#6604066

*2.083-2. 2783

del Marmol, $\mathrm{p}$.

DELAYEED-NFUTRON PRECURSORS

Nuclear Data Tables, A6:141-151

June $196 \overline{9}$

For delayed-neutron precursors, i.e., nuclei whose beta-decay populates neutron-emitting states of their daughters, experimental data on the following properties are compiled and evaluated: half-life, neutron-emission probability, charge and mass assignment, methods of production, beta-decay properties leading to the neutron-emitting states, neutron spectra, measured or estimated hetadisintegration energy of the parent, and neutronseparation energy of the daughter nucleus.

$\# 6500006$

*1.1153

Marton, L.; Marton, C.; Hali, W. G.

ELECTRON PHYSICS TABLES

NBS Circular 571, National Bureau of Standards, Washington, D. C.

March 1956

$83 \mathrm{p}$

Available from Superintendent of Documents, GPO

The tables presented here are intended as replacement of "Miscellaneous Physics Tables - Part 11. Electronic Functions" (Mathematical Tables 17, National Bureau of Standards, 1941). Eight parameters have been calculated on the Standards Electronic Automatic Computer (SEAC) and are tabulated with an eight digit accuracy.
\#6912007

$\star 2.2223$

der Mateosian, E.: McKeown, M

TABLE OF GAMMA-RAYS EMITTED BY RADIOACTIVE NUCLE 1 ARRANGED IN ORDER OF INCREASING ENFRGY

BNL -605 , Brookhaven National Laboratory, Upton, New York

May 1960

$216 p$

Available from CFSTl

Data were taken principally from two sources: the Table of Isotopes by $D$. Strominger and the Nuclear Data Sheets To facilitate comparison of the data, aIl gamma rays reported in these sources were plotted on an energy scale under the names of the experimentalists responsible for the measurements. only a very limited critical evaluation of the data was attempted.

\#6910044

*2.25.53

Mathur, S. C.; Ashe, J. B.; Morgan, 1. L.

COMPILÁTION OF NEUTRON RFÁCTION AND TOTAL CROSS SECTION AT $14 \mathrm{MeV}$

Technical Documentary Report No. ASD-TDR-62-1038, Texas Nuclear Corporation, Austin, Texas Apri 11964

$75 p$

Available from CFST1, AD 617126

A list of cross section data and related information has been compiled for total neutron cross sections and the reaction cross sections, $(n, p), \quad(n, a l n h a)$, and $(n, 2 n)$ in the energy region around $14 \mathrm{MeV}$. This compilation includes published data through June 1963 . A gamma ray spectral atlas containing 54 sets of spectra of some of the more common elements in the Periodic Table was obtained by activation analys is with $14.8 \mathrm{MeV}$ neutrons. A one and a five minute irradiation of each element was made and spectra recorded at various times after irradiation to emphasize decay characteristics of the radioactive products from the irradiated element. Gamma ray peaks from the reaction products were identified as well as radioactive decay products from trace impurities in each element whenever possible. Carbon and nitrogen surface contamination of several high purity beryllium samples were measured by proton irradiation. The results seem to indicate that a large percentage of this contamination is in surface layers suggesting that these contaminants may have been added during a machining process.

$\# 6601001$

$* 2.1153$

Mat tauch, J. H. E.; Thiele, W.; Wapstra, A. H. ADJUSTMENT OF RELATIVE ATOMIC MASSES

Nuclear Physics, 67:73-120

May 1965

An extensive computer program has been written for and applied to, the calculation of "hest values" for the masses of atoms from measured values of mass spectroscopi.c doublets and those of nuclear reaction energies. The program also serves for the calculation of reaction and decay energies derived from these best values. The experimental input data are tabulated, discussed and compared with the adjusted values.

\#6601003 *2.1153

Mattauch, J. II. E.; Thiele, W.; Wapstra, A. H.

CONSISTENT SET OF Q-VALUES

Nuclear Physics, $67: 32-72$

May 1965

A list of alpha decay energies, binding energies of some combinations of protons and neutrons and reaction energies of various nuclear ruactions for a large number of nuclides are derived by least squares adjustment of a large set of experimental data. 
$\# 6601002$

*2.1153

Mattauch, J. H. E.; Thiele, W.; Wapstra, A. H.

1964 ATOMIC MASS TABLE

Nuclear Physics, $67: 1-31$

May 1965

This is a completely new mass tahle derived from experimental data. Data given for each element include $N, Z$, atomic number, mass excess, binding energy, and beta-decay energy. A list of references to the literature is included.

\#6500179 *2.012

MEASUREMENT OF ABSORBED DOSF: OF NEUTRONS, AND OF MIXTURES OF NEUTRONS AND GAMA RAYS

NBS Handbook 75, National Bureau of Standards, Washington, D. C.

February 1961

$86 \mathrm{p}$

Available from Superintendent of Documents, GPO

This handbook contains a brief discussion on the interaction of neutrons and gamma rays with matter and a discussion of various techniques used in the measurement of doses produced in the material.

$\# 6508049$

$* 2.482$

MEASUREMENT OF NEUTRON FLUX AND

PIIYSICAL AND BIOLOGICAL APPLICATIONS

NBS Ilandbook 72, National Bureau of Standards, Washington, D. C.

July 15,1960

$92 \mathrm{p}$

Available from Superintendent of Documents, GPO

The measurement of neutron flux and spectra is discussed, various methods are compared, and results of intercomparisons are given. Methods of measurement are discussed for the emission rate of radioactive neutron sources, thermal neutron flux, intermediate neutron flux, fast neutron flux, and neutron energy spectra, Neutron radiation instruments for area survey and personnel monitoring involving flux and snectrum measurements are included. Typical spectra of various neutron sources are shown.

$\# 6911025$

$* 2.2553$

Meek, M. E. ; Rider, B. F.

SUMARY OF FISSION" PRODUCT YIELDS FOR U-235, U-238, Pu-239, AND Pu-241 AT THERMAL, FISSION SPECTRIM AND $14 \mathrm{MeV}$ NEUTRON ENERGIES

APED-5398, General Electric Company, Nucleonics Laboratory, Pleas anton, California

March 1, 1968

$106 \mathrm{p}$

Available from AEC Dep. Lihraries

A tabulation of experimental data, extrapolated chain yields, calculated charge distributions and a recommended value for all known fission products is presented. The fissionable nuclides covered were $\mathrm{U}=235$ at thermal, fission spectrum, and $14 \mathrm{MeV}$ neutron energies: $\mathrm{Pu}-239$ at thermal and fission spectrum energies; and Pu-241 at thermal energies.

$\# 6604066$ *2. 083-2.2483

Melkanoff, M. A.; Sawada, T.; Cindro, N. DEUTERON ABSORPTION COEFFICIENTS AND REACTION CROSS SECTIONS CALCULATED WITH OPTICAL MODEL POTENTIALS Nuclear Data, A2:263-279

September 1966

Tables of deuteron absorption coefficients and reaction cross sections are presented for C, $\mathrm{Mg}$, $\mathrm{Ni}, \mathrm{Rh}, \mathrm{Nd}$, and $\mathrm{Au}$ at deuteron energies of 4 to 28 MeV. These were obtained from optical model calculations using parameters previously published by the authors.
$\$ 6509033$

*2.2453

Menichella, E. S.; Tonolini, F.; Severgnini, L. T. ANALYSIS OF THE ( $\mathrm{n}, \mathrm{alpha}$ ) NUCLFAR REACTIONS Energia Nucleare, 11:324-336

June 1964

In Italian

Experimental data on $(\pi, a l p h a)$ reactions, particularly at $14 \mathrm{MeV}$, are reviewed and analyzed by statistical model formulas.

\#6609033

Miller, S. M.; Parker, K.

LIST OF DATA FILES AVÁILABLE IN THE UKAEA NUICLEAR DATA LIBRARY AS OF 15 APRIL 1965

AliRE Report No. $0-55 / 65$, Atomic Weapons Research Establishment, Aldermas ton, England

July 1965

$31 \mathrm{p}$

Available from AEC Dep. Lihraries

Brief details are given of the data files in the UKAEA Nuclear Nata Library at 15 th April 1965 . The data consist principally of cross sections and related data for neutrons at energies hetween $0.0001 \mathrm{eV}$ and $15 \mathrm{M} / \mathrm{eV}$ hut some photon interaction cross sections are includer. A companion report currently AWRE Report No. $0-70 / 63$ - describes the conventions of the Lihrary which stores convenientiy vast amounts of data required in the preparation of input data for neutron and photon transport computer codes. The list of data files has been prepared by a computer program and can easily be updated.

*6500013 *2.1053-2.2053

Nakasima, R.; Ogata, H. (Editors)

NUCLEAR THEORY REFERENCE BOOK FOR 1961 AND 1.962 , COMPILATION OF NUCLEAR THEORY INDEX CARDS

National Academy of Sciences-National Research Council, Nuclear Data Project, Washington, D. C. December 1963

$169 \mathrm{p}$

Available from Superintendent of Documents, GPO

This hook is a guide to theoretical papers on nuclear-structure physics appearing in journals and reports during 1961 and 1962, compiled and previously appearing on index cards. The book has been made by photographing the cards themselves. The major categories for papers are: nuclear structure, nuclear reactions, electromagnetic radiation, beta decay and parity, alpha decay, tables and computational aids, and special topics. Each reference contains the following information: title, author(s), affiliation of authors, journal name, volume, page number, and year of puhilication. Papers that fall into more than one classification are listed under each classification. An author index is included.

\#6907025 *1.051-2.051

National Neutron Cross Section Center ENIJF NEWSLETTER VOLUME 3 , NUMBER 2

Brookhaven National Laboratory, Upton, New York October 2, 1967

About 100 p

This newsletter contains a sumary of Rentron cross section data, information and references for $H, D$, $\mathrm{Li}-6, \mathrm{Li}-7, \mathrm{C}-12, \quad 0-16, \mathrm{Mg}, \mathrm{Ti}, \mathrm{V}, \mathrm{Mn}-55$, Mo Xe135, Sm-149, Eu-151, Eu-153, Gd, Dy-164, Lu-175, Lu-176, Au-197, U-235, Pu-238, Pu-239, Pu-240, Pu$242, \mathrm{Cm}-244, \mathrm{Na}-23, \mathrm{Hf}-176, \mathrm{Hf}-177$, Hf- $178, \mathrm{Hf}-179$, $\mathrm{HF}-180, \mathrm{Hf}-174$. 
Nelms, A. T.

GRAPISS OF THE COMPTON ENERGY-ANGLE RELATIONSIIP AND TIIE KLEIN-NISHINA FORMULA FROM $10 \mathrm{KeV}$ TO $500 \mathrm{MeV}$

NBS Circular 542, National Bureau of Standards, Washington, D. C.

August 28,1953

$89 \mathrm{p}$

Available from Superintendent of Documents, GPO

The Compton energy versus angle relationship and the differential and integral Klein-Nishina cross sections are presented graphically as functions of the energy and direction of the scattered photon and of the recoil electron. These graphs are intended to serve the purpose of tables. Unpolarized primary gamma rays in an energy range from $10 \mathrm{keV}$ to $500 \mathrm{MeV}$ are considered. The accuracy of all curves is estimated at 1 percent. The advantage of this form of presentation is the convenience and accuracy of two-way interpolation. In general, interpolated values may he obtained with an accuracy of 2 percent.

\section{\#6509003 *2.2553}

Neuert, H.; Pollehn, H.

TABLES OF CROSS SECTIONS OF NUCLEAR REACTIONS WITH NEUTRONS IN THE 14-15 MeV FNERGY RANGE

EUR-122.e, University of Hamburg, Ilamburg, Germany 1963

235 p

Available from AEC Dep. Libraries

During the last few years many measurements of nuclear reactions with neutrons $(n, 2 n),(n, p)$, $(n, n p),(n, d),(n, H e-3),(n, t),(n, a) p h a)$, and $(n, n$ alpha) in the energy range $E_{n}=14-15 \mathrm{MeV}$ were carried out. These tables are a compilation of the cross-sections of these reactions, and provide a basis for special theoretical considerations. In some cases, they point up the need for making further investigations on more precise measurements in this field.

\#6912031-6912037.

*2. $2552-2.1452$

\#6905036,6910005

NEWSLETTER NO. 1 THROUGH NO, 9

CCDN-NI/1 to 9, European Nuclear Energy Agency, Centre de Cómpilation de Donnes Neutroniques (CCDN), Saclay, France

May, June, Oct., Dec. 1966; Apr. Sept, 1967; Mar. 1968; Mar., May 1969

$51 \mathrm{p}, 95 \mathrm{p}, 31 \mathrm{p}, 239 \mathrm{p}, 70 \mathrm{p}, 33 \mathrm{p}, 72 \mathrm{p}, 384 \mathrm{p}, 10 \mathrm{p}$

Newsletter No. 1 gives detailed information on the activities of the Centro de Compilation de Donnes Neutroniques (CCDN) during May 1964 to May 1966 and explains the exact services provided by the Centre. News letter Nos. $2,5,7$, and 9 contain a 1 isting of the evaluated reference data held on the SCISRS Master Data Tape in June 1966, April 1967, March 1968, March 1969 respectivély; Newslettér Nos. 4 and 8 present a computerized index on neutron dat at $\bar{D}$ ecember 1966 and March 1969 , respectively. Newsletter No. 3 contains a comparison of existing data on the Li-6(n,alpha)ll-3 reaction cross section. Newsletter No. 6 presents a compilation of existing data on the He-3(n,p)T and the He $-3(n, d) D$ reaction cross sections and the total and scattering cross sections.

\section{\#6500056}

Nonaka, I.; et al.

NUMERICAL 'DIFFERENTIAL CROSS SECTIONS OF THE SCATTERING OF ALPHA PARTICLES, III. C-12 - Part I. INSJ -60 , University of Tokyo, Institute for Nuclear Study, Tokyo, Japan

August 1963

$10 \mathrm{p}$

Elastic and inelastic $(Q=-4.43,-7.656$, and -9.63 $\mathrm{MeV}$ ) cross sections for alpha particle scattering at 28.4 to $33.6 \mathrm{MeV}$ on carbon- 12 (carbon foil, 2.38 $\mathrm{mg} / \mathrm{sq} \mathrm{cm}$ in thickness) are tabulated.
Nonaka, I.; et al.

NUMERICAL DIFFERENTIAL CROSS SECTIONS OF THE SCATTERING OF ALPHA PARTICLES, IV. C-12 - Part II. INSJ-61, University of Tokyo, Institute for Nuclear Study, Tokyo, Janan

August 1963

$25 p$

Elastic and inelastic cross sections for the scattering of alpha particles at 27 to $35.5 \mathrm{MeV}$ on carbon-12 (natural carbon foil, $2.38 \mathrm{mg} / \mathrm{sa} \mathrm{cm}$ in thickness) are tabulated.

\#6912014

$$
\text { *2.753 }
$$

Norton, D. S.

THE U.K.A.E.A. NUCLEAR DATA LIBRARY, FERRUARY 1968 AEEW-M 824, Atomic Energy Establishment, Winfrith, England

May 1968

$25 \mathrm{p}$

Available from AEC Dep. Libraries

Details are given of the UKAEA Nuclear Data Lihrary as of February 1968. The updating of the library included the incorporation of several new data files, together with the extension of ranges of some older files.

\# 6708006

* 4.1252-2.1552

NUCLEAR SCIENCE AND TECHNOLOGY FOR CERAMISTS, PROCEEDINGS OF A SYMPOSIUM, APRIL 7-12, 1966

NBS Miscellaneous Publication 285, National Bureau of Standards, Washington, J. C.

May 26,1967

$75 \mathrm{p}$

Available from Superintendent of Documents, GPO

An introductory survey of those properties involved in the choice and use of materials is provided. The radiation fields present in reactors and the features of these fields most important to the development of nuclear materials, touching upon the principal physical effects induced in materials hy the radiation; the solid state physics of radiation damage processes, with examples drawn partly from ceramics; and the effects these processes have on physical properties are described. The thermodynamics of non-stoichiometry, a particularly significant problem encountered with nuclear fuels is discussed. Finally, the problems encountered in the development of nuclear fuels on the one hand, and structural and moderator materials on the other are taken up.

\#6604066

$$
\text { *2.083-2.2783 }
$$

O'Conne11, R. F.; Carrol1, C. 0.

INTERNAL CONVERSION COEFFICIENTS AT GAMMA-RAY THRESHOLD ENERGIES

Nuclear Data, A3:287-325

october $\frac{1967}{196}$

Internal conversion coefficients, for threshold values of the gamma-ray energy, are presented for the K-she11, L-subshells, and M-subshe 11 s for all values of $z$ in the range $6 \leq z \leq 95$, and for the first five electric and magnetic multipoles. These numbers are based upon the exact analytical results previously obtained by the authors for the internal conversion coefficients for any shell and for any energy for a point nucleus with no screening.

\#6508029

O'Conne 11, R. F.: Carroll, C. O.

INTERNAL CONVERSION COEFFICIENTS: GENERAL FORMULATION FOR ALL SHELLS AND APPLICATION TO LOWENERGY TRANSITIONS

Physical Review, 138:B1042-1054

गuा 7, 1965

Exact analytical results (in the case of a point nucleus with no screening) for the internal conversion coefficients for any shell are presented. These results simplify considerably at 
threshold values of the gamma-ray energy. Numerical results, at threshold, are obtained for the $K, L\left(L_{I}\right.$, LII, LIII) and M(MI, MIT, "MIII, MIV, $M_{V}$ ) shelis for 19 values of $Z$ in the range than or equal to $z$ less than or equal to 95 , anc
for the first five electric and magnetic multipoles. The results for the $K$ shell agree with those ohtained by Sninrad. It is shown that the threshold results are actually correct to order $\mathrm{P}$ (momentum of electron in units of its mass). The effects of finite nuclear size are also considered.

\section{$\# 6500051$}

$\star 2.2163$

felt, G. S.

AN EVALUATION OF KACSER'S SFCOND ORDER BORN APPROXIMATION TO TIIE BREMSSTRAHLUNG DIFFERENT IAL CROSS SECTTON

NBS Technical Note 81 , National Bureau of Standards, Washington, D. C.

June 1961

$20 \mathrm{p}$

Available from the Superintendent of Documents, GPO, PB 161582

The second order term, as derived hy C. Kacser (Proc. Roy. Soc. A253 (1959)), of the Born approximation se ries for the bremsstrahlung cross section differential with respect to photon energy, photon direction, and final electron direction averaged over initial and summed over final polarization states has been put in a form that admits to numerical evaluation for the coplanar case. The results are valid for relativistic as well as non-relativistic incident electrons. Completely general (non-coplanar) expressions are included for the first order (Bethe-lleitler) and second order terms. However, the latter has not been given in a form admissible to numerical evaluation. Tabulated values are given for the case of the incident electron kinetic energy $=500$ keV, photon energy $=450 \mathrm{keV}$, and photon direction $20^{\circ}$ from the incident electron direction. For this case the second order term increases the differential cross section by about 50 percent for $\mathrm{Al}$ and about 200 percent for $\mathrm{Au}$ for the various angles of electron emission. A rough approximation to the integrated (over final electron direction) cross section is included and a comparison is made with experimental values. The inclusion of the second order term brings the theoretical cross section closer to the experimental values.

\#6910036

Ohba, I.; Kobayashi, T.

Pi-N INELASTIC PROCESSES

Progress of Theoretical Physics (Kyoto) Supplement, प1-42:90- -218

1967

Experimental data on $\pi^{2} p$ inelastic processes published from 1964 to 1966 are compiled. Attention is given to data on the quasi-two-hody processes which involve the meson resonance and/or the baryon resonance. Nonresonant direct process (10 $\mathrm{GeV} / \mathrm{C})$ and strange particle production are treated separately from the quasi-two-body processes. The incident momentum, reaction, observed quantities, and method are given with each of the 122 references.

$\# 6512001$

$$
\text { *2. } 2463
$$

Okada, $M$.

GAMMA SPECTRA OF NATURAL ELFMENTS IRRADIATED WITH

PILE NEUTRONS

Radioisotopes (Tokyo), 12:234-252

May 1963

In Japanese

$\# 6509022$

Okada, M.

SIIORT-LIVED NUCLIDES FORMED BY NEUTRON ACTIVATION

Nucleonics, 22:110-111

Augus t 1964
In neutron activation analysis various types of neutron reactions are likely to occur simultancously. To analyze an element in a sample it is necessary to consider all of these interfering reactions. A table is presented that gives the original element, the type of neutron reaction, the nuclide formed, half Iife, gamna energies, and other decay particles present.

\section{\#6509021}

*2. 2663

Okada, M.

22 GAMMA SPECTRA OF SHORT-I,IVED NUC.I.IDF:S

Nucleonics, 21:62-63

November 1063

A supplement is presented to Data Sheet No. 47 (NSA 17: 9549) that contains gamma spectra for nuclictes with half lives of 0.8 sec. to $24 \mathrm{~min}$.

40012024

otter, J. M. ; et al.

EVALUATED NEUTRON CROSS SECTIONS FOR COPPER-63. COPPER-65, AND NATIJRAL COPPER

AI-AEC-12741, ENDF-124, Atomics International, Canoga Park, California

December 15,1968

$125 \mathrm{p}$

Available from C.FSTI

Evaluated neutron cross-section data for copper-63. copper-65, and naturally occurring copper have been prepared for the ENDF/B nuclear data file. Calculations, based on nuclear systematics, were used to augment availahle experimental data, where required. A complete set of neutron cross-section data was prepared for each nuclide, for incident neutron energies between $10^{-3}$ and $1,5 \times 10^{\circ} \mathrm{eV}$.

\# 6608022

Owen, G. E.; et al.

NUCLEAR REACTIONS IN LIGHT NUCLEI AND MSSSBAUER STUDIES

The Johns Ilopkins University, Baltimore, Marvland November 1965

$108 \mathrm{p}$

Available from CFSTI, TID2240.3

Angular distributions of ground and first excited state neutrons in the reaction $\mathrm{F}-19(\mathrm{~d}, \mathrm{n}) \mathrm{Ne}-20$ have been measured at $E_{d}=0.97,1.47,1.97,2.17$, and $2.47 \mathrm{MeV}$, at laboratory anglés from $0^{\circ}$ to $165^{\circ}$. Differential neutron yield curves at $0^{\circ}$ and $120^{\circ}$ (Iaboratory) were measured from 0.9 to $2.7 \mathrm{MeV}$. Integrated cross-section yields based on the angular distributions measured in this work as wel1 as others show peaking for the first excited state in the neighborhood of $2.0 \mathrm{MeV}$. The neutrons were detected using a single stilbene crystal spectrometer employing pulse shape discrimination. The absolute differential cross-section at $0^{\circ}$ for the first excited state neutrons was measured at $1.97 \mathrm{MeV}$ to be $7.4 \pm 0.8 \mathrm{mb} / \mathrm{sr}$, using a CaF, target. The ground state distributions at energies other than $1.0 \mathrm{MeV}$ do not exhibit typical stripping patterns. The first excited state distributions exhibit broad peaks around $120^{\circ}$ (1aboratory), and the $1.97,2.17$, and $2.47 \mathrm{MeV}$ curves also exhibit peaking at $0^{\circ}$. The angular distributions are in the process of being analyzed with the plane-wave, dual mode theory of Edwards.

$\# 6500066$

$* 2.2463$

Parker, $\mathrm{K}$

NEUTRON CROSS SECTIONS OF U-235 AND U-238 IN THE ENERGY RANGE I $\mathrm{keV}$ - $15 \mathrm{MeV}$. PART I: BEST CROSSSECTIONS FOR U-238 BASED ON MICROSCOPIC EXPERIMENTAL AND THEORETICAL DATA AVAII.ABLE AT DECEMBER 1961

AWRE Report No, 0-79/63, Atomic Weapons Research Es tablishment, Aldermaston, England January 1964

$127 \mathrm{p}$

Available from AEC Dep. Libraries 
Recommended values are given for neutron total and partial cross-sections of $U-2.39$ in the energy range $1 \mathrm{keV}$ - $15 \mathrm{MeV}$. The available experimental data are supplemented by estimates based on nuclear theory. Details of energy and angular distributions are given so that the data are complete for the purposes of neutronics calculations. Experimental and theoretical data which have become available since the best values were determined are discussed as far as possible. 215 references are included.

H6500041

$* 2.2463-2.463$

Parker, $\mathrm{K}$

A REVIEW OF EVALUATIONS OF NEUTRON CROSS

AVAILABLE AT SEPTEMBER 1963

EANDC (UK) -26 "U", Atomic Weapons Research Establishment, Aldermaston, England

September 1963

$23 p$

Available from AEC Dep. Libraries

A survey of evaluation work by laboratories is given, followed by a tabular summary. Some discussion is given of the position for each chemical element and certain isotopes and chemical compounds.

\#6500076

*2. 2463

Pattender, N. J.; Ilarvey, J. A.

TABULATION OF THE NEUTRON TOTAL CROSS SECTION OF U233 FROM 0.07 TO $10,000 \mathrm{eV}$ MEASURED WITH THE ORNL FAST CHOPPER

ORNL-TM-556, Oak Ridge National Laboratory, Oak Ridge, Tennessee

Apri1 1963

$35 \mathrm{p}$

A tabulation of the neutron total cross section of $\mathrm{U}-233$ as a function of neutron energy from 0.07 to $10,000 \mathrm{eV}$, measured with the ORNL fast chopper time-of-fiight neutron spectrometer is given.

\#6604066

$\star 2.083-2.2783$

Paul, I1.

SIIAPES OF BETA SPECTRA

Nuclear Data, A2:281-298

September 1966

A compilation of information on the measured shapes of beta spectra is given. It contains, whenever possible, the numerical coefficients describing the "correction factor," $\mathrm{C}(\mathrm{W})=\mathrm{N}_{\mathrm{m}}\left(\mathrm{W}^{*}\right) / \mathrm{N}$ (V) , where $\mathrm{N}_{\mathrm{m}}(\mathrm{W})$ is the measured energy spectrum and $\mathrm{N}_{\text {th }}(\mathrm{W})$ is an appropriate theoretical energy spectrum. The information was collected from publications that date roughly between 1957 and July 1966.

\# 6908044

*2. $2763-3.163$

Pauli, H. C

TABLES OF INTERNAL CONVERSION COEFFICIENTS AND PART ICLE PARAMETERS

COO-1420-137, Purdue University, Department of Physics, Lafáyette, Indiana

August ig67

$206 p$

Available from CFSTI

The internal conversion coefficients and particle parameters of the $K$ and $L$ subshells have been recalculated for the ten nuclei, $2=60,64,68$, $72,76,80,84,88,92$, and 96 and ten energies, $k$ $=0.02,0.05,0.10,0.15,0.20,0.30,0.40,0.50$, $0.70,1.00 \mathrm{mec}^{2}$. The four lowest orders of electric and magnetic multipole transitions have been considered. The internal conversion coefficients and particle parameters of the $M$ subshells have been computed for the seven nuclei, $2=72,76,80,84,88,92$, and 96 and for ten energies listed above. The screening of the atomic electrons has been taken into account on the bas is of the Thomas-Fermi-Dirac model. All static and dynamic effects of the finite nuclear size have been taken into account, assuming a sharp nuclear edge.

\#5509001

Paxton, H. C.

LOS ALAMOS CRITICAL-MLSS DATA

LAMS-3067, Los Alamos Scientific Laboratory, Los Alamos, New Mexico

April 1964

$61 \mathrm{p}$

Tabulated are critical masses of simple systems, which have been measured at Los Alamos through the year 1963.

A 6510014

$* 2.463$

Paxton, H. C.; et al.

CRITICAL DIMENSIONS OF SYSTFMS CONTAINING U-235, $\mathrm{Pu}-239$, AND $\mathrm{U}=233$

TID-7028, Los Alamos Scientific Laboratory and Oak Ridge National Laboratory, Los Alamos, New Mexico and Oak Ridge, Tennessee

June 1964

$145 \mathrm{p}$

Available from CFSTI

A compilation of critical data obtained from experiments performed since 1945 is presented. The report supplements the Nuclear Safety Guide (TID$7016(\operatorname{ReV} .1))$ and shows the bases of the recommendations that appear in the Guide. Conversions to standard geometries is shown. Results are given. for single units and multipleunit arrays. 130 references are provided.

\#6604066

Pearlstein, $S$.

AN EXTENDED TABLE OF CALCULATED $(\mathbf{n}, 2 n)$ CROSS

SECTIONS

Nuclear Data, A3:327-341

october $\frac{1967}{1967}$

The table presents, for some 500 stable and unstable targets, computed values of the $(n, 2 n)$ cross section for three neutron energies in the neighborhood of $14 \mathrm{MeV}$ and for a fission neutron spectrun. Cross sections for $(n, 3 n)$ for fission neutrons are also given. The calculations are based on the statistical model and on empirical expressions for inelastic cross sections and level density.

\# 6912025

Pennington, E. M.

ENDF/B NEUTRON CROSS SFCTION DATA FOR NATURAL HE L IUM

ANL-7426, (ENDF-125), Argonne National laboratory, Argonne, Illinois

October 1968

$33 p$

Available from CFSTI

This report describes the compilation of neutron cross-section data for natural helium. The elastic scattering cross section, $\overline{\mathrm{mu}}_{\mathrm{L}}, \mathrm{xi}, \mathrm{gamma}$, and elastic scattering Legendre coefficients are included for $\mathrm{He}-4$. Since $\mathrm{He}-3$ has a natural abundance of merely $0.00013 \%$, only its $(n, p)$ cross section, which is large at low energies, is considered. A listing of the data in the ENDF/B format is presented, as are graphs of much of the data.

\#6908006

$* 2.063-0.3163$

Penny, S, K.: Trubey, D.K.; Gurney, J.

BIBLIOGRAPHY, SUBJECT INDEX, AND NUTHOR INDFX OF THE LITERATURE EXAMINED BY THE RADIATION SIIILDING INFORMAT ION CENTER

ORNL-RSIC-5 (Rev, 1), Radiation Shielding Information Center, Oak'Ridge National Iaboratory, Oak Ridge, Tennessee

May 1966

$603 \mathrm{p}$. 
This bibliography is the fourth cumulative edit of the literature concerned with nuclear reactors, nuclear weapons, and radioisotopes which has heen selected hy the Radiation Shielding Information Center. It supercedes ORNL-RSIC- 2 and ORNL-RSIC-5 (j)ecember r964). The key Word Index, which should serve as an aid in locating biblingraphic references to a given suhject, has also heen revised. The pages associated with a word in the index will contain a list of documents that might he relevant to the word. This bibliography is synchronized with abstracts published as ORNL-RSIC- 6 in a looseleaf hinder. New abstracts, corresnonding to accessions since the orginal issue, are now availahle. The two major sections in this hibliography are a list of documents by subject category and an author index. The document list covers nine major suhjects with a total of 147 categories. If a document applies to more than one category, it is listed under each. The author index includes cross references to the categories under which the author's puhlications appear. Precening the bibliography is a list of the subject categories, their corresponding category numbers, and the pages on which they appear in the bibliography.

\#6604066

$\approx 2.083-1.083$

Perez, R.; Flores, J.

REDUCTION OF U, REPRESENTATIONS INTO SU,

IRREDUCI HLE COMPONENTS

Nuclear Data, A4:265-270

May 1968

A table of the $\mathrm{SU}_{3}$ representations contained in a given Young diagram specifying the irreducihle representations of the orbital group $U_{6}$ is constructed for all possible partitions in the $2 \mathrm{~s}$ ld shell.

\#6911022

$\div 2.2263$

Perneczky, G.; Quittner, $\Gamma$.

TABLES FOR FAST IDENTIFICATION OF GAMA-EMITTERS FROM THE COMBINED HALF-LIFE AND ENERGY DATA KFKI-5/1967, Magyar Tudomanyos Akademia Kozponti Fizikai Kutato Intezete, Budapest, Hungary April 5, 1967

$6 \mathrm{p}$

Available from the AEC Dep. Libraries

Tables are given for the rapid identification of gamma emitters, produced by ( $\mathrm{n}, \mathrm{gamma}$ ) and fastneutron reactions, from their approximately known half lives and energies.

\#6509008

$* 2.2463-2.463$

Persiani, P. J.; Kaganove, J. J.; McArthy, A. E. NEUTRON RESONANCE INTEGRAL AND AGE DATA

TID-18590, Reactor Physics Constants Center Newsletter No. 10, Argonne National Laboratory, Argonne, Illinois

April 1963

$40 \mathrm{p}$

Available from CFSTI, TID- 18590

Results of new calculations and measurements of neutron resonance integrals for various isotopes are presented. Most of the integrals for various isotopes are presented. Most of the integrals involved calculations taking into consideration negative energy levels to describe more accurately low energy neutron cross sections. Ages of fission and non-fission neutrons in various mixtures, elements, and compounds are also included.

$\# 6604051$

$* 2.2181-2.2211$

Photonuclear Data Group (Compilers)

PIIOTONUCLEAR DATA INDEX

NBS Miscellaneous Publication 277, National Bureau of Standards, Washington, D. C.

April 1966

$96 \mathrm{p}$

Available from Superintendent of Documents, GPO
An index to experimental data on photonuclear reactions is presented. Organized hy element and is otope, each entry in the index supplies quantitative information for a specific reaction on the ranges of excitation energy, source energy, detected particle energy, and emission angles covered in each reference. Information is also given on the type of measurement and the detector us ed.

$\# 6712008$

$* 2.2181-2.2211$

Photonuclear Data Group (Compilers)

PHOTONUCLEAR DATA INDLX, JANUARY 1965 THROUGII APRIL 1967

NBS Miscellaneous Publication 277, Supplement I, National Bureau of Standards, Washington, D. C.

October 1967

$66 \mathrm{p}$.

Available from Superintendent of Documents, GPO

This index, a supplement to NBS Miscellaneous Publication 277 , primarily covers data puhlished in the period dated January 1, 1965 through the middle of April 1967. Organized by element and isotope, each entry in the index supplies quantitative information for a specific reaction on the ranges of excitation energy, source energy, detected particle energy, and emission angles for reaction produced covered in each reference. Information is also given on the type of measurement and detector used.

\#6912012

$* 2.2563$

Pitterle, T. A.; Yamamoto, M.

PVALUATED NEUTRON CROSS SECTIONS OF Pu- 240 FOR THE ENDF/B FILE

APDA-218 (ENDF-122), Atomic Power Development Associates, Inc., Detroit, Michigan

June 1968

$66 \mathrm{p}$

Available from CFSTI

This report describes an evaluation of Pu-240 neutron cross section data carried out for the ENDF/B file, llata were evaluated from $10^{-4}$ to 15 $\mathrm{MeV}$ for the following neutron reactions: total, nganma, fission, $(n, 2 n),(n, 3 n)$, elastic scattering including Legendre polynomial expansions of the angular dependence, nonelastic, and inelastic scattering including resolved levels. Graphs of the evaluated data are included in the report.

\#6912013

$* 2.2563$

Pitterle, T. A.

EVALUATED NEUTRON CROSS SECTIONS OF SODIUM- 23 FOR THE ENDF/B FILE

APDA-217 (ENDF-121), Atomic Power Development Associates, Inc., Detroit, Michigan

June 1968

$50 \mathrm{p}$

Available from CFSTI

This report describes an evaluation of sodium-23 neutron cross section data carried out for the ENDF/B file. Data were evaluated from $10-4 \mathrm{eV}$ to $15 \mathrm{MeV}$ for the following neutron reactions: total, elastic scattering including Legendre polynomial expansions of the angular dependence, nonelastic, inelastic including resolver levels, (n,gamma), $(n, p), \quad(n, a) p h a)$, and $(n, 2 n)$. Graphs of the evaluated data are compared with experimental data in the report.

\#6908056

Post, J. C.

TABLE OF ALPHA-EMITTING NUCLIDES ACCORDING

ALPIHA-ENERGY

$\frac{\text { Actinides }}{\text { September }} \frac{\text { Review }}{1967}, 1: 55-69$

A tahle of alpha-emitting nuclides arranged according to alpha-energy is presented as an aid in the interpretation of alpha spectra for analytical purposes. The table includes the energy $\mathrm{F}_{\text {alpha }}$ of 
the emitted alpha particle, relative abundance of the alpina emission of energy Ealpha, element and mass number, half-life of the nuclide, branching fraction of alpha enission of the nuclide, and year and symbol of first author of publication with a tabulation of author symbols given at the end of the table.

\#6608023

Pressly, R. S.

LOI ENERGY GAMMA AND X-RAY FROM RADIOACTIVF. SOURCES ORVL-P-1928, Oak Ridge National Laboratory, Oak Ridge, Tennessee

February 1966

310

Available from CFSTI, Conf 660207-2

The methods used in the fabrication of low-energygamma, brensstrahlung and X-ray sources are described. The spectrums of the radiation from several sources are given with efficiency measurements. The newer radio-isotopes which are being investigated and their availability are discussed.

\#6912003

$\div 2.2563$

Price, L. R.; et al.

A COMPILATION OF $\mathrm{K}^{+} \mathrm{N}$ REACTIONS

UCRL $=20000 \mathrm{~K}^{+} \mathrm{N}$, Lawrence Radiation laboratory, University of California, Berkeley, California Septeraber 1969

$240 \mathrm{p}$

Available from the Particle Data Group, Lawrence Radiation Laboratory, University of California, Berkeley, California

Ninety papers reporting $\mathrm{K}^{+} \mathrm{N}$ and $\mathrm{K}^{+} \mathrm{d}$ interactions. Cross section, angular distributions, and polarizations are displayed. Included are indices to the papers, as we 11 as a complete listing of the selected $\mathrm{K}^{+} \mathrm{H}$ data, and the results of the fits to some of the data. The cutoff date for this report was July 15, 1969 .

\section{H6911026}

$* 2.2563$

Prosdocini, $A$

NEUTRON CROSS SECTIONS AND FISSION PARAMETERS OF PU-240, PU- 241, AND PU-242

EANDC(E)-74(U), European Atonic Energy Comnunity, Centra1 Nuclear Measurements Bureau, EuropeanAmerican Nuclear Data Comittee, Geel, Belgium

August 25,1960

$18 \mathrm{p}$

Available from AEC Dep. Libraries

A cornilation of neutron resonance integrals and parameters, values for the numbers of prompt neutrons enitted in neutron and spontaneous fission, and neutron fission and total cross sections is presented for $\mathrm{Pu}-240, \mathrm{Pu}-241$, and $\mathrm{Pu}-$

242.

\section{\#6500634 *2.372-0.2172}

RADIATION QUANTITIES AND UNITS, ICRU RFPORT $10 \mathrm{~A}$

NBS Handbook 84, National Bureau of Standards, Washington, D. C.

October 1964

$17 \mathrm{p}$

Available from Superintendent of Documents, GPO

This handbook presents recommendations agreed upon at the meeting of the International Connission on Radiological Units and Measurements held in Montreux, Switzerland, in April 1962. Quantities, units, and the names of radiation are given. MEASUREMENTS (1962) (ICRU) REPORT 10C

NBS Handbook 86, National Bureau of Standards, Washington, D. C.

November 1963

$53 p$

Available from Superintendent of Documents, GPO

This publication contains information on the following subjects: (1) direct and relative measurements of the activity of radioactive sources; (2) low-level radioactivity in materials and its relation to radiological measurements; (3) availability of radioactivity standards - present and future requirements; (4) techniques for measuring radioactivity in samples and living subjects. Appendix I contains standards and measurement of radioactivity for radiological use and Appendix II contains radiation quantities and units.

\#6500149 *2.2672

THE RADIOCHEMICAL MANUAL PART 1: PHYSICAL DATA The Radiochemical Centre, Amersham, England

January 1962

$102 p$

Available from AEC Dep. Libraries

A table of radioisotopes which are commonly used and readily accessible is presented. The table is arranged alphabetically, and for each radioisotope the basic physical data is given. An indication of the usual method of their production and reference to the physical and chemical forms which are readily available are presented. In other tables the principal radioisotopes are classified by halflife, radiation energy, and other physical characteristics. The re are also select lists of isotopes which are suitable for special purposes, such as radiography, thickness gauging, the calibration of instruments and as sources of neutrons.

\section{\#6510031 \\ *2.2673}

Rakow, A.

TABELLE 2UR IDENTIFI 2 IERUNG UNBEKANNTER GAMRASPEKTREN (TABLE FOR THE IDENTIFICATION OF UNKNONN GAMMA SPECTRA)

Munich, Germany: Verlag Karlthiemig KG, ThiemigTas chenbuecher Band 2 .

1962

$70 p$

In German

Data on gamma-emitting isotopes are tabulated, including half lives, gamma energies, and the number of quanta of each energy emitted per 100 decays.

\#6912027

*2.2073

Raman, S.; Miller, J.

DATA SUMMARIES (NUCLEAR-STRUCTURE PHYSICS)

ORNL-4317-1, Oak Ridge National Laboratory, Oak Ridge, Tennessee

September 1968

$212 \mathrm{p}$

Available from CFSTI

Tabular and graphical summaries of experimental results reported in The physical Review, Physical Review Letters, Nuclear Physics, and Physics

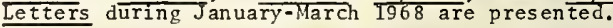

\#6508043,6508042

$* 2.082$

REACTOR HANDBOOK, VOLUME I. PHYSICS, VOLUME II. ENGINEERING, FIRST EDITION

AECD-3645, AECD-3646, Atomic Energy Commission, Washington, D. C.

February 1965, May 1965

$790 \mathrm{p}, 1075 \mathrm{p}$

Available from Superintendent of Documents, GPO 
A condensed source of reliable data and reference information for those working in the reactor field is presented. Volume I of the Handbook is divided into two sections. Section 1, Reactor Physics and Section 2, Radiation Shielding. Section 1 contains information on experimental methods, nuclear physics, kinetic theory of neutrons, reactor statics and reactor dynamics. Section 2 gives information on sources of radiation, permissible levels of radiation, gamma-ray attenuation, neutron attenuation, geometry, ducts through shields, heat generation in shields, shield optimization with respect to weight, and shield materials. Volume 2 contains information of the following subjects: light- and heavy-water-cooled systems, liquidmetal-cooled systems, gas-cooled systems, aqueous fuel systems, liquid-metal-fuel systems, fused-salt systems, handling and control, and reactor designs. An Appendix and Index are also given.

$\# 6508041 \quad * 2.082-4.082$

THE REACTOR HANDBOOK, VOLUME 3, SECTION 1: GENERAL PROPERTIES OF MATERIALS

AECD-3647, Atomic Energy Commission, Washington, D. C.

February 1955

$610 \mathrm{p}$

Available from Superintendent of Documents, GPO

This Handbook is a comprehensive and critical compilation of nuclear engineering data issued by the Atomic Energy Commission's Technical Information Service for reference use by scientists and engineers engaged in AEC reactor projects. The material presented represents the efforts of specialists in the various areas of reactor science and technology, and summarizes the accomplishments of the Commission's nuclear reactor program to date. Important nuclear reactor data are given in graphical and tabular form for the following metals and alloys: aluminum and its alloys; beryllium and its alloys; beryllia; beryllium carbide; bismuth carbides; cements and concretes; graphite; hydrides; lithium and its alloys; magnesium, molybdenum, nickel, plutonium, thorium, titanium, uranium, vanadium, zirconium, and their alloys: cobalt-base alloys; rare earths; silicon carbide; stainless steels; tungsten; and high-cross-section materials.

\#6508044

*2.2572-2.483

REACTOR PHYSICS CONSTANTS, SECOND EDITION

ANL-5800, Second Edition, Argonne National Laboratory, Argonne, Illinois

July 1963

$850 \mathrm{p}$

Available from Superintendent of Documents, GPO

This book of Reactor Physics Constants is a compilation of the latest and "best" values of constants, recipes, formulae, etc., which are necessary to calculate reactor characteristics. This edition is based on data available through March 15, 1961 . The values presented include data associated with the fission process; selected cross section data; the statics of thermal homogeneous and heterogeneous reactors; information pertinent to the analysis of control effectiveness, long-term changes, reactor kinetics, stability, and excursions in thermal reactors; multigroup calculation methods for intermediate and fast reactors. Also included are tables of representative multigroup constants, integral fast neutron physics data with emphasis on parameters for simple system analysis, and corresponding data for more complex reactor prototypes; basic shielding constants; the constants necessary for the interpretation of counting data, and the properties of neutron sources. Spectra and yields of both artificial and spontaneous fission neutron sources are presented in tabular form. Computer programs used in reactor physics and reactor design are described. A subject index is included.
Reynolds, J. T.; et al.

EVALUATED CROSS SECTIONS FOR THE HAFNIUM ISOTOPES

KAPL-3327, ENDF-112, Knolis Atomic Power

Laboratory, Schenectady, New York

August 17,1967

$54 \mathrm{p}$

Available from CFSTI

Evaluated libraries of cross sections have been prepared for natural hafnium and its isotopes Hf174, Hf-176, Hf-177, $\mathrm{Hf}-178, \mathrm{Hf}-179$, and $\mathrm{Hf}-180$. The libraries contain total, elastic, capture, inelastic, $(n, p)$, and $(n, 2 n)$ cross sections and elastic scattering Legendre moments below $15 \mathrm{MeV}$. The most recent experimental data were used in the evaluation, and whenever data were not available, theoretical calculations were made.

\#6908020 *2.2773-4.173-8.173-7.173

Rimshaw, S. J.; Ketchen, E. E.

CESIUM- 137 DATA SHEETS

ORNL-4186, Oak Ridge National Laboratory, Oak

Ridge, Tennessee

December 1967

$27 \mathrm{p}$

Available from CFSTI

Data on CS-137 are tabulated. Properties of the fuel forms CsCl, Cs2SO4 and cesium borosilicate glass are given. Twenty-seven references are included.

\section{$\# 6908021$}

*2.2773-4.173-8.173-7.173

Rimshaw, S. J.; Ketchen, E. E.

CURIUM DATA SHEETS

ORNL-4187, Oak Ridge National Laboratory,

Ridge, Tennessee

December 1967

$52 \mathrm{p}$

Available from CFSTI

Data on the fuel forms of $\mathrm{Cm}-244$ are presented. properties of $\mathrm{Cm}=244$ metal, Cm203, Cm202S, CmF3, Cm-242, $242 \mathrm{Cm} 203$ cermet are tabulated. Forty-nine references are given.

\#6908022

$\star 2.2773-4.173-8,173-7.173$

Rimshaw, S. J.; Ketchen, E. E.

STRONT IUM-90 DATA SHEETS

ORNL-4188, Oak Ridge National Laboratory, Oak Ridge, Tennessee

December 1967

$45 \mathrm{p}$

Available from CFSTI

Data are given on composition, specific power, radiation, critical mass, container compatability, thermophysical properties, mechanical properties, chemical properties, biological tolerance, and shielding for strontium-90 metal, titanate (SrTi03), oxide (ST03), fluoride (ŚF2), and orthotitanate (Sr2Ti04): The data are indexed to 53 references which are also included.

$\# 6910039$ $* 2.2273$

Rose H, J. Brink, D. M.

ANGULAR DISTRIBUTIONS OF GAMMA RAYS IN TERMS OF PHASE-DEFINED REDUCED MATRIX ELEMENTS Reviews of Modern Physics, 39:306-347 Apri1 1967

The theory of angular distributions of gamma-rays is developed systematically, aiming at a phase consistent derivation of angular distribution formulas for gamma rays emitted in the decay of an aligned initial state. The development starts from first principles, that is, the angular distribution formulas are derived directly from perturbation theory and all quantities introduced are carefully and explicitly defined. In particular the mixing ratios are phase consistently related to reduced matrix elements of interaction multipole operators which again are well defined in phase. Hence the 
mixing ratios become physical quantities which can be extracted from angular distribution measurements and then compared in both magnitude and sign with the predictions of nuclear models (especially the independent particle model). Critical stages in the theoretical development at which either a choice of phase convention has to be made or transformation properties enter are emphasized. In the last section, reduced matrix elements of the interaction multipole operators are given explicitly for one- and two-particle states and one- and two-hole states of the independentparticle model.

\section{\#6903037}

Rotenberg, M.; et al.

THE 3-j AND 6-j SYMBOLS

Cambridge, Massachusetts: The Technology Press, Massachusetts Institute of Technology

1959

$498 \mathrm{p}$

This book contains the tabulation of the $3-j$ and 6-j symbols, which are the Wigner modified clebschGordan coefficients in the case of two particles and the Racah $W$-coefficients in the more complicated cases of quantum mechanical problems. Their high symmetry allows a tremendous abbreviation in the length of the table, with none of the concomitant hardships in finding a particular symbol. At most, only the sign of the $3-j$ symbol is changed under permutations of the parameters. A complete table is compiled in the sense that a large-scale digital computer would probably be necessary for problems requiring symbols beyond the range that is tabulated. The $3-j$ and $6-j$ coefficients are tabulated in steps of $1 / 2$ up to any angular momentum whose magnitude is less than or equal to 8 units. The special $3-j$ symbol with al1 magnetic quantum numbers zero is tabulated for $j \leqq 16$ units. The introduction gives a collection of formulas involving the $3-j$ and $6-j$ symbols, with brief mention of the $9-j$ and 12-j symbols.

\section{\#6912004}

Sadoulet, B.

DATA COMPILATIONS OF ANTIPROTON-PROTON REACTIONS INTO ANT IHYPERON-HYPERON

CERN-HERA 69-2, HERA Group (High Explosive Rocket Assisted Group), CERN European Organization for Nuclear Research, Geneva, Switzerland

April 20, 1969

$80 \mathrm{p}$

Available from Tech. Info. Div., University of California, Berkeley, California

This compilation presents existing data on the differential cross sections for the reactions antiproton-proton into ten different antihyperonhyperon channels.

\section{\# 6908027}

*2. 573

Sargis, D.; Baxter, A.; Archibald, R.

A BENCHMARK STUDY 'OF '2PR-III ASSEMBLY 48 USING ENDF/B CROSS SECTIONS

GA-8847, Gulf Genera1 Atomic, Inc., San Diego,

California

August 22, 1968

$35 \mathrm{p}$

A study of the fast critical reactor 2 PR-III Assembly 48 has been performed using ENDF/B cross sections. Minor alterations of the ENDF/B data were made to conform with the format requirements of the cross section preparation programs. comparison was made of experimental and calculated results for various integral quantities, including central reactivity worths, fission and capture ratios, and Doppler and sodium-void coefficients. The comparison indicated some possible areas of uncertainty in the basic cross section data. Real and adjoint neutron spectra were also computed for comparison with calculated and measured results The study was performed primarily to test the unmodified ENDF/B data file in cooperation with the Cross Section Evaluation Working Group's evaluation program. For this reason modifications to the ENDF/B data were limited to minor alterations and energy range corrections.

\#6911024

Sasse, S.

TABLES OF TOTAL GAMMA ATTENUATION COEFFICIENTS OF THE ELEMENTS AT $0.01 \mathrm{MeV}$ TO $100 \mathrm{MeV}$

ABS-THH-1040, Technische Hochschule, Arbeitsgruppe fuer Bautechnischen Strahlenschutz, Hanover, Germany

July 1967

$28 \mathrm{p}$

In German

Available from AEC Dep. Libraries

The total attenuation coefficients were extrapolated from the values for 22 elements experimentally determined by Hubbel1 and Berger. The results are tabulated.

\#6510007

*2. 1023

Schint lmeister, $J$

DER ISOSPIN VON ATOMKERNEN (THE ISOSPIN OF ATOMIC NUCLEI)

Berlin: Akademie-Verlag

1960

$163 p$

In German

Isobaric spin and similar states of atomic nuclei, isobaric spin and the hypothesis of the density independence of nuclear forces, and the isotopic spin of light nuclei are discussed. Topics covered include comparison of the forces which act between nucleons, the concept of isobaric spins, selection rules, level displacement in isobaric nuclei on account of the coulomb energy and the mass difference between the neutron and proton, isobaric spin of several nuclei, unambiguity of isobaric spins, isobaric spin and gamma radiation and effects on nuclear excitation, isobaric spin and photoreactions of the types (gamma, alpha) and (gamma,d), isobaric spin of mesons and light particles, the quantum mechanical formulation of the density independence, energy levels of isobaric nuclei, selection rules for isobaric spin and nuclear reactions, the isotopic spin of nucleons, and levels of light nuclei.

\# 6911002 * 2.1273

Schirmer, W.; Waechter, N.

TABLE OF" SPECIFIC ACTIVITIES OF THE NUCLIDES WITH $2=88$ TO $2=194$

Actinides Reviews, 1:125-134

1968

The specific activities of a 11 the nuclides of the elements from $\mathrm{Ra}$ through $\mathrm{Ku}$ were calculated on the basis of the decay data published by A. H. Wapstra (Actinide Reviews, $1: 39-53,1967$ ). Data for shortlived nuclides are included mainly in view of their genetic relation with longer-lived nuclides.

\#6911028

Schmidt, J. J.

NEUTRON CROSS SECTIONS FOR FAST REACTOR MATERIALS PART I. EVALUATION

KFK-120 (Part 1) (EANDC-E-35"U"), Kernforschungszentrum, Institut fuer Neutronenphysik und Reaktortechnik, Karlsruhe, West Germany

February 1966

$1317 \mathrm{p}$

Available from AEC Dep. Libraries

A compilation of neutron cross sections and resonance parameters for $\mathrm{C}, \mathrm{Cr}, \mathrm{He}, \mathrm{H}, \mathrm{Fe}, \mathrm{Mo}, \mathrm{Ni}$, 0 , $\mathrm{Pu}=239, \mathrm{Na}, \mathrm{U}-235$, and $\mathrm{U}-238$ is presented. The techniques that were used in the analyses of the data are described. Neutron energies in.the range from $0.01 \mathrm{eV}$ to $10 \mathrm{MeV}$ are covered. 
\#6608014

*2.1673

Seeger, P. A.

A TABLE OF BINDING ENERGIES AND PARTICLE SEPARATION ENERGIES FROM A MASS LAW

LA-3380-MS Los Alamos Scientific Laboratory, Los Alamos, New Mexico

September 1, 1965

$108 p$

Available from CFSTI

A table of approximately 10,000 masses for nuclei with $22 \leqq z \leqq 120$ and $22 \leqq N \leqq 184$ is presented. The mass law used is one developed for application to the astrophysical problem of nucleosynthesis by rapid neutron capture.

\# 6604066

*2.083-2.2583

Seth, K. K.

S-WAVE NEUTRON STRENGTH FUNCTIONS

Nuclear Data, A2:299-332

september 1966

The concept of neutron strength functions and the experimental methods of measuring them are reviewed briefly. S-wave neutron strength functions, $S(0)$, obtained from experimental data on neutron total cross sections at energies below $700 \mathrm{keV}$ are presented in tabular form. Publications and private communications published between 1954 and July 1966 have been covered. Recommended values of $S(0)$ are listed and plotted as a function of atomic weight. Theoretical predictions based on the nuclear optical model are also presented for purposes of comparison.

$\$ 6910009$

$* 2.1673$

Shirley, D. A.

TABLE OF HYPERFINE FIELDS

Appendix $B$ in Hyperfine Structure and Nuclear Radiation, International Conference on Hyperfine Interactions Detected by Nuclear Radiation Pacific Grove, California, A Agust 25-30, 1967, edited by E. Matthias and D. A. Shir

1968

$6 \mathrm{p}$

Hyperfine fields are tabulated for impurities in $\mathrm{Fe}, \mathrm{Co}$, and $\mathrm{Ni}$; a few entries are also given for $\mathrm{G}$ as host. References are not exhaustive. Columns 2 and 5 are, respectively, the impurity and host elements. Column 3 gives the isotope number. Column 4 gives the solute concentration in atomic percent. The fields appear in Column 7 . No hyperfine-anomaly corrections have been made.

\section{\#6910010}

Shirley, V. S.

TABLE OF NUCLEAR MOMENTS

Appendix C from llyperfine Structure and Nuclear Radiation, International Conference on Hyperfine Interactions Detected by Nuclear Radiation, Pacific Grove, California, August $\frac{25-30}{1967}$, edited by $E$. Matthias and D. A. Shirley, pp 985-1041

New York: John Wiley \& Sons, Inc.

1968

$57 \mathrm{p}$

Small differences between the mean squared charge radii of excited states and ground states of nuclei have been deduced from isomer shifts of recoilless gamma-rays. The results of such experiments to data are summarized in this table.

\#6510009,6510003 *2.2673

Singru, R. M.; Steffen, R. M.

TABLES FOR TIIE ANALYSIS OF BETA-GAMMA ANGULAR CORRELATION DATA INVOLVING FIRSTT-FORBIDDEN BETATRANSITIONS IN A $1-(B) \rightarrow 2^{+}(\gamma) \rightarrow 0^{+}$CASCADE

TID-19714, Purdue University, Department of Physics, Lafayette, Indiana

August 1963

$626 \mathrm{p}$

Available from CFSTI
Formulas are presented for the shape factors, anisotropy coefficients, gamma circular polarizations, and parameters representing the contributions of the various nuclear matrix elements for $1^{-}$(beta) $\rightarrow 2^{+}$(gamma) $\rightarrow 0^{+}$transitions. The first three quantities are tabulated for a variety of parameters and energies. Volume I contains values for $\mathrm{Tm}-170$, $\mathrm{Re}-186$, and $\mathrm{Ir}-194$; values for $\mathrm{Pr}-144$ and Eu-152 are tabulated in Volume II.

\#6604066

$\star 2.083-2.2783$

Skorka, S.J.; Hertel, J.; Retz-Schmidt, T.W.

COMPILÁTION OF ELECTROMAGNETIC TRANSITION RATES IN

LIGHT NUCLEI (A $\lesssim 40$ )

Nuclear Data, A2:347-401

November 1966

Data on absolute electromagnetic transition rates in nuclei of the $1 p-$ and $2 s-1 d-s h e l l s$ are presented. Table $I$ lists the experimental results for partial widths with quoted errors, transition strengths, and reduced transition probabilities. This table also contains averages and mean errors of the averages in cases where more than one experimental result on a certain transition is available. Table II contains additional information, mainly mixing ratios which were used to calculate transition strengths in mixed transitions, as well as widths or lifetimes which could not be further reduced. Branching ratios are not listed. Table III presents a survey of mean transition strengths and of standard deviations from the mean values for the various multipole types of transitions, subdivided according to the isospin selection rules. The distributions of the transition strengths are presented in a number of histograms. The dependence of the transition strengths on the mass number and excitation energy, as well as $T$-forbiddeness factors, are discussed and in some cases graphically illustrated.

$\# 6500022$

$\star 2.2373,2.2573$

Smith, D.B. (Editor)

CHARGED PART ICLE CROSS SECTIONS - NEON TO CIIROMIUM

LA-2424, Los Alamos Scientific Laboratory, Los Alamos, New Mexico

June 1960

$137 \mathrm{p}$

Available from CFSTI

Plots of cross sections for charged particle reactions are presented for $\mathrm{Ne}: 20$ through $\mathrm{Cr}-53$. A bibliography is included.

\#6908052

*2. 2573

Smi th, J.R.; Grimesey, R.A.

AN EVALUATION AND COMPILATION OF NEPTUNIUM-237 CROSS SECTION DATA FOR THE ENDF/B FILE

IN-1182, Idaho Nuclear Corporation, I daho Falls, I daho

May 1969

$85 \mathrm{p}$

Available from CFSTI

This evaluation of $\mathrm{Np}-237$ neutron cross-section data was undertaken as part of the effort by the Cross Section Evaluation Working Group (CSEWG) to assemble the first version of the Evaluated Nuclear Data File B (ENDF/B). Graphs of calculated and experimental data from which the various files for this isotope were constructed are included along with complete documentation over the energy range from $0.00001 \mathrm{eV}$ to $15 \mathrm{MeV}$.

$\# 6604066$

Sood, P.C.

$* 2.083-2.1183$

COMPARATIVE STUDY OF TWO-PARAMETER MODELS FOR PREDICTING ROTATIONAL ENERGIES IN EVEN-EVEN NUCLEI

Nuclear Data, A 4:281-300

An objective comparison of the various twoparameter models proposed for the description of rotational states in even-even nuclei is carried 
out to assess the reliability of their extrapolation for prediction of as yet unobserved levels. The comparison involving energy ratios $E(I) / E(2)$ contains no arbitrarily adjustable parameters. The semi-empirical formula

$$
E(I)=A\left\{1-\frac{(B / A) I(I+1)}{I+N(B / A) I(I+1)}\right\} I(I+1)
$$

is seen to give the best fit. A least-squares fit to observed levels with this formula gives a rms deviation of better than 0.5 in nearly all cases. The calculated values of the rotational constants $A$ and $B$, and the predicted energies of rotational levels with spin up to $I=16$ are tabulated.

$\# 6509018$

$$
* 2.473
$$

Spinrad, B.I.; Meier, R.W.

FIRST BIENNIAL REPORT OF THE ACTIVITIES OF THE EUROPEAN AMERICAN COMMITTEE ON REACTOR PHYSICS

EACRP-U-17, European Nuclear Energy Agency, Paris, France

June 1964

$30 \mathrm{p}$

The activities of the committee are sumnarized including comments concerning the status and accomplishments of the committee at the end of the period. Information is included on comnittee initial formation and on functions and organization. The committee was concerned with heavy water lattice physics, lattice evaluation, thermal reactor neutron spectra, thermal reactor burnup physics, criticality predictions, irradiation experiments, nuclear data, computer codes, detector standardization, and critical experiments.

\section{$\# 6500083$}

*2.2683

Stegun, I.A. (Editor)

TABLES FOR THE ANALYSIS OF BETA SPECTRA

NBS Applied Mathematics Series No. 13, National Bureau of Standards, Washington, D. C.

June 1952

$62 \mathrm{p}$

Available from Superintendent of Documents, GPO

The primary purpose of these tables is to assist in the theoretical analysis of experimental data on beta-ray spectra. This analysis involves the use of the CAMMA function of a complex argument, a function which cannot be readily evaluated and which has not yet been extensively tabulated. It is hoped that the present tables can be effectively utilized for most of the current and of the forseeable needs of analysis of beta-spectra.

\section{\#6508059,6604018}

$$
\text { * } 2,2573
$$

Stehn, J.R.; et al

NEUTRON CROSS SECTIONS VOLUME I, $2=1$ to 20 , VOLUME II I, $2=88$ to 98

BNL 325, Second Edition, Supplement No. 2, Brookhaven National Laboratory, Upton, New York

May 1964, February 1965

$300 \mathrm{p}, 300 \mathrm{p}$

Available from CFSTI

The neutron cross section data for the elements $Z$ = 1 to 20 and $2=88$ to 98 are presented. The volumes are arranged strictly by chemical elements and by their isotopes. Thermal cross sections, resonance parameters, and cross section curves for one element or isotope are together on successive pages, separate from the next element or isotope. The format is fluid, varying from one page to the next to suit the information to be presented. The reference sources are listed as close to the data as is feasible.
\#6510013

Steige1mann, W.H.

RADIOISOTOPE SHIELDING DESIGN MANUAL

NYO-10721, Frank1in Institute, Laboratories for Research and Development, Philadelphia, Pennsylvania

July 1963

$138 \mathrm{p}$

Available from CFSTI

Forty-two radioisotope sources, 5 additional equilibrium combinations of parent-daughter isotopes, and mixed fission products with 5 decay times are considered. Graphs are given of unshielded dose rate vs. distance from a point source, for gamma, $X$, and neutron radiations. Dose attenuation vs. Shielding thickness is plotted for 5 shielding materials - water, concrete, steel, lead, and uranium. Methods of calculation are described.

\# 6608021

Stelson, P.H.

*2. 2673

INFORMATION ON CONVERSION COEFFICIENTS FROM COULOMB EXCITATION AND LIFE-TIME MEASUREMENTS

ORNL-P-1419, Oak Ridge National Laboratory, Oak Ridge, Tennessee

July 1965

$22 \mathrm{p}$

Available from CFSTI, Conf-650525-5

This is a paper presented on the International Conference on the Internal Conversion Processes, Vanderbilt University, Nashville, Tennessee, May $10=13$, 1965. It reviews the available information on total E2 conversion coefficients for even-even $2^{-} \rightarrow 0$ transitions for rare-earth and very heavy nuclei. Also, tables of conversion coefficient data, both experimental and theoretical, are presented.

$\# 6604066$

Stelson, P.H.; Grodzins, L

NUCLEAR' TRANSITION PROBABILITY, B(E2), FOR $0^{+g_{S} \rightarrow 2}+2^{+}$irst TRANSITIONS AND DEFORMÁTION PARAMETER, beta

Nuclear Data, Al:21-102

Decenber $\frac{\text { ata }}{1965}$

Adopted values for the reduced electromagnetic transition probability, B (E2) from the ground to the first-excited $2^{+}$-state of even-even nuclei are given in Table I. Values of beta, the quadrupole deformation parameter, and of tau, the mean life of the $2^{+}$state, are also listed there. Table II presents the data on which Table I is based, namely the experimental results for $B(E 2)$ values with quoted errors. The literature has been covered to January 1965. The ratio of the value of beta, to values expected from the single-particle model ${ }^{2}$ is presented graphically as a function of neutron number. As an indication of the extent of collective features in nuclear structure, this ratio seems a better measure than the value of beta ${ }_{2}$ itself.

\#6907012

$$
\text { *2. } 2573
$$

Stephenson, T.; Prince, A.; Pearlstein, S.

CROSS SECTION EVALUATION CENTER: EVALUATION OF THE NEUTRON CROSS SECTION OF MANGANESE FOR THE ENDF/B L IBRARY

BNL-50060(T-463), ENDF 101, Brookhaven National Laboratory, Upton, New York

June 1967

$54 \mathrm{p}$

Available from CFSTI

This report describes the collection and choice of Mn-55 data placed in the proper format for the Evaluated Nuclear Data File - Version B, ENDF/B. These data are subject to further examination and possible revision and do not necessarily constitute the data for $\mathrm{Mn}-55$ which are contained in the current ENDF/B Library, although any changes that occur should be minor. The description of how the 
evaluation was performed is contained in Sections III and IV. Section III deals with the manganese cross section below $80 \mathrm{keV}$, and Section $1 \mathrm{~V}$ deals with the manganese cross section from $80 \mathrm{keV}$ to 20 $\mathrm{MeV}$. A complete listing of the $\mathrm{Mn}-55$ data in the ENDF/B format is presented at the end of this Report.

\#6908009

$$
\text { *2. } 2573
$$

Stewart, L.

EVALUATED NEUTRON CROSS SECTIONS FOR TRITIUM

LA-3270, Los Alamos Scientific Laboratory, Los

Alamos, New Mexico

April 1965

$73 p$

Available from CFSTI

Neutron cross sections on $T$ are tabulated from $0.025 \mathrm{eV}$ to $20 \mathrm{MeV}$. Graphs and tables are presented of the total, elastic, nonelastic, ( $n$, gamma), $(n, 2 n)$, and $(n, 3 n)$ excitation functions, as well as for the angular distributions of the neutron elastic scattering from $T$.

\#6908024

$$
\text { * } 2.2273
$$

Storm, E.; Israel, H.I.

PHOTON CROSS SECTIONS FROM 0.001 TO $100 \mathrm{MeV}$ FOR ELEMENTS 1 TIIROUGH 100

LA-3753, Los Alamos Scientific Laboratory, Los Alamos, New Mexico

June 1967

$257 \mathrm{p}$

Available from CFST 1

The report contains tables of photon cross sections in the range 0.001 to $100 \mathrm{MeV}$ for $2=1$ to 100 . Supplementary tables of mass attenuation coefficients for air, water, concrete, and sodium iodide; $x$-ray atomic energy levels; line energies; average energies; relative intensities; and fluorescence yields are also presented.

\section{\#6509006}

Strain, J.E. ; Ross, W.J.

$14 \mathrm{MeV}$ NEUTRON REACTIONS

ORNL-3672, Oak Ridge National Laboratory, Oak Ridge, Tennessee

January 1965

$50 \mathrm{p}$

Available from CFSTI

Reactions observed when pure, naturally occurring elements $(2=1$ to $2=30)$ are irradiated with unmoderated $14 \mathrm{MeV}$ neutrons produced by the $\mathrm{T}-3(\mathrm{~d}, \mathrm{n}) \mathrm{He}-4$ reaction are listed. Cross sections are calculated where possible, and normalized standard gama spectra are collected. These standard spectra are equivalent to the gamma radioactivity that will be produced when $1 \mathrm{~g}$ of the pure element is irradiated for a given length of time at a $14 \mathrm{MeV}$ neutron flux of $5 \times 10^{\circ}$ neutrons per sq. cm per sec. If more than one radionuclide is produced, a spectral decay is presented. These spectra may be used to predict accurately the sensitivity of nondestructive spectral analysis of almost any sample.

$\# 6500085$

*2.173-2.2673

Strominger, D.; Hollander, J.M.; Seaborg, G.T.

TABLE OF 1SOTOPES

Reviews of Modern Physics, 30:585-904

Apríl 1958

The $t a b l e$ represents a complete list of all the radioactive and stable isotopes of the elements, together with a number of their salient features, as recorded in the literature or by private communication as of approximately February 1958. The system of references developed for the last edition of the $\mathrm{T}$ able of Isotopes has been retained. The format of the table is as follows: The first column contains the atomic numbers, chemical symbols, and mass numbers of the nuclear species. An individual entry has been made for each nuclear state whose half-life is long enough for the species to have been separated chemically or mechanically from other activities; the shortest half-life so listed is approximate $1 y$ o.01 sec. Information about metastable states with half-lives shorter than $\sim 0.01 \mathrm{sec}$. is included with the other gamma-ray transition data. An exception to this policy is made when the mass assignment of a shortlived metastable state is not known; in this case a separate entry is made for the activity regardless of the half-life. Metastable excited states lister. in this column are denoted by the superscript " $\mathrm{m}$ " following the mass number and in those cases in which two isomeric states are listed, they are distinguished by use of superscripts " $m$ " and " $m$ ". Half-life values are listed without qualification where the determination has been a direct measurement of decay rate. The half-lives of nuclear states not included as separate entries are included in column four. An attempt has been made to list the most precise value or values first. The entry denoting mode of decay is preceded by the special symbol for radiation. The degree of certainty of each isotopic assignment is indicated by a letter code. The means by which the mass assignments were made are tabulated. Data on genetic relationships are located in column three underneath the class-assignment information. The relative isotopic abundances for the elements are given in accordance with the "best values" listed in the report by K.T. Bainbridge and A.O. Nier, except as supplemented by more recent values. Unless otherwise stated, the measured value of the nuclear moment is that of the ground state. The energies of particles and photons are included together in column four. Energy-level diagrams have been drawn in many cases; these are not necessarily complete representations of the data, but include only those features which seem to be reasonably we1l established and unambiguous.

$\# 6910037$

Sumi, $Y$.

$* 1.2073$

MESON-NUCLEON ELASTIC AND CHARGE EXCHANGE PROCESSES progress of Theoretical physics (Kyoto) Supplement, $41-42: 3-5 \frac{1}{2} 1967$

An extensive survey of experimental data on elastic piN and $K N$ interactions up to $30 \mathrm{GeV} / \mathrm{c}$ is systematically arranged and presented. The dat include total and total elastic cross sections as a function of the incident laboratory momentum $\left(p_{1}\right)$, elastic differential cross sections, polarization of recoil proton, and charge-exchange scattering. Values of $p$, observed quantities, and the experimental technique are presented for the 113 references, which represent the main part of the experimental information published from 1960 to 1966.

\#6910038

*1. 2073

Sumi, Y.; Yoshida, T

NUCLEON-'AND ANTINUCLEON-NUCLEON ELASTIC AND CHIARGE EXCHANGE PROCESSES

Progress of Theoretical Physics (Kyoto) Supplement, $41-42: 53-89$

1967

Experimental data on nucleon-nucleon and nucleonantinucleon elastic interactions in the high energy region are summarized and presented. The data include total and total elastic cross sections: singular distribution of elastic and charge. exchange cross section; and recoil proton polarization, together with the ratio of the real to imaginary forward amplitude in the pp elastic scattering. In the appendix several kinematical quantities are tabulated. Values of the incident laboratory momentum, observed quantities, and the method used are presented for the 67 references which cover a main part of the experimental information published from 1960 to 1966. 
\#6511005

* $8.172-2.772$

TABLES OF DATA,

THERMODYNAMICS OF NUCLEAR MATERIÁLS

vienna, Austria: International Atomic Energy Agency, STI/DOC/10/38

December 1964

$95 \mathrm{p}$

Available from UN Headquarters

A compilation is presented of data on the thermodynamic properties of materials of interest for nuclear technology. The data were released in the Symposium on the Thermodynamics of Nuclear Materials, which was held in May 1962. The properties covered include: heats of formation, free energies of formation, entropies of formation, and standard entropies at $298^{\circ}$ and at other temperatures; enthalpy increments, free energy functions, entropy increments; enthalpies, free energies, entropies of transitions; heats, free energies, entropies of reaction; heat capacities: vapor pressures; thermodynamic properties of mixtures; melting points, densities; surface tensions; and lattice parameters.

\section{\#6511015}

Tajima, E.

GAMMA-RAY TABLE

Radioisotopes (Tokyo), 12:206-233

May 1963

In Japanese

(No abstract)

\# 6911004

*1. 1173

Taylor, B.N.; Parker, W.H.; Langenberg, D.N.

DETERMINATION OF $\mathrm{e} / \mathrm{h}$, USING MACROSCOPIC QUANTUM PHASE COHERENCE IN SUPERCONDUCTORS: IMPLICATIONS FOR QUANTUM ELECTRODYNAMICS AND THE FUNDAMENTAL PHYSICAL CONSTANTS

Reviews of Modern Physics, 41:375-496

The implications of the new determination of e/h using the ac Josephson effect in superconductors for both quantum electrodynamics(QED) and our knowledge of the fundamental physical constants are analyzed in detail. The implications for QED are investigated by first deriving a value of the fine structure constant alpha from experimental input data which do not require the use of QED theory for their analysis. These include the Josephson-effect value of $e / h$, the Faraday constant, the gyromagnetic ratio of the proton, the magnetic moment of the proton in untis of the nuclear magneton, the ratio of the ampere as maintained by the United States National Bureau of Standards to the absolute ampere, and certain accurately known auxiliary constants.

\section{\#6511006 *8.172-2.772}

TIIERMODYNAMICS OF NUCLEAR MATERIALS, PROCEEDINGS OF THE SYMPOSIUM ON THERMODYNAMICS OF NUCLEAR MATERIALS HELD BY THE INTERNATIONAL ATOMIC ENERGY AGENCY IN VIENNA, 21-25 May 1962

Vienna, Austria: International Atomic Energy Agency, STI / PUB / 58

September 1962

$822 \mathrm{p}$

Available from IAEC

The International Atomic Energy Agency initiated a project to assist in disseminating and assessing data on important nuclear materials. Thus, the symposium was organized in an attempt to present and appraise the results of the work on the rmodynamics of nuclear material; to assess their reliability and further to discuss the application of the results and principles of thermodynamics to the solution of current problems in nuclear technology. Particular emphasis was placed on the thermodynamics of actinides and vaporization processes.
$\# 6604066$

$* 2.083-2.2083$

Thomps on, W.J

PENETRATION FACTORS AND RESONANCE MIXING PHASES FOR ISOBARIC ANALOG STATES

Nuclear Data Tables, A6:129-140 June 1969

Graphs are presented of penetration factors, $P_{n}$, to enable neutron spectroscopic factors, $S$, to be evaluated directly from proton elastic- and inelastic-scattering is obaric-analog-resonance partial widths, gamma , according to the relation

$$
S=(N+1-Z) \text { GAMMA } p^{p_{n}},
$$

where $N$ and $Z$ are the numbers of target neutrons and protons, respectively. Also presepted are graphs of the resonance mixing phase, phic which is required in the extraction of gamma from data. The $Z$-range is 16 to 82 in varying steps convenient for interpolation. The angular momentum range is $L$ $\mathrm{L}=0$ (1) $4, \mathrm{~J}=\mathrm{L} \pm \mathrm{I} / 2(\mathrm{~J}>0)$. A standard set of optical-model parameters is used.

\section{\#6604066}

Towner, I.S.: Hardy, J.C.

TABLES OF FRÁCTIONAL PARENTAGE COEFFICIENTS IN THE ISOSPIN FORMALISM FOR THE $j=3 / 2$ AND $5 / 2$ SHELLS

Nuclear Data Tables, A6:153-234

Values of one- and two-particle coefficients of fractional parentage in the isospin formalism have been computed for the $j=3 / 2$ and $5 / 2$ sheils. These values are tabulated for the first half of each shel1. For the second half particle, hole formulae are quoted by which the coefficients of fractional parentage may be readily obtained.

\#500077 $\star 2.2473$

Troubetzkoy, E. : Goldstein, H.

COMPILATION' OF 'INFORMATION ON GAMMA-RAY SPECTRA RESULTING FROM THERMAL-NEUTRON CAPTURE

ORNL-2904, Oak Ridge National Laboratory, Oak Ridge, Tennessee

January 18,1961

$75 p$

Available from CFSTI

Thermal neutron capture gamma-ray spectra are compiled for various nuclei that are useful for reactor shielding applications. The bulk of the data is taken from a Chalk River group and a group at the Institute of Atomic Energy of the Academy of Sciences, USSR. A11 the data are presented in tabular form for seven energy intervals from 0 to above $9 \mathrm{MeV}$. In addition, figures illustrating the appearance of the spectra are given, including continuous differential spectra for 50 nuclei and line spectra for 15 nuclei. The line spectra consist of vertical lines having heights proportional to intensities. Eighty references are provided.

\#6508054 * 2.573

Trower. W.P.

HIGH-ENERGY PARTICLE DATA, VOLUME III . KINEMATICS OF PARTICLES AS A FUNCTION OF NOMENTUM

UCRL-2426 Vol. III (1965 Rev.), California University, Lawrence Radiation Laboratory, Berkeley, California

January ig65

$112 \mathrm{p}$

Avai lable from CFSTI

This volume is a revision of the 1963 UCRL-2426 Volume III and is divided into two sections. The first section displays the kinematic quantities $T$, (gamma-1), H. beta, or (1-beta), and $P_{\text {pet }}$ as functions of particle momentum for the mostetommon elementary particles. The second part presents the dynamic quantities pi lambda squared, nuM, beta or (1-beta), nu, (gamma-1) and $w$ as functions of incident-particle momentum for the most common 
elementary particles interacting with a proton. The particles SIGMA $^{-}$, SIGMA $^{\circ}, \mathrm{CHI}^{\circ}$ and $n$ were not plotted, being so close in mass to one of the particles that has been included that they cannot be distinguished graphically. Mass, energy, and momentum are expressed in terms of energy.

\section{\# 6500650}

$$
\text { *2. } 2573
$$

Trubey, D.K.; Emmett, M.B.

A COMPARISON OF FIRST- AND LAST-FLIGHT EXPECTATION VALUES USED IN AN 05R MONTE CARLO CALCULATION OF NEUTRON DISTRIBUTIONS IN WATER

ORNL-RSIC- 3, Oak Ridge National Laboratory, Oak Ridge, Tennessee

1965

$25 p$

Available from the RSIC, ORNL, Oak Ridge, Tennessee

The distributions in water of fast neutrons from isotropic fission and $14 \mathrm{MeV}$ point sources have been calculated with OSR code. Two methods of scoring the same case histories were used to estimate the energy distribution and the dose rate. The estimators employed were the "first-" and "last-" flight expectation, or statistical estimation, values. The first-flight estimator was found to be somewhat better due to the importance of the first flight in the total transport of the neutrons. The results are in good agreement with RENUPAK moments method results and with experimental results.

\section{\#6911021}

Uken, E.A.; Steele, T.W.

$$
\text { *2. } 2283
$$

TABLE OF THE ISOMERIC STATES OF STABLE NUCLIDES

NIM-41, National Institute for Metallurgy, Johannesburg, South Africa

June 15,1966

$29 \mathrm{p}$

Available from AEC Dep. Libraries

This set of $t$ ables lists all known isomeric transitions of the stahle nuclei, obtained by the nuclear reactions marked with a cross in "Table of Neutron Cross Sections." The reacting stable nuclide and its isotopic abundance in nature (quoted as percentages) are cited in increasing order of atomic number. Opposite each reaction are listed the half-life and the gamma-ray energies observed for the transition of the isomeric state as well as of the ground state. Gamma-ray energies are shown in order of decreasing intensity. In the last column the calculated Q-value, in $\mathrm{MeV}$, is cited to indicate the energy balance of the reaction.

\section{$\# 6511003$}

$\star 8,182-2,782$

URANIUM-CARBON AND PLUTONIUM-CARBON SYSTEMS, A THERMOCHEMICAL ASSESSMENT, A REPORT FROM THE PANEL ON THERMOUYNAMIC PROPERTIES OF THE URANIUM-CARBON AND PLUTONIUM-CARBON SYSTEMS HELD IN VIFNNA, 8-12 OCTOBER 1962

Vienna, Austria:International Atomic Energy Agency, ST I DOC $/ 10 / 14$

February 1963

$48 \mathrm{p}$

Available from International Atomic Energy Agency

Thermodynamic data reported by various laboratories on U-C and Pu-C systems are reported. Efforts were made to resolve various discrepancies. Sections are included on preparation of uranium carbides, phase diagrams, heat capacities and thermal properties of the actinide carbides, heat of formation of the uranium carbides, vaporization studies, high-temperature equilibrium measurements, and the $\mathrm{Pu}-\mathrm{C}$ system.
\#6911009

$\star 2.2583$

Vidal, R. ; Roullier, F.

TABLE OF RESONANCE INTEGRALS

Preprint No. $\mathrm{CN}-23 / 73$, International Atomic Energy Agency, Vienna, Austria

1966

$14 \mathrm{p}$

Available from the International Atomic Energy Agency

In French

Tables of resonance integrals are presented which have been reduced to a common definition using sigma $(B-10)=38406$, Sigma $(\mathrm{Au})=98.86, \mathrm{E}_{0}=$ $0.025 \mathrm{eV}, \quad I(A u)=15406$ as standard values and corrected to a lower cutoff of $\mathrm{E}=0.55 \mathrm{eV}$. The corrected resonance integrals are tabulated for several elements, references are given for each measured value and an adopted value recommended. A table of adopted resonance integrals for the natural elements and for several isotopes is also presented.

$\# 6604066$

Viola, V.E., Jr.

$* 2.083 \cdot 2.2483$

CORRELATION OF FISSION FRAGMENT KINETIC ENERGY DATA Nuclear Data, A1:391-410

July 1966

Recent measurements of the most probable kinetic energy release in fission are compared in order to study the dependence on nuclear species. Appropriate corrections to data obtained with semi-conductor detectors, particularly with regard to calibration procedures, are discussed and applied. Corrected results are listed in Table I and plotted in Fig. 1. It is found that the most probable kinetic energy release in fission can be simply expressed by the function

$$
\mathrm{E}_{\mathrm{k}} \quad=0.1071 \mathrm{z}^{2} / \mathrm{A}^{1 / 3}+22.2 \mathrm{MeV} \text {. }
$$

The corrected <Ek > values are compared with recent liquid drop calculations and good agreement is found for $Z^{2} / A$ less than 35 , but not above this value. Effects of mass asymmetry are also briefly discussed. Analysis of the data indicates that the most probable kinetic energy release is essentially independent of excitation energy. Use was made only of results published since 1961. Prior data can be found in the review of Huizenga and Vandenbosch.

$\# 6509005$

$\star 2.2083$

Wallin, A.; et a1.

OPTICAL MODEL OF THE NUCLEUS. AN INDEXED AND

ABSTRACTED LITERATURE REVIFIV

FOA 4 rapport A 4374-411, Foersvarets Forsknings anstalt, Stockholm, Sweden

April 1964

$108 \mathrm{p}$

Available from Foersvarets Forskningsanstalt, Stockholm 80, Sweden

Al1 available literature, dealing with optical mode 1 calculations, was reviewed to aid in assessing suitable well parameters and to indicate when the model is successful in predicting various nuclear data. The material investigated was indexed according to nuclide, and short abstracts were prepared to provide a concise summary of the contents and main results. Three hundred forty-two references are examined.

$\# 6908055$

$$
* 2.2183
$$

Wapstra, A.H.

ACT INIDE FINGERPRINTS

Actinides Review, 1:39-53

September 1967

Tables and charts are presented in which known data are conveniently arranged for rapid identification of the actinide nuclides from their nuclear radiations. 
$\$ 6604066$

*2. $183-2.1233$

Wapstra, A.H.

TWO-PROTON AND TWO-NEUTRON BINDING-FNERGY

SYSTEMATICS AND ALPHA-DECAY ENERGIES

Nuclear Data, A1:1-19

December $\frac{\text { ata }}{1965}$

Graphs are presented of the binding energy of two neutrons as a function of $\mathrm{N}$, of two protons as a function of $Z$, and of alpha-particle decay energy as a function of $\mathrm{N}$. The range covered is $\mathrm{Z}=10$ to 102 and $N=10$ to 154 . Values shown are based on a 1964 mass adjustment.

\#6604066

$\star 2.083-2.2483-2.2583$

Watson, D.D.; Harris, G. I

TABLES OF COEFFICIENTS FOR ANGULAR CORRELATIONS OF RADIATIVE TRANSITIONS FROM AL IGNED NUCLEI

Nuclear Data, A3:25-122

Basic formulas and tabulations of numerical coefficients are provided for the analysis of data on angular correlations of successive radiative transitions from aligned nuclei. The formulas are extracted from an earlier published development of the angular-correlation formalism. This development is based upon a factored version of the equations which is used to extend the application from the usual two-step cascade to general multiple-step cascades. The resulting flexibility and greater efficiency of the formalism are utilized to provide an extensive tahulation of coefficients for a variety of specific applications. Coefficients are provided for analysis in terms of either the population parameters or the statistical tensors of the aligned state. The coefficients can he used for the analysis of angular distributions, linear polarizations, and triple correlations ínvolving one or more intermediate unobserved radiations. In contrast to previous tabulations of coefficients for angular-correlation analysis, the range of the present tables has been extended to include multipolarities through octupole. The overall length of the tables, however, remains about the same as previous tables because of the greater efficiency of the factored formalism. The range of spins covered by the tables are $J \leq 11 / 2$ for the initial aligned state and $J \leqq 21 / 2$ for the final state of cascade.

\section{\# 6604066}

Way, K.

NUCLEAR DATA, SECTION B, 1-1-1 to $1-6-132$ and 2-1-1 to $2-6-136$

February 1966 - October 1968

Nuclear Data, Section B, presents the experimental results on radioactivity, nuclear moments, and nuclear reactions which give information about nuclear energy levels. The results for all nuclei of one mass number (A-number) are grouped together into an "A-chain." Each issue gives an up-to-date revision of five to ten A chains, depending on their complexity. One issue a year is devoted to Recent References, covering all A-chains and bringing the work up to date with references to articles published since the last issue of Recent References. The material for an A-chain consists of three parts: the level schemes, compiler's analysis sheets, and the data sheets. From the collected data listed on the data sheets, the compilers construct the level scheme which gives a consistent and plausible interpretation of the experimental determinations. The types of data included on the data sheets are ground state: best values of $J$, mu, Q, OMEGA, natural abundance and thermal neutron absorption cross section; ground state decay: data on half-lives, energies and intensities of alpha's, beta's, gamma's, coincidences, angular correlatións, etc.; metastable state: data on J, mu, Q, OMEGA; metastable-state decay: same as ground-state decay; reaction data on resonances, capture gamma's, coulomb excitation, and other reactions; and references to mass-doublet measurements. The drawings present energy levels and the known transitions between them. This information includes energy, half-life, spin and parity, Nilsson model quantum numbers, nuclear reactions known to excite the level, the various transition properties, and mass differences (total betadisintegration energies and neutron and proton separation energies). Adjusted Mass Differences are also given. The various issues are listed below along with the corresponding A-chains found in each:

\section{Volume}

A-chain, $A=$

Vol. 1, No. 1, Feb ruary 1966

Vo1. 1, No. 2, June 1966

Vol. 1, No. 3, July 1966

Vol. 1, No. 4, September 1966

Vo1. 1, No. 5, November 1966

Vo1. 1, No. 6, December 1966

Vol. 2, No. 1, July 1967

Vol. 2, No. 2, August 1967

Vol. 2, No. 3, October 1967

Vol. 2, No. 4, December 1967

Vol. 2, No. 5, March 1968

Vol. 2, No. 6, October 1968

182 to 185

180 to 189

Recent References

21 to 212

77 to 83

213 to 228

70 to 76

142 to 145

Recent References

21 to 228

62 to 64

146 to 148

59 to 61

65 to 69

Other compilations of nuclear data for which the values have not as yet been revised are also included in the NSRDS Library, making a complete set of information for $A=5$ to $A=228$. The earlier sets were published during the 1962 to 1965 period.

$\# 6500009$

Way, K. (Edi tor)

1959 NUCLEAR DATA TABLES

National Academy of Sciences-National Research Council, Nuclear Data Project, Washington, D. C. April 1959

$151 p$

Available from Superintendent of Documents, GPO

Nuclear information is presented which has been systematized according to some nuclear property, such as isotopic abundance, or some special topic such as neutron strength function. Ten tables are included which are self sufficient; explanations of abbreviations used and policies followed in each compilation are given at the beginning, and all relevant references at the end of that compilation.

\#6902010

Way, K. ; et al

NUCLEAR LEVEL SCHEMES $\mathrm{A}=40$ TO $\mathrm{A}=92$ (COVERING ELEMENTS $\mathrm{Ca}$ TO $\mathrm{Z} \mathbf{r}$ )

National Academy of Sciences-National Research Council, Nuclear Data Project, Washington, D. C. September 1955

221 p

Available from DDC

A collection of diagrams showing positions and properties of nuclear energy levels, characteristics of radioactive decay and nuclear reactions, together with a tabular compilation of the experimental data and bibliographic references to the original papers is presented.

\# 6903001,6903002

*2.0181

Way, $K$.

WORLD-WIDE NEWS OF COMPILATIONS IN NUCLEAR PHYSICS Volumes 1 , Numbers 1 and 2

September 1963, June 1964

$9 \mathrm{p}, 10 \mathrm{p}$

A listing is made of groups and individuals preparing compilations in nuclear physics. 
Way, K.; Hurley, F,W.

DIRECTORY TO TABLES AND REVIEWS OF ANGULAR-MONENTUM AND ANGULAR-CORRELATION COEFFICIENTS

Nuclear Data, A1:473-486

\section{July 1966}

Brief descriptions of comprehensive tables of Clebsch-Gordan, Racah, Wigner, and related coefficients are presented in the first section and, in the second, of tables of coefficients of Legendre polynomials in angular-distribution expressions. The present lists, which cover material available to us up to June 1966, supersede those in Section 3 of the 1958 Directory to Nuclear Data Tabulations and its supplements. Bibliographies of some abridged tables and review articles in the same field are included in the third and fourth sections.

\#6509011 *2. 2483

Weaver, L.E.; Strom, P.0.; Killeen, P.A. ESTIMATED TOTAL CHAIN AND INDEPENDENT YIELDS FOR SEVERAL NEUTRON-1NDUCED PROCESSES

USNRDL-TR-633, Naval Radiological

Laboratory, San Francisco, California

March 5, 1963

$46 \mathrm{p}$

Available from CFSTI

Calculated estimates are presented of unmeasured total chain yields and of independent fission yields for the fission products resulting from fission-spectrum and $14 \mathrm{MeV}$ neutron bombardment of $U-233, U-235, U-238$, and $\mathrm{u}-239$ and from thermal neutron bombardment of U-233, U-235, and pu-239.

\section{\#6908037}

*2.2573

Wes cott, C.H. i et al.

A SURVEY OF VALUES OF THE $2200 \mathrm{~m} / \mathrm{s}$ CONSTANTS FOR FISSILE NUCLIDES

Atomic Energy Review, 3:3-60 1965

A study of the most probable values of the $2200 \mathrm{~m} / \mathrm{s}$ constants for the fissile nuclides U- 233, U-235, $\mathrm{Pu}-239$, and $\mathrm{Pu}-241$ has been undertaken, using a least-squares fitting method. The various experimental data have been carefully reviewed. A detailed discussion of the problems involved, especially in the accuracy of the individual measurements and of the final output values, is given. The errors shown are quoted as standard deviations.

$\# 6711019$

Wils on, B.S. (Editor)

$* 2.6183-3.183-0.2183$

THE RADIOCHEMICAL MANUAL, SECOND EDITION

Amersham, England: Radiochenical Centre

1966

$327 \mathrm{p}$

The manual is intended as a guide for all who use radioactive substances professionally; its aim is to help the individual user choose the right material for his purposes and to make effective use of it. The text portion of the book includes information on preparation of radioisotopes: radiation sources; problems of using radioactive materials; radioactive chemicals

pharmaceuticals; quantification of radioactive substances; radiological safety and waste disposal; packaging and carrying radioactive materials; ownership and use regulations and a guide to literature. The tabulated data includes: physical data on the more common radioisotopes, including physical characteristics, availability, beta and gamma decay energies and half-lives; radiation sources, synthetic routes to labelled compounds, decomposition rates of labelled compounds, measurement and safety.
Wing, J .

A COMPARISON OF NUCLIDIC MASS EQUATIONS WITH EXPERIMENTAL DATA

ANL-6814, Argonne National Laboratory, Argonne, I 11 inois

January 1964

$85 \mathrm{p}$

Available from CFSTI

Existing mathematical expressions for the systematics of the nuclidic masses, binding energies, and nucleon separation energies are summarized. The calculated values of the nuclidic mass equations of Fermi, Roman, Bussetti, Cameron, Tsen, Findler, Seeger, Ayres et al., Kummel et al., Thieberger et al., Zeldes, Green, Levy, Baker, and Wing-Fong (a new mass equation) are compared with the available experimental data. Improvements are suggested for some of the observed deviations in these equations. Some expressions for the isobaric parameters $B$ and 2 (nuclear charge of the most stable isobarf, and for the pairing and shell effects are compared with the experimental evidence. An improved empirical formula for 2 and empirical equations for the deformation parameter beta are presented.

\#6509017

$* 2.2383-2.2483$

Wing, $\mathrm{J}$

ISOMERIC-YIELD RATIOS IN NUCLEAR REACTIONS (WITH A COMPILATION OF EXPERIMENTAL DATA)

ANL-6598, Argonne National Laboratory, Argonne, I11inois

September 1962

$143 p$

Available from CFST1

Experimenta1 procedures are briefly described for the determination of the ratios of cross sections for the formation of isomeric states in nuclear reactions. Much available experimental data on isomeric yield ratios in nuclear reactions is compiled. Some of the collected data are discussed in terms of angular-momentum effects in nuclear reactions.

$\# 6908042$

* 2.2583

Wittkopf, W.A.; Roy, D.H.; Livolsi, A.2.

U- 238 NEUTRON CROSS-SECTION DATA FOR THE ENDF/B

ENDF-103, BAW-316, The Babcock \& Wilcox Company, Lynchburg, Virginia

May 1967

$108 \mathrm{p}$

Available from CFSTI

As part of the cooperative effort of the Cross Section Evaluation Working Group organized at Brookhaven National Laboratory in June 1966, the nuclear data on U-238 for use in the Evaluated Nuclear Data File $B$ (ENDF/B) are presented. The data cover the energy range from $0.001 \mathrm{eV}$ to 15 MeV. Data sources are referenced, and the theoretical methods used in evaluating certain data are described. A complete listing of the data in the ENDF/B format is provided.

\#6604066

Wong, C.Y.

$* 2.083-2.1083$

EFFECTIVE MASS $\mathrm{B}_{2}$ AND STIFFNESS PARAMETER $\mathrm{C}_{2}$ FOR QUADRUPOLE VIBRATION IN EVEN-EVEN SPHERICAL NUCLEI Nuclear Data, A4:271-280

May 1968

Values of the effective mass $B_{2}$ and the stiffness parameter $\mathrm{C}_{2}$ for quadrupole vibration of even-even spherical nuclei have been computed from the $B(E 2)$ values and energies of first $2^{+}$states adopted by Stelson and Grodzins. A comparison of these with values predicted by a liquid-drop model shows $B_{2}$ an order of magnitude larger than the predictions. The value of $\mathrm{C}_{2}$ is usually less than the prediction but at magic numbers is many times greater. 
Yamada, M. (Editor)

NEW NUCLEAR DATA, 1957 CUMULATION

National Academy of Sciences-National Research

Council, Nuclear Data Group, Washington, D. C.

1958

$167 \mathrm{p}$

Available from Superintendent of Documents, GPO

Nuclear data collected from 44 journals from about November 1956 to August 1957 is cumulated. The data from Nuclear Science Abstracts, Vol. 11, Nos. $6 \mathrm{~B}$ and $12 \mathrm{~B}$ are included. A separate 1 ist of data on elastic and inelastic scattering of charged particles is a new feature of this cumulation, and it covers experimental data appearing since 1950 . The tabular presentation of previous cumulations is retained.

\section{\#6604066}

Yamazaki, T.

CLEBSCH-GORDAN COEFFICIENTS FOR NUCLEAR TRANSITION PROBABILITIES FOR EVEN-A DEFORMED NUCLE1

Nuclear Data, Al:453-471

Jily 1966

This table has been prepared in order to help calculate alpha-, beta-, and gamma-ray transition probabilities for deformed nuclei. A similar table for odd-mass deformed nuclei was issued before by Lum, Light, and Asaro. In addition to the ClebschGordan coefficients, the squares of the coefficients are also tabulated, since the transition probabilities are proportional to the squares. The values have been computed with IBM7094.

\section{$\# 6604066$}

Yamazaki, T.

TABLES OF COEFFICIENTS FOR ANGULAR DISTRIBUTION OF GAMMA RAYS FROM ALIGNED NUCLEI

Nuclear Data, A3:1-23

August 1967

The present paper has been prepared to provide a simplified formulation of angular distribution functions and tables of coefficients for the angular distribution of gamma rays which the author hopes will be useful in the analyses of experimental data. The theory has been well established.

\section{\#6911023}

$* 2.2593$

Yiftah, S.; et al

NUCLEAR DATA FOR Pu-240, Pu-241, AND Pu-242

IA-1152, 1srael Atomic Energy Commission, Soreq Nuclear Research Center, Yavne, Israel

December 1967

$100 \mathrm{p}$

Available from AEC Dep. Libraries

An evaluation of the basic nuclear data for Pu- 240 , $\mathrm{Pu}-241$, and $\mathrm{Pu}-242$ in the range $0.01 \mathrm{eV}$ to $15 \mathrm{MeV}$ was made. Partial cross sections are constructed for the thermal and fast neutron ranges; they are presented in graphical and tabular form. Resonance parameters and average parameters are recommended for the resonance region; they are presented in tabular form. The cross sections constructed are: total, nonelastic, elastic scattering, radiative capture, fission, total and partial inelastic scattering, $(n, 2 n)$, and $(n, 3 n)$. Other nuclear data considered are: the average parameters above $1 \mathrm{keV}$, the average number of prompt neutrons per neutroninduced fission, the average scattering cosine in the $1 \mathrm{ab}$ system, and the energy spectrum of secondary neutrons from fission.
Young, J.C.: Huffman, D.

EXPERIMENTAL AND THEORETICAL NEUTRON SPECTRA

GA-5319, General Dynamics Corporation, General Atomic Division, San Diego, California

May 22, 1964

$370 \mathrm{p}$

Available from CFSTI

Experimental and theoretical values of neutron spectra for a number of moderators and "poisons" in several geometries and at different temperature are presented graphically. The theoretical values are normalized to give "best fit" at the higher energies and the parameters then used to generate the spectra given. The experimental values of the neutron spectra are also presented in tabular form. This book serves as a guide to reactor-design groups and is presented in 100 se-leaf format to facilitate up dating and additions.

$\# 6509024$

$* 2.2493$

2011 er, L, K.

FAST-NEUTRON-REMOVAL CROSS SECTIONS

Nucleonics, 22:128-129

August 1964

A complete and consistent set of calculated fast neutron removal cross sections is provided for use in point-kernel and moments-method shielding calculations. The cross sections were calculated from empirical expressions that permit the determination of fast neutron effective removal cross section for any element. Cross sections are presented for 83 elements.

\# 6510005

*2. 493

Zumwalt, L.R.

FISSION PRODUCT ELEMENT YIELDS AND CHARACTERISTICS GAMD-891-REV-1, General Dynamics Corporation, General Atomic'Division, San Diego, California

February 1964

$3 p$

Available from CFSTI

In considering the possible physical and chemical effects of fission product elements at high temperatures on graphites and other materials, a table of data on the fission yields of the elements taken as the sum of the yields of all mass chains ending with a stable nuclide of the given element or a nuclide that has a half-life of one year or more was compiled. This gives a distribution of the elements, which corresponds approximately to that obtained from U-235 where a fuel element has run for an extended period of time (order of a year). The fission yields were taken from Schmitt and 2 umwalt who have compiled a reasonably up-todate summary of thermal neutron, U-235 fission yields. Also included in the table are the hoiling points of the elements and qualitative data on the carbides. The volatility category of the elements is listed.

$\# 6601007$

*2. $2493-2.493$

Zysin, Yu. A.; Lhov, A.A.; Sel'chenkov, L.I

FISSION PRODUCT YIELDS AND THEIR MASS DISTRIBUTION New York:Consultants Bureau

1964 (original 1963)

$124 p$

Authorized translation from the Russian

Experimental data accumulated from 1939 to 1962 on fission yields and mass distributions are presented. Fission induced by neutrons, gammarays, and $100-\mathrm{MeV}$ charged particles and spontaneous fission are discussed. 
Atomic and Molecular Properties 
6907023

*3. 113

Anding, D.

BAND-MODEL METHODS FOR COMPUTING ATMOSPHERIC SLANTPATH MOLECULAR ABSORPTION

Report. No 7142-21-T. Michigan University, Infrared and Optical Sensor Laboratory, Ann Arbor, Michigan February 1967

$292 \mathrm{p}$

Available from CFSTI, AD 815481

The general transmissivity equation for computing slant-path molecular absorption spectra is developed and two methods for evaluating this equation, the direct integration and that which assumes a model of the band structure are discussed. Five band models are discussed and twelve methods for computing molecular absorption based on these band models are presented. Spectra computed by band-model methods are compared with spectra calculated by direct integration of the general transmissivity equation and with open-air field measurements of absorption spectra. Conclusions concerning the capability of band-model methods for predicting slant-path absorption spectra are stated and recommendations for future research are outlined. A summary of open-air field measurements and laboratory measurements of absorption spectra for homogeneous paths is presented, and a computer program for computing the equivalent sea-level path, the Curtis-Godson equivalent pressure, and the absorber concentration for atmospheric slant paths of any model atmosphere is given in appendix I.

"6500154 *2.2413-3.113

Aron, W. A.; Hoffman, B. G.; Williams, F. C.

RANGE ENERGY CURVES, SECOND REVISION, 1949

UCRL-121 (AECU-663), California University, Lawrence Radiation Laboratory

Berkeley, California

1949

$99 \mathrm{p}$

Two tables are presented, one for protons and one for electrons, which enable the computation of the rate of energy loss, given the value of the mean excitation potential. While the value of this latter constant has been taken, in this paper, as being given by

$I=\alpha Z=11.52 \mathrm{eV}$

the validity of such a simplistic formulation has been increasingly questioned, several recent experiments yielding results considerably different from those expected. The range-energy curves therefore are tentative, pending a better understanding of the mean excitation potential. The energy-loss considered in this paper derives only from collision effects. The losses due to bremsstrahlung and meson and pair production result from an essentially different process. The calculation of the Fermi effect is ill-advised pending a better determination of the constants.

6907020

*3.113-4.113-8.113

Ballard, S. S. : McCarthy, K. A. ; Wolfe, W. L

OPTICAL MATERIÁLS FOR INFRARED INSTRUMENTATION

IRIA Report ?389-11S, Michigan University, Willow Run Laboratories. Anr Arbor, Michigan

January 1959

$113 p$

Available from DDC, AD 217367

The properties of approximately fifty materials which are useful in optical instrumentation in the infrared portion of the electromagnetic spectrum are described and compared. Each material is discussed in detail in terms of its optical, chemical, thermal, and mechanical properties Tables and figures are given so that the properties of the various materials can be readily compared.
66907021 *3.113-4.113-8.113

Ballard, S, S, McCarthy, K. A, Wolfe, W. L.

OPTICAL MATERIALS FOR INFRARED INSTRUMENTATION, SUPPLEMENT

Report 2389-11-S, Michigan University, Infrared Laboratory, Ann Arbor, Michigan Apri 1961

$18 \mathrm{p}$

Available from CFSTI, AD 255699

In this supplement, additional data are compiled on the optical materials, which are useful for infrared instrumentation, treated in 2389-11-S, as well as data on three new materials, Irtran-1, Irtran-2, and diamond. In addition, several errors and ambiguities in the original report are corrected and clarified.

"6910016

*3. 213

Barnett, C. F.; Ray, J. A.; Thompson, J. C.

ATOMIC AND MOLECULAR COLLISION CROSS SECTIONS OF INTEREST IN CONTROLLED THERMONUCLEAR RESEARCH ORNL-3113 Revised, Oak Ridge National Laboratory, Oak Ridge, Tennessee

January 1965

$316 \mathrm{p}$

Available from CFSTI

A graphical compilation is presented of atomic and molecular cross sections of interest to controlled thermonuclear research. The present compilation is a revision of ORNL-3113, published in 1960. The cross sections are shown, as a function of energy, for collision processes involving molecular ion dissociation, charge exchange, excitation, ionization, photoionization, scattering, energy loss. secondary products and recombination. Pertinent nuclear cross sections are also included. A bibliography is given covering the literature since 1950

낸 $6811011,6811012,6811018$,

$6811021,6811022,6902013$,

6902047,6906024

Barnett, C. F.; et al.

BIBLIOGRAPHY OF ATOMIC AND MOLECULAR PROCESSES FOR 1963, 1964, JULY-DECEMBER 1965, JULY-DECEMBER 1966 , JANUARY-JUNE 1967 , JULY-DECEMBER 1967, JANUARY-JUNE 1968, JULY-DECEMBER 1968

ORNL-AMPIC-1, 3, 6, 8, 9, 10, 11, 12, Oak Ridge National Laboratory, Oak Ridge, Tennessee

July 1965, March 1966, September, October, January, June 1967, June 1968, January, June 1969

$133 \mathrm{p}, 208 \mathrm{p}, 257 \mathrm{p}, 176 \mathrm{p}, 225 \mathrm{p}, 264 \mathrm{p}, 288 \mathrm{p}, 243 \mathrm{p}$

Available from CFSTI

These annotated bibliographies contain references of interest to atomic and molecular processes research. Bibliographical sources consist of more than eighty-five scientific journals and five abstract journals. The references are classified into categories with appropriate sub-categories. Each entry in the respective category is entered alphabetically by author and includes the reactants or the atomic molecular system of interest.

\#6500171 $\star 3.313$

Bass, A. M.; Broida, H. P.

A SPÉCTROPHOTOMETRIC ATLAS OF THE SPECTRUM OF $\mathrm{CH}$ FROM $3000 \AA$ TO $5000 \AA$

NBS Monograph 24, National Bureau of Standards, Washington, D, C.

February 14, 1961

$20 \mathrm{p}$

Available from Superintendent of Documents, GPO

The near ultraviolet and visible emission spectrum of $\mathrm{CH}$ was recorded and presented in the form of a spectrophotometric atlas. The spectrum was recorded photoelectrically from an acetylene-oxygen flame in the region 4900 to $3000 \AA$ by use of a highresolution grating monochromator. Each. of the lines in the $\mathrm{CH}$ spectrum is identified. 
46508057

$\star 3.213-5.213$

Bates. P. R。 (Editor)

ATOMIC AND MOLECULAR PROCESSES

New York: Academic Press

1962

$904 \mathrm{p}$

This compilation, which is designed primarily as a reference book for research scientists, is concerned with radiative and collisional processes involving atoms or molecules. It provides surveys covering the following topics: forbidden and allowed lines and bands, photoionization, photodetachment; recombination, at tachment; elastic and inelastic scattering of electrons, energy loss by slow electrons; collision broadening of spectral features; encounters between atomic systems including range, energy loss, excitation, ionization, detachment, charge transfer, elastic scattering, mobility, diffusion, relaxation in gases, and chemical reactions. A chapter is devoted to the use of high temperature shock waves and accounts are given of the other main experimental methods. The relevant theoretical work is also described, detailed mathematics being avoided as far as possible.

\section{9}

$3.113-4.113$

Bearden, J. A.

$X-R A Y$ WAVELENGTHS

NYO-10586, Atomic Energy Commission, Oak Ridge, Tennessee

1964

533 p

Avaílable from CFSTI

This book is the result of a four-year survey by the $\mathrm{X}-\mathrm{ray}$ Laboratory at the Johns Hopkins University. The primary objectives are to: (1) recompute all published wavelengths on a consistent basis; (2) include data published subsequent to the Sandstroem survey of 1957 and, in particular, reference wavelengths measured in the Johns Hopkins Laboratory; (3) convert these wavelengths to an absolute basis; (4) tabulate the data on which the recommended values are based; and (5) include explicitly probable errors for all adopted wavelength values. The $W K_{a l p h}$, line peak was taken as a wavelength standard. The first part of the tabulated data includes wavelength, wavenumber, energy values arranged according to series and by element. The $K, L, M, N$ and 0 series, as well as absorption edges are given. The second part of the tables presents wavelength and energy values in order of increasing wavelength. The appendix contains an evaluation of wavelength data and presents for each line a literature reference, a published, adjusted and recommended value, and reas on for making the adjustment, if any adjustment was made.

16809012

$* 3.113-4.113$

Bearden, J. A.

$X$ - RAY WAVELENGTHS AND $X-R A Y$ ATOMIC ENERGY LEVELS NSRDS-NBS-14, National Bureau of Standards, Washington, D. C.

September 25,1967

$66 \mathrm{p}$

Available from Superintendent of Documents, GPO

This publication contains two critical compilations: 1) X-Ray Wavelengths. Inconsistencies in accepted values (in $x$ units) of $x$-ray reference lines have recently been demonstrated, al though all are supposedly based on "good" calcite crystals. Factors supporting the selection of the $W$ Kalpha critically discussed. A review is given of the experimental measurements which are used to establish the wavelength of this line on an absolute angstrom basis. Its value is $W K_{a l p h a l}$ $=(0.2090100 \div 5 \mathrm{ppm}) \mathrm{A}$. This may be used to define a new unit, dēnoted by $A^{*}$ such that the $W K_{a l p h a 1}$ wavelength is exactly $0.2090100 \mathrm{~A}^{*}$; hence $1 \mathrm{~A}^{\mathrm{t}}=1 \mathrm{~A}^{+}+$ $5 \mathrm{ppm}$. The wavelengths of the Ag $K_{\text {alpha, }} \overline{\mathrm{c}}$ $K_{a l p h a}$, Cu Kalpha, and the Cr Kalpha have been established as secondary standards with probable error of approximately one part per million. Sixty-one additional $x$-ray lines have been used as reference values in a comprehensive review and reevaluation of more than 2700 emission and absorption wavelengths. The recommended wavelength values are listed in $A^{*}$ units together with probable errors; corresponding energies are given in $\mathrm{keV}$. A second table lists the wavelengths in numerical order, and likewise includes their energies in keV. 2) Reevaluation of X-Ray Atomic Energy Levels. All of the $x$-ray emission wavelengths have recently been reevaluated and placed on a consistent $A^{*}$ scale. For most elements these data give a highly overdetermined set of equations for energy level differences which have been solved by least-squares adjustment for each case. This procedure makes "best" use of all $x$-ray wavelength data, and also permits calculation of the probable error for each energy difference. Photoelectron measurements of absolute energy levels are more precise than $x$-ray absorption edge data. These have been used to establish the absolute scale for eighty-one elements and, in many cases, to provide additional energy level difference data. The $x$-ray absorption wavelengths were used for eight elements and ionization measurements for two; the remaining five we re interpolated by a Moseley diagram involving the output values of energy levels from adjacent elements. probable errors are listed on an absolute energy basis. In the original source of the present data, a table of energy levels in Rydberg units is given. Difference tables in volts, Rydbergs, and milli-A* wavelength units, with the respective probable errors, are also included there.

A 6605016

Bearden, J. A.: Burr, A. F.

ATOMIC ENERGY LEVELS

NYO-2543-1, Final Report, Atomic Energy Commission, Oak Ridge, Tennessee

1965

$244 \mathrm{p}$

Available from CFSTI

This book contains the erergy levels of atoms, and the differences between those levels, as computed from a table of $x$-ray wavelengths. The first set of tables contain values and probable errors of the energies of all occupied levels of each element. presented both in Rydbergs and in electron volts. The second set of tables contain differences between all the energy levels presented in the first set, in units of Rydbergs, electron volts, and milliangstroms.

$\$ 6807004$ *3.213

Bentley, F. F.; Smithson, L. D.; Rozek, A. L.

INFRARED SPECTRA AND CHARACTERISTIC FREQUENCIES $700-300 / \mathrm{cm}$, A COLLECTION OF SPECTRA INTERPRETATION AND BI BLIOGRAPHY

New York: Interscience Publishers

1968

$779 \mathrm{p}$

This book is a reference for use in the analysis of organic and inorganic compounds through infrared spectra. The subject matter is confined to the cesium bromide region. The book is divided into four major parts: Part I discusses the characteristic frequencies and the conditions which must exist, the types of vibrations occurring in the infrared, some uses of the cesium bromide region, calibration standards, and sources of spectra in the cesium bromide region. Part II presents a complete review of the known characteristic frequencies in the cesium bromide region. Most of the common organic classes and some of the inorganic classes are covered. Part III contains the collection of 1566 infrared spectra covering the cesium bromide region. part IV is a chemical class bibliography consisting of over 2000 references to literature on infrared data below $700 / \mathrm{cm}$ through 1966 . The book also contains 
a numerical and chemical class index to the collection of spectra, a molecular formula index, table for the conversion of microns to wavenumber in reciprocal centimeters, a table of reciprocals, an author index and a subject index.

8500166 *3.213

Bentley, F. F.; Wolfarth, E. F.

ANALYTICAL APPLICATIONS OF FAR INFRARED SPECTRA II SPECTRA-STRUCTURE CORRELATIONS FOR ALIPHATIC AND AROMATIC HYDROCARBONS IN THE CESIUM BROMIDE REGION

WADC Technical Report No. 58-198, wright Air Development Center, Wright-Patterson Air Force Base, Ohio

May 1958

$50 \mathrm{p}$

Available from DDC, AD 155566

The infrared absorption spectra of some 400 aliphatic and aromatic hydrocarbons have been investigated from 15 to 35 microns and the characteristic absorption frequencies incorporated into spectra-structure correlation charts. The classes of compounds studied were alkanes, alkenes, cyclopropanes, cyclobutanes, cyclopentanes, cyclohexanes, substituted benzenes, naphthalenes and biphenyls. The skeletal bending frequencies of the alkanes and alkenes and the nonplanar bending frequencies of cycloalkanes and aromatics are the most useful. The wavelength and intensity of the out-of-plan ring frequencies of aromatic molecules give some indication of the nature of the substituents. Typical infrared spectra of the hydrocarbons are presented.

\#6500167 3.213

Bentley, F, F.; Wolfarth, E. F.

ANALYTICAL APPLICATIONS OF FAR INFRARED SPECTRA II SPECTRA-STRUCTURE CORRELATIONS FOR ALIPHATIC AND AROMATIC HYDROCARBONS IN THE CESIUM BROMIDE REGION, SUPPLEMENT I

WADC Technical Report No. 58-198, Supplement 1, Wright Air Development Center, Wright-Patters on Air Force Base, Ohio

December 1958

$49 \mathrm{p}$

Available from DDC, AD 207796

The infrared absorption spectra of some 400 aliphatic and aromatic hydrocarbons have been investigated from 15 to 35 microns and the characteristic absorption frequencies incorporated into spectra-structure correlation charts. The classes of compounds studied were alkanes, alkenes, cyclopropanes, cyclopentanes, cyclohexanes, substituted benzenes, naphthalenes and biphenyls. The skeletal bending frequencies of the alkanes and alkenes and the non planar bending frequencies of the aromatic hydrocarbons are the most useful for qualitative analysis in this region. The wavelength and intensity of the out-of-plane ring frequencies of aromatic molecules give some indication of the nature of the substituents. Typical infrared spectra of the hydrocarbons are presented.

\#6500165

Bentley, F. F.; et al

$$
\text { *3. } 213
$$

ANALYTICAL APPLICATIONS OF FAR INFRARED SPECTRA, I HISTORICAL REVIEW, APPARATUS AND TECHNIOUES

WADC Technical Report No. 57-359, Wright Air Development Center, Wright-Patterson Air Force

Base, Ohio

Septémber 1957

$55 \mathrm{p}$

Available from DDC, AD 142010

A review of the work accomplished in the far infrared region is presented, and most of the available literature is cited. A double beam double pass spectrophotometer equipped with cesium bromide optics is described. This instrument extends the useful spectral range to 35 microns. The general features of the instrument's construction and its performance capabilities are discussed. Conventional infrared techniques were used, and in some instances simplified in obtaining infrared spectra in the cesium bromide region. Many common solvents such as carbon disulfide furan, benzene and dioxane, have large "open windows" in the long wavelength region, and the infrared spectra of these and other solvents are given. The advantages of far infrared spectra, and some of the special techniques of obtaining infrared spectra in the cesium bromide region, are also discussed.

$\# 6902005$

*4.113-3.113-8.113

Berezhnoi, A. S.

SI I ICON AND ITS BINARY SYSTEMS

New York: Consultants Bureau

1960

$275 \mathrm{p}$

Translated from Russian

This text, translated from Russian, is a review of the properties of silicon and its binary compounds. The text discusses the preparation of binary silicon compounds, properties and uses of $\mathrm{H}-\mathrm{Si}$ and Halogen-Silicon systems, systems formed by silicon with elements of Groups IA, IB, IIA, IIB, III, IVB, VB, VIB, transition metals of Groups IV, V', VI', VII, and VIII, and with metals of the iron subgroup, palladium subgroup, platinum subgroup, lanthanides and actinides. Ternary and quaternary systems are also included. There is a list of 716 references and tables of data accompany the systems discussed.

\#6605002

$* 3.313-5.313$

Berlman, I. B.

HANDBOOK OF FLUORESCENCE SPECTRA OF AROMATIC MOLECULES

New York: Academic press

1965

$258 \mathrm{p}$

Spectra of approximately 100 aromatic molecules, varying in size, shape, and structure, are assembled in the handbook. The list of molecules begins with the simple aromatic molecule benzene and includes progressively larger and more complicated systems. Although a special effort has been made to include practically all of the currently popular organic scintillators, the choice of the other molecules has been rather arbitrary. The luminescence characteristics of each aromatic molecule have been measured in a systematic fashion. Fluorescence and absorption spectra are plotted for each molecule and additional data related to the fluorescence process are given in each graph. The additional data include the fluorescence decay time, the fluorescence quantum yield, the natural lifetime as computed from the absorption spectrum, the Stokes loss, the wavelength of the center of gravity of the fluorescence spectrum, and the average wavelength of the fluorescence spectrum. Such supplementary material as topical bibliographies, tables to convert wavelength to wave number and energy, a table of values of the index of refraction of cyclohexane as a function of wave number, and a table of values of oscillator strengths have been placed in the Appendix.

$\# 6910029$

$\star 3.123-5.123$

Berry, R, S.

SMALL FREE NEGATIVE IONS

Chemical Review, 69:533-542

August $19 \%$

This article surveys the restricted subject of properties of isolated atomic and small molecular negative ions. Included in the discussion are electron affinities, electronic states, optical properties, and the experimental and theoretical methods for studying these properties. Excluded from the discussion is the vast group of problems associated with large polyatomic negative ions and 
with most of the rich subject of collision processes involving negative ions, such as detachment and transfer of electrons in collisions.

" 6500168

*3.313

Beynon. J. H. ; Willians, A. E

MASS AND ABUNDANCE TABLES FOR USE IN MASS SPECTROMETRY

New York: Elsevier Publishing Company

1963

570 p

These tables are intended to bring up to date those previously published as an Appendix to "Mass Spectrometry and its Applications to organic Chemistry," by J. H. Beynon, 1960, and to extend their range. They have been calculated and printed on a Ferranti Pegasus Computer and are based on the mass $s$ tandard $12 \mathrm{C}=12 \mathrm{u}$ exactly. The list has been restricted to the four most common elements of organic chemistry, carbon, hydrogen, nitrogen and oxygen, and the combinations listed have been restricted to containing at least one atom of carbon and six or fewer atoms of nitrogen or oxygen. The introduction to the tables is given in English, German, French and Russian.

6500138

Bhalla, C. P.

TABLES OF ELECTRON RADIAL FUNCTIONS AND TANGENTS OF PHASE SHIFTS FOR LIGHT NUCLEI ( 2 - 1 THROUGH 10)

NBS Monograph 81, National Bureau of Standards, Washington, D. C.

August 6 , 1964

$393 p$

Available from Superintendent of Documents, GPO

To facilitate the theoretical analyses of betadecay experiments in light nuclei, electronic radial wave functions, evaluated at the nuclear radius, and tangents of phase-shifts are tabulated for total angular momentum equal to $1 / 2$ and $3 / 2$. Separate tables for electrons and positrons are given for ten values of 2 , starting from $z$ equal to one, in steps of unity and for beta momentum values from 0.1 mc to $42.0 \mathrm{mc}$ in steps of $0.1 \mathrm{mc}$. The nucleus is represented as a sphere with uniform charge distribution. The nuclear radius, rho, is taken to be $1.2 \mathrm{~A}^{1 / 3} \times 10^{-13} \mathrm{~cm}$ in the major body of these tables. However, additional tables for 2 - 6 and $2=7$ are given for tho $=1.1 \mathrm{~A}^{1 / 3} \times 10^{-13} \mathrm{~cm}$ and rho $=1.3 \mathrm{~A}^{1 / 3} \times 10^{-13} \mathrm{~cm}$.

\#6508024 2.1113-3.113

Bhanot, V. B.; Gupta, S. C.

MASS-SPECTROSCOPIC ATOMIC MASS DIFFERENCES

Nuovo Cimento, 10:1011-1023

June 1963

New mass-spectroscopic data on atomic mass differences in the medium and heavy mass regions were compiled and tabulated. Corresponding values in the new scale based on $\mathrm{C}-12$ as the standard are also given. Unadjusted experimental values for nuclidic masses in the new scale were listed. For a large number of $\mathrm{stab}$ le nuclides in the region from strontium to antimony the newer values obtained from the data of Demirkhanov et al are more accurate than the corresponding values $i$ isted in the recent exhaustive mass table of Koenig et al. Attention is drawn to the advantages of the isotopic doublets over the published conventional mass doublets in the heavy mass region.

66711002

3.313

Bielski, B. H. J.; Gebicki, J. M.

ATLAS OF ELECTRON SPIN RESONANCE SPECTRA

New York: Academic Press

1967

$665 \mathrm{p}$

This atlas is made up of electron spin resonance spectra recorded and published by several hundred scientists during the last eighteen years. The aim of the book is to make available those spectra of interest to experimental spectroscopists, including all spectra which could be assigned wi th reasonable certainty to specific paramagnetic atoms, molecules or ions. Spectra are made up of overlapping signals due to two or more paramagnetic materials unless the individual components could be identified and the spectra assigned. A few complicated spectra were omitted because the only available pictures we re not clear enough for the resolution of some individual lines. All spectra of oriented single crystals, which would require much additional information, have been omitted. Published results through late 1964 and some unpublished spectra are included. The following data are included with each spectrum: paramagnetic species, irradiated material, formula for paramagnetic species, method of preparation of sample, medium, g-factor, temperature, total number of lines observed, relative line intensities, splitting constants, spin density, and reference to original publications.

Birnbaum, G.; Maryott, A. A.

LINE SHAPES, WIDTHS, AND SHIFTS IN MICROWAVE SPECTRA OF GASES

Research Report No. 228, Hughes Research Laboratories, Malibu, California December 1961

$34 \mathrm{p}$

This report is a survey of results obtained in recent years concerning the shape, width, and frequency shift of microwave lines in dilute and compressed gases. The dilute gas results are concerned with relatively sharp lines associated with molecular transitions and atomic hyperfine transitions in hydrogen and optically pumped alkali metal vapors in various buffer gases. The compressed gas results are concerned with rather broad lines or absorption bands and, in particular, with the shape and width of the nonresonant spectra of symmetric top molecules, frequency shifts in the microwave spectrum of $02, \mathrm{NH}_{3}$, and ND3, collisioninduced absorption in nonpolar molecules, and absorption in the microwave wings of the infrared rotational lines of $\mathrm{HCl}$ and $\mathrm{DC}$.

" 6908045

$4.213-8.213-3.213$

Bondi, A. A.

PHYSICAL PROPERTIES OF MOLECULAR CRYSTALS, LIQUIDS, AND GLASSES

New York: John Wiley \& Sons, Inc.

1968

$502 \mathrm{p}$

This book developes a methodology enabling chemists and chemical engineers to relate certain physical properties of condensed phases to molecular structure. The states of matter treated are molecular crystals, liquids, and glasses. The physical properties covered are $\mathrm{p}-\mathrm{V}-\mathrm{T}$ and related thermal properties, and certain transport properties. Energy of vaporization, van der Waals dimensions, molar refractivity, dipole moment data and dielectric loss are used but not discussed thoroughly. Tables 14.1 to 14.16 contain the requisite molecular structure increments. Background material presented is a guide to the literature.

\#6805003

$* 3.213$

Bovey, F. A.

NMR DÁTA TABLES FOR ORGANIC COMPOUNDS, VOLUME I

New York: Interscience Publishers

1967

$610 \mathrm{p}$

The purpose of this book is to provide a convenient source of high resolution NMR data on a large number and variety of organic compounds and a few related inorganic compounds. These data can be useful in two general ways: 
1. to assist in the identification of new compounds and the recognition of known compounds by matching of spectral data;

2. to provide data on known compounds, in order. to test new correlations, hypotheses and calculations.

This volume covers the literature from 1959 to 1962. The data for each compound are arranged in a horizontal series of entries which form columns for compound number, empirical formula, structural formula and name, solvent and concentration, temperature and reference compound, chemical shift and multiplicity, spin-spin coupling, spectrum type and/or analysis, observing frequency, and remarks and bibliographic reference.

\section{\#6805001}

Brams on, M. A.

Rodman, R. B. (Translator)

Wolfe, W. L. (Editor of Translation)

INFRARED RADIATION, A HANDBOOK FOR

WITH A COLLECTION OF REFERENCE TABLES

New York: Plenum press

1968

$623 \mathrm{p}$

This is the first volume of a series by M. A. Bramson which includes discussions on the basic principles of optics and the use of planck's law for radiant intensity, for radiant existence and for radiance in terms of wavelength, frequency and photon number. The Wien and Rayleigh-Jeans expressions and modifications of the StefanBoltzmann law are derived. Chapters contain discussions on radiation, color, brightness temperatures, two-color emissivity-independent temperature-measurement methods, measurements of flames, bodies cooler than the radiometer, partially transparent bodies, effects on measurements, and give tables of blackbody functions. This handbook includes procedures for computing the properties of infrared radiation over a temperature range from 100 to $6000^{\circ} \mathrm{K}$

collection of "Reference Tables for the Infrared Radiation from Heated Bodies" comprises the second portion of the book and includes tabulations of the Planck function, formulas derived from it, and the corresponding integrals. The book is aimed as reference source and as a text for senior or graduate courses in infrared technology.

\section{\#6607004}

B rown, S. C.

BASIC DATA OF PLASMA PHYSICS

Cambridge, Massachusetts: The M.I.T. Press

1959

$336 \mathrm{p}$

This text consists of a combination of the class notes and the Basic Data technical report for graduate course in gas discharges at M.I.T. put into book form. Its chapters deal with such topics as elastic-collision cross sections, chargetransfer cross sections, diffusion and mobility, inelastic collisions of electrons, diffusioncontrolled breakdown, electron attachment, breakdown controlled by electron attachment, recombination, secondary electron emission by electrons, breakdown controlled by secondary electron emission, the second Townsend coefficient, $\mathrm{D}-\mathrm{C}$ breakdown, the corona discharge, glow discharges, steady-state microwave discharges and arc discharges. The text has an author index as well as a subject index.

\#6806002

$\star 3.213$

Bruege1, W. (Compiler)

NUCLEAR MAGNETIC RESONANCE SPECTRA AND CHEMICAL STRUCTURE, VOLUME 1 , THE SPECTRAL NMR PARAMETERS OF COMPOUNDS WITH ANALYZED SPECTRA

New York: Academic Press

1967

$235 p$
These tables contain the spectral nuclear magnetic resonance (NMR) parameters, i.e. the values of the chemical shift (magnetic shielding) and of the coupling constants of chemical compounds whose spectra have been analyzed. Section 1 of the tables deals with those cases where the atoms capable of resonance are either hydrogen or hydrogen together with other resonating nuclei, such as F-19 and P-31 (if they are present in a state where the spectrum can be described in a simple way, e.g. by reporting a single chemical shift of a nucleus other than hydrogen). All analyzed compounds of this type known to the reviewer are included with the exception of certain organic molecules containing $\mathrm{Si}, \mathrm{Ge}$ and $\mathrm{Sn}$, for which only some model-compounds are shown. Section 2 of the tables summarizes data on nuclei other than hydrogen. All inorganic compounds have been omitted.

$\$ 6908057$

Bucci, p.

PLUTONIUM IN FAST NUCLEAR REACTORS, VI. APPENDIX Ingegneria Nucleare, $8: 33-77$

November-December 1967

Tables, graphs and phase diagrams on the properties are presented. The characteristics of the systems $\mathrm{Pu}-\mathrm{Al}$, Pu-Be, Pu-Cr, Pu-Fe, Pu-H, Pu-Hg, Pu-Mo, Pu-

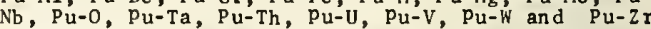
are discussed and shown in graphic form.

$\# 6512044$ *3. 313

Buckley, F.; Maryott, A. A

TABLES OF 'DIELECTRIC DISPERSION DATA FOR PURE LIQUIDS AND DILUTE SOLUTIONS

NBS Circular 589, National Bureau of Standards, Washington, D. C.

November 1, 1958

Available from the Superintendent of Documents, GPO

Primary dielectric dispersion data and characteristic dispersion parameters are tabulated for almost 200 substances in the liquid state and for dilute aqueous and non-aqueous solutions with more than 150 solutes. There are 6 tables and 1 section of graphs. There are 4 tables for pure liquids, 2 containing summaries of the derived dispersion parameters and 2 containing the primary data. The section on graphs supplements the tables for pure liquids and contains reproductions of pertinent data that are available only in the form of graphs.

\# 6509014

Buckmas ter, H. A.

TABLES OF ANGULAR MOMENTUM TRANSFORMATION MATRIX ELEMENTS $d$, $(j)$ (beta) $(j=2,4,6)$

Canadian Journal of physics, $42: 386-391$

February 1964

Crystalline electric field angular momentum operator equivalent transforms are given.

$\# 6704004$

*3.123

Candler, C.

ATOMIC SPECTRA AND THE VECTOR MODEL, SECOND EDITION Princeton, New Jersey: D. Van Nostrand Company, Inc.

1964

$412 \mathrm{p}$

This second edition of Atomic Spectra compiles data and atomic spectral information through the use of the vector model. Where quantum mechanics has refined the predictions of the model, these predictions are compared with experimental results. MKS units are used entirely for all equations and data. The book contains chapters dealing with the hydrogen atom data, alkali doublets, the alkaline earths, absorption spectra, the zeeman effect, the Paschen-Back effect, the periodic system, the doublet laws, displaced terms, combination to several electrons, elements of the short periods, 
long periods, the lanthanides, the actinides, line intensities, the sum rules and ( $j j$ ) coupling, series limit, hyperfine structure and quadrupole radiation. Tables of protonic, neutronic and deutonic nuclei, natural atomic units, the Grotrian diagrams, Rydberg term tables, and a bibliography are also included.

$\# 6906023 \quad$ *.213-3.213

Carter, R. S.; Rush, J. J. (Editors)

MOLECULAR DYNAMICS AND STRUCTURE OF SOLIDS

NBS Special Publication 301, National Bureau of St andards, washington, D. C.

June 1969

$571 \mathrm{p}$

Available from Superintendent of Documents, GPO

This volume is based on material presented at the Second Materials Research Symposium of the National Bureau of Standards, held 0ctober 16-19, 1967. It provides a review of the application of spectroscopic and diffraction techniques to the study of the structure and dynamics of molecular solids. Invited papers on the theory and practice of the major experimental methods, including neutron and $x$-ray diffraction, neutron inelastic scattering, infrared and Raman spectroscopy and nuclear magnetic-resonance, serve as a background for the more detailed presentation and discussion of results which follows. The topics covered in a series of invited and contributed papers include the lattice dynamics of molecular crystals, the spectroscopy and crystal structure of organic and inorganic solids and the dynamics of polymers.

\section{$\# 6910023-6910024$}

$$
\star 3.123
$$

Capellen, J.; Svec, H. J.; Sage, C. R.

BIBLIOGRAPHY OF 1966 MASS SPECTROSCOPY LITERATURE COMPILED BY A COMPUTER METHOD

IS-1611 and Addendum, Ames Laboratory, Iowa State University, Ames, Iowa

July 1967, June 1967

$14 \mathrm{p}, 637 \mathrm{p}$

'vailable from CFSTI

About 2800 articles of interest to mass spectroscopists are listed as a result of computer searching of 270,000 scientific articles published during 1966. The 149 papers presented at the 14 th Annual Conference on Mass Spectrometry and Allied Topics, ASTM E-14, May 22-27, 1966, are also included. In addition to the bibliography, an author index and permuted title index (KWIC) are included.

$\# 6500126$

*3. 113

Catalan, M. A.; Meggers, W. F.; Garcia-Riquelme, 0. THE FIRST SPECTRUM OF MÁNGANESE, Mn I

Journal of Research of the National Bureau of Standards. A. Physics and Chemistry, 68A: 9-59 January-Feb rūary 1964

In 1894, two short series of threefold spectral terms were discovered in the arc spectrum of manganese, and in 1922 other regularities involving fivefold and sixfold terms were discovered by Catalan who coined the word "multiplet" for the group of related lines resulting from combinations of such complex terms. Multiplet analyses of complex spectra promptly led to the present formal quantum interpretation of all such phenomena, but comparable progress in the analysis of the Mn I spectrum was handicapped by the paucity of experimental data. New observations of about 2500 wavelengths and intensities plus 440 Zeeman patterns made available in 1948-49 have now been completely exploited to derive additional atomic energy levels and thereby explain more of the observed Mn I lines. The result is that a total of 42 even terms with 125 levels and $60 \mathrm{~g}$-values have now been designated and allocated to electron configurations, and 94 odd terms with 266 levels, $164 \mathrm{~g}$-values, plus $13 \mathrm{miscellaneous} l e v e 1 s$. These terms are distributed among four multipiicities (doublets, quartets, sextets, octets), and transitions between even and odd terms account for more than 2030 lines ranging in wavelength from $1785 \AA$ to $17608 \AA$.

\#602007

*3.213

Cauchois, Y.; Hulubei, $H$.

TABLES OF CONSTANTS AND NUMERICAL DATA, VOLUME 1. SELECTED CONSTANTS, WAVELENGTH OF $X$-RAY EMISSIONS AND OF DISCONTINUITY OF X-RAY ABSORPTION

Paris: Hermann \& Cie, Editeurs

1947

$199 \mathrm{p}$

In French

This book contains a table of $x$-ray spectral emission line wavelengths taken from literature published between 1926 and 1946. The range of wavelengths covered is 0.1 to 400 angstroms. $\mathrm{K}, \mathrm{L}$, $M, N$, and 0 series, plus absorption edges, are included. The data included for each line are: wavelength of the line, the element and series producing that line, and the frequency and square root of the frequency of Rydbergs. References to the literature are also given for each line.

\section{\#6908051 \\ $\star 3.321$}

The Coblentz Society (Editors)

EVALUATED INFRARED REFERENCE SPECTRA

Philadelphia, Pennsylvania: Sadtler Research Laboratories

1969

$1100 \mathrm{p}$

Available from the Sadtler Research Laboratories, Philadelphia, Pennsylvania

The present collection of 1000 infrared spectra was initiated with the intent of improving and adding to the sources that already exist in a variety of forms. The criteria set up to provide specifications for the evaluation of infrared spectra include three classes of spectra: critically defined physical data, research quality analytical spectra, and approved analytical spectra. The collection includes four sets of indices: alphabetical index, chemical classes index, molecular formula index, and a numerical index. The Chemical Abstracts system of nomenclature $h$ as been employed in naming the compounds and additional names have been added as cross-references to facilitate location.

\# 6811017 * 3.171

The Coblentz Society Board of Managers SPECIFICATIONS FOR EVALUATION OF INFRARED REFERENCE SPECTRA

Analytical Chemistry, 38: 27A-38A

August 1966

For purposes of evaluation, infrared spectra can be classified into three categories: Class $I$, standard spectra; Classes II and III, reference spectra. Class I spectra are designated as "standard spectra" because they are of a sufficiently high quality to be acceptable as physical constants of the substances under precisely defined conditions of measurement, and further refinements in spectrophotometric technique will not be expected to change them significantly. Few, if any, spectra of this quality have as yet been produced. Class II spectra are reference spectra obtained on the best currently available commercial infrared grating spectrophotometers. operated at maximum efficiency under conditions consistent with acceptable laboratory practice. The purity of the compounds must be rigidly specified with respect to the type and quantity of any spectroscopically detectable impurity present. Class III are the absorption spectra of individual compounds, polymers, and resins of known composition that have been obtained with sufficient accuracy to be useful in the identification of unknown materials. Class III is to be regarded primarily as a repository for usable data recorded prior to the general availability of high resolution grating spectrophotometers. The Board 
of Management of the Coblentz Society recommends that spectra in Classes II and III be called "reference spectra" and that they be so designated in the published charts and in associated literature. It strongly recommends that the use of the word "standard" be reserved solely for spectra of Class I.

\section{$\$ 6500160,6500159$}

*3. 223

Coleman, C. D.; Bozman, W. R.; Meggers, W. F.

TABLE OF WAVENUMBERS, VOLUME 1 : 2000 ANGSTROMS TO 7000 ANGSTROMS, VOLUME 2: 7000 ANGSTROMS TO 1000 MI CRONS

NBS Monograph 3, National Bureau of Standards. Washington, D. C.

May 1960

$500 \mathrm{p}, 534 \mathrm{p}$

Available from the Superintendent of Documents, GPO

A two-volume table for converting wavelengths in standard air to wavenumbers in vacuum was computed by using the equation

$$
\sigma_{\text {vac }}=1 /\left(n \lambda_{\text {air }}\right)
$$

where eta was computed from Edlen's 1953 equation for the refractive index of air. Wavenumbers are given to the nearest $0.001 K\left(\mathrm{~cm}^{-1}\right)$ for wavelengths from 2000 to $7000 \&$ in volume $I$, and $7000 \&$ to 1000 microns in volume II. Proportional tables are given for linear interpolation between entries of lambda. Also included are the vacuum increase in wavelength, (eta-1) and the refractivity of standard air in the form (eta-1) $\times 1000$.

\section{A 6908015

$$
\text { * } 4.123-3.123-8.123
$$

Connolly, T. F. Copenhaver, E. D.

BIBLIOGRÁPHY OF MAGNETIC MATERIALS AND TABULATION OF MAGNETIC TRANSITION TEMPERATURES

ORNL-RMIC- 7 (Revised, 1969, preprint), Research Materials Information Center, Oak Ridge National Laboratory, Oak Ridge, Tennessee

1969

$78 \mathrm{p}$

ORNL-RMIC-7 (March 1968) has been corrected, revised, and expanded to include about 1000 additional measurements of Curie and Neel points of magnetic materials. As in the original, the materials are arranged alphabetically by chemical formula and appear in two lists - one for ferromagnetic (or ferrimagnetic) and one for antiferromagnetic (or metamagnetic) substances. The tabulation is based on papers and reports received by the RMIC through January 1969.

\author{
\#6500.148 *3.223-5.223
}

Cook, C. J.: Lorents, D, C

ELECTRON COLLISION FREQUENCIES AND SCATTERING CROSS SECTIONS IN THE IONOSPHERE, A

Final Technical Report No, 6, Stanford Research Institute, Menlo Park, California

August 1961

$54 \mathrm{p}$

Available from DDC AD 291591

The propagation of electromagnetic waves through an ionized, gaseous medium can be described in terms of a set of macroscopic parameters that are a function of the physical properties of the medium, the permittivity, conductivity, and permeability. This report calculates the propagation parameters for the normal ionosphere above $50 \mathrm{~km}$, and from these parameters calculates theoretical values for electron collision frequencies and scattering cross sections in the ionosphere.
H6607033

*8. $223-3.223-5.223$

Cooper, J. (Editor)

PROCEEDINGS OF WORKSHOP CONFERENCE ON THE LOWERING OF THE IONI ZATION POTENTIAL AND RELATED PROBLEMS OF THE EQUILIBRIUM PLASMA

JILA Report No. 79 , Joint Institute for Laboratory Astrophysics, Boulder, Colorado July 1966 $225 \mathrm{p}$

This workshop was sponsored by the Joint Institute for Laboratory Astrophysics. Its concern was statistical mechanics of plasma both from experimental and theoretical points of view. There were many recent theoretical advances in the treatment of such problems as the plasma partition function and the lowering of the ionization potential, although there were strong disagreements between different workers in this field. The proceedings were taped and should be considered as unpublished material. It was the object of this workshop to bring together active theorists and experimenters in this field to resolve some of the differences. Included in the Appendix is a Bibliography on Research on Lowering of the Ionization Potential.

* 6811007

$\star 3.123$

Cord, M. S.; et al.

MICROWAVE SPECTRAL TABLES, VOLUME IV, POLYATOMIC MOLECULES WITHOUT INTERNAL ROTATION

NBS Monograph 70, Volume IV, National Bureau of Standards, Washington, D. C.

October 1968

$418 \mathrm{p}$

Available from the Superintendent of Documents, GPO

Measured frequencies, assigned molecular species, and assigned quantum numbers are given for about 14,000 spectral lines of polyatomic molecules without internal rotation observed by coherent radiation techniques. Molecular data, such as rotational constants, dipole moments and various coupling constants determined by such techniques, are also tabulated. References are given for all included data.

* 6811008

* 3.123

Cord, M. S.; Lojko, M. S.; Petersen, J. D.

MICROWAVE SPECTRAL TABLES, VOLUME $V$, SPECTRAL LINE LISTING

NBS Monograph 70, Volume $v$, National Bureau of Standards, Washington, D. C.

June 1968

$533 p$

Available from the Superintendent of Documents, GPO

This volume is a listing of the spectral lines reported in Volumes I, I I , and IV of the Microwave Spectral Tables. These lines are listed according to ascending magnitude of frequency and provide a ready reference.

\#6903018

*3. 123

Corliss, C. H.

REVISION OF THE NBS TABLES OF SPECTRAL-LINE INTENSITIES BELOW 2450 ANGSTROMS

NBS Monograph 32 Supplement, National Bureau of Standards, Washington, D. C.

July 7,1967

$37 \mathrm{p}$

Available from Superintendent of Documents, GPO

A calibration is applied to the intensity measurements of the 1400 lines below 2450 angstroms in the NBS Tables of Spectral-Line Intensities. Tables of the new values are presented with the ines arranged by elements and by wavelengths. 
$\$ 6500156$

*3. 123

Corliss, C. H.; Bozman, W. R.

EXPERIMENTAL TRANSITION PROBABILITIES FOR SPECTRAL LINES OF SEVENTY ELEMENTS DERIVED FROM THE NBS TABLES OF SPECTRAL-LINE INTENSITIES

NBS Monograph 53, National Bureau of Standards, Washingtor, D. C.

July 20,1962

$562 \mathrm{p}$

Available from Superintendent of Documents, GPO

Relative intensities of 39,000 spectral lines with wavelengths between 2000 and 9000 angstroms have been determined on a uniform energy scale for seventy elements. The light source was an arc between copper electrodes to which a single element was added in the ratio of one atom of element to 1000 atoms of copper. The temperature of the arc was determined, by comparison of the observed intensities with published relative gf-values, to be $5100 \pm 110 \mathrm{~K}$. The degree of ionization of eleven elements in the arc was determined by comparison of intensities in spectra of neutral and ionized atoms with known absolute gf-values. With the aid of Saha's ionization equation, the electron density in the arc was found to be $2.4 \times 10^{14} \mathrm{~cm}^{-3}$. The ionization of seventy elements separately added to the arc was then calculated with Saha's equation. A correction was made for diffusion of atoms from the arc stream. With this information, relative values of $\mathrm{g}$ on a uniform scale can be computed for the 25,000 lines which have been classified. By calibration with known absolute gfvalues, the scale is put on an absolute basis. Absolute transition probabilities for 25,000 lines of 112 spectra have been calculated in this way and the results are tabulated by spectrum. The wavelength in angstroms, energy levels to the nearest kayser, gA in $10^{8}$ per second, $g f$, and $10 \mathrm{~g}$ gf are given for each line.

6612001

$\star 3.323$

Cornu, A. Massot, R.

COMPILATION OF MASS SPECTRAL DATA

London: Heyden \& Son Limited

1966

$617 \mathrm{p}$

Introduction in French and English

This work presents several classifications of the principal parameters of about 5000 mass spectra and facilitates the identification and analys is of organic compounds by mass spectrometry. The compilation has been sorted and arranged by computer techniques from a punched card index. The 5000 spectra have been sorted and indexed in four different ways: by reference number, by molecular weight, by molecular formula, and by fragment ion values (including the molecular ion). The exact origins of the spectra are also listed.

$\$ 6500485 \quad * 3.213$

CorTuccini, R. J

DIELECTRIC CONSTANT OF LIQUID PARAHYDROGEN

NBS Technical Note 144, National Bureau of Standards, Boulder, Colorado

April 1962

$11 p$

Available from CFSTI, PB 161645

This study shows that the available data on the dielectric constant of hydrogen conform to the Clausius-Mossotti equation within the probable experimental errors. The published data cover temperatures from the triple point (approximately 14 K) to well above room temperature and a thousandfold range of densities. Tables of the dielectric constant of the liquid have been computed for temperatures from the triple point $(13.803 \mathrm{~K})$ to $32 \mathrm{~K}(58 \mathrm{R})$ and pressures from saturation to 340 atmospheres, by using an average value of the specific polarization.
\#6004008 *3.223

Coulson, K. L.; Dave, J. V.; Sekera, 2.

TABLES RELATED TO RADIATION EMERGING FROM

PLANETARY ATMOSPHERE WITH RAYLEIGH SCATTERING

Los Angeles and Berkeley: University of California

Press

1960

$548 \mathrm{p}$

The present volume provides tables giving the exact distribution and polarization of the reflected and transmitted light in a plane-parallel atmosphere scattering in accordance with Rayleigh's laws. These data make it possible to determine by subtraction (from the observed distributions) the nonmolecular component of skylight.

\#6509019 \$3.123

Dame row, R. A.; Ries, R. R.; Johnson, W. H., Jr. ATOMIC MASSES FROM RUTHENIUM TO XENON

physical Review, 132: 1673-1681

November 15,1963

A sixteen-inch double-focusing mass spectrometer employing the peak matching method of measurement has been used to measure the atomic masses of all stable isotopes in the region ruthenium to xenon. Atomic masses of 53 radioactive nuclei have been calculated from mass differences derived from nuclear reaction and beta-decay energies. Nucleon binding and pairing energies have been calculated from the resulting mass table. The effect of the shell closure at $2=50$ on the systematics of nucleon binding and pairing energies has been investigated in greater detail than has previously been possible. The discontinuity in proton binding energy is shown to be caused by a decrease in binding energy of protons beyond $2=50$. The main result of the study of nuclear systematics in this region seems to be that the binding energies of both neutrons and protons exhibit smooth behavior except for discontinuities at a shell closure. The presence of doubly charged, diatomic tellurium satellites which interfere with the singly charged ion peaks is also noted.

\#7001019

$* 8.123-3.123$

Darwent, B. deB.

BOND DISSOCIATION ENERGIES IN SIMPLE MOLECULES

NSRDS-NBS-31, National Bureau of Standards, Washington, D. C.

January 1970

$52 \mathrm{p}$

Available from the Superintendent of Documents, GPO

Bond dissociation energy values (kcal/mol) and (kJ/mol) of simple compounds are tabulated from a literature review covering the year 1962-1966 inclusively. Some selected values which appeared in the years 1956-1962 are also included. Organic compounds are excluded except those containing one carbon atom. The groups $>\mathrm{CO}$ and $-\mathrm{CN}$ are not considered to be organic. The values are quoted usually at $0 \mathrm{~K}$ OT $298 \mathrm{~K}$ and refer to the gaseous state. They represent the energy required to break $a$ bond at the specified temperature with all substances in the zero vibrational state of the ground electronic state. The experimental method for the energy value listed is given and referenced in the table. A value recommended by the author is listed as the final value for each reaction.

\#6604007

Davis S. Phillips *3.123

THE RED SYSTEM (A2 $\pi-X 2$ E) OF THE CN MOLECULE

Los Angeles, California: University of California

Press

1963

$214 \mathrm{p}$

This book presents the first results of a program designed for the production and analysis of the spectra of molecules of astrophysical interest. The red system appears prominently in the spectra of carbon stars and sunspots and extends over a 
wide spectral region, from about 4370 angstroms to beyond the photographically accessible infrared. Fifty-four bands have been identified in the photographically accessible region. The tables list the wavelengths of the molecular lines in standard air the intensities of the lines normalized to 100 for the strongest line in the band, the relative intensities on the arc and discharge tube plates, the wave numbers of the lines in vacuum and the rotational quantum numbers of the various branches.

6905034

Dicks on, P. F, Jones, M. C

INFRARED SPECTRAL REFLECTANCES OF METALS AT LOW TEMPERATURES

Cryogenics, 8:24-29

February 1968

This paper graphically displays values, derived from the literature of the normal spectral reflectances ( $P, \rho_{\text {) }}$ of ten pure metals and an alloy at low temperatufes. The metals and alloy included are aluminum, antimony, bismuth, copper, brasses, gold, silver, lead, tin, nickel and sodium.

\section{$\$ 6704042$}

$\$ 8.323-3.323$

Dub, M. (Edi tor)

ORGANOMETALLIC COMPOUNDS, METHODS OF SYNTHESIS PHYSICAL CONSTANTS AND CHEMICAL REACTIONS, VOLUME I : COMPOUNDS OF THE TRANSITION METALS, SECOND EDITION, COVERING THE LITERATURE FROM 1937 TO 1964 New York: Springer-Verlag New York, Inc.

1966

$856 \mathrm{p}$

Volume I of the series Organometallic Compounds covers the derivatives of the transition metals of Groups III through VIII of the Periodic Table. It is a comprehensive, noncritical source of information concerning organometallic compounds containing at least one carbon-metal bond. The information includes methods of preparation, properties, chemical reactions, applications, addition compounds and biological properties. This volume covers the literature from 1937 to 1964. In addition to the tables of data and information given, a bibliography of over 2300 references and a listing of review publications and monographs are included.

\section{0}

$8.323-3.323$

Dub, M. : Weiss, R. W. (Editors)

ORGANOMETALLIC COMPOUNDS, METHODS OF SYNTHESIS PHYSICAL CONSTANTS AND CHEMICAL REACTIONS, VOLUME II : COMPOUNDS OF GERMANIUM, TIN AND LEAD INCLUDING BIOLOGICAL ACTIVITY AND COMMERCIAL APPLICATION SECOND EDITION, COVERING THE LITERATURE FROM 1937 TO 1964

New York: Springer-Verlag New York, Inc.

1967

$727 \mathrm{p}$

Volume II of the series organometallic Compounds covers organic derivatives of germanium tin and lead. It is a comprehensive, noncritical source of information concerning organometallic compounds. The scope is limited to compounds containing at least one carbon-metal bond. The information includes methods of preparation, properties, chemical reactions, applications, addition compounds and biological properties. This volume covers the literature from 1937 to 1964. In addition to the tables of data and information given, a bibliography of over 2700 references and a listing of review publications and monographs are included.
6903046

3.021

Dukelskii, V. M. (Edi tor)

Cobble, D. M. (Translator)

ELECTRONIC AND ATOMIC COLLISIONS BIBLIOGRAPHY OF

RUSSIAN LITERATURE FOR THE YEARS 1946-1966

Atomic and Molecular processes Information Center,

Oak Ridge National Laboratory, Oak Ridge, Tennessee October 1968

$69 \mathrm{p}$

This bibliography is based on a machine translation of a Russian edition. The original Russian references and their translations are both listed. The references are grouped into major categorical processes. The numerical order is unchanged from the original Russian bibliography. The major categories include general collisions, electronatomic collisions, collisions of atomic particles and their applications. An author index is provided.

6906009

Eisenberg, D.; Kauzmann, $W$.

*3.123-8.123-0.2523

THE STRUCTURE AND PROPERTIES OF WATER

New York and Oxford: Oxford University Press 1969

$296 p$

In this book the authors summarize the many experimental observations on water and present theoretical discussions which effectively correlate the data. The topics covered include the water molecule and forces between water molecules; the thermodynamic properties of steam; the structures of the ices; the thermodynamic, electrical, spectroscopic, and transport properties of the ices and of liquid water; hydrogen bonding in ice and water; and models for liquid water. The authors emphasize the relation of the properties of $i c e$ and water to their structures, and discuss properties such as infrared and Raman spectra because they reveal a great deal about these structures. The inclusion of some background material in physical chemistry makes the book accessible to the biologist, biochemist, and geologist as well as the chemist and physicist. An addendum listing a number of recent articles on the structures and properties of water completes the volume.

\#6508065 *3.133-4.133

Foex, G.; Gorter, C.-J.; Smits, L.-J.

TABLES OF CONSTANTS AND NUMERICAL DATA, VOLUME 7 . SELECTED CONSTANTS, DIAMAGNETISM AND PARAMAGNETISM, PARAMAGNETIC RELAXÁTION

Paris: Masson $\xi$ cie, Editeurs

1957

$318 \mathrm{p}$

In French, introduction in French and English

These tables list constants and numerical data for materials which exhibit diamagnetism or paramagnetism. These materials include the elements, binary alloys, mineral compounds, double or complex salts, organic compounds, free radicals, hydrides, oxides hydracids, acids of minerai oxides, organic acids, anhydrous single and double salts, hydrated single and double salts, complex cations in order of decreasing valency, complex anions in order of increasing valency, non-ionic and metallo-organic compounds. The properties given for these materials are magnetic moments, Curie points and Curie constants, mean atomic susceptibility, moments calculated according to Weiss law, magnetic anisotropy of crystalline compounds, molecular susceptibilities, effective moments, atomic moments, and other related data. The study of paramagnetic relaxation gives information both on the interactions between paramagnetic ions and on the interactions between the ions and the crystalline lattice. These data give magnetic techniques required for the production of very low temperatures by adiabatic demagnetization of paramagnetic substances. Cross references and bibliographical references are included. 
16906004

$* 5.133-3.133$

Franklin, J. L. i et al.

IONIZATION POTENT IALS, APPEARANCE POTENTIALS, AND HEATS OF FORMATION OF GASEOUS POSITIVE IONS

NSRDS-NBS-26, National Bureau of Standards, Washington, D. C.

June 1969

290p

Available from Superintendent of Documents, GPO

This is a compilation of ionization and appearance potentials of positive ions published from 1955 through June 1966. The compilation lists the ion formed, the parent species from which it was formed, the other products of the process, the threshold energy for the formation of this ion, and the method by which this data was obtained. Where feasible, the heat of formation at $298 \mathrm{~K}$ of the positive ion has been computed for each entry using auxiliary thermochemical data. From these computed values "best" values have been chosen.

16904009

*3.133-2.2633

Furnish, J.

ALPHA-ALPHA ELASTIC SCATTERING: A BIBLIOGRAPHY

LA-4011-MS, Los Alamos Scientific Laboratory, Los Alamos, New Mexico

October 1968

$16 \mathrm{p}$

Nuclear Science Abstracts was searched from Volume 7 (1955) through Volume 22, Number 15 (August 15, 1968) for material on the elastic scattering of alpha particles on alpha particles. The references are arranged numerically within each volume with the abstracts from the most recent volumes presented first.

6500048

$* 3.133$

Grodstein, G. W.

$X$-RAY ATTENUATION COEFFICIENTS FROM $10 \mathrm{keV}$ TO 100 $\mathrm{MeV}$

NBS Circular 583, National Bureau of Standards, Washington, D. C.

April 30,1957

$54 \mathrm{p}$

Available from the Superintendent of Documents, GPO

A tabulation of at tenuation coefficients of $X$-rays and gamma-rays from 0.01 to $100 \mathrm{MeV}$ for 29 materials is presented. A summary of information on the probability of the basic interaction processes of photons with matter and a detailed analysis of experimental and theoretical evidence are included. Present information on the basic processes is adequate for many applications; however, improved theory and additional experimental data are needed in certain areas. A comparison of calculated and experimental coefficients points up this need.

\section{3}

*4.133-3.133-8.133

GROUPS IV, $\mathrm{V}$, AND VI TRANSITION METALS, SELECTED REFERENCES 'ON PREPARATION, STRUCTURE', PHYSICAL PROPERT IES, METALLOGRAPHY, AND TRANSPORT PROPERTIES ORNL-RMIC-3 (Rev.), Research Materials Information Center, Oak Ridge National Laboratory, Oak Ridge, Tennessee

April 1965

$94 \mathrm{p}$

This bibliography contains lists of selected references on preparation methods, crystal structure, transport properties, and physical and mechanical properties for the Groups IV, V, and VI transition metals. An abstract is included along with each reference entry.
\#6902097

*4.133-3.133-8.133

GROUPS IV, V, AND VI TRANSITION METALS, SUPPLEMENT NO. 1, SELECTED REFERENCES ON PREPARATION STRUCTURE, PHYSICAL PROPERTIES, METALLOGRAPHY, AND TRANSPORT PROPERTIES

ORNL-RMIC-8, Research Materials Information Center, Oak Ridge National Laboratory, Oak Ridge, Tennessee April 1965

$27 p$

Available from CFSTI

This bibliography is a supplement to ORNL-RMIC- 3 (Rev.) of April 1965, and contains lists of selected references on preparation methods, crystal structure, transport properties, and physical and mechanical properties for the Groups IV, V, and VI transition metals.

16500116

Guthrie, J. W. (Editor)

TABLE OF ATOMIC MASSES

Sandia Corp. Monograph SCR-245A Corporation, Aibuquerque, New Mexico

April 1963

$302 \mathrm{p}$

Available from CFSTI

This table is a compilation of the atomic masses, symbols, atomic numbers, nominal weights, square roots of masses, relative abundance and absolute abundance of the is otopes of nuclides. It contains over 2400 entries.

\#6908043

*2. 2733-3.133

Hager, R. S.; Seltzer, E. C.

INTERNAL CONVERSION TABBLES. PART I. K, L, M-SHELL CONVERSION COEFFICIENTS

CALT-63-60, California Institute of Technology, Pasadena, California

June 1967

$160 \mathrm{p}$

Available from CFSTI

Calculations of $\mathrm{K}-, \mathrm{L}_{-}$, and $\mathrm{M}$-shell conversion coefficients were performed for nuclei with $Z$ from 30 to 103 taking into account the four lowest electric and magnetic multipoles. The energies of the continuum electron ranged from 1 to $1500 \mathrm{keV}$ for the M-shell. The coefficients are presented as a function of the continuum electron energy, the continuum electron momentum, and the gamma-ray transition energy.

\# 6905027

*8.133-3.133

Hanley, H. J. M. ; Klein, M.

ON THE SELECTION OF THE INTERMOLECULAR POTENTIAL FUNCTION: APPLICATION OF STATISTICAL MECHANICAL THEORY TO EXPERIMENT

NBS Technical Note 360 , National Bureau of Standards, Boulder, Colorado

November 20,1967

$88 \mathrm{p}$

Available from the Superintendent of Documents, GPO

A method has been developed to evaluate quantitatively the relationship between model intermolecular potential functions and macroscopic experimental properties. Specifically, the following function families have been studied: m- 6 , Kihara, exp:6 and Morse; the properties studied are: viscosity coefficient, diffusion coefficient, second virial and Joule-Thoms on coefficients. The principal conclusions from this work are: 1 . a temperature range exists (around room temperature for most substances) in which data are completely insensitive to the potential function; 2 . the function families studied are essentially equivalent; 3 . more than one function is required to represent data over a range of about $1000^{\circ} \mathrm{K} ; 4$. the experimental error which can be tolerated if experimental data are to be used to select a potential function has been estimated. The conclusions are experimentally verified for both transport and equilibrium properties. 
$\# 6811016$

$\star 5.031-3.031$

Harllee, F. N.; Rosenstock, H. M.; Herron, J. T. A BIBLIÓGRAPHY ON ION-MOLECUI.E REACTIONS, JANUARY 1900 TO MARCH 1966

NBS Technical Note 291, National Bureau of Standards, Washington, D. C.

June 28, 1966

$38 \mathrm{p}$

Available from Superintendent of Documents, GPO

This document is a bibliography on ion-molecule reactions of chemical interest. The bibliography is listed in a chronologic-alphabetic arrangement and includes 431 titles and references taken from the published literature since 1900. The coverage is limited to reactions between ions and molecules leading to changes in the atomic composition of either the projectile ion or target molecule. No attempt has been made to completely cover resonant charge exchange or elastic scattering.

\section{\#6500133 \\ Harrison, G.R. (Compiler)

$$
\text { *3. } 153
$$

AVELENGTH TABLES WITH INTENSITIES IN ARC, SPARK, OR DISCHARGE TUBE OF 100,000 SPECTRUM LINES MOS $t$ Strongly Emitted by the Atomic Elements Under Normal Conditions of Excitation between 10,000 and $2000 \mathrm{~A}$ Arranged in Order of Decreasing Waveleng ths

Cambridge, Massachusetts: MIT Press

April 1963

$429 \mathrm{p}$

These tables contain the wavelengths and intensities over 100,000 spectral lines measured and compared with literature values by the M.I.T. Spectroscopy Laboratory. In general, when the measured wavelength of a line agreed with the literature value within a reasonable tolerance, the measured wavelength has been compiled; this was the case for $75 \%$ of the lines. For other lines, the literature value that the authors considered most accurate has been quoted, and a reference to the original paper given. Wavelengths between 10,000 and 2,000 angstroms have been tabulated. For each line, the wavelength, intensity, and element producing the line has been listed, with a reference to the literature where necessary.

\# 6606014

*3.133

Heinrich, K. F. J .

AN OUTLINE FOR COOPERATIVE ACTION ON THE DETERMINATION OF X-RAY MASS ATTENUATION COEFFICIENTS IN THE WAVELENGTH RANGE FROM 0.5 TO 100 ANGSTROMS (25 TO $0.12 \mathrm{keV}$ )

NBS Technical Note 284, National Bureau of Standards, Washington, D. C.

February i966

$12 \mathrm{p}$

Available from the Superintendent of Documents, GPO

Sources of information on $x$-ray mass attenuation coefficients and the present status of data in this area are reviewed. Many gaps are observed in the existing data and some values derived by interpolation may be of questionable accuracy. Because of the importance of accurate mass attenuation coefficients, the extension and improvement of these measurements is proposed together with a plan for coordination of effort and exchange of information.

\#6500134 * 3.133

Heinz, 0.; Feinler, E. J

TABULÁTION OF ATOMIC AND MOJJECULAR CHARGE-TRANSFER CROSS SECTIONS

Interim Technical Report No. 1, Stanford Research Institute, Stanford, California

January 20,1961

$44 \mathrm{p}$

Available from DDC, AD 252518

Experimental results pertaining to atomic and molecular charge-transfer processes reported in the literature from 1950 through 1960 have been collected and tabulated on IBM punched cards. The parameters 1 isted for each reaction are: the energy interval of the measurement, the maximum charge-transfer cross section, and the energy at which this maximum cross section occurs. Inasmuch as the Massey criterion (a $\triangle E / v h$ approxinates 1 for maximum a) still represents the best method for correlating existing data and for making at least rough predictions for other charge-transfer events, calculated values of $\Delta \mathrm{E}$ have been included. The report also contains a fairly complete bibliography of the charge-transfer literature, including both experimental and theoretical papers. The punched cards from which the tables in this report were printed are available for duplication.

\#6510002 *3. 133

Herrmann, R.; Alkemade, C. T. J.

Gi lbert, P. T., Jr. (Translator)

CHEMICAL ANALÝSIS BY FLAME PHOTOMETRY, SECOND REVISED EDITION

New York: Interscience Publishers

1963

$644 \mathrm{p}$

This monograph deals with the principles and applications of chemical analysis by flame photometry, and data obtainable by or useful for flame analysis. The topics dealt with in the text section are: basis of flame analysis, apparatus and methods of measurement, control of the flame and introduction of the sample, optics and electronics, analytical technique, use of equipment, the flame emissions, standardization and measurement and flame analysis errors. The applications dealt with include agricultural applications, medical and biological applications, applications to geology, industry and other fields. The data in the appendix include the intensities of wavelengths of 3400 Iines and bands of 145 atoms, ions and molecules representing 70 elements that can be determined by flame photometry. This information was taken from results of spectrographic analyses published in the literature. The lines are arranged by emitting species in one table, and by wavelength in the other.

\#6605009

$$
\star 3.333
$$

Hershenson, H. M.

INFRARED ABSORPTION SPECTRA INDEX FOR 1958 - 1962

New York: Academic Press

1964

$173 \mathrm{p}$

This volume provides a five-year supplement to the original Infrared Absorption Spectra: Index for 1945-1957. The Ii te rature coverage of this Index includes 66 journals and one collection of spectra in book form. It contains approximately 20,000 references to infrared absorption spectra published during 1958-1962. As in the previous volume of this series, each reference indicates the journal, volume and page where an actual spectrum of the compound is reproduced.

$\# 6602002$ $\star 3.333-3.131$

Hershenson, H. M.

NUCLEAR MÁGNETIC RESONANCE AND ELECTRON RESONANCE SPECTRA INDEX FOR 1958-1963

New York: Academic Press

1965

$95 \mathrm{p}$

This volume provides aid in the form of an index for locating nuclear magnetic resonance (NMR) and electron spin resonance (ESR) spectroscopy data in the literature. It includes more than 8000 references to NMR and ESR spectra published in sixty-seven worldwide journals and in two collections of spectra during 1958-1963. Each reference in this index indicates the journal, volume, and page where an actual spectrum of the compound mentioned may be found. The index is arranged alphabetically according to the compounds whose spectra are given. 
\#6508012

$\star 3.233$

Vierzberg, G.

MOLECULAR SPECTRA AND MOLECULAR STRUCTURE,

I. SPECTRA OF DIATOMIC MOLECULES, SECOND EDITION

New York: D. Van Nos trand Company, Inc.

1950

$658 \mathrm{p}$

This textbook covers the field of diatomic molecule spectra, and contains tabulations of the electronic levels and the vibrational and rotational constants of all known diatomic molecules, plus photographs of certain important molecular spectra. The topics covered in the text portion are: a review of the elements of atomic spectra, observed molecular spectra, rotation and víbration of diatomic molecules, discussion of electronic states and transitions, details of electronic states and transitions, building up principles, electron configurations, continuous and diffuse molecular spectra, examples, results, and applications. The data tabulated include the electron term, yibrational constants, rotational constants, dissociation energy, internuclear distance, and observed transitions in every known state of every diatomic molecule.

f 6704016

$\star 3.233$

Herzberg, G.

MOLECULAR SPECTRA AND MOLECULAR STRUCTURE II INFRARED AND RAMAN SPECTRA OF POLYATOMIC MOLECULES New York: D. Van Nostrand Company, Inc.

Augus t 1966

$632 p$

This text is the second volume in a series of three on Molecular Spectra and Molecular Structure and deals with the information obtainable from infrared and Raman spectra. A large number of illustrations and tables have been included in which theoretical results are summarized or observed data are collected. The tables contain critically reviewed data published through 1945. The chapters in this text deal with rotation and rotational spectra, vibrations, vibrational energy levels, vibrational eigenfunctions, vibrational infrared and Raman spectra, interaction of rotation and vibration, rotation-vibration spectra and applications. The book contains an appendix of physical constants and conversion factors, a bibliography of almost a thousand references, and an author and subject index.

\section{$\# 6706037$}

$\star 3.233$

Herzberg, G.

MOLECULAR SPECTRA AND MOLECULAR STRUCTURE III. ELECTRONIC SPECTRA AND ELECTRONIC STRUCTURE OF POLYATOMIC MOLECULES

New York: D. Van Nostrand Company, Inc.

1967

745 p

This book presents a comprehensive review of theory and experimental results of studies dealing with electronic spectra of polyatomic molecules. The text deals with the following subjects: (1) electronic states, electronic energy, vibrational and rotational structure of states, effects of electric and magnetic fields on energy levels; (2) electronic transitions: types of transitions, rotational and vibrational transitions, Zeeman and Stark effect; (3) building-up of principles: correlation of electronic states, electron configurations, stability of molecular electronic states; (4) dissociation, predissociation and recombination, continuous and diffuse spectra; (5) electronic spectra of individual molecules and their interpretation for molecules of three to twelve atoms. The data presented include energylevel diagrams and individual molecular spectra as we 11 as tables of molecular constants of most polyatomic molecules (up to 12 atoms) for which discrete spectra have been analyzed. Constants tabulated for each molecule are: point group,
electronic levels, vibrational frequencies, rotational constants, structural parameters, observed transitions, and literature references.

\#6711013

$\star 3.333$

Hi ray ama, $K$.

HANDBOOK OF ULTRAVIOLET AND VISIBLE ABSORPTION SPECTRA OF ORGANIC COMPOUNDS

New York: Plenum Press Data Division

1967

$642 \mathrm{p}$

Ultraviolet and visible absorptions of organic compounds are characteristic of absorbing systems that consist of a so-called chromophore group and the auxochromic groups bonded to it. For this reason, the data in this volume are organized from the point of view of the absorbing system. Data contained in $\mathrm{Table} I$ include: absorbing system, compound name, solvent, wavelength of maximum absorption, mólar absorptivity, and reference to the literature. Table II is a ist of absorbing systems arranged by wavelength of maximum absorption which can be used for identifying compounds by absorption maxima.

\# 6908038

Hogg, B. G.; et al.

$* 3.133$

TABLE OF POSITRON ANNIHILATION DATA

Atomic Energy Review, 6:149-212

lay 1968

A portion of this table was compiled and circulated privately by B. G. Hogg as part of the Positrons Programs International in 1965. The table has been updated to mid-year 1967. The compilers of the table have a primary interest in chemical physics and hence there may be certain omissions of data in areas not directly concerned with this field, for example, data on the gamma-ray angular correlation measurements on metals and alloys. A further list of references is given at the end of the paper Physical Chemistry of the Positron and Positronium, by v. I. Goldanski in Atomic Ene $\overline{r g y}$ Review, Volume 6, May 1968.

\#6711005,6711006 *3.333

Howe 11, M. G.; Kende, A. S.; Webb, J. S. (Editors) FORMULA INDEX TO NMR LITERÁTURE DATA, VOLUME 1 AND VOLUME 2

New York: Plenum Press

1965,1966

$206 p, 516 p$

The information in these indices was taken by abstractors from searches through the relevant organic and physical chemistry journals as we 11 as through appropriate review articles and books. References were catalogued if they described data for a single compound (rather than a mixture) and if they met any of the following requirements: ful1 NMR curve was reproduced in the reference; part of an NMR curve was reproduced with proper calibration; precise chemical shift or spincoupling data were reported for one or more specified peaks. For each compound, indexing formula, structural formula and reference to articles in the literature are given. The indexing formula is the molecular formula with the element sequence written in the order $\mathrm{P}, \mathrm{S}, \mathrm{N}, \mathrm{O}, \mathrm{I}, \mathrm{Br}$, $\mathrm{Cl}, \mathrm{F}, \mathrm{C}, \mathrm{H}, \mathrm{D}$, then alphabeticaliy; this tends to group all' related compounds together. Volume 1 contains references prior to 1961; Volume 2 contains references from 1961-1962. 
\#6909064

*3.133

Hubbe11, J. H.

PHOTON CROSS SECTIONS, ATTENUATION COEFFICIENTS AND ENERGY ABSORPTION COEFFICIENTS FROM $10 \mathrm{keV}$ TÓ $100 \mathrm{GeV}$

NSRDS-NBS-2 $\dot{9}$, National Bureau of Standards, Washington, D. C.

August 1969

$85 \mathrm{p}$

Available from the Superintendent of Documents, GPO

This report updates and extends previous NBS tabulations. Section 1 contains the information of most immediate practical use: (a) a tabulation of the attenuation coefficient for 23 elements (H-1 to (1-92) between $10 \mathrm{keV}$ and $100 \mathrm{GeV}$ and (b) a tabulation of the energy absorption coefficient for air, water, and 18 elements between $10 \mathrm{keV}$ and 10 $\mathrm{MeV}$, and for air, water, and 7 elements up to 100 MeV. Section 2 contains detailed information on the predominant processes (photoelectric absorption, Compton scattering and pair production) and a brief discussion of other processes which combine to give the attenuation coefficient. Theoretical and experimental data are reviewed, and auxiliary tables and approximation formulas are given. Section 3 contains tabulations of cross sections for the predominant processes between 10 $\mathrm{keV}$ and $100 \mathrm{GeV}$ for 23 elements and for 13 compounds and mixtures.

- 6904007

$\star 2.2233,3.133$

Hubbe11, J. H. ; Berger, M. J.

PHOTON ÁTTENUATION

Engineering Compendium on Radiation Shielding, Vol. 1. Chapter 4 pages $167-202$

Berlin: Springer-Verlag

1968

"Photon Attenuation" is a section of the Engineering Compendium on Radiation Shielding which discusses and tabulates narrow-beam attenuation coefficients and energy-absorption coefficients. The mass energy-absorption coefficient is only one of many quantities that can be used to compute the transfer of energy to the medium. The mass energytransfer coefficient and the mass absorption coefficient are also tabulated. The principal results for attenuation coefficients and for the energy-absorption and related coefficients are listed in tables. The present compilation is based on a new analysis of all available information. Discussions of the atomic cross sections underlying the interaction coefficients are included.

\#6712007

*8.133-3.133

Hughes, R. L.; Smith, I. C.; Lawless, E. A. Holzmann, R. T. (Editor)

PRODUCTION OF THE BORANES AND RELATED RESEARCH

New York, London: Academic Press

1967

$533 \mathrm{p}$

This is a review and analysis of borane chemistry. The topics covered include: precursors to the boranes; formation of the boron-hydrogen bond and diborane; preparation and properties of the boranes, alkylboranes, and carboranes; kinetics and mechanism studies; preparation and properties of pentaborane and decaborane from diborane; separation and purification; analytical methods; boronnitrogen chemistry; acid-base chemistry and appendices on toxicology; infrared spectra, molecular structures, physical properties of boranes and their derivatives.
A 6811020

$* 3.042$

INTERNATIONAL DIRECTORY OF WORKERS IN THE FIELD OF ATOMIC AND MOLECULAR COLLISIONS, MAY 1967

ORNL-AMPIC-5, Atomic and Molecular processes Information Center, Oak Ridge National Laboratory, Oak Ridge, Tennessee

June 1967

$87 \mathrm{p}$

This is a directory of international scientists engaged in or interested in the field of electronic, atomic and molecular collisions; it is a revision of the 1965 directory and is a publication of the Atomic and Molecular processes Information Center.

\#6902026

*3.271

International Union of Pure and Applied Chemistry, Commission on Molecular Structure and Spectroscopy TABLES OF WAVENUMBERS FOR THE CALIBRATION OF INFRARED SPECTROMETERS

London: But terworths

1961

$112 \mathrm{p}$

These tables are reprinted from pure and Applied Chemistry, 1:537-699(1961) and contain a set of provisional wavelength standards presented by the Conmission on Molecular Structure and Spectroscopy. This report is in two parts: Part I contains $t a b l e s$ for the calibration of grating spectrometers of resolution $0.1-1 \mathrm{~cm}^{-1}$ in the range $4300-600 \mathrm{~cm}^{-1}$. Part II tabulates wavenumbers for the calibration of prism and small grating spectrometers of resolution $0.5-10 \mathrm{~cm}^{-1}$ in the range $3950-600 \mathrm{~cm}^{-1}$.

\#6509027

Johns on, W. H., Jr. (Edi tor)

NUCLIDIC MASSÉS, PROCEEDINGS OF THE SECOND INTERNATIONAL CONFERENCE ON NUCLIDIC MASSES, VIENNA, AUSTRIA, JULY 15-19, 1963

New York: Springer-Verlag

1964

$480 \mathrm{p}$

Separate abstracts were prepared for 30 of the 37 papers included. Six other papers were previous 1 y abstracted for NSA $17: 31406,32998,33950,36639$; 18:1057; and 19:14607. The remaining paper discusses the interest of the International Union of Pure and Applied Chemistry in the values of the fundamental constants.

$\# 6902078$

Jones, R. N.; et al.

$* 3.043-5.043$

COMPUTER PROGRAMS FOR ABSORPTION SPECTROPHOTOMETRY NRC Bulletin No. 11, National Research Council of Canada, Ottawa, Canada

1968

$159 \mathrm{p}$

Nine "building block" computer programs for performing the basic numerical computations of absorption spectrophotometry are described and listed. They are written in FORTRAN IV for card input and output. The input and output formats are standardized to permit easy interfacing to produce more complex data processing systems. Though developed for infrared spectrophotometry these programs are adaptable for use in the visible and ultraviolet. The operations covered include scale change and scale interval change, location of band maxima and minima, smoothing and differentiation, slit function convolution and deconvolution, single band generation, profile analysis and asymmetry analysis of single bands from the truncated moments, and ordinate addition. 
\# 6610010

*3. 143

Jordan, S.: Kaplan, S. A.; Pickelner, S. B.

AERODYNAMIC PHENOMENA IN STELLAR ATMOSPHERES-SUPPLEMENTARY BIBLIOGRAPHY COVERING THE PERIOD $1960-1964$

JILA Report No. 25, Joint Institute for Laboratory Astrophysics, Boulder, Colorado

December 2, 1964

$66 \mathrm{p}$

This bibliography is a supplement to NBS Technical Note 30 which contains a working bibliography of papers which appeared in the astronomical literature covering aerodynamical phenomena is stellar atmospheres for the period 1920-1959. This supplement updates that coverage through 1964.

\#6500172,6500173

$\star 3.343$

6500174

Kamlet, M.J. (Editor of Volume I)

Ungnade, H.E. (Editor of Volume II)

phillips, J.R.; Nachod, F.C. (Editors of Volume IV) ORGANIC ELECTRONIC SPECTRAL DATA,

VOLUME I: $1946-1952$;

VOLUME II: 1953-1955;

VOLUME IV: 1958-1959

New York: Interscience Publishers

$1960,1960,1963$

$1222 \mathrm{p}, 929 \mathrm{p}, 1184 \mathrm{p}$

These three volumes contain data on measurements of ultraviolet and visible spectra published in the literature. The authors have abstracted spectra for over 60 journals. Data were included if the investigated compounds were definable by a molecular formula and of sufficient purity to satisfy the usual analytic requirements; also, compounds of unidentifiable structure were included if they would be identifiable without recourse to the original reference. Also, both the wavelengths of maximal absorption and the corresponding molar absorptivity must have been stated or determinable from the original paper. Each reference contained the following information: compound rame and molecular formula, solvent, wavelength of maximum absorption, maximum molar absorptivity, Iiterature reference and year of publication. Volume (6500172) contains abstracts from articles published between 1946 and 1952, inclusive. Volume II (6500173) contains abstracts from I953-1955 and Volume IV (6500174) contains abstracts from 1958 and 1959 .

\# 7001017

Kelley, R. L.

ATOMIC EMISSION LINES BELOW 2000 ANGSTROMS TYYDROGEN THROUGH ARCON

NRL Report 6648, Naval Postgraduate School, Monterey, California and Naval Research Laboratory, Washington, D. C.

February 1968

$356 \mathrm{p}$

Available from the Superintendent of Documents, GPO

A tabulation of observed spectral lines below 2000 angstroms has been prepared from the published literature up to January 1967. It is intended principally as an aid to the astrophysicist and to the plasma spectroscopist who deals with the spectra of highly stripped atoms. This report includes the first 18 elements, hydrogen through argon. The tabulation is divided into two main sections: the emission lines by spectrum, and a finding list. The entries give the element and spectrum, vacuum wavelength, intensity, and classification. A reference list is included.

\section{$\# 6500150$}

$* 3.143$

Ke1ley, R. L.

A TABLE OF EMISSION LINES IN THE VACUUM ULTRAVIOLET FOR ALL ELEMENTS ( 6 ANGSTROMS TO 2000 ANGSTROMS)

UCRL 5612, Stanford Research Institute, Menlo Park, California

1959

$528 \mathrm{p}$
This report contains all the published atomic emission lines contained in the literature up to January 1959. Each line of data contains the vacuum wavelength in angstroms, the element and spectroscopic notation for ionization, the intensity, and reference numbers which refer to the list of references at the end of the report. The tabulation is divided into four sections. Section I is a list of intense Iines by ion; Section II is a finding list of these intense lines; Section II is a list of all the lines arranged by element: Section IV is a finding list for all elements. Sections I and II are those lines of section II which are reported as having high intensities. Section III is the most complete list, containing 41,000 lines. Section IV has many of the weaker lines of Section III removed and contains 23,000 lines. The lines marked with an asterisk in Sections III and IV are those listed in Sections I and II. Sections I and III include the data on ionization potentials (I.P.) given in Charlotte Moore's Atomic Energy Levels (National Bureau of Standards Circular No. 467). The intensities given in the tables are those published for spark sources. Throughout this table, the original author's estimates for intensity are used.

\#6603027

Kieffer, L, J.

*3.143-5.143

A BIBLIOGRAPHY OF LOW ENERGY ELECTRON

CROSS SECTION DATA

JILA Report No. 4, Joint Institute for Laboratory As trophysics, Boulder, Colorado

January 1,1964

$76 \mathrm{p}$

Available from Superintendent of Documents, GPO

This bibliography was compiled by the JILA Data Center for the purpose of providing a list of published reports which contain information on collisions between electrons, ions, atons, and molecules. The publication includes a bibliography of the experimental and theoretical low energy data, a molecule list, journal list, and author list. This bibliography is updated at yearly intervals.

A6811010

$\star 5.143-3.143$

Kieffer, L. J.

BIBLIOGRAPHY OF LOW ENERGY ELECTRON COLLISION CROSS SECTION DATA

NBS Miscellaneous publication 289, National Bureau of Standards, Washington, D. C.

March 10,1967

$87 p$

Available from Superintendent of Documents, GPO

A bibliography of Iow energy electron collision cross section data is presented. Only references which report original measurements or calculations of electron collision cross sections are included. The cross section data for each process are listed by atomic species in order of their atomic number. The data for molecules are listed in arbitrary order.

\#6810007

*5.143-3.143

Kieffer, L. J.

BIBLIOGRAPIIY OF PHOTOABSORPTION CROSS SECTION DATA JILA Report No. 5, Joint Institute for Laboratory Astrophysics, Boulder, Colorado

April 15, 1968

$32 \mathrm{p}$

This bibliography provides references to comprehensive collections of low energy atomic collision data. The data sought are those for wave-length regions of continuous absorption. Absorption and iorization coefficients, from which cross sections can be derived, are included, as well as references to molecular absorption in wavelength regions where there is continuous absorption even though there are line absorptions superimposed. The report contains a section which describes the data in the references of the 
bibliography; a section listing the title, authors, and complete reference for the papers cited; and a section consisting of an alphabetical author index.

\# 6500144

$* 5.143-3,143$

Kieffer, L. J

COMPILATION OF CRITICALLY EVALUATED ELECTRON IMPACT IONIZATION CROSS SECTION DATA FOR ATOMS AND DIATOMIC MOLECULES

JILA Report No. 30, Joint Institute for Laboratory As trophysics, Boulder, Colorado

Feb ruary 5, 1965

$73 \mathrm{p}$

This compilation of critically evaluated data presents basically two kinds of data. The first is "gross" of "total" ionization cross-sections, which correspond to the probability of producing a single positive change from any atomic or molecular species regardless of the mass or multiplicity of change on the ions produced. The other kind is that which gives the relative probability of producing ions with a specific charge and mass (in the case of molecules where dissociation can take place). The tables of "total" cross-section data are grouped by their $z$-number. The data for seven molecular species are ordered by their atomic weights. After these tables, the absolute crosssection data for single ionization by electron impact are presented. There then follows a section of cross sections for the dissociative ionization of diatomic molecules, and finally a section giving relative numbers of multiple-charged ions. Accuracy of data is in all cases at best $5 \%$

\section{$\# 6902064$}

Kieffer, L. J.

$* 5.143-3.143$

COMPILATION OF LOW ENERGY ELECTRON COLLISION CROSS SECTION DATA, PART I, IONIZATION, DISSOCIATIVE PROCESSES, AND VIBRATIONAL AND ROTATIONAL EXCITATION

JILA Report No, 6, Joint Institute for Laboratory Astrophysics, Boulder, Colorado

January 10,1969

$95 \mathrm{p}$

A comprehensive compilation of low energy electron collision cross-section data is presented. This is the first of three parts and is limited to experimental measurements, including data for all atomic species and for those molecules which are important in aeronomy, astrophysics and plasma physics. Literature data through September of 1968 are included. The compilation is divided into three main categories: ionization, including all processes which result in electron ejection from atomic or molecular targets; dissociation, including processes which lead to ionized fragments; and vibrational and rotational excitation. The data are organized by species in the order: atoms, diatomic molecules, triatomic molecules, etc. The atoms are ordered by the $Z$ number, and the molecules are ordered according to the atom with the largest $Z$ in the molecule.

$\# 6910045$

Kieffer, L. J

COMPILATION OF LOW ENERCY ELECTRON COLLISION CROSS SECTION DATA, PART II: LINE AND LEVEL EXCITATION JILA Report No. 7, Joint Institute for Laboratory Astrophysics, Boulder, Colorado

September 22, 1969

$177 \mathrm{p}$

This is the second part of a comprehensive compilation of low energy electron collision cross section data. The compilation is limited to experimental measurements and includes data for all atomic species and those molecules which are important for aeronomy, astrophysics, and plasma physics. The data included were taken from literature published through December 1968. However, reference to some materials is obtained from abstracting journals, so this compilation probably does not include all data published in the late fall of 1968. All of the cross section data in this volume of the compilation are for electronic excitation. The data are organized by initial species in the following order: atoms, diatomic molecules, triatomic molecules, etc. The atoms are ordered by the $z$ number of the nucleus, the smallest $Z$ 's first. For molecules, the $Z$ number of the atom with the largest $z$ in the molecule is taken to characterize the molecule. The molecules are then ordered by these characteristic $z$ 's, the smallest $z$ first. If necessary the second highest $Z$ atom in the molecule is used in the same way to determine the order. If the line emitted is characteristic of the ionized species, the data are located following the neutral species.

\#6811005

Kieffer, L. J.; Dunn, G. H.

ELECTRON IMPACT IONIZATION CROSS-SECTION DATA FOR ATOMS, ATOMIC IONS, AND DIATOMIC MOLECULES: I. ATPERIMENTAL DATA

Reviews of Modern Physics, 38: 1-35 January 1966

This review includes a compilation and critical evaluation of absolute cross sections for ionization of atoms and diatomic molecules by electron impact. Experimental techniques used for ionization are surveyed. Selected relative cross sections for production of multiply charged ions and a brief discussion of relative cross-section data near threshold are presented. Absolute limits are not set on the size of probable systematic error in the various experiments.

\#6811009

Krauss, Morris

COMPENDIUM OF ab initio CALCULATIONS OF MOLECULAR ENERGIES AND P ROPERTIES

NBS Technical Note 438, National Bureau of Standards, Washington, D. C.

December 1967

Available from the Superintendent of Documents, GPO

The number of ab initio molecular electronic calculations has increased dramatically in the last few years. Both the practitioners and other interested students of the results of the calculations have found it increasingly difficult to determine the present status of these calculations. This compendium references the work from 1960 to the present and abstracts from the mas of data the best values for several observable properties including the total energy, dissociation energy, electron affinity, spectros copic constants, electric moments, field gradients, polarizabilities, and magnetic constants. In order to provide an insight into molecular electronic structure, tables of orbital energies are also included. These tables are meant to direct attention to the successes and failures of the calculations by compiling a large percentage of the best results in a reasonably compact form. Its usefulness will be limited in time by rapid advance in the field.

\#6608025

$* 3.143$

Krupenie, P. H.

THE BAND SPECTRUM OF CARBON MONOXIDE

NSRDS-NBS-5, National Bureau of Standards, Washington, D. C.

July 8,1966

$87 \mathrm{p}$

Available from the Superintendent of Documents, GPO

This is an exhaustive review of the literature and a critical compilation of the observed and predicted spectroscopic data on $\mathrm{CO}, \mathrm{CO}^{+}$, and $\mathrm{CO}_{2}^{+}$ in the gas phase. 
$\# 6508002$

*3. 313

Kuentzel, L. E.

INDEX OF MASS SPECTRAL DATA LISTED BY MOLECULAR WEIGHT AND TIEE FOUR STRONGEST PEAKS

ASTM Special Technical Publication No. 356 , American Society for Testing and Materials, Phi ladelphia, Pennsylvania

July 1963

$244 \mathrm{p}$

This index contains data on 3200 mass spectra. The data tabulated for each spectrum are the compound name, molecular weight, peak mass/charge ratios and the intensities of these peaks. The spectra were taken from the American Petroleum Institute Project 44 files, the ASTM files, and the Manufacturing Chemists Association Project. Each entry contains the serial number by which the spectra may be found in the files. Compounds are arranged by molecular weight and by the mass/charge ratio of the four highest peaks.

\section{\#6508006,6508005}

$$
\text { *3. } 343
$$

MOLECULAR FORMULA LIST OF COMPOUNDS, NAMES, AND REFERENCES TO PUBLISHED INFRARED SPECTRA

FIRST SUPPLEMENT TO MOLECULAR FORMULA LIST OF COMPOUNDS, NAMES, AND REFERENCES TO PUBLISHED INFRARED SPECTRA

ASTM Special Technical Publication No. 331 and No. 331-A, American Society for Testing and Materials, philadelphia, Pennsylvania

1962,1963

$312 \mathrm{p}, 112 \mathrm{p}$

These two volumes serve as an index to 43,500 infrared spectra of organic compounds, published in the literature and abstracted by ASTM. The compounds are arranged by molecular formula. Each entry contains the molecular formula, compound name, and a literature reference giving journal name, volume, page and date. Where several spectra have been published for a given compound, all are listed.

\section{$\# 6508004$}

Kuentzel, L. E.

$$
\text { *3. } 313
$$

MOLECULAR FORMULA LIST OF COMPOUNDS, NAMES, AND REFERENCES TO PUBLISHED ULTRAVIOLET AND VISIBLE SPECTRA

ASTM Special Technical Publication No. 357, American Society for Testing and Materials, Philadelphia, Pennsylvania

1963

$153 n$

This list includes the 16,130 compounds, the ultraviolet or visible spectra of which have been indexed by the Wyandotte - ASTM Punched-Card Index as issued through the fifth supplement. The seriaI numbers given provide a reference to the location of the original spectra as published in the literature or in standard catalogues of spectra. Arrangement is by the order of the numbers of atoms in the molecular formulas. A journal and book reference appendix is included. CODEN has been used for journal references.

\section{$\# 6508003$}

Kuentzel, L. E.

SERIAL NUMBER LIST OF COMPOUND NAMES AND REFERENCES TO PUBL ISHED INFRARED SPECTRA

ASTM Special Technical publication No. 358 , American Society for Testing and Materials, Philadelphia, Pennsylvania

October 1963

$436 \mathrm{p}$

This list includes 55,665 compounds, the infrared spectra of which have been indexed hy the Wyandotte-ASTM Punched-Card Index as issued through the Tenth Supplement to the Infrared Data Index. of these spectra, 51,389 are regular infrared (2 to 6 microns), 2221 are near-infrared (0.7 to 3.6 microns) and 2055 are far-infrared (1i to 36 microns). The serial numbers provide a reference to the location of the original spectra as published in the literature or in standard catalogues or collections. This hook is companion to ASTM No. 331 , Molecular Formula List of Compounds, Names and References to publisher Infrared Spectra, whi ch Tists these same compounds in numerical order of their molecular formulae. STP No. 331 enables one to locate the published spectra of known compounds. This book provides the identity of unknown compounds from the spectrum serial number as obtained via some search mechanism involving the spectral or structural data.

\#6906001 *3.153-5.153 Laborie, P.; Rocard, J. -M.; Rees, J. A. ELECTRONIC CROSS-SECTIONS MACROSCOPIC COEFFICIENTS: 1. IIYDROGEN AND RARE GASES Paris: Dunod

1968

In French and English

The catalogue contains some of the principal experimental data concerning the interaction of electrons with atoms and molecules. It is a noncritical selection of data from the most accurate and most recent measurements available. The crosssections are all expressed in $\mathrm{cm} 2$ per atom or molecule, and given as a function of the electron energy expressed in electron volts. Curves are reproduced where the original article gives data in that manner. Tables of data are given when available. Atomic or molecular energy levels and macroscopic coefficients are included. The book includes data for hydrogen, helium, neon, argon, krypton, and xenon.

$\# 6500525$

$*_{0} 0.1151-3.151$

LANDOLT - BORVSTEIN, VUMERICAL DATA AND FUNCTIONAI RELATIONSHIPS IN' PHYSICS, CHEMISTRY, ASTRONOMY, GEOPIIYSICS AND TECINNOLOGY, SIXTH EDITION,

VOLUME I: ATOMIC AND MOLECULAR PHYSICS,

PART 1: ATOMS AND IONS

Eucken, A.; Hellwege, K.-H. (Editors)

Berlin, New York: Springer-Verlag

1950

$441 \mathrm{p}$

In German

This section of the Landolt-Bornstein tables contains data on the fundamental physical constants and the properties of atoms and ions. Information for the use of the tables, including data on systems of measurement and units, has also been included. A discussion of atomic spectra, including wavelength standards, electron terms, important spectral line wavelengths, ionization potentials, electron affinities, X-ray spectra, Zeeman and Stark effect data, pressure broadening and pressure displacement of spectral lines, and oscillator strengths and lifetimes of excited states is presented. Other properties of atoms and ions compiled include electron distribution, X-ray scattering and absorption, cross sections, magnetic moments, magnetic and electric polarizability, and Faraday effect data.

\#6500526

$* 0.1151-3.151-5.151-8.151$

LANDOLT-BORNSTEIN, NUMERICAL DATA AND FUNCTIONAL RELATIONSIIPS IN PHYSICS, CHEMISTRY, ASTRONOMY, GEOPHYSICS AND TECHNOLOGY, SIXTII EDITION,

VOLUME I: ATOMIC AND MOLECULAR PIIYSICS,

PART 2: MOLECULES I (NUCLEAR STRUCTURE)

Eucken, A.; Hellwege, K.-H. (E di tors)

Berlin, New York: Springer-Verlag

1951

$571 \mathrm{p}$

In German

This section of the Landolt-Bornstein tables contains atomic physical data of molecules which describes the structure and dynamics of their nuclear framework. The following properties have been tabulated: atomic distances and structures, 
valence energies of chemical bonds, dissociation energies of chemical honds, and molecular vibrations and rotations.

$\# 6500527$

$* 0.1151-2.2051-3.151$

LANDOLT-BORNSTEIN, NUMERICAL DATA AND FUNCTIONAL RELATIONSHIPS IN PIYSICS, CHENISTRY, ASTRONOMY, GEOPIIYSICS AND TECHNOLOGY, SIXTII EDITION,

VOLUME I: ATOMIC AND MOLFCULAR PIIYSICS,

PART 3: MOLECULISS II (ELECTRON SHELL) IVITH AN APPENDIX TO VOLIME I, PARTS 1,2 , AND 3

Eucken, A.; Ilellwege, K.-H. (Éditors)

Berlin, New York: Springer-Verlag

1951

$724 \mathrm{p}$

In German

This section of the Landolt-Bornstein tables contains data on the electron shells of molecules. The volume includes data published through September 1951. The following properties have been tabulated: band spectra of diatomic molecules, electron band spectra of multiatomic molecules, light absorption of solutions in the infrared and visible region, ionization energies of molecules, optical rotary power of molecules, electrical and optical polarizability of molecules, magnetic moments of molecules, diamagnetic polarizability of molecules, and quanta output of photochemical reactions.

$\# 6500528$ - NORNSTEIN, NUMERICAL DATA AND FUNCTIONAL RELATIONSHIPS IN' PHYSICS, CIIENISTRY, ASTRONOMY, GEOPHYSICS, AND TECHNOLOGY, SIXTII EDITION,

VOLUME I: ATOMIC AND MOLECULAR PIYYSICS,

PART 4: CRYSTALS

Hellwege, K.-H. (Edi tor)

Berlin, New York: Springer-Verlag

1955

$1007 \mathrm{p}$

In German

This section contains data on the atomic physical interpretation of crystal physics as of November 1954. The following properties have been tabulated: the characteristics of symmetry, elements, the 32 crystal classes, space groups, lattice types, structures, crystal dimensions of inorganic and organic crystals, atomic and ionic radii, lattice energies, internal vibrations of crystals, electron emission, energy bonds, X-ray spectra and bonding states, electron spectra of crystals, and high frequency spectra of crystals.

A 6500529

*0.1151-2.2051-3.151

LANDOLT-BORNSTEIN, NUMERICAI, DATA AND FUNCTIONAL RELATIONSIIIPS IN PHYSICS, CHEMISTRY, ASTRONOMY, GEOPHYSICS AND TECINOLOGY, SIXTH EDITIÓN,

VOLUME I: ATOMIC AND MOLECULAR PIIYSICS,

PART 5: ATOMIC NUCLEI AND ELEMENTARY PARTICLES

Hellwege, K.-H. (Editor)

Berlin, New York: Springer-Verlag

1952

$470 \mathrm{p}$

In German

This section of the Landolt-Bornstein tables contains tables on atomic nuclei and elementary particles. These tables were compiled by the authors from literature on these subjects published through November 1951. The properties tabulated are: the hyperfine structure of atomic terms and atomic lines, a table of all atomic nuclei and their properties, mass spectrographic measurements, nuclear reaction energies, electron line spectra of artificially radioactive atoms, the radioactive families, nuclear level schemes of radioactive transformations, energy schemes of the light atomic nuclei, beta-spectra, common naturally radioactive atoms and their alpha- and beta-spectra, scattering of charged particles by atomic nuciei, nuclear transition products, passage of alpha-particles, electrons, protons and gamma-rays through matter and cosmic radiation.
$\# 6500535$

$*_{0} .1151-3.151=4.151-8.151$

LANDOLT-BORNSTEIN, NUMERICAL DATA AND FUNCTIONAL RELATIONSHIPS IN PIIYSICS, CIIEMISTRY, ASTRONOMY, GEOPIIYSICS AND TECINOLOGY, SIXTH EDITIÓN,

VOLUNE II: PROPERTIES OF' MATTER IN ITS AGGREGATED STATES,

PART 8: OPTICAL CONSTANTS

Hellwege, K. -H.: Hellwege, A.M. (Editors)

Berlin, New York: Springer-Verlag

1962

$901 \mathrm{p}$

In German

This section of the Landolt-Bornstein tables contains tables and figures for the optical properties of substances. Data are compiled from literature published through June 1962. Data are compiled for: the optical constants and reflection of metals, alloys, and thin metal layers; refractive indices of inorganic and organic materials as a function of wavelengths; optical constants of selected solid materials in broad wavelength regions; rotation of the polarization plane in crystals; double refraction; piezooptical and electrooptical constants of piezoelectric crystals; light refraction by glasses and transparent plastic; light permeability, double refraction, Faraday effect and Kerr effect on glasses; refractive index of crystalline fluids: refractive index and optical rotatory power of pure liquids and solutions; Cotton-Mouton effect of liquids; double refraction due to current in liquids; Kerr constants of liquids; refractive indixes of gaseous elements and compounds; refraction and dispersion of air and of hydrogen compounds; Cotton-Mouton effect of gases and Kerr constants for gases.

\#6500537

*0.1151-2.2051-3.151

LANDOLT-BORNSTEIN, NUMERICAL DATA AND FUNCTIONAL RELATIONSHIPS IN PHYSICS, CHEMISTRY, ASTRONOMY, GEOPHYSICS AND TECINOLOGY, SIXTH EDITION, VOLUNE III: ASTRONONY AND GEOPIYYSICS

Bartels, J.; Bruggencate, P. T. (Editors)

Berlin, New York: Springer-Verlag 1952

$795 p$

In German

This volume of the Landolt-Bornstein series contains data on astronomy and geophysics. The material has been taken from literature published through 1951. The astrononical section includes data on astronomical instruments, position and time determination, abundance of the elements in the universe, the sun, mechanics and physics of the planets, moons, comets and meteors, stellar radiation, stellar spectra, stellar positions and movements, double stars, variable stars, novae, the galaxy, star clusters and extra-galactic nebulae. The section on geophysics contains data on the earth's gravity and tidal forces, radioactivity density; elastic, electrical, magnetic and thermal properties of minerals and rocks; seismicity and earthquake waves; properties of the earth's interior, surface and magnetism; physical and chemical properties of sea water; sea tides, hydrography, meteorology and climatology; and upper atmosphere physics.

$\# 6500539$

LANDOLT - BORNSTEIN, NUMERICAL DATA AND FUNCTIONAL RELATIONSHIPS IN' PHYSICS, CIENISTRY, ASTRONOMY, GEOPIIYSICS AND TECINOLOGY, SIXTH EDITIÓN,

VOLUME IV: TECHNOLOGY,

PART 2a: MATERIAL VALUES AND PROPERTIES OF INDUSTRIAL METALS, BASIC PRINCIPLES, TFSTING PROCESSES, INDUSTRIAL STEELS

Borchers, H.; Schmidt, E. (Edi tors)

Berlin, New York: Springer-Verlag

1963

$888 \mathrm{p}$

In German

This section of the Landolt-Bornstein tables includes data on metallographic principles and 
concepts, testing properties, and the properties of iron and its alloys. Data are given on diagrams of state, strength testing and destruction free materials. The physical properties of iron tabulated are lattice constants, density, thermal expansion, diffusion, atomic heats, specific heats, enthalpies, entropies and free enthalpies, pliase diagrams and transition points, thermal conductivity, electrical properties, magnetic properties, optical constants and excitation crosssections. Composition, treatment, strength values and special chemical properties of pure iron, unalloyed steels, highly alloyed steels, special steels, and cast iron are presented.

\section{$\# 6500540 \quad * 0.1151-3.151-4.151$}

LANDOLT-BORNSTEIN, NUMERICAL DATA AND FUNCTIONAL RELATIONSI]IPS IN PIIYSICS, CHEMISTRY, ASTRONOMY, GEOPIIYSICS AND TECHNOLOGY, SIXTH FDITIÓN,

VOLUME IV: TECHNOLOGY,

PART 3: ELECTROTECINOLOGY, OPTICS, X-RAY TECHNOLOGY Schmidt, E. (Editor)

Berlin, New York: Springer-Verlag

1957

$1076 \mathrm{p}$

In German

This section of the Landolt-Bornstein tables deals with the technological aspects of electricity, light and $X$-rays. It is compiled from the literature published through 1956. Wherever possible, the compilers have drawn, from several values available for a given quantity, the value they feel is most accurate. The properties tabulated in the electrical section are electrical conductivity, resistance and temperature coefficient of resistance; conductivity of molten metals; effect of pressure, tension, and cold machining on electrical resistance; resistance as functions of frequencies; resistance of thin layer semiconductors; thermoelectric emf of metal pairs and thermocouples in use; effects of pressure and cold treatment on the rmocouples; conduction of electricity in gases, breakdown potentials of gases; insulating liquids, filling and impregnating media, fibrous material insulators, insulating foils, nica insulators, rubber insulators; resin, plastics, quartz, glass, ceramic materials and rocks as insulators; and magnetic materials. The quantities tabulated in the light section are: sensitivity of the human eye, illuminating materials, light sources, light filters and photographic films. The $\mathrm{X}$-ray section includes data on measurements and units of X-ray technology.

\#6500524

$* 0.1151-2.2651-3.151$

LANDOLT-BORNSTEIN, NUMERICAL DATA AND FUNCTIONAL RELATIONSHIPS IN SCIENCE AND TECHNOLOGY, NEW SERIES

GROUP I: NUCLEAR PHYSICS AND TECHNOLOGY,

VOLIME 1: ENERGY LEVELS OF NUCLEI: $A=5$ TO $A=257$

Hellwege, A.M.; Hellwege, K.-H. (Editors)

Berlin, New York: Springer-Verlag

1961

$813 \mathrm{p}$

In German and English

This first volume of the New Series of LandoltBornstein contains the known energy levels and transitions for all nuclei from $\mathrm{A}=5$ to $\mathrm{A}=257$. The essential parts of the material are the level and decay schemes. These are supplemented by tables and special data. The tables list the following parameters for each level: excitation energy above ground state; the total angular momentum quantum number and the parity; the isobaric spin quantum number; the half life or mean life of the state, its half-width; the known modes of decay of the states; binding energies for various nucleon combinations, and the $Q$-values for the relevant nuclear reactions. Much of the same information is incorporated in diagrams in which the energy levels of the various nuclei are exhibited schenatically, together with the reactions by which they are formed. Recent references to papers dealing in a more general way with the properties of the nucleus in question are included. This compilation includes the data available through May 1960 with a few references to work done since that time.

\# 6802002 $*_{0.1151-2.1351-3.151}$

LAVDOLT-BORNSTEIN, NUNERICAL DATA AND FU:CTIONAL RELATIONSHIPS IN SCIENCE AND TECINOLOGY, NEW SERIES,

GROUP I: NUCLEAR PHYSICS AND TECINOLOGY,

VOLUME 2: NUCLEAR RADI I

Schopper, II. (Editor)

Berlin, New York: Springer-Verlag

1967

$54 \mathrm{p}$

In German and English

The volume contains nuclear radii and other characteristic parameters of atomic nuclei which are deduced from electron scattering experiments and the observation of mesic X-rays. The first section contains data on nuclear charge distributions derived from sources other than scattering, primarily mu-mesic atons for spherical nuclei. Data are given on energy levels and radius parameters for mu-mesic X-ray transitions; also for neighboring nuclides, deforned nuclei, and radii of isomers. The second section deals with nuclear radii determined by electron scattering. Both electric charge and magnetic radii are given. Because a variety of nuclear models are available, each nucleus is discussed separately, in addition to listing the size parameters.

\#6902044

$* 0.1151-2.2051-3.151$

LANDOLT-BORNSTEIN, NUNERICAL DATA AND FINCTIONAL RELATIONSHIPS IN SCIENCE AND TECHNOLOGY, NEW SERIES,

GROUP I: NUCLEAR PHYSICS AND TECHNOLOGY

VOLUME 3: NUMERICAL TABLES FOR ANGULAR CORRELATION COMPUTATIONS IN alpha-, beta-, AND gammaSPECTROSCOPY: $\quad 3 j-, 6 j-, g_{j-S Y M B O L S}, F-$ AND GAIRICOEFFICIENTS

Appe 1, H.

Schopper, H. (Edi tor)

Berlin, New York: Springer-Verlag

1968

$1206 \mathrm{P}$

In German and English

The various types of angular correlation coefficients have been tabulated in the literature. These tables provide the coefficients for a complete calculation of the basic angular correlations in alpha-, beta-, and gamma-spectroscopy. In chapter 2, the angular correlation formalism is given in a - necessarily incomplete- collection of relations involving the tabulated coefficients, i.e., 3j-symbols, 6j-symbols, 9j-symbols, F-coefficients, and GAMM-coefficients. In the computer tables these symbols are given in upright capital letters: $\mathrm{J}=\mathrm{j}, \mathrm{J}_{1}=\mathrm{j}_{1}$ etc. and GAMAA $=\Gamma$. These quantities are given solely hy angular momentum conservation for the total system in consideration. When answering the question of how the angular momenta of the various subsystems of particles may combine to fulfill the conservation condition, one ends up with Clebsch-fordan-coefficients for two angular momenta and with Racah-coefficients for more than two To achieve a higher degree of symmetry, Wigner has rewritten hoth types of coefficients as the $3 j-$ and $6 j$-symhols, respectively. In more compilicated correlations (e. triple correlations, where the intermediate transition also is observed) a new combination of $6 j-s y m b o l s$ appears which is usually called the 9j-symbol. For alpha-, beta-, and gamma-spectroscopy a combination of one $3 j-$ and one $6 j-s y m b o l$ with statistical factors is frequently used and is called the F-coefficient. The GAMA-coefficient is a slightly modified form of this F-coefficient useful for beta-spectros copy. The coefficients mentioned above are extensively tabulated in this volume. For the 9j-symbol, however, only selected ones are listed. In addition, there is a chapter dealing with angle functions. Since angular 
correlations are generally developed in a series of Legendre polynomials, these functions should be very useful.

\section{$\# 6605007$}

$* 0.1151-3.151$

LANDOLT-BORNSTEIN, NUNERICAL DATA AND FUNCTIONAL RELATIONSHIPS IN SCIENCE AND TECHNOLOGY, NEW SERIES,

GROUP II: ATOMIC AND MOLECULAR PIIYSICS,

VOLUME 1: MAGNETIC PROPERTIES OF FREE RADICALS

Fischer, $\mathrm{H}$.

Hellwege, K.-H.; Hellwege, A.M. (Editors)

Berlin, New York: Springer-Verlag

1965

$154 \mathrm{p}$

In German and English

This book contains data on the magnetic properties of free radicals, including inorganic and organic uncharged free radicals and radical ions. These data were taken from published results of ESR and atomic beam resonance experinents. The data compiled include: name and structural formula of a free radical compound, method of generation, crystal matrix or solvent used for the radical, temperature of measurenent, frequency of measurement, g-factor, hyperfine splitting parameter, primary references for the values quoted, and further references on the same subject.

$\# 6706020$

* $0.1151-3.151-4.151$

LANDOLT-BORNSTEIN, NUNERICAL DATA AND FUNCTIONAL RELATIONSHIPS IN SCIENCE AND TECHNOLOGY, NEW SERIES

GROUP III: ATOMIC AND MOLECULAR PIIYSICS,

VOLUME 2: MAGNETIC PROPERTIES OF COORDINATION AND ORGANO-METALLIC TRANSITION METAL COMPOUNDS

Koenig, E.

Hellwege, K.-II.; llellwege, A.M. (Editors)

Berlin, New York: Springer-Verlag

1966

$578 \mathrm{p}$

In German and English

Magnetic properties are listed for coordination and organo-metalic transition metal compounds. The magnetic susceptibility section lists for each compound gram susceptibility and molar susceptibility at a particular temperature: magnetic moments and paramagnetic Curic temperatures, and the experimental method used to obtain data. The electron paramagnetic resonance section lists for each compound the spectroscopic splitting g-factor, fine structure and hyperfine structure parameters, half-width of the EPR 1 ine, and temprerature and frequency of measurement.

\section{\#6706036}

$* 0.1151-3.151-5.151$

LANDOLT-BORNSTEIN, NUMERICAL DATA AND FUNCTIONAL RELATIONSHIPS IN SCIENCE AND TECHNOLOGY, NEW SERIES

GROUP II: ATOMIC AND MOLECULAR PHYSICS

VOLUME 3: LUMINESCENCE OF ORGANIC SUBSTANCES

Schmillen, A.; Legler, R.

Hellwege, K.-H.; Hellwege, A.M. (Editors)

Berlin, New York: Springer-Verlag

1967

$416 \mathrm{p}$

In German and English

The most important data on the luminescence of over 1000 luminescent organic substances investigated have been assembled into a main table. Most substances are shown note only in a pure state (liquid or solid) but diluted in several solvents as well. On compiling the literature, it was noticed that not all data were of equal reliability, e.g. owing to insufficient purity of the substances investigated or to the application of theories which had hecome out of date in the meantime. For these reasons the main table only contains the physical magnitudes which are known with sufficient reliability for the majority of substances investigated. Data appearing in the main table for each compound include: its state, solvent used, if any, temperature, wavelength used for stimulation, absorption and emission wavenumbers for fluorescent and phosphorescent emission, quantum efficiency, decay time, and literature reference. Following the main table, a selection of typical luminescence spectra are given. Tables of the wavenumbers of very sharp single bands are given for some important spectra. Data are given concerning applications of luminescence by radiation damage. The tables were taken from literature dating from 1949 to 1964.

\#6708004

$*_{0.1151-3.151}$

LANDOLT-BORNSTEIN, NUMERICAL DATA AND FUNCTIONAL RELATIONSIIIPS IN SCIENCE AND TECHNOLOGY, NEIY SERIES,

GROUP II: ATOMIC AND MOLECULAR PIYSICS,

VOLUME 4: MOLECULAR CONSTANTS FRON' MICROINAVE SPECTPOSCOPY

Stark, B.

Hellwege, K.-H.; Hellwege, A.M. (Edi tors)

Berlin, New York: Springer-Verlag

1967

$813 \mathrm{p}$

In German and English

This collection provides data on the rotation of molecules in the gaseous state obtained by microwave spectroscopy. The following molecular constants are presented: rotational constants centrifugal distortion constants, rotationvibration interaction constants, beta-type doubling constants, and isotopic masses or mass ratios. These constants are separately tabulated for diatomic molecules, linear molecules, symmetric top molecules and asymmetric top molecules. Also presented are electric dipole moments, nuclear quadrupole constants, and hindered rotation constants. There are also diagrams of the structura1 arrangement, including the position of the electric dipole moment, for those molecules whose structure could be deduced from the microwave measurements with sufficient reliability. References are tabulated with a special table for magnetic constants determined by zeeman effect.

$\# 6802001$

*0.1151-4.151-8.151

LANDOLT-BORNSTEIN, NUMERICAL DATA AND FUNCTIONAL RELATIONSHIPS IN SCIENCE AND TECHNOLOGY, NEW SERIES,

GROUP II. ATOMIC AND MOLECULAR PHYSICS, VOLUME 5: MOLECULAR ACOUSTICS

Schaaffs, $W$.

Hellwege; K.-H.; Hellwege, A.M. (Editors)

Berlin, New York: Springer-Verlag

1967

$286 \mathrm{p}$

In German and English

This volume lists data on acoustics based essentially on the molecular properties of matter, and values of sound velocity, absorption coefficients, and dispersion data. The measurements were made at frequencies ranging from $10 \mathrm{kc}$ to $200 \mathrm{Mc}$. Preference was generally given in compiling data to measurements for which statements on density, chemical purity, and the temperature coefficient were added, often, however, several statements can be found for a measured value. Generally, the limit of accuracy is $1 \%$ to $2 \%$. Data are given on sound velocity in gases and liquids : for pure gases and liquids as a function of temperature, pressure, and structure; also for mixtures of gases, mixtures of liquids, and solution. Data are compiled on sound absorption and dispersion in gases; sound absorption in homogeneous liquids, in solution, and on sound propagation in polymers, also on sound propagation in isotropic or quasi-isotropic solids (metals, inorganic non-metals, organic solids). There are special sections on sound propagation in liquid helium and shock wave velocity. 
$\# 6605010$

$* 0.1151-3.151$

LANDOLT-BORNSTEIN, NUMERICAL DATA AND FUNCTIONAL RELATIONSIIIPS IN SCIENCE NND TECHNOLOGY, NEW SERIES,

GROUP, VI : ASTRONOMY, ASTROPIIYSICS AND SPACE RESEARCH,

VOLUME 1: ASTRONOMY AND ASTROPHYSICS

Voigt, H.H. (Editor)

Berlin, New York: Springer-Verlag

1965

$711 \mathrm{p}$

In German and English

This volume of the Landolt-Bornstein New Series contains data useful in the fields of astronomy and astrophysics. The following topics are covered: optical instruments, radio-astronomical devices, performance of telescopes, photoelectric photonetry, position and time determination, astronomical constants, abundances of the elements in the universe; physical and mechanical properties of the sun, planets, satellites, comets, meteors, artificial earth satelites, interplanetary space, stellar positions and motions, stellar spectra, star colors and magnitudes, physics of stellar atmospheres, stellar structure and evolution, double stars, variable stars, novae and supernovae, planetary nebulae, star clusters and association, properties of the nearest stars, galactic structure, kinematic and hynamic properties of the galaxy, interstellar space, galaxies, and clusters of gálaxies.
\#6704009
$* 3.353$

Lang, L. (Editor)

ABSORPTION SPECTRA IN THE ULTRAVIOLET AND VISIBLE REGION A THEORETICAL AND TECHNICAL INTRODUCTION, FOURTH EDITION

New York: Acadenic Press, Inc. 1966

$80 \mathrm{p}$

This book introduces a continuing series of collections of absorption spectra in the ultraviolet and visible region for a wide range of compounds, all constructed to the logarithmic scale. Observed $\log$ I / I values have been entered into tables containing the original measured data. Spectrum characteristics unobtainable with the necessary degree of accuracy from the graph are thereby made accessible. Although the work was started in Hungary, subsequent volumes contain spectra by authors of several countries. This introductory book presents the fundamental theoretical information required for the interpretation of the spectra, as we 11 as conversion $t a b l e s:$ wavelengths - wave numbers, wave numbers - wavelengths, wave lengths - fresnels, wavelengths - Kca1/mole, transmittancy - absorbancy -absorptancy, list of the formulas used, and spectroscopical conversion table for energies. The book includes discussions on structure of spectra, application of the Franck-Condon principle to polyatomic molecules, anharmonicity, dissociation and predissociation, the form of electronic band systens, chromophores, complexes, the role of the medium effect, and a bibliography.

\#6704008,6609001-6609005

*3. 353

$6706048,6708010,6912055$

7001018,7001045

Lang, L. (Editor)

ABSORPTION SPECTRA IN TIIE ULTRAVIOLET AND VISIBLE REGION, VOLUMES 1 THROUGII 11

New York: Academic Press, Inc.

1963-1969

$414 \mathrm{p}, 408 \mathrm{p}, 424 \mathrm{p}, 414 \mathrm{p}, 528 \mathrm{p}, 412 \mathrm{p}, 412 \mathrm{p}, 416 \mathrm{p}$, $429 \mathrm{p}, 424 \mathrm{p}, 423 \mathrm{p}$

These volumes contain a description of the absorption spectra between 2000 and $5000 \mathrm{~A}$ of recently synthesized compounds, of compounds known in the literature, of data observed for individual compounds in different solvents, of spectra compiled for the investigation of $\mathrm{pH}$ relationships of spectra of isomer pairs and of a few vapor spectra. Absorption spectra are given for 2065 compounds. The logarithm of the molar extinction coefficient is plotted as a function of wavelength. A tabulation of optical densities is also given for each compound as a function of wavelength. Volume 1 is the fourth edition, Volumes 2 and 3 are the second edition, and Volunes 4 through 11 are the first edition.

$\# 6601004$

Lebedev, Ya.S.; Voevodskii, V.V.; Tikhomirova, N.N. ATLAS OF ELECTRON SPIN RESONANCE SPECTRA, 2 THEORETICALLY CALCULATED MULT ICOMPONENT SYMETRICAL SPECTRA

New York: Consultents Bureau

1964

$195 \mathrm{p}$

Translated from Russian

This book contains series of theoretically calculated multicomponent symmetrical ESR spectra. These spectra are for systems of 4 to 9 components, having binomial or certain internediate intensities, for both lorentzian and Gaussian line shapes of the components.

\#6508007 *3.353

Lewis, J. IN.

CONPILATION OF GAS CHROMATOGRAPIIC DATA

ASTN Special Technical Publication No. 343, American Society for Testing and Materials, Philade1phia, Pennsylvania

1963

$625 \mathrm{p}$

This compilation is a collection of results of gas chromatographic data taken from the literature on the subject. The tables can be used, either for identification of unknown materials, or for selecting a column for separating a known mixture. Data have heen tabulated on the basis of the liquid phase used and the serial number of the reference material.

\section{\#6606021 $* 3.253-5.253$}

Lipmann, B. A.; et al.

SYSTEMS ANALYSIS OF BALLISTIC MISSILE REFFNSE CONCERTS (U) VOLUME II: CALCULATION OF ATOMIC ANM MOLECULAR REACTION CROSS SECTIONS

DRC 66-7589, Defense Research Corporation, Santa Barbara, California

March 15, 1966

$121 \mathrm{p}$

Available theoretical and experimental data on the reactions that control the electron density in missile wakes and in the environment surrounding nuclear bursts have been reviewed. Calculations of cross sections (reaction rates) for specific reactions of importance in these prohlem areas have been made. The ain of the work here is also to develop general computational techniques designed to improve the accuracy and reliability of calculations.

\#6906028 $* 3.153$

Lucken, E. A. C.

NUCLEAR QUADRUPOLE COUPLING CONSTANTS

New York: Academic Press

1969

$360 \mathrm{p}$

This book of nuclear quadrupole coupling constants is divided into two sections. In the first, the basic theory of quadrupole coupling is presented the methods available for the experimentai measurement of the coupling constants reviewed and the relationship between them and a molecule's electronic structure discussed both from the rigorous and approxinate view-points. In the second, the experimental data are reviewed and correlated by means of the approximate methods developed in the first section. This includes the halogen coupling constants of organo-halogen 
compounds, N 1 it quadrupole coupling constants, constants of compounds of the typical elements of the Periodic Table, compounds of the transition and rare-earth elements, and charge transfer complexes. Author and subject indexes are included.

\section{" 6500184}

$\star 3.253$

IcCle1lan, A. I.

TABLES OF EXPERIMFNTAL DIPOLE MOMENTS

San Francisco: W. II. Freeman and Company

1963

$713 p$

This book is a non-critical compendium of experinental dipole moments taken from literature published from the 1920 's through 1961. These dipole moments were derived principally from measurements of the dielectric constants of materials or microwave measurements. The materials are divided into three classes; compounds without carbon, compounds containing carbon, compounds of unspecified formula. The second class is the largest by far and contains about 6000 entries. For each compound, the formula, compound name, and one or more measured values for the dipole moment are given. For each measurement, the state of the sample and/or type of solvent used, temperature of measurement, method of measurement, and a reference number referring to the book's bibliography are given. The appendices contain a list of average dipole moments of 75 common chemical compounds, the available moments for twelve homologous alkane series, and the criteria and treatment of data applied during the computation of the recommended values.

\section{\#6500047}

$\star 3.153$

McGinnies, R. T.

$X$-RAY ATTENUATION COEFFICIENTS FROM $10 \mathrm{keV}$ TO 100 $\mathrm{MeV}$

NBS Circular 583, Supplement, National Bureau of Standards, Washington, D. C.

October 30, 1959

$10 \mathrm{p}$

Available from the Superintendent of Documents, GPO

A revision is given of the $X-r a y$ attenuation coefficients presented in National Bureau of Standards Circular 583. Table 4 of that publication is eliminated, and a new table is given for each material for photon energies less than 100 $\mathrm{keV}$. The uncertainties in the estimates of attenuation coefficients at low energies are from 3 to 5 percent, which is the same as was previously given at higher energies. The cross sections for scattering are unchanged. Two values are listed for the photoelectric cross section, one calculated from the Sauter-Stobbe formulas and the other derived from new experimental evidence. The procedures for smoothing experimental data are described and are generally the same as were used in Circular 583. In addition to the systematic coverage of the region from $10 \mathrm{keV}$ to $100 \mathrm{MeV}$, some data are included for a number of elements based on experimental measurements below $10 \mathrm{keV}$ and above $100 \mathrm{MeV}$. A comparison is made between calculated and experimental total attenuation coefficients at energies above $10 \mathrm{MeV}$.

\section{\#6608027} *3.353

McLafferty, F. W.

MASS SPECTRAL CORRELATIONS

Advances in Chemistry Series No. 40, Mmerican Chemical Society, Washington, D. C.

1963

$117 \mathrm{p}$

This table provides the empirical and structural formulas of ions that might be found at a particular mass/charge ratio in a mass spectrum, plus an indication of how each such fragment ion might have arisen. An indication of the probability of each such classification of ions occurring is also given. Thus, the table indicates possible ion structures and precursor molecules for each of the prominent ions in the mass spectrum of an unknown compound, with a further indication of the general probability of their occurrence. Over 4000 spectra are listed.

\#6706033

McMaster, W. H. ; et al.

COMPILATION OF $X$-RAY CROSS SECTIONS SECTION II

UCRL-50174, California University, Lawrence Radiation Laboratory, Livermore, California

January 1967

$96 \mathrm{p}$

Preliminary graphs and tables of $\mathrm{X}$-ray cross sections in the range of $1 \mathrm{keV}$ to $1 \mathrm{MeV}$ are given. The total cross sections presented are the sum of computed scattering cross sections and the fit to derived experimental photoelectric cross sections. Form factors available in the literature were used to compute the scattering cross sections. Where solutions to the Ilartree-Foch equation were unavailable, Bewilogua's values for the ThomasFermi model were used. $L$ and $\|$ shell jump ratios were calculated from the theoretical values of Rakavy and Ron. The graphs for each element show the experimental data points used to obtain the fit.

\#6508060 *3.253

Mallemann, R. de; Suhner, $F$.

TABLES OF CONSTANTS AND NUMERICAL DATA, VOLUME 3. SELECTED CONSTANTS, MAGNETIC ROTÁTORY POIVER (FARADAY EFFECT) KERR MAGNETO-OPTIC EFFECT

Paris-Ve: Hermann \& Cie, Depositaires

1951

$138 \mathrm{p}$

In French

This set of tables gives data on gases and vapors of sirple compounds, mineral compounds, and organic compounds; on liquids of simple compounds, mineral compounds, and organic substances including hydrocarbons, esters of inorganic acids, alcohols, phenols, ethers, aldehydes, ketones sugars, acids, chloride acids, amides, anhydrides, nitrites, nitrates, azoimide, hydrazines, quaternary ammonium salts, heterocyclic compounds, and organo-metalic compounds; on electrolytic solutions of acids, bases, and mineral salts; and on molecular rotations of pure substances and electrolytic solutions. The Kerr magnetooptic effect section includes tables of rotation of ferromagnetic substances, graphs of rotation versus wavelength, dispersion of the rotation of visible light, dispersion of rotation of infrared, ellipticity, and the rotation of non-ferro-magnetic metals. The book contains formula indexes as well as bibliographical references.

\section{\#6500457 $\$ 3.353$ \\ Mary ott, A. A.; Buckley, F.}

TABLE OF DIELECTRIC CONSTANTS AND ELECTRIC DIPOLF MOMENTS OF SUBSTANCES IN THE GASEOUS STATE

NBS Circular 537, National Bureau of Standards, Washington, D. C.

June 25,1953

$29 \mathrm{p}$

Available from the Superintendent of Documents, GPO

Values of the dipole moments and other pertinent information are tabulated for approximately 350 substances in the vapor state. All values derived from measurements of dielectric constants have been recalculated by one of two systematic procedures in order to place the work of various investigators in a more comparable basis than exists in the literature. Values obtained independently from microwave spectroscopy and other methods are also included. values of the dielectric constants recommended for reference purposes are listed for helium, hydrogen, oxygen, argon, air, nitrogen and carbon dioxide. These selected values were derived from a consideration of radio frequency, microwave, and optical data. Much of the data given in this 
table have been superseded by $\# 6809016$ (NSRDS-NBS10).

\#6512043

TABLE OF DIELECTRIC CONSTANTS OF PURE LIOUIDS

NBS Circular 514, National Bureau of Standards, Washington, D. C.

August 1951

$44 \mathrm{p}$

Available from the Superintendent of Documents, GPO

The "static" dielectric constants of more than 800 substances in the liquid state were critically examined and tabulated in concise form. The table consists of three sections: A, Standard Liquids: $B$, Inorganic Liquids; and $C$, Organic Liquids. An indication of the probable accuracy of the data is given. Therever feasible, a simple analytical function is employed to express the variation of dielectric constant with temperature.

\#650\$063 *3.253

Mathieu, J. P.; Desnuelle, P.; Roche, J.

TABLES OF CONSTANTS AND NUNIERICAL DATA, VOLUME 10 , SELECTED CONSTANTS, OPTICAL ROTATORY POWER 3,' AMINO-ACIDS

London: Pergamon Press

1959

$61 p$

Preface and Introduction in French and English

This volume compiles optical rotatory power data from results published in the literature for amino acids, listed according to their molecular formulae. The data given include the chemical formula, structure, specific rotatory power, name and concentration of solvent and the temperature at which the measurement took place. An alphabetical substances index and list of hibliographica1 references are included.

$\$ 6605001$ *3.253

Mathieu, J. P.; Janot, M. M.

TABLES OF CONSTANTS AND NUMERICAL DATA, VOLUME 11 , SELECTED CONSTANTS OPTICAL ROTATORY POHER 4. ALKALOIDES

New York: Pergamon Press

1959

$211 p$

Preface and Introduction in French and English

Volume IV of Optical Rotatory Power is a compilation of physical constants of alkaloids. In the Table of Rotatory Powers the entries are made each alkaloid is the empirical formula. The alkaloid is printed in bold face whenever the rotatory power and the empirical formula have been seeningly reliably determined. The second colum contains the molecular weight. In the following columns are given: the solvent, concentration, temperature, wavelength and finally the specific rotatory power corresponding to those experimenta1 condition. Melting point limits follow the empirical formula and cannot be considered as physical constants but they are given in order to facilitate the identification of the alkaloid. The table is followed by the bibliography, an index of authors and formulae.

$\# 6508064$

*3.253

Mathieu, J.P.; Ourisson, G.

TABLES OF CONSTANTS AND NUMERICAL DATA VOLUME 9. SELECTED CONSTANTS, OPTICAL ROTATORY PONER 2. TRITIRPENOIDS

New York: Pergamon Press

1958

$302 \mathrm{p}$

Preface and Introduction in French and English

This volume compiles optical rotatory power data from results published in the literature for triterpenoids, listed according to their molecular formulae. The data given include the chemical formula, structure, specific rotatory power, name and concentration of solvent, temperature at which the measurement took place, and wavelengths. An alphabetical index of substances, author index, and list of bibliographical references are included.

\# 6503062

Mathieu, J. P.; Petit, A.

TABLES ÓF CONSTMNTS NYD NUMERICAL DATA VOLU:E 6 : SELECTED CONSTANTS, OPTICAL ROTATORY POWER, I. STEROIDS

Paris: Masson \& $\mathrm{C}^{i e}$

1956

$507 \mathrm{p}$

Preface and Introduction in French and English

This volume compiles optical rotatory power data from results published in the Iiterature for steroids, listed according to their molecular formulae. The data given include the chemical formula, structure, specific rotatory power, name and concentration of solvent, temperature at whicl the measurement took place, and wavelengths. An alphabetical index of substances, author index, and list of bibliographical references are included.

\#6811015

Mavrodineanu, R.

BIBLIOGRAPIYY' ON FLAME SPECTROSCOPY,

APPLICATIONS 1800-1966

NBS Miscellaneous Publication 281, National Bureau of Standards, Washington, D. C.

February 23,1967

$155 \mathrm{p}$

Available from the Superintendent of Documents, GPO

This collection consists of 5,113 references to works on flame spectros copy, selected with emphas is toward analytical measurements. It covers the period from 1300 to 1966. Subject indexes, keyed by number to the references cited, precede most of the sections.

\#6512004,6512005

Mavrodineanu, $R$.

BIBLIOGRAPHY ON ANALYTICAL FLANE SPECTROSCOPY,

VOLUNES 1 AND 2

Ellicott City, Maryland: Central Library of

Spectros copy

1963,1962

$64 \mathrm{p}, 54 \mathrm{p}$

The collection of references on analytical flame spectrometry presented in these two volumes cover the periods 1848 to 1956 with 925 references (Volume 1) and 1956 to March 1959 with 766 references (Volume 2). In addition to references on instrumentation and methods using the flame as an excitation source, a large number of works where flame spectrophotometry was used as an analytical tool were included. The references are indexed as follows: books on analytical flame spectroscopy; basic and theoretical papers; bibliographies on analytical flame spectroscopy in scientific books; reviews; theses; instrumentation; procedures; air and $\mathrm{CO}^{2}$; alcoholic beverages; hiological materials; cement; refractories and glass; chenicals; dairy products; food; fuels, oils and coal; metals, alloys and catalysts; piants; rubber, plastics and paper; soil, fertilizers, minerals and rocks; water; and the following elements: $A g, A 1, B, B a$, $\mathrm{Br}, \mathrm{C}-12$ and $\mathrm{C}-13, \mathrm{Cs}, \mathrm{Cu}, \mathrm{Fe}, \mathrm{Ga}, \mathrm{I}, \mathrm{In}, \mathrm{K}, \mathrm{Li}$, $\mathrm{Mg}, \mathrm{Mn}, \mathrm{N}, \mathrm{Na}, \mathrm{Ni}, \mathrm{Pb}$, Rare Earths, $\mathrm{Rb}$, Só, $\mathrm{Sn}$, Sr, $v, 2 n$. The papers are arranged chronologically and in alphabetical order by author and wherever possible, the Chemical Abstracts or Biological Abstracts reference is added. All of the references are indexed on punched tab cards for use with the "peek-a-boo" retrieval systen. 
Yavrodineanu, R. ; Boiteux, II.

FLA'IF SPECTRÓSCOPY

New York: John Wiley \& Sons, Inc.

1965

$721 \mathrm{p}$

This text discusses analytical spectroscopy by flame. Topics emphasized range from the principles important to the proper selection, design, and construction of excitation sources, to the identification, interpretation, and understanding of the various radiations excited by flares, and to the significance of certain reactions involved in the radiation processes. A large volume of data from the literature has been included with proper references. The book is divided into three parts. The first part consists of the analysis of various properties of flames, and of the description of the elements involved' in the construction of the excitation source and of its function conditions. The second part explains the mechanism of spectra of the chemical species excited in the acetylene flames, and discusses some of the reactions occurring in flames. The third part comprises reproductions of spectra from various chemical species excited in the acetylene flames, followed by wavelength tables and two complementary bibliographies.

\# 6500129,6500132 *3.153

Meggers, W. F.; Corliss, C. II.; Scribner, B. F. TABLES OF SPECTRAL LINE INTENSITIES, PART I. ARRANGED BY ELEMENTS; PART II. ARRANGED BY WAVFLFNGTIS

NBS Monograph 32, Part I and Part II, National Bureau of Standards, Washington, D. C.

December 29, 1961; Óctober 2, 1961

$473 p, 272 p$

Available from the Superintendent of Documents, GPO

The relative intensities or radiant powers of 39,000 spectral lines with wavelengths hetween 2000 and 9000 angstroms have been determined on a uniform energy scale for seventy chemical elements. This was done by mixing 0.1 atomic percent of each element in powdered copper, pressing the powdermixture to form solid electrodes which were burned in a 10 ampere, 220 volt direct-current arc, and photographing the spectra with a stigmatic concave grating while a step sector was rotating in front of the slit. The sectored spectrograms facilitated the estimation of intensities of all element lines relative to copper lines which were then calibrated on an energy scale provided by standardized lamps, and all estinated line intensities were finally adjusted to fit this calibration. Comparisons with other intensity neasurements in individual spectra indicate that the National Bureau of Standards spectral-1ine intensities may have average errors of 20 percent but first of al1, they provide uniform quantitative values for the seventy chemical elements commonly determined by spectrochemists. These data are presented by element in part $I$, and al1 39,000 observed lines are given in order of wavelength in part II.

\# $7003005 \quad$ *3.153

Miles, B.M.; Wise, W.L.

BIBLIÓGRAPIYY ON ÁTONIC TRANSITION PROBABILITIES, JANUARY 1916 THROUGI JUNE 1969

NBS Special Publication 320, National Bureau of Standards, Washington, D.C.

Feb ruary 1970

111 p

Available from Superintendent of Documents, GPO

A revised and updated bibliography on atomic transition probabilities including all new references up to July 1,1969 is presented. The papers, except for a number of comprehensive articles, are arranged according to elements and stages of ionization. In addition to the literature reference, the employed experimental or theoretical method is indicated. For the 20 lightest elements and $\mathrm{Ba} I$ and II the literature is presented on a critically selected basis. To keep the bibliography to a compact size, a number of comprehensive papers which contain numerical results throughout extended isoelectronic sequences are 1 isted in a separate section. Also included in this bihliography is a supplementary list of selected important papers dealing with the subject of transition probabilities from a general point of view.

里 6500429

$* 3.153$

Milik, J. T.

FLUOROCARBON GASES, DATA SIIEETS

DS-142, Ilughes Aircraft Company, Electronic Propertics Information Center, Culver City, California

November 1964

$111 \mathrm{p}$

A compilation of the electrical properties of various halocarbon or halogenated hydrocarbons known as Freons, Genetrons, Arctons, etc., is presented. A master identification chart relating the trade names and numbers to the chemical name is included for easy reference. Detailed electrical properties include corona effects, dielectric constant, dielectric strength and dissipation factor. Each property is compiled over the widest possible range of pressure, temperature, electrode geometry effects and types of electrodes from references obtained in a thorough literature search. Physical and chemical property data are also included as well as electrical and electronic applications.

$\$ 6500431$

Milek, J. T.

SULFUR HEXAFLUORIDE DATA SIIEETS

DS-140, Electronic Properties Information Center, Hughes 'Aircraft Company, Culver City, California October 1964

$68 \mathrm{p}$

A compilation of the electrical properties of sulfur hexafluoride, a dielectric gas, is presented. Electrical properties include corona, dielectric constant, dissipation factor and dielectric strength. The latter property data section is segregated into parameter effects as follows: pressure, gap distance, temperature, electrode configurations and gas mixtures. Fach property is compiled over the widest possible range of parameters ohtained in a thorough search of the world's literature.

\# $6907006 \quad * 3.153$

Miles, B. M.; Wiese, W. L.

CRITICALIY EVALUATED TRANSITION PROBABILITIES FOP $\mathrm{Ba}$ I AND I Tectical Note 474 , National Bureau of Standards, Washington, D. C.

January 1969

$22 n$

Available from the Superintendent of Documents, fro

Critically evaluated transition protabilities, in order of increasing quantum numbers, are comoiled for $\mathrm{Ba}$ I and II from available literature sources.

\# 6511002

Miller, R. V.; Golden, S. A.

RADIATIVE PROṔERTIES ÓF GASES, VOLUME 1. GENERAL DISCUSSIONS

R-6272, Rocketdyne, Canoga Park, California August 12 , 1965

$222 \mathrm{p}$

This report describes theoretical studies of the basic radiative properties of high temperature gases. The primary emphasis of this work has been devoted to methods of calculating spectral absorption coefficients of electronic transitions in diatomic molecules. The smeared line model has been used to calculate absorption coefficients in 
fifty such transitions. Ancillary work has included studies of methods of calculating numerical potential functions of the Rydberg-KIcinRees type, solutions to the Schroedinger equation with such potential functions, and computational procedures for evaluating Franck-Condon and r-centroid factors.

H 6500146

$* 3.153$

Mohler, F. L.

P!IOTOIOH:IZATION OF ATOHS AND IIOLECULISS

NBS Technical Note 131, National Bureau of Standards, Washington, D. C.

January 1962

$43 \mathrm{p}$

Available from CFSTI, PB 161632

This is a review of experimental results on photoionization of atoms and some molecules. There are some quantitative data on all the alkalies. magnesium, calcium and thalliurn and all rare gases except xenon. Results are given for the common gases: hydrogen, nitrogen, oxygen, $\mathrm{CO}, \mathrm{CO}$, NO, $\mathrm{N}_{2} \mathrm{O}$, NO ${ }_{2}, \mathrm{H}_{2} \mathrm{O}$ and $\mathrm{CH}_{4}$. Autoionization - excitation to a state above the ionization threshold followed by transition to the ionized state - can be an important factor. Often the broad autoionization lines mask the true continuum. There are some mass spectrometric measurements of photoionization products for most of these molecules.

\#6811003

$* 5.153 \cdot 3.153$

Noiseiwits ch, B. L. ; Smith, S. J.

ELECTRON IMPACT EXCITATION OF ATOIS

NSRDS-NBS-25, Nationa1 Bureau of Standaris, Washington, D. C.

August 1968

$120 \mathrm{p}$

Available from Superintendent of Documents, GPO

The experimental and theoretical literature about the electron impact excitation of atoms is reviewed. Theoretical methods ranging from the Bethe and Born approximations to the close coupling. approximations are discussed and intercompared. Where possible, on theoretical grounds or through intercomparison, the reliability of the various methods is discussed. A general critique of the optical method of measuring excitation functions is given, with the objective of promoting higher quality future experimental work. A critical study of existing experimental work leads to the conclusion that most workers have ignored inportant physical and instrumental effects, and it may he presumed that the data in the literature are subject to many unrecognized systematic errors. The literature on the alkalis, heavy rare gases, mercury, cadmitum and zinc is surveyed but the quality of the literature does not support critical review heyond some general comments about the physics of these atons.

\section{$\# 6500118,6500119$ \\ $* 3.153$}

6500120

Moore, C. F.

ATONIC ENERGY LEVELS, VOLUME I, 1 II TO $23 \mathrm{~V}$, VOLINIE II. $24 \mathrm{Cr}$ TO $41 \mathrm{Nb}$,

VOLUME III. $42 \mathrm{MO}$ TO $57 \mathrm{La}, 72 \mathrm{Hf}$ TO $89 \mathrm{AC}$

NBS Circular 467, National Bureau of Standards, Washington, D. C.

Jure 15, 1949; August 15, 1952 ; Nay 1, 1958 $309 \mathrm{p}, 227 \mathrm{p}, 245 \mathrm{p}$

Available from Superintendent of Documents, GPO

This series of three volumes is a critical compilation of atomic energy levels preparer at the National Bureau of Standards from the analyses of optical spectra. Volume I contains data on the spectra of hydrogen, deuterium, tritium, helium, lithium, heryliium, boron, carbon, nitrogen, oxygen, fluorine, neon, sodium, magnesium, aluninum, silicon, phosphorus, sulfur, chlorine, argon, potassium, calcium, scandium, titánium, and vanadium ( 111 to $23 \mathrm{~V}$ ). Volume II analyzes the spectra of chromiun, manganese, iron, cobalt, nickel, copper, zine, gallium, germanium, arsenic, selenium, bromine, krypton, rubiclium, strontium, yttrium, zirconium, and niobium (24Cr to $41 \mathrm{Nb})$. Volume II includes the spectra of molybclenur, technetium, ruthenim, rhodium, palladium, silver, cadmiun, indium, tin, antimony, tellurium, iodine, xenon, cesiun, barium, lanthanum, hafnium, tantalun, tungsten, rhenium, osmium, iridium, platinum, gold, mercury, thallium, lead, bismuth, polonium, radon, radium, and actinium (42\%lo to $57 \mathrm{La}, 72 \mathrm{Iff}$ to $89 \mathrm{Ac}$ ).

\#6508023

Moore, C. E.

THE ATOMIC SPECTRA OF TIIE RARE EARTIJS: TIIEIR PRESENCE IN THE SUN

$\frac{\text { Applied }}{\text { July } 196 \frac{\text { ptics }}{3}}, 2: 665-674$

A summary of the present state of analysis of the first and second spectra of the rare-earth elements is presented with an extensive bibliography. The astrophysical importance of these spectra is stressed. Counts are given of the number of lines of each rare-carth spectrum identified at present in the accessible solar spectrum. Most of then appear only in the singly ionized state; exceptions are Eu I, Tm I?, and Yh I. The possible presence of the strongest laboratory line of Ce III in the solar spectrun is suggested. If correct, this is the first evidence of a third spectrum line in the accessible range of the solar sspectrum $(\lambda)$ $30009)$.

$\# 6902038,6905015$

$* 3.153$

6906025,6910015

Noore, C. E.

BIBLIOGRAPIIY ON TIEE ANALYSIS OF OPTICAL ATONIC SPECTRA,

SECTION 1: 1II T0 $23 \mathrm{~V}$

SECTION 2: $24 \mathrm{Cr}$ TO $4 \mathrm{i} \mathrm{Nb}$,

SECTION 3: $42 \mathrm{NO}$ TO $57 \mathrm{La}$ AND 72 IIf TO $89 \mathrm{AC}$,

SECTION 4: La-57La TO $71 \mathrm{Lu}$ AND 89AC TO OดEs

NBS Special Publications 306-1, 2, 3, 4, National Bureau of Standards, Washington, D. C.

September 1968; February, May, Aurust 1969

$90 \mathrm{p}, 63 \mathrm{p}, 43 \mathrm{p}, 55 \mathrm{p}$

Availahle from the Superintendent of Docunents, GPO

The three published volumes on "Atomic Energy Levels", N3S Circular 467, contain for each spectrum the bibliography that was used in compiling the data. The present work is a continuation of these bibliographies arranged in the sane form. The time interval is the span from the respective dates of the earlier publications to the present. The selection of references is restricted to those needed for the preparation of revised tables of atomic energy levels and multiplets. The hibliography is heing published in four sections; each covers the same elenents as the respective volumes of AFL. Section I contains reference lists for the elements 1 if through $23 \mathrm{~V}$, corresponding to Volume I. The spectra of a given element are listed in order of increasing stage of ionization. Listings are included for spectra of the 23 elements. The original papers have been examined for nearly all of the quoted references. A selected list of general literature references is also included. Section II is similarly arranged, giving references to the spectra of the elements $24 \mathrm{Cr}$ through $41 \mathrm{Nb}$, corresponding to AEL Volume II. For a given element the spectra are listed in order of increasing stage of ionization. The original papers have been examined for nearly all of the quoted references. Section III is similarly arranged giving references to the spectra of the elements, $42 \mathrm{Mo}$ through $57 \mathrm{La}$ and $72 \mathrm{IIf}$ through $89 \mathrm{Ac}$, similar to AEI. Volume III. For a given element the spectra are listed in order of increasing stage of ionization. The original papers have heen examines for nearly all of the quoted references. Section IV concludes this puhlication, and completes the coverage of the Periodic Table. The references listed have been selected on a general hasis as those needed for the preparation of VoIume IV of 
"Atonic Fnergy Levels". Since rare-earth structure is revealed in the neighboring spectra, 57J.a and $89 A C$, the references for these elements aro repeated in the present Section, which contains the two groups of elements: $57 \mathrm{La}$ to $71 \mathrm{Lu}$ and $89 \mathrm{Ac}$ to $99 \mathrm{Es}$. A selected list of general literature references is also included. Nearly all of the quoted references have been checked from the original papers.

\section{$\# 6500125$}

$\div 3.153$

Noore, C. F.

OILT IPLTT TAISLE OF ASTROPIIYSICNL INTEREST

PART 1: TABLE OF IULTIPLETS ;

PART 2: FINDING LIST OF ALL LINFS IIS TAE TABLF OF MULT I PLITS

NBS Technical Note 36, National Bureau of Standards, Washington, D. C.

Noventyer 1959

$140 \mathrm{p}$

Available from CFSTI, PI 151395

Pending the completion of a current edition, the 1945 fultiplet $\mathrm{Table}$ is heing reprinted here to meet continuing denands. The leading lines in 196 atomic spectra of 85 chemical elements are listed in related groups called multiplets. Estimated intensities, excitation potentials and multiplet designations are given for the indiviclual lines, and each multiplet is assigned a number. An extensive bibliography covers the source material used for the compilation. The Table is presented in two parts: Part I inclues the multiplets, with the spectra of eacb element being given in order of increasing ionization, and the elements in order of increasing atomic number. Part II is a Finding List in which all the lines in Part I are entered in order of increasing wavelength, with their multiplet numbers. The range of the $\mathrm{Tab}$ le is from $2951 \mathrm{~A}$ to $13164 \AA$. A supplementary table of "Forbidden Lines" extends from $2972 \AA$ to $12645 \AA$.

$\# 6500161$ $* 3.153$

Moore, C. E.

SELECTED TABLES OF ATOMIC SPECTRA, ATONIC ENERGY LEVTLS AND MULTIPLET TABLES, Si II, Si II Sure Si IV
NSRDS-NBS -3 , Section 1 , National Standards, Washington, D. C.

June 25,1965

$38 \mathrm{p}$

Available from the Superintendent of Documents, GPO

The compilation contains tables of atomic energy levels and multiplet tables for the spectra of Si II, Si II, and Si The data in these tables II,
were
tafen fron through 1965. Section A, Atomic Energy Levels, contains tables of energy levels of each atom or ion. The data given for each level include its electron configuration, spectroscopic notation, the $v a l u e$ of the quantum number $J$, the energy expressed in $\mathrm{Cm}^{-1}$, and the interval in $\mathrm{cm}^{-1}$ hetween levels of the same configuration and different values of $\mathrm{J}$. The Multiplet Table, Section B, contains the wavelength, intensity and multiplet numer of each multiplet found. Also shown are the excitation potentials, spectroscopic configurations, and values of $J$ for the two levels hetween which the transition takes place. Literature references are given for hoth tables.

\#6809020 $* 3.153$

Moore, C. E.

SELECTED TABLES OF ATOMIC SPECTRA, ATOMIC ENERGY LEVET,SLS, AND MLTIPLET TABLES, Si

NSRDS-NBS-3, Section 2, National Bureau of Standards, Washington, D. C.

Novenber 30,1967

$22 \mathrm{p}$

Available from the Superintendent of Documents, GPO

The present publication is the second section of a series being prepared in response to the increasing demand for a current revision of two sets of tables containing data on atomic spectra as derived from analyses of optical spectra. Both the atomic energy levels and the multiplet table are included in the same puhlication, as parts $\Lambda$ and $B$, respectively. The sections are heing prepared at irregular intervals for these spectra whose analyses are essentially complete. A flexible paging system permits the arrangement of the various sections by atonic number, regardless of the order in which the spectra are published in this series. Section 1 included three spectra of silicon, $Z=14:$ Si II, Si III, Si IV, The present section contains similar data for Si I. The form of presentation is described in detail in the text to Section 1, and need not he repeated here.

$\# 6611020$

*3. 153

loore, C. E.; Minnaert, M. F. J.; Houtgast, J.

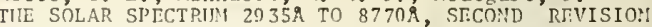
OF ROHLAND'S PRELIMINARY TABLF, OF SOLAR SPECTRUM WAVELENGTHS

NBS Monograph 61, National Bureau of Standards, Washington, D. C.

December 1966

$340 \mathrm{p}$

Available from the Superintendent of nocuments, GP

The present compendiun of solar spectrum wavelengths and intensities is essentially a second revision of Rowland's Tahle, corrected and supplemented by material from the Utrecht Photonetric Atlas. Approxinately 24,000 lines are listed. In a numher of cases new wavelengths were determined. Measured equivalent widths from the Atlas records replace the Rowland estinated line intensities recorded in the 1928 revision. From these directly measured equivalent widths have been derived reduced widths, which, if necessary, we re corrected for disturbing influences. The intensity behavior of atomic lines in the spot spectrum as compared with the spectrum of the solar disk is indicated hy letters denoting strengthening, weakening, and the like. Atomic lines present only in the spot spectrun are also included, 223 in all. Revised identifications of the lines, as to chemical origin, are given for both atomic and molecular lines. For classified atomic lines the lower excitation potential and multiplet numher are listed. For molecular lines the rotation branch and quantur number, and the vibration band are indicated. Wote numbers refer to notes in which the complete designation of the band is given. An introductory text gives a detailed description of each colurin of the solar.ledgex. Figures are included to illustrate the procedure used to derive the observed equivalent widths $\Delta \lambda(\mathrm{mA})$ and the reduced widths $\Delta \lambda / \lambda(F)$. Tables include counts of lines of each spectrum recorded in the identification colum, leading lines in the first and second spectra, and surraries of molecules and elements present in the sun. About 73 percent of the lines are wholly or partially identified. Sixty-three elements are recorded at present. A number need further study. The numher of molecules identified in the sun totals 11.

\#6500121-6500124

$* 3.153$

loore, C. F.

AN ULTRAVIOLET MULTIPLIT TABLE, SECTION $1:$ IH TO $23 \mathrm{~V}$; SECTION 2: $24 \mathrm{Cr}$ TO $4 I \mathrm{Nh}$; SLCTION $3: 42 \mathrm{MO}$ TO $57 \mathrm{La}$ AND $72 \mathrm{IIf}$ TO $88 \mathrm{Ra}$; SECTION 4: FINDING IIST FOR SPECTRA OF THE ELEIIENTS ITYDROCEN TO NIOBIUN $(Z=1$ TO 41); SECTION 5: FINDING LIST FOR SPECTRA OF THE ELEMENTS MOLYEDENUM TO LANTILNU: $(Z=42$ TO 57) AND HAFNIUM TO RADON ( $Z=72$ TO 88)

NBS Circular 488, Sections $I$ \& $2,3,4$ and 5 , Nationa 1 Bureau of Standards, Washington, D. C. April 1950, August 1952, April 1962

$86 \mathrm{p}, 120 \mathrm{p}, 98 \mathrm{p}, 70 \mathrm{p}, 34 \mathrm{p}$

Available from the Superintendent of Docurients, GPO

An Ultraviolet Multiplet Table, NBS Circular 488, is a table of multiplet spectral lines, with wavelengths of $300 \mathrm{~A}$ or less. These tables were taken from results of spectral observations, published in the literature. Sections 1 and (6500121) contain spectra of neutral and ionized 
atoms of $z=1$ to 41 . Section 3 contains spectra of atoms of $Z=42$ to 57 and 71 to 89 . These tables are arranged, first hy atomic number then by ionization series, and finally hy wavelength. The data included in these tahles are the wavelength and intensity of the multiplet line, the excitation potentials for the upper and lower states of the transition involved, the J-values and multiplet designation of the lines, and a literature reference. Sections 4 and 5 are finding lists for the multiplet $t$ ahles, arranged in order of wavelengths. Section 4 covers $z=1$ to 41 and Section 5 covers $Z=42$ to 57 and $z=72$ to 80 . These lists give for each line its wavelength, the radiating atom or $i$ on, and the multiplet number.

$\# 6809007$ *3.153

Moore, C. E.; Merrill, P. $\%$.

PARTIAL GROTRIAN DIAGRAMS OF ASTROPHYSICAL INTEREST NSRDS-NBS-23, National Bureau of Standards, Washington, D. C.

June 1968

650

Availahle from the Superintendent of Documents, GPO

This publication is a reprint of Appendix $A$ of the book by paul $W$. Merrill entitled, "Lines of the Chemical Elements in Astronomica? Spectra" (Carnegie Inst. Wash. Puhl. 610, 1956). It contains partial Grotrian Diagrams of selecter spectra of astrophysical interest, which give wavelengths, multiplet numhers and key letters for the transitions shown. The diagrams are accompanied by tahular keys in which the key letters indicate related lines in spectra similar in structure to those illustrated in the diagrams. There are 39 diagrans of spectra hetween hydrogen and nickel and tabular keys for 90 spectra hetween lithium and rhenium.

$\# 6604027$

Mork, K.; 01sen, II.

RADIÁTIVÉ CORREĆTIONS I. III GH-ENERGY

STRAILUNG AND PAIR PRODUCTION

Physics Review, 140:B1661-1674

December $\frac{\text { rev }}{1965}$

The radiative corrections to the high-energy Brenssstrahlung and pair-production spectra are calculated in the Weizsacker-williams approximation. Results for the soft-photon radiative correction to the spectra are given, and for the case of pair production the soft-plus-hard-photon radiative correction is also obtained. The radiative correction to the total pair-production cross section is found to be practically independent of the plioton energy and of the atomic number of the target material. Comparison with available experimental data shows essential agreement within the relatively wide experimental limits.

\section{\#6902008}

Muetterties, E. L.

THE CHEMISTRY OF BORON AND ITS COMPOUNDS

New York: John Wiley \& Sons, Inc.

1967

$699 \mathrm{p}$

This is a text on the properties of boron and horon compounds containing ten chapters, each written by experts in the different phases of boron chemistry. The chapters discuss the element horon, compounds of high boron content, borates, boric acid, boron hydrides and halides, horon-nitrogen, carbon, phosphorus compounds, and sulfur and selenium compounds of boron. Each chapter contains a large listing of references as well as tables of the chemical properties of boron compounds.
\#6706030 * $4.153-3.153-8.153$

Mui r, A. I1., Jr.; Ando, K. J.; Coogan, II. M. MOSSBAUER EFFECT DATA INDEX 1958-1965

New York: John ililey \& Sons, Inc.

1966

$351 p$

The Mosshauer Effect Data Index is an organized index to experimental research results relating to the Mossbauer Effect published through 1965. The index groups experiments first hy nuclear transition (daughter isotope and gama-ray energy), then hy source material or host, and then by absorber (or scatter) naterial or host. Each experiment is descrihed and summarized on an $80-$ column IBM card. The data listed for each experiment are: "Yossbauer isotope and gamma-ray transition energy, host material in which the source atoms arc inhedded, source temperature, absorber, absorber temperature, line width, dip of the spectrum (percentage change in counting rate from on-resonance to off-resonance), shift of the absorption spectrum, quadrunole splitting energy (for transitions between spin $3 / 2$ and $1 / 2$ levels), shape of the absorption spectrun and a code for the bibliography reference. A summary sheet is presented for each isotope presenting simplified decay scheme ganma-ray and $x$-ray energies, halflife and total internal conversion coefficient of the Mosshauer transition, natural isotopic abundance, and magnetic and quadrupole moments. There is an index to references hy topic and author.

$\# 6500049$

Nelms, A. T.

$$
* 2.2153-3.153
$$

GRAPIIS OF THE COMPTON ENERGY-ANGLE RELAATIONSHIP AND TIIE KLEIN-NISIIINA FORMULA FROM $10 \mathrm{KeV}$ TO $500 \mathrm{MeV}$

NBS Circular 542, National Bureau of Standards, Washington, D. C.

August 28,1953

Available from Superintendent of Documents, GPO

The Compton energy versus angle relationship and the differential and integral Klein-Nishina cross sections are presentcd graphically as functions of the energy and direction of the scattered photon and of the recoil electron. These graphs are intended to serve the purpose of tables. Unpolarized primary gamma rays in an energy range from $10 \mathrm{keV}$ to $500 \mathrm{MeV}$ are considered. The accuracy of all curves is estimated at 1 percent. The advantage of this form of presentation is the convenience and accuracy of two-way interpolation. In general, interpolated values may be obtained with an accuracy of 2 pcrcent.

\section{\#6809016}

$$
\text { *3. } 153-8.153
$$

Ne1son, R.D., Jr.; Lide, D.R., Jr.; Mary ott, A.A. SELECTED VALUES ÓF ELECTRIC DIPOLE MOMENTS FOR MOLECULES IN THE GAS PI!ASE

NSRDS-NBS-10, National Bureau of Standards,

Washington, D. C.

Available from the Superintendent of Documents, GPO

This table revises, brings up to date, and extends the coverage on numerical values of dipole moments which was included in NBS Circular 537, Tables of Dielectric Constants and Electric Dipole Moments of

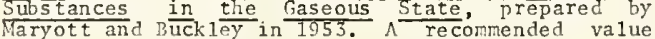
with an estimate of accuracy is presented for more than five hundred organic and inorganic compounds. Extensive comrents are given on the definition of dipole moment and principal rethods of dipole moment measurement, as we 11 as an exposition of the criteria employed in selecting the tabulated data. 
\#6500176

*3. 153

Nicholls, R. W.

INTENSITY MEASURE!IENTS ON MOLECUIAR SPECTRA

AFOSR Report No. 4971, estern Ontario University, London, Ontario, Canada

1963

$4 \mathrm{p}$

Available from DDC, AD 414136

This paper reviews some aspects of a continuing research progran in which intensity measurements have been made on a number of important and common diatomic molecular band systems, including the calculation of Franck-Condon factors, the $\gamma$-centroids, and the integrated emission intensity.

$\$ 6903051$

$* 3.153$

Nielson, C. W.; Koster, G. F.

SPECTROSCOPIC COEFFICIENTS FOR TII: $\mathrm{p}$, dn, AND fn CONFIGURATIONS

Cambridge, Massachusetts: The M.I.T. Press 1963

$275 p$

This book consists of a set of tables presenting reduced natrix elements and related quantities for al1 possible configurations of equivalent $p, d$, and $f$ electrons. part of the material is a recalculation of results already available in the literature, but most of the results on $f$ electrons are new. The tables contain listings of states, fractional parentage coefficients, electrostatic energy matrices, and unit tensor operator matrices.

\#7001042

$* 3.153-5.153-8.153$

Niles, F. T:

FORMATION OF IONS IN IONI 2T:D AIR

BRL-R-1458, Ballistic Research

Aberdeen Proving Grounds, Maryland

Novenber 1969

$54 \mathrm{p}$

Available from DDC

Schematic representations are given for both the fornation of positive ions and the formation of negative ions in ionized air. The formation of positive ions can be divider into three regions. Region I contains the ionization of neutral constituents and two-body reactions in iry air. Region II contains reactions involving ionic species which are formed by three-body reactions. Region III contains reactions requiring water vapor and shows the formation of the hydrated hydronium ions. The forration of negative ions proceeds predoninanty through $\mathrm{O}_{2}^{-}$. The clustering of neutral molecules to $0^{-}$opens arditional channels for the formation of No, an ion which is not attacked by atomic oxygen. Rate constants are given, incluring the experirental values on wich they are based, for the reactions in the schematic representations.

\section{\#6500158 *3.153}

Norris, J. A.

WAVELENGTII TABLIF OF RARF-EARTII ELEMENTS AND ASSOCIATED ELEYENTS INCLUDING 2 IRCONIUM, TIIORIUM, HAFNIUM, RHENIUM, AND TELLURIUM

ORNL-2774, Oak Ridge National Laboratory, nak Rirge, Tennessee

February 1960

$739 \mathrm{p}$

Available from CFSTI

A table of the wavelengths of the rare-earth elements and several associated elements has been compiled for the usc of spectroscopists working in this field. Data on the rare-earth elements, zirconium, hafnium, thorium, rhenium, and tellurium, have been collected from various sources and set forth in this table. A descending order of wavelength number is the basic format of the table. Included also are data on intensity and wave nunber.
\#6510001

O'Brier, C. J.

BOHR THEORY RADII OF TIIE ELEMIENTS

In Conference on Noble Gas Compounds Argonne, I 11 inois, Aprii $1, \overline{22}-2 \overline{3}, \overline{1963}$ Argonne, I

$9 \mathrm{p}$

Atonic radii of the elements have been calculater by the use of the Bohr rarius equation for the hydrogen atom and assuming that the energy levels of an aton are determined by the energy levels of its hydrogen-like ion. The measurer atomic radii for the elements $\mathrm{Li}, \mathrm{Be}, \mathrm{B}$, and $\mathrm{C}$ are from 0.0 ? to $0.02 \mathrm{~A}$ less than the radii calculated. The average deviation of the calculated radii fron the experinental radii for all elements is approximately 0.089 .

$\# 6902063$

$* 3.163$

Odabasi, H.

SELECTED TOPICS IN THE TIIEORY OF ATOMIC SPECTRA

JILA Report No. 97, Joint Institute for Laboratory Astrophysics, Boulder, Colorado

Noventer 15,1968

30 Ip

The numerical procedure is outlined. The theoretical ionization potentials, term and level energies in SLJM and intermeriate coupling schene, for the five lowest lying configurations of silicon, phosphorus and sulfur isoelectronic sequences are calculated using non-relativistic Hartree-Fock-Slater basis sets. In the process of evaluation, different interactions are adred one at a tirie to give an idea of their effects. At each step the theoretical results are compared with the observed ones. To exhibit the mixing of terms or levels for typical cases the "mixing coefficients," as defined in the text, are tabulated. In the beryllium isoelectronic sequence, with the exception of $\mathrm{Be}$ and $B$. the observations show that the $2 \mathrm{~s} 3 \mathrm{p}$ ' $\mathrm{p}$ term lies higher than $2 \mathrm{~s} 3 \mathrm{p}$ ip term, contrary to the theoretical expectation. This fact is explainer by configuration mixing. other configuration mixing calculations are carried out for the $3 p 4 p{ }^{3} n,{ }^{3} p,{ }^{3} S,{ }^{1} n,{ }^{1} p$, and $S$ terms in the silicon isoelectronic sequence. The behavior of line strengths in the extreme mixing of the levels due to spin-orbit interaction is shown by calculating intermediate coupling line strengths for $3 s-3 p$ and $3 p-3 d$ transitions in the carbon isoelectronic sequence.

$\# 6908044$

$* 2.2763-3.163$

Pauli, It. C.

TABLES OF INTERNAL CONVERSION COEFFICIENTS AND PARTICLE PARAYETERS

COO-1420-137, Purdue University, Department of Plysics, Lafayette, Indiana

August 1967

$206 \mathrm{p}$

Available from CFSTI

The internal conversion coefficients and particle paraneters of the $K$ and $L$ subshells have been recalculater for the ten nuclei, $2=60,64,68$, $72,76,80,84,88,92$, and 96 and ten énergies, $k$ $=0.02,0.05,0.10,0.15,0.20,0.30,0.40,0.50$, $0.70,1.00 \mathrm{~m} \mathrm{c}^{2}$. The four lowest orders of electric and mağnetic multipole transitions have been considered. The internal conversion coefficients and particle parameters of the $M$ subshells have been computed for the seven nuclei, $z=72,76,80,84,88,92$, and 96 and for ten energies listed above. The screening of the atomic electrons has been taken into account on the basis of the Thomas-Fermi-Jirac model. A11 static and dynamic effects of the finite nuclear size have been taken into account, assuming a sharp nuclear edge. 
\#6500178 *3.163

Pearse, R. 13 .

New York: John Wiley \& Sons, Inc.

1963

$347 \mathrm{p}$

The tables for the Identification of Polecular Spectra are divided into two sections. The first section consists of a list of the strongest heads of the nore persistent and better known band systems of each nolecule, in order of wavelength. The second section consists of individual lists of band heads for each systen of each molecule, accompanied hy notes about the occurrence and appearance of the system, the nature of the clectronic transition involved, the vibrational assignment of the bands in the system, and references to the source of the data. The lists are arranged in alphabetical order of the chenical formula of the molecules. All recorded systems of diatonic nolecules found hy the authors have been included, as well as those triatomic and more complex molecules which show well defined band structure and are of frequent occurrence in spectroscopic investigations. The wavelength region consiclered is from 10,0009 to 20009 . An appendix containing twelve photographic plates of spectra of the most frequently encountered systems is included.

\#6500252

Pears on, W. B.

A HANDBOOK OF LATTICE SPACINGS ANI STRUCTURSS OF METALS AND ALLOYS

New York: Pergamon Press, NRC-430

1958

$1044 \mathrm{p}$

This Ilandhook contains data on lattice spacings and structures of metals and alloys, and the information that can be determined from these quantities. The author presents data for elements and binary and ternary alloys, taken from literature published through the end of 1756 . The applications considered include applications to equilibrium diagrams, magnetic properties and alloy formation theory. The data includes crystallographic data on "Strukturbericht" types tabulated lattice spacings, structure types, density of the elements and intermediate phases of alloy systems, and a review of work done on metals and alloys, including graphs of lattice spacings as functions of composition.

\#6906010

Pears on, H. B.

A IIANDBOOK OF LATTICE SPACINGS AND STRUCTURES OF METALS AND ALLOYS, VOLUME

New York: Pergamon press, NRC No. 8752

1967

$1446 \mathrm{p}$

This second volume of Lattice Spacings and Structures of Metals and Alloys presents data for elements and binary and ternary alloys taken from literature published through June of 1965 . The applications considered include equilihrium diagrams, magnetic properties and alloy formation theory. The data include crystallographic data, tabulated lattice spacings, structure types density of the elements and intermediate phases of alloy systens, and a review of work done on metals and alloys, including graphs of lattice spacings as functions of composition. This volume seeks to present on ly the best data for X-ray methods, alloy purity and exact knowledge of composition. Brief preparative details and notes on the methol of $X$ ray examination accompany descriptions of $X$-ray data on alloy phases.
\#6711001

$* 3.263$

Phillips, J, P.

PVIITTRA-STRUCTURE CORRELATION

Mew York: Academic press

1964

$172 p$

This volume gives the salient feature of the absorption spectra of various compounds hetween 1750 angstroms and $35 \mathrm{microns}$, broken down into 5 spectral divisions; far ultraviolet, ultravioletvisible, near infrared, infrared and far infrared. The following classes of compounds are considered: hydrocarbons; compounds with oxygen function; compounds with nitrogen functions; heterocyclics; organic compounds containing halogen, sulfur, phosphorus, silicon or boron; inorganic compounds; complex materials. The data presented arc wavelengths of maximum absorption for typical compounds in each class.

$\# 6902077$

Pitha, J.; Jones, R. N.

OPTIMIZATION YETIIODS FOR FITTING CIJRVES TO INFRARED BAND ENVELOPES COIPUTRR PROGRMNS

NRC Bulletin No. 12, National Research Council of Canada, Ottawa, Canada

1968

$133 \mathrm{p}$

Three computer programs for fitting infrared band contours are described and listed. These programs utilize Cauchy (Lorentz), Gauss, Canchy-Gauss product, and Cauchy-Gauss sum functions, and will optimize the paraneters for fitting single bands or multiplc overlapping band systems using a nonlinear least squares algorithm. Supplementary programs are included for the evaluation of the half-widths and shape characteristics of the synthesized component bands, also for the conputation of the ordinates of the synthesized band envelope, and for an analysis of the misfit with the true spectrum. This latter program also serves as a general purpose program for subtracting spectral ordinates. The programs are written in FORTRAN IV. They are designed for card input and output in a standardized format that facilitates their interfacing with other programs developed in our laboratory for the numerical analysis of infrared and ultraviolet spectrophotometric absorption curves.

\#6500253-6500257,6603002-6603003*4.263-3.263 Forter, II. W.; Spiller, R. C. (Volunes 1 and 2) Porter, II. W.; Codd, L. W. (Volume 3)

TIE BARKER INDEX OF CRYSTALS, A MITIIOD FOR THE IDENTIFICATION OF CRYSTALLINE SUBSTANCES, VOLUNT: I : PARTS I AND 2; VOLUMT II: PARTS 1, 2 N:D 3; VOLUMT I II: PARTS 1 AND

Cambridge, England: $W$. Ileffer \& Sons, Ltd.

$1951-1964$

$300 \mathrm{p}, 1000 \mathrm{p}, 383 \mathrm{p}, 600 \mathrm{p}, 500 \mathrm{p}, 375 \mathrm{p}, 400 \mathrm{p}$

The Barker Index of Crystals provides a means of identification of crystals through the reflection of $1 \mathrm{ight}$ from their faces, as well as a source of structural data for known crystals. Volume I, Part 1 contains an introduction to and explanation of the Barker lndex, and is arranged by barker classification angle, by refractive index, hy densities, and alphabetically by chemical name. The Barker Index is based on the angles between the normals to the major planes and also indices to tetragonal, hexagonal, trigonal and orthorhomhic crystals. Volume 1, part 2 contains the following data for tetragonal, hexagonal trigonal and orthorhomhic crystals: classification angles, axial ratios, interfacial angles; symmetry; physical properties, such as cleavage, specific gravity, color and melting point; indices of refraction and strong lines in the $X$-ray powder diffraction spectrum. Volume II, Part 1 contains indexes for monoclinic crystals arranged by classification angle, density and specific gravity; Part 2 and 3 contain monoclinic crystal data of the types 
inclucled in Volume I. Volume III, parts 1 and 2 contain similar information for triclinic crystals.

\#650026.

$* 4.163-3.163$

Prather, J." L.

ATONIC ENERGY LEVELS IN CRYSTALS

NBS Monograph 19, National Bureau of Standards,

Vashington, D. C

February $24,196 i$

$84 p$

Available from the Superintendent of Documents, GPO

Discrete energy levels ohserved within certain crystals arc treated as due to perturbations of the energy levels of the free ion by an electrostatic ficld arising from the crystal lattice. The analytic procedures for determining the field from the charge configuration are given, and the resulting fields are classified according to their symmetry. After a general survey of grouptheoretical ideas, the applicahle groups are analyzed in detail, and characters appropriate for both integral and half-integral angular momenta of the free ion are tabulated. These are applied to the determination of the number and type of levels arising from a frec ion level with $J=3$. The results of this analysis are tahulated, as are the selection rules for electric dipole, magnetic dipole, and electric quadrupole transitions. Calculation of the perturbation matrix elements hy the use of Wigner and Racah coefficients is discusscd. Examples of the application of these several techniques to specific problems are given.

\section{$\# 650182$}

$$
* 3.373
$$

RandalI, H. M.; et aI.

IXFRARED DETLRNINATION OF ORGANIC STRUCTURES

Princeton, New Jersey: D. Van Nostrand Company, Inc. 1949

$244 p$

In Infrared $\frac{\text { Determination }}{\text { approach have }}$ of organic $\frac{\text { Structures, }}{\text { out }}$ possibilities inherent in the nethod have been explored, and currently established correlations between structure and absorption spectrum have been itemized. The instruments and tochniques have heen described. In Chapter III, a unique tabulation is marle of the assirnmerts of absorption bands for a large number of compounds containing douhle bonds. In Chapter IV, the results of years of work on molecular structure are compiled ir a form easily usable by nontheoretical chemists and physicists. Chapter $V$ offers illustrations of the modus operandi for proceeding from the reasured absorption spectrum to its interpretation in terms of molecular structure. Finally, the actual recoris of a great variety of compounds are reproduced for their reference value as a library.

\section{\#6510034 *3.273-3.273}

Reedy, T. L. (Liditor)
ICRPG PROCTEDINGS OF THIRD MEETING MARCH 17-13, 1965, VOLUME 1

CPIA Publication No. 32, Johns liopkins Applied Pliysics Laboratory, Chemical Propulsion Information Agency, Silver Spring, Maryland

July 1965

$172 \mathrm{p}$

Availab le from DDC

This volume contains the unclassified presentations given at the Third Neeting of the Vorking Group on Thermochemistry, and is concerned with data of interest for rocket motor performance calculations. The 22 papers include reviews of the status of thermochenical data for the heavy element compounds and ionized species. Recent experimenta1 progress is reported in the areas of matrix spectroscopy mass spectroscopy, calorimetry, and high temperature vapor pressure measurements of $1 \mathrm{ight}$ element compounds.
Reid, R. C. ; She rwood, T. K.

TIIE PROPERTIES OF GASES AND LIQUIDS ESTIMATION AND CORRELATION, SECOND EDITION New York: MeGraw-lli11 Book Company, Inc. 1966

$646 \mathrm{P}$

This book presents a critical review of the various estimation procedures for a limited number of properties of gases and liquids. These include critical properties, $\mathrm{P}-\mathrm{V}-\mathrm{T}$ and thermodynamic properties, vapor pressures, latent heats, heats of formation, free energies of formation, heat capacities, surface tensions, viscosities, thermal conductivities, diffusion coefficients, and equilibrium properties of mixtures. Comparisons of experimental and estimated values are shown in the form of tables for indication of the degree of reliahility of the methods discussed. The book is designed to he used as a text for molecular physics or a reference source for engineers. A set of Appendixes summarizes material referred to in the chapters and contains a large volume of tabulated data as well as references to the Iiterature.

" 6509020

* 3.173

Ries, R. R.; Dame row, R. $\Lambda$.; Jolinson, "v. Il. , Jr. ATOIIC MASSES FROM GÁLLIUM TO MOLYBDENUM Physical $\frac{\text { Review, }}{15,1961}$

The 16-inch double-focusing mass spectrometer at the University of linnesota has been employed to measure the atomic mass of $42 \mathrm{stable}$ isotopes in the region $A=69$ to 100 . The standard error associated with these results is approxinately parts in $10 \%$. Improvenents in the instrument are descrihed that result in an increase of useful resolution of a factor of 2 to 3 . A set of 64 radioactive masses is calculated from the stable mass data together with a heta-decay energies and nuclear reaction $n$ values. The resultant tahle of masses is used to calculate total nuclear binding energies, separation energies and pairing energies for a number of nuclei in the region near $N=50$. The systematics of the separation energies display very smooth characteristics except at the shell closure. Neutron pairing energies show a marked decrease in value following the shell closure.

$\# 6509025$

$* 3.173$

Rose, R. G.

GANIÁ-RAY ATTINUATION CORFFICIENTS

Nucleonics, $12: 126-127$

Rugust 1964

Gama-ray attenuation coefficients are presented for several shielding materials for gama energies from 0.1 to $10 \mathrm{MeV}$.

$\# 6508061$

Barrow, R. F.; et al.

Rosen, B. (Editor)

TABLES OF CONSTANTS AND NUNERICAL DATA, VOLUME: 5. SELECTED CONSTANTS, ATLAS OF WAVELENGTH CIIARACTERISTICS OF EMISSION AND ABSORPTION BANDS OF DIATONIC MOLECULES

Paris: Ilermann \& Cie, Depositaires

1952

$390 \mathrm{p}$

In French

This book contains tables of characteristic wavelengths of diatomic molecular bands (indicating for each band, the method of observation, the direction of degradation, the vibrational classification, etc.), the characteristic wavelengths of the bands of $\mathrm{C}_{2}, \mathrm{CIl}, \mathrm{CII}^{+}, \mathrm{CN}, \mathrm{CN}, \mathrm{CO}^{+}$, $\mathrm{N}_{2}, \mathrm{~N}_{2}^{+} \mathrm{NI}, \mathrm{NH}^{+}$, NO, ${ }^{+}{ }_{2}, \mathrm{O}_{2}^{+}, \mathrm{OH}, \mathrm{CI}^{+}$, and the molecules found in parts 4 and 5 are. included as well as a list of bibliographical references. 
$\# 6510035$

$* 3.273$

kosen, B. (Editor)

TABLES OF CONSTAMTS AND NUMIRRICAL DATA, YOLUME 4. SELECTED CONSTANTS, SPECTROSCOPIC MATA CONCFRNING DIATOI!IC MOLECULES

Paris: Ilermann \& Cie, Depositaires

1951

$361 p$

In French

These tables contain spectroscopic data for diatonic molecules. The data includec for each molecular species includes: observed transitions, limits of observations, wavelengths of characteristic bands, vihrational and rotational frequencies and other molecular constants.

\section{$\$ 6904008 \quad * 3.173$}

RUdiEe, M. R. II' INIPACT

Reviews of Hodern Physics, 40:564-590

A review of the quantur theory of ionizing collisions is presented, with particular regard to recent theoretical developments. A discussion is given of quantal and classical approxinations and their predictions compared with experimental data. Sone useful empirical formulas are listed and compared, and in the conclusion mention is made of the present inadequacies in the theory.

\section{\#6508053}

$$
* 3.273-5.273
$$

Schexnayder, C. J. Jr.

TABULATED VÁLUES OF BOND DISSOCIATION ENERGIES, IONIZATION POTENTIALS, AND ELECTRON AFTINITIFS FOR SONE MOLECULES FOUND II: IIIGII-TTEMPRATURE CIIINII CAL REACT IONS

NASA TiN D-1791, Langley Research Center, Langley Station, Ilampton, Virginia

May 1963

$62 \mathrm{p}$

Available from CFSTI

Values of the bond dissociation energies, ionization potentials, and electron affinities that were taken from the literature are presented in $\mathrm{tables}$ for some monatomic, diatomic, and polyatomic molecules which are found in many high-temperature chenical reactions including combustion reactions. Much of the information came from literature published after 1950 which either reported experimental and theoretical energy values or gave a review of previous literature on the subject.

\section{$\# 6809014$}

$* 3.173$

Schwendenan, R. II.

TABLES FOR TIE RIGID ASYMETRIC POTOR: TRANSFORMATION COEFFICIENTS FROM SYMPETRIC TO ASY:METRIC BASES AND EXPECTATION VALUTS OF $\mathrm{P}_{2}^{2}, \mathrm{P}_{2}^{4}$, AND $p \frac{6}{2}$

NSRDS-NBS-12, National Bureau of Standards,

Washington, D. C.

June 1968

$98 \mathrm{p}$

Available from Superintendent of Documents, GPO

Tables of computed quantities associated with the rigid asymetric rotor are presented. The first group of tahles gives transformation coefficients from symetric to asymetric rotor bases. These coefficients permit the eigenfunctions of the asymetric rotor to be written in terms of symetric-rotor eigenfunctions. In Part II the angular-momentum expectation values ( $\left(\frac{2}{2}\right),(\mathrm{P} \xi)$, and $\left(P{ }^{6}\right)$ are tahulated. In hoth sets of tables values are given at intervals of 0.1 in the asynnetry parameter $k$ and for $j \leq 15$. The tabulated quantities find use in the analysis of microwave rotational spectra and the rotational fine structure in vibrational and electronic band spectra.
$\# 6809019,6309015,6809010 \quad * 3.173$

Shimanouchi, T.

TABLES OF MÓLECULAR VIBRATIONAL FRT:QUENCITS, PARTS 1,2 , Ain 3

NSRDS-IBS-6, 11 and 17, National Bureau of Standards, Washington, D. C.

Narch 1, 1967, October 1967, 2larch 1968

$56 \mathrm{p}, 33 \mathrm{p}, 39 \mathrm{p}$

Availahle from the Superintendent of Documents, G?

A compilation of vibrational frequency data for selected molecules is being conducted at the University of Tolyo in cooperation with the National Standard Reference Data Program of the National Bureau of Standards as a part of an international effort to compile and evaluate physical and chemical clata. These tables contain fundamental vibrational frequencies of 167 molecules together with vihrational assignments sources of data, brief coments and citations of references. The fundamental frequencies are obtained mainly from the infrared and Ranan spectra. When these are not available, other experimental data such as microwave results are taken into account. The selection of vibrational fundamentals from observed spectral data is hased upon careful studies of the spectral data and comprehensive mathenatical analyses. These tables were designed to provide a concise sumary needed for the conputation of ideal gas thermodynamic properties. They may also provide a convenient source of information to those who require vibrational energy levels and related properties in molecular spectroscopy, analytical chemistry, and other fields of physics and chemistry.

\#6602013 *3.37.3-3.373

Somayajulu, G. R.; Zwolinski, 3. J.

ADDITIVITY OF THE POTENTIAL BARRIFRS ABOUT TOUBLF BONDS IN IIMLOGEIIATED ETITYLENTSS

Chenical The rmodynamic Properties Center, Texas AGM University, College Station, Texas

Septemher 1, 1965 9p

Potential harriers about double bonds have heen found to be additive of the contributions from pairs of honds separated by $\mathrm{C}=\mathrm{C}$ hond in substituted ethylenes. On the basis of this work, the torsional frequency of ethylene has been found to be $325 \mathrm{~cm}^{-1}$. New torsional assignments have been suggested for some molecules.

46510037

Somayajulu, G. R.; Zwolinski, B. J

A GENERALIZED BOND FNERGY SCIIXYE INCLUDING BARRIERS TO FREL ROTATION AND CORRECTIONS FOR ROTATIONAL I SOMERISM

Chenical Thermodynamic Properties Center, Texas AGM University, College Station, Texas 1965

$21 \mathrm{p}$

A generalized procedure for predicting the energies of the paraffinic hydrocarbons is deduced on the assumption of (i) interactions hetween pairs of bonds attached to a carhon atom, (ii) interactions between trios of bonds attached to a carhon ator and (iii) interactions between pairs of bonds separated by a $\mathrm{C}-\mathrm{C}$ bond. The derived equation justifies Tatevskii's empirical approach and is shown to be not very different from Al1en's equation without the steric terms. Under defined conditions this equation reduces exactly to Allen's equation. Barriers to free rotation were considered in deriving the new equation. This new equation is further modified by including corrections for rotational isomerisn in place of trigonal interactions. The new relation is hetter founded in principle, correctly estimates enthalpies of atomization for normal and isomeric alkanes including those molecules without rotational isomers, and furthermore provides more refined steric terms due to higher order interactions. 
$\$ 6603012,6603013,6603014$

*. $373-5.373-3.373$

Somayajulu, G. R.; Zwolinski, R. J.

RELATIONSHÍ RETUIEN ROW THERGY AND BOND DISSOCIATION ENERGY,

II. ENTHALIIES OF' FORUTION OF THE, SUBSTITUTED DETIIAVES, SUBSTITUTEN ITETIYL RADICALS ANI SUBSTITUTED IIETIANES,

III. MAG:ITUDES OF TIIF BOND A:ID PAIR BOND CONTRIBUTIOIS TO TUH: EMERTIES OF TIE MOLECULTS IV. ENTIALPIES OF FORHAION OF SURSTITUTID ETITYLE: ES

Clemical Thermodynamic Properties Center, Texas Ach University, College Station, Texas

August 1965, August 1965, September 1965

$12 \mathrm{p}, 15 \mathrm{p}, 3 \mathrm{p}$

In part II, the enthalpies of formation of the substituted riethanes have been calculated on the bas is of Zahn's model. A method has heen developed for the calculation of the tissociation energy of tlee $R^{\prime}-R^{\prime \prime}$ hond in a substituted ethane. Based on the dissociation energy of the $R^{\prime}-R^{\prime \prime}$ bond, the enthalpics of formation of the substituted methy radicals and the substituted ethanes have been calculated. The enthalpies of formation of the substituted methyl raclicals can also be calculated on the basis of Zalın's model. In Part II methods have bcen developed for the calculation of the magnitudes of the bond and pair bond contributions based on relationslips between hond energy and hond dissociation energy. The pair hond contributions for the pair of bonds $\mathrm{C}_{<x}^{x}$ in $\mathrm{CX}_{4}, \mathrm{CX}_{3}$, and $\mathrm{CX}_{2}$ have been determined. Also determined were the enthalpies of formation of $\mathrm{CX}_{2}$ and $\mathrm{CS}$ radicals, $\mathrm{X}$ being II, $\mathrm{F}, \mathrm{Cl}, \mathrm{Br}, \mathrm{I}$ or ${ }^{2} \mathrm{Cll}$. In Part IV enthalpies of formation of the CX, type radicals obtained in Part III have been used to obtain the enthalpies of formation of the CXY type radicals. Based on a relationship between bond energy and bond dissociation energy, the dissociation energies of the $R^{\prime}-R^{\prime \prime}$ bonds in substituted ethylenes have been calculated. Using the bond dissociation energies and the enthalpies of formation of the substituted riethylene radicals, enthalpies of formation of a few substituted ethylenes have been calculatei. The calculated values have been four to be in good agreement with the observed values.

\section{$\# 6906006$}

$* 5.173-3.173$

Stevens, B.

COLLISIONAL ACTIVATION IN CASES

New York, London: Pergamon Press

1967

$236 \mathrm{p}$

The International Encyclopedia of Physical and current account of al 1 aspects of the domain of science between chemistry and physics, written for the graduate and research worker. The subject matter is grouped in twenty topics. This third volume on the topic gas kinetics deals with energy transfer systems in gas reactions, relaxation techniques, rate measurements of competing processes, low energy level studies, and higher vibrational level energy transfer. Tahles give vibrational relaxation times and collision numbers for diatomic molecules; relative collisional deactivation probabilities for diatomic molecules $M$ in presence of additive $X$; vilurational relaxation times, reduced relaxation times, and collision numbers for polyatonic molecules; and relative probabilities for collisional deactivation of polyatonic nolecules by alditives $x$.

H6902007 *3. 173

Striganov, A. R.; Sventitskii, N. S.

TABLES OF' SPICTRAL LINES OF NEITRAL AND IONIZEN ATONG

Wew York, Washington: IFI/Plenum

1968

$399 \mathrm{p}$

Trans laterl from Russian
This book contains the emission spectra of 22 elements dealt with most frequently in the investigations of all forms of plasmas. For each spectral line, the wavelength, intensity, and classification are given. The book contains the spectra of all the gaseous elements except radon, of all the alkali metals, except rubidium and radioactive francium, and of carbon, magnesium, aluminum, silicon, calcium, titanium, iron, and copper. For each element, in addition to the spectrum of the neutral atom, the spectra of several of its ions are given. In all, the tables contain over 30,000 lines in the IP, visible, nearUV and far-UV regions of the spectrum.

\#6500 Z99, 6609026 *3. 213

Sutton, L. E. : et al. (Scientific Editors)

TABLES OF INTERATOMIC DISTANCES AND CONFIGURATION IN MOLECULES AND IONS, AND SUPPLEMENT 1956-1959

Special Publications No. 11 and No. 18, The Chemical Society, London

1958,1965

$385 \mathrm{p}, 288 \mathrm{p}$

These $t a b l e s$ include information obtained by spectroscopy and electron, neutron and $x$-ray diffraction about interatomic distances and bond angles. The information in the main volume was compiled from literature published through the end of 1955, and the Supplement from literature published 1956-59. Interatomic distances and bond angles are compiled for gaseous and solid-state molecules, and complex ions in the solid. Typical values of certain important interatomic distances are listed in a separate table.

\section{\#6500180} *3.373

Szymanski, H. A.

INFRARED BAND HANDBOOK

New York: Plenum Press

1963

$484 p$

The Infrared Band Handbook presents data on absorption band spectra of various organic molecules arranged by wavenumber in steps of $1 \mathrm{~cm}^{-1}$ from 3610 to $617 \mathrm{~cm}^{-1}$. The data for this compilation were taken from the American Petroleum Institute project 44 series, including spectra published since 1943, and other data published between 1957 and 1963 . The data included for each band are: wavenumber in $\mathrm{cm}^{-1}$; intensity of the band (as: strong, medium, weak, etc.); the physical state in which the spectrum was measured or the solvent used; the structural group to which the vibration was assigned in the original reference (if one was assigned) and the mode of vibration, where pertinent; and a reference number pertaining to the list of source material. There is an index to compounds arranged alphabetically by empirical formulas. The appendix contains correlation tables for methyl deformation frequencies and $\mathrm{C}-\mathrm{N}$ stretching frequencies.

\#6711014-6711016

$\star 3.373$

Szymanski, II. A.

INTERPRETED INFRARED SPECTRA, VOLUMES 1,2 , AND 3 New York: plenum Press

$1964,1966,1967$

$293 p, 304 p, 275 p$

The interpretation of infrared spectra is based on the so-called "group frequencies" which related molecules have in common. A group frequency can be defined as a vibration which can be associated with an isolated structural group in a molecule. Each class of compounds is introduced by first presenting the vibrational analysis of a few compounds of that class, then the group frequencies found for that class, and finally the interpreted spectra. The vibrational analys is of a molecule is accomplished by calculating a series of force constants which are reasonable and lead to the observed frequencies. Substances included in Volume I are alkanes, alkenes, benzene ring 
compounds, cyclopropane, cyclobutane, cyclopentane, and cyclohexane derivatives. Substances included in Volume II are alkynes, alcohols, and diols. Substances included in Volume III are phenols, ethers and peroxices.

\#6512008 *0.2172-3.172

TABLES OF COULOMB WAVE FUNCTIONS, VOLUME I

NBS Applied Mathenatics Series No. 17, National Bureau of Standards, Washington, D. C.

April 1952

$141 \mathrm{p}$

Available from Superintendent of Documents, GPO

This report presents numerical values of the Coulomb wave functions in somewhat peculiar notation. However, an introduction by $M$. Abramowitz defines all quantities and it is possible to reconstruct the actual Coulomb wave functions from the tables presented herein. In addition, there is a discussion of the significance of these wave functions in a preface by G. Breit.

$\# 6910001$ $* 3.143$

Thomas, R. N. (Editor)

AERODYNAYIC PHENOMENA IN STELLAR ATMOSPHERES, A BIBLIOGRAPHY

NBS Technical Note 30, National Bureau of Standards, Washington, D. C.

Sep tember 1959

$93 \mathrm{p}$

Available from Superintendent of Documents, GPO

This bibliography covers the period from 1914 to 1959. It was prepared as a working bibliography for the Fourth Symposium on Cosmical Gas Dynamics: Aerodynamic Phenomena in Stellar Atmospheres.

\#6902027 *3.373

Tips On, R. S. INFRARED SPECTROS

NBS Monograph 110, National Bureau of Standards, Washington, D. C.

June 1968

$21 \mathrm{p}$

Available from Superintendent of Documents, GPO

A survey has been made of the literature on the infrared spectros copy of carbohydrates, in order to assemble and systematize information in this field. The Monograph discusses principles and instrumentation, sampling techniques, comparis on of samples, the interpretation of the spectra. particularly as regards to functional groups of carbohydrates and their derivatives, correlations for the fingerprint region and beyond, and conformational studies. In addition, examples are discussed of the use of infrared spectra for qualitative and quantitative purposes and for the determination of structure. Special techniques are briefly described including use of plane-polarized radiation, the technique of attenuated total reflection and Raman spectra.

\#6706031,6706032

$\star 3.363$

6801006,6809029

UV ATLAS OF ORGANIC COMPOUNDS, VOLUMES $1,2,3$ AND 4 New York: plenum Press

$1966,1967,1968$

$430 p, 238 p, 317 p$

The Atlas consists of a collection of the ultraviolet and visible spectra of compounds containing typical chromophoric groups, supplemented by tables showing the effects of substitutents and solvents. The units chosen for plotting the spectra are $\mathrm{cm}^{-1}$ for abscissae and a logarithmic plot of molar absorptivity. The wave numbers covered are either 55,000 to 20,000 or 45,000 to $10,000 \mathrm{~cm}^{-1}$, depending on the spectrum. The value, wave number and wavelength of maximus absorptivity are indicated explicitly on each spectrum, together with details of the compound and the conditions under which the spectrum was obtained. The sections contained in Volumes 1 to 4 include: a. compounds containing multiply bonded $C$ atoms only; b. compounds containing $\mathrm{C}=0$ and $\mathrm{C}=\mathrm{S} ; \mathrm{c}$. compounds containing multiply bonded N atoms; $d$. monocyclic aromatics; e. polycyclic aromatics; $f$. aromatic compounds containing non-benzenoid rings; g. monocyclic heterocyclics; h. polycyclic heterocyclics; i. derivates of saturated organic compounds; $j$. selected compounds of biochemical and clinical interest; $k$. inorganic systems; and $m$. solvents. A Molecular Formula Index covers all compounds contained in Volumes 1-4.

\#6610009

Veldre, V. I.

EFFECTIVE CROSS SECTIONS FOR COLLISIONS OF ELECTRONS WITH ATOMS, ATOMIC COLLISIONS III

JILA Report 3, Joint Institute for Laboratory As trophysics, Boulder, Colorado

May 20,1966

$153 \mathrm{p}$

This report consists of a series of articles on effective cross sections for collisions of electrons with atoms, as calculated theoretically by the original authors. The calculations include collisions of slow electrons with atoms of alkali metals, elastic scattering of electrons on atoms of alkali metals, excitation of neon atoms by electron bombardment, computation of excitation cross sections of excited hydrogen atoms, and computing lower bounds for phases of électron-hydrogen scattering.

$\# 6611001$

$* 3.183-5.183$

Veldre, V. I a.

IONIZATION OF ATOMS BY ELECTRON BOMBARDMENT

Electron-Aton Collisions RIGA

1965

$100 \mathrm{p}$

This is a review of the ionization of atoms by electron impact and of the theory of ionization. The article contains a bibliography of 103 references as well as 54 graphs and 5 tables of data. The article reviews the classical and quantum theory of ionization, the asymptotic behavior of the wave function, Born's Approximation, exchange calculations, calculation of distortion, momentum approximation, ionization from excited states, ionization of the helium atom and of complex atoms and ions, threshold behavior, and empirical formulas.

\#6500143 *3.183

Wacker, P. F.; pratto, M. R.

MICROWÁVE SPÉCTRAL TABLES, LINE STRENGTHS OF ASYMMETRIC ROTORS

NBS Monograph 70, Volume 2, National Bureau of St andards, Washington, D. C.

December 1964

$338 \mathrm{p}$

Available from Superintendent of Documents, GPO

Microwave Spectral Tables, Volume 2 is the result of a computer calculation of line strengths of asymmetric rotors; the line strength is defined as a sum of squares of absolute values of direction cosine matrix elements over both laboratory-fixed Cartesian coordinates and Zeeman componentss. These line strengths are useful for the calculations of rotational lines in microwave and other spectra, determination of dipole moments froom Stark effects or vice versa, and determination of nuclear quadrupole coupling coefficients from hyperfine splittings or vice versa. The line strengths have been tabulated as a function of the rotational quantum number $J$, for values of $\mathrm{J}$ from 0 to 35 , and Ray's asymetry parameter K. Procedures for interpolation as a function of $k$ and extra rotation to larger $J$ values are discussed. 
Wacker, p. F.; et al.

MICROWÁVE SPEĆTRAL TABLES, DIATOIIC MOLECULES

NBS Monograph 70, Volume 1, National Bureau of Standards, Washington, D. C.

December 1, 1964

Available from Superintendent of Documents, GPO

For about 1500 spectral lines of diatomic molecules observed by coherent radiation techniques, measured frequencies, assigned molecular species, assigned quantum numbers, and newly computed intensities are given. Molecular data, such as rotational constants, dipole moments, and various coupling constants, determined by such techniques, are also tabulated, as are other molecular constants used in the intensity computations. References are given for all included data. For determination of hyperfine spectra, both Casimir's function and the intensity splitting factors are given for both integral and half-integral quantum numbers $J$ and $I$, permitting application to additional molecules with unclosed electronic shells and with hyperfine splitting produced by more than one nucleus.

\section{\#6907001}

$* 3.183$

Wacker, p. F.; et a1.

MICROWAVE SPECTRAL TABLES, VOLUMF. III. POLYATOMIC MOLECULES WITH INTERNAL ROTATION

NBS Monograph 70, Volume III, National Bureau of Standards, Washington, D. C.

June 1969

$265 p$

Available from Superintendent of Documents, GPO

Measured frequencies, assigned molecular species, and assigned quantum numbers are given for about 9,000 spectral lines of polyatomic molecules with internal rotation observed by coherent radiation techniques. Molecular data such as rotational constants, dipole moments, and various coupling constants, deternined by such techniques, are also tabulated. References are given for ail included data.

\section{\# 6607011}

*3. 183

Wiese, W. L.; Smith, M. W.; Glennon, B. M.

ATOMIC TRANSITION PROBABILITIES, VOLUME 1. IIYDROGEN THROUGII NEON

NSRDS-NBS-4, Volume 1, National Bureau of Standards, Washington, D.'C.

May 20, 1966

$153 \mathrm{p}$

Available from Superintendent of Documents, GPO

Atomic transition probabilities for about 4,000 spectral lines of the first ten elements, based on all available literature sources are critically compiled. The data are presented in separate tables for each element and stage of ionization. For each ion the transitions are arranged according to multiplets, supermultiplets, transition arrays, and increasing quantum numbers. Allowed and forbidden transitions are listed separately. For each line the transition probability for spontaneous emission, the absorption oscillator strength, and the line strength are given along with the spectroscopic designation, the wavelength, the statistical weights, and the energy levels of the upper and lower states. In addition, the estinated accuracy and the source are indicated. In short introductions, which precede the tables for each ion, the main justifications for the choice of the adopted data and for the accuracy rating are discussed. A general introduction contains a critical review of the major data sources.
\# 6911005

* 3.183

Wiese, W. L.; Smith, M. W.; Miles, B. M.

ATOMIC TRANSITION PROBABILITIES, VOLUME II SODIUM TIIROUGI CALCIUM A CRITICAL DATA' COMPILATION

NSRDS-NBS-22, National Buresu of Standards, Washington, D. C.

october 1969

$306 \mathrm{p}$

Avalilable from Superintendent of Documents, GPO

Atomic transition probabilities for about 5,000 spectral lines of the second ten elements, based on all available literature sources, are critically compiled. The data are presented in separate tables for each element and stage of ionization. For each ion the transitions are arranged according to multiplets, supermultiplets, transition arrays, and increasing quantum emission; the absorption oscillator strength, and the line strength are given along with the spectroscopic designation, the wavelength, the statistical weights, and the energy levels of the upper and lower states. In addition, the estimated accuracy and the source are indicated. In short introductions which precede the tables for each ion, the main justifications for the choice of the adopted data and for the accuracy rating are discussed. $A$ general introduction contains a detailed discussion of the critica 1 factors entering into each major experimental and theoretical method. It also includes a general critical assessment of the widely used coulomb approximation, and a number of illustrative examples for the exploitation of regularities or systematic trends among oscillator strengths.

\section{\#6711019}

*2. $683-3.183-0.2183$

Wilson, B. S. (Editor)

TIIE RADIOCIEMICAL MANUAL, SECOND EDITION

Amersham, England: Radiochenical Centre

1966

$327 \mathrm{p}$

The manual is intended as a guide for all who use radioactive substances professionally; its ain is to help the individual user choose the right material for his purposes and to make effective use of it. The text portion of the book includes information on preparation of radioisotopes: radiation sources; problems of using radioactive materials; radioactive chemicals as pharmaceuticals; quantification of radioactive substances; radiological safety and waste disposal; packaging and carrying radioactive naterials; ownership and use regulations and a guide to literature. The tabulated data includes: physical data on the more common radioisotopes, including physical characteristics, availability, beta and gamma decay energies and half-lives; radiation sources, synthetic routes to labelled compounds, decomposition rates of labelled compounds, measurement and safety.

\section{\# 6500248}

Winche11, A. N.

$$
\star 8.383-3.383
$$

THE OPTICAL PROPERTIES OF ORGANIC COMPOUNDS, SECOND EDITION, ENLARGED AND COMPLETELY REVISED

New York: Academic Press

1954

$515 \mathrm{p}$

This second edition of The Optical Properties of Organic Compounds describes more than twenty-five hundred substances and nearly two thous and are placed on one or both of the two diagrams which are enclosed with. the book for the determination of compounds. All organic compounds whose optical properties were described prior to october 1,1952 are included. 
\#6704017

$\star 3.083$

Wo $1 \mathrm{fe}$, W. L. (Editor)

HANDBOOK OF MILITARY INFRARED TECINOLOGY

office of Naval Research, Department of the Navy, Washington, D. C.

1965

$906 \mathrm{p}$

Available from Superintendent of Documents, GPO

This Handbook reviews the basic radiation laws, blackbody simulators, the properties of targets and of backgrounds, radionetrics, atmospheric absorption, scattering and scintillation, optics and optical design, detectors, coolers for infrared detectors, film and preamplifiers, and other special features of infrared engineering. Numerous graphs and tables of data are included.

\section{$\# 6907024$}

* 3.183

Wolfe, W.; Limperis, T. (E di tors)

INFRARED OUANTUM DETECTORS

IRIA State-of-the-Art Report 2389-50-T, Michigan University, Institute of Science and T'echnology, Ann Arbor, Michigan

July 1961

$348 \mathrm{p}$

Availlable from CFSTI, AD 326487

The theory of the detection process is described, and data such as NEP, $D^{*}$, noise spectrum, time constant, and resistance are given for the following infrared detectors: the lead salts, impurity-activated germanium, germanium-silicor alloys, tellurium, indium arsenide, and indium antimonide. Appendixes include a description of test procedures, immersion techniques and cooling devices.

\#6907022 *3.193-8.193

Working Group on Infrared Backgrounds

INFRARED TARGET AND BACKGROUND MEASUREMENTS, CONCEPTS, UNITS, AND TECHNIQUES

IRIA Report 2389-64-T, Michigan University, Institute of Science and Technology, Ann Arbor, Michigan

January 1962

$43 p$

Available from DDC

This report discusses concepts, units, and techniques for making and describing measurements of radiation from targets and backgrounds. Graphs, figures, and references are included.

\# 6905006 *3.183

Wurster, W. H. ; Marrone, p. V.

FURTHER MEASUREMENTS OF' THE NEAR IR SPECTRUM OF SHOCK-IIEATED AIR

CAL Report No. QM-1756-A-1, Cornel1 Aeronautical Laboratory, Inc., Buffalo, New York

August 1966

$41 \mathrm{p}$

Available from DDC

Quantitative spectroscopic measurements have been made of the infrared spectrum of shock-heated air and nitrogen between 0.9 and 1.3 micron. The measurements for air were ohtained in the reflected shock region of a shock tube, covering the temperature range $6500-7200^{\circ} \mathrm{K}$. The nitrogen data were obtained behind incident shock waves for temperatures between $4600-5700^{\circ} \mathrm{K}$, and in the reflected shock region for temperatures from 6800 $7500^{\circ} \mathrm{K}$. In a previous study it was shown that air radiates much more significantly than nitrogen in this special range, and that the radiation could be attributed to transitions between excited electronic states of the nitric oxide molecule. The present measurements confirm these results and also show that the observed excitation energy of the radiation is inconsistent with the energy levels in nitrogen. The data from both studies are reviewed, and it is concluded that the NO hypothesis is consistent with the experimental evidence.
$\# 6510004$

$X$-RAY WAVELENGTIS FOR SPECTROMETER, BRAGG ANGLES FOR CRYSTALS, ADP, EDT, QUART $26.68, \mathrm{Si}, \mathrm{NaC} 1, \mathrm{LiF}$, QUARTZ 2.75; TOPAZ, GYPSUM, PET 'INCLUNING' X-RAY' ABSORPTION EDGES AND ALL PRINCIPLE X-RAY EMISSION LINES, THIRD EDITION

New York: General Electron Company

1964

$209 \mathrm{p}$

These tables contain data helpful in the use of an $x$-ray spectrometer. The first section includes tables of the Bragg angles of various crystals for the first five orders of reflection, arranged hy increasing angles. The data included in this section are: excited element, x-ray wavelength, order of reflection and Bragg angle for ADP, EDT, quartz 6.68 , silicon, sodium chloride, gypsum, PET, LiF, quartz 2.75 , and topaz. The second section inciudes a table of Bragg angles for each crystal mentioned above, arranged hy elements in order of increasing atomic number.

$\# 6500127$

$$
\text { *3.193-4.193 }
$$

Yakowitz, H.; Cuthill, J. R.

ANNOTATED BIBLIOGRAPHY ON SOFT X-RAY SPECTROSCOPY

NBS Monograph 52, National Bureau of Standards, Washington, D. C.

June 1962

$109 \mathrm{p}$

Availab le from Superintendent of Documents, GPO

About 550 references are contained in this bibliography which includes, it is believed, a complete coverage of the soft X-ray literature since 1950 and through 1960 . Some references to earlier work are given, but the general review references, listed separately, give an adequate coverage of the earlier work. The emphas is is on the application of soft X-ray spectroscopy in the study of valence band electronic states in metals and alloys, and therefore, the spectral region of 25 to 800 angstroms involving ruled glass grating spectrometers is of principal interest. However, a wealth of data have been gathered, primarily by the Russians, on valence electronic states by means of high energy transitions where crystal spectrometers are satisfactory. These references and any $\mathrm{X}$-ray work leading to the distribution of valence electronic states are included regardless of the transitions employed. In addition to soft X-ray data, references on all pertinent aspects of the apparatus and experimental problems are included. Also listed separately are references of value in corroborating soft X-ray data with other results, such as energy band calculations. Subject, author, $X$-ray band, material, and other indices are included.

$\# 6508056 \quad$ *3.193

Zaide1', A. N.; Prokof'ev, V. K.; Raiskii, S. M.

TABLES ÓF SPECT́RUM LINES

New York: The Macmillan Company

1961

$600 \mathrm{p}$

This book is a compilation of spectral lines wavelengths taken from Harrison's 1939 compilation of 100,000 lines. The first part contains the wavelengths of 43,000 lines from 60 elements, which are the most useful for identifying unknown elements. These lines are arranged by wavelength and cover wavelengths from 8,000 to $2,000 \AA$. The second part contains the wavelengths of 23,000 lines of 93 elements, arranged hy element. The last part contains auxiliary tables, including ionization potentials, molecular weights, and melting and boiling points. 
Zaidel', A. N.; et al.

TABLITSÝ SPEKTRA NYKH LINII (TABLES OF SPECTRAL L INES)

Moscow: Izsatel'stvo Nauk, Glavnaya Redaktsiya, Fiziko-Mathematicheskoi Literatury

1969

$784 \mathrm{p}$

In Russian

This volume is a compendium of spectral lines arranged in three parts. The first is a finding list of 349 pages in which the lines of the various spectra are arranged in the order of decreasing wavelength, from 8000 to $2000 \%$. The spectrum is indicated in most cases, and arc and spark intensities are given. The second part contains lists of lines by element; it includes fainter lines and covers a longer spectral range than part 1. The reference source, intensity and higher excitation potential are listed for each line. The third part contains selected tables of leading lines arranged by element, standard wavelengths, transition probabilities, and ionization potentials for the first five spectra. It concludes with a brief table giving melting and boiling temperatures for elements and some molecules.

$\# 6611019$

$$
\text { * 4.293-3.293 }
$$

Zeitlin, A. (Compiler)

ANNOTATED BIBLIOGRAPHY ON HIGH-PRESSURE TECHNOLOGY New York: The American Society of Mechanical Engineers

Washington and London: Butterworths

1964

$304 \mathrm{p}$

The bibliography is presented in four principal parts: Part I is a general bibliography of 4500 entries arranged alphabetically by authors, but not including the writings of P. W. Bridgman. Part II is an index of the material of Part I, arranged by subject. Part III is a complete bibliography of the 310 writings of $P$. W. Bridgman. Part IV is an index of the material in Part III, arranged by subject.

$\# 6500188$

$* 3.393$

Zwolinski, B. J.; et al.

CATALOG OF INFRÁRED SPECTRAL DATA, SERIAL NOS. 1 TO 451 AND INDEX

Chemical Thermodynamic Properties Center, Texas A\&M University, College Station, Texas

December 1968

451 loose-leaf pages

This volume contains infrared spectra of organic compounds collected from various contributing laboratories by the staff of the Thermodynamic Research Center at Texas AogM University. It contains 451 spectra presented as a plot of absorbance versus wavelength, together with the compound name, structural formula, state, temperature of measurement, concentration, and the instrument used in taking the spectrum.

\#6500189

$$
\text { *3. } 393
$$

Zwolinski, B. J.; et al.

CATALOG OF MASS SPECTRAL DATA, SERIAL NOS. 1 TO 149 AND $1 \mathrm{~m}$ TO $74 \mathrm{~m}$ IVITH INDEXES

Chemical Thermodynamic properties Center, Texas A\&M University, College Station, Texas

June 1968

223 loose-leaf pages

This volume contains mass spectral data for 223 organic compounds presented in tabular form, with the relative intensity for each value of the mass/charge ratio tabulated for different values of the ionizing voltage. Peaks are labeled and the type of peak given. Other information given include molecular weight, formula, boiling point, structure, source of spectra, type mass spectrometer used, ionizing current, purity, relative and total intensity, sensitivities and temperatures of operation.

\#6500190-6500191

*3.393

Zwolinski, B. J.

CATALOG OF NUCLEAR MAGNETIC RESONANCE SPECTRAI. DATA, SERIAL NOS. 1 TO 611 , Ic TO $71 \mathrm{C}$ AND INDEXFS

Chemical Thermodynamic Properties Center, Texas AçM University, College Station, Texas

December 1968

682 loose-leaf pages

These two volumes contain nuclear magnetic resonance spectra of organic compounds collected by the staff of the Thermodynamics Research Center at Texas A\&M University. They contain 682 spectra, none of which are hydrocarbons. The spectra are presented graphically, each on one sheet. Information on the compound (rame, chemical and structural formula, state, solvent and temperature) and the instrument type and the frequency used are also included. The set of volumes contains a numerical index and an index of compounds.

$\# 6602006$

Zwolinski, B. J.; et al.

*3. 393

CATALOG OF RAMAN SPECTRAL DATA, SERIAL NOS. 1 TO 54 AND INDEX

Chemical Thermodynamic Properties Center, Texas A\&M University, College Station, Texas

December 1966

54 loose-leaf pages

This volume contains Raman spectra collected by the staff of the Thermodynamics properties Center at Texas AEM University from the contributing laboratories that measured spectra for the Manufacturing Chemists Association. The line spectra are presented in tabular and graphical form with intensity of the line plotted or tabulated against the Raman shift in $\mathrm{cm}^{-1}$. The intensity scale is arbitrary, with the intensity of the strongest line set equal to 100 .

\#6500192

Zwolinski, B. J.; et al.

CATALOG OF ULTRAVIOLET SPECTRAL DATA, SERIAL NO. 1 TO 103 AND INDEX

Chemical Thermodynamic Properties Center, Texas AGM University, College Station, Texas

December 1965

103 loose-leaf pages

This volume contains ultraviolet spectra of organic compounds collected from various contributing laboratories by the staff of the Thermodynamic Research Center at Texas AEM University. It contains 103 spectra presented as a plot of absorbance versus wavelength over a range of wavelengths from 2000 to $4500 \mathrm{~A}$ Also presented with each spectrum are the structural and chemical formulas for each compound, the state of the compound and the measurement temperature.

$\# 6905041$ *3. 393-8. 393

Zwolinski, B. J.; Wilhoit, R. C.

COMPREHENSIVE INDEX OF API 44-TRC SELECTED DATA ON THERMODYNAMICS AND SPECTROSCOPY

Publication 100, Chemical Thermodynamic Properties Research Center, Texas A\&M University, College Station, Texas

1968

$507 \mathrm{p}$

The API-TRC Comprehensive Index is a key to the contents of publications presenting critically evaluated numerical property values and selected spectral data that are prepared, compiled, published and distributed by the Thermodynamics Research Center of Texas A\&M University. This index contains in codified form the contents of twelve distinct loose leaf data sheet compilations of physiocochemical, thermodynamic and 
spectroscopic data. Included are a compound name index and a formula index.

\#6500200-6500205 *3.393

Zwolinski, B. J.

SELECTED INFRARED SPECTRAL DATA, SERIAL NOS. 132791

Chemical Thermodynamic properties Research Center, Texas A\&ll University, College Station, Texas

October 1967

3000100 se-1eaf pages

"Infrared Spectral Data," Volumes 1 to 8 contain infrared spectra contributed by 33 laboratories, including government, industrial, and university laboratories, which measured these spectra. Over 2600 spectra are included, nearly all are of organic compounds. Each spectrum appears on a separate page, together with the compound name, structural formula, state, temperature of measurement, and concentration. The instrument used and the laboratory taking the spectrum are also given.

\#6500193-6500199

*3. 393

Zwolinski, B. J.

SELECTED MASS SPECTRAL DATA

Chemical Thermodynamic Properties Research Center,

Texas A\&M University, College Station, Texas

April 1966

2300 10ose-1eaf pages

This collection of mass spectral data was compiled by the American Petroleum Institute Research Project 44 staff from mass spectra obtained experimentally from 16 laboratories. A description of the instrument and procedures used by each laboratory is given. Each mass spectrum is presented in tabular form, with the relative intensity for each value of the mass/charge ratio tabulated for different values of the ionizing voltage. Peaks are labelled and the type of peak given. Additional information concerning metastable ion transition and relative intensities for a n-butane are given, as well as data on the spectrometer sensitivity, current, voltages and temperatures of operation. Six volumes contain 1933 spectra. Volume 7 contains 154 spectra presented in matrix to the catalog.

\section{$\# 6500208,6500209$

$$
\text { *3. } 393
$$

Zwolinski, B. J.

SELECTED NUCLEAR MAGNETIC RESONANCE DATA SERIAL NOS. 1 TO $594,1 \mathrm{c}$ TO $124 \mathrm{c}$

Chemical Thermodynamic properties Research Center, Texas Aक्षM University, College Station, Texas April 1968

$900100 s e-1 e a f$ pages
These volumes contain nuclear magnetic resonance spectra of organic compounds collected by the staff of the The rmodynamics Research Center at Texas AGM University. The two volumes prepared for the American petroleum Institute project 44 contain about 600 spectra, largely of hydrocarbons. The spectra are presented graphically, each on one sheet. Information on the compound (name, chenical and structural formula, state, solvent and temperature), the instrument type and the frequency used are also included. Each set of volumes contains a numerical index and an index of compounds.
\#6500210,6500211
$* 3.393$

Zwolinski, B. J.

SELECTED RAMAN SPECTRAL DATA, SFRIAL NOS. 1-409

Chemical Thermodynamic properties Research Center, Texas AGM University, College Station, Texas

October 1964

520 loose-leaf pages

These volumes contain Raman spectra collected by the staff of the Thermodynamics properties Center at Texas AqM University from the contributing

laboratories that measured those spectra for the American Petroleum Institute Research project 44. The line spectra are presented in tabular and graphical form with intensity of the line plotted or tabulated against the Raman shift in $\mathrm{cm}^{-1}$. The intensity scale is arbitrary, with the intensity of the strongest line set equal to 100 .

\#6500212-6500213

Zwolinski, B. J.

*3.393

SELECTED UILTRAVIOLET SPECTRAL DATA, SERIAL NOS, 11075

Chemical Thermodynamic Properties Research Center, Texas AGM University, College Station, Texas

April 1967

1300 loose-leaf pages

These volumes contain ultraviolet spectra of organic compounds collected from various contributing laboratories by the staff of the Thermodynamic Research Center at Texas AGM University, The two volumes prepared for the American petroleum Institute, Project 44 contain about 1,000 spectra. Each spectrum is presented as a plot of absorbance vs. wavelength over a range of wavelengths from 2000 to $4500 \AA$. A1so presented with each spectrum are the structural and chemical formulas for each compound, the state of the compound and the measurement temperature. 
Solid State Properties 
\#6910007

$* 4.113-8.113$

Abrikosov, N. Kh.; et al.

Tylbulewicz, A. (Translator)

SEMICONDUCTING II-VI, IV-VI, AND V-VI COMPOUNDS

New York: plenum Press

1969 (1967)

$260 \mathrm{p}$

Translated from Russian

This monograph deals with the semiconducting conpounds formed from elements of groups II, IV, or $V$ and sulfur, selenium, or tellurium. The monograph describes the crystal structure of these compounds, and presents the published data on their polymorphic transitions, including transitions observed at high pressures. The monograph includes published $\mathrm{P}-\mathrm{T}-\mathrm{X}$ diagrams for the compounds discussed and describes a method for plotting the diagrams. The more important physiochemical properties of the compounds are also given: the forbidden band width, the carrier mobility, the effective masses of carriers, the electrical conductivity, and the thermoelectric power. The monograph consists of three chapters. The first deals with chalcogenides of elements in group II: zinc, cadium, and mercury. The second discusses semiconducting compounds of elements in group IV: germanium, tin, and lead. And the third describes investigations of compounds of elements of group V: arsenic, antimony, and bismuth. The monograph does not cover chalcogenides of elements in group II. Also excluded are data on semiconducting glasses. The monograph includes the published data up to the second half of 1966 on the crystal structure, physicochemical properties and phase diagrams of important semiconducting compounds which are used in various branches of engineering.

\section{\#6909067-6909068}

$\star 8.113-4.113$

Ageev, N. V. (Editor)

Schmorak, J. (Translator)

HANDBOOK' OF BINARY METALLIC SYSTEMS, STRUCTURE AND PROPERTIES, PHYSICOCHEMICAL PROPERTIES OF THE ELEMENTS

VOLUME I! SYSTEMS OF ACTINIUM, ALUMINUM, AMERICUM, BARIUM, BERYLLIUM, BORON, AND NITROGEN,

VOLUME II: SYSTEMS OF BISMUTH, DYSPROSIUM, EUROPIUM, GADOLINIUM, GALLIUM, GERMÁNIUM, HAFNIUM, HOLMIUM, HYDROGEN, IRON, TUNGSTON, AND VANADIUM

IPSAT cat.-nos. 1711 and 1712, Israel program for Scientific Translations, Jerusalem, Israel

1967 (1959) - Vol. 1; 1967 (1962) - Vol. 2

$645 \mathrm{p} ; 887 \mathrm{p}$

Available from CFSTI, TT 66-51149 and TT 66-51150

Translated from the Russian

These books are the first two volumes of a reference source containing information on phase diagrams, crystalline structure and physical and chemical properties of binary metallic systems. The first volume includes phase diagrams of systems constituted by actinium, aluminum, americum, barium, beryllium, boron, and nitrogen; the second volume includes phase diagrams of the systems constituted by bismuth, dysprosium, europium, gadolinium, gallium, germanium, hafnium, holmium, hydrogen, iron, tungsten, and vanadium. The second constituent is each element of the periodic system (except for noble gases) in alphabetical order. Subject to the extent of the present knowledge of each system, the following data are given: 1) phase diagrams and crystaliine structures; 2) physical properties (heat of melting or heat of mixing, surface tension and viscosity, boiling point, density, mechanical properties, heat expansion, heat capacity, thermal conductivity, electrical resistance and its temperature coefficient, emf, magnetic properties); 3) chemical properties (resistance to corrosion under atmospheric conditions, and in various corrosive media and in gases); 4) a list of literature references.
\# 6902037

$*^{*} .113$

Anderson, 0 . L

PROPERTIES OF POLYCRYSTALLINE AGGREGATES OF CERTAIN MINERALS CALCULATED FROM ELASTIC CONSTANTS OF SINGLE CRYSTALS

Columbia University, LaMount Laboratory, New York, New York

(No Date)

$310 \mathrm{p}$

This is a computer printout compilation of critically evaluated data, giving properties of polycrystalline aggragates of minerals containing 74 elements and anions. Literature sources of each elastic constant used in calculating properties are given. The properties tabulated for each entry include: crystalline structure; composition. density; elastic, bulk, shear, Young, and Long moduli; the Poisson ratio; and the Debye temperature.

\#6907020

*3. $113-4.113-8.113$

Ballard, S. S.; McCarthy, X. A.; Wolfe, W. L.

OPTICAL MATERIALS FOR INFRARED INSTRUMENTATION

IRIA Report 2389-11S, Michigan University, Willow Run Laboratories, Ann Arbor, Michigan

January 1959

$113 p$

Available from DDC, AD 217367

The properties of approximately fifty materials which are useful in optical instrumentation in the infrared portion of the electromagnetic spectrum are described and compared. Each material is discussed in detail in terms of its optical, chemical, thermal, and mechanical properties. Tables and figures are given so that the properties of the various materials can be readily compared.

$\# 6907021 \quad * 3.113-4.113-8.113$

Ballard, S. S.; McCarthy, K. A.; Wolfe, W. L.

OPTICAL MATERIALS FOR INFRARED INSTRUMENTATION, SUPPLEMENT

Report 2389-11-S, Michigan University, Infrared Laboratory, Ann Arbor, Michigan

Apri1 1961

$18 \mathrm{p}$

Available from CFSTI, AD 255699

In this supplement, additional data are compiled on the optical materials, which are useful for infrared instrumentation, treated in 2389-11-s, as well as data on three new materials, Irtran-1, Irtran-2, and diamond. In addition, several errors and ambiguities in the original report are corrected and clarified.

\# 6500495

Baurer, T.

$$
* 8.113-4.113
$$

SOME EXPPERIMENTS ON THE DEPOSITION OF GASES AT $4.2 \mathrm{~K}$ NBS Technical Note 73 , National Bureau of Standards, Washington, D. C.

October 1960

$32 \mathrm{p}$

Available from CFSTI

In order to develop some preliminary information concerning the very basic problems of gaseous deposition at extremely low temperaturës, samples of argon, nitrogen, oxygen, and hydrogen were deposited at $4.2 \mathrm{~K}$, and variations in some of the parameters associated with these depositions were observed. In addition, samples of the same gases were passed through an excitation zone prior to deposition, and differences in the deposition behavior were recorded. The pressure downstream of the deposition region and the temperature within the deposited solids were found, in general, to increase with flow rate of the depositing substances, and to vary with time in a manner depending on the density, flow rate, and state of excitation of the incoming gases. Arrhenius curves were plotted using warm-up data observed in these experiments and solid-phase transition data determined elsewhere. Variations in the positions 
of these plots were sometimes the result of differences in the conditions of deposition, and at other times an effect of the chemical nature of the gas studied. The experimental behavior of hydrogen, relative to that of the other gases studied, was observed to be strongly dependent upon its much higher thermal conductivity.

\#6500139

$\star 3.113-4.113$

Bearden, J, A.

$X-R A Y$ WAVELENGTHS

NYO-10586, A tomic Energy Commission, Oak Ridge,

Tennessee

1964

533 p

Available from CFSTI

This book is the result of a four-year survey by the $x$-ray Laboratory at the Johns Hopkins University. The primary objectives are to: (1) recompute a11 published wavelengths on a consistent basis: (2) include data published subsequent to the Sandstroem survey of 1957 and, in particular, reference wavelengths measured in the Johns Hopkins Laboratory; (3) convert these wavelengths to an absolute basis; (4) tabulate the data on which the recommended values are based; and (5) include explicitly probable errors for all adopted wavelength values. The $W K_{a l p h}$, line peak was taken as a wavelength standard. Phe first part of the tabulated data includes wavelength, wavenumber, energy values arranged according to series and by element. The $K, L, M, N$ and 0 series, as well as absorption edges are given. The second part of the tables presents wavelength and energy values in order of increasing wavelength. The appendix contains an evaluation of wavelength data and presents for each line a literature reference, a published, adjusted and recommended value, and reason for making the adjustment, if any adjustment was made.

\section{\# 6809012}

$* 3.113-4.113$

Bearden, J. A.

$X$-RAY WAVELENGTHS AND X-RAY ATOMIC ENERGY LEVELS NSRIS-NBS-14, National Bureau of Standards, Washington, D. C.

September 25,1967

$66 \mathrm{p}$

Available from Superintendent of Documents, GPO

This publication contains two critical compilations: 1) $X$-Ray Wavelengths. Incons is tencies in accepted values (in $x$ units) of $x$-ray reference lines have recently been demonstrated, although all are supposedly based on "good" calcite crystals. Factors supporting the selection of the $W K_{a l p h a}$ line as the $X$-Ray Wavelength Standard are critically discussed. A review is given of the experimental measurements which are used to establish the wavelength of this line on an absolute angstrom basis. Its value is $\lambda \mathrm{W} \mathrm{K}_{\mathrm{alphal}}$ $=(0.2090100 \pm 5 \mathrm{ppm}) \mathrm{A}$. This may be used to depine a new unit, denoted by $A^{*}$ such that the $W K^{\text {a }} l_{p h} p_{A}$ $5 \mathrm{ppm}$. The wavelengths of the $\mathrm{Ag} \mathrm{K}$. $K_{a l p h a} \mathrm{Cu} \mathrm{K} \mathrm{K}_{\mathrm{alpha}}$, and the $\mathrm{Cr} \mathrm{K}_{\mathrm{alph}}$ have been established as secondary standards with probable error of approximately one part per million. Sixty-one additional $x$-ray lines have been used as reference values in a comprehensive review and reevaluation of more than 2700 emission and absorption wavelengths. The recommended wavelength values are 1 isted in $A^{*}$ units together with probable errors; corresponding energies are given in keV. A second table lists the wavelengths in numerical order, and likewise includes their energies in keV.' 2) Reevaluation of X-Ray Atomic Energy Levels. All of the $x$-ray emission wavelengths have recently been reevaluated and placed on a consistent $A^{*}$ scale. For most elements these data give a highly overdetermined set of equations for energy level differences, which have been solved by least-squares adjustment for each case. This procedure makes "best" use of all x-ray wavelength data, and also permits calculation of the probable error for each energy difference. Photoelectron measurements of absolute energy levels are more precise than $x-r a y$ absorption edge data. These have been used to establish the absolute scale for eighty-one elements and, in many cases, to provide additional energy level difference data. The $x$-ray absorption wavelengths were used for eight elements and ionization measurements for two; the remaining five were interpolated by a Moseley diagram involving the output values of energy levels from adjacent elements. Probable errors are listed on an absolute energy basis. In the original source of the present data, a table of energy levels in Rydberg units is given. Difference tables in volts, Rydbergs, and milli-A* wavelength units, with the respective probable errors, are also included there.

$\# 6605016$

$* 3.113-4.113$

Bearden, J. A.; Burr, A. F.

ATOMIC ENERGY LEVELS

NYO-2543-1, Final Report, Atomic Energy Commission, Oak Ridge, Tennessee

1965

$244 \mathrm{p}$

Available from CFSTI

This book contains the energy levels of atoms, and the differences between those levels, as computed from a table of $x-r a y$ wavelengths. The first set of tables contain values and probable errors of the energies of all occupied levels of each element, presented both in Rydbergs and in electron volts. The second set of tables contain differences between all the energy levels presented in the first set, in units of Rydbergs, electron volts, and milliangstroms.

\#6902005

Berezhnoi, A. S.

SILICON AND ITS BINARY SYSTEMS

New York: Consultants Bureau

1960

$275 \mathrm{p}$

Translated from Russian

This text, translated from Russian, is a review of the properties of silicon and its binary compounds. The text discusses the preparation of binary silicon compounds, properties and uses of $\mathrm{H}-\mathrm{Si}$ and Halogen-Silicon systems, systems formed by silicon with elements of Groups IA, IB, IIA, IIB, III, IVB, VB, VIB, transition metals of Groups IV, V, VI, VII, and VIII, and with metals of the iron subgroup, palladium subgroup, platinum subgroup, lanthanides and actinides. Ternary and quaternary systems are also included. There is a list of 716 references and tables of data accompany the systems discussed.

\#6907014 *8.172-4.172

BIBLIOGRAPHY, LOW-TEMPERATURE SPECIFIC HEAT Research Materials Information Center, Oak Ridge National Laboratory, Oak Ridge, Tennessee September 1968

$42 \mathrm{p}$

This bibliography contains 452 references of $10 \mathrm{ow}$ temperature, specific heat data and information for elements, compounds or alloys, of all elements having atomic numbers 1 to $84,90,92$, and 94 , with the exception of the inert gases and technetium. The bibliography is accompanied by an index of elements and compounds. 
\$6907036

$* 4.113$

Billington, D. S.; Danielson, G. C.

STATUS OF CRYSTAL GROWTH IN THE UNITED STATES

ORNL-RMIC-10, Research Materials Information

Center, Oak' Ridge National Laboratory, Oak Ridge,

Tennessee

December 1968

$11 \mathrm{p}$

Available from CFSTI

This report gives data and information about the development and progress made in crystal growth in the United States for a variety of compounds. The demand, availability, location, and purity data are included in the information given. This report covers the year 1968 .

\#6500152 $* 4.113$

Biondi, M. A.; et al.

EXPERIMENTAL EVIDENCE FOR AN ENERGY GAP IN SUPERCONDUCTORS

Reviews of Modern Physics, 30: 1109-1136

Uctober 1958

This paper is a critical review of evidence from (a) thermal conductivity, specific heat and critical fields, (b) nuclear resonance and relaxation, and (c) microwave and infrared studies of bulk metal and of thin films. About 80 references are included.

\#6908045

$* 4.213-8.213-3.213$

Bondi, A. A.

PHYSICAL PROPERTIES OF MOLECULAR CRYSTALS, LIQUIDS, AND GLASSES

New York: John Wiley \& Sons, Inc.

1968

$502 \mathrm{p}$

This book developes a methodology enabling chenists and chemical engineers to relate certain physical properties of condensed phases to molecular structure. The states of matter treated are molecular crystals, liquids, and glasses. The physical properties covered are P-V-T and related thermal properties, and certain transport properties. Energy of vaporization, van der Waals dimensions, molar refractivity, dipole moment data and dielectric loss are used but not discussed thoroughly. Tables 14.1 to 14.16 contain the requisite molecular structure increments. Background material presented is a guide to the literature.

\section{\#6811025}

*4. 123

Carter, G.C.; et al.

THE NBS ALLOY DATA CENTER: FUNCTION, BIBLIOGRAPHIC SYSTEM, RELATED DATA CENTERS AND REFERENCE BOOKS NBS Technical Note 464, National Bureau of Standards, Washington, D. C.

August 1968

$168 \mathrm{p}$

Available from Superintendent of Documents, GPO

The Alloy Data Center, part of the National Standard Reference Data System, has two primary functions. One is to stimulate cooperation and coordination among the existing data centers in the area of the physical properties of well characterized alloys. The other purpose is the collection (from publications as well as private communications), evaluation, and publication of data in areas of special competence in the NBS Alloy Physics Section. Of interest to the center are metals, semimetals, intermetallic compounds, and alloys. Excluded are those materials which have ill-defined constitutions and heat treatments. An automated system has been developed to meet the bibliographic needs of the center. This system is described, as well as the specific properties of interest. The system presently contains a complete annotated file on papers dealing with NMR Knight shift measurements. The soft $X$-ray spectroscopy compilation is being kept up to date with the same system.
\#6906023 $* 4.213-3.213$

Carter R. S.; Rush, J. J. (Editors)

MOLECULAR DYNAMICS AND STRUCTURE OF SOLIUS

NBS Special Publication 301, National Bureau of Standards, Washington, D. C.

June 1969

$571 \mathrm{p}$

Available from Superintendent of Documents, GPO

This volume is based on material presented at the Second Materials Research Symposium of the National Bureau of Standards, held October 16-19, 1967. It provides a review of the application of spectroscopic and diffraction techniques to the study of the structure and dynamics of molecular solids. Invited papers on the theory and practice of the major experimental methods, including neutron and $x$-ray diffraction, neutron inelastic scattering, infrared and Raman spectroscopy and nuclear magnetic-resonance, serve as a background for the more detailed presentation and discussion of results which follows. The topics covered in a series of invited and contributed papers include the lattice dynamics of molecular crystals, the spectroscopy and crystal structure of organic and inorganic solids and the dynamics of polymers.

\#6500509 $* 8.223-4.223$

Cezairliyan, A.; Touloukian, Y. S.

CORRELATION AND PREDICTION OF THERMAL CONDUCTIVITY OF METALS THROUGH THE APPLICATION OF THE PRINCIPLE OF CORRESPONDING STATES

In Advances in Thermophysical Properties at Extreme Temperatures and Pressures, S. Gratch, Editor, pp301-313

New York: American Society of Mechanical Engineers 1965

It is shown that the Principle of Corresponding States is applicable to thermal conductivity of metals. Two correlative relations are presented; one at low temperatures $(0<\mathrm{T}<1.5 \mathrm{Tm})$, and another at moderate and high temperatures $\left(0.3 \theta_{<}<<\right.$ $3 \theta$ ), where Tm is the temperature corresponding to the thermal conductivity maxima and $\theta$ is the Debye temperature. A universal relation for the reduced thermal conductivity is obtained in the low temperature region based on the solid state physical theory of metals and the proposed Principle of Corresponding States.

$\$ 6907026$

Clark, P. V.; Dean, C. 0.

FUSED SALT MIXTURES: EUTECTIC COMPOSITION

MELTING POINTS BIBLIOGRAPHY 1907-1968

SC-R-68-1680, Sandia Laboratories, Sandia

Corporation, Albuquerque, New Mexico

December 1968

$792 \mathrm{p}$

Available from CFSTI

Data on eutectic compositions and melting points of fused salt mixtures are presented in two complementary tables arranged by systems and by melting points. Over 5,000 references are given through December 30,1968 .

$\# 6908015$

$* 4.123-3.123-8.123$

Connolly, T. F.; Copenhaver, E. D.

BIBLIOGRAPHY OF MAGNETIC MATERIALS AND TABULATION OF MAGNETIC TRANSITION TEMPERATURES

ORNL-RMIC 7 (Revised, 1969, preprint), Research Materials Information Center, Oak Ridge National Laboratory, Oak Ridge, Tennessee

1969

$78 \mathrm{p}$

ORNL-RMIC-7 (March 1968) has been corrected, revised, and expanded to include about 1000 additional measurements of Curie and Nel points of magnetic materials. As in the original, the materials are arranged alphabetically by chemical formula and appear in two lists - one for ferromagnetic (or ferrimagnetic) and one for antiferromagnetic (or metamagnetic) substances. 
The tabulation is based on papers and reports received by the RMIC through January 1969.

6605019

COPPER WIRE TABLES

NBS llandbook 100, National Bureau of Standards, Washington, D, C.

February 21, 1966

$41 \mathrm{p}$

Available from Superintendent of Documents, GPO

This flandbook is a revision of the Copper Wire Tables previously published as NBS Circular 31. It reflects changes in the nominal diamenters of gages 45 and smaller and extends the tables to 56 gage. The Handbook provides data for the weight and length per ohm, weight per unit length, length per unit weight of copper wire, resistivity, temperature coefficient, density, and conductivity of copper wire from 0000 to $56^{\circ}$ gage as a function of temperature from 0 to $200 \mathrm{C}$.

甘 6500304,6500305

$$
\text { * } 8.123-4.123
$$

Corruccini, R.J.; Gniewek, J.J

THERMAL EXPPANSION OF TECHNICAL SOLIDS AT LOW TEMPERATURES, A COMPILATION FROM TIE LITERATURE

NBS Monograph 29, and Supplement, National Bureau of Standards, Washington, D. C.

May 19,1961

$22 \mathrm{p}, 2 \mathrm{p}$

Available from Superintendent of Documents, GPO

Tables are given of the 1 inear contraction relative to $293^{\circ} \mathrm{K}\left(\mathrm{L}_{293}-\mathrm{L}_{\mathrm{T}}\right) \mathrm{L}_{293}$, and the 1 inear expansion coefficient $\mathrm{dL}^{2} \mathrm{~L}_{29} \mathrm{dT}$, of thirty elements, fortyfive alloys, twenty-two other inorganic substances and twenty plastics and elastomers in the temperature range, 0 to $300^{\circ} \mathrm{K}$.

\section{\# 6906033}

$* 6.023-4.023$

Deitz, V.R.

BIBLIÓGRAPHY OF SOLID ADSORBENTS 1943 TO 1953, AN ANNOTATIVE BIBLIOGRAPHICAL SURVEY

NBS Circular 566, National Bureau of Standards, Washington, D. C.

March 1956

$1528 \mathrm{p}$

Available from Superintendent of Documents, GPO

This book contains abstracts to 13763 references which give lata and information on solid adsorbents published primarily between 1943 and 1953. Some references to publications before 1943 have been added from the fields of heterogeneous catalys is and surface reactions on textile materials. The coverage is restricted to heterogeneous phenomena at solid-liquid and solid-gas interfaces. The patent literature is not covered. The book is divided into chapters on the general topics of adsorption of gases and vapors on solid adsorbents, adsorption from solutions on solid adsorbents, thermal effects in adsorption processes, theories of adsorption, refining of sugar and other applications of adsorbents, general information on adsorbents and special methods of investigation, and preparation of adsorbents. A subject index, author index, and list of commercial solid adsorbents are included.

$\# 6500269$

$* 4,223$

Donnay, J.J.H. (Editor)

CRYSTÁ DATA, DETERMINATIVE TABLES, SECOND EDITION ACA Monograph 5, American Crystallographic Association, Oak Ridge, Tennessee

April 1, 1963

$1302 \mathrm{p}$

Available from Polycrystal Book Service, Brooklyn, New York

This volume presents a compilation of crystal structure data taken from literature published $1912+1960$. The data can be used for identifying an unknown substance whose lattice constants have been measured or for finding structural data of a known compound. The tables are arranged by crystal class, and by axial ratio within each class (by cell length for cubic crystals). The following data are presented for each compound: axial ratios, name and chemical formula, lattice dimensions and angles (where appropriate), number of formula units per unit cell, space group, structure information, and measured and calculated specific gravity. For some compounds, melting point, color, cleavage, and optical properties are also included. The reference to the literature for each compound includes the author's name, journal name, volume, pages, and data. Both inorganic and organic compounds have been included. Indexes arranged by chemical formula and name have been included.

\# 6500476 *8.123-4.123

Eldridge, E. A.; Deen, Il. W.

REPORT ON PHYSICAL PRÓPERTIİS OF METALS AND ALLOYS FROM CRYOGENIC TO ELEVATED TEMPERATURES

ASTM Special Technical Publication 296, American Society for Testing and Materials, Philadelphia, Pennsylvania

Apri 11961

$206 \mathrm{p}$

This report is a compilation of the physical properties of the metals aluminum, cobalt, iron, magnesium, molybdenum and nickel, and their alloys. The properties included are thermal conductivity, linear thermal expansion, specific heat, electrical resistivity, density, emissivity, diffusivity and magnetic permability. Data are compiled as a function of temperature, where possible, over the range $-457^{\circ} \mathrm{F}$ to $4500^{\circ} \mathrm{F}$. The data contained in this report came principaily from data sheets supplied hy companies and organizations who responded to an ASTM-ASMI survey, trade bulletins, published technical literature and U.S. Government publications.

\#6707009

Eyring, L. (Editor)

PROGRESS IN TIIE SCIENCE AND TECHNOLOGY OF TIIE RARF: EARTIIS, VOLUME I

New York: The Macmillan Company

1964

$532 \mathrm{p}$

This volune is a survey, the first of a series, of research done in the field of rare earth elements, written by a group of specialists, each eminent in his own general discipline and also in the application of that discipline to the development of knowledge of the rare earths. This volume covers work done hetween 1955 and 1961. The topics covered are: geochemistry of the rare earths, mass extraction and separation, separation of rare earths hy ion exchange, liquid-liquid extraction of the rare earths, fractionation of rare earths by liquid-liquid extraction using phosphorus-hased extractants, solution chemistry, recent Soviet research on the chemistry of rare earth complexes, phase equilibria studies in mixed systems of rare earth and other oxides, crystal chemistry of rare earth sesquioxides, aluminates and silicates, structural and physical properties of alloys and intermetallic compounds, thermodynamic and magnetic properties of the rarc earth chalcogenides, Soviet research on analytical chemistry of rare earths, uses and applications.

\#6508065 *3.133-4.133

Foex, G. ; Gorter, C. -J.; Snits, I., -J.

TABLES OF CONSTANTS AND NUMERICAL DATA, VOLUME 7 . SELECTED CONSTANTS, DIAMAGNET ISM AND PARAMAGNETISM, PARAMAGNETIC RELAXATTION

Paris: Mass on \& $c^{1 e}$, Editeurs

1957

$318 \mathrm{p}$

In French, introduction in French and English

These tahles list constants and numerical data for materials which exhibit diamagnetism or 
paramagnetism. These materials incluce the elements, binary alloys, mineral compounds, double or complex salts, organic compounds, free radicals hydrides, oxides, hydracids, acids of mineral oxides; organic acids, anhydrous single and double salts, hydrated single and double salts, complex cations in order of decreasing valency, complex anions in order of increasing valency, non-ionic and metallo-organic compounds. The properties given for these materials are magnetic moments, Curie points and Curie constants, mean atomic susceptibility, moments calculated according to Weiss law, magnetic anisotropy of crystalline compounds, molecular susceptibilities, effective moments, atomic moments, and other related data. The study of paramagnetic relaxation gives information both on the interactions between paramagnetic ions and on the interactions between the ions and the crystalline lattice. These data give magnetic techniques required for the production of very low temperatures by adiabatic demagnetization of paramagnetic substances. Cross references and bibliographical references are included.

$\# 6607035$

$* 8.143-4.143$

Fomenko, V.S.

Sams onov, G. V. (Editor)

HANDBOOK OF THERMIONIC PROPERTIES, ELFCTRONIC WORK FUNCTIONS AND RICIIARDSON CONSTANTS OF ELEMENTS AND COMPOUNDS

New York: Plenum Press Data Division

1966

$151 \mathrm{p}$

The experimental and theoretical calculation of the work functions of particular materials (elements and compounds) are done by various methods: thermionic, photoelectronic, field-emission, effusion, contact potential difference, calorimetric and empirical relationships linking the work function to various physical characteristics which are utilized in the theoretical computation of the work function. Richardson constants are also given and references are listed at the end of the book.

\section{\#6807008}

* 4.133

Fulkerson, W.; et al.

THE RMAL CONDUCTIVITY, ELECTRICAL RESISTIVITY, ANI SEEBECK COEFFICIENT ÓF SILICON FROM 100 TO $1300^{\circ} \mathrm{K}$

Physical Review, 167:765-782

\section{March $15, \frac{1968}{196}$}

Results are presented of measurements of the thermal conductivity $(90-1328 \mathrm{~K})$, the electrical resistivity $(300-1273 \mathrm{~K})$, and the Seebeck large-grained polycrystalline specimens of $99.99+\%$ pure silicon. The thermal conductivity above $387 \mathrm{~K}$ was measured by an absolute radial-heat-flow technique; below $350 \mathrm{~K}$, by an absolute longitudinal technique. Some intermediate thermal-conductivity measurements from $300-400 \mathrm{~K}$ were made on the polycrystaline material using a comparative longitudinal-heat-flow apparatus. The estimated errors of these three thermal-conductivity methods were $\pm 2 \% \pm$ ? $\mathrm{K}, \pm 1.2 \% \pm 0.1 \mathrm{~K}$, and $\pm 4.0 \% \pm 1 \mathrm{~K}$, respectively. The estimated error for the electrical-resistivity measurements was $\pm 1.4 \% \pm 2 \mathrm{~K}$, and for the Seebeck measurements $\pm 1.6 \frac{2}{1} \pm 2 \mathrm{~K}$. The therma1-conductivity values were compared with conflicting data from the literature, and they corroborate the higher-temperature results obtained by Glassbrenner and slack. Therefore, we agree with their conclusion that the electronic contribution is reasonably close to theoretical estimates which include a large ambipolar-diffusion term. The temperature dependence of the lattice thermal resistance has been compared to various theoretical models but no approach seems to explain the data in detail. An abrupt slope change in the thermal resistivity at about $670 \mathrm{~K}$ is a major cause of the difficulty.
$\# 6512006$

* 4.133

French, J. C.

BIBLIOGRAPHY ON THE MEASUREMENT OF BULK RESISTIVITY OF SEMICONDUCTOR MATERIALS FOR ELECTRON DEVICES

NBS Technical Note 232, National Bureau of Standards, Washington, D. C.

October 21,1964

$118 \mathrm{p}$

Available from Superintendent of Documents, GPO

This bibliography contains the references important to the measurement of bulk resistivity of semiconductor materials for electron devices. The publication contains a subject index, an index to authors, and a list of references. The subject index is devided into thirteen categories. An abstract is included for each item listed.

\#6510036

* 4.133

Gertsriken, S.D. ; Dekhtyar, I. Ya

SOLID STATE DIFFUSION IN MÉTALS AND ALLOYS

AEC-tr-6313, AEC Division of Technical Information Extension, Oak Ridge, Tennessee

April 1964

$528 \mathrm{p}$

Available from the CFSTI

Translated from the Russian

This book discusses the progress made from 19541958 in the investigation of solid state diffusion in metals and alloys. It describes the investigation of diffusion using radioactive isotopes and reviews interatomic interaction and laws established for diffusion in the solid phase. A majority of the work presented was developed in the USSR. An extensive list of references is included as well as tables of diffusion properties. The volume includes information on the theory of diffusion, laws of diffusion and calculation for formulas, methods of investigating diffusion in metals, diffusion and interatomic interaction in alloys, defects in the crystal structure and diffusion in metals and alloys, diffusion and resistance to heat, and the use of diffusion phenomena in certain fields of technology.

\#6500477-6500481 (inclusive)

$* 8.133-4.133$

Goldsmith, A. ; Waterman, T.E. ; Hirshhorn, II.J.

HANDBOOK OF THERMOPHYSICAL PROPERTIES OF SOLID MATERIALS, REVISED EDITION,

VOLUME I: ELEMENTS,

VOLUME II: ALLOYS,

VOLUME III : CERAMI CS

VOLUME IV: CERMETS, INTERMETALLICS, POLYMFRICS, AND COMPOSITES

VOLUME V: APPENDIX

New York: The Macmillan Company

1961

I : $752 \mathrm{p} ;$ I I $: 1270 \mathrm{p} ;$ I I I :1162p; IV: $798 \mathrm{p} ; \mathrm{V}: 286 \mathrm{p}$

This set of books is an evaluated compilation of all data on the the rmophysical properties of solid materials published during the period 1940-1957. Materials with melting points above $1000^{\circ} \mathrm{F}$ are included. The following physical properties are included: density, melting point, latent heat of fusion, latent heat of vaporization, latent heat of sublimation, specific heat, thermal conductivity, thermal diffusivity, emissivity, reflectivity, linear thermal expansion, vapor pressure and electric resistivity. Volume I contains data on the elements; Volume II, alloys; Volume III ceramics; Volume IV, cermets, intermetallics, polymeric materials, and composite materials. Volume $V$, the Appendix, contains the Materials Index, the Author Index, and an aIphabetic list of materials. Whe rever possible, a recommended value is given. 
Grigsby, D.L.; Velles, S.J.

A BIBLIÓGRAPIYY ON SELÉCT PROPERTIES OF ALUMINUM

Electronic Properties Information Center, Hughes Aircraft Company, Culver City, California

Apri1 1969

$59 \mathrm{p}$

This bibliography on pure aluminum and dilute aluminum alloys contains references on the following select properties: macroscopic structure, energy band structure, electrical resistivity, emission properties, magnetic properties, optical properties, thernal properties, Debye temperature and attenuation properties.

\section{\#6706012}

*4.133-7.133

Grigsby, D. L.

VANADIUM SILICIDE

Data Sheet DS-154,Electronic Properties Information Center, Hughes Áircraft Company, Culver City,

California

April 1967

$45 \mathrm{p}$

These data sheets present a compilation of the electrical and electronic properties of vanadiumsilicon compounds, especially $V_{3}$ Si. These properties include transition temperature, specific heat and Debye temperature, criticai field, electrical resistivity, penetration depth and absorption. other properties included are: superconducting energy gap, magnetic hysteresis and susceptibility, critical current and current density, Ha11 coefficient and thermoelectric power. Three additional sections of this report cover the crystallographic and mechanical properties of V3Si as well as the nature of magnetic flux characteristics in the mixed state. Fach of these properties is compiled over the widest possible range of parameters from references obtained in a thorough literature search.

\#6712005,6712006 *4.137

Grigsby, D. L. (Editor)

ELECTRONIC PROPERTIES OF MATERIALS, A GUIDE TO THE

LITERATURE VOLUME 2, PART 1 AND PART 2

New York: Plenum Press

1967

$1065 \mathrm{p} ; 734 \mathrm{p}$

This volume provides an updated index to the literature on the electrical and electronic properties of materials. The documents listed in the bibliography and the index represent the acquisitions of the Electronic Properties Information Center since January 1965 , based on a search of 63 journals and the use of eight abstracting services. part 1 is alphabetically arranged by the name of a material, then by property. For each property, one or more accession numbers referring to a publication (if data on that material and property has been published) is 1isted. The specific properties tabulated are: general, absorption cross section, Curie constant, Curie temperature, Debye temperature, dielectric constant, domain structure, effective mass, electrical conductivity, electrical resistivity, electroacoustic properties, electron field emission, electromechanical properties, electron photoemission, electron secondary emission, electron specific heat, energy bands, energy gap, energy levels, flux characteristics, gyromannetic properties, Hall coefficient, insulation resistance, irradiation properties, lifetime, magnetic hysteresis, magnetic susceptibility magneto-electric properties, magneto-mechanicaí properties, microwave emission, mobility, photoelectron properties, photon electroluminescence, photon emissivity, photon 1 uminescence, reflection coefficient, piezólectric properties, refractive index, superconductor transition temperature, thermal conductivity, thermoelectric properties, thermomagnetic properties, transport properties, and work function. part 2 contains the bibliography arranged by accession number. For each publication there is listed the author's name, title of the publication, name, volume, date and page number of the journal.

\#6500427

* 4.133

Grigsby, D. L.

NIOB IUM DATA SIIEETS

DS-141, Electronic Properties Information Center, Hughes Aircraft Company, Culver City, California November 1964

$106 \mathrm{p}$

These data sheets present a compilation of a wide range of electrical and magnetic properties for niobium of various purities in bulk and film form. Electrical properties include conductivity, resistivity, Hall coefficient and transition temperature. Magnetic properties include susceptibility, hysteresis and threshold field. Emission and energy data include thermionic properties, photon emissivities, absorption and energy gap. Debye temperature and therma1 conductivity data are also given. Each property is compiled over the widest possible range of parameters from references obtained in a thorough literature search.

\#6804011

* 4.133

Grigsby, D. L.

NIOBIUM TIN, PART I

DS-159, Electronic Properties Information Center, Hughes Aircraft Company, Culver City, California January 1968

$159 \mathrm{p}$

These data sheets, as Part I of two parts, represent a compilation of the structure-sensitive properties of niobium-tin compounds. These properties include thermal conductivity, critical current and current density and solenoid device applications. Finally, the properties of niobiumtin - X ternary compounds are presented. Each of these properties is compiled over the widest range of parameters from the references obtained in a thorough 1 iterature search.

\# 6810008

Grigsby, D. L.

$$
\text { * } 4.133
$$

NIOBIUM'TIN, PART II

DS-160, Electronic Properties Information Center, Hughes Aircraft Company, Culver City, California July 1968

$174 \mathrm{p}$

These data sheets, as Part II of two parts, represent a compilation of the structurally insensitive properties of niobium-tin compounds. These properties include phase diagrams, crystallographic properties, specific heat, transition temperature, magnetic properties, penetration depth and electrical resistivity. Each of these properties is compiled over the widest range of parameters from the references obtained in a thorough 1iterature search.

\#6902019

Grigsby, D. L.

NIOBIUM-ZI I RCONIUM

DS-152, Electronic Properties Information Center, Ilughes Aircraft Company, Culver City, California November 1966

$198 \mathrm{p}$

These data sheets present a compilation of the electrical and electronic properties on niobiumzirconium alloys. These properties include transition temperature, specific heat and Debye temperature, critical field, electrical resistivity, electrical and thermal conductivities and ultrasonic attenuation. Other properties included are: superconducting energy gap, magnetic hysteresis and susceptibility, critical current and current density, hall voltage and thermoelectric 
power. Three additional sections of this report cover the crystallographic and mechanical properties of $\mathrm{Nb}-\mathrm{Zr}$ alloys as well as the nature of magnetic flux characteristics in the mixed state. The use of these alloys in solenoid applications is also discussed. Each of these properties is compiled over the widest possible range of parameters from references obtained in a thorough literature search.

\# 6500653

* $4.133-3.133-8.133$

GROUPS IV, V, NND VI TRANSITION METALS, SELECTED REFERENCES 'ON PREPARATION, STRUCTURE, PHYSICAL PROPERTIES, METALLOGRAPHY, AND TRANSPORT PROPERTIES ORNL-RMIC-3 (Rev.), Research Materials Information Center, Oak Ridge National Laboratory, Oak Ridge, Tennessee

April 1965

$94 \mathrm{p}$

This bibliography contains 1 ists of selected references on preparation methods, crystal structure, transport properties, and physical and mechanical properties for the Groups IV, V, and VI
transition metals. An abstract is included along with each reference entry.

\#6902097

* $4.133-3.133-8.133$

GROUPS IV, V, AND VI TRANSITION METALS, SUPPLEMENT NO. 1 , SFLLECTED REFERENCES ON PREPARATION STRUCTURE, PHYSICAL PROPERTIES, METALlOGRAPIIY, AND TRANSPORT PROPERTIES

ORVL-RMIC-8, Research Materials Information Center, Oak Ridge National Laboratory, Oak Ridge, Tennessee April 1965

$27 \mathrm{p}$

Available from CFSTI

This bibliography is a supplement to ORNL-RMIC-3 (Rev.) of April 1965, and contains lists of selected references on preparation methods, crystal structure, transport properties, and physical and mechanical properties for the Groups IV, V, and VI transition metals.

\#6500465

*8.133-4.133

Gubareff, G.G.; Janssen, J.E.; Torborg, R.H.

TIIERMAL RADIATION PROPERTIES SURVEY, A REVIEW OF TIIE LITERATURE, SECOND EDITION

Minneapolis, Minnesota: Honeywell Regulator Company

1960

$293 \mathrm{p}$

Thermal radiation property values for more than 40 metals and their alloys are given. The properties reported are enissivity, absorptivity, reflectivity, and transmissivity at various surface conditions, temperatures, and wavelengths. In addition, radiation properties of building materials, paints, glasses, papers, cloths, and other miscellaneous solid materials have been compiled, and solar absorptivity and equilibrium temperatures for various materials have been tabulated. These values have been gathered from about 200 references in English, German, French, and Russian and have been carefuily analyzed to obtain, in the author's opinion, the most trustworthy data for practical application. Recommended data for technically important materials are also presented graphically. An extensive survey was conducted to compile values for Stefan-Boltzmann's, P1 anck's, and Wien's constants, and the most reliable values for practical application are 1 isted in a separate table.
\# 6806004

Ha11, L. A.

SURVEY OF ELECTRICAL RESISTIVITY MEASUREMENTS ON 16 PURE METALS IN THE TEMPERATURE RANGE 0 TO $273^{\circ} \mathrm{K}$

NBS Technical Note 365, National Bureau of Standards, Washington, D. C.

February 1968

$111 \mathrm{p}$

Available from Superintendent of Documents, GPO

Experimental electrical resistivity data for 16 pure metals have been compiled, tabulated, and graphically illustrated for a temperature range of 0 to $273^{\circ} \mathrm{K}$. A section has been prepared for each particular metal which includes references, brief comments concerned with preparation of sample, purity, other pertinent information, tabulated data, and graphs. Data are presented for aluminum, beryilium, cobalt, copper, gold, indium, iron, lead, magnesium, molybdenum, nickel, niobium, platinum, silver, tantalun and tin.

\$ 6809011 * $8,233 \cdot 4,233$

Ho, C.Y.; Powe11, R.W.; Liley, P.E.

THERMAL CONDUCTIVITY OF SELECTED MATERIALS, PART 2 NSRDS-NBS-16, National Bureau of Standards, Washington, D. C.

February 1968

$146 \mathrm{p}$

Available from the Superintendent of Documents, GPO

The work presented in this report comprises the critical evaluation, analysis, synthesis of the available thermal conductivity data, and the generation of recommended values for twelve metallic elements, mainly for the solid state, for a range of graphites and for three fluids in the gaseous state. These are cadmium, chromium, lead, magnesium, molybdenum, nicke1, niobium, tantalum, tin, titanium, zinc, zirconium, Acheson graphite, ATJ graphite, pyrolytic graphite, 8755 graphite, $890 \mathrm{~S}$ graphite acetone, ammonia and methane. For each of the materials recommended values are given over a wide range of temperature.

\#6500393

$* 8.133-4.133$

Hultgren, R.; et al.

SELECTED VALUES OF THERMONYNAMIC PROPERTIES OF METALS AND ALLOYS

New York, London: John Wiley \& Sons, Inc. 1963

$974 \mathrm{p}$

This volume contains the results of evaluations for all the metallic elements and binary alloy systems for which thermodynamic data were available. Results of the evaluations in the form of discussions and analyses of the data and tables of selected values are given for 63 elements and 168 alloy systems. Some of the properties covered include freezing point, vapor pressure, transition temperature phase diagrams, entropy, heat capacity, free energy function, heat content; heat of transition, sublimation, fusion and vaporization; entropy of transition, fusion and vaporization; free energy of sublimation and vaporization; free energy of formation; free energy - excess integral, relative partial molar and relative partial molar excess; heat of formation - relative partial molar; entropy of formation - excess integral, relative partial molar and relative partial molar excess; heat capacity - relative partial molar.

\# 6511007 *8.133-4.133

Hultgren, R.; Orr, R.L.; Kelley, K.K.

SUPPLEMENT TO SELECTED VALUES OF THERMONYNAMIC PROPERTIES OF METALS AND ALLOYS

California University, College of Engineering and Lawrence Radiation Laboratory, Berkeley, California June 1970

$500+100$ se-leaf pages

This Supplement contains the results of evaluations for 56 metallic elements and 178 binary alloy systems for which thermodynamic data were 
available. Reevaluation and extension of the selected values will be a continuing process as nel data become available. Prior to the publication of a revised edition of the book, additiona 1 evaluations, as they are completed, will be issued in loose-leaf form at frequent intervals for inclusion with this Supplement. Description of the methods of evaluation and forms of presenting the data may be found in the parent volume of "Selected Values." Results of the evaluations in the form of discussions and analyses of the data and tables of selected values are given. Some of the properties covered include freezing point, vapor pressure, transition temperature, phase diagrams, entropy, heat capacity, free energy function, heat content; heat of transition, sublimation, fusion and vaporization; entropy of transition, fusion and vaporization; free energy of sublimation and vaporization; free energy of formation; free energy - excess integral, relative partial molar and relative partial molar excess; heat of formation relative partial molar; entropy of formation. excess integra1, relative partial molar and relative partial molar excess; heat capacity relative partial molar.

\#6500445-6500447 $\quad * 4.143$

INSULATION MATERIALS - POLYETHYLENE TEREPHTIIALATE, POLYTETRAFLUOROETHYLENE PLASTICS, POLYTRIFLUORO CHLOROETHYLENE PLASTICS

DS-105, 106, 107, Electronic Properties Information Center, Hughes 'Aircraft Company, Culver City, California

June 1962

$32 p, 31 p, 14 p$

These reports contain data sheets on the properties of three insulation plastics: polytrifluorochloroethylene, polytetrafluoroethylene and polyethylene terephthalate. Each report contains data on some or all of the following properties: arc resistance, corona effects, dielectric constant, dielectric strength, dissipation factor, insulation resistance, power factor, surface resistivity, and volume resistivity.

\# 6500463

$\star 8.143-4.143$

Janssen, J.; Luck, J.; Torborg, R.

REFLECTANCE OF ANODIZED TITANIUM AND BERYLLIUM

Aeronautical Systems Division, Wright-Patterson Air Force Base, Ohio

No date)

$32 \mathrm{p}$

Selection of materials having the desired thermal radiation properties is of primary importance for space applications, since heat transfer to and from an object in space occurs only by radiation. The effect of anodizing variables on the reflectance of anodized titanium and beryllium was studied over the range of 0.4 to 22 microns in a vacuum of $10^{-5}$ $\mathrm{mm} \mathrm{Hg}$ with specimen temperatures of 100 to $1300 \mathrm{~F}$. of the anodizing variables studied, the various anodizing solutions generally had the greatest effect on the reflectance. A number of surfaces investigated had high alpha/epsilon ratios, indicating usefulness as potential solar collector surfaces.

\section{\#6500464}

$\star 8.143-4.143$

Janssen, J.; Juck, J.; Torborg, R.

THERMAL REFLECTAŃCE OF ANÓDIZED METALS FOR SPACECRAFT

Aeronautical Systems Division, Wright-Patterson Air Force Base, Ohio

(No date)

$33 p$

Selection of materials having proper thermal radiation properties permits the engineer to achieve passive temperature control in spacecraft. The effect of anodizing variables on the reflectance of anodized aluminum and magnesium was studied over the range of 0.4 to 22 microns in vacuum of $10^{-5} \mathrm{~mm}$ of $\mathrm{Hg}$ with specimen temperatures of 100 to $825 \mathrm{~F}$. In general, the anodizing process had a greater effect on the reflectance than did alloying elements in the metal. The pressure of water in some coatings, which was driven off at elevated temperatures in the vacuum, gave lower reflectance in the infrared.

\# 6902030

Kennard, 0.; Watson, D. G.

CRYSTAL STRUCTURE LIBRARY, VOLUME I. BIBLIOGRAPHY OF ORGANIC COPIPOUNDS 1960' 1966

The Office for Scientific and Technical Information, British Data and Information Program, Cambridge, England

March 1968

$275 p$

This bibliography of organic crystal structures covers the period 1960-1966 and provides references to about 2,000 compounds whose structures were determined by $X$-ray or neutron diffraction. The bibliography forms part of a computer-oriented structural Library which contains reference material, numerical data including atomic coordinates and thermal parameters, and descriptive information. The bibliography includes compound name, author and journal references.

\section{\#6500399 *8.143-4.143}

Kolsky, H. G.

THE THERNODYNAYIC PROPERTIES OF 54 ELEMENTS CONSIDERED AS IDEAL MONATOMIC GASES

LA-2110, Los Alamos Scientific Laboracory, Los Alamos, New Mexico

March 15, 1957

$138 \mathrm{p}$

Available from CFSTI

Tables of the thermodynamic properties of fiftyfour elements have been calculated considering each as an ideal monatomic gas. The energy levels as determined by the National Bureau of Standards Spectroscopy Section were prepared on IBM cards for one of the LASL IBM 704 computers. The partition functions were calculated for each element and the thermodynamic properties were evaluated from them for 150 temperatures between $10^{\circ} \mathrm{K}$ and $8000^{\circ} \mathrm{K}$. A short table of the harmonic oscillator functions was also done in the same format as the other tables for use in evaluating the vibrational contributions to the thermorynamic properties. A brief discussion of the theory and calculational details is given.

\#6807001

Krishnan, R. S.

$* 4.243$

PROGRESS IN CRYSTAL PHYSICS, VOLUME I: THERMAL, ELASTIC AND OPTICAL PROPERTIES

Madras, India: S. Viswanathan

1958

$198 \mathrm{p}$

This volume contains data and a bibliography on the physical properties of crystals current as of December 1957, for general reference use in research on crystal physics. Seven authors review thermal expansion, thermal conductivity, elastic properties, photo-elastic properties, thérmo-optic behavior, Faraday Effect and dielectric behavior of crystals. Metal crystals and polycrystalline materials are excluded. Each chapter discusses the theory, experimental methods, measurements, and results. The available data on each property for single crystals have been collected and presented in a tabular form at the end of the chapter. 
$\# 6500528$

$*_{0.1151-2.2051-3.151-4.151}$

LANDOLT - BORNSTEIN, NUMERICAL DATA AND FUNCTIONAL RELATIONSHIPS IN PIYSICS, CHEMISTRY, ASTRONOMY, GEOPIIYSICS, AND TECHNOLOGY, SIXTH EDITION,

VOLUME I: ÁTONIC AND MOLECULAR PIYSICS,

PART 4: CRYSTALS

Hellwege, K. $-\mathrm{H}$. (Editor)

Berlin, New York: Springer-Verlag

1955

$1007 \mathrm{p}$

In German

This section contains data on the atomic physical interpretation of crystal physics as of November 1954. The following properties have been tabulated: the characteristics of symetry, elements, the 32 crystal classes, space groups, lattice types, structures, crystal dimensions of inorganic and organic crystals, atomic and ionic radii, Iattice energies, internal vibrations of crystals, electron emission, energy bonds, X-ray spectra and bonding states, electron spectra of crystals, and high frequency spectra of crystals.

\#6500530

$$
\text { *0.1151-4.151-8.151 }
$$

LANDOLT-BORNSTEIN, NUMERICAL DATA AND FUNCTIONAL RELATIONSHIPS IN PHYSICS, CIIEMISTRY, ASTRONOMY, GEOPHYSICS AND TECHNOLOGY, SIXTH EDITION,

VOLUME II: PROPERTIES OF MATTER IN ITS AGGREGATED STATES,

PART 2b: EQUILIBRIA EXCEPT FUSION EQUILIBRIA, EQUILIBRIA OF SOLUTIONS I

Schaefer, K.; Lax, E. (Edi tors)

Berlin, New York: Springer-Verlag

1962

$982 \mathrm{p}$

In German

This section of the Landolt-Bornstein tables contains information on solution equilibria of gaseous substances in liquids, solid and liquid metals and alloys, as well as the solubilities of solid and Iiquid substances in liquid solution media. Except for the section "gases in metals," only solution equilibria in the temperature region of about $-200^{\circ} \mathrm{C}$ to $300^{\circ} \mathrm{C}$ are presented. The data were taken from literature published through 1962. Solution equilibria are presented for gases with water, aqueous solutions, non-aqueous inorganic liquids, liquids and mixtures of organic and inorganic liquids. Also, solution equilibria of hydrogen, oxygen and nitrogen in pure metals are presented. Solution equilibria are presented for inorganic materials in water and other inorganic liquids, organic liquids, organic materials in water, and solutions with organic solvents.

\section{$\# 6500531$}

*0.1151-4.151-6.151-8.15I

LANDOLT-BORNSTEIN, NUMERICAL DATA AND FUNCT IONAL RELAT IONSIIIPS IN PIIYSICS, CHEMISTRY, ASTRONOMY, GEOPHYSICS AND TECHNOLOGY, SIXTH EDITIÓN,

VOLUME II: PROPERTIES OF MATTER IN ITS AGGREGATED STATES,

PART 3:

FUSION EQUILIBRIA AND INTERFACIAL

Schaefer, K.; Lax, E. (Edi tors)

Berlin, New York: Springer-Verlag

1956

$535 p$

In German

This section of the Landolt-Bornstein tables contains data on fusion equilibria and interfacial tensions. The section includes data from literature published through September 1955. Where several values for one measurement were found, the average was presented. Data on fusion equilibria are presented for binary and ternary systems of inorganic compounds, silicate systems, organic systems, and organic-inorganic systems. Interfacial tension data are presented for pure liquids towards their vapors or air, solutions towards air, two non-miscible systems, and interfacial films on water. Data are also presented on absorption from the gas phase and the liquid phase, and on paper chromatography.
\#6500533

*0.1151-4.151-8.151

LANDOLT-BORNSTEIN, NUMERICAL DATA AND FUNCTIONAL RELATIONSHIPS IN PHYSICS, CHEMISTRY, ASTRONOMY, GEOPHYSICS, AND TECHNOLOGY, SIXTH EDITION, VOLUME II: PROPERTIES OF MATTER IN ITS ÁGGREGATED STATES,

PART 6: ELECTRICAL PROPERTIES I

Hellwege, K.-H.; Hellwege, A.M. (Editors)

Berlin, New York: Springer-Verlag

1959

$1018 \mathrm{p}$

In German

This section of the Landolt-Bornstein tables contains data on the electrical properties of matter (except electrochenical systems) published through February 1959. The properties tabulated include the conductivities of pure metals and certain alloys at $0{ }^{\circ} \mathrm{C}$ and their temperature variations, resistance ratios, effect of magnetic fields on resistance, super-conducting metals and alloys and their transition temperature, magnetic field effects on super conductivity, galvanomagnetic and thermomagnetic effects, ionic conductivity in crystals, transfer numbers in solids. Also tabulated are the constants of homogeneous semiconductors; photoelectric conduction; piezoelectric equations; and the elastic, piezoelectric and dielectric constants of piezoelectric crystals; dielectric properties of inorganic and organic crystals; crystalline fluids and pure fluids; dielectric properties at high frequencies; temperature and pressure dependence of dielectric constants; and the dielectric properties of gases. Thermionic emission and work functions, thermopotentials, Peltier and Thomson heats, and photoemission data are also listed.

\section{\#6500536 *0.1151-4.151}

LANDOLT-BORNSTEIN, NUNERICAL DATA AND FUNCTIONAL RELATIONSIIIPS IN PHYSICS, CHEMISTRY, ASTRONOMY, GEOPHYSICS AND TECHNOLOGY, SIXTH EDITION, VOLUME II: PROPERTIES OF'MATTER IN ITS ÁGGREGATED

STATES, MAGNETIC PROPERTIES
PART 9:

Hellwege, K.-H.; llellwege, A.M. (Editors)

Berlin, New York: Springer-Verlag

1962

$900 \mathrm{p}$

In German

This section of the Landolt-Bornstein tables contains tables and curves of magnetic data for paramagnetic, ferromagnetic, ferrinagnetic and antiferromagnetic substances, taken from the literature published through September 1962. The following quantities have been tabulated: fundamental magnetic properties (dia- and paramagnetic susceptibility, spontaneous magnetization, and Curie temperatures) for metallic elements and alloys, exchange energy, crystal energy, magnetostriction, magnetocaloric effects, Faraday effect, magnetooptical effect, Kerr effect, gyromagnetic effects and resonance in ferromagnetic metals and alloys, properties of ferrospinels, magnetic garnets, perovskites and hexagonal ferrites, magnetic properties of transition element compounds, lanthanide series compounds and actinide series compounds, paramagnetic centers in crystals, paramagnetic ions as impurities, magnetic susceptibility, cyclotron resonance, de Has-Schubnikow effect and Faraday effect in semi-conductors, paramagnetic relaxation and nuclear magnetic relaxation. 

VOLUME II: PROPERTIES OF MATTER IN ITS AGGREGATED STATES,

PART 10: MAGNETIC PROPERTIES II

Hellwege, K.-H.; llellwege, A.M. (Editors)

Berlin, New York: Springer-Verlag

1967

$173 p$

In German

This section of the Landolt-Bornstein tables gives the paramagnetic and diamagnetic properties of substances. The compiler has listed one or more values for each substance, and marked with an asterisk those considered acceptable and reliable. These, and other selected values considered worthy of inclusion, are listed under "Ised References" and values far outside the others under "Further References." The values were taken from literature up to April 1966. Molar magnetic susceptibilities are listed for compounds, and magnetic mass susceptibilities for elements. Diamagnetic susceptibilities are listed for isotropic organic and inorganic substances, and principal susceptibilities for anisotropic substances. Paramagnetic susceptibilities are listed for organic compounds only.

\section{$\# 6500538$}

$* 0.1151-4.151-7.151-8.151$

LANDOLT-BORNSTEIN, NUMERICAI DATA AND FUNCTIONAL RELATIONSIIIPS IN PHYSICS, CHEMISTRY, ASTRONOMY, GEOPHYSICS AND TECHNOLOGY, SIXTH EDITION,

VOLUNE IV: TECIINOLOGY,

PART 1: MATERIAL VALIJES AND MECIHANICAL PROPERTIES OF NON-METALS

Schmidt, E. (Edi tor)

Berlin, New York: Springer-Verlag

1955

$881 \mathrm{p}$

In German

This section of the Landolt-Bornstein tables contains material values and mechanical behavior of non-metallic substances, and their technical applications. Data are compiled from literature published through autumn 1964. The following general information is included: measuring systems, units and conversion tables, atomic weights, reduction to standard states, densities of water and mercury. Physical and mechanical properties of non-metallic solids are given for natural and synthetic building materials, organic natural materials (wood, paper, cellulose, wood pulp) fibrous materials, ceramics, giasses, synthetic materials, and natural synthetic materials. Also presented are data on friction and rolling resistance of non-metallic and metallic substances, viscosity, flow through tubes, circuitous flow of bodies during two dimensional flow and rough flow, flotation, speed of sound, sound radiation and absorption, the human ear sound spectra, vibrational numhers, spatial and building acoustics, sound recording and hiological effects of ultrasonics.

\#6500539

*0.1151-3.151-4.151-7.151-8.151 LANDOLT-BORNSTEIN, NUMERICAL DATA AND FUNCTIONAL RELATIONSHIPS IN' PHYSICS, CIEMISTRY, ASTRONOMY, GEOPIIYSICS AND TECHNOLOGY, SIXTH EDITION,

VOLUNE IV: TECHNOLOGY

PART 2a: MATERIAL VALIJES AND PROPERTIES OF INDUSTRIAL METALS, BASIC PRINCIPLES, TESTING PROCESSES, INDUSTRIAL STEELS

Borchers, H.; Schmidt, E. (Editors)

Berlin, New York: Springer-Verlag

1963

$888 \mathrm{p}$

In German

This section of the Landolt-Bornstein tables includes data on metallographic principles and concepts, testing properties, and the properties of iron and its alloys. Data are given on diagrams of state, strength testing and destruction free materials. The physical properties of iron tabulated are lattice constants, density, thermal expansion, diffusion, atomic heats, specific heats, enthalpies, entropies and free enthalpies, phase diagrams and transition points, thermal conductivity, electrical properties, magnetic properties, optical constants and excitation crosssections. Composition, treatment, strength values and special chemical properties of pure iron, unalloyed steels, highly alloyed steels, special steels, and cast iron are presented.

\#6510029

$$
\text { *0.1151-4.151-7.151 }
$$

LANDOLT-BORNSTEIN, NUMERICAL DATA AND FUNCTIONAL RELATIONSHITS IN PHIYSICS, CILEMISTRY, ASTRONOMY, GEOPHYSICS AND TECINOLOGY, SIXTII EDITIÓN, VOLUME IV: TECHNOLOGY,

PART 2b: MATERIAL VALUES AND PROPERTIES OF INDUSTRIAL NETALS, SINTERED INDUSTRIAL MATERIALS, HEAVY METALS (TVITHOUT SPECIAL INDUSTRIAL MATERIALS) Borchers, H.; Schmidt, E. (Editors) Berlin, New York: Springer-Verlag 1964

$1000 \mathrm{p}$

In German

This section of the Landolt-Bornstein series deals with numerical data and behavior of both metallic and powdered industrial materials. The materials covered include the metals tungsten, tantalum, niobium, vanadium, chromium, cobalt, nicke1, manganese, gold, silver, copper, antimony, zinc, cadmium, lead, bismuth, tin, and their alloys. Data are provided for the physical and mechanical properties of these materials, their corrosion behavior and some of their applications to technology.

$\# 6605008$

$* 0.1151-4.151-7.151$

LANDOLT - BORNSTEIN, NUMERICAL DATA AND FUNCTIONAL RELATIONSHIPS IN PHYSICS, CHEMISTRY, ASTRONONY, GEOPIIYSICS AND TECHNOLOGY, SIXTH EDITION, VOLUME IV: TECHNOLOGY,

PART 2C: MATERIAL VALUES AND PROPERTIES OF INDUSTRIAL METALS, ALKALINE EARTHS, SPECIAL INDUSTRIAL MATERIALS, SEIIICONDUCTORS, CORROSION Borchers, H.; Schmidt, E. (Editors)

Berlin, New York: Springer-Verlag

1965

$976 \mathrm{p}$

In German

This section is the third part in a series of Landolt-Bornstein tables presenting the physical properties and behavior characteristics of most of the 75 elements in the periodic system exhibiting metallic characteristics as well as data concerning their alloys. Special emphasis is directed toward light metals, special work metals, binding materials, enamel properties, corrosive behaviors, reactor metals, metals of the rare earths, semiconductors, and current developments in binary alloy systems. The volume is divided into sections entitled: titanium, beryllium, aluminum, magnesium, lithium, rubidium, cesium, liquid metals, general survey of reactor metals, uranium, piutonium, zirconium, hafnium, thorium, rare earths, semiconductors, bearing metals, enamels, solder, welding and cutting metals, metal adhesives, and corrosive behavior of materiais.

\#6500540

*0.1151-3.151-4.151

LANDOLT-BORNSTEIN, NUNERICAL DATA AND FUICTIONAL RELATIONSHIPS IN PIIYSICS, CHEIISTRY, ASTRONONY, GEOPHYSICS AND TECHNOLOGY, SIXTII EDITION,

VOLUME IV: TECHNOLOGY

PART 3: ELECTROTECHNOLOGY, OPTICS, X-RAY TECHNOLOGY Schmidt, E. (Edi tor)

Berlin, New York: Springer-Verlag

1957

$1076 \mathrm{p}$

In German 
This section of the Landolt-Bornstein tables deals with the technological aspects of electricity, Iight and $X$-rays. It is compiled from the literature published through 1956. Wherever possible, the compilers have drawn, from several values available for a given quantity, the value they feel is most accurate. The properties tabulated in the electrical section are electrical conductivity, resistance and temperature coefficient of resistance; conductivity of molten metals; effect of pressure, tension, and cold machining on electrical resistance; resistance as functions of frequencies; resistance of thin layer semiconductors; thermoelectric emf of metal pairs and thermocouples in use; effects of pressure and cold treatment on thermocouples; conduction of electricity in gases, breakdown potentials of gases; insulating liquids, filling and impregnating media, fibrous material insulators, insulating foils, mica insulators, rubber insulators; resin, plastics, quartz, glass, ceramic materials and rocks as insulators; and magnetic materials. The quantities tabulated in the light section are: sensitivity of the human eye, illuminating materials, light sources, light filters and photographic films. The $X$-ray section includes data on measurements and units of X-ray technology.

\section{\#6804008 *0.1151-4.151-8.151}

LANDOLT-BORNSTEIN, NUMERI CAL DATA AND FUNCTIONAL RELATIONSHIPS IN PHYSICS, CHEMISTRY, ASTRONOMY, GEOPHYSICS AND TECHNOLOGY, SIXTH EDITIÓN,

VOLUME IV: TECHNOLOGY

PART 4a: HEAT TECHNOLOGY, HEAT TECHNOLOGY ENGINEERING MEASURING METHODS, THERMODYNAMIC PROPERTIES OF HOMOGENOUS MATERIALS

Haus en, H. (Edi tor)

Berlin, New York: Springer-Verlag

1967

$944 \mathrm{p}$

In German

This section of Landolt-Bornstein is a compilation of data dealing with the technology of thermal meas urements and the thermodynamic properties of gases, vapors, liquids, and solid materials. Data are included for such areas as the basis of temperature measurement; the temperature measurement with contact thermometers: optical temperature measurement (Pyrometry); hygrometry; thermodynamic properties of homogeneous materials, gases, water and steam, refrigerating agents; thermal expansion of solids and liquids; and the specific thermal properties of technically important materials.

$\because 6706020$

$\star 0.1151-3.151-4.151$

LANDOLT-BORNSTEIN, NUMERICAL DATA AND FUNCTIONAL RELATIONSHIPS IN SCIENCE AND TECHNOLOGY, NEW SERIES,

GROUP II: ATOMIC AND MOLECULAR PHYSICS,

VOLUME 2: MAGNETIC PROPERTIES OF COORDINATION AND ORGANO-METALLIC TRANSITION METAL COMPOUNDS

Koenig, E.

Hellwege, K.-H.; Hellwege, A.M. (Editors)

Berlin, New York: Springer-Verlag

1966

$578 \mathrm{p}$

In German and English

Magnetic properties are listed for coordination and organo-metallic transition metal compounds. The magnetic susceptibility section lists for each compound gram susceptibility and molar susceptibility at a particular temperature; magnetic moments and paramagnetic Curie temperatures, and the experimental method used to obtain data. The electron paramagnetic resonance section lists for each compound the spectroscopic splitting g-factor, fine structure and hyperfine structure parameters, half-width of the EPR line, and temperature and frequency of measurement.
\#6802001

*0.1151-4.151-8.151

LANDOLT-BORNSTEIN, NUMERICAL DATA AND FUNCTIONAL RELATIONSHIPS IN SCIENCE AND TECHNOLOGY, NEW SERIES

GROUP II. ATOMIC AND MOLECULAR PHYSICS,

VOLUME 5: MOLECULAR ACOUSTICS

Schaaffs, $w$.

Hellwege, K.-H.; Hellwege, A.M. (Editors)

Berlin, New York: Springer-Verlag

1967

$286 \mathrm{p}$

In German and English

This volume lists data on acoustics based essentialiy on the molecular properties of matter, and values of sound velocity, absorption coefficients, and dispersion, data. The measurements were made at frequencies ranging from $10 \mathrm{Kc}$ to $200 \mathrm{Mc}$. Preference was generally given in compiling data to measurements for which statements on density, chemical purity, and the temperature coefficient were added. Often, however, several statements can be found for a measured value. Generally, the limit of accuracy is $1 \%$ to $2 \%$. Data are given on sound velocity in gases and liquids. for pure gases and liquids as a function of temperature, pressure, and structure; also for mixtures of gases, mixtures of liquids, and solution. Data are compiled on sound absorption and dispersion in gases; sound absorption in homogeneous liquids, in solution, and on sound propagation in polymers, also on sound propagation in isotropic or quasi-isotropic solids (metals, inorganic non-metals, organic solids). There are special sections on sound propagation in liquid helium and shock wave velocity.

$\$ 6608030$

*0.1151-4.151

LANDOLT-BORNSTEIN, NUMERICAL DATA AND FUNCTIONAL RELATIONSHIPS IN SCIENCE AND TECHNOLOGY, NEW SERIES

GROUP III: CRYSTAL AND SOLID STATE PHYSICS,

VOLUME 1: ELASTIC, PIEZOELECTRIC, PIEZOOPTIC AND ELECTROOPTIC CONSTANTS OF CRYSTALS

Bechmann, R.; Hearmon, R.F.S.

Hellwege, K.-H.; Hellwege, A.M. (Editors)

Berlin, New York: Springer-Verlag

1966

$160 \mathrm{p}$

In German and English

This volume of the new Landolt-Bornstein series gives the elastic, piezoelectric, piezooptic, and electrooptic constants of crystals. The data included were taken from data published in the literature through 1965. For materials where one or two sets of measurements of a given constant were found, they are quoted as published; for more than two sets of measurements, the mean and coefficient of variation are given. For nonpiezoelectric constants, the elastic stiffness and compliance constants, together with their temperature and pressure variations, are given. For piezoelectric constants, the elastic, piezoelectric, dielectric, piezooptic, and electrooptic constants, plus the electromechanical coupling factors are given. The temperature coefficients of the elastic stiffness, compliance, piezoelectric stress and strain constants are also given.

$\$ 7001036$

*0.1151-4.151-8.151

LANDOLT-BORNSTEIN, NUNERICAL DATA AND FUNCTIONAL RELATIONSHIPS IN SCIENCE AND TECHNOLOGY, NEK SERIES

GROUP III: CRYSTAL AND SOLID STATE PHYSICS, VOLUME 2: ELASTIC, PIEZOELECTRIC, PIEZOOPTIC, ELECTROOPTIC CONSTANTS, AND NONLINEAR DIELECTRIC SUSCEPTIBILITIES OF CRYSTALS

Bechmann, R. ; Hearmon, R.F.S.; Kurtz, S.K.

Hellwe ge, $K_{0}-H_{0}$; Hellwege, A.M. (Editors)

Berlin, New York: Springer-Verlag

1969

$242 p$

In German and English 
Volume III/2 of the New Series of Landolt-Bornstein continues Volume III/1 only three years after publication of the 1atter. All the tables contained in III/1 are brought up to date by supplements in III/2. In addition, the elastic and electrooptic constants of higher order and the nonlinear optical susceptibilities have now been included. Readers should note that the Index of Substances at the end of III/2 refers to both volumes.

\#6511001 $*^{*} .133$

LOW-TEMPERATURE PHYSICS, PROCEEDINGS OF THE NBS SEMICENTENNIAL SYMPOSIUM ON LOW-TFMPERATURE PHYSICS HELD AT THE NBS ON MARCH 27, 28 AND 29, 1951

NBS Circular 519, National Bureau of Standards, Washington, D. C.

October 6, 1952

$29 \mathrm{lp}$

Available from Superintendent of Documents, GPO

The papers published in this book were presented at the NBS Semicentennial Symposium on Low-Temperature Physics and contain sixty-one articles representing scientific fields in which the Bureau is active. The book examines the superconductivity of various elements and isotopes and the low-temperature effects on atomic heat, resistance, specific heat, heat capacity, Eddy currents and supercurrents of elements. The book includes discussions of liquid properties of gases and the solidification of gases and gives optical and spectral data of the lowtemperature effects.

\section{\# 6500220}

Lyman, T. (Edi tor)

$* 4.153$

METALS HANDBOOK, VOLUME 1: PROPERTIES AND SELECTION OF METALS, EIGHTH EDITION

ASM Metals Handbook Committee, American Society for Metals, Metal Park, Ohio

1961

$1300 \mathrm{p}$

This book is a comprehensive monograph covering alloy steels, aluminum alloys, carbon steels, copper alloys, magnetically soft materials, permanent magnet materials, stainless steels and tool steels. Data are presented for magnetic electrical and special purpose materials nickei and nickel alloys, tin and tin alloys, zinc and zinc alloys, and precious metals. Physica property data are included.

\section{\#6611022}

Lyman, T. (Editor)

METALS HANDBOOK, VOLIME 2: HEAT TREATING, CLFANING AND FINISHING, EIGHTH EDITION

ASM Metals Handbook Committee, American Society for Metals, Metal Park, Ohio

1964

$708 \mathrm{p}$

This is the second in a series of five volumes that supersede and expand the 7 th edition of Metals Handbook. The aim has been to provide information helpful in selecting and controlling processes for heat treating, cleaning and finishing of ferrous and nonferrous metals. The material on heat treating is organized into eight principal sections, four of which deal largely with processes for heat treating carbon and low-alloy steels, and four of which deal with the heat treating of metals other than carbon and low-alloy steels. Articles on heat treating of stainless steel, heat-resisting alloys, and various nonferrous metais are included. The subject matter on cleaning and finishing is organized into seven principal sections, five of which deal with iron and steel, and two of which deal with other metals.
\# 6500418

* 4.113

LaGuillaume, $C$.; et al.

TABLES OF CONSTÁNTS AND NUNERICAL DATA, VOLUME SELECTED CONSTANTS RELATIVE TO SEMI CONDUCTORS

New York, Paris: Pergamon Press

1961

$76 \mathrm{p}$

In French, preface in French and English

This "Table of Selected Constants" 1ists constants and numerical data for materials which exhibit a semi-conducting character. It gives the semiconducting and physicochemical properties for germanium, silicon, tellurium, selenium, and diamond and for the I - VIII compounds. For germanium, silicon and the III-V compounds, extrinsic properties are also given (activation energy, diffusion coefficient and solubility of impurity elements). The numerical data have associated references to the original work from which they were taker. The physical quantities listed for each include symmetry group and crystal parameters, refractive index, dielectric constant, effective ionic charge, work function, photoemission work function, piezoresistance coefficient, elastic coefficients, Phonon and Debye temperatures, magnetic susceptibility, coefficient of linear expansion, fusion temperature, sublimation temperature, specific heat, latent heat of fusion and sublimation, thermal conductivity. disorder factor, and density. In addition to data specific to band structure, the Table also includes mobilities of electrons and their variation with temperature.

\#6609015

LISTS OF AVAILABLE AND DESIRED MATERIALS AND AVAILABLE CRYSTAL-GROWTH REFERENCES (ULTRAPURE INORGANIC RESEARCH MATERIALS)

ORNL-RMIC-5, Research Materials Information Center, Oak Ridge National Laboratory, Oak Ridge, Tennessee August 1966

$45 p$

Available from the CFSTI

The materials in the Available Materials list are preferably single crystals selected for highest purity and largest dimensions. Other forms (wires, powder, foils, etc.) and lower purities and smaller sizes are usually available. Those entries marked with an asterisk were produced in the course of research, and samples might or might not be available for loans or exchange. All others are commercially produced for sale. Purities and other characteristics are in most cases those claimed by the producer.

\#6902095

* 4.172

SUPPLEMENTAL LISTS OF AVAILABLE AND DESIRED MATERIALS AND AVAI LABLE CRYSTAL-GROWTH REFERENCES (ULTRAPURE INORGANIC RESEARCH MATERIALS)

ORNL-RMIC-6, Research Materials Information Center, Oak Ridge National Laboratory, Oak Ridre, Tennessee Augus $t 1967$

$18 \mathrm{p}$

Available from CFSTI

The materials in this list are single crystals selected for highest purity and largest dimensions. other forms (wires, powder, foils, etc.) and lower purities and smaller sizes are usually available. Those entries marked with an asterisk were produced in the course of research, and samples might or might not be available for loans or exchange. All others are commercially produced for sale. Purities and other characteristics are in most cases those claimed by the producer. This Iist is a supplement to the Available Materials List in ORNL-RMI C- 5 . 
$\$ 6704026$

* 4.253

MeClintock, F. A.; Argon, A. S. (Editors)

MECHANICAL BEHAVIOR OF MÁTERIALS

Reading, Massachusetts: Addison-Wesley Publishing Company, Inc.

1966

$770 \mathrm{p}$

Review of various kinds of mechanical behavior is presented, including theory of stress, strain and elasticity; theory of dislocations; deformation of polymers; constitutive relations, tensile and compressive deformation, bending and torsion, approximate stress analysis, stress and strain concentrations, residual stress; applications including modes of fracture, hardness, damping, creep, friction, and wear and composite materials. Data are compiled concerning creep, damping, elongation, fatigue, fracture (under increasing load), hardness, modulus of shear and elasticity, reduction of area at fracture stress and strain curves, tensile strength, and yield strength. Properties are listed for metallic elements, alloys and certain othur materials.

\#6603023

*0.2573-4.173

Michaelis, R.E.: Wyman, L.L.; Fletsch R.

STANDARD REFERENCE MATERIALS: PREPARÁTION OF NBS COPPER-BASE SPECTROCHEMICAL STANDARDS

NBS Miscellaneous Publication 260-2, National Bureau of Standards, Washington, D.C.

Oct ober 15,1964

$36 \mathrm{p}$

Available from Superingendent of Documents, GPO

A procedure has been developed for the preparation of copper alloys in both the chill-cast and wrought forms, sufficiently uniform in composition and microstructure for calibration and use as standard samples in spectrochemical analysis. The method involves casting the molten metal on a massive water-cooled copper plate producing unidirectional cooling. A large disk casting 27 in. in diameter and about 4 in. high is obtained which is split laterally into two pieces, the lower section providing standards in the chill-cast condition and the upper section material for subsequent fabrication into standards for the wrought condition. Details of the planning, preparation and testing of the standards material for 21 compositions are given, and application of the samples in calibration for optical and $x$-ray spectrochemical analysis is described.

$\# 6500423$

$* 4.153$

Mi lek, J. T .

ALUMINUM OXIDE DATA SHEETS

DS-136, Electronic Properties Information Center, Hughes Aircraft Company, Culver City, California March 1964

$161 \mathrm{p}$

A compilation of the electrical properties of a wide range (80-100\%) aluminum oxide ceramic composition is presented herein. Included are sapphire, sintered single crystal and polycrystalline material. Detailed electrical properties for the individual alumina material data sheet include dielectric constant, dielectric strength, dissipation factor, electrical conductivity, electrical resistivity, loss factor and $\mathrm{Te}$ value. Each property is compiled over the widest possible range of temperatures and frequencies from references obtained in a thorough literature search. The crystal structure, applications and phase diagrams are briefly reviewed.

\#6500424

Mi lek, J. T.

4.153

BOROSILICATE GLASSES DATA SHEETS

DS-138, Electronic Properties Information Center, Hughes Aircraft Company, Culver City, California June 1964

$115 \mathrm{p}$
A compilation of the electrical properties of various borosilicate glasses is presented. Arrangement of the data sheets are by corning code or number with a Master ldentification Chart relating the known chemical composition of each glass. Detailed electrical properties include corona effects, dielectric constant, dielectric strength, dissipation factor, 105 factor, power factor, surface and volume resistivity. Each property is compiled over the widest possible range of temperature and frequencies from references obtained in a thorough literature search. The structure, applications and phase diagrams are briefly reviewed.

\#6809023

Milek, J. T.

EPITAXIAL SILICON AND GALLIUM ARSENIDE THIN FILMS ON INSULATING CERAMIC SUBSTRATES, A STATE-OF-THEART REPORT

S-9, Electronic Properties Information Center, Hughes Aircraft Company, Culver City, California August 1968

1460

Available from DDC, AD 675578

A state-of-the-art literature survey is presented on the success of epitaxial deposition of silicon and gallium arsenide thin films on ceramic substrates for device applications. The electrical, thermal, chemical and crystallographic properties of sapphire, spinel, beryllium oxide, magnesium oxide, diamond, quartz, silicon carbide, aluminum silicate glass, glazed ceramic and other miscellaneous ceramic substrate materials are presented. A master flow chart is developed to identify each variable at each stage of the film/substrate preparation and resultant device fabrication processes. A series of charts identifying each of these variables as reported in the literature for each substrate material is presented. A complete bibliographic review of silicon epitaxial deposition, independent of substrate, is also presented.

\#6500435

Milek, J. T

FORSTERITE DATA SHEETS

DS-129, Electronic Properties Information Center, Hughes Aircraft Company, Culver City, California Augus t 1963

$28 \mathrm{p}$

This report contains a compilation of the insulation properties of Forsterite (2 MgO.SiO,). This information was taken from literature published through August 1963. The following quantities are tabulated: dielectric constant, dielectric strength, electrical resistivity, loss factor, power factor and $\mathrm{T}_{e}$ value.

$\# 6500434$

$* 4.153$

Milek, J. T

PYROCERAM DATA SHEETS

DS-130, Electronic Properties Information Center, Hughes Aircraft Company, Culver City, California Augus t 1963

$37 \mathrm{p}$

This report contains a compilation of the insulating properties of Pyroceram, a new family of glass materials developed by Corning Glass Works. Seven types of Pyroceram are discussed; of these however, only two were commercially available as of August 1963. The properties tabulated include dielectric constant, dielectric strength, dissipation factor, loss factor and volume resistivity. 
Milek, J.T.

SULFUR HEXAFLUORIDE DATA SHEETS

DS-140, Electronic Properties Information Center, Hughes Aircraft Company, Culver City, California October 1964

$68 \mathrm{p}$

A compilation of the electrical properties of sulfur hexafluoride, a dielectric gas, is presented. Electrical properties include corona, dielectric constant, dissipation factor and dielectric strength. The latter property data section is segregated into parameter effects as follows: pressure, gap distance, temperature, electrode configurations and gas mixtures. Each property is compiled over the widest possible range of parameters obtained in a thorough search of the world's literature.

\#6500430

$\star 4.153$

Mi lek, J.T

A SURVEY MATERIALS REPORT ON TFTRAFLUOROETHYLENE (TFE) PLASTICS

S-3, Electronic Properties Information Center, Hughes Aircraft Company, Culver City, California

September 1964

$104 \mathrm{p}$

A survey of the following properties of tetrafluoroethylene plastics was made: mechanical, electrical, thermal, chemical and irradiation behavior. Each property set of data represents the greatest range of parameters: temperature, frequency, pressure, voltage and environmental conditions (vacuum, radiation, etc.) found in the literature. The effects of fillers on every property are also detailed where such data were available. Applications such as space, mechanical, electrical and cryogenic, are treated in some detail for the various fabricated forms of tetrafluoroethylene. A brief discussion of forming and fabrication, joining and fastening methods, and sources of specifications to aid the designer in selecting tetrafluoroethylene plastics for various applications is presented.

\#6706013 *4. $153-8,153$

Milek, J. T.; Welles, S. J.

BORON

DS-151. Electronic Properties Information Center, Hughes Aircraft Company, Culver City, California 1967

$251 \mathrm{p}$

A state-of-the-art survey and a data sheet compilation of elemental boron are presented. The thorough literature search uncovered 304 references which were evaluated for information and data on the (1) reduction preparation methods, (2) zonerefining and melting techniques, (3) crystal structures, (4) electrical properties, (5) optical properties, (6) thermal and thermodynamic properties, (7) physical and chemical properties, (8) metailurgy and mechanical properties, (9) applications, and (10) availability and suppliers. Major emphasis was the characterization of boron's semiconductive and acoustic properties with respect to potential semiconductor and delay-line devices. Detailed information on the various methods of preparing pure boron and the resulting crystal structures plus properties are summarized in tables.

\#6905016 *4.153-8.153

Milek, J. T.; Welles, S. J.

CHEMICAL COMPOSITION' AND ELECTRICAL RESISTIVITY OF ALUMINUM ALLOYS

DS-161, Electronic Properties Information Center, Hughes Aircraft Company, Culver City, California April 1969

$27 \mathrm{p}$

All commercially available aluminum alloys are identified by means of their standard designations.
A11 available chemical composition and electrical resistivity data for these alloys are presented in tabular form for each alloy class. Electrical resistivity data for pure aluminum are reviewed.

\#6500268

$\star 4.353$

Miller, R. L.

CRYSTALLOGRAPHIC DATA FOR VARIOUS POLYMERS IV.

Chemstrand Research Center, Inc., Durham, North Carolina

May 1963

$53 \mathrm{p}$

This report contains data on the crystallographic properties of various polymers. The data for the polymers were taken from examination of the literature and entered onto IBM cards which were printed to yield the report. For each polymer, the following data are included: name of the polymer, molecular weight of the monomer from which it is formed: crystal system and space group; unit cell lattice parameters and angles; number of monomer units per unit cel1; density in crystal and amorphous states; reported melting points in ${ }^{\circ} \mathrm{C}$, and heats of fusion per monomer unit; chain conformation and literature references.

A 6902008

$* 4.153-8.153-3.153$

Muetterties, E. L.

THE CHEMISTRY OF BORON AND ITS COMPOUNDS

New York: John Wiley \& Sons, Inc.

1967

$699 \mathrm{p}$

This is a text on the properties of boron and boron compounds containing ten chapters, each written by experts in the different phases of boron chemistry. The chapters discuss the element boron, compounds of high boron content, borates, horic acid, boron hydrides and halides, boron-nitrogen, carbon, phosphorus compounds, and sulfur and selenium compounds of boron. Each chapter contains a large listing of references as well as tables of the chemical properties of boron compounds.

\#6706030

* $4.153-3.153-8.153$

Muir, A. H. Jr. Ando, K. J.; Coogan, H. M. MOSSBAUER EFFECT DATA INDEX 1958-1965

New York: John Wiley \& Sons, Inc.

1966

$351 \mathrm{p}$

The Mossbauer Effect Data Index is an organized index to experimental research results relating to the Mossbauer Effect published through 1965. The index groups experiments first by nuclear transition (daughter isotope and gamma-ray energy), then by source material or host, and then by absorber (or scatter) material or host. Each experiment is described and summarized on an 80 column IBM card. The data listed for each experiment are: Mossbauer isotope and gamma-ray transition energy, host material in which the source atoms are imbedded, source temperature, absorber, absorber temperature, line width, dip of the spectrum (percentage change in counting rate from on-resonance to off-resonance), shift of the absorption spectrum, quadrupole splitting energy (for transitions between spin $3 / 2$ and $1 / 2$ levels), shape of the absorption spectrum and a code for the bibliography reference. A summary sheet is presented for each isotope presenting simplified decay scheme gamma-ray and x-ray energies, halflife and total internal conversion coefficient of the Mossbauer transition, natural isotopic abundance, and magnetic and quadrupole moments. There is an index to references by topic and author. 
$\# 6500437$

* 4.153

Neuberger, $\mathrm{N}$.

CADMIUN SÉLENIDE DATA SHEETS

DS- 134, Electronic Properties Information Center, Hughes Aircraft Company, Culver City, California November 1963

$54 \mathrm{p}$

This report contains a compilation from literature puhlished through November 1963 of the semiconductor properties of cadmium selenide. The properties of cadmium selenide considered are: absorption, cross-sections, Debye temperature, dielectric constant, effective mass, electrical conductivity, electrical resistivity, energy bands, energy gap, energy levels, Hall coefficient, irradiation effects, mobility, photoelectronic properties, photon luninescence, diezoelectric properties, reflection coefficient, refractive index, thermal conductivity and thermoelectric properties.

\#6706011

* 4.153

Neuberger, $M$.

CADMIIUM SULFIDE

DS-124/2E, Electronic Properties lnformation Center, Hughes Aircraft Company, Culver City, California

March 1967

$242 \mathrm{p}$

Available from DDC

These data sheets present a compilation of a wide range of electronic properties for cadmium sulfide. Electrical properties include conductivity, dielectric constant, Ilall coefficient and mobility. Emission data have been hroken down into the varied electron and photon emissions which result from application of electromagnetic energy over a wide spectrum and a wide variety of photoelectronic phenomena is shown. Energy data include energy bands, energy gap and energy levels as well as effective mass tables and work function. The optical properties include ahsorption, reflection and refractive index. Magnetic data are presented, as we 11 as several other physical phenomena, such as Debye temperature. Thermoelectric and thermomagnetic properties are shown. Each property is compiled over the widest possible range of parameters including bulk and film. form from references obtained in a thorough literature search. A sumary of crystal structure and phase transitions has been included.

\section{$\# 6500436$}

$* 4.153$

Neuberger, $M$.

CADMIIIM TELLURIDE DATA SIIEETS

DS-101, Electronic Properties Information Center, Hughes Ai rcraft Company, Culver City, California June 1962

$49 \mathrm{p}$

This report is a compilation of the semiconductor properties of cadmium telluride, taken from literature published through June 1962. The quantities tabulated include absorption, Debye temperature, dielectric constant, effective mass, electrical conductivity, emission, energy hands, energy gap, Hall coefficient, irradiation effects, magnetoelectric properties, mobility, photoelectronic properties, reflection, refractive index, resistivity and thermoelectric properties.

\section{$\# 6903043$}

* 4.153

Neuberger, $M$.

CADMIUM TELLURIDE AND THE CADMIUM TELLURIDE-MERCURY TELLURIDE SYSTEM

DS-157, Electronic Properties Information Center, Hughes Aircraft Company, Culver City, California August 1967

$200 \mathrm{p}$

Available from the DDC

These data sheets present a compilation over a wide range of electronic properties for cadmium telluride. These properties are compiled over the widest possible range of parameters and are then agglomerated in several large groups as follows: optical properties include absorption, reflection and refraction. Transport properties include electrical conductivity, Jall coefficient, mobility, effective mass, lifetime and magnetoresistance. Energy band structure includes energy gap and levels, as well as work function values. Phonon branch distribution appears separately. Both photon and electron emission data are represented. Thermal properties include nebye temperature, thermal conductivity, and thermal enf. There are other individual properties and effects included, such as dielectric constant, piezoelectric values, irradiation effects and magnetic susceptibility. A large number of graphs illustrate the various photoelectronic phenomena. A section on general properties includes crystal, phase diagrams, vapor-pressure data, and preparation of single crystals and films. A table of the best values available for all physical and electronic properties is given. A separate section is given for the cadmium telluride-mercury telluride system and device applications for both the cadrium telluride crystals and the mixed crystals are appended.

\section{$\# 6706010$}

$* 4.153-8.153$

Neuberger, $M$.

DIAMOND

DS-153, E1ectronic Properties Information Center, llughes Aircraft Company, Culver City, California

1967

$146 \mathrm{p}$

Tables of electrical properties of diamond are presented as follows: conductivity, resistivity, dielectric constant, llall coefficient mobility and thermoelectric power. Emission data are shown as well as a wide variety of irradiation effects. Energy data given are energy bands, energy gap, energy levels, effective mass tables, lifetimes anc phonon energies. Optical properties included are absorption, reflection, refractive index, magnetoelectric properties, Debye temperature and thermal conductivity. A summary of crystal structure, phase transitions, and diamond types is included.

\# 6604012

* 4.153

Neuberger, $M$

GERMANIUM, DATA SHEETS

DS-143, Electronic Properties Information Center, Hughes Aircraft Company, Culver City, California February 1965

$236 \mathrm{p}$

These data sheets present a compilation of a wide range of electrical, optical and energy values for pure and variously-doped germanium in hulk and film form. Electrical properties include conductivity, resistivity, dielectric constant, Hall coefficient, mobility, lifetime, thermoelectric and thermomagnetic effects. Emission data has been broken down into the varied electron and photon emissions which result from application of energy in the electromagnetic spectrum. Energy data include energy bands, energy gap and energy levels for variously-doped germanium, as well as, effective mass tables, work function, carrier diffusion and cross sections. The optical properties include absorption, reflection and refractive index. Other magnetic data and irradiation effects are included, as well as several bordering physical phenomena, such as piezoelectric properties, Debye temperature and thermal conductivity. Each property is compiled over the widest possible range of parameters from references obtained in a thorough literature search. A crystallographic sumary has been added. 
DS-155, Electronic Properties Information Center, llughes Aircraft Company, Culver City, California May 1967

$7 \mathrm{lp}$

These data sheets present a compilation of a wide range of electronic properties for lead oxide. Electrical properties include conductivity, resistivity and dielectric constant. A wide variety of photoelectronic phenomena are shown. Energy data include energy bands, energy gap and energy levels, as well as effective mass tables, phonon energy dispersion and work functions. The optical properties include ahsorption, reflection and refractive index. Data on several physical phenomena, such as thermal conductivity, Debye temperature, magnetic susceptibility and Richardson's Constant are presented. Thermoelectric data is given. Each property is compiled over the widest possible range of parameters including bulk and film form, from references obtained in a thorough literature search. A sumary of crystal structure and phase transitions has been included.

$\# 6500433$

* 4.153

Neuberger, $M$

MAGNESIUM' STANNIDE

DS-114, Electronic Properties Information Center, Hughes Aircraft Company, Culver City, California October 1962

$23 \mathrm{p}$

This report contains data sheets on magnesium stannide compiled from the literature, giving values for absorption, effective mass, electrical conductivity, energy gap, energy level, Jall coefficient, magnetoelectric properties, magnetic susceptibility, mobility, photoelectronic properties, resistivity, the rmal conductivity and thermoelectric properties. This compilation deals only with magnesium stannide as a semiconductor. A list of references to the literature is included. Data are given graphically and in tabular form.

$\# 6912052$ $* 4.153$

Neuberger, $M$.

II-VI SEMICONDUCTING COMPOUNDS DATA TABLES

S-11, Electronic Properties Information Center, Hughes Aircraft Company, Culver City, California

October 1969

$156 \mathrm{p}$

Available fron $D D C$

These tables include the most reliable information available to date for every property .. mechanical, crystallographic, physical, thermal, magnetic, electronic and optical .. of each of the 2-6 binary semiconducting compounds.

\section{\#6907033}

Neuberger, $M$

SILICON CARBIDE DATA TABLE AND SUPPLEMENTARY BI BLIOGRAPIIY

DS- 145 (S-1), Interim Report No, 62, Electronic Properties Information Center, llughes Aircraft Company, Culver City, California

August 1968

$46 \mathrm{p}$

This interim report represents a supplement to the Electronic Properties Information Center Data Sheets on Silicon Carbide, published in June 1955 (DS- 145 by $M$. Neuberger, AD 465161 ). Almost 100 new documents concerned with silicon carbide are 1isted. Selected graphs and/or tables are presented from those articles containing data of particular interest. A data table of physical and electronic property values has heen added to allow for a rapid review of the important properties of the alpha- and heta- crystalline forms of silicon carbide.
DIIEETS

DS-133, Electronic Properties Information Center, Ilughes Aircraft Company, Culver City, California October 1963

$44 \mathrm{p}$

This report is a compilation of the semi-conductor properties of zinc oxide. The information was compiled from literature through october 1963. The report contains data on: absorption, Dehye temperature, dielectric constant, effective mass, electrical conductivity, electrical resistivity, energy bands, energy gap, energy levels, Hall coefficient, irradiation effects, lifetime, magnetoelectric properties, mobility, photoelectronic properties, photon luminescence, photon thermoluminescence, piezoelectric properties, reflection coefficient, thermal conductivity, thermoelectric properties, and work function.

\#6500439 * 4.153

Neuberger, $M$.

2INC SELENIDE DATA SHEETS

DS-132, Electronic Properties Information Center, IJughes Aircraft Company, Culver City, California September 1963

$26 \mathrm{p}$

This report is a compilation of the semi-conductor properties of zinc selenide. The information was compiled from literature published through September 1963. The report contains data on: absorption, Debye temperature, dielectric constant, effective mas, electrical conductivity, electrical resistivity, elactroacoustic properties, energy bands, energy gap, energy levels, mobility, photoelectronic properties, photon electroluminescence, piezoelectric properties, reflectivity, refractivity, refractive index and thermal conductivity:

\#6912050 *4.153

Neuberger, M.; Carter, D. B. MAGNESI UM OXIDE

DS-163, Electronic Properties Information Center, Ilughes Aircraft Company, Culver City, California October 1969

$99 \mathrm{p}$

Available from DDC

A wide range of electrical and optical properties are covered in these data sheets on magnesium oxide. Particular emphas is has been placed on the dielectric properties and the energy band structure. A data tahle containing the best available information on the crystallographic, thermal, mechanical, physical, electronic and photoemissive properties is included.

\#6500440 *4.153

Neuberger, M.; Grigsby, D. L.

ZINC SULFIDE DATA SHEETS

DS-135, Electronic properties Information Center, Hughes Aircraft Company, Culver City, California December 1963

$72 \mathrm{p}$

This report contains a compilation of the seniconductor properties of zinc sulfide. The information was compiled from literature published through December 1963. The report contains data on: absorption, cross sections, Dehye temperature, dielectric constant effective mass, electrical conductivity, electrical resistivity, energy bands, energy gap, energy levels, irradiation properties, lifetime, magnetic susceptihility, mobility, photoelectronic properties, photon electroluminescence, piezoelectric properties, reflection coefficient and refractive index. 
\# 5912051

* 4.153

Neuberger, M.: ivelles, S. T.

SILICON

DS-162, Electronic Properties Information Center, Ilughes Aireraft Company, Culver City, California

October 1969

$264 p$

Availlable from DDC

These data sheets present a compilation of a wide range of electronic properties for silicon. The energy hand structure is thoroughly reviewed and included are effective mass, solubility and diffusion with resulting values for energy levels and the dielectric constant. Electrical properties include mobility, resistivity, lifetime and piezoresistance. The various thermal and nagnetic properties such as Dehye temperature, thermal conductivity, phonon dispersion and the Seeheck, Nernst and Ettingshausen coefficients are reported.' The g-factor, magnetic susceptibility and electron spin resonance are given. The optical properties, absorption, refractive index and reflectivity are reported as well as both photon and electron emission, including work function data. Each property is compiled over the widest possible range of parameters, including bulk and film samples. $\Lambda$ data table containing a wide range of mechanical, physical and thermal properties is included, as well as, a sumary of crystal structure and phase transitions. There is also a general discussion of silicon technology.

$\# 6708006$

*4.1253-2,15:53

NUCLEAR SCIENCE AND TECINOLOGY FOR

PROCEEDINGS OF A SYMPOSIUM, APRIL 7-12, 1966

NBS Miscellaneous Publication 285, National Bureau of Standards, Washington, D. C.

May 26, 1967

$75 \mathrm{p}$

328

Available from the Superintendent of Documents, GPO

The purpose of this symposium was to provide an introductory survey of those properties involved in the choice and use of materials. In the first chapter, Dr. C. O. Mueh 1hause describes the radiation fields present in reactors and the features of these fields most important to the development of nuclear materials, touching upon the principal physical effects induced in materials by the radiation. A. B. Lidiard, in the second chapter, discusses the solid state physics of radiation damage processes, with examples drawn partiy from ceramics. The effects these processes have on physical properties are then described by P. Levy. Turning to chenical problens, R. J. Thorn and $G$. H. Wins low discuss the the rmodynamics of non-stoichiometry, a particularly significant problem encountered with nuclear fuels. Finally, in the 1 ast two chapters, N. W. Readey and J. H, Handwerk, and D. R. dellalas, W. C. Morgan, and $M$. D. Freshley take up the problems encountered in the development of nuclear fuels on the one hand, and structural and moderator materials on the other, thus displaying the applications of the basic ideas presented in the first four chapters.

\#6500281-6500283

*4.163

Palache, C.; Berman, H.; Frondel, C.

THE SYSTEM ÓF MINERALOGY OF JAMES DIVIGITT DANA AND EDWARD D. SALISBURY DANA, YALE UNIVERSITY 18371892, SEVENTII EDITION

VOLUME I: ELEMENTS, SULFIDES, SULFOSALTS, OXIDES;

VOLUME II: HALIDFS, NITRATES, BORATES, CARBONATES, SULFATES, PIIOSPHÁTES, ARSENATES, TUNGSTATES, MOLYBDATÉS, ETC.

VOLUNE III: SILICA MINERALS

New York: John Wiley \& Sons, Inc.

$163,1963,1962$

$84 \mathrm{p}, 1124 \mathrm{p}, 334 \mathrm{p}$

These three volumes are collections of mineralogical data, the latest revision of a collection dating from 1837 ; the last edition was
completed in 1892. The data were taken from earlier editions of the work, articles in mineralogical, chenical and physical journals, and independent books. Volume I $(6500281)$ contains an introduction to the three volumes and data on rative elements and sulfide, sulfosalt and oxide minerals; Volume II $(6500282)$ contains data on halides, ritrates, borates, sulfates, phosphates, arsenates, tungstates, and molybdates. Volume III $(6500283)$ is by C. Frondel only. It contains information on, and a discussion of silica materials. The mineral data sections are arranged by the elemental composition of the minerals. The following properties are listed for each material: crystal structure and class space group, axial ratios and angles, structure of unit cell, crystal habits, cleavage, hardness, specific gravity and color. Also, chenical analyses of specimens are presented, and data are provided on natural occurrence, artificial preparation of the mineral and the origin of its name.

$\# 6500252$

$* 4.163-3.163$

Pearson, W. B.

A HANDBOOK OF LATTICE SPACINGS AND STRUCTURES OF METALS AND ALLOYS

New York: Pergamon press, NRC-430

1.958

$1044 p$

This Handbook contains data on lattice spacings and structures of metals and alloys, and the information that can be determined from these quantities. The author presents data for elenents and binary and ternary alloys, taken from literature published through the end of 1956. The applications considered include applications to equilibrium diagrams, magnetic properties and alloy formation theory. The data includes crystallographic data on "Strukturbericht" types, tabulated lattice spacings, structure types, density of the elements and intermediate phases of alloy systems, and a review of work done on metals and alloys, including graphs of lattice spacings as functions of composition.

\#6906010

$* 4.163-3.163$

Pearson, W. B.

A IIANDBOOK OF LATTICE SPACINGS AND STRUCTURES METALS AND ALLOYS, VOLUME 2

New York: Pergamon Press, NRC No, 8752

167

$1446 p$

This second volume of Lattice Spacings
Structures of Metals and Alloyspresents data Structures of Metals and Alloys presents data for literature published through June of 1965 , The applications considered include equilibrium diagrams, magnetic properties and alloy formation theory. The data include crystallographic data, tabulated lattice spacings, structure types, density of the elements and intermediate phases of alloy systems, and a review of work done on metals and alloys, including graphs of lattice spacings as functions of composition. This volume seeks to present only the best data for X-ray methods, alloy purity and exact knowledge of composition, Brief preparative details and notes on the method of $X$ ray examination accompany descriptions of X-ray data on alloy phases.

\#6907016

$* 4.163-8,163$

Pomerance, $\mathrm{H}$.

BIBLIOGRAPIIY OF SECOND AND THIRD ORDER ELASTIC CONSTANTS

ORNL-RMIC-9, Research Materials Information Center, Oak Ridge Nátional Laboratory, Oak Ridge, Tennessee December 1968

$27 \mathrm{p}$

Available from CFSTI

This bibliography of second and third order elastic constants of monocrystalline materials was compiled from the files of the Research Materials Information Center and from Physics Abstracts. The 
literature since 1964 has been surveyed. The references are arranged first by the year of publication, next by the first author's initial, last by a serial number. The tables do not show numerical values. The first table is the second order elastic constants, the second table is the third order constants; in each the substances are arranged alphabetically by chemical symbol. In the first table, the second column shows the crystalline form where it is not cubic. The third column shows the temperature range of the measurements, the pressure range or other parameters. Where the full set of constants is reported, the letters $c$ and $s$ do not appear, but incomplete sets of derivatives are noted. The fourth colum shows the format, whether the data are graphical or tabular or by algebraic formula. The last column is the reference number.

$\# 6500253-6500257,6603002-6603003 * 4.263-3.263$ porter, II. W.; Spiller, R. C. (Volumes 1 and 2) porter, H. W.; Codd, L. W. (Volume 3)

THE BARKER INDEX OF CRYSTALS, A METHOD FOR THE IDENT IF ICAT ION OF CRYSTALLINE' SUBSTANCES, VOLIME I: PARTS 1 AND 2; VOLUTE II: PARTS 1, 2 AND 3; VOLUM I I : PARTS 1 AND 2

Cambridge, England: W. lieffer \& Sons, Ltd.

$1951-1964$

$300 p, 1000 p ; 383 p, 600 p, 500 p ; 375 p, 400 p$

The Barker Index of Crystalsprovides a means of identification of crystals through the reflection of light from their faces, as well as a source of structural data for known crystals. Volume I, Part 1 contains an introduction to and explanation of the Barker Index, and is arranged by Barker classification angle, by refractive index, by densities, and alphabetically by chemical name. The Barker Index is based on the angles between the normals to the major planes and also indices to tetragonal, hexagonal, trigonal and orthorhombic crystals. Volume I, Part 2 contains the following data for tetragonal, hexagonal, trigonal and orthorhombic crystals: classification angles, axial ratios, interfacial angles; symmetry; physical properties, such as cleavage, specific gravity, color and melting point; indices of refraction and strong lines in the X-ray powder diffraction spectrum. Volume II, Part 1 contains indexes for monoclinic crystals arranged by classification angle, density and specific gravity; part 2 and 3 contain monoclinic crystal data of the types included in Volume I. Volume III, Parts 1 and contain sinilar information for triclinic crystals.

\# 6500419

*8.163-4.163

Powe 11, R. L.; Blanpied, W. A.

THERMAL CONDUCTIVITY OF METALS AND ALLOYS AT LOW

TEMPERATURES, A REVIEW OF THE LITFRATURE

NBS Circular 556, National Bureau of Standards, Washington, D. C.

September 1, 1954

$68 \mathrm{p}$

Available from the Superintendent of Documents, GPO

An extensive compilation is given of the measured values of thermal conductivity for metals and alloys from room temperature down to approximately $0^{\circ} \mathrm{K}$. The more extensive and important data are plotted in 48 graphs. The tables and graphs for the metalic elements and alloys are essentialiy complete for literature reference from 1900 to early 1954. For comparison, several graphs and tables are given for some representative dielectrics.

\section{\#6500494} *8.163-4.163

Powe 11, R. L.; Roder, H. M.; Hal1, W. J. LOW-TEMPERATURE TRANSPORT PROPERTÍES OF COPPFR AND ITS DILUTE ALLOYS: PURE COPPER, ANNEALED AND COLDDRAWN

Physical Review, $115: 314-323$

Ju1y 15, T959
Experimental results between $4^{\circ}$ and $300^{\circ} \mathrm{K}$ are given for (1), the themal conductivity, electrical resistivity, and thermoelectric force and power of two high-purity coppers, one annealed and one colddrawn 26\%; and (2), the electrical resistivity of a series of seven samples of high-purity copper colddrawn between $0 \%$ and $20 \%$ elongation. The total electronic thermal resistivities each consist of three terms: the intrinsic resistivity, $W_{*}$; the imperfection resistivity, W; and a deviation term, Wig! indicating the departure from strict additivity of $W_{i}$ and $W_{0}$. The intrinsic thermal resistivity and intrinsic electrical resistivity vary as $T^{2} \cdot B$ and $T^{7} .5$, respectively, contrary to the predictions of the usual transport theory using: Bloch approximations and assumptions. The resistivity of pure copper is $1.545 \mathrm{ohm} \mathrm{cm}$ at $0^{\circ} \mathrm{C}$. The increase in imperfection electrical resistivity is approxinately linear with increase in cold-drawn elongation. Ilowever, the added resistivity is not independent of temperature (Matthiessen's rule), but about twice as great at the ice point as it is at $4^{\circ} \mathrm{K}$. The change in thermoelectric power with drawing is positive at the lower temperatures, but negative above $38^{\circ} \mathrm{K}$. The Lorenz number does not approach the Somnerfeld value at the lowest temperatures, but flattens out to a value considerahly smaller. A discussion of each of the various effects is provided.

\section{\#6809018 $* 8.163-4.163$}

Powe 11, R. I. ; Ho, C. Y.; Liley, P. E.

THERMAL CONDUCTIVITY OF SELECTED MATERIALS

NSRDS-NBS-8, National Bureau of Standards, Washington, D. C.

November 25,1966

$168 \mathrm{p}$

Avallable from the Superintendent of Documents, GPO

This compilation consists of the critical evaluation and analysis of the available thermal conductivity data on eleven metals and nine nonmetals mainly for the solid state, on seven fluids for both the liquid and gaseous states and two for the liquid state on $1 \mathrm{y}$. The materials studied were selected primarily for their potential applicability as reference standards or because of their technical importance. The temperature range for which values are given often exceeds that for which these values are known with a high degree of certainty. The metals included are aluminum (solid and liquid state), copper, gold, iron (Armco and pure), manganin, mercury, platinum, platinum alloyed with 40 percent rhodium, silver and tungsten. The nonmetallic solids treated include aluminum oxide, beryllium oxide, Corning code 7740 glass, diamond, magnesium oxide, pyroceran brand code 9606 glass, quartz, thorium dioxide and titanium dioxide. Data in the literature for the following substances in both liquid and gaseous state were examined and evaluated in this compilation: argon, carbon tetrachloride, diphenyl, helium, nitrogen, m-terpheny 1, p-terpheny 1 , toluene and water. Graphs, tables and references are given for each substance.

\#6500264

*4.163-3.163

Prather, J. L.

ATOMIC ENERGY LEVELS IN CRYSTALS

NBS Monograph 19, National Bureau of Standards, Washington, D. C

February 24,1961

$84 \mathrm{p}$

Available from the Superintendent of Documents, GPO

Discrete energy levels observed within certain crystals are treated as due to perturbations of the energy levels of the free ion by an electrostatic field arising from the crystal lattice. The analytic procedures for determining the field from the charge configuration are given, and the resulting fields are classified according to their symmetry. After a general survey of grouptheoretical ideas, the applicable groups are analyzed in detail, and characters appropriate for both integral and half-integral angular momenta of 
the free ion are tabulated. These are applied to the determination of the number and type of levels arising from a free ion level with $J \leq 8$. The results of this analysis are tabulated, as are the selection rules for electric dipole, magnetic dipole, and electric quadrupole transitions. Calculation of the perturbation matrix elements by the use of wigner and Racah coefficients is discussed. Examples of the application of these several techniques to specific problems are given.

$\# 6609012$

* $8.173-4.153$

Rand, N. H.; et al.

PLUTONIUM: 'PIIYSICO-CHFNICAL PROPERTIES OF ITS COMPOUNDS AND ALLOYS

Atomic EnergyReview, 4:1-112

June 106

This issue covers plutonium and its compounds and includes a critical evaluation of the data on thermodynamic properties, densities, crystallographic structures, compounds with non-metals, intermetallic phases, phase diagrams, equilibrium diagrans and diffusion rates in the condensed states. This assessnent includes all the data published up to the end of 1964 .

\# $6508041 \quad * 2.082-4.082$

TIIE REACTOR IIANDBOOK, VOLUMI 3 , SECTION 1: GENERAL PROPERT IES OF MATERIÁLS

AECD-3647, Atomic Energy Comission,

Washington, D. C.

February 1955

$610 p$

Available from Superintendent of Documents, GPO

This Handbook is a comprehensive and critical compilation of nuclear engineering data issued by the Atomic Energy Connission's Technical Information Service for reference use by scientists and engineers engage in $_{\mathrm{AEC}}$ reactor projects. The material presented represents the efforts of specialists in the various areas of reactor science and technology, and summarizes the accomplishments of the Comnission's nuclear reactor program to date. Important nuclear reactor data are given in graphical and tabular form for the following metals and alloys: aluninum and its alloys; beryllium and its alloys; beryllia; beryllium carbide; bismuth; carbides; cements and concretes; graphite; hydrides; lithium and its alloys; magnesium, molybdenum, nicke1, plutonium, thorium, titanium, uranium, vanadium, zirconium, and their alloys; cobalt-base alloys; rare earths; silicon carbide; stainless steels; tungsten; and high-cross-section materials.

\#6803010 * 4.173

Reed, R. P.; likese 11, R. P.

LOW TEMPERATURE MECHANICAL PROPERTIES OF COPPER AND SELECTED COPPER ALLOYS, A CONPILATION FROM THE L ITERATURE

NBS Monograph 101, National Bureau of Standards, Washington, D. C.

December 1967

$161 p$

Available from the Superintendent of Documents, GPO

This compilation presents the mechanical properties of copper at temperatures up to $500^{\circ} \mathrm{K}$. The specific properties compiled are yield and tensile strength, elongation, impact energy, creep rate, reduction of area, hardness, fatigue behavior and moduli of elasticity and rigidity. The compilation is dividied into four parts. The first section is intended for quick reference use for those who are interested in average values. The second section includes data from most of the investigators who have published results on the mechanical properties of copper and its alloys. The third section is composed of $t a b l e s$ classifying the investigations which were not included in section two. These usually involve investigations in which data were obtained only at one temperature, such as room temperature. The fourth section lists, in alphabetical order, all references used.

\#6906011

* 4.073

Reiss, H.

PROGRESS IN SOLID STATE CILMISTRY VOLU:IE 3

Oxford, New York: Pergamon Press

167

$510 \mathrm{p}$

This third volume of Progress in Solid State Chemistry discusses the scientific understanding $\overline{0^{t}}$ the pronising new techniques that have evolved for the preparation of solids in unusual states exemplified by single crystal epitaxial layers, solids at high pressure, and solids which are so disordered that they are essentially amorphous. The chapters include discussions on silicon heteroepitaxy on oxides by chemical vapor deposition; physical and chemical properties of semiconductor surfaces; solid state electrochenistry; lattice defects, ionic conductivity, and valence change of rare-earth impurities in alkaline-earth halides; non-stoichiometry in binary semiconductor compounds; lattice energies and related topics; phase relations and structures of solids at high pressures; metastahle phases obtained by rapid quenching from the liquid state; and glass transitions in polymers. An author index, subject index, tables of data and references are included.

$\# 6908020$

Rimshaw, S. J.; Ketchen, F. E.

CESIUN-137 DATA SHEFTS

ORNL-4186, Oak Ridge National Lahoratory, Oak Ridge, Tennessee

Decenber 1967

$27 \mathrm{p}$

Available from CFSTI

Data on CS-137 are tabulated. Properties of the fuel forms CsCl, Cs, so and cesium borosilicate glass are given. ${ }^{2}$ Tैenty-seven references are included.

\#6908021

*2.2773-4.173-8.173-7.173

Rimshaw, S. J.; Ketchen, E. F.

CURIUM DATA SHEETS

ORNL-4187, Oak Ridge National Laboratory,

Ridge, Tennessee

December 1967

$52 \mathrm{p}$

Available from CFSTI

Data on the fuel forms of $\mathrm{Cm}-244$ are presented. Properties of $\mathrm{Cm}-244$ metal, $\mathrm{Cm}_{2} \mathrm{O}_{3}, \mathrm{Cm}_{2} \mathrm{O}_{2} \mathrm{~S}, \mathrm{CmF}_{3}$, Cm-242, $242 \mathrm{Cm}_{2} \mathrm{O}_{3}$ cermet are tabulated. Forty-nine references are given.

\# 6908022

*2.2773-4.173-8.173-7.173

Rimshaw, S. J.; Ketchen, E. E.

STRONTIUM-90 DATA SIIEETS

ORNL-4188, Oak Ridge National Laboratory, Oak Ridge, Tennessee

Decemher 1967

$45 \mathrm{p}$

Available from CFSTI

Data are given on composition, specific power, radiation, critical mass, container compatability, thermophysical properties, mechanical properties, chemical properties, biological tolerance, and shielding for strontiun-90 metal, titanate $\left(\mathrm{SrTiO}_{3}\right)$, oxide $\left(\mathrm{SrO}_{3}\right)$, fluoride $\left(\mathrm{SrF}_{2}\right)$, and orthotitanate ( $\left.\mathrm{Sr}_{2} \mathrm{TiO}_{4}\right)$. The data are indexed to 53 references which are also included. 
Rittenhouse, J. B. ; Singletary, J. B. SPACF MATERIALS HANDBOOK, TIIIRD EDITION AFML-TR-68-205, Air Force Materials Lahoratory, lVright-Patters on Air Force Base, Ohio July 1968

$742 p$

Available from the $\mathrm{DDC}$

This edition is the result of an extensive revision and reworking of the second edition of the Space Materials Ilandbook along with the incorporation of entirely new suhject matter coverage and new materials data. A11 of the most significant material, phenomena, properties and principles covered in the original Handhook are presented and expanded in this revised and updated version. However, treatment of theoretical aspects has heen condensed in order that more emphasis could he placed on the extensive new materials knowledge and data obtained from the design and successful launching of a wide variety of space systems. The Handhook is organized into four parts, namely: space environment, effect of space environment on materials, materials in space and biological interaction with spacecraft materials. Information on mechanical, physical and chemical properties and characteristics is given for a wide variety of metallic and nonmetallic materials. The effects of natural and induced environments on materials are appraised. Materials categories include coverage of thermal control materials, optical materials, adhesives, organic structural materials, inorganic structural materials, electronic components and materials, materials for sealing applications and lubrication materials. In addition, a comprehensive multiple citation index is incorporated which gives ready access to information on specific subject areas with regard to their locations within the liandbook.

\section{\#6905025}

$* 4.173-8.173$

Roberts, B. iv.

SUPERCONDUCTIVE MATFRIALS AND SOME OF THEIR PROPERT IES

NBS Technical Note 482, National Bureau of Standards, Washington, D. C.

May 1969

$129 \mathrm{p}$

Avallable from the Superintendent of Documents, GPO

This is a noncritical compilation of data on superconductive materials that has been extracted from a portion of the literature published up to early 1968. The properties concerned are composition, critical temperature, cri+ical magnetic field, crystallographic data, and lowest temperature tested for superconductivity. The compilation also includes a bibliography, general reference review articles and a special tabulation of high magnetic field superconductors. This compilation supersedes NBS Technical Note 408 having the same title.

\section{$\# 6807009$}

$$
\text { * } 4.173
$$

Rosenberg, s. J

NICKEL AND ITS ÁLLOYS

NBS Monograph 106, National Bureau of Standards, Washington, D. C.

May 1968

$156 p$

Available from the Superintendent of Documents, GPO

This monograph provides current information of the production, properties, and uses of high-purity and commercial forms of nickel and its important ferrous and nonferrous alloys. It is a revision of National Bureau of Standards Circular 592, issued in 1958. It gives 1100 references as well as tables and graphs of data.
\#67060 40

*8.173- 4.173

Roth, R. S.

PHASE EQUILIBRIA STUDIES ON MIXFD SYSTEMS OF RARF EARTH AND OTHER OXIDES

From progress in the Science and Technology of the Rare Farthi, pages T67-202

New York: Pergamon Press

164

$36 \mathrm{p}$

This report consists of a review of work done on phase equilibria of mixed systems of rare earth and other oxides. "Rare earth" here includes the elements yttrium and scandium as well as the lanthanide series; only trivalent oxides have been considered. The results are presented in the form of phase diagrams in temperature and percentage compositon and as tables of the lattice constants of oxide crystals.

\#6603017 *8.173-4.173

Samsonov, G. V. HANDBOOKS OF HTG

MATERIALS NO. 2. PROPFERTIES INDFX

New York: Plenum Press

164

430 p

Authorized translation from the Russian

This book contains data on the physical, technical, mechanical, chemical, and refractory properties of refractory compounds which are currently most widely used in technical developments and which offer the most promise of further application in solving the problems of modern engineering. Chapters I-V of the reference book give information of a general character on refractory compounds, data on their crystal structure, specific gravity, thermochemical, thermal, electrical and magnetic, optical, mechanical, chenical and refractory properties. In Chapters VI-VII, the author has mainly attempted to provide some idea of the resistance of refractory compounds to the action of different chenical reagents and molten media, and to oxidation. Chapter VIII gives concise tahles of information on current and prospective fields of application of refractory compounds in different branches of industry. The data are organized according to the properties of more than 600 different materials such as borides, carbides, nitrides, silicides, etc. (oxides are excluded). In each chapter, the following sequence in order of classes of compounds has heen adopted: metal-like borides, carbides, nitrides, silicides, phosphides, sulfides, and non-metaliic compounds. The reference book gives the most reliable data and indicates the literature sources in which duplicate values have been obtained by different investigators. Included are 1337 references.

\#6500460

BASIC MAGNETIC QUANTITIES AND THE MEASURENENT OF THE MAGNETIC PROPFRTIES OF MATERIALS

NBS Monograph 47, National Bureau of Standards, Washington, D. C.

May 1962

$36 \mathrm{p}$

Available from the Superintendent of Documents, GPO

This paper gives general information regarding the two basic quantities, magnetic induction, $B$, and magnetizing force, 11 , and also the magnetic constant $T$ (often designated by the symbols $\mu_{\nu}$ and $\left.\mu_{0}\right)$. Information is also given regarding the magnetic properties of various materials, and methods and apparatus commonly used in the Magnetic Measurements Section for measuring these properties by means of reversed direct current or alternating currents of low frequency. Magnetic measurements peculiar to high frequencies are not discussed. In view of the gradual adoption of the rationalized mksa system of units, this system is included as well as the classical egs electromagnetic system. 
6500223,6500224

*7.173- 4.173

Schmidt, F. F.; Ogden, II. R.

FNGINEERING PROPERTIFS OF TUNGSTEN AND TUNGSTFN ALLOYS ENGINEERING PROPERTIFS OF :IOLYBDENUM AND MOLYBDENIM. ALLOYS

DMIC Reports 191 and 190, Defense Metals Information Center, Battelle Nemorial Institute, Columbus, Ohio

Septemher 1963; Septemher 1963

$133 \mathrm{p}, 288 \mathrm{p}$

Available from DDC

These two reports present the results of a stateof-the-art survey covering tungsten, molyhdenum and 19 of their alloys. All data are given in tabular and graphical form covering some of the more important physical, mechanical and metallurgical properties for each material. For both reports, the physical properties included are melting point, density, thermal expansion, thermal conductivity and electrical resistivity. The mechanical properties included are tensile properties (ultimate tensile strength, yield tensile strength, elongation, reduction in area, modulus of elasticity) and their temperature dependence, notched tensile properties, creep and stress-rupture properties, and other selected mechanical properties. Metallurigcal properties include: fahricability, transition temperature, weldahility, stress-relief temperature and recrystallization temperature. The ten alloys considered in the tungsten properties report include "doped" tungsten and various alloys with niobium, thorium dioxide, tantalum carbide, molybdenum and rhenium. The nine alloys considered in the molybdenum properties report include alloys with niobium, zirconium, titanium, tungsten and carbon. References are given at the conclusion of each materials section.

\section{$\# 6909061$}

Seddon, B. J. (Compiler)

PIIYSICAL PROPERTIES OF

COMPOUNDS: A DATA MANIIAL

\section{*7.173-4.173}

TRG-Report 1601, United Kingdom Atomic Energy Authority, Reactor froup, Risley, England

December 1967

$54 \mathrm{p}$

Available from CFSTI

This report is a compilation of the hest available data on the physical properties of some of the plutonium compounds which are, or may he, of interest as fuel materials. These are the dioxide, monocarbide, mononitride, monosulfide and monophosphide of plutonium, uranium-plutoniun dioxide, uranium-plutonium monocarhide, uraniumplutonium mononitride, and the plutonium silicides.

\section{\#6603018}

$$
\text { * } 8.173-4.173
$$

Shaffer, P.T.B.

PLENUM PRESS IIANDBOONS OF

MATERIALS NO, 1 MATERIALS INDEX

New York: Plenum Press

1964

$760 \mathrm{p}$

Bata on the properties of more than 520 different materials, such as carbides, borides, nitrides, sulfides, phosphides, silicides, oxides, as well as mixed oxides and mixed carbides are presented. The data are taken from 698 references, with the large majority from the U.S. Government report literature. The data concern general, chemical, electrical, mechanical, nuclear, optical, structural, and thermal properties. The references are listed by number as they appear in the tables, as well as alphabetically by name of author. The extensive table of contents on pages vii to $x x$ makes it easy to find the various materials in the tables. preprint, Electronic Properties Information Center, Ilughes Aírcraft Company, Culver City, California

1969

$300 \mathrm{p}$

This set of data sheets on silicon gives data for energy band structure, effective mass, dielectric constant, solubility and diffusion, energy levels, electrical properties (electrical resistivity, mobility, lifetime, and piezoresistance), optical properties (absorption, refractive index, reflectivity, spectral emissivity, luminescence and photoconductivity), thermal and lattice properties (thermal conductivity, Bebye temperature, Phonon dispersion, Seebeck coefficient and the rmomagnetic properties), magnetic properties (magnetoresistance, magnetic susceptibility and electron spin resonance), electron enission (photoemission, thermionic emission, work function, and secondary electron emission), and crystalographic properties. A bibliography is included.

$\# 6500216$

Si lve rman, A.; et al.

DATA ON 'CHEMIICALS FOR CFRAMIC USE FORNUIAAS, MOLECULAR IVEIGITS, COLORS, CRYSTAL FORMS, DENSITIES, REFRACTIVE INDICES, MELTING POINTS, BOILING POINTS, TRANSITION POINTS, DECOHPOSITION TEMPERATURES

Bulletin of the National Research Council No. 118 , University of Pittsburgh, Pittsburgh, Pennsylvania June 1249

$193 p$

The data contained in Data on Chemicals for Ceramic Use are a revision of Bulle tin $\frac{\text { 107, whi ch was taken }}{107}$ from the Report of the Comittee on Chemical Data for Ceramists submitted to the Division of Chemistry and Chemical Technology of the National Research Council, 1942. Elenents and compounds are arranged aIphabeticaliy according to names. All available data as of 1949 on density melting point, transition point, hoiling point, suhlimation point, decomposition temperature, refractive index, crystal form, colors, formulas and molecular weights is listed. This revised edition brings the report up to June 1949 .

\section{\#6500217 *8.373-4.373}

Simonds, 17. R.; Church, J. il.

A CONCIŚ GUINE TO PLASTTICS, SECOND EDITION

New York: Reinhold Publishing Corporation

1963

$404 \mathrm{p}$

This second edition brings up to date the text of the first edition of A Concise Guide to Plastics expansion of the plastics industry itself. An objective of this book was to answer the questions asked at the 1961 Plastics Exhibit. The suhjects discussed include basic chemistry of plastics manufacturing techniques, stereospecific catalysts, ablative action, borderline materials, forks of plastics, resin manufacture, compounding, processing, applications, production and prices, selection factors, and the business of plastics. One chapter is devoted to plastic manufacturers; statements and contains information about the company's materials, trade names and sales for fifty-two producing firms. Over fifty pages of tables of the United States Plastics Trade Names are included, as well as numerous graphs and tahles of data concerning plastic properties. 
$\# 6500250,6500251$

$\star 4.273$

Smith, J. V. (Eititor)

$X-R A Y$ POIIDFR DATA FILE, SETS 1-5 RTVISFD, ORGANIC and IMORGA'IC

AST:1 Special Technical publication 48-J, American Society for Testing and Materials, Philadelphia, pennsylvania

1960

$685 \mathrm{p}, 374 \mathrm{p}$

These two books contain data on the X-ray powsler diffraction patterns for many compounds. The Inorganic and Organic volumes were prepared by

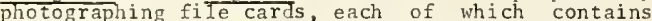
data on one compound. The data given for each compound includes the interplanar spacings corresponding to the three strongest lines in the spectrum and the largest interplanar spacing found for the specimen; the intensities of these lines; wavelength, source and filter of the $X$-rays used; crystallographic system, lattice parameters and interaxial angles; indices of refraction, density, melting point and color of the specimen; chemical and structural formulas; interplanar spacing, intensities and Miller Indices for all lines found; and the reference to the literature from which the data were taken.

\#6711018

$* 4.173-6.173$

Stern, K.1F。

BIBLIOGRAPIYY OF LIESEGANG RINGS (SECOND EDITION)

NBS Miscellaneous publication 292, National Bureau of Standards, Washington, D.C.

September 1967

$61 \mathrm{p}$

Available from Superintendent of Documents, GPO

A bibliography of Liesegang rings (periodic precipitation figures produced hy diffusion) and other periodic structures within the period $1855-$ 1965 is listed alphahetically according to author. An author index and an index to applications of Liesegang rings to extra-chemical fields are also given. This second edition has added new references, which concern only true liesegang rings, to the original hibliography of 681 references, many of which dealt with other kinds of periodic structures. The subject and author indices have been appropriately amended. Errors found in the first edition have been corrected. All journal article titles and rearly all book titles have been translated into Finglish. Abbreviations of journal titles are those given in the issue of Chemical Abstracts in whicl the given reference first appeared.

\section{\#6711012}

Storms, E. K.

THE REFRACTORY CARBIDES

New York: Academic press, 1967

$285 \mathrm{p}$

This book is a critical review of data collected between 1957 and 1967 on the properties of the metal-carbide systems of the following elements: $\mathrm{Ti}, \mathrm{Zr}$, IIf, $\mathrm{V}, \mathrm{Nb}, \mathrm{Ta}, \mathrm{Cr}, \mathrm{Mo}, \mathrm{W}, \mathrm{Th}, \mathrm{U}$, and $\mathrm{P}_{\mathrm{u}}$ Each system is discussed individually in terms of its preparation, phase relationship, lattice parameter and structure, appearance, chemical reactivity, hardness, and thermochemical properties.

$\# 6500285-6500294$ * 4.173

Swanson, H. E.; et al

STANDARD DIFFRÁCTION POIDER PATTERNS, VOLUMES 1 TO 10, DATA FOR INORGANIC SUBSTANCES

NBS Circular 539, Volumes 1 - 10, National Bureau of Standards, Washington, $\mathrm{N}$. C.

June 1953, June 1954, March, October 1955, September 1956, September 1957, April 1959, February, Sep tember 1960

$97 p, 67 p, 75 p, 77 p, 77 p, 63 p, 72 p, 79 p, 68 p, 63 p$

Available from the Superintendent of Documents, GPO
These volumes represent a continuing MIsS-AST project for inproving a file of $X$-ray diffraction patterns. Reports are made on substances for each pattern prepared, which updates and add to the files. The ASTr patterns are tabulated for comparison with additional patterns from the literature and one prepared at the NBS. Miller indices are derived from the calculation of spacings by desk calculator or the electronic computer SEAC. Interplanar spacings in angstroms (except where otherwise noted) and relative intensities from 1 to 100 are tahulated. For the NBS pattern the three strongest lines are given, as well as the lattice constants and the computer density. The index of refraction of the sample is noted if it could he determined. Crystal-structure data from the literature are noted. There fiftyfour sulstances covered in Volume I; Volume II includes thirty substances; Volume III includes thirty-four suhstances; fifty-two suhstances are covered in Volume IV; forty-five suhstances in Volume V; forty-four suhstances in Volme VI; Volume VII covers fifty-three substances; Volume VII covers sixty-one suhstances; Volume IX includes forty-three suhstances; and volume $x$ includes forty suhstances. A Cunulative Index to Volumes $I-X$ is located at the end of volume $X$.

\#6500295-6500297,6902072-6902073, \#4.173 6810001,6910012

Swanson, II. F., ; et al.

STANDARS $X$-RAY' DIFFRACTION PONDER PATTERNS

NBS Monograph 25, Sections 1-7, National Bureau of Standards, Washington, D. C.

March 1962, May 1963, July i964, June 1966,

August 1967, June 1968. Septemher 1969

$56 \mathrm{p}, 50 \mathrm{p}, 68 \mathrm{p}, 87 \mathrm{p}, 94 \mathrm{p}, 101 \mathrm{p}, 190 \mathrm{p}$

Availahle from the Superintendent of Documents, GPO

The X-Ray Powder Data File is a many volumed compilation of diffraction patterns from many sources and is used for the identification of unknown crystalline materials hy matching spacing and intensity measurements. The patterns were made with a Geiger counter X-ray diffractometer using samples of high purity. The d-values were assigned Miller indices determined by comparison with calculated interplanar spacings and from space group considerations. The densities and lattice constants were calculated and the refractive indices were measured whenever possihle. The calculated $X$-ray powder diffraction patterns were obtained from single crystal structure data and from published crystal structure data. Accurate cell determination measurements for the internal standards were obtained hy using a flat-plate back reflection focusing camera. The reported peak height intensities for calculated patterns were converted from integrated intensities. Reference intensity values based upon the strongest line of corundum (113) in a 50 percent weight mixture are given for some materials. Included in Section 1 are data for 60 substances, in section 2 for 37 substances, in Section 3 for 51 substances, in Section 4 for 103 substances, in Section 5 for 80 substances, in Section 6 for 60 suhstances, and in Section 7 for 81 substances. A cunulative index to Circular 539, Volumes 1 through 10 and Monograph 25. Sections 1 through 7 is included at the end of Monograph 25, section?.

$\# 6500147$

* 4.173

Swanson, II, E.

TABLES FOR CONVERSION OF X-RAY DIFFRACTION ANGLES TO INTLRPIAANAR SPACING

NBS Applied Mathematics Series 10, National Bureau of Standards, Washington, D. C.

September 20,1950

$164 \mathrm{p}$

Available from the Superintendent of Documents, GPO

These tahles give spacing values $d$ in angstrom units corresponding to the angles $\theta$ which are usually neasured when diffraction patterns are used for chemical identification and crystal structure determination. The angles are related to 
interplanar spacings $d$ hy the formula $d=\lambda / 2 \sin \theta$, where $\lambda$ is the wavelength of incident $x$-radiation and $\theta$ is half the angle between the incident and diffracted rays. The first six tables give the spacing values for the angles $\theta$ from $0^{\circ}$ to $90^{\circ}$ at intervals of $0.01^{\circ}$; these were calculated by using the $\kappa$ wavelengths for $\mathrm{x}$-ray targets of molybdenu, copper, nickel, cobalt, iron and chromiun, respectively. The wavelengths used in the calculations are those adopted at the International Conference sponsored by the British Institute of Physics in London, July 1046. The last two tables contain a rearrangement of the data for copper and iron, giving spacing values for the argument $2 \theta$ from $0^{\circ}$ to $180^{\circ}$ at intervals of $0.02^{\circ}$.

\#6601026 *4.173

Taylor, A.; Kagle, B. J. (Compilers)

CRYSTALLOGRAPIIIC DATA ON YIFTAL AND ALLOY STRUCTURES New York: Dover Publications, Inc.

1963

$263 p$

The data asserhled in these tables include information on alloys and intermetallic compounds, borides, carbides, hydrides, oxides, and nitrides, as well as crystai structures of the elements. The information has heen compiled from the original literature, the powder Data File of the ASTi!, Pearson's Ilanthook of Lattice Spacings and Structures of Tetals and Alloys, Stracture Reports, Strukturberich t, and the LandoIt Bornstein Tahles. As an aid to identification, the spacings and relative intensities of the three strongest Iines of the Debye-Scherrer powder diagrams have been included where availahle. All spacing data are uniformly expressed in absolute Anestrom units.

\#6907013

$* 8.172-4.172$

TIIERMAL FXPANSION BIBLIOGRAPYIY

Research "aterials Information Center, Oak Ridge National Laboratory, Oak Ridge, Tennessee

Feb ruary 1969

$57 p$

This is a hibliography of thermal expansion information sources based on papers, reports, and abstracts received by the RMIC. It also contains a materials index. Over 350 references are given covering material through February 1969.

\#6500170 *8.183-4.183

Van Kranendonk, J.; Van Vleck, J. H.

SPIN WAVES

Reviews of Nodern Physics, 30:1-23

January $\frac{1}{19} 5$

This article reviows the spin waves method of calculating the magnetization for a crystal composed of regularly spaced atoms at low temperatures, where magnetization differs only slightly fron that at absolute zero. This article assembles in one place and in a unified fashion many of the results which are rather scattered in the literature, and introduces a simplified version of the quantum-mechanical theory. The approach is based on approximating the magnetic spin system by a system of harmonic oscillators. The treatment is based on the Ileisenberg, or localized-spin model for a magnetic solid, which is analogous to the Heitler-London model of chemical bonds. The magnetism is regarded as coming entirely from electronic spins regularly spaced in the crystal.

\#6903057 * 4.183

Warren, K. A.; Reed, R. P.

TENSILE AND IMPACT PROPIRTIFS OF SELECTED MATERIALS FROM 20 TO $300^{\circ} \mathrm{K}$

NBS Monograph 63, National Bureau of Standards, Washington, D. C.

June 28,1963

517

Availahle from Superintendent of Documents, GPO
The tensile and impact properties of structural materials were experimentally determined at temperatures from 20 to $300^{\circ} \mathrm{K}$. Tensile properties of a few naterials were also determined at $4^{\circ} \mathrm{K}$. The materials included forty-two comercial alloys of iron, aluninum, titanium, copper, nickel, and cobalt, and two metal-bonded carbides. The properties experimentally determined were the yield strength, tensile strength, elongation, and reduction of area, the stress versus strain curve, and the impact energy. The test equipment and procedures are described. The individual data are presented in tables, and the average results are displayed in graphs.

H 6707011

$\div 4.183$

Welles, S. J. COPPER

DS-156, Electronic Properties Information Center Hughes Aircraft Company, Culver City, California 1967

$328 \mathrm{p}$

These data sheets present a compilation of the electronic, thermal and opcical properties of copper over the widest possible range of parameters from references obtained in a thorough literature search. These properties are correlated with each other and with the theoretical and experimental description of the Fermi surface of copper. An extensive cvaluation of the electrical resistivity of pure copper is presented. Data are given on magnetoresistance, llall effect, superconductivity, thermal conductivity, Lorenz number, thermoelectric power, specific heat, optical properties and electron emission. Representative data on many copper alloys and the anomalies observed in the properties of dilute copper-transition metal alloys are considered. A detailed index to the data is presented.

\#6903048

* 4.183

White, E. W. ; et al.

$X-R A Y$ WAVELEITGTIS AND CRYSTAL INTERCIIANGE SETTINGS FOR WAVELENGTH GEARED CURVED CRYSTAL SPECTROMITERS, SECOND EDITION

Mineral Industries Experiment Station Special Publication No. 3-64, The Pennsylvania State University, University Park, Pennsylvania August 1965

$195 \mathrm{p}$

This second edition includes $\mathrm{X}$-ray emission line wavelengths in angstrom units for the range 0.25 to 100 angstroms. The table is especially designed for use with curved crystal spectrometers that are geared to read directly in wavelength. The table is presented in two sections. Section one lists all the first order lines including satellite and low intensity diagram lines for each element (boron and above), arranged in order of increasing atomic number with lines sorted on the hasis of decreasing wavelength with in each element. Section two lists all lines sorted on the basis of increasing wavelength. Odoneter settings for commonly interchanged crystals have been computed and listed for each line in both sections. The two sections are separated by a periodic table of the elenents which lists the most intense lines for each element.

\#6704035, 6611010

7001040,7001041

$* 4.183-8.183$

Willardson, R. K.; Beer, A. C. (Fditors)

SEMICONDUCTORS AND SEMIMETALS,

VOLUME 1: PHYSICS OF II I - V COMPOUNDS;

VOLUME 2: PIIYSICS OF III-V COMPOUNDS;

VOLUNE 3: OPTICAL PROPERTIES OF III-V CONPOUNDS;

VOLUME 4: PHYSICS OF III-V COMPOUNDS

New York: Academic Press

$1966-1968$

$500 \mathrm{p}, 432 \mathrm{p}, 581 \mathrm{p}, 524 \mathrm{p}$

This set of four volumes deals with the semiconductor, semimetal and optical properties of 
the III-V compounds. Contributions from ten to fifteen scientists are included in each volume. The books are designed for reference work as we 11 as texts on the graduate leve1. Volume 1 reviews key properties of the III-V compounds, with special emphas is on band structure, magnetic field phenonena, and plasma effects. In volume 2 , the emphas is is on physical properties, thermal phenomena, magnetic resonances, and photoelectric effects, as well as radiative reconbination and stimulated erission. Volume 3 is concerned with optical properties, including lattice effects, intrinsic absorption, free carrier phenomena and photoelectronic effects. Volune 4 includes thermodynamic properties, phase diagrans, diffusion, hardness, and phenomena in solid solutions as well as the effects of strong electric fields, hydrostatic pressure, nuclear irradiation and nonunifornity of impurity distribution on the electrical and other properties of III-V compounds.

$\# 6500270$ to \#6500280 inclusive * 4.283

ivilson, A. J. C.; Pearson, H. B. (Editors)

STRUCTURE REPORTS FOR 1940-1941, 1942-1944, $1945-$ 1946,1949 , SUPPLEMENTARY VOLUNE ANI) CUNMLATIVE INDEX FOR 1940-1950,1952, 1953, 1954, 1955, 1956, VOLUIES $8,9,10,12,14,15,16,17,18$, î, \& 20 Utrecht, Netherlands: N.V.A. Oosthoek's Uitgevers Mi j

$1956,1955,1953,1952,1959,1957,1959,1963$, $1961,1963,1963$ respectively for volunes 8 - 20 $392 \mathrm{p}, 452 \mathrm{p}, 333 \mathrm{p}, 486 \mathrm{p}, 223 \mathrm{p}, 596 \mathrm{p}, 659 \mathrm{p}, 891 \mathrm{p}$, $853 \mathrm{p}, 699 \mathrm{p}, 736 \mathrm{p}$ in volumes $8-20$ respectively.

"Structure Reports" contain reports on all papers published on the structural characteristics of crystals. Each report presents all the data of structural interest found by the authors in the original papers. Each volume of "Structure Reports" contains reports on papers in one year (since 1948) or two or three years $(1940-47)$. Reports are presented on the structure of metallic elements, alloys, inorganic compounds and organic compounds. Each section is arranged by substance with all the papers found for a given substance reported together. Subject, formula, and author indices are provided for each volune. The scope of coverage of the reports is variable depending upon the papers considered. Each report includes sone or all of the following topics: unit cell, space group atomic positions and parameters, interatomic and internolecular distances, and details of analysis. References are provided for each paper including the title of a paper, name of the author journal name, volume, page and date. Volume 8 of "Structure Reports" covers 1940-41; Volume 9 covers 1942-44; Volume 10 covers 1945-46; Volume 12 covers 1949: Volume 14 includes a cumulative index for 1940-1950; Volumes 15-20, respectively, each cover one year for 1951-56.

\section{\#6500248} $* 4.383$

Winche11, A. N.

OPTICAL PROPFRTIES OF ORGNIC COMPOUNDS, SECONI EDITION, ENLARGED AND COMPLTELY REVISED

New York: Academic Press, Inc.

1954

$487 \mathrm{p}$

This volume is a collection of the optical properties of over 2500 organic substances. This information may be used either in the identification of unknown compounds or the finding of properties of known compounds. The following information is compiled for each compound: crystal class and space group, lattice constants, density, melting point, indices of refraction, color and literature references to a11 papers which were found to contain data for that compound. An alphabetical index to conpounds is included. For the purposes of identification of unknown compounds, two large separate-sheet diagrams are included, which represent each compound as a plotted point on a chart of optic axis angle vs. refringence or of birefringence vs. refringence.
Each noint has a key number which identifies the compound.

$\# 6500258$

$* 4.183$

Winchel1, A. N.; "Vinchel1, II.

ELEMENTS OF OPTICAL NINERALOGY AN" I!TRONICTION TO MICROSCOPIC PETROGRAPIIY, PART 2, DFSCRIPTIOIS OF MINERALS, FOURTII EDITION

New York: John Viley \& Sons, Inc.

1961

$551 p$

This book is a compendins of data on the physical and crystalline properties of minerals taken fron literature published through 1949 . The authors have presented data on the properties of these materials as a function of their composition in elements and simple molecules. The data included for each entry are: mineral name; chemical formula with known natural variations, if any; crystal structure symetry and space group; lattice constants and axial angles; hardness, specific gravity, melting point; indices of refraction along principal axes and optical angles. Graphs or tables of these properties as a function of composition are provided for materials of variable composition. Data are provided for native element materials and for halides, sulfides, oxides. carbonates, borates, sulfates, phosplates and silicates.

\#6500265,6500266,6500267 *4.183

Winche11, A. iN. ; "Winche11, II.

THE MICROSCOPICAL CIARACTERS OF

ART IFICIAI

INORGANIC SOLID SUBSTANCES: OPTICAL PROPERTIES OF ART I FICIAL :IINERALS, TIIIRD EDITION

New York: Academic Press, Inc.

1964

$439 \mathrm{p}$

This book contains descriptions of inorganic solid substances whose properties are known and a set of deterninative tables and charts for identifying an unknown compound from its optical properties. The following data are given for each substance: chenical formula and name; dimensions and axial angles of the unit cell; data on crystal liabits, cleavage, twinning, etc.; hardness; specific gravityy; optical properties, including optic orientation, principal refractive indices, optic sign, optic axial angle, dispersion, color, and absorption. The spacings for the substance's $x$-ray powder diffraction pattern are also given. The determinative tables are presented as polar plots of optic angle vs, birefringence with each plot covering a narrow range of index of refraction variation. Each compound is represented as a point on such a plot, and a table accompanying each plot gives a page number for the text where the compound can be found. The following classes of compounds are included: elements, carbides, nitrides, sulfides, selenides, teílurides, sulfosalts, halides, hydrides, amides, cyanides and cyanates, oxides, carbonates, nitrates, halates, borates, sulfates, molybdates, tungstates, phosphates and silicates.

\#6500247

Winchell, H.

OPTICAL PROPERTIES OF MINERALS: A DETER'IINATIVI: TABLE

New York: Acaderic Press, Inc.

1965

$91 \mathrm{p}$

This compilation presents data useful for the optical identification of minerals. The data include the refractive indices, birefringence, and optic axial angle for over 1000 minerals. The data are presented graphically on a polar plot of optic angle against birefringence for materials falling within a narrow range of refractive indices. Each mineral is represented by a point on such a graph. Also listed for each material are the strongest 
lines in its $X$-ray powder diffraction spectrum and a literature reference.

\section{$\# 65002$ r 9}

$* 8.383-4.383$

Wood, L. A.

VALUES OF. THE PIIYSICAL CONSTANTS OF RUBBER

In "Rubber Technology Conference, London, May 2325, 1938, First Proceedings," pp. 933-953

Cambridge, England: W. Ileffer and Sons, Ltd. 1938

$21 \mathrm{p}$

A critical survey has been made of published values for sixteen of the principal physical constants of rubber in the fields of mechanics, heat, optics and electricity, namely: dimensions of unit cell, density, expansivity, thermal conductivity, specific heat, heat of fusion of crystalline rubber, heat of conbustion, volume compressibility, Poisson's ratio, velocity of sound, refractive index and dispersion, stress-optical coefficient, dielectric constant, power factor, and conductivity. The value for each constant which seems to be the most reliable has been indicated, and, when necessary, corrected to the standar? conditions of normal atmospheric pressure an a temperature of $25^{\circ} \mathrm{C}$. Temperature coefficients are given when posible. The values are given for rubber in four different forms: the purifier hydrocarbon, commercial raw rubber, soft vulcanised rubber containing $2:$ combined sulfur, and hard rubber containing $32 \%$ comined sulfur. Relationships between expansivity and change of density with temperature, expansivity and change of refractive index with temperature, and refractive index and diclectric constant are discussed.

\#6603019 *8.183-4.183

Wood, W. D.; Deen, II. W.; Lucks, C. F.

PLENUYI PRESS ILNDBOOKS OF IIIGII-TEMPFRATURE MATERIALS NO. 3. TIIERMAL RADIATIVT PROPERTIES Now York: Plenum Press

1964

$474 \mathrm{p}$

This report is a compilation of data on thermal radiative properties. It also includes a brief discussion of the basic fundamentals of thermal radiation and of the methods of measuring these properties. Much of the information has previous $1 y$ been distributed in DMIC memoranda; however, it is consolidated in this report for the benefit of those with a broad interest in radiant heat transfer. Thermal radiative data are included for the following materials: titanium and its alloys; stainless steels; iron-, nickel-, and cobalt-base superalloys; the refractory metals (chromium, columbium, molybdenum, tantalum, and tungsten) and their alloys; coated materials for elevatedtemperature service; and ceramics and graphite. Forty-one references are included.

$\# 6500259-6500263$

Wy ckoff, R. W. G

CRYSTAL STRUCTURES, VOLUMES 1 TO 5, ORGANIC NND INORGANIC

New York: Interscience Publishers, Inc. 1960

$528 \mathrm{p}, 706 \mathrm{p}, 814 \mathrm{p}, 318 \mathrm{p}, 960 \mathrm{p}$

These volumes provide collections of data taken from the literature on the structure of crystals. Information is provided in the form of text tables and illustrations. Data provided in the tables for each crystal include: crystal type and class, and its lattice constants, For each crystal type, data on symetry and positions of atoms within the lattice are provided in the text section, and illustrations of the typical compounds are given. The volumes are divided into chapters according to the chemical type of the compounds. Volume 1 contains clements and compounds of the type $R X$ and RX. Volume I I contains complex binary compounds $R_{m} x_{n}$, and structures of the type $R\left(M X_{2}\right)_{n}$, $R_{n}$ (NIX ${ }^{2} p$, ammoniates, silicates and miscellaneous inorganic compounds, plus an inorganic formula index and a mineralogical name inclex. Volume IV contains structures of aliphatic compounds, while Voluse $V$ contains structures of benzene derivatives and alicyclic and heterocyclic compounds and of carbohydrates, and an index to organic compounds. 172]

\#6605006 *4.283

Wyckoff, R. W. G.

CRYSTAL STRUCTURES, SECOND EDITION, VOLUNF 1

New York: Interscience Publishers, Inc.

1965

$467 \mathrm{p}$

The book is livided into four chapters: preface and introduction; structures of the elenents ; structures of the compounds RX; structures of the compounds $R x_{2}$. A bibliography is given at the end of each chapter. A name indes and formula index are provided at the end of the hook.

\section{\#6605004}

Wyckoff, R. W. G

CRYSTAL, STRUCTURES, SECONI) EDITION, VOLUME

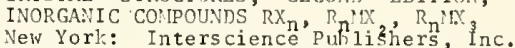

1964

$583 \mathrm{p}$

This book is divided into three chapters: structures of complex binary compounds R ; structures of the compounds $R\left(M X_{2}\right) n$ and componnts the end of each chapter. A name index and formula index are provided at the end of the book. $\Lambda$ s this attempt to provide a unified statement of the atonic positions in all analyzed crystals progresses, improvements become possihle. The principal one introduced with this volume is a nore reliable bibliography.

\#6605005

Ty cloff, R. W. G.

CRYSTAL, STRUCTURES, SECOND EDITION, VOLUINE 3 , AND MMONI $A T E S$

New York: Interscience Publisliers, Inc.

1960

$931 p$

This third volume contains three chapters giving crystal structure for inorganic compounds of the type $R_{x}\left(M X_{4}\right) y$, type $R_{x}\left(M_{n} X_{p}\right)_{y}$, and hydrates and ammoniates. $\Lambda$ bibliography is given at the end of each chapter. The book also includes name and formula indexes.

46500127 $* 3.193-4.193$

Yakowitz, II. ; Cuthi11, J. R.

ANYOTATED BIBLIOGRAPIYY ON SOFT X-RAY SPECTROSCOPY

NBS Ilonograph 52, National Bureau of Standards, Washington, D. C

June 1962

$109 \mathrm{p}$

Available from Superintendent of Documents, GPO

About 550 references are contained in this bibliography which includes, it is believed, a complete coverage of the soft X-ray literature since 1950 and through 1960 . Some references to earlier work are given, but the general review references, listed separately, give an adequate coverage of the earlicr work. The emphasis is on the application of soft X-ray spectroscopy in the study of valence band electronic states in metals and alloys, and therefore, the spectral region of 25 to 800 angstrons involving ruled glass grating spectrometers is of principal interest. Ilowever, a wealth of clata have been gathered, primarily by the Russians, on valence electronic states by means of high energy transitions where crystal spectrometers are satisfactory. These references and any X-ray work leading to the distribution of valence 
electronic states are included regardless of the transitions enployed. In addition to soft $X$-ray data, references on all pertinent aspects of the apparatus and experimental problems are included. Also listed separately are references of value in corroborating soft $x$-ray data with other results, such as energy hand calculations. Subject, author, $x$-ray band, material, and other indices are included.

ก 6611019

Zeitlin, A. (Compiler)

$\star 4.293-3.293$

ANNOTATED BIBLIOGRAPHY ON III GI-PRESSURE TECINOLOGY

New York: The American Society of Mechanical
Engineers

Washington and London: Butterworths 1964

$304 \mathrm{p}$

The bibliograply is presented in four principal parts: Part $I$ is a general bibliograplhy of 4500 entries arranged alphabetically by authors, but not including the writings of $\mathrm{P}$. W. Bridgman. Part II is an index of the material of Part I, arranged by subject. Part III is a complete bibliography of the 310 witings of P. W. Bridgman. Part IV is an index of the material in Part II, arranged hy subject. 
Chemical Kinetics 
SOLVENT EFFECTS ON REACTION RATES AND MECHANISMS

New York: Academic Press

1966

$328 \mathrm{p}$

Theoretical explanations are given for the solvent effects on reactions between various charge types of reactants. These include ion-ion, ion-dipolar molecule, dipolar molecule-dipolar molecule, exchange, and some charge type reactions which do not fit in the above classes. Hypothetical solvent effects as well as experimentally observed effects are explained on a theoretical basis.

\section{\# 6508057}

Bates, P. R. (Editor)

$$
\star 3.213-5.213
$$

ATOMIC AND MOLECULAR PROCESSES

New York: Academic Press

1962

$904 \mathrm{p}$

This compilation, which is designed primarily as a reference book for research scientists, is concerned with radiative and collisional processes involving atoms or molecules. It provides surveys covering the following topics: forbidden and allowed lines and bands, photoionization, photodetachment; recombination, attachment; elastic and inelastic scattering of electrons, energy loss by slow electrons; collision broadening of spectral features; encounters between atomic systems including range, energy loss, excitation, ionization, detachment, charge transfer, elastic scattering, mobility, diffusion, relaxation in gases, and chemical reactions. A chapter is devoted to the use of high temperature shock waves and accounts are given of the other main experimental methods. The relevant theoretical work is also described, detailed mathematics being avoided as far as possible.

\#6810014,6903055,6908033

$$
\star 5.213
$$

Baulch, D. L.; et al.

HIGH TEMPERATURE RATE DATA, CRITICAL EVALUATION OF RATE DATA FOR HOMOGENEOU', GAS-PHASE REACTIONS OF INTEREST IN HIGH-TEMPERATURE SYSTEMS

Report Nos. 1, 2, 3, Department of Physical Chemistry, The University of Leeds, England

May 1968, December 1968, April 1969

$25 \mathrm{p}, 34 \mathrm{p}, 54 \mathrm{p}$

These reports are a series of critical evaluations on gas phase reaction data. They contain discussions of the values listed and include bibliographies. Report No. 1 treats the following reactions :

$$
\begin{aligned}
& \mathrm{CO}+\mathrm{OH}=\mathrm{CO} 2+\mathrm{H} \\
& \mathrm{CO}+\mathrm{OD}+\mathrm{CO} 2+\mathrm{D} \\
& \mathrm{CO}+\mathrm{O}=\mathrm{CO} 2+\mathrm{hnu} \\
& \mathrm{CO}+\mathrm{O}=\mathrm{CO} 2 \\
& \mathrm{CO}+\mathrm{O}+\mathrm{M}=\mathrm{CO} 2+\mathrm{M} \\
& \mathrm{CO}+\mathrm{O} \rightarrow \mathrm{CO}+\mathrm{O} 2
\end{aligned}
$$

Report No. 2 treats the following reactions:

$$
\mathrm{H}_{2}+\mathrm{O}+\mathrm{OH}+\mathrm{H}
$$$$
\mathrm{OH}+\mathrm{H}+\mathrm{H} 2+\mathrm{O}
$$$$
\mathrm{H} 2+\mathrm{OH} \rightarrow \mathrm{H} 2 \mathrm{O}+\mathrm{H}
$$$$
\mathrm{H}+\mathrm{H} 2 \mathrm{O}+\mathrm{H} 2+\mathrm{OH}
$$$$
\mathrm{H} 2 \mathrm{O}+\mathrm{O} \rightarrow \mathrm{OH}+\mathrm{OH}
$$$$
\mathrm{OH}+\mathrm{OH} \rightarrow \mathrm{H} 2 \mathrm{O}+\mathrm{O}
$$$$
\mathrm{H} 2 \mathrm{O}+\mathrm{M} \rightarrow \mathrm{H}+\mathrm{OH}+\mathrm{M}
$$$$
\mathrm{H} 2+\mathrm{OH}+\mathrm{M} \rightarrow \mathrm{H} 2 \mathrm{O}+\mathrm{M}
$$

Report No. 3 treats the following reactions:

$$
\begin{aligned}
& \mathrm{O} 2+\mathrm{H}=\mathrm{O}+\mathrm{OH} \\
& \mathrm{O}+\mathrm{OH}=\mathrm{O} 2+\mathrm{H} \\
& \mathrm{O} 2+\mathrm{H}+\mathrm{M}=\mathrm{HO} 2+\mathrm{M} \\
& \mathrm{HO} 2+\mathrm{M}=\mathrm{O} 2+\mathrm{H}+\mathrm{M} \\
& \mathrm{H} 2 \mathrm{O} 2+\mathrm{H}=\mathrm{H} 2+\mathrm{HO} 2 \\
& \mathrm{H} 2+\mathrm{HO} 2=\mathrm{H} 2 \mathrm{O}_{2}+\mathrm{H} \\
& \mathrm{H} 2 \mathrm{O} 2+\mathrm{H}=\mathrm{H} 2 \mathrm{O}+\mathrm{OH} \\
& \mathrm{H} 2 \mathrm{O}+\mathrm{OH}=\mathrm{H} 2 \mathrm{O} 2+\mathrm{H} \\
& \mathrm{H} 2 \mathrm{O} 2+\mathrm{OH}=\mathrm{H} 2 \mathrm{O}+\mathrm{H} 2 \mathrm{O}_{2} \\
& \mathrm{H} 2 \mathrm{O}+\mathrm{HO} 2=\mathrm{H} 2 \mathrm{O} 2+\mathrm{OH} \\
& \mathrm{H} 2 \mathrm{O} 2+\mathrm{M}=\mathrm{OH}+\mathrm{OH}+\mathrm{M} \\
& \mathrm{OH}+\mathrm{OH}+\mathrm{M}=\mathrm{H} 2 \mathrm{O} 2+\mathrm{M}
\end{aligned}
$$

Berlman, I. B.

HANDBOOK OF FLUORESCENCE SPECTRA OF AROMATIC MOLECULES

New York: Academic Press

1965

$258 \mathrm{p}$

Spectra of approximately 100 aromatic molecules, varying in size, shape, and structure, are assembled in the handbook. The list of molecules begins with the simple aromatic molecule benzene and includes progressively larger and more complicated systems. Although a special effort has been made to include practically all of the currently popular organic scintillators, the choice of the other molecules has been rather arbitrary. The luminescence characteristics of each aromatic molecule have been measured in a systematic fashion. Fluorescence and absorption spectra are plotted for each molecule and additional data related to the fluorescence process are given in each graph. The additional data include the fluorescence decay time, the fluorescence quantum yield, the natural lifetime as computed from the absorption spectrum, the Stokes loss, the wavelength of the center of gravity of the fluorescence spectrum, and the average wavelength of the fluorescence spectrum. Such supplementary material as topical bibliographies, tables to convert wavelength to wave number and energy, a table of values of the index of refraction of cyclohexane as a function of wave number, and a table of values of oscillator strengths have been placed in the Appendix.

$\$ 6910029$

$$
\text { *3. } 123-5.123
$$

Berry, R. S

SMALL FREE NEGATIVE IONS

Chemical Review, 69:533-542

August 1969

This article surveys the restricted subject of properties of isolated atomic and small molecular negative ions. Included in the discussion are electron affinities, electronic states, optical properties, and the experimental and theoretical methods for studying these properties. Excluded from the discussion is the vast group of problems associated with large polyatomic negative ions and with most of the rich subject of collision processes involving negative ions, such as detachment and transfer of electrons in col1isions.

$\$ 6905039$

* 5.371

BIBLIOGRAPHY ON THE RADIATION CHEMISTRY OF AQUEOUS SOLUTIONS - I. AMINO ACIDS 1952-1968

C00-38-641, Radiation Chemistry Data Center, Radiation Laboratory, University of Notre Dame, Notre Dame, Indiana

November 1968

$16 \mathrm{p}$

Available from CFSTI

This bibliography, covering the literature from 1952 to 1968 , contains radiation data for amino acids. Abstract references are included for foreign language articles and technical reports. The papers are listed by year, with each year's papers listed alphabetically by first author.

$\# 6905040$

* 5.371

BIBLIOGRAPHY ON THE RADIATION CHEMISTRY OF AQUEOUS SOLUTIONS - II. CARBOHYDRATES 1952-1968

COO-38-642, Radiation Chemistry Data Center, Radiation Laboratory, University of Notre Dame, Notre Dame, Indiana

December 1968

$11 \mathrm{p}$

Available from CFSTI

This bib1iography covering the literature from 1952 to 1968 contains radiation data for carbohydrates. Abstract references are included for most foreign language articles and technical reports. The 
papers are listed by year, with each year's papers listed alphabetically by first author. At the end of each reference the Radiation Chemistry Data Center serial number is given.

\#6905038 $\$ 5.371$

BIBLIOGRAPHY ON THE RADIATION CHEMISTRY OF ALCOHOLS AND PHENOLS

C00-38-643, Radiation Chemistry Data Center, Radiation Laboratory, University of Notre Dame, Notre Dane, Indiana

November 1968

$34 p$

Available from CFSTI

This bibliography covering the literature from 1953 to 1968 contains radiation data for alcohols and phenols. Abstract references are included for foreign language articles and technical reports. Investigations of aqueous solutions of alcohols and phenols are not included; polyhydraxy compounds (g1ycols) are also not included. The papers are listed under the categories by serial number which is approximately chronological. If an article is pertinent to more than one category it is listed under each. The compound index includes the alcohols or phenols and additives, and refers to the serial number of the paper.

6712004 $\star 5.213$

Blank, C. A. (Editor)

DASA REACTION RATE HANDBOOK

Santa Barbara, California: DASA Information and Analysis Center, TEMPO

October 1967

$410 \mathrm{p}$

Chemical rate processes are of extreme importance in the deionization and recovery of the highly ionized atmosphere created by a nuclear burst. The Handbook presents what it considers to be the best available chemical kinetic data and related unclassified information, required to calculate the kinetics of atmosphere deionization. The Handbook is divided into four major sections, each comprising several chapters of related information. The first of these sections deals with general background materials. The conditions of temperature, density and composition which are needed to determine the importance of specific processes are discussed for the normal and disturbed atmosphere. Basic data, energy levels and equilibrium constants are given and the general types of processes which need to be considered are discussed. In the second section, various aspects of the different methods employed to obtain data on the processes of importance are discussed. The nature and scope of such data are considered in the third section of this Handbook, where the state of knowledge of various types of reactions is examined and evaluated. The fourth section contains chapters dealing with the proper utilization, status, uncertainties and central problem areas associated with the determination of reaction rates as well as a detailed summary of values recommended for use in atmospheric deionization calculations.

$\# 6906003$

$\star 5.113$

Bortner, M. H.

A REVIEW $W$ OF RATE CONSTANTS OF SELECTED REACTIONS OF INTEREST IN RE-ENTRY FLOW FIELDS IN THE ATMOSPHERE NBS Technical Note 484, National Bureau of Standards, Washington, D. C.

May 1969

$62 \mathrm{p}$

Available from Superintendent of Documents, GPO

The major reactions normally encountered in flow field re-entry calculations for the oxygen-nitrogen system are reviewed and a rate constant value for each reaction is recommended. Collisional ionization processes, charge-exchange reactions and attachment-detachnent reactions are included. Rate constant coefficients to fit the equation

$$
k=a T^{b} e^{-c / T}
$$

are reported in tabular form for the selected rate constant. The data are also graphed as $10 \mathrm{~g}$ vs $T(K)$. In a11, over twenty forward and reverse reactions are reviewed. Additionally, a large number of rate constant values for reactions which also effect flow field calculations are given.

$\# 6500368$

Cohn, C. E.; Golden, G. H.

A TABLE OF THERMODYNAMIC PROPERTIES OF HYDROGEN FOR TEMPERATURES FROM 100 TO $3000^{\circ} \mathrm{K}$ AND PRESSURES FROM 1 TO 50 ATMOSPHERES

ANL-6673, Argonne National Laboratory, Argonne, Illinois

January 1963

$267 \mathrm{p}$

Available from CFSTI

This report presents a table of thermodynamic properties of gaseous hydrogen covering the temperature range from 100 to $3000^{\circ} \mathrm{K}$ and the pressure range from 1 to 50 atmospheres. The calculations have been done by means of a computer routine. The table has entries for temperature at $10^{\circ}$ increments, for pressure at 1 atmosphere and at 5 a tmosphere increments from 5 through 50 atmospheres, and for $25 \%, 50 \%, 75 \%$, and $100 \%$ of the gas initially in the para state. The first of these latter values corresponds to normal hydrogen, whereas the remaining values apply to vapor from liquid which has been stored for varying lengths of time. For each entry, the table gives the density in $\mathrm{gm} / \mathrm{liter}$, the enthalpy in joules/gm, the entropy in joules/(gm) $\left({ }^{\circ} \mathrm{K}\right)$ and the fraction of the gas molecules which are dissociated.

*6500148

Cook, C. J.; Lorents, D. C.

ELECTRON COLLISION FREQUENCIES AND SCATTERING CROSS SECTIONS IN THE IONOSPHERE, A

Final Technical Report No. 6, Stanford Research Institute, Menlo Park, California

August 1961

$54 \mathrm{p}$

Available from UDC AD 291591

The propagation of electromagnetic waves through an ionized, gaseous medium can be described in terms of a set of macroscopic parameters that are a function of the physical properties of the medium, the permittivity, conductivity, and permeability. This report calculates the propagation parameters for the normal ionosphere above $50 \mathrm{~km}$, and from these parameters calculates theoretical values for electron collision frequencies and scattering cross sections in the ionosphere.

\#0607033

Cooper, J. (Editor)

PROCEEDINGS OF WORKSHOP CONFERENCE ON THE LOWERING OF THE IONI2ATION POTENTIAL AND RELATED PROBLEMS OF THE EQUILIBRIUM PLASMA

JILA Report No. 79, Joint Institute for Laboratory Astrophysics, Boulder, Colorado

July 1966

$225 p$

This workshop was sponsored by the Joint Institute for Laboratory Astrophysics. Its concern was statistical mechanics of plasma both" from experimental and theoretical points of view. There were many recent theoretical advances in the treatment of such problems as the plasma partition function and the lowering of the ionization potential, although there were strong disagreements between different workers in this field. The proceedings were taped and should be considered as unpublished material. It was the object of this workshop to bring together active theorists and experimenters in this field to resolve some of the differences. Included in the Appendix is a Bibliography on Research on Lowering of the Ionization Potential. 
$\$ 6906026$

$\star 8.123-5.123$

Diamond, J. J. (Editor)

BIBLIOGRAPHY ON THE HIGH TEMPERATURE CHEMISTRY AND PHYSICS OF MATERIALS, October, November, December 1968; January, February, March 1969; April, May, June 1969; July, August, September 1969; October, November, December 1969

NBS Special Publication 315, 315-1, 2, 3 \& 4, National Bureau of Standards, Washington, $\nu . C$. Jan., April, July, Oct. 1969, Jan. 1970

$72 \mathrm{p}, 81 \mathrm{p}, 82 \mathrm{p}, 90 \mathrm{p}, 85 \mathrm{p}$

Available from Superintendent of Documents, GPO

These bibliographies consist of references to research involving temperatures above $1000^{\circ} \mathrm{C}$, which were noted by the contributors during the abovementioned three-month periods. Since these are intended primarily as current-awareness bibliographies, there is no cross-referencing or indexing. These issues contain about 700 references each, roughly grouped under fifteen subject headings.

\#6908036 *5. 123

Dragoo, A. L.

DIFFUSION RATES IN INORGANIC NUCLEAR MATERIALS Journal of Research of the National Bureau of March, April 1968

The tracer diffusion coefficient, the selfdiffusion coefficient, the intrinsic diffusion coefficient and the interdiffusion coefficient are briefly described. Grain boundary and lattice (volume) diffusion are contrasted. The frequency factors $\left(\mathrm{B}_{0}\right)$ and activation energies (Q) are tabulated $\frac{0}{\text { I }}$ diffusion in the borides, carbides, and oxides of Be, iff, Mo, Nb, Ta, Th, Ti, and $\mathrm{Zr}$ and for diffusion of $C, N$, and $O$ in these metals. The purity of the solvent media, the preparation and properties of the samples, the method, the type of diffusion coefficient measured and the temperature range are also specified.

Bt 6500417

Flynn, J. H.

RAPID DETERMINATION OF THE ORDER OF CHEMICAL REACTIONS FROM TIML-RATIO TABLES

NBS Technical Note 62 , National Bureau of Standards, Washington, D. C.

August 1960

$19 \mathrm{p}$

Available from Superintendent of Documents, GPO

A table of ratios of reaction times corresponding to $15 \% / 5 \%, 20 \% / 10 \%, \ldots, 95 \% / 85 \%$ reaction for orders of chemical reaction from -10 to +10 is presented. Use of this table allows the rapid calculation of the order of chenical reactions that are kinetically simple and involves only one subjective step. Methods are discussed for interpreting deviations from constant order resulting from errors in the initial times and concentrations, from errors in stoichiometry, and from the reaction following a more complicated rate expression.

\#6906004

$$
* 5.133-3.133
$$

Franklin, J, L, et al.

IONIZATION POTENTIALS, APPEARANCE POTENTIALS, AND HEATS OF FORMATION OF' GASEOUS POSITIVE IONS

NSRLS-NBS-26, National Bureau of Standards, Washington, D. C.

June 1969

$290 \mathrm{p}$

Available from Superintendent of Documents, GPO

This is a compilation of ionization and appearance potentials of positive ions published from 1955 through June 1966. The compilation lists the ion formed, the parent species from which it was formed, the other products of the process, the threshold energy for the formation of this ion, and the method by which this data was obtained. Where feasible, the heat of formation at $298 \mathrm{~K}$ of the positive ion has been computed for each entry using auxiliary thermochemical data. From these computed values "best" values have been chosen.

\#6808004

$\star 5.233$

Garvin, D. (Editor)

A COMPENDIUM OF EVALUATED AND ESTIMATED RATE COEFFICIENTS

NBS Report 9884, National Bureau of Standards, Washington, D. C.

July 1968

$316 \mathrm{p}$

This report is an annotated handbook on rates of elementary gas phase reactions. It is 1 imited to rate evaluations. No attempt has been made to record all research papers pertinent to a particular reaction. There are two principal parts to the report: a compilation and a set of rate evaluations. The compilation merges the information presented in several recent journal articles and reports that are devoted to the evaluation or estimation of rate coefficients for elementary reactions. A single table, arranged by reaction, lists the various recommended rates in a common format. Notes to the table show what data sources were used. by each evaluator, and, where possible, how his recommended value was determined. The person requiring a rate for a specific reaction may compare the recommendations, compare their data bases and also extract a bibliography of the pertinent work. The rate evaluations, which are presented in full, stand independent of the main table. They are for reactions of some importance to combustion and atmospheric chemistry. They are presented in a form suited to the reaction-byreaction evaluation of rate data as opposed to that used in monographs. It is hoped that they will serve as models, albeit ones to be improved upon, for future rate data examinations.

$\# 6811016$

*5.031-3.031

Harllee, F. N.; Rosenstock, H. M.; Herron, J. T.

A BIBLIOGRAPHY ON ION-MOLECULE REACTIONS, JANUARY 1900 TO MARCH 1966

NBS Technical Note 291, National Bureau of Standards, Washington, D. C.

June 28,1966

$38 \mathrm{p}$

Available from Superintendent of Documents, GPO

This document is a bibliography on ion-molecule reactions of chemical interest. The bibliography is listed in a chronologic-alphabetic arrangement and includes 431 titles and references taken from the published literature since 1900. The coverage is limited to reactions between ions and molecules leading to changes in the atomic composition of either the projectile ion or target molecule. No attempt has been made to completely cover resonant charge exchange or elastic scattering.

$\# 6902099$

Henley, E. J.; Johnson, E. R

THE CHEMISTRY AND PHYSICS OF HIGII ENERGY REACTIONS Washington, D. C.: University Press 1969

$475 \mathrm{p}$

This is a text on radiation chemistry on the graduate level, discussing radiation effects on complex systems. The book includes discussions on general nomenclature; interaction of radiation with matter; radiation sources and dosimetry; chenical consequences of the absorption of high energy radiation; modern techniques in radiation chemistry, radiation chemistry of gases, water, and of aqueous and solid systems; effect of radiation on liquid hydrocarbons and polymers; radiationinduced polymerizations; and industrial applications. 
r 6512007

*5. 133

Harrick, S.

TABLES FOR ROCKET AND COMET ORBITS

NBS Applied Mathematics Series 20, National Bureau of Standards, Washington, D. C.

March 9, 1953

$124 \mathrm{p}$

Available from Superintendent of Documents, GPO

Tables of $\sin E$ and $1-\cos E$ with argument $E-s$ in $E$, with parallel tables in the hyperbolic functions, are given for the determination of position and velocity from the time, for rectilinear orbits. Direct interpolation is possible, without the aid of series expansions or successive approximations. The tables may also be used in connection with nearly rectilinear motion, that is, motion in ellipses and hyperbolas whose excentricities are near unity. The application of the tables is set forth extensively in the Introduction.

$\$ 6908017$ $\star 5.153$

Hochstim, A. R. (Editor)

Berman, $M$, ; et al.

BIBLIOGRAPHY OF CHEMICAL KINETICS AND COLLISION PROCESSES

New York, Washington: IFI/Plenum

1969

$962 \mathrm{p}$

This book contains about 20,000 entries, each specifying the study of a collision process, drawn from about 7000 selected articles. The entries cover the following: reaction rates of atoms, ions, and small molecules in the gas phase; inelastic scattering cross sections of atoms, ions, and smal1 molecules in the low-energy range ( $t \leqslant 100 \mathrm{eV}$ ); low-energy elastic electron collision cross sections; lifetimes of excited states of various species; and reaction rates involving solid carbon. In the context of this book, "small molecules" means species with four atoms or less. Only occasionally are larger molecules included.

\# 6809013

Horiuti, J.; Miyahara, K.

HYDROGENATION OF ETHYLENE ON METALLIC CATALYSTS

NSRDS-NBS-13, National Bureau of Standards, Washington, D. C.

June 1968

$62 \mathrm{p}$

Available from Superintendent of Documents, GPO

Reaction rate data for the catalyzed hydrogenation of ethylene, primarily in the presence of unsupported metallic catalysts, are critically reviewed. Reaction mechanisms are discussed in detail, and a statistical mechanical treatment of the reaction is given, according to the generalized theory of reaction starting from the well-known procedure of Glasstone, Laidler, and Eyring. Data for single-element catalysts and alloys are included and interpreted, as are data illustrating differences due to the physical form of the catalyst (film, foil, wire, powder, and some supported systems). Problems are' discussed concerning reproducibility of experimental results over repeated runs, and as a function of catalyst pretreatment. The lata are analyzed in 29 graphs and 29 tables, some of which are very extensive. The bibliography includes 141 references.

\#6811002

Johnston, Harold S.

GAS PHASE REACTION KINETICS OF NEUTRAL OXYGEN SPECIES

NSRDS-NBS-20, National Bureau of Standards, Washington, D. C.

September 1968

$49 \mathrm{p}$

Available from Superintendent of Documents, GPO

The available data for reactions among neutral oxygen species, oxygen atoms, oxygen molecules and ozone, have been reviewed. Selected data have been

reanalyzed and used to establish values for the rates of these reactions:

$$
\begin{aligned}
& 02+M+0+0+M \\
& O+O+M+02+M \\
& O 3+M+0+02+M \\
& 0+02+M+03+M \\
& 0+03+02+02 \\
& 0+02+0+02
\end{aligned}
$$

$(M=02)$

$(M=02,03, \mathrm{He}$, $\mathrm{N} 2, \mathrm{CO} 2$ )

$(M=02,03, \mathrm{He}, \mathrm{Ar}$, $\mathrm{N} 2, \mathrm{CO} 2$ )

(isotopic exchange)
\# 6906005

Johns ton, H. S.

GAS PHASE REACTION RATE THEORY

New York: The Ronald Press Company

1966

$362 \mathrm{p}$

This is a college text which reviews the theories of chemical kinetics based on molecular mechanics and chemical kinetics. The algebraic structure of simple two-atom collision rates and three-atom metathetical reactions is presented in detail. Considerable use is made of graphical presentation in terms of three-atom models. More complex methods are briefly outlined and reference is given to advanced publications. Several unpublished studies are included. Experimental data are compared with theory whenever possible. Only a few chemical reactions are discussed in detail. Tables of chemical kinetics data and a large number of references are included.

\#6902078

Jones, R. N. i et a1.

$\star 3.043-5.043$

COMPUTER PROGRAMS FOR ABSORPTION SPECTROPHOTOMETRY NRC Bulletin No. 11, National Research Council of Canada, Ottawa, Canada

1968

$159 \mathrm{p}$

Nine "building block" computer programs for performing the basic numerical computations of absorption spectrophotometry are described and listed. They are written in FORTRAN IV for card input and output. The input and output formats are standardized to permit easy interfacing to produce more complex data processing systems. Though developed for infrared spectrophotometry these programs are adaptable for use in the visible and ultraviolet. The operations covered include scale change and scale interval change, location of band maxima and minima, smoothing and differentiation, slit function convolution and deconvolution, single band generation, profile analysis and asymmetry analysis of single bands from the truncated moments, and ordinate addition.

$\# 6805004$

$\star 5.243$

KerI, J.A.

BOND DISSOCIATION ENERGIES BY KINETIC METHODS

Chemical Reviews, 66: 465-500

September 26, 1966

The main aim of this review is to present an assessment of bond dissociation energies, which have recently been determined for the first time. The review is centered on polyatomic molecules, and deals with modifications and expansions of the methods which have been developed over the past few years. The bond dissociation energy or bond strength of a chemical bond A-B is usually taken to mean the enthalpy change of the reaction $A-B=A+B$ in the ideal gas state referred to $298^{\circ} \mathrm{K}$. Experimental methods are given for pyrolytic reactions, metathetical reactions, shock-tube reactions, and estimated errors. Data on bond energies are given for hydrocarbon, organic nitrogen-oxygen-, sulfur- and halogen-containing compounds, and metal alkyls. Data are given for heats of formation of atoms and radicals, bond dissociation energies and best available values of bond dissociation energies. 
H 6603027

kieffer, L. J.

A BIBLIOGRAPHY OF LOW ENERGY ELECTRON COLLISION

CROSS SECTION DATA

JILA Report No. 4, Joint Institute for Laboratory Astrophysics, Boulder, Colorado

January 1,1964

$76 \mathrm{p}$

Available from Superintendent of Documents, GPO

This bibliography was compiled by the JILA Data Center for the purpose of providing a list of published reports which contain information on collisions between electrons, ions, atoms, and molecules. The publication includes a bibliography of the experimental and theoretical low energy data, a molecule list, journal list, and author list. This bibliography is updated at yearly intervals.

\# 6811010

*5.143-3.143

Kieffer, L. J

BIBLIOGRAPHY OF LOW ENERGY ELECTRON COLLISION CROSS SECTION UATA

NBS Miscellaneous Publication Z89, National Bureau of Standards, Washington, D. C.

March 10,1967

$87 \mathrm{p}$

Available from Superintendent of Documents, GPO

A bibliography of low energy electron collision cross section data is presented. Only references which report original measurements or calculations of electron collision cross sections are included. The cross section data for each process are 1 isted by atomic species in order of their atomic number. The data for molecules are listed in arbitrary order.

\# 6810007

*5.143-3.143

Kieffer, L. J.

BIBLIOGRAPHY OF PHOTOABSORPTION CROSS SECTION DATA JILA Report No. 5, Joint Institute for Laboratory Astrophysics, Boulder, Colorado

Apri1 15,1968

$32 \mathrm{p}$

This bibliography provides references to comprehensive collections of low energy atomic collision data. The data sought are those for Absorption and ionization coefficients, from which cross sections can be derived, are included, as we 11 as references to molecular absorption in wavelength regions where there is continuous absorption even though there are line absorptions superimposed. The report contains a section which describes the data in the references of the bibliography; a section listing the title, authors, and complete reference for the papers cited; and a section consisting of an alphabetical author index.

\#6500144

Kieffer, L. J.

*5.143-3.143

COMPILATION OF CRITICALLY EVALUATED ELECTRON IMPACT IONIZATION CROSS SECTION DATA FOR ATOMS AND DIATOMIC MOLECULES

JILA Report No. 30, Joint Institute for Laboratory Astrophysics, Boulder, Colorado

February 5, 1965

$73 \mathrm{p}$

This compilation of critically evaluated data presents basically two kinds of data. The first is "gross" of "total" ionization cross-sections, which correspond to the probability of producing a single positive change from any atomic or molecular species regardless of the mass or multiplicity of change on the ions produced. The other kind is that which gives the relative probability of producing ions with a specific charge and mass (in the case of molecules where dissociation can take place). The tables of "total" cross-section data are grouped by their $z$-number. The data for seven molecular species are ordered by their atomic weights. After these tables, the absolute crosssection data for single ionization by electron impact are presented. There then follows a section of cross sections for the dissociative ionization of diatomic molecules, and finally a section giving relative numbers of multiple-charged ions. Accuracy of data is in all cases at best $5 \%$.

\#6902064

* $5.143-3.143$

Kieffer, L. J .

COMPILATION OF LOW ENERGY ELECTRON COLLISION CROSS SECTION DATA, PART I. IONIZATION, DISSOCIATIVE PROCESSES, ÁND VIBRATIONAL AND ROTATIONAL EXCITATION

JILA Report No. 6, Joint Institute for Laboratory Astrophysics, Boulder, Colorado

January 10,1969

$95 \mathrm{p}$

A comprehensive compilation of low energy electron collision cross-section data is presented. This is the first of three parts and is limited to experimental measurements, including data for all atomic species and for those molecules which are important in aeronomy, astrophysics and plasma physics. Literature data through September of 1968 are included. The compilation is divided into three main categories: ionization, including all processes which result in electron ejection from atomic or molecular targets; dissociation, including processes which lead to ionized fragments; and vibrational and rotational excitation. The data are organized by species in the order: atoms, diatomic molecules, triatomic molecules, etc. The atoms are ordered by the $Z$ number, and the molecules are ordered according to the atom with the largest $Z$ in the molecule.

\#6910045

Kieffer, L. J .

*5.143-3.143

COMPILATION OF LOW ENERGY ELECTRON COLLISION CROSS SECTION DATA, PART II: LINE AND LEVEL EXCITATION

JILA Report No. 7, Joint Institute for Lahoratory Astrophysics, Boulder, Colorado

September 22, 1969

$177 \mathrm{p}$

This is the second part of a comprehensive compilation of low energy electron collision cross section data. The compilation is limited to experimental measurements and includes data for a11 atomic species and those molecules which are important for aeronomy, astrophysics, and plasma physics. The data included were taken from literature published through December 1968. However, reference to some materials is obtained from abstracting journals, so this compilation probably does not include all data published in the late fall of 1968. All of the cross section data in this volume of the compilation are for electronic excitation. The data are organized by initial species in the following order: atoms, diatomic molecules, triatomic molecules, etc. The atoms are ordered by the $Z$ number of the nucleus, the smallest $Z$ 's first. For molecules, the $Z$ number of the atom with the largest $Z$ in the molecule is taken to characterize the molecule. The molecules are then ordered by these characteristic $Z$ 's, the smallest $Z$ first. If necessary the second highest $z$ atom in the molecule is used in the same way to determine the order. If the line emitted is characteristic of the ionized species, the data are located following the neutral species.

\#6811005 *5.143-3.143

Kieffer, L. J *; Dunn, G. H.

ELECTRON IMPACT IONIZATION CROSS-SECTION DATA FOR ATOMS, ATOMIC IONS, AND DIATOMIC MOLECULES: I. EXPERIMENTAL DATA

Reviews of Modern Physics, 38: 1-35 January 1966

This review includes a compilation and critical evaluation of absolute cross sections for 
ionization of atoms and diatomic molecules by electron impact. Experimental techniques used for ionization are surveyed. Selected relative cross sections for production of multiply charged ions and a brief discussion of relative cross-section data near threshold are presented. Absolute limits are not set on the size of probable systematic error in the various experiments.

\#6811009

*5.143-3.143

Krauss, Morris

COMPENDIUM OF ab initio CALCULATIONS OF MOLECULAR ENERGIES AND PROPERTIES

NBS Technical Note 438, National Bureau of Standards, Washington, D. C.

December 1967

Available from the Superintendent of Documents, GPO

The number of ab initio molecular electronic calculations has increased dramatically in the last few years. Both the practitioners and other interested students of the results of the calculations have found it increasingly difficult to determine the present status of these calculations. This compendium references the work from 1960 to the present and abstracts from the mass of data the best values for several observable properties including the total energy, dissociation energy, electron affinity, spectroscopic constants electric moments, field gradients, polarizabilities, and magnetic constants. In order to provide an insight into molecular electronic structure, tables of orbital energies are also included. These tables are meant to direct attention to the successes and failures of the calculations by compiling a large percentage of the best results in a reasonably compact form. Its use fulness will be limited in time by rapid advance in the field.

$\# 6906001$

*3.153-5.153

Laborie, P.; Rocard, J.-M.; Rees, J. A.

ELECTRONIC CROSS-SECTIONS AND ATS

COEFFICIENTS

1968

$199 \mathrm{p}$

In French and English

The catalogue contains some of the principal experimental data concerning the interaction of electrons with atoms and molecules. It is a noncritical selection of data from the most accurate and most recent measurements available. The crosssections are all expressed in $\mathrm{cm}^{2}$ per atom or molecule, and given as a function of the electron energy expressed in electron volts. Curves are reproduced where the original article gives data in that manner. Tables of data are given when available. Atomic or molecular energy levels and macroscopic coefficients are included. The book includes data for hydrogen, helium, neon, argon, krypton, and xenon.

\#6500526

$* 0.1151-3.151-5.151-8.151$

LANDOLT-BORNSTEIN, NUMERICAL DATA AND FUNCTIONAL RELATIONSHIPS IN' PHYSICS, 'CHEMISTRY, ASTRONOMY, GEOPHYSICS AND TECHNOLOGY, SIXTH EDITION,

VOLUME I: ATOMIC AND MOLECULAR PHYSICS,

PART 2: MOLECULES I (NUCLEAR STRUCTURE)

Eucken, A.: Hellwege, K.-H. (Editors)

Berlin, New York: Springer-Verlag

1951

$571 p$

In German

This section of the Landolt-Bornstein tables contains atomic physical data of molecules which describes the structure and dynamics of their nuclear framework. The following properties have been tabulated: atomic distances and structures, valence energies of chemical bonds, dissociation energies of chemical bonds, and molecular vibrations and rotations.
H6909060 $\star 0.1151-5.151-8,15]$

LANDOLT-BORNSTEIN, NUMERICAL DATA AND FUNCTIONAL RELATIONSHIPS IN PHYSICS, CHEMISTRY, ASTRONOMY, GEOPHYSICS AND TECHNOLOGY, SIXTH EDITION,

VOLUME II: PROPERTIES OF MATTER IN ITS AGGREGATED STATES

PART $5 \mathrm{~b}:$ TRANSPORT PHENOMENA II, KINETIC HOMOGENOUS GAS EQUILIBRIA

Schaefer, K. (Editor)

Berlin, New York: Springer-Verlag

1968

$397 \mathrm{p}$

In German

Part 5b of Volume II contains some kinetic properties of the elements and selected compounds. The first series of tables lists diffusion constants for elements and ions in metals, salts, and alloys, both liquid and solid. A short table of values for diffusion constants of some gases in selected metals is included. The second series of tables deals with thermal conductivity data for gases, liquids and solids. The constants are reported as a function of temperature, pressure and composition. The next series of tables deals with thermal diffusion constants for binary and ternary gas mixtures. Constants are also reported in liquid mixtures and solutions (Ludwig-soret Effect). The next section lists a series of kinetic rate constants for reaction in gas phase and includes: dissociation, atom-radical and ionmolecule reactions. Another series of tables lists rate constants and equilibrium constants for reactions in solid and mixed phase. Several addenda to the main sections are appended.

$\# 6706036$

*0.1151-3.151-5.151

LANDOLT-BORNSTEIN, NUMERICAL DATA AND FUNCTIONAL RELATIONSHIPS IN SCIENCE AND TECHNOLOGY, NEW SERIES

GROUP II: ATOMIC AND MOLECULAR PHYSICS,

VOLUME 3: LUMINESCENCE OF ORGANIC SUBSTANCES

Schmillen, A.; Legler, R.

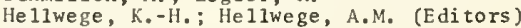

Berlin, New York: Springer-Verlag

1967

$416 \mathrm{p}$

In German and English

The most important data on the luminescence of over 1000 luminescent organic substances investigated have been assembled into a main table. Most substances are shown note only in a pure state (1iquid or solid) but diluted in several solvents as well. On compiling the literature, it was noticed that not all data were of equal reliability, e.g. owing to insufficient purity of the substances investigated or to the application of theories which had become out of date in the meantime. For these reasons the main table only contains the physical magnitudes which are known with sufficient reliability for the majority of substances investigated. Data appearing in the main table for each compound include: its state, solvent used, if any, temperature, wavelength. used for stimulation, absorption and emission wavenumbers for fluorescent and phosphorescent emission, quantum efficiency, decay time, and literature reference. Following the main table, a selection of typical luminescence spectra are given. Tables of the wavenumbers of very sharp single bands are given for some important spectra. Data are given concerning applications of luminescence by radiation damage. The tables were taken from literature dating from 1949 to 1964.

\#6606021

Lipmann, B. A. ; et al.

SYSTEMS ANALYSIS OF BALLISTIC MISSILE DEFENSE CONCEPTS (U) VOLUME II: CALCULATION OF ATOMIC AND MOLECULAR REACTION CROSS SECTIONS

DRC 66-7589, Defense Research Corporation, Santa Barbara, California

March 15, 1966

$121 p$ 
Available theoretical and experimental data on the reactions that control the electron density in missile wakes and in the environment surrounding nuclear bursts have been reviewed. Calculations of cross sections (reaction rates) for specific reactions of importance in these problem areas have been made. The aim of the work here is also to develop general computational techniques designed to improve the accuracy and reliability of calculations.

\#6902032

Matula, R. A.

COMBUSTION KINETICS OF TETRAFLUOROETHYLENE

Final Technical Report, Fluid Dynamics Laboratory, Department of Mechanical Engineering, University of Michigan, Ann Arbor, Michigan

July 1968

410

The pyrolysis of C2F4, C3F6, and CF2O have been studied in the temperature and pressure ranges $300-$ $455^{\circ} \mathrm{C}, 25-760$ torr; $550-675^{\circ} \mathrm{C}, 50-410$ torr; and $330-480{ }^{\circ} \mathrm{C}, 25-600$ torr, respectively. The rate equations and appropriate Árrhenius rate constants for these three reactions are reported. The oxidation kinetics of $\mathrm{C} 2 \mathrm{~F} 4$ are also being investigated and preliminary results in the temperature and pressure ranges $175-300{ }^{\circ} \mathrm{C}$ and $25-$ 200 torr are discussed. Finally the application of gas-solid chromatography techniques to the separation of low molecular weight fluorocarbons and the analysis of the C2F4 oxidation products are discussed.

\section{$\# 6906029$}

$\star 5.153-8.153$

Mickle, E. A.; Clauson, W. W.; Szego, G. C. FREE RADI CALS AS HIGH ENERGY PROPELLANTS

General Electric Company, Flight Propulsion Laboratory, Cincinnati, Ohio

1956

$1062 \mathrm{p}$

This compilation gives data concerning the use of free radicals as high energy propellants as well as discussions on rocket propulsion metastable states, and assumptions and methods of calculation. Tabulations and figures are included for the $\mathrm{H}, \mathrm{H} 2$; $\mathrm{F}, \mathrm{F} 2, \mathrm{H} 2 ; \mathrm{N}, \mathrm{N} 2$, $\mathrm{He} ; \mathrm{N}, \mathrm{N} 2, \mathrm{H}_{2} ; \mathrm{CH}_{4}, \mathrm{CH} 3, \mathrm{H}$; and ionic systems. Included in the thermodynamic properties given for each system are: speed of sound in a gaseous mixture in which chemical equilibrium is maintained at all times, speed of sound in a gaseous mixture in which the chemical composition remains fixed, specific heat capacity at constant pressure for a gaseous mixture in chemical equilibrium, mole ratio of free radical to stable "parent" species in reactant mixture, mole ratio of diluent to stable parent species in reactant mixture, molal enthalpy, equilibrium constants, mass of a particle, molecular weight, specific or total entropy, specific volume, and alpha and beta emissions. Nozzle performance parameters and equilibrium composition in mole fractions are also listed. These data are listed for a broad range of temperatures and pressures for each syster.

\section{\#6811003}

*5.153-3.153

Moiseiwitsch, 8. L.; Smith, S. J.

ELECTRON IMPACT EXCITATION OF ATOMS

NSRDS-NBS-25, National Bureau of Standards, Washington, D. C.

August 1968

120 p

Available from Superintendent of Documents, GPO

The experimental and theoretical literature about the electron impact excitation of atoms is reviewed. Theoretical methods ranging from the Bethe and Born approximations to the close coupling approximations are discussed and intercompared. Whe re possible, on theoretical grounds or through intercomparison, the reliability of the various methods is discussed. A general critique of the optical method of measuring excitation functions is given, with the objective of promoting higher quality future experimental work. A critical study of existing experimental work leads to the conclusion that most workers have ignored important physical and instrumental effects, and it may be presumed that the data in the literature are subject to many unrecognized systematic errors. The 1iterature on the alkalis, heavy rare gases, mercury, cadmium and zinc is surveyed but the quality of the literature does not support critical review beyond some general comments about the physics of these atoms.

$\# 6609009$

National Academy of Sciences - National Research Council. Comittee for the Survey of Chemistry CHEMICAL DYNAMICS - A CURRENT REVIEW

Publication 1292-B, National Academy of Sciences, Washington, D. C.

1966

$68 \mathrm{p}$

This report is a review of the current theory and experimental findings in chemical dynamics. It summarizes heterogeneous catalysis and electrochemical dynamics in surface chemistry, and includes reactions in solution as well as reactions in biological systems.

$\# 7001042$

Niles, F. E.

FORMATION OF IONS IN IONIZED AIR

BRL-R-1458, Ballistic Research

Aberdeen proving Grounds, Maryland

November 1969

$54 \mathrm{p}$

Available from DDC

Schematic representations are given for both the formation of positive ions and the formation of negative ions in ionized air. The formation of positive ions can be divided into three regions. Region I contains the ionization of neutral constituents and two-body reactions in dry air. Region II contains reactions involving ionic species which are formed by three-body reactions. Region II contains reactions requiring water vapor and shows the formation of the hydrated hydronium ions. The formation of negative ions proceeds predominantly through $0-$ The clustering of neutral molecules to $0_{\overline{2}}$ opens additional channels for the formation of $\mathrm{NO}_{3}^{-}$an ion which is not attacked by atomic oxygen. Rate constants are given, including the experimental values on which they are based, for the reactions in the schematic representations.

\#6907039

0 'Neal, H. E. B Berson, S. ${ }^{*} .363$

THE BIRADICAL MECHANISM IN SMALL RING COMPOUND REACT IONS

The Journal of physical Chemistry, 72: 1866-1887 June 1968

The kinetics of dissociation, structural isomerization, and geometric isomerization in three- and four-membered cyclic compounds are discussed in terms of the biradical mechanism. . With the exceptions of the cyclobutene isomerizations and the cis-1,2- alky 1viny 1cyclopropane isome rizations, all small ring compound reactions to date are shown to be both qualitatively and quantitatively consistent with the biradical mechanism. Methods for making a priori transition-state estimates of the activation energies and Arrhenius $A$ factors for these reactions are outlined. Estimates are shown to be reliable to about $0.3 \log$ unit in $A$ and about $1.5 \mathrm{kcal} / \mathrm{mol}$ in $\mathrm{E}$, on the average. The thermochemistry of fluorine substitution in cyclopropanes and in cyclobutanes along with the effect of such substitution on the mechanism and kinetics of fluorinated small ring compound reactions is also discussed. 
*6907040

$\star 5.363$

O'Neal, H. E.; Benson, S. W.

A METHOD FOR ESTIMATING THE ARRHENIUS A FACTORS FOR FOUR- AND SIX-CENTER UN1MOLFCULAR REACTIONS

The Journal of Physical Chemistry, 71: 2903-2921 A ugust 1967

The Arrherius A factors for gas-phase, unimolecular reactions which proceed through a cyclic transition state are examined from the point of view of transition-state theory and are shown to be compatible with a structure which is looser than the ring prototypes. On the basis of a few simple rules for assigning bending, stretching, and torsion frequencies it is shown that quantitative estimates of these A factors can be made to within the average experimental uncertainty of \pm 0.3 unit in $\log \mathrm{A}$ (units of $\mathrm{sec}^{-1}$ ). The extreme range of $\mathrm{A}$ values extends over 6.0 log units. In a few isolated cases the discrepancy between estimated and observed A factors exceeds 1.0 log unit and the weight of evidence suggests that the experimental values may not be reliable. The reactions include dehydrohalogenations which go through four-center transition states, ester pyrolyses, "ene" reactions, which go through six-center transition states, and Cope rearrangements which do the same. The aralysis confirms previous suggestions that the most important contributions to $\Delta S^{\dagger}$ come from losses in hindered internal rotations (about -4.0 gibbs/mole per rotor) in forming the loose cyclic structure. However, other coupled changes in structure can also he important.

$\# 6902077$

$\star 3.163-5.163$

Pith a, J ; Jones, R. N.

OPTIMIZATION METHODS FOR FITTING CURVES TO 1NFRARED BAND ENVELOPES COMPUTER PROGRAMS

NRC Bulletir No. 12, National Research Council of Canada, Ottawa, Canada

1968

$133 p$

Three computer programs for fitting infrared band contours are described and 1 isted. These programs utilize Cauchy (Lorentz), Gauss, Cauchy-Gauss product, and Cauchy-Gauss sum functions, and will optimize the parameters for fitting single bands or multiple overlapping band systems using a nonlinear least squares algorithm. Supplementary programs are included for the evaluation of the half-widths and shape characteristics of the synthesized component bands, also for the computation of the ordinates of the synthesized band envelope, and for an analysis of the misfit with the true spectrum. This latter program also serves as a general purpose program for subtracting spectral ordinates. The programs are written in FORTRAN IV. They are designed for card input and output in a standardized format that facilitates their interfacing with other programs developed in our laboratory for the numerical analysis of infrared and ultraviolet spectrophotometric absorption curves.

\#6704027-028-031,6904010 *5.263

Porter, G. (Editor)

PROGRESS IN REACTION KINETICS, VOLUMES 1 TO 4

London: Pergamon Press

$1961,1964,1965,1967$

$276 \mathrm{p}, 391 \mathrm{p}, 515 \mathrm{p}, 523 \mathrm{p}$

This series reviews most of the principal branches of chemical kinetics and presents an up-to-date compilation of rate constants of current interest and importance. The chemical reactions are tabulated, rate constants given, the methods of measurement discussed, and the reliability of these values is assessed. The emphasis throughout is or the quantitative aspects of the subject and most of the articles contain original work not published elsewhere. The later volumes include cumulative indexes and extensions and amendments of the tables in previous volumes. The topics reviewed in the first volume range from elementary gas reactions and combustion through diffusion kiretics to physical and chemical rate processes in solution and finally to biochemical kinetics. The articles in the second volume cover some of the major themes of chemical kinetics -- the reactions of halogen atoms and methylenes, mercury photosensitized reactions, anionic polymerization, cis-trans isomerization and protolytic reactions. Quantitative rate studies in biochemical systems are well illustrated by Gibson's account of haem reactions and the reaction index tabulates 1000 reactions for which rate data are given in the first two volumes of the series. The third volume begins with a theoretical review of biomolecular reactions which includes an original discussion of some of the more obscure points of rate theory. There are articles on hydrogen atom reactions and hot atom chemistry and on the experimental technique of pulse radiolysis. Kinetic data obtained by this method are included. Gas kinetics are represented by a review of the fundamental physical process of vibrational relaxation and inhibition of radical chain reactions. A discussion of linear energy transfer in radiation processes and a review of recent work on the spectra and kinetics of aromatic radicals are given. There are two reviews of the kinetics of polymerization processes in solution; the first deals with polymerization by lithium alkyls and the second with the kinetics of radical polymerization processes. Volume four contains review articles on the reactions of nitrogen atoms and on free radical reactions, dealing with halogenomethyl and alkoxyl radicals. Information is given on electronically excited states, including descriptions of recent progress made in this field. Proton transfer and kiretics of electrode processes are also discussed.

$\# 6500636$

*5.173

Romanoff, M.

UNDERGROUND CORROSION

NBS Circular 579, National Bureau of Standards, Washington, D. C.

April 1957

$231 \mathrm{p}$

Available from the Superintendent of Documents, GPO

This Circular is a final report on the studies of underground corrosion conducted by the National Bureau of Standards from 1910 to 1955. Up to 1922, the studies were confined to corrosion due to stray-current electrolysis and its mitigation. After it became apparent that serious corrosion occurred in soils under conditions that precluded stray-currents as an explanation, a field burial program was initiated in order to obtair information pertaining to the effect of soil properties on the corrosion of metals. More than 36,500 specimers, represerting 333 varieties of ferrous, nonferrous, and protective coating materials, were exposed in 128 test locations throughout the United States. During this time the electrical and electrochemical aspects of underground corrosion have been continuously studied in the laboratory. Results from both field and laboratory investigations are presented.

\# 6508053

Schexnayder, C. J. Jr.

TABULATED VALLUES OF BOND DISSOCIATION ENERGIES, IONIZATION POTENTIALS, AND ELECTRON AFFINITIES FOR SOME MOLECULES FOUND IN HIGH-TEMPERATURE CHEM1CAL REACT IONS

NASA TN D-1791, Langley Research Center, Langley Station, Hampton, Virginia

May 1963

$62 \mathrm{p}$

Available from CFSTI

Values of the bond dissociation energies ionization potentials, and electron affinities that were taken from the literature are presented in tables for some monatomic, diatomic, and polyatomic molecules which are found in many high-temperature chemical reactions including combustion reactions. Much of the information came from literature published after 1950 which either reported 
experimental and theoretical energy values or gave a review of previous literature on the subject.

46910046

Sinnot t, G. A.

BIBLIOGRAPHY OF ION-MOLECULE REACTION RATE DATA

JILA Report No. 9, Joint Institute for Laboratory Astrophysics, Boulder, Colorado

August 15, 1969

$88 \mathrm{p}$

This bibliography extends the JILA Information Center coverage of low energy atomic collision phenomena to include ion-molecule reaction rate data. It was compiled by a search of the open, published literature for those papers that contain original experimental data on ion-molecule reaction cross sections or rate constants. The bibliography is divided into three parts. The first is a listing by reaction equation under the several headings. The second section is a list of references by citation number. The third section is an author index (a11 authors, not only the first).

\#6510037

* $8.373-5.373-3.373$

Somayajulu, G. R. ; Zwolinski, B. J.

A GENERALIŹED BOND ENERGY SCHEME INCLUDING BARRIERS TO FREE ROTATION AND CORRECTIONS FOR ROTATIONAL ISOMERISM

Chemical Thermodynamic Properties Center, Texas AEMM University, College Station, Texas 1965

$21 \mathrm{p}$

A generalized procedure for predicting the energies of the paraffinic hydrocarbons is deduced on the assumption of (i) interactions between pairs of bonds attached to a carbon atom, (ii) interactions between trios of bonds attached to a carbon atom and (iii) interactions between pairs of bonds separated by a $\mathrm{C}-\mathrm{C}$ bond. The derived equation justifies Tatevskii's empirical approach and is shown to be not very different from Allen's equation without the steric terms. Under defined conditions this equation reduces exactly to Allen's equation. Barriers to free rotation were considered in deriving the new equation. This new equation is further modified by including corrections for rotational isomerism in place of trigonal interactions. The new relation is better founded in principle, correctly estimates enthalpies of atomization for normal and isomeric alkanes including those molecules without rotational isomers, and furthermore provides more refined steric terms due to higher order interactions.

$\# 6603012,6603013,6603014 \quad \star 8.373-5.373-3.373$

Somayajulu, G. R.; Zwolinski, B. J.

RELATIONSHIP BETWEEN BOND ENERGY AND BOND DISSOCIATION ENERGY,

II. ENTHALPIES OF" FORMATION OF THE SUBSTITUTED METHANES, SUBSTITUTED METHYL RADICALS AND SUBSTITUTED ETHANES,

III. MAGNITUDES OF THE BOND AND PAIR BOND CONTRIBUTIONS TO THE ENERGIES OF THE MOLECULES,

IV. ENTHALPIES OF FORMATION OF SUBSTITUTED ETHYLENES

Chemical Thermodynamic Properties Center, Texas AGM University, College Station, Texas

August 1965, August 1965, September 1965

$12 \mathrm{p}, 15 \mathrm{p}, 8 \mathrm{p}$

In Part II, the enthalpies of formation of the substituted methanes have been calculated on the basis of Zahn's model. A method has been developed for the calculation of the dissociation energy of the $\mathrm{R}^{\prime}-\mathrm{R}^{\prime \prime}$ bond in a substituted ethane. Based on the dissociation energy of the $R^{\prime}-R^{\prime \prime}$ bond, the enthalpies of formation of the substituted methy 1 radicals and the substituted ethanes have been calculated. The enthalpies of formation of the substituted methyl radicals can also be calculated on the basis of Zahn's mode1. In Part III methods have been developed for the calculation of the magnitudes of the bond and pair bond contributions based on relationships between bond energy and bond dissociation energy. The pair bond contributions for the pair of bonds $C X$ in $C X, C X$, and $C X$, have been determined. Also determined were ${ }^{2}$ the enthalpies of formation of $\mathrm{CX} 2$ and $C S$ radicals, $X$ being $\mathrm{H}, \mathrm{F}, \mathrm{Cl}, \mathrm{Br}, \mathrm{I}$ or $\mathrm{CH}$. In Part IV enthalpies of formation of the $C X$, type radicals obtained in Part III have been used to obtain the enthalpies of formation of the CXY type radicals. Based on a relationship between bond energy and bond dissociation energy, the dissociation energies of the $R^{\prime}-R^{\prime \prime}$ bonds in substituted ethylenes have been calculated. Using the bond dissociation energies and the enthalpies of formation of the substituted methylene radicals, enthalpies of formation of a few substituted ethylenes have been calculated. The calculated values have been found to be in good agreement with the observed values.

\section{\#6500415 *5.213}

Stauffer, C. H. (Project Director)

ALPHABETICAL INDEX TO TABLES OF CHEMICAL KINETICS HOMOGENEOUS REACTIONS

NBS Circular 510, Supplement 2, National Bureau of St andards, Washington, D. C.

August 5, 1960

$37 \mathrm{p}$

Available from Superintendent of Documents, GPO

This Supplement to the Tables of Chemical Kinetics: Homogeneous Reactions, provides a subject index, an alphabetical index according to class of reaction, and an alphabetical index according to class of compound. As in Circular 510, each table is designated by a six-digit number, the first two of which refer to the type of reaction, the third to the phase of the homogeneous reaction, gaseous, liquid, or solid. The second three-digit group of the table number refers to the types of substances involved.

\#6500414,6500416 *5.213

Stauffer, C. H. (Project Director)

TABLES OF CHEMICAL KINETICS - HOMOGENEOUS RFACTIONS (SUPPLEMENTARY TABLES) VOLUME 1 AND VOLUME 2

NBS Monograph 34, Volumes 1 and 2 to accompany NBS Circular 510, Supplements 1 and 2, National Bureau of Standards, Washington, D. C.

Sept. 15, 1961, Volume 1; July 1, 1964, Volume 2 $450 \mathrm{p}, 300 \mathrm{p}$

Available from Superintendent of Documents, GPO

These tables form Supplement 3 to NBS Circular 510 , Tables of Chemical Kinetics: Homogeneous Reactions covering the period 1955-1959, for chemical reactions of the exchange-substitution and elimination type. The tables serve as a critically evaluated compilation of available factual numerical data on rates and rate constants obtained experimentally. The information compiled for each reaction is: amount of each reactant used and name of solvent; amount and name of catalyst used, if any, the rate constant, in terms of natural logarithms, temperature at which the measurement was taken, and a literature reference. For some reactions, the activation energy and Arrhenius constant are also given.

Af 6906006

$$
\text { *5.173-3.173 }
$$

Stevens, B.

COLLISIONAL ACTIVATION IN GASES

New York, London: Pergamon Press

1967

$236 \mathrm{p}$

The International Encyclopedia of Physical Chemistry and Chemical Physics is a comprehensive and current account of alT aspects of the domain of science between chemistry and physics, written for the graduate and research worker. The subject matter is grouped in twenty topics. This third volume on the topic gas kinetics deals with energy 
transfer systems in gas reactions, relaxation techniques, rate measurements of competing processes, low energy level studies, and higher vibrational level energy transfer. Tables give vibrational relaxation times and collision numbers for diatomic molecules; relative collisional deactivation probabilities for diatomic molecules $M$ in presence of additive $X$; vibrational relaxation times, reduced relaxation times, and collision numbers for polyatomic molecules; and relative probabilities for collisional deactivation of polyatomic molecules by additives $x$.

\#6500411,6500413

$\star 5.273$

Thon, N. (Editor)

TABLES OF CHEMICAL KINETICS HOMOGENEOUS REACTIONS

NBS Circular 510 and Supplement 1, National Bureau of Standards, Washington, D. C.

September 28, 1951; November 14, 1956

$750 \mathrm{p}, 450 \mathrm{p}$

Available from Superintendent of Documents, GPO

Circular 510 and Supplement contain a critically evaluated compilation of the available numerical data on rates and rate constants of homogeneous chemical reactions. The emphasis is placed on experimentally ascertained facts, and data depending on interpretations are not generally included. These tables represent a critically evaluated compilation of all the available factual numerical data on rates and rate constants of homogeneous chemical reactions. Stress is laid on experimentally ascertained facts. Data largely contingent on interpretations have generally not been included. In order to put the tabulations in uniform units, original data have been systematically recalculated wherever necessary. Reactions are arranged and numbered in the order of increasing complexity of the key reactant, thus in the case of organic compounds, in the order of increasing numbers of carbon atoms with brached isomers placed after the normal-chain isomers. Literature references and comments are appended to each table. Supplement 1 includes new tables, additions to the published tables, and revised sheets canceling and replacing parts of the present tables. The tables are issued in the form of punched loose sheets for use in suitable loose-leaf binder.

\section{\#6809017 *5.271}

Trotman-Dickenson, A. F. ; Milne, G. S. TABLES OF BIMOLECULAR GAS REACTIONS

NSRDS-NBS-9, National Bureau of Standards, Washington, D. C.

October 27,1967

$129 \mathrm{p}$

Available from Superintendent of Documents, GPO

This survey covers the kinetics of bimolecular and termolecular gas reactions that do not involve atoms or molecules in electronically excited states. Bimolecular reactions are here defined as reactions in which two molecules are involved as reactants, that yield two or more molecules as products. Those reactions in which two molecules combine to form one molecule are most usefully considered as the reverse of unimolecular reactions which will be dealt with in another survey. Reactions of oxygen and nitrogen atoms have been omitted as they will also form the subject of another survey. The literature surveyed covers the period from 1954 to December 31, 1965. The survey of earlier work has been based on one of the writers' books on "Gas Kinetics" which covered the literature to 1954. Use of the book for over ten years has revealed few omissions and these have been included in these tables. Data for the period January to August 1966 have been included where possible.
$\# 6611001$

$\star 3.183-5.183$

Veldre, V. Ia.

IONIZATION OF ATOMS BY ELECTRON BOMBARDMENT

Electron-Atom Collisions RICA

1965

$100 \mathrm{p}$

This is a review of the ionization of atoms by electron impact and of the theory of ionization. The article contains a bibliography of 103 references as well as 54 graphs and 5 tables of data. The article reviews the classical and quantum theory of ionization, the asymptotic behavior of the wave function, Born's Approximation, exchange calculations, calculation of distortion, momentum approximation, ionization from excited states, ionization of the helium atom and of complex atoms and ions, threshold behavior, and empirical formulas.

\section{\#6904006 *5.183}

Venugopalan, M. ; Jones, R. A.

CHEMISTRY OF DISSOCIATED WATER VAPOR AND RELATED SYSTEMS

New York: Interscience publishers

1968

$481 \mathrm{p}$

This text reviews some of the latest $s$ tudies and advances in knowledge of the oxygen-hydrogen system and explains experimental methods of dissociation, trapping, and analysis of dissociated and trapped products from water vapor and related systems. The book includes studies and reaction mechanisms for discharged water and hydrogen peroxide vapors, reaction of discharged hydrogen with oxygen and ozone, and for the products condensed at low temperatures from these systems. Thermal, photolytic, and radiolytic dissociations and mass spectrometric investigations of water and hydrogen peroxide vapors are summarized. The text describes reactions of hydrogen and oxygen pertinent to the chemistry of dissociated water vapor. It includes a discussion on superoxides of hydrogen and present knowledge of the methods of production of hydrogen and oxygen atoms, and of hydroxy and hydroperoxy radicals, their properties, and kinetic information on their reactions. Attention is focused mainly on gas-phase investigations.

\#6905001,6904014

*6.183-5.183

Warner, P. O.; Barry, H. F. (Compilors)

Losey, E.N.; Means, D.K. (Compilors of Supplement) MOLYBDENUM CATALYST BIBLIOGRAPHY (1950-1964) AND SUPPLEMENT NO. 1 (1964-1967)

New York: Climax Molybdenum Company of Michigan, Inc.

1968,1969

$347 \mathrm{p}, 189 \mathrm{p}$

The Molybdenum Catalyst Bibliography and Supplement 1 compile available references to over twenty different types of reactions involving various molybdenum catalysts. The broad types of reactions included in this compilation are hydroforming and reforming; cracking and hydrocracking; hydrotreating, hydrofining and desulfurization; hydrogenation; dehydrogenation; oxidation; reduction; amination and NH3 reactions; hydration, dehydration, hydroxylation, dehydroxylation and hydrolysis; carbonylation-decarbonylation; FischerTropsch synthesis; polymerization; isomerization; isotope exchange; condensation; chlorination; alkylation - dealkylation; catalyst preparation, activation, regeneration and physical properties: sulfurization-sulfonation; and others. Within each section the material is listed according to individual process and type of catalyst used and gives the appropriate reference for such information. 
Colloid and Surface Properties 
\#5906033

*6.023-4.023

Deitz, V.R.

BIBLIOGRAPHY OF SOLID ADSORBENTS 1943 to 1953 AN

ANNOTATIVE BIBLIOGRAPHICAL SURVEY

NBS Circular 566, National Bureau of Standards, Washington, D.C.

March 1, 1956

$1528 \mathrm{p}$

Available from Superintendent of Documents, GPO

This book contains abstracts to 13,763 references which give data and information on solid adsorbents published primarily between 1943 and 1953. Some references to publications before 1943 have been added from the fields of heterogeneous catalysis and surface reactions on textile materials. The coverage is restricted to heterogeneous phenomena at solid-liquid and solid-gas interfaces. The patent literature is not covered. The book is divided into chapters on the general topics of adsorption of gases and vapors on solid adsorbents, adsorption from solutions on solid adsorbents, thermal effects in adsorption processes, theories of adsorption, refining of sugar and other applications of adsorbents, general information on adsorbents and special methods of investigation, and preparation of adsorbents. A subject index, author index, and list of commercial solid adsorbents are included.

\section{\#807003}

*6.233

Gaines, G.L., Jr.

INSOLUBLE MONOLAYERS AT LIQUID-GAS INTERFACES

New York: Interscience Publishers

1966

$386 \mathrm{p}$

This text is the first volume in a series of monographs on physical chemistry designed as a reference source for experimental techniques, data, and ways to interpret results obtained in the study of monolayer behavior. The text includes discussions on the properties of liquid surfaces, experimental methods, properties of monolayer films, mixed monolayers, reactions in monolayers, transfer of monolayers to solids, multilayers, and scientific and technological applications. References to original reports are provided.

\#6609035

Gushee, D.E. (Editor)

CHEMISTRY AND PHYSICS OF INTERFACES

Washington, D.C.: Anerican Chemical Society

Publications

1965

$177 \mathrm{p}$

The papers published in this book were presented at the Symposium on Interfaces sponsored by the Division of Industrial and Engineering Chemistry of the American Chemical Society and Industrial and Engineering Chemistry. The text presents a selection of physicochemical phenomena of broad industrial significance. Chapters include topics on attractive forces at interfaces, gas-liquid and liquid-liquid interface phenomena, solid-liquid phenomena, and solid-gas interface phenomena.

\#6809013

Horiuti, J.; Miyahara, K.

HYDROGENATION OF ETHYLENE ON METALLIC CATALYSTS NSRDS-NBS-13, National Bureau of Standards, Washington, D.C

June 1968

$62 \mathrm{p}$

Available from Superintendent of Documents, GPO

Reaction rate data for the catalyzed hydrogenation of ethylene, primarily in the presence of unsupported metallic catalysts, are critically reviewed. Reaction mechanisms are discussed in detail, and a statistical mechanical treatment of the reaction is given, according to the generalized theory of reaction starting from the well-known procedure of Glasstone, Laidler, and Eyring. Data for single-element catalysts and alloys are included and interpreted, as are data illustrating differences due to the physical form of the catalyst (film, foil, wire, powder, and some supported systems). Problems are discussed concerning reproducibility of experimental results over repeated runs, and as a function of catalyst pretreatment. The data are analyzed in $29 \mathrm{graphs}$ and 29 tables, some of which are very extensive. The bibliography includes 141 references.

$\# 6903047$

$\star 6.143$

Kratohvil, J.P.

LIGHT SCATTERING

Analytical Chemistry Annual Reviews, 38:517R-526R

This is a review of the recent literature giving information on the developments and advances made in respect to the fundamental aspects of scattering of light. It examines theoretical developments, instrumentation and experimontal techniques, basic experimental studies on gases, liquids, and solids, determination of molecular dimensions and solution properties, particle size analysis, turbidimetric titrations of polymers, and nephelometry and turbidimetry. An extensive 1 ist of 338 references is provided.

$\# 6500531$

$* 0.1151-4.151-6.151-8.151$

LANDOLT-BORNSTEIN, NUMERICAL DATA AND FUNCTIONAI RELATIONSHIPS IN PHYSICS, CHEMISTRY, ASTROMOMY, GEOPHYSICS AND TECHNOLOGY, SIXTH EDITION

VOLUME II: PROPERTIES OF MATTER IN ITS AGGREGATED STATES,

PART 3:

FUSION EQUILIBRIA

AND INTERFACIAL

Schaefer, K.; Lax, E. (Editors)

Berlin, New York: Springer-Verlag

1956

535 p.

In German

This volume of the Landolt-Bornstein tables contains data on fusion equilibria and interfacial tensions. The volume includes data from literature published through September 1955. Where several values for one measurement were found, the average was presented. Data on fusion equilibria are presented for binary and ternary systems of inorganic compounds, silicate systems, organic systems, and organic-inorganic systems. Interfacial tension data are presented for pure liquids towards their vapors or air, solutions towards air, two non-miscible systems, and interfacial films on water. Data are also presented on absorption from the gas phase and the liquid phase, and on paper chromatography.

$\$ 6805005$ $* 6.253$

McClellan, A.L.; Harnsberger, H.F.

CROSS-SECTIONAL' AREAS OF MOLECULES ABSORBED ON SOLID SURFACES

Journal of Colloid and Interface Science, 23:577.

599
1967

This article is a tabulation of the cross-sectional area of gas molecules absorbed on solid surfaces. There are basically three ways to calculate these areas - molecular models, van der Waals constants derived from critical data, and 1 iquid density, based on assumption of spherical shape and hexagonal close packing. In general, each method yields a different result for each molecule. The data compiled for each molecule includes a recommended value for the area, calculated by each method, followed by individual results calculated for different absorbents, together with the appropriate literature reference. 
SECOND EDITION)

NBS Miscellaneous Publication 292, National Bureau of Standards, Washington, D.C.

September 1967

$61 \mathrm{p}$

Available from Superintendent of Documents, GPO

A bibliography of Liesegang rings (periodic precipitation figures produced by diffusion) and other periodic structures within the period 18551965 is 1 isted alphabetically according to author. An author index and an index to applications of Liesegang rings to extra-chemical fields are also given. This second edition has added new references, which concern only true Liesegang rings, to the original bibliography of 681 references, many of which dealt with other kinds of periodic structures. The subject and author indices have been appropriately amended. Errors found in the first edition have been corrected. Al1 journal article titles and nearly all book titles have been translated into English. Abbreviations of journal titles are those given in the issue of Chemical Abstracts in which the given reference first appeared.
Losey, E.N.; Means, $\mathrm{K}$. (Compliers of supplement 1 ) MOLYBDENUM CATALYST BIBLIOGRAPHY (1950-1964) and SUPPLEMENT NO. 1 (1964-1967)

New York: Climax Molybdenum Company of Michigan Inc.

1968,1969

$347 \mathrm{p}, 189 \mathrm{p}$

The Molybdenum Catalyst Bibliography and Supplement 1 compile available references to over twenty different types of reactions involving various molybdenum catalysts. The broad types of reactions included in this compilation are hydroforming and reforming; cracking and hydrocracking; hydrotreating, hydrofining and desulfurization; hydrogenation; dehydrogenation; oxidation; reduction; amination and NH3 reactions; hydration, dehydration, hydroxylation. dehydroxylation and hydrolysis; carbonylation-decarbonylation; Fischer Tropsch synthesis; polymerization; isomerization; isotope exchange; condensation; chlorination; alkylation-dealkylation; catalyst preparation, activation, regeneration and physical properties; sulfurization-sulfonation; and others. Within each section the material is listed according to individual processes and type of catalyst used and gives the appropriate reference for such 
Mechanical Properties 
\#6500225

*8, $113-7,113$

American Society for Testing and Materials

ASTM STANDARDS ON THERMOSTAT METALS

Philadelphia, Pennsylvania: American Society for

Testing and Materials

January 1963

$51 \mathrm{p}$

This is an authorized reprint from copyrighted publications of the American Society for Testing and Materials of the Specifications for Thermostat Metal Sheet and Strip (B 388-62 T); Methods of Test for: Flexivity of Thermostat Metals (B 106-56), Modulus of Elasticity of Thermostat Metals (Cantilever Bean Method) (B 223-56), Maximum Loading Stress at Temperature of Thermostat Metals (Cantilever Beam Method) (B 305-56), Resistivity of Metalically Conducting Resistance and Contact Materials (B 63-49), Change of Resistance with Temperature of Metalic Materials for Electrical Heating (B 70-56), Mechanical Torque Rate of Spiral Coils of Thermostat Metal (B 362-62 T), Thermal Jeflection Rate of Spiral and Helical Coils of Thermostat Metal (B 389-62 T), Mean Specific Heat of Thermal Insulation (C 35i-61), and Diamond pyramid Hardness of Metal1ic Materials ( $\mathrm{E}$ 92-57)

\author{
\# 6500221
}

Beatty, G. If.; Ogden, H. R.

STATISTICAL ANALYSIS OF TENSILE PROPERTIES OF HEAT -

TREATED Ti-4Al-3M0-1V AND Ti-2.5Al-16V SHEET

DMIC Report 46J, Defense Metals Information Center, Battelle Memorial Institute, Columbus, Ohio

June 6,1961

$42 \mathrm{p}$

Available from CFSTI, $\mathrm{pB}_{B} 171425$

In connection with the Titanium Alloy Sheet-Rolling program, DMIC has collected and processed tensileproperty data submitted by producers of titanium sheet alloys. These data result from experimental production aimed at speeding the development of high-strength, heat-treated sheet alloys for airframes and missiles. Ultimate strength, yield strength, and total elongation in $2^{\prime \prime}$ of $\mathrm{Ti}-4 \mathrm{Al}-3 \mathrm{MO}-$ IV and $\mathrm{Ti}-2.5 \mathrm{Al}-16 \mathrm{~V}$ are examined in this report. The producers are Crucible Steel Company of America (CSC), Titanium Metals Corporation of America (TMCA), and Mallory-Sharon Metals Corporation (MS).

\# 7001023

$* 7.123-8.123$

Campbe 11, J. E.

PLANE-STRAIN FRACTURE-TOUGHNESS DATA FOR SELECTED METALS AND ALLOYS

DMIC Report S-28, Defense Metals Information Center, Battelle Memorial Institute, Columbus, Ohio June 1968

$26 \mathrm{p}$

Available from $D D C$

plane-strain fracture-toughness ( $\mathrm{K}_{\mathrm{c}}$ ) parameters may be used to estimate critical flaw sizes in structural metals subjected to known stresses at specified temperatures. Previous toughness parameters for evaluating high-strength alloys provided only empirical data that could not be used directly in design. This report contains the first compilation of available $K_{I c}$ data and is the result of considerable interest during the past few years in developing test methods for obtaining these data. The report is divided into sections on aluminum alloys, high-strength alloy steels, intermediate - and low-strength steels, precipitation-hardening stainless steels, titanium alloys, nickel-base Alloy 718 , and beryilium. Data on the aluminum alloys are limited to the 2000 - and 7000 -series alloys. The high-strength alloy steels include the maraging steels, 9Ni-4Co steels, and lower alloy steels such as AISI 4340 , D6ac, $300 \mathrm{M}$, and $H-11$. The intermediate-strength steels include those that have been considered for submarine hulls, atomic-reactor vessels, and steam-turbine rotors. Data for the stainless steels are limited to the precipitation-hardening grades. it 6907030

* 7.122

DATA STORAGE CONTENT OF THE MECHANICAL DATA FILE

Inventory Report 652, Mechanical properties Data Center, Traverse City, Michigan

January 1969

$2 \mathrm{p}$

Available from Mechanical Properties Data Center

This inventory presents an updated accounting of mechanical properties data and related information available from the Center. The data storage file consists of more than 750,000 mechanical properties test records, including associated material and test variables of more than 4000 structural metals and alloys. The chart is constructed listing the quantity of retrievable test results by test type. Types of supplementary descriptive information and results are also tabulated.

\# 6500220

$* 7.123$

Everhart, J. L.; et al.

MECHANICAL PROPERTIES OF METALS AND ALLOYS

NBS Circular C447, National Bureau of Standards, Washington, D. C

December 1, 1943

$481 \mathrm{p}$

Available from Superintendent of Documents, GPO

This circular is a summary of the results of comprehensive survey of the technical literature on the strength and related properties, thermal expansion, and thermal and electrical conductivities of ferrous and nonferrous metals and alloys at normal, high, and low temperatures. In general, the data are presented in tabular form, although graphical representation is often used to indicate the effects of changing composition or conditions on the properties. Data on aluminum, copper, iron and steel, lead, magnesium, nickel, tin, zinc, a number of miscelianeous metals and their alloys are included. The circular is not limited to conventional engineering materials but contains data on the properties of many materials not usually classed as such. Literature references to the sources of the data are included.

\# 6907029

* 7.132

FATIGUE OF MECHANICAL AND WELD JOINTS OF TITANIUM $8-1-1$

Search No. 2169, Mechanica1 properties Data Center, Traverse City, Michigan (No date)

$9 \mathrm{p}$

Available from Mechanical properties Data Center

This folder contains graphs for the fatigue of Ti-8Al-1Mo-V triplex annealed bolted lap joint specinens, for fatigue of Ti-8AI-1Mo-1V annealed and welded sheet specimens, for fatigue of Ti-8Al1 Mo-1V duplex annealed and TIG welded $0.20^{\prime \prime}$ sheet specimens, and for the fatigue of Ti-8Al-1Mo-1V duplex annealed and electron beam welded $0.20^{\prime \prime}$ sheet specimens plotting maximum stress versus cycles of life. Two pages of diagrams of fatigue specimens and a supplementary reference list are included.

\# 6706012

$$
* 4.133-7.133
$$

Grigsby, D. L.

VANADIUM SILICIDE:

Data Sheet DS-154 Electronic properties Information Center, Hughes Aircraft Company, Culver City, California

April 1967

$45 \mathrm{p}$

These data sheets present a compilation of the electrical and electronic properties of vanadiumsilicon compounds, especially $\mathrm{V}_{3} \mathrm{Si}$. These properties include transition temperature, specific heat and Debye temperature, critical field, electrical resistivity, penetration depth and absorption. Other properties included are: superconducting energy gap, magnetic hysteresis and susceptibility, critical current and current 
density, Hall coefficient and thermoelectric power. Three additional sections of this report cover the crystallographic and mechanical properties of $\mathrm{V}_{3} \mathrm{Si}$ as well as the nature of magnetic flux characteristics in the mixed state. Each of these properties is compiled over the widest possible range of parameters from references obtained in a thorough literature search.

\#6907038

$* 7.033-8.033$

Hamer, W, J.

RESUME OF VALUES OF THE FARADAY Journal of $\frac{\text { Research }}{\text { Standards }}$ of A. Pliysics $\frac{\text { the National }}{\text { and }}$ Bureau of July-August $\overline{19} 6 \overline{8}$

A resume is given of the determinations of the value of the Faraday. Values obtained by silver deposition, iodide oxidation, oxalate oxidation, the omegatron, and silver dissolution are reviewed. All values are converted to the unified $\mathrm{C}_{12}$ international scale of atomic weights using the international atomic weights of 1967. Values of the Faraday are given in terms of both the NBS (legal) and absolute units of electrical measure. In the latter the new value for the acceleration due to gravity is used in computing the absolute value of electric current. On this basis and using the atomic weight of silver determined by Shields, Craig, and Dibeler, and converting to the $\mathrm{C}_{12}$ scale, the value of the Faraday is $96,486.9 \pm 1.6$ absolute coulombs per gram-equivalent which differs by only 1 part per million from the value recommended by the National Academy of SciencesNational Research Council. If the atomic weight of silver recommended in 1967 by the International Atomic Weight Commission is used, the Faraday on the new gravity value is $96,486.5 \pm 1.6$ absolute coulombs per gram-equivalent which differs by 5 parts per million from that recommended by the National Academy of Sciences-National Research Council. No change in the value of the Faraday adopted by the NAS-NRC Committee is recommended.

\section{\#6909032}

$* 7.133-2.733$

Hammond, J. P.

PHYSICAL, MECIIANICAL, AND IRRADIATION PROPLRTIES OF THORIUM AND THORIUM ÁLLOYS

ORNL-4218, Oak Ridge National Laboratory, Oak Ridge, Tennessee

April 1968

$71 \mathrm{p}$

Available from CFSTI

Physical and mechanical property data on thorium and thorium alloys were reviewed and information pertinent to nuclear reactor application is compiled.

\section{$\# 6909059$}

Jurevic, W. G.

STRUCTURAL PLASTICS

SUPPLEMENT I. TEXT METHODS

Technical Report AFML-TR-67-332, Supplement I, Air Force Materials Laboratory, Wright-patterson Air Force Base, Ohio

June 1969

$43 \mathrm{p}$

The procedures used for characterizing and evaluating structural plastics are described. Included are methods for determining physical, chemical, thermal, electrical, and mechanical properties.
$\$ 6500538$

* $0.1151-4.151-7.151-8.151$

LANDOLT-BORNSTEIN, NUMERICAL DATA AND FUNCTIONAL RELATIONSHI PS IN PHYSICS, CHEMISTRY, ASTRONOMY, GEOPHYSICS AND TECHNOLOGY, SIXTH EDITION,

VOLUME IV: TECHNOLOGY

PART 1: MATERIAL VALUES AND MECHANICAL PROPERTIES OF NON-METALS

Schmidt, E. (Editor)

Berlin, New York: Springer-Verlag

1955

881 p

In German

This section of the Landolt-Bornstein tables contains material values and mechanical behavior of non-metallic substances, and their technical applications. Data are compiled from literature published through autumn 1964. The following general information is included: measuring systems, units and conversion tables, atomic weights, reduction to standard states, densities of water and mercury. physical and mechanical properties of non-metallic solids are given for natural and synthetic building materials, organic natural materials (wood, paper, cellulose, wood pulp), fibrous materials, ceramics, glasses, synthetic materials, and natural synthetic materials. Also presented are data on friction and rolling resistance of non-metallic and metallic substances, viscosity, flow through tubes, circuitous flow of bodies during two dimensional flow and rough flow, flotation, speed of sound, sound radiation and absorption, the human ear, sound spectra, vibrational numbers, spatial and building acoustics, sound recording and biological effects of ultrasonics.

$\# 6500539$

$* 0.1151-3.151-4.151-7.151-8.151$

LANDOLT-BORNSTEIN, NUMERICAL DATA AND FUNCTIONAL RELATIONSHIPS IN PHYSICS, CHEMISTRY, ASTRONOMY, GEOPHYSICS AND TECHNOLOGY, SIXTH EDITION,

VOLUME IV: TECHNOLOGY,

PART 2a: MATERIAL VALUES AND PROPERTIES OF INDUSTRIAL METALS, BASIC PRINCIPLES, TESTING PROCESSES, INDUSTRIAL STEELS

Borchers, H.: Schmidt, E. (Editors)

Berlin, New York: Springer-Verlag

1963

$888 \mathrm{p}$

In German

This section of the Landolt-Bornstein tables includes data on metallographic principles and concepts, testing properties, and the properties of iron and its alloys. Data are given on diagrams of state, strength testing and destruction free materials. The physical properties of iron tabulated are lattice constants, density, thermal expansion, diffusion, atomic heats, specific heats, enthalpies, entropies and free enthalpies, phase diagrams and transition points, thermal conductivity, electrical properties, magnetic properties, optical constants and excitation crosssections. Composition, treatment, strength values and special chemical properties of pure iron, unalloyed steels, highly alloyed steels, special steels, and cast iron are presented.

\#6510029
LANDOLT-BORNSTEIN, NUMERICAL DA1.A AND FUNCTIONAL

*0.1151-4.151-7.151 RELATIONSHIPS IN PHYSICS, CHEMISTRY, ASTRONOMY, GEOPHYSICS AND TECHNOLOGY, SIXTH EDITION,

VOLUME IV: TECHNOLOGY

PART 2b: MATERIAL VALUES AND PROPERTIES OF INDUSTRIAL METALS, SINTERED INDUSTRIAL MATERIALS, HEAVY METALS (WITHOUT SPECIAL INDUSTRIAL MATERIALS) Borchers, H.; Schmidt, E. (Editors) Berlin, New York: Springer-Verlag

1964

$1000 \mathrm{p}$

In German

This section of the Landolt-Bornstein series deals with numerical data and behavior of both metallic and powdered industrial materials. The materials covered include the metals tungsten, tantalum, nio- 
bium, vanadium, chromium, cobalt, nickel, manganese, gold, silver, copper, antimony, zinc, cadmium, lead, bismuth, tin, and their alloys. Data are provided for the physical and mechanical properties of these materials, their corrosion behavior and some of their applications to technology.

\#6605008

${ }^{*} 0.1151-4.151-7.151$

LANDOLT - BORNSTEIN, NUMERICAL DATA AND FUNCTIONAL RELATIONSHIPS IN PHYSICS, CHEMISTRY, ASTRONOMY, GEOPHYSICS AND TECHNOLOGY, SIXTH EDITION,

VOLUME IV: TECHNOLOGY,

PART 2C: MATERIAL VALUES AND PROPERTIES OF INDUSTRIAL METALS, ALKALINE EARTHS, SPECIAL INDUSTRIAL MATERIALS, SEMICONDUCTORS, CORROSION

Borchers, H.: Schmidt, E. (Editors)

Berlin, New York: Springer-Verlag

1965

$976 \mathrm{p}$

In German

This section is the third part in a series of Landolt-Bornstein tables presenting the physical properties and behavior characteristics of most of the 75 elements in the periodic system exhibiting metallic characteristics as we 11 as data concerning their alloys. Special emphasis is directed toward light metals, special work metals, binding materials, enamel properties, corrosive behaviors, reactor metals, metals of the rare earths, semiconductors, and current developments in binary alloy systems. The volume is divided into sections entitled: titanium, beryllium, alùminum, magnesium, lithium, rubidium, cesium, liquid metals, general survey of reactor metals, uranium, plutonium, zirconium, hafnium, thorium, rare earths, semiconductors, bearing metals, enamels, solder, welding and cutting metals, metal adhesives, and corrosive behavior of materials.

$\# 6611002$

$\star 7.153$

Larson, M. L.

EXPER IMENTAL MOLYBDENUM CHEMICALS

Chemical Data Sheets EX-1 to EX-14, Chemical Data Bulletins Cdb-1 to 13, Climax Molybdenum Company, New York, New York

March 1964 -September 1966

$200 \mathrm{p}$

This is a collection of bulletins giving experimental data, physical properties, and references for various molybdenum compounds: The period covered is from March 1964 to September 1966.

$\# 6500227$

$\star 7.253$

McClintock, R. M.; Gibbons, H. P.

MECHANICAL PROPERTIES OF STRUCTURAL MATERIALS AT LOW TEMPERATURES, A COMPILATION FROM THE LITERATURE NBS Monograph 13, National Bureau of Standards, washington, D. C.

June 1960

180 p

Avallable from Superintendent of Documents, GPO

This compilation was taken from about 100 journal articles and technical reports on the mechanical properties of structural materials at low temperature. It is published as an aid in the design of cryogenic equipment. The tensile strongth, yield strength, tensile elongation and impact energy of about 200 materials, metallic and nonmetallic, are given graphically as functions of temperature between $4^{\circ}$ and $300^{\circ} \mathrm{K}$. The classes of materials considered are: aluminum, copper, nickel, titanium and their alloys; magnesium alloys, stainless steels, construction steels, super alloys, brazing and soldering alloys, miscellaneous alloys and pure metals, and non: metallic materials.
\$6706021,6805006,6708017,6903040 $\star 7.211 \cdot 8.211$ MATERIALS PROPERTIES DATÁ BOOK

Report 2275, Aerojet-General Corporation, Azuza, California

Ju1y 31,1968

$713 \mathrm{p}$

The Materials Properties Data Book provides noncritical reference data of materials properties for use by persons involved with the analysis, design, fabrication or test responsibilities of the NERVA Project. The Data Book includes physica1, mechanical and chemical properties of interest for many materials of significance to the NERVA Program and, in addition, data on the effects of irradiation on materials. Information is also provided concerning material processing, with special emphasis on specifications. Material suppliers are given. The Data Book has been organized for the most effective use of the data by the design engineer. Volume I contains light metal alloys (aluminum, titanium, etc.) data. Volume I-A contains nicke1-base alloys, refractory metals and alloys, and other nonferrous metals and alloys (cobalt, copper, etc.) data. Volume 2 contains ferrous alloys data. Volume 3 contains nuclear materials (fuels, neutron controls, beryllium, etc.) graphites, refractory ceramics, adhesives and plastics data. The section for each individual material contains descriptive basic data sheets with pertinent data relative to properties, characteristics and other related information, together with curve sheets for graphic presentation of the parameters.

\section{$\$ 6500222 \quad * 7.163$}

Ogden, H. R.; Beatty, G. H.; Mace, A. E.

STAT IST ICAL ANALYS IS OF TENSILE PROPERT IES OF HEAT TREATED Ti-4Al-3Mo-1V SHEET

DMIC Report 46I, Defense Metals Information Center, Columbus, Ohio

September 16, 1960

$40 \mathrm{p}$

Available from CFSTI, PB 151095

The Defense Metals Information Center has collected data generated in Phase I of the Titanium-Alioy Sheet-Rolling Program. The program is designed to develop the commercial production of four titanium alloys. This report presents the first of the analyses made to determine relationships between factors affecting the properties of the titanium alloys. The report is confined to the tensile properties of only one of the four alloys in the program, Ti-4A1-3Mo-1V. The report is divided into two parts. The first part examines the variance in tensile properties of the alloy. The second part examines some of the correlations between properties and processing variables.

\# 6908020

Rimshaw, S. J.; Ketchen, E. E.

CESIUM-137 DATA SHEETS

ORNL-4186, Oak Ridge National Laboratory, Oak Ridge, Tennessee

December 1967

27p

Available from CFSTI

Data on CS-137 are tabulated. Properties of the fuel forms $\mathrm{CsCl}, \mathrm{Cs}_{2} \mathrm{SO}_{4}$ and cesium borosilicate glass are given. Twenty-seven references are included.

\#6908021

*2.2773-4.173-8.173-7.173

Rimshaw, S. J,; Ketchen, E. E.

CURIUM DATA SHEETS

ORNL-4187, Oak Ridge National Laboratory, Oak Ridge, Tennessee

December 1967

$52 \mathrm{p}$

Available from CFSTI

Data on the fuel forms of $\mathrm{Cm}-244$ are presented. Properties of Cm-244 metal, $\mathrm{Cm}_{2} \mathrm{O}_{3}, \mathrm{Cm}_{2} \mathrm{O}_{2} \mathrm{~S}, \mathrm{CmF}_{3}$, 
$\mathrm{Cm}_{\mathbf{m}}-242, \quad 242 \mathrm{Cm}_{2} \mathrm{O}_{3}$ cermet are tabulated. Forty-nine references are given.

6908022 $\star 2.2773-4.173-8.173-7.173$

Rimshaw, S. J.; Ketchen, E. E.

STRONTIUM- 90 DATA SHEETS

ORNL-4188, Oak Ridge National Laboratory,

Ridge, Tennessee

December 1967

$45 \mathrm{p}$

Available from CFSTI

Data are given on composition, specific power, radiation, critical mass, container compatability, thermophysical properties, mechanical properties, chemical properties, biological tolerance, and shielding for strontium-90 metal, titanate $\left(\mathrm{SrTiO}_{3}\right)$, oxide $\left(\mathrm{SrO}_{3}\right)$, fluoride $\left(\mathrm{SrF}_{2}\right)$, and orthotitanate $\left(\mathrm{Sr}_{2} \mathrm{TiO}_{4}\right)$. The data are indexed to 53 references which are also included.

6909007

$* 7.173-4.173$

Rittenhouse, J. B. : Singletary, J. B.

SPACE MATERIALS HANDBOOK, THIRD EDITION

AFML-TR-68-205, Air Force Materials Laboratory, Wright-Patterson Air Force Base, Ohio

July 1968

$742 \mathrm{p}$

Available from the DDC

This edition is the result of an extensive revision and reworking of the second edition of the Space Materials Handbook along with the incorporation of entirely new subject matter coverage and new materials data. All of the most significant material, phenomena, properties and principles covered in the original Handbook are presented and expanded in this revised and updated version. However, treatment of theoretical aspects has been condensed in order that more emphasis could be placed on the extensive new materials knowledge and data obtained from the design and successful launching of a wide variety of space systems. The Handbook is organized into four parts, namely: space enviromment, effect of space environment on materials, materials in space and biological interaction with spacecraft materials. Information on mechanical, physical and chemical properties and characteristics is given for a wide variety of metallic and nonmetalic materials. The effects of natural and induced environments on materials are appraised. Materials categories include coverage of thermal control materials optical materials, adhesives, organic structural materials, inorganic structural materials, electronic components and materials, materials for sealing applications and lubrication materials. In addition, a comprehensive multiple citation index is incorporated which gives ready access to information on specific subject areas with regard to their locations within the Handbook.

$\$ 6500223,6500224$

$\star 7.173-4.173$

Schmidt, F. F.; Ogden, H. R.

ENGINEERING PROPERTIES OF TUNGSTEN AND TUNGSTEN ALLOYS, ENGINEERING PROPERTIES OF MOLYBDENUM AND MOLYBDENUM ALLOYS

DMIC Reports 191 and 190, Defense Metals Information Center, Battelle Memorial Institute, Columbus, Ohio

September 1963; September 1963

$133 \mathrm{p}, 288 \mathrm{p}$

Available from DDC

These two reports present the results of a stateof-the-art survey covering tungsten, molybdenum and 19 of their alloys. All data are given in tabular and graphical form covering some of the more important physical, mechanical and metallurgical properties for each material. For both reports, the physical properties included are melting point, density, thermal expansion, thermal conductivity and electrical resistivity. The mechanical properties included are tensile properties (ultimate tensile strength, yield tensile strength, elon- gation, reduction in area, modulus of elasticity) and their temperature dependence, notched tensile properties, creep and stress-rupture properties, and other selected mechanical properties. Metallurigcal properties include: fabricability, transition temperature, weldability, stress-relief temperature and recrystallization temperature. The ten alloys considered in the tungsten properties report include "doped" tungsten and various alloys with niobium, thorium dioxide, tantalum carbide, molybdenum and rhenium. The nine alloys considered in the molybdenum properties report include alloys with niobium, zirconium, titanium, tungsten and carbon. References are given at the conclusion of each materials section.

\#6809025-6809028

Schwartzberg, F. R.; et al

CRYOGENICS MÁTERIALS DATA HANDBOOK

Technical Documentary Report No. ML-TDR-64-280 and Supplements $1,2, \& 3$; The Martin Company, Denver Colorado

Aug. 1964, Feb. 1965, July 1965, March 1966

$400 \mathrm{p}, 130 \mathrm{p}, 130 \mathrm{p}, 130 \mathrm{p}$

Available from CFSTI; AD $609562, A D 611$ 165, AD $618065, \mathrm{AD} 633388$

This is a revision of the previously published "Cryogenic Materials Data Handbook" and its supplements with the CFSTI designation PB 171809. It is organized into sections of aluminum, stainless steel, titanium, superalloys, alloy steels, miscellaneous metals and alloys, polymeric materials and fiber-reinforced plastics containing mechanical and physical property data on 62 metallic and nonmetallic materials. The properties measured include yield, tensile, notch tensile, weld tensile, impact, compressive, fatigue, shear and flexural strengths; elongation; reduction of area; fracture toughness; stress-strain diagram; modulus of elasticity and rigidity; hardness compressive, shear and flexural moduli; thermal expansion; Poisson's Ratio; thermal conductivity; and resistivity. The Handbook contains material, property and accumulative indices and a reference 1ist. Progress reports are issued semiannually to update and maintain the Handbook. The first Supplement to the Cryogenic Materials Data Handbook updates the publication to February 1965. The second Supplement updates it to July 1965, and the third to March 1966.

$\# 6909061$

Seddon, B. J. (Compiler) PHYSICAL PROPERTIES OF COMPOUNDS: A DATA MANUAL

TRG-Report 1601, United Kingdom Atomic Energy Authority, Reactor Group, Risley, England December 1967

$54 \mathrm{p}$

Available from CFSTI

This report is a compilation of the best available data on the physical properties of some of the plutonium compounds which are, or may be, of interest as fuel materials. These are the dioxide, monocarbide, mononitride, monosulfide and monphosphide of plutonium, uranium-plutonium dioxide, uraniumplutonium monocarbide, uranium-plutonium mononitride, and the plutoniun silicides.

$\$ 6907032$

*7.171

SELECTED BIBLIOGRAPHY, MECHANICAL PROPERTIES OF Ti6AL - 4 V REVISED

Chapter 3707, Ti-6Al-4V Revised, Mechanical Properties Data Center, Traverse City, Michigan 1969

$102 \mathrm{p}$

Available from Mechanical Properties Data Center

This is a set of computer printout sheets giving the titles and sources of selected references to the literature for data and information on the mechanical properties of $\mathrm{Ti}-6 \mathrm{Al}-4 \mathrm{~V}$. 
\#6907031

$\star 7.171$

TENSILE PROPERTIES OF UDIMET 700

Search Number 2180, Mechanical Properties Data

Center, Traverse City, Michigan

1969

$12 \mathrm{p}$

Available from Mechanical Properties Data Center
This report contains computer printout sheets of data on the tensile properties of Udimet 700 at room and elevated temperatures including the primary operation, secondary operation, heat treatment, specimen configuration, specimen thickness or diameter, notch configuration, orientation, test environment amount, ultimate tensile strength, yield stress, gage length, percent elongation, percent reduction in area, along with the reference source. 
The rmodynamic and Transport Properties 
\#6910007

$* 4.113=3.113$

Abrikos ov, I. lih.; et al.

Ty lbulewicz, A. (Translator)

SEMI CONDUCTIMC II-VI, IV-VI, AND V-VI COMPOUNDS

New York: Plenum Press

1969 (1967)

$260 p$

Translatec fron Tussian

This monograph deals with the semiconducting compounds formed from olements of groups II, IV, or $V$ and sulfur, selenimi, or tellurium. The monograph describes the crystal structure of these compounds, and presents the pulblished data on their polynorphic transitions, including transitions obscrved at ligh pressures. The monograph includes published $\mathrm{P}-\mathrm{T}-\mathrm{X}$ diagrans for the compounds discussed and describes a method for plotting the diagrams. The more important physiocherical properties of the compounds are also given: the forbidden band width, the carrier mobility, the effective masses of carriers, the electrical conductivity, and the themoelectric power. The monograph consists of tliree chanters. The first deals with chalcogenides of elements in group II: zinc, cadium, and mercury. The second discusses semiconducting compounds of elenents in group IV: germanium, tin, and lead. And the third descrihes investigations of compounds of elenents of group $V$ : arsenic, antimony, and bisnuth. The monograph does not cover chalcogenides of elements in group II . Also excluded are data on scmiconducting glasses. The nonograph includes the published data up to the second half of 1966 on the crystal structure, physicochemical propertios and phase diagrams of important seniconducting compounds which are used in various hranches of engineering.

\section{\#6909067-6900068}

$* 3.113-4.113$

Ageev, N. V. (Jiditor)

Schnorak, J. (Translator)

ILANDBOOK OF BINARY PIETALLIC SYSTEMS, STRUCTURT AND) PROPERTIES, PIYSICOCFEMICAL PROPERTIES OF TIL ELE!IENTS,

VOLUME I: SYSTEMS OF ACTINIUM, ALUMINUM, AMERICUM, BARIUM, BERYLLIUM, BORON, ARD NITROGEN,

VOLUME II: SYSTEMS OF BISMUTH, DYSPROSIUM, EUROPIUM, GADOLINIUP, GAILIUM, GERMANIUM, ILFNIUM, HOLMIUY, IYDROGEN, IRON, TUNGSTON, AND VN'NADIJN

IPSAT cat. nos. 1711 and 1712, Israel program for Scientific Translations, Jerusálem, Israel 1967 (1959) - Vol. 1; 1967 (1962) - Vol. 2 $645 \mathrm{p} ; 887 \mathrm{p}$

Available from CFSTI, TT 66-51149 and TT 66-51150 Translated from the Russian

These books are the first two volumes of a reference source containing information on phase diagrans, crystalline structure and physical and chemical"properties of binary metallic systers. The first volume includes phase diagrans of systems constituted hy actinium, aluminum, arericum, harium, beryllium, horon, and nitrogen; the second volume includes phase diagrams of the systems constituted hy bismutl, dysprosiun, europium, gadolinium, gallium, gérmaniun, hafníu, holmium, hydrogen, iron, tungsten, and vanalium. The second constituent is eacl elenent of the periodic systen (except for noble gases) in alphabetical order. subject to the extent of the present knowledge of each system, the following data are given: 1) phase diagrans and crystalline structures; 2) physical properties (heat of melting or heat of mixing, surface tension and viscosity, boiling point, density, mechanical propertiés, heat expansion, heat capacity, thermal conductivity, electrical resistance and its temperature coefficient, enf, magnetic properties); 3) chemical properties (resistance to corrosion under atmospheric conditions, and in various corrosive media and in gases); 4) a list of literature references.
\#6902012,6902051,6902052 $\div 0.5413-3.213$

Ane rican Institute of Chenical Engineers

AIChE PIISICAI, PROPERTY ESTIILATION SYSTRM, VOLUME I: CIADTISR 8: SURFACE TENSION (ROUGH), CIAAPTER 9: VISCOSITY; VOLUMII: II: USER'S VAVUAL; VOLUIE III : SYSTEYS MANUAL

New York: Arthur D. Little, Inc.

September 1965

$600 \mathrm{p}$

These loose-leaf documents, the AIChE Piysical Property Istination System, are manuals directed primarily at computer oriented staff who are familiar with the operation of the computer system on which prograns are compiled and executed. Volume II instructs the user on how he can commicate with the estination syster. The input requirenents, alternatives, and options are described together with thie various outputs that the system can generate. Volume II further describes how the system programs are delivered and how they can be compiler, assembled into a system, and executed on a computer. Volume II discusses the process of updating the system, system table requirements, the suhject of transfer function link construction, and the passing of information from program to program through a pass tape system. This volume contains a functional descrintion and flow charts for the main system programs. The layout of common storage for cach main progran is also shown. Chapter 8 discusses the reliable techniques to estimate surface tensions of pure liquids, the variation of surface tension with tcmperature and pressure, and surface tension estination methods for non-aqueous mixtures and aqueous mixtures. Interfacial tensions are briefly considered. Chapter 9 deals with the viscosity of gases and liquids. Methods are recomended for correlating low-pressure viscosity data with temperature estinating viscosities when no experimental data are available, estinating the effect of pressure on viscosity, and estimating the viscosities of rixtures. The rolecular theory of viscosity is also considered.

\section{\#6912056}

*8. 212

American Society of Ileating, Refrigerating, and Air-Conditioning Engineers, Inc.

ASIIRAE TIIERIODYNNIC PROPERTIJS OF REFRIGTRNTS

New York: American Society of Teating, Refrigerating, and Air-Conditioning Ingineers, Inc. 1969

$329 \mathrm{p}$

This new puhlication, ASIRNE, Thermodynamic Properties of Refrigerants, has been compiled to provide comprehensive coverage of the thermodynamic properties of a broad selection of refrigerants in a single authoritative reference source. It includes hoth saturation and superheat tables together with appropriate thernodynamic charts. The tables cover the ranges of temperature and pressure of normal interest for each fluid. Refrigerants are identified hy numer in accordanco with ASIIRAE Standard 34-67 USA Standard B79.1. The refrigerants are in four principal classifications: (a) commonly used commercial refrigerants, (h) specialized fluorocarbon refrigerants, hydrocarbons, (d) cryogenic fluids.

\section{\#6500225 *8.113-7.113}

American Society for Testing and latcrials

AST:! STANDARDS ON TIERMOSTAT MTSTLS

Philadelphia, Pennsylvania: Arerican Society for Testing and Materials

January 1963

$51 \mathrm{p}$

This is an authorized reprint from copyrighted publications of the American Society for Testing and Materials of the Specifications for Thermostat Metal Sheet and Strip ( $3388-62 \mathrm{~T}$ ); liethods of Test for: Flexivity of Thermostat Metals (B 106-56), Modulus of Elasticity of Thermostat Metal's (Cantilever Beam Method) (B 223-56), Maxinum Loading Stress at Temperature of The rmostat Metals 
(Cantilever Beam Method) (13 305-56), Resistivity of lietalically Conducting Resistance and Contact Materials (B 63-49), Change of Resistance with Temperature of ifetallic llaterials for Electrical Heating (3 70-56), Mechanical Torque Rate of Spiral Coils of Thernostat Metal (B 362-62 T), Thermal Deflection Rate of Spiral and llelical Coils of Thermostat lletal (B 389-62 T), Mean Specific lleat of Thermal Insulation (C 35i-61), and Diamond Pyranid !lardness of letallic Waterials ( $E^{92-57)}$.

\#6500458 *8.311

American Society for Testing Materials

AST:1 VISCOSITY INDEX CALCULATEN FROM KINEMATIC VISCOSITY

ASTil Special Technical publication No. 168 , American Society for Testing :Aaterials,

philacielphia, Pennsylvania

June 1955

$832 \mathrm{p}$

The tables in this publication permit direct reading of the Viscosity Inclex of a petroleum or Iubricant if its kinenatic viscosities at $100 \mathrm{~F}$ anc $210 \mathrm{~F}$ are known. Use of these tables eliminates the calculation required for determining Viscosity Index.

\#6500302

$$
\text { * } 8.311
$$

American Society for Testing "Iaterials Committee $\mathrm{D}-2$ on Petroleur Products and Lubricants PIYSICAL CONSTANTS OF INDROCARBONS C- I TO C-10

AST:1 Special Technical puhlication No. 109 , American Society for Testing and Materials, Philadelphia, Pennsylvania

July 1963

$69 \mathrm{p}$

The tabulation furnishes constants in both the English engineering and metric systens of measurement. The major sources of data for this tabulation are the findings of Research Project 44, Data on Ilydrocarbons and Related Compouncs, and Research Project 45, Synthesis, Purification, and properties of llydrocarbons of Low Molecular Weight, of the American Petroleum Institute. These data are either critically selected, precisely calculated or deened to be of good experimental quality. Nata included from other sources are shown in italics. Data calculated for this compilation are shown in parentheses. The atomic weights used are hased on oxygen $=16.000$, carbon $=$ 12.01 , and hydrogen $=1.00 \mathrm{~s}$. The recent changes in the atoric weight scale are so small that for enginecring use the change in the atomic weight scale is not important.

\#6910013

$$
* 8.113
$$

Aroeste, II.; Magee, J. L.

THER'AAL RANIATION PIIENORTIA, VOLUIE 3. TABLES OF PADIATIVE PROPIRTIES OF AIR

DASA 1971-3, Lockheed Missiles and Space Cornany, palo $\Lambda 1$ to Research Laboratories, Palo M1to, California

May 1967

6330

Available from CFSTI, AD 654773

This volune presents tables of the equilibriur radiative properties of air and its constituents for a wide range of temperatures and densities. The work is divided into five parts: part $A$ is a sumary section desimed to give an overview of the field. Some of the data are presented in semischeratic form and should not he used for quantitative work. part $B$ gives basic data and references used in the preparation of the subsequent parts. Finally, parts C, D, and $D$ contain the absorption coefficients in the ranges $1000^{\circ} \mathrm{K}$ to $24,000^{\circ} \mathrm{K}$ (Part C), $1 \mathrm{eV}$ to $20 \mathrm{eV}$ (Part D), and above $20 \mathrm{eV}$ (Part E).
Bain, R.

STEAII TABLES 1964 PII'SICAL PROPERTIES OF WATER AUID STEA:I $0-300^{\circ} \mathrm{C} \cap-1000$ BARS

Edinburgh, Scotland: Iler 'lajesty's Stationery office

1964

$147 p$

These tables, prepared at the National Engineering Laboratory of the Nepartment of Scientific and Industrial Research, provide physical properties of water and stean. The tables are divided into three parts: Table 1 contains values of the saturation pressure and of the specilic volume, specific enthalpy and specific entropy of the saturate liquid and vapor at round values of temperature. Table 2 contains the sare quantities at round values of pressure. Table 3 contains values of the specific volume, specific enthalpy and specific entropy of both liquid and yapor at equally spaced intervals in the temperature range $0-300^{\circ} \mathrm{C}$ and at varyingly spaced intervals in the pressure range 0 1000 bars. The international units nsed are those accepted for the international skeleton tahles.

\#6512015

Ballard, B. E.; Nelson, E.

ABSORPTION OF IIIPLANTED SOLID DRUG

Journal of Pharmaceutical Sciences, $51: 915-924$

Factors that clearly affect absorption rates of implants are their surface area and solubility in body fluids. Factors proposed that do not affect absorption rate greatly (if at all), with the present information, are pellet density (when area corrections are made), crystal size used in implant preparation, phagocytosis, physiological need or sex of the aninal, encapsulation, "ghost" formation, and the age of the aninal (if it is not young). Factors such as site of inplantation an hody movement and diluents do have an effect, hut little quantitative information is available concerning the magnitude of the effect.

\#6907020 *3.113-4.113-8.113 Ballard, S. S.; McCarthy, K. A.; Wolfe, N. L. IRIA Report 2389-11S, Michigan University, Willow Run Laboratories, Ann Arbor, Michigan January 1959

$113 p$

Available from DDC, AD 217367

The properties of approximately fifty materials Which are useful in optical instrumentation in the infrared portion of the electronagnetic spectrun are describer and compared. Each material is discussed in detail in terns of its optical, chenical, thermal, and mechanical properties. Tables and figures are given so that the properties of the various materials can he readily compared.

\#6907021

*3.113-4.113-8.113

Ballard, S. S.; McCartly, K. A,; Nolfe, w. L.

OPTICAI, MATTRIALS FOR INFRARFD INSTRUIENTATION, SUPPLEMTINT

Report 2389-II-S, Michigan Iniversity; Infrared Laboratory, Ann $\Lambda$ rbor, Michigan

ApriI 1261

$18 \mathrm{p}$

Available fron CFSTI, AN 255699

In this supplement, additional data are compiled on the optical materials, which are useful for infrared instrumentation, treated in 2339-11-S, as well as data on three new materials, Irtran-1, Irtran-2, and diamond. In addition, several errors and anbiguities in the original report are corrected and clarified. 
Bauer, T. "..; Bookett, C. W.; Johnston, 11.

CALORIMETRY AT LOW TEYPI:RATIJRES

Technical Report No. F-TR-2262-IA, Ohio State University, Cryogenics Laboratory, Columbus, Ohio April 1949

$200 \mathrm{p}$

This paper is a review of seven hundred and ten articles on low temperature calorimetry covering the period from 1763 to 1948 . A historical review is given: the main calorimeter types are described briefly; and the thermal data on all substances measured have been collected in tahular form. Author and formula indexes have been constructed.

\#6500495

*8. $113-4.113$

Baure $\mathrm{r}, \mathrm{T}$.

SOME EXPERIMENTS ON TIIE DEPOSITION OF GASFS AT $4.2 \mathrm{~K}$ NBS Technical Note 73, National Bureau of Standards, Washington, D. C.

October 1960

$32 \mathrm{p}$

Available from CFSTI

In order to develop some preliminary information concerning the very basic problens of gaseous deposition at extremely low temperatures, samples of argon, nitrogen, oxygen, and hydrogen were deposited at $4.2 K$, and variations in some of the parameters associated with these depositions were observed. In addition, samples of the same gases were passed through an excitation zone prior to deposition, and differences in the deposition behavior were recorded. The pressure downstream of the deposition region and the temperature within the deposited solids were found, in general, to increase with flow rate of the depositing substances, and to vary with time in a manner depending on the density, flow rate, and state of excitation of the incoming gases. Árrhenius curves were plotted using warm-up data observed in these experiments and solid-phase transition data determined elsewhere. Variations in the positions of these plots were sometimes the result of differences in the conditions of deposition, and at other times an effect of the chemical nature of the gas studied. The experimental behavior of hydrogen, relative to that of the other gases studied, was observed to be strongly dependent upon its much higher thermal conductivity.

\#6500306

*8.113

Blake, C.

TABLES FOR TIIE ISENTROPIC EXPANSION OF IDFAL GASES WITH CONSTANT RATIO OF SPECIFIC IIT:ATS, GAMIA: PART I. GAMMA $=1,150$

Téchnical Memorandum 283, Rocket Propulsion Establishment, Westcott, England

June 1963

$45 \mathrm{p}$

Data relating to the isentropic one-dimensional expansion of an ideal gas with a constant ratio of specific heats are presented in tabular form. In part 1, the tables are calculated for the value $Y=1.150$.

\#6902005

* 4.113-3.113-8.113

Berezhnoi, A. S.

SILICON AND ITS BINARY SYSTTMS

New York: Consultants Bureau

1960

$275 \mathrm{p}$

Translated from Russian

This text, translated from Russian, is a review of the properties of silicon and its binary compounds. The text discusses the preparation of binary silicon compounds, properties and uses of II-Si and Halogen-Silicon systems, systems formed hy silicon with elements of Groups IA, IB, IIA, IIB, III, IVB, VB, VIB, transition metals of 'Groups IV, V', VI, VII, and VIII, and with metals of the iron subgroup, palladium suhgroup, platinum subgroup, lanthanides and actinides. Ternary and quaternary systers are also included. There is a list of 716 references and tahles of data accompany the systems discussed.

\# 6907014 *3.172-4.172

BIBLIOGRAPIYY, LOW-TEMPERATURF SPECIFIC HTAT

Research Materials Information Center, Oak Ridge National Laboratory, Oak Ridge, Tennessee Septeriber 1968

$42 p$

This bibliography contains 452 references of $10 \mathrm{~W}$ temperature, specific heat data and information for elements, compounds or alloys, of all elements having atomic numbers 1 to $84,90,92$, and 94 , with the exception of the inert gases and technetium. The bibliography is accompanied by an index of elements and compounds.

A 6908045

*4.213-8.213-3.213

Bondi, A. A.

PIYSICAL PROPERTIES OF NOLTCULAR CRYSTALS, LIQUIDS, AND GLASSES

New York: John Wiley\& Sons, Inc.

1968

$502 \mathrm{p}$

This book developes a methodology enabling chemists and cherical engineers to relate certain physical properties of condensed phases to molecular structure. The states of matter treated are molecular crystals, liquids, and glasses. The physical properties covered are $\mathrm{P}-\mathrm{V}-\mathrm{T}$ and related thermal properties, and certain transport properties. Energy of vaporization, van der Waals dimensions, molar refractivity, dipole moment data and dielectric loss are used but not discussed thoroughly. Tables 14.1 to 14.16 contain the requisite molecular structure increrents. Background material presented is a guide to the literature.

\#7001037 *8.113

Brahinsky, II. S.; Neel, C. A.

TABLES OF EQUILIBRIUM THIERMODYNAMIC PROPTRTIES OF AIR VOLUME I. CONSTANT TEMPERATURE

AEDC-TR-69-39 Volume 1, Arnold Engineering Development Center, ARO, Inc., Arnold Nir Force Station, Tennessee

Apri 11969

$152 \mathrm{p}$

Available from DDC

Equilibrium thermodynamic data are presented for air from a temperature of 100 to $15,000^{\circ} \mathrm{K}$ and from a $\log \rho$ of -7.0 to 3.0 , where $p$ is density in amagats. These data are tabulated at constant temperature with density incremented. The dependent parameters are $Z, E / R T, I I / R T, S / R, \log P$, and $z^{*}$. Several sources were used in assembing the data.

\section{\#7001038 $\quad$ *3.113}

Brahinsky, !. S.; Nee1, C. A.

TABLES OF' EQUILIB́RIUM THERMODYNAMIC PROPERTIES OF AIR VOLUME II. CONSTANT PRESSURE.

AEDC-TR-69-39 Volume 2, Arnold Engineering Development Center, ARO, Inc., Arnold Air Force Station, Tennessee

April 1969

$268 \mathrm{p}$

Available from DDC

Equilibrium themodynamic data are presented for air from a $\log p$ of -7.4 to 4.8 and from a temperature of 100 to $15,000^{\circ} \mathrm{K}$, where $\mathrm{p}$ is pressure in atmospheres. These data are tabulated at constant pressure with temperature incremented. The dependent paraneters are $\log H / R, \log \rho, Z$, $S / R, H / R T$, and $Z$ *. Several sources were used in assembling the data. 
7001033

$\star 8.113$

Brahinsky, H. S.; Neel, C. A.

TABLES OF EQUILIBRIUY THER'IODYNAIIC PROPERTIES OF AIR VOLUIIE III. CONSTANT ENTPOPY

AEDC-TR-69-39 Volume 3, Arnold Engineering Development Center, ARO, Inc., Arnold Air Force Station, Tennessee

April 1969

$434 p$

Available from DDC

Equilibrium thermodynamic data are presented for air from an $S / R$ of 13.2 to 133.0 and from a temperature of 100 to $15,000^{\circ} \mathrm{K}$, where $S / R$ is dimensionless entropy. These data are tabulated at constant entropy with temperature increnented. The dependent parameters are log $\log \mathrm{P}, \log$ !I/R, gamsa $\mathrm{E}, \mathrm{A} / \mathrm{AA}, \mathrm{Z}, \mathrm{II} / \mathrm{RT}$, and' ${ }^{*}$. Several sources were used in assenbling the data.

\# 7001039

$* 8.113$

Brahinsky, I1, S.: Veel, C. A.

TABLES OF EQUILIBRIUII THERIODYNAMIC PROPERTIFS OF AIR VOLUIIE IV. CONSTANT TFIIPERATURE WITH SPECIFIC IIEAT ANID SPEED OF SOUND DATA

AEDC-TR-69-39, Volume 4, Arnold Fngineering Development Center, ARO, Inc., Arnold $\Lambda$ ir Force Station, Tennessee

April 1969

$152 \mathrm{p}$

Available from DDC

Equilibriun thermodynamic data are presented for air from a temperature of 100 to $15,000^{\circ} \mathrm{K}$ and from $a \log \rho$ of -7.0 to 3.0 , where $\rho$ is density in anagats. These data are tabulated at constant terperature with density incremented. The dependent parameters are $\log P, 2, R, C_{p} / R, C v / R$, gamna, gamma ${ }_{E}$, and $A / A A$. Several sources were used in assembling the data.

$\$ 7001029$

* 8.113

Brahinsky, II. S.; Neel, C. A

TABLES OF EQUILIBRIUI TIERMONYNAMIC PROPERTIIS OF ARGON VOLURIE I. CONSTANT TEYIPERATIJRE

AEDC-TR-69-19 Volume 1, Arnold Engineering Development Center, ARO, Inc., Arnold Air Force Station, Tennessee

March 1969

$122 \mathrm{p}$

Available from DDC

Equilibriun thermodynamic data are presented for argon from a temperature of 300 to $35,000^{\circ} \mathrm{K}$ and from a $10 \mathrm{~g} \rho$ of -5.0 to 2.4 , where is density in anagats. These data are tabulated at constant temperature with density incremented. The dependent parameters are $2, E / R T, I I / R T, S / \dot{R}, \log P$. and $2 *$. Several sources were used in assembling the data.

\# 7001030

Brahinsky, H. S.; Neel, C. A.

TABLES OF 'EQUILIBRIUM TIIERMODYNAMIC PROPERTIES OF ARGON VOLUR:IE II . CONSTANT PRESSURE

AEDC-TR-69-19 Volume 2, Arnold Fngineering Development Center, $A R O$, Inc., Arnold Air Force Station, Tennessee

Iarch 1969

$211 p$

Avaîlable from DDC

Equilibrium thermodynamic data are presented for argon from a $\log F$ of -4.9 to 4.2 and from $a$ temperature of 300 to $35,000^{\circ} \mathrm{K}$, where $\mathrm{P}$ is pressure in atmospheres. These data are tabulated at constant pressure with temperature incremented. The dependent paraneters are $\log \mathrm{H} / \mathrm{R}, \log p, 2$, $S / R, I / R T$, and $2 *$. Several sources were used in assembling the data.
\#7001031

$* 8.113$

Bralinsky, H. S.; iveel, C. A.

TABLES OF EQUILIBRIUNI THENIODYNAMIC PROPERTIRS OF ARGON VOLUNE III, CONSTANT ENTRORY

AEDC-TR-69-19 Volume 3, Amold Engineering Development Center, ARO, Inc., Arnold Air Force

Station, Tennessee

March 1969

$394 p$

Available fron DDC

Equilibrium thermodynamic data are presented for argon from an S/R of 12.6 to 101 . $n$ and from a temperature of 300 to $35,000^{\circ} \mathrm{K}$, where $S / R$ is dimensionless entropy. These data are tabulated at constant entropy with temperature incremented. The dependent parameters are $\log \rho, \log P, \log H / R$, gama $E, A / A A, 2$, II/RT, and $Z^{\star}$. Several sources were used in assenbling the data.

\# 7001032

*\$. 113

Brahinsky, II. S.; NeeI, C, A.

TABLES OF EQUILIBRIUN THIPMODYNAIIC PRORERTIES OF ARGON VOLUNE IV. CONSTANT TEMIPERATURE VITH SPECIFIC IIEAT AND SPEED OF SOUND DATA

AEDC-TR-69-19 Volune 4, Arnold Engineering Development Center, ARO, Inc., Arnold Air Force Station, Tennessee

March 1969

$122 \mathrm{p}$

Available from DDC

Equilibriun thermodynamic data are presented for argon from a temperature of 300 to $35,000^{\circ} \mathrm{K}$ and from a log $\rho$ of -5.0 to 2.4 , where $\rho$ is density in anagats. These data are tabulated at constant temperature with density incremented. The dependent parameters are $\log P, 2, C / R, C \gamma^{\prime} R$, gamma, gama, and A/AA. Several sources $p_{\text {were }}$ used in assembling the data.

\# 7001025 $* 8.113$

Brahinsky, II. S.; Neel, C. A.

TABLES OF EQUILIBRIUM THERMODYNAMIC PROPERTIES

OF NITROGEN VOLUME I, CONSTANT TEMPERATURE

AEDC-TR-69-126 Volume 1, Arnold Engineering Development Center, ARO, Inc., Arnold Air Force Station, Tennessee

August 1969

$153 \mathrm{p}$

Available from DDC

Equilibriun thernodynamic data are presented for nitrogen from a temperature of 100 to $15,000^{\circ} \mathrm{K}$ and from $a \log \rho$ of -7.0 to 3.0 , where $\rho$ is density in amagats. These data are tabulated at constant temperature with density incremented. The dependent parameters are $2, E / R T, I / R T, S / R, \log P$, and $2 *$. Several sources were used in assembling the data.

$\# 7001026$ * 8.113

Brahinsky, H. S.; Neel, C. A.

TABLES OF EQUILIBRIUY THER IODYNAMIC PROPERTIES OF NITROGEN VOLUME I I. CONSTANT PRESSURE

AEDC-TR-69-126 Volume 2, Arnold Engineering Development Center, ARO, Inc., Arrold Air Force Station, Tennessee

August 1969

$279 p$

Available from $D D C$

Equilibrium thermodynamic data are presented for nitrogen from a $\log \mathrm{P}$ of -7.4 to 4.8 and from a temperature of 100 to $15,000^{\circ} \mathrm{K}$, where $\mathrm{p}$ is pressure in atmospheres. These data are tabulated at constant pressure with temperature incremented. The dependent parameters are $\log H / R, \log \rho, 2$, $S / R, H / R T$, and $2^{*}$. Several sources were used in assembling the data. 
\# 7001027

* 3.113

Brahinsky, II. S.; Nee1, C. A.

TABLES OF EQUILIBRIUI THERMODYNMIC PROPERTIES OF NITROGEN VOLUNE III, CONSTANT ENTROPY

ALDC-TR-69-126, Volume 3, Arnold Engineering Development Center, ARO, Inc., Arnold Mir Force Station, Tennessee

August 1969

455 p

Available from DDC

Equilibrium the rmodynamic data are presented for nitrogen from an S/R of 11.6 to 133.0 and from a temperature of 100 to $15,000^{\circ} \mathrm{K}$, where $\mathrm{S} / \mathrm{R}$ is dimensionless entropy. These data are tabulated at constant entropy with temperature incremented. The dependent paraneters are $\log \rho, \log \mathrm{p}, \log [1 / \mathrm{R}$, gamia $, A / A A, Z, I I / R T$, and $Z *$. Several sources were Esed in asseribling the data.

\section{\#7001028 \\ $\div 8.113$}

Brahinsky, H. S.; Neel, C. $\mathrm{A}$.

TABLES OF EOUILIBRIUM THER'IODYNAMIC PROPERTIES OF NITROGEN VOLURIE IV. CONSTANT TEITERATURE WITI SPECIFIC IIEAT AND SPEED OF SOUND DATA

AEDC-TR-69-126 Volume 4, Arnold Engineering Development Center, ARO, Inc., Arnold Air Force Station, Tennessee

August 1969

153 p

Available from DDC

Equilibrium thermodynamic data are presented for nitrogen from a tempterature of 100 to $15,000^{\circ} \mathrm{K}$ and from a log $\rho$ of -7.0 to 3.0 , where $\rho$ is density in amagats. These data are tabulated at constant temperature with density increnented. The dependent parameters are $\log p, z, C_{p} / R, C_{v} / R$, ganna, gamas, and A/AA. Several soufces were used in assembing the data.

\#6706017 *8.113

Britske, F. V.; et al. (Compilers)

THERLAL CONSTANTS OF INORGANIC SUBSTANCES

Moscow and Leningrad: Academy of Sciences 1949

$1014 \mathrm{p}$

In Russian

This reference book is divided into three parts: thermal effects, entropies of inorganic substances and heat capacity equations of inorganic substances at elevated temperatures. The first part gives data for heats of dilution, heats of solution, and heats of formation. References to the original literature are also listed. The second part contains data including the experimental evaluations of entropies of hydrated ions. Literature through January of 1941 is included. part 3 lists elements according to the periodic system giving the heat capacities of each entry. Each section has a condensed introduction presenting the theoretical background of the data given. A limited amount of data on organic compounds is included.

$\# 7001034 \quad * 8.113$

Brush, S. G.; Kraft, R.; Senkin, J.

HIGII-PRESSURE EQUATION OF STATE BIBLIOGRAPIY AND INDEX $(1925-1962)$

UCRL-7160, California University, Lawrence Radiation Laboratory, Livermore, California

January 22,1963

313 p

Available from CFSTI

The bibliography lists about 1500 references on the equation of state of materials at high pressure. These are listed chronologically; each citation is accompanied hy descriptors listing the materials or theories investigated. The descriptors have been indexed alphabetically according to the "keyword" system. Thus it is possible to locate references dealing with any substance or theory, or with various other keywords, by looking in the keyword index (Part I), and then finding the reference itself in the bibliography (part 2) by its serial number. The indexing is more complete for elements, inorganic compounds, minerals, and explosives than for organic compounds and corposite materials. llost mixtures have heen excluded. For gases, "high pressure" is taken to mean pressures above 50 atm, or virial coefficients higher than the second; but for liquids and solids, all compressibility measurements are included when found. Topics such as shock-wave compression, melting curves, quantur theory of the electron gas, and Thomas-Fermi theory have also heen included.

\#6601011

Buresh, M. K.; et al.

A SURVEY OF TIIERMODYNAYIC PROPERTIES OF TIIE CONPOUNDS OF THE ELEMTNTS $\mathrm{C} \mathrm{H} \mathrm{N} \mathrm{O} \mathrm{p} \mathrm{S}$

NBS Report 8992, National Bureau of Standards, Tashington, D. C.

october 1, 1965

$26 \mathrm{p}$

A systematic compilation of a bibliography on heatcapacity data of ammonia, carbon dioxide, and water is being made. The literature data examined thus far are listed. The list is probably 30 percent complete.

\section{3}

Canpbel1, J. E.

PLAIE-STRAIN FRACTURE-TOUGINESS DATA FOR SELECTED MIETALS AND ALLOYS

DMIC Report S-28, Defense Metals Information Center, Battelle Memorial Institute, Columbus, Ohio June 1968

$26 p$

Available from DDC

plane-strain fracture-toughness ( $K$ ) parameters may he used to estimate critical flaw sizes in structural metals suhjecter to known stresses at specified temperatures. previous toughness paraneters for evaluating high-strength alloys provided only enpirical data that could not be used directly in design. This report contains the first compilation of available $\mathrm{K}_{\mathrm{Ic}}$ data and is the result of considerable interest during the past few years in developing test methods for obtaining these data. The report is divided into sections on aluminum alloys, high-strength alloy steels, intermediate- and low-strength steels, precipitation-hardening stainless steels, titanium alloys, nickel-base Alloy 718 , and berylilium. Data on the aluminum alloys are limited to the 2000 - and 7000-series alloys. The high-strength alloy steels include the maraging steels, 9Ni-4Co steels, and lower alloy steels such as AISI 4340, D6ac, $300 \mathrm{~N}$, and II-11. The intermediate-strength steels include those that have been considered for submarine hulls, atomic-reactor vessels, and stean-turbine rotors. Data for the stainless steels are limited to the precipitation-hardening grades.

\#6906015 *8.113

Canjar, L. N.; Nanning, F. S. TIIERMODYNAMIC PROPERTIES AND REDUCED CORRELATIONS FOR GASES

Houston, Texas: Gulf Publishing Company 1967

$212 p$

This compilation contains tables and detailed charts on twenty comnon gases. Reduced correlation charts permit the estimation of the thermodynanic properties of some 100 other compounds. A universally applicable reference state of datum plane is employed. The properties include critical temperature, pressure and volume, vapor pressure, specific volumes, enthalpies, entropies, fugacity coefficients and compressed liquid regions. Substances covered are methane, ethane, propane, $n$ butane, n-pentane, i-butane, n-hexane, ehtylene, propylene, 1-butene, acetylene, benzene, ammia, 
carbon dioxide, carbon monoxide, hylrogen, nitrogen, oxygen, sulfur dioxide and water.

$\# 6500509^{\circ}$

$* 8.223-4.223$

Cezairliyan, A.; Touloukian, Y. S.

CORRELATION AND PREDICTION OF TIIERLAL CONDUCTIVITY OF IIETALS TIIROUGII TIIE APPLICATIO: OF TIIF PRIICIRLF OF CORRESPONDING STATES

In Advances in Thermophysical properties at Fxtreme pps01-313

New York: Anerican Society of Mechanical Engineers 1965

It is shown that the Principle of Corresponding States is applicable to thermal conductivity of metals. Two correlative relations are presented; one at low temperatures $(0<\mathrm{T}<1.5 \mathrm{~T})$, an another at moderate and high temperatures $(0.3 \theta<\mathrm{T}<$ 30), where $\mathrm{T}_{\mathrm{m}}$ is the temperature corresponding to the thermal conductivity maxima and $\theta$ is the Debye temperature. A universal relation for the reduced thernal conductivity is obtained in the low temperature region based on the solid state physical theory of metals and the proposed Principle of Corresponding States.

\#6508066

$\div 8.123$

Charlot, G.

TABLES OF CONSTANTS AND NUNIFICAL DATA \&. SELECTED

CONSTANTS OXYDO-REDUCTION POTENTIALS

New York, London, Paris: Perganon Press

1958

$41 \mathrm{p}$

Introduction in French and English

These tables have heen compiled as a convenient tool for finding quickly the best or most proballe value of the normal oxidation-reduction potentia1, or of the formal or apparent potential, of a given oxidation-reduction systen. The tables are arranged as follows: (1) oxidation numbers, (2) the actual syster, (3) value of the potential, (4) temperature, (5) total concentration of the redox compounds, (6) nature and composition of the medium and (7) rethod of determination.

\#6905032

*3. 123

Childs, G. E.; llanley, II. J. X.

APPLICÁBILITY OF DILUTE GAS TRANSPORT PROPERTY TABLES TO REAL GASES

Cryogenics, 8:94-97

April 1968

The pressure range for which puhlished 'clilute' or ' low pressurc' transport property tables may he used is defined experimentally. The method is based on the fact that, in a well-defined temperature range, transport properties can be computed from kinetic theory expressions. The method is general hut specific examples are given for argon, neon, krypton, xenon, nitrogen, and oxygen.

\#6905028 *8.123

Chueh, P. L.; Prausnitz, J. M.

THI RD VIRIAL COEFFICIENTS OF NONPOLAR GASES AND TIIEIR MIXTURES

AIChE Journal, 13:896-902

september 1967

This work presents a correlation of third virial coefficients within the framework of the corresponding states principle. The correlation is useful for estimating third virial coefficients of pure and mixed nonpolar gases, including the quantur gases helium, hylrogen, and neon. The importance of third virial cross coefficients in phase equilibrium predictions is illustrated with calculations for the solid-gas, methane-hydrogen system at $76^{\circ} \mathrm{K}$. Brief attention is given to the pressure series form of the virial equation. Because of fortuitous cancellations it is shown that for reduced temperatures above 1.4 , the pressure series, truncated after the second term, is applicable to a wider range of density than the density series truncated after the second term. Ilowever, when both series are truncated after the third term, the density series appears to be superior regardless of reduced temperature.

\#6510041

Clague, L. J. ; et al.

1964 BIBLIOGRÁPIY NND SUBSTANCF - PROPERTY INDEX O: PIIYSICAL AND TIIER"IODYNAYIC PROPERTIJS OF ORGANIC SUDSTANCES ANT MIXTIIRES

API Institute Research Project 44 and MCA Research project, Chemical Thermodynamic Properties Center, Texas AG!I University, College Station, Texas May 1, 1965

$84 \mathrm{p}$

This report consists of an index by substance and associated physical and thermodynamic property (Substance-Property Index) and bibliography covering the literature on organic substance for the year 1964. As a further aid to users of this index, a new "Guide to the Substance-Property Index," based on the standard order of arrangement of the elenents and compounds has heen included on this report. The index, though the abstracts, summarizes work in progress throughout the world dealing with physical, thermochenical and thermodynamic measurements on organic substances and organic nixtures.

\# 7001016 $\star 8.123$

Clark, $\Gamma$. V.

FUSED SALT MIXTURES, SPECIFIC CONDUCTIVITY TABLES SC-R-69-1386, Fused Salts Information Center, Sandia Laboratories, Albuquerque, New Mexico Decerber 1969

433 p

Available from CFSTI

This work was undertaken to meet a need in Sandia Laboratories for ready access to the data in the literature of fused salt chemistry. The coverage includes all apparently reliable data found in the literature through August 1968. In these tahles specific conductance values for some 270 fused salt systems are presented as a function of composition and temperature. In a majority of cases, a system will have been reported only once. Ilowever, in instances of multiple reports, data from each seeningly reliahle report has been included. The systems are written in alphabetical order by formula and all two-component systems report the percentage of the first component only.

$\# 6704019$

$$
\text { *8. } 123
$$

Clark, P. V.

PIIYSICAL PROPERTIES OF FUSED SALT MIXTURES VOLUME 1 SC-R-65-930, Volume 1, Sandia Laboratory, Sandia Corporation, Albuquerque, New Mexico

June 15,1965

293 p

Available from CFSTI

This volume contains tahulated data on the physical properties of fused salt mixtures. Articles in the literature covering the period 1907 to 1962 were reviewed and critically evaluated. The data consist of a list of fused salt mixtures with eutectic compositions and melting points presented in two complementary tables: hy fused salt systems and in order of eutectic melting points. The composition of all systens is reported in mole vercent. A complete list of references is included for consultation of the original literature. A coordinate index is included for rapidly locating system components and systens covered. 
Clark, $\Gamma$. V.

PIIYSICAL PROPERTIES OF FUSED SALT NIXTHRES, EUTECTIC COMPOSITIOYS AND IELTING POINTS, VOLUME 2 $S C-R-65-930, V .2$, Fused Salts Information Center, Sandia Laboratory, Albuquerque, New "lexico Noveriber 1966

$423 \mathrm{p}$

Available from the CFSTI

This volume is a continuation of "Physical properties of Fused Sa1t "ilxtures," SC-R-65-930, Volume 1 , in which eutectic melting point data from the literature, 1907-1963, were compiled. The present work includes additional data, extending the coverage to June 30,1965 . The references from volume one are repeated, and the index is cumulative.

\#6907026

Clark, P. V.; Dean, C. O.

FUSED SALT MIXTURES: EUTECTIC COMPOSITION

I.ELTING POINTS BIBLIOGRAPHY 1907-1963 SC-R-68-1680, Sandia Laboratories, Sandia
Corporation, Albuquerque, New :lexicn

Decenber 1963

$792 \mathrm{p}$

Available from CFSTI

Data on eutectic compositions and melting points of fused salt mixtures are presented in two complenentary tables arranged by systems and by melting points. Over 5,000 references are given through Decenber 30,1968 .

$\# 6906034$

$* 3.223$

Clark, R.G.; llyman, F.L.; Wilson, G.:.

LITERATURE SURVEY ON REFRIGERANTS ESSENTIAL TO CRYOCOOLER TECINOLOGY

Technical Report AFML-TR-66-136, Mir Products and Chemicals, Inc., Air Force Yaterials Laboratory, Wright-Patters on Air Force Base, Ohio

April 1966

$486 \mathrm{p}$

The literature prior to November 1965 was searched for cryogenic properties of the following pure refrigerants and mixtures: heliun-4; para-, orthoand normal hydrogen; deuterium; hydrogen deuteride; neon; nitrogen; argon; oxygen; methane; krypton; refrigerant-13; ethane; refrigerant-23. refrigerant-14; carbon dioxide; helium-3 - helium4; hydrogen-neon; nitrogen-argon; nitrogen-methane; methane-ethane; argon-methane; refrigerant-14. refrigerant-23; and refrigerant-13 refrigerant 23. The properties of interest were: PVT (including compressibility, density and virial coefficients); equation of state; phase equilibrium data; enthalpy; entropy; heat capacity; latent heat; Joule-Thoms on coefficient; thermal pressure; coefficients of compression an expansion; thermal conductivity; viscosity; velocity of sound; and surface tension. Phase equilibria data were sought for the following binary mixtures: heliumhydrogen; helium-neon; hydrogen-nitrogen; hydrogenargon; neon-nitrogen; neon-argon; neon-oxygen; helium-carbon monoxide; hydrogen-carbon nonoxide; neon-carbon monoxide; carbon dioxide in each of nitrogen, argon, oxygen, methane, and ethane; and hydrocarbons in oxygen. A survey was made of the research activities of all other laboratories known to be measuring the the rmodynamic and thermophysical properties of cryogenic materials.

\#6500368

$$
\text { *8. } 223-5.223
$$

Cohn, C. E.: Golden, G. I1.

A TABLE OF THERIODYNAMIC PROPERTIIS OF IYYROGEN FOR TEMPERATURES FROM 100 TO $3000^{\circ} \mathrm{K}$ AND PRESSURES FROM I TO 50 ATI'tOSFHERES

ANL-6673, Argonne National Laboratory, Argonne, Illinois

January 1963

$267 p$

Available from CFSTI
This report presents a table of thermodynamic properties of gaseous hydrogen covering the temperature range from in to $30 n 0^{\circ} \mathrm{K}$ and the pressure range from 1 to 50 atmospheres. The calculations have been done by means of a computer routine. The table has entries for temperature at $10^{\circ}$ increnents, for pressure at 1 atmosphere and at 5 atmosphere increments from 5 through 5 ? atmospheres, and for $25 \%, 50 \%, 75 \%$, and $100 \%$ of the gas initially in the para state. The first of these latter values corresponds to normal hydrogen, whereas the remaining values apply to vapor from liquic which has heen stored for varying lengths of time. For each entry, the table gives the density in $\mathrm{gm} / \mathrm{liter}$, the enthalpy in joules/gm, the entropy in joules/(gm) $\left({ }^{\circ} \mathrm{L}\right)$ and the fraction of the gas molecules which are Jissociated.

\#6607013

$$
\text { *3. } 323
$$

Cohn, E. J.; Jidsa11, J. T.

PROTEINS, AUINO ACIDS AND DEPTIDES AS IONS AVID DIPOLAR IONS

New York, London: llafner Publishing Corpany

1965

$404 \mathrm{p}$

In this book an atterpt has been made to characterize amino acids, peptides, and proteins. The evidence concerning the size and shape of these molecules and the number and distribution of the electric charges which they bear has been examined. The implications of the molecules' charged structure by their physical properties, and the physiochemical interaction with other molecules is considered. This hook is not a systematic treastise on all aspects of the chenistry of the proteins, or of their physical chenistry, hut is aimed at subjects which are of primary concern. Experimental techniques have been discussed only when it has been necessary to do so in order to make clear the operational basis on the principles which are involved.

\#6500461

Collins, F. C.

TUEORETICAL AND EXPERIMENTAL STIIDIES OF LIQUID VISCOSITY

WADC Technical Report 57-413, Aeronautical Research Laboratoory, Wright-Patterson Air Force Base, Ohio July 1957

$35 \mathrm{p}$

Available from DDC, AD 130971

A review of the theoretical hackground is presented together with an analysis of the outstanding difficulties of the various theories of liquid viscosity. The present status of the rigid sphere model of liquid viscosity and its relationship to sonic velocity is reviewed. The experimental data relating to the viscosity and sonic velocity in a number of typical liquids are presented and tentatively evaluated in terms of the rigid sphere model of liquid viscosity. A brief description is given of the apparatus for measuring sonic absorption in liquids.

\section{\#6908015} Connolly, T. F.; Copenhave r, E. D. OF MAGNETIC TRNNSITION TEMPERATURFS

ORNL-RIIC 7 (Revised, 1969, preprint), Research Materials Information Center, Oak Ridge National Laboratory, Oak Ridge, Tennessee

1969

$78 p$

ORNL-RIIC-7 (March 1968) has been corrected, revised, and expanded to include about $10 n$ n additional measurements of Curie and Nel points of magnetic materials. As in the original, the materials are arranged alphabetically by chemical formula and appear in two lists one for ferromagnetic (or ferrinagnetic) and one for antiferromagnetic (or metamagnetic) substances. 
The tabulation is hased on papers and reports received by the RiIC through January 1969.

\#6500671

*8. $222-0.3322$

CONSOLIDATEN INDEXX OF SELECTED PROPERTY VALUES, PHYSICAL CIIEYISTRY NND TIIERIODYNAIICS

NAS-VRC Publication 976, National Academy of Sciences-National Research Council, Office of Critical Tables, Washington, D. C.

1962

$275 p$

Available from Superintendent of Documents, fro

The Consolidated Index is a key to the contents of publications that present critically evaluated numerical property values. This initial volune contains in codified form the contents of the following six compilations of physicochemical and thermodynamic data: Selected Values of Properties of Ilydrocarbons and Related Compounds (American Fetroleun Institute Research Project 44), Selected Values of Properties of Chemical Compounds (Manufacturing Chenists Association Research Project). Selected Values of Chenical Thermodynamic Pronerties (NBS Cireular 500), The rmodynamic Properties of the Elements (D.R. Stull and G.C. Sinke), Contributions to the Data on Theoretical Metallurgy (Bureau of ilines Bulletins 383,384, $393,496,407,477,542,584)$, and Selected'Values for the Themodynanic Properties of letals and Alloys (linerals Research Laboratory, University of California). Each elenent, compound, or system of pure substances appears as a separate index entry. For a given substance, all properties that appear in one or nore of the compilations are listed separately under each. A given property, if appearing in several, is repeated under each publication.

\#ี 6500185

$* 3.223$

Conway, B.E.

ELECTRÓCHEMICAL DATA

Ansterdam, London, New York: Elsevier Puhlishing Company

1952

$374 p$

A series of more than 300 tables of electrochemical data selected from the hest quantitative information available is presented. The range of subject matter includes universal constants, properties of colloids and macronolecules of biological inportance, conductivities and potentials in fused salts, and parameters of electrode reaction kinetics, as well as the nore usual compilations of conductances, standard electrode potentials and transport numbers. The author is meticulous in giving references to the original sources and his extensive bibliography may he almost as useful as the data thenselves. Thenever possible he states the precision of the values given and in many cases indicates the experimental or theoretical method by which they were obtained. The hook is well organized, beginning with universal constants and general physical properties, progressing through the thermodynanics of solutions to ionic transport. The author then deals with the electric double layer at interfaces. After a diversion into the field of fused and solid ionic conductors, he returns to the logical order with a chapter on reversible electrode reactions. The concluding section, of which Dr. J.O'M. Brockris is the author, is an attenpt to collect data on electrode kinetics, giving tahles of the Tafel parameters. Most of the material deals with hydrogen evolution but there are tables on the deposition of metals, redox processes and anode reactions such as the evolution of exygen and the halogens. There is a 14-page index to provide ready reference to specific information.
月 660703.3

Cooper, J. (Editor)

PROCEEDINGS OF HORKSIOP CONFERENCE O: TIIE LONTRING OF TIIE IONIZATION: POTENTIAL AND RTLATED PROBLIMIS OF TIIE EOUILIBRIUM PLAS:I

JILA Report No. 79, Joint Institute for laboratory Astrophysics, Boulder, Colorado

July 1966

$225 \mathrm{p}$

This workshop was sponsored hy the Joint Institute for Laboratory Astrophysics. Its concern was statistical mechanics of plasma both fror experinental and theoretical points of view. There were many recent theoretical advances in the treatment of such problens as the plasma partition function and the lowering of the ionization potential, although there were strong disagreements between different workers in this field. The proceedings were taped and should he considered as unpublished material. It was the object of this workshop to hring together active theorists an experinenters in this field to resolve some of the differences. Included in the Appendix is Bibliography on Research on Lowering of the Ionization Potential.

$\# 6605019$

$* 8.122-4.122$

COPPER WIRE TABLES

NBS llandbook 100 , vashington, D. C.

February 21, 1960

$41 \mathrm{p}$

Available from Superintendent of Documents, GPO

This Ilanchook is a revision of the Copper Wire Tables previously published as NIBS Circular 31. It reflects changes in the nominal dianenters of gages 45 and snaller and extends the tahles to 56 gage. The llandhook provides data for the weight and length per ohm, wight ner unit length, length per unit weight of copper wire, resistivity, temperature coefficient, density, and conductivity of copper wire from 0000 to 56 gage as a function of temperature from 0 to $200^{\circ} \mathrm{C}$.

\# 6500354 *8.233

Corruccini, R. J.; Gniewel;, J. J.

SPECIFIC UIAATS AUD ENTIALPIES OF TECIMICAL SOLIDS AT LOW TENPERATURES, A COMPILATION FRON TIIE LITERATURE

NBS Monograpl 21, National Bureau of Standards, Washington, D. C.

October 3, 1960

$20 \mathrm{p}$

Available from Superintendent of Documents, GPO

A compilation of heat capacity data on cryogenic materials for designers of cryogenic equiprent is given. Tables are given of the specific heat, $C$ and tice enthalpy of 28 metals, 3 alloys, 8 other inorganic substances and 8 organic substances in the temperature range $1^{\circ}$ to $300^{\circ} \mathrm{K}$. The data presented are hased on selected sources listed under each table, including references which did not influence the tabulated data but are of current interest. The 170 references cover the period 1930 to 1958. The literature search was based on Physics Abstracts and Chemical Abstracts for those years not covered hy bibliographies of Kelley (1950) and Schiffman (1952).

\#6500304,6500305 *3.123-4.123

Corruccini, R.J.; Gniewek, J.J.

THERMAL EXPANSION OF TECINICAL SOLIDS $A T$ LOW TEMTERATURES, $A$ COMILILATION FROR THE LITERATURE

NBS Monograph 29, and Supplement, National Bureau of Standards, Washington, D. C.

May 19,1961

$22 \mathrm{p}, 2 \mathrm{p}$

Available from Superintendent of Documents, GPO

Tables are given of the linear contraction relative to $293^{\circ} \mathrm{K}\left(\mathrm{L}_{29},-\mathrm{L}_{\mathrm{T}}\right) \mathrm{L}_{29}$, , and the linear expansion coefficient $\mathrm{dL} / \mathrm{L}_{2} \mathrm{~g}_{3} \mathrm{dT}$, of thirty elements, forty- 
five alloys, twenty-two other inorganic substances and twenty plastics and elastomers in the temperature range, 0 to $300^{\circ} \mathrm{K}$.

\#6500358

$* 8.223$

Coughlin, J. P.

CONTRIBUTIONS TO THE DATA ON TTEORETICAL RTTALIURGY XII. IIEATS AND FREE ENERGIES OF FORYATION OF INORGANIC OXIDES

Bulletin 542, Bureau of Mines, Washington, D. C. 1954

$30 \mathrm{p}$

Available from Superintendent of Documents, GPO

This bulletin compiles heat and free energy of formation data for inorganic oxides. A survey of worldwide literature to September 1953 permitted assembly of data for over 170 inorganic oxides. These data are presented by three separate methods: (1) tables of heat and free energy of formation values at $298.16^{\circ} \mathrm{K}$, at phase-change temperatures and at even $100^{\circ}$ intervals from $400^{\circ}$ to a maximum of $2000^{\circ} \mathrm{K}$, (2) equations giving free energies of formation as functions of temperature, utilizing in their derivation the best available high temperature heat-content values, and (3) equations of more approxinate nature giving free energies of formation as linear functions of temperature.

\section{\#6510008 *8.273}

CRYOGENIC DATA MEMORANDUM: FILF NUMBRS 1 - M7

Cryogenic Data Center, National Bureau of Standards, Boulder, Colorado

1965

$30 \mathrm{p}$

These cryogenic data memoranda contain thermodynamic data and reference sources for properties of several substances, including normal deuteriun liquid vapor pressure, normal hydrogen solid vapor pressure, equilibriun deuterium heat of vaporization, normai deuterium saturation density of liquid, solid helium density and compressibility, solid methane, ethane, propane, butane, pentane density and compressibility, and solid hydrogen density and compressibility.

\#7001019 *8.123-3.123

Darwent, $B$. deB.

BOND DISSOCIATION ENERGIFS IN SIMPLE MOLECULES

NSRDS-NBS-31, National Bureau of Standards, Washington, D. C.

January 1970

$52 \mathrm{p}$

Available from the Superintendent of Documents, GPO

Bond dissociation energy values (kcal/mol) and $(\mathrm{kJ} / \mathrm{mol})$ of simple compounds are tabulated from a literature review covering the year 1962-1966 inclusively. Some selected values which appeared in the years 1956-1962 are -1so included. Organic compounds are excluded except those containing one carbon atom. The groups $=\mathrm{CO}$ and $-\mathrm{CN}$ are not considered to be organic. The values are quoted usually at $0^{\circ} \mathrm{K}$ or $298^{\circ} \mathrm{K}$ and refer to the gaseous state. They represent the energy required to break a bond at the specified temperature with all substances in the zero vibrational state of the ground electronic state. The experimental method for the energy value listed is given and referenced in the table. A value recommended by the author is listed as the final value for each reaction.

\#6500347

$* 8.223$

De an, J. W.

A TABULATION OF THE THERMODYNAMIC PROPERTIES OF NORMAL IYYDROGEN FROM LOW TEMPERATURES TO $300^{\circ} \mathrm{K}$ AND FROM 1 TO 100 ATMOSPHERES

NBS Technical Note 120, National Bureau of Standards, Boulder, Colorado

November 1961

$71 \mathrm{p}$

Available from the Superintendent of Documents, GPO
Pressure, volume, temperature, internal energy, enthalpy, and entropy of normal hydrogen gas have been tabulated along isobars in $1^{\circ} \mathrm{K}$ temperature steps. The range covered is from the saturation temperature to $300^{\circ} \mathrm{K}$ and from a pressure of 1 to 100 atmosplieres. The source of data is the Research Paper 1932 of the National Bureau of Standards Journal of Research. The method is described by which the data presented in Research Paper 1932 is reduced to properties directly useful for engineering calculations. A method is also described for estimating the effect of ortho-para conpositions upon the tabulated properties. Tabular values are presented in the dimensional units of the metric system. The tabulations are a1so available in the dimensional units of the British system in Supplement A of the Technical Note 120.

\section{\#6500349}

*8.223

Dean, J. H.

A TABULATION OF TIIE TIIERMODYNAIIC PROPERTIFS OF NORMAL INDROGEN FRON LOW TEMPERATURES TO $540^{\circ} \mathrm{R}$ AND FROH 10 TO 1500 PSIA, SUPPLEMENT A (BRITISII UNITS) NBS Teclinical Note 120A, National Bureau of Standards, Boulder, Colorado

June 1962

$75 \mathrm{p}$

Available fron the Superintendent of Documents, GPO

Pressure, volune, temperature, internal energy, entha1py, and entropy of norma1 hydrogen gas have been tabulated along is obars in $2^{\circ} \mathrm{R}$ temperature steps. The range covered is from the saturation temperature to $540^{\circ} \mathrm{R}$ and from a pressure of 10 to 1500 psia. The source of data is Research Paper 1932 of the National Bureau of Standaris Journal of Research. The method is described by which the data presented in Research Paper 1932 is reduced to properties directly useful for engineering calculations. A method is also described for estimating the effects of ortho-para compositions upon the tabulated properties. Tabular values are presented in the dimensional units of the British system. The tabulations are also available in the dimensional units of the metric system as Technical Note No. 120 .

\#6608015 *8.123

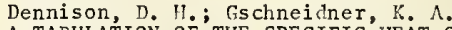

A TABULATION OF TIE SPECIFIC IIEAT CONTRIBUTION DUF, TO TIIERNAL EXCITATION (SCIOTTIYY TFETCT) OF TIIE 4F ELECTRONS OF SOIE DI- ANI TRI-VALENT LANTIIANIDE ELEMENTS

IS-1156, Ames Laboratory, Ames, Iowa

May 1965

$37 \mathrm{p}$

Available frora CFSTI

The $4 \mathrm{~F}$ electronic specific heat contribution for divalent samarium and for tfe trivalent lanthanide elements Ce, Pr, Nd, Pm, Sr, Eu, To, Dy, Ilo, Er, Tn and $\mathrm{Yb}$ has been calculated and is tabulated at $25^{\circ} \mathrm{K}$ intervals from $50^{\circ}$ to $2000^{\circ} \mathrm{K}$, at $50^{\circ} \mathrm{K}$ intervals from $200^{\circ}$ to $400^{\circ} \mathrm{K}$, and at $100^{\circ} \mathrm{K}$ intervals from $400^{\circ}$ to $2000^{\circ} \mathrm{K}$.

\section{\#6906026 \\ *8.123-5.123}

Diamond, J. J. (Editor)

BIBLIOGRAPIIY ON TIE IIIGII TEATERATURE CIEN'ISTRY N N PHYSICS OF MATERIAIS, October, November, Necember 1968; January, February, March 1969; April, May, June 1969; July, Nugust, September 1069; OCtober, Novenber, December 1969

NBS Special Publication $315,315-1,2,3$ \& 4 , National Bureau of Standards, Washington, D. C. Jan., Apri1, July, Oct. 196?, Jan. 1970

$72 \mathrm{p}, 81 \mathrm{p}, 82 \mathrm{p}, 90 \mathrm{p}, 35 \mathrm{p}$

Available from Superintendent of Documents, GPO

These bibliographies consist of references to research involving temperatures above $1000^{\circ} \mathrm{C}$, which were noted by the contributors during the abovementioned three-month periods. Since these arc intended primarily as current-awareness 
bibliographies, there is no cross-referencing or indexing. These issues contain about 700 references each, roughly grouped under fifteen subject headings.

$\# 6905034$

$\star 3.123-3.123$

Dickson, P. E. ; Jones, M. C.

INFRARED SPECTRAL REFLECTANCES OF METALS AT LOW

TESTERATURSS

Cryogenics, $8: 24-29$

February 1968

This paper graphically displays values, derived from the literature of the nomal spectral reflectances ( $P, P$ ) of ten pure netals and an alloy at low temperatutes. The metals and alloy included are aluminum, antimony, bismuth, copper, brasses, gold, silver, lead, tin, nickel and sodium.

$\$ 6902048$

* 3.123

Dijkhuis, C.; Dijkhuis, R.; Janz, J.

MOLTEN SALT ELECTROMOTIVF FORCE FORIATION CELLS Chemical Reviews, 68:253-275

Tay 24,1968

Cells with C1-graphite electrode, $\mathrm{Br}_{2}|\mathrm{C},| \mathrm{I}, \mathrm{C}$ and NOO J 0 Pt as anion electrodes and glass as cation indicator electrode are discussed. The theoretical principles and methods for calculating excess free energies of binary mixtures, with comnon ion including charge-symetrical and chargeunsymetrical mixtures is critically reviewed For comparison of the free-cnergy values, "the enf data in the literature are systematically recalculated. The free-energies in these nixtures are small as conpared with the total lattice energy and their application for evaluation of structure and properties of the molten salts are linited. Some regularities exist hetween mixtures, and their excess free energies. One hundred eight references are included.

\#6903011 $* 8.123$

Diller, D. E. ; Mas on, E. A.

LOW-TEIPERATURE TRANŚPORT PROPFRTIES OI- GASFOUS $\mathrm{Il}_{2}$, D AND IID

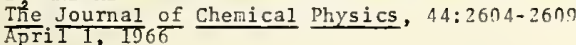

A conplete quantal phase-shift calculation of the transport properties of gaseous $\|_{2}, \mathrm{D}_{2}$, IID, and sone of their mixtures is presented 'from $10^{6}$ to $300^{\circ} \mathrm{K}$, based on the Lennard-Jones (12-6) potential. It is found that $\mathrm{H}_{2}$ and $\mathrm{D}_{2}$ have siightly different intermolecular potentials, in agreenent with evidence from other sources. Agreement between calculation and experisent is generally good, but there are some snall but clear indications that the (12-6) potential model is oversinplified, such as the necessity to use slightly different potential parameters for the transport properties than for the second virial coefficients.

\#6500376,6500377,6500378 *8.223

Din, F. (Editor)

THERIODYNAYIC FUNCTIONS OF GASES,

VOLUNE 1: AYONIA, CARBON DIÓXIDE, AID CARBON MONOXIDE,

VOLUIE 2: AIR, ACETYLINE, ETIYLENE, PROPANE AND ARGON,

VOLUME 3: METIIANE, NITROGEN, ETIIANE

London: Butterworths

$1962,1956,1961$

The following thermodynamic properties are given for ammonia, carbon dioxide, carbon nonoxide, air, acetylene, ethylene, propane, argon, methane, nitrogen and ethane: enthalpy and entropy for the solid, liquid and gaseous states; volume; specific heat at constant pressure and at constant volume; and temperature-entropy diagrams. A survey of the existing data is made and bibliographies are given.
\#6906032

Dobbins, T. 0

TIIERMODYNAMIICS OF ROCKET PROPULSION AND THEORETICAL EVALUATION OF SOME PROTOTYPE PROPELLANT COMBINATIONS

WADC-TR-59-757, Wright Air Development Center, Wright-Patters on Air Force Base, Ohio

December 1959

$711 p$

This report presents a concise summary of the thermodynamics of rocket propulsion and the evaluation of 100 prototype propellant combinations. Emphasis is placed on the thermodyramics of chemical propulsion and on flow through the converging-diverging nozzle. Two methods are developed for conducting theoretical performance calculations. One utilizes thermodynamic equilibrium constants while the second utilizes completely new concept of minimizing the total free energy of the combustion products. Sample calculations are presented for each method. Thermodynamic data are presented and discussed for several reactants and all important products of combustion consisting of the elements carbon, hydrogen, oxygen, nitrogen, chlorine, fluorine, lithium, boron, beryllium, aluminum, and magnesium. Specific impulse, chamber temperature, exhaust temperature, characteristic exhaust velocity, and chamber molecular weight are plotted as a function of the propellant composition. Also, complete performance data are tabulated for selected fuelto-oxidizer ratios.

\#6704030

Dorsey, N. E. (Compiler)

PROPERTIES-OF ORDINARY WATER-SUBSTANCE IN ALL ITS PHASES: WATER-VAPOR, WATER, AND ALL THE ICES Ann Arbor, Michigan: University Microfilms, Inc. New York: Reinhold Publishing Corporation 1967 673 p

This compilation gives information regarding the properties of pure, ordinary water-substance in all $i$ ts phases, water-vapor, water, and the several ices, and the phenomena and data pertaining to its synthesis and dissociation and to its transition from phase to phase. The effect of the presence of air and the solubilities and diffusivities in water of the atmospheric and noble gases, of hydrogen, air, hydrogen, and carbon dioxide, and through few solids is considered. The data given are inclusive of the literature through 1937.

\#6500400

* 8.233

Douglas, T. B.

HIGH-TEMPERATURE THERMODYNAMIC FUNCTIONS ZIRCONIUM AND UNSATURATED ZIRCONIUN HYDRIDES Journal of Research of the National Bureau of Standards -1

This report gives greatest weight to the experimentally measured highes $t$ decomposition pressures and the enthalpies in one-phase fields. Interconsistent integral and differential enthalpies (heat contents), heat capacities, entropies, and Gibbs free energies are the rmodynamically derived for the crystalline oneand two-phase fields of the zirconium-hydrogen system for all stoichiometric compositions from $\mathrm{ZT}$ to $\mathrm{ZrH}$ and over the temperature range 298.15 to $1200^{\circ} \mathrm{K}^{1} \cdot T^{5}$ ese properties are derived in analytical form, and in most cases are represented by numerical equations, with tabulation for zirconium and $\mathrm{H} / \mathrm{Zr}$ atom ratios of $0.25,0.50,0.57,0.75$, 1.00 and 1.25 . Most of the unique phasefield boundaries which are consistent with the derived properties are located and are compared with those previously reported. In the $\mathrm{Zr}-\mathrm{H}$ system the enthalpies are shown to relate certain properties at different compositions as well as at different temperatures. Some of the various data show good interconsistence, while others reveal discrepancies which are discussed critically. 
Douslin, D. R.; et al.

THERMODYNAMIC PROPERTIES OF ORGANIC DERIVATIVES OF THE LIGHTER ELEMENTS. ANNUAL TECHNICAL SURMARY REPORT, MARCH 1,1966 TO MARCH 1,1967

Bartlesville Petroleum Research Center, U. S. Bureau of Mines, Bartlesville, Oklahoma

March 1967

$13 \mathrm{p}$

Available from DDC

Thermochemical research was carried out on ethylerimire, four aliphatic diamines, and two organic fiuorine compounds. Results for ethylenimine include an experimental determination of its thermodynamic properties by means of lowtemperature calorimetry, combustion calorimetry and vapor pressure measurements. The measured third-law entropy is discussed in light of a recent Russian value calculated from spectroscopic information. Work on the the rmochemistry of aliphatic diamines includes purification of 5 amples of 1,2-diaminoethane, 1,2-diaminopropane, 1,2diaminobutane, and 1,2-diamino-2-methylpropane; heats of combustion of 1,2diamino-propane and 1,2diamino-2-methylpropane; and some low-temperature thermal measurements on 1,2-diaminopropane. P-V-T relations determined for hexafluoroethane include vapor pressures up to the critical state, critical constants, $P, T_{C}$ and $V_{C}$, and compressibility of the gas up to $350^{\circ} \mathrm{C}$ and 400 atmospheres. preliminary values for the vapor heat capacity of hexafluorobenzene are presented.

$\# 6905013$

* 8.323

Douslin, D. R. ; et al

THERMODYNAMIC PROPERTIES OF ORGANIC DERIVATIVES OF THE LIGHTER ELEMENTS ANNUAL TECHNICAL SUMMARY REPORT FOR THE PERIOD MARCH 1, 1967 TO MARCH 1, 1968

Bartlesville Petroleum Research Center, U. S. Bureau of Mines, Bartlesville, Oklahoma

March 1968

20 p

Available from DDC

Continued thermochemical research was carried out on ethylenimine as a substance which showed high ring strain energy and as the parent compound of possible derivatives containing the ethylenimine ring which might show promise as high energy fuels. Programs of thermochemical research on aliphatic diamines were continued in order to better define the $\mathrm{C}-\mathrm{N}$ thermochemical bond energy. Determinations of the state properties of the fluorocarbons were continued as a means for deriving their intermolecular energies. Results for ethylenimine include thermodynamic functions in the ideal gas state to $1500^{\circ} \mathrm{K}$ calculated from spectroscopic parameters; vapor pressure measurements; and derived enthalpies of vaporization. Research on the aliphatic diamines include enthalpies of combustion and formation, the $\mathrm{C}-\mathrm{N}$ thermochemical bond energy, low-temperature heat capacities, third-law entropies, vapor pressures, and enthalpies of vaporization. Research on the state properties and intermolecular energies of fluorine compounds includes correlated values of the vapor pressure of hexafluorobenzene up to the critical, second and third virial coefficients of hexafluoroethane, and reduction of the $\mathrm{P}-\mathrm{V}-\mathrm{T}$ values for hexafluoroethane to standard data forms.

\# $6906013 \quad$ *8.323

Douslin, D. R.; et al.

THERMODYNAMIC PROPERTIES OF ORGANIC DERIVATIVES OF THE LIGHTER ELEMENTS ANNUAL TECHNICAL SUMMARY REPORT FOR THE PERIOD MARCH 1, 1968 TO MARCH 1 , 1969

Bartlesville Petroleum Research Center, U. S. Bureau of Mines, Bartlesville, Oklahoma

March 1969

$14 \mathrm{p}$

Available from DDC
Measurement of the thermal properties of a mixture of ethylenimine and its dimer near the eutectic compositon has provided needed information for interpreting the premelting region of pure ethylenimine. Tables of values for enthalpy of formation, Gibbs energy of formation, and logarithm of the equilibrium constant of formation of ethylenimine were calculated. Molal thermodynamic functions for the condensed phases of 1,2diaminoethane and 1,2-diaminopropane were calculated from low-temperature calorimetric measurements. The thermochemical properties of is obutylamine were measured in order to further define $\mathrm{C}-\mathrm{N}$ bond energy in amines arid diamines. Correlations of the thermodynamic properties of hexafluorobenzene were completed from 0 to $1500^{\circ} \mathrm{K}$.

\#6500490

*8.223

Drayer, D. E.

A COMPILATION OF THE PHYSICAL EQUILIBRIA AND RELATED PROPERTIES OF THE HYDROGEN-METHANE SYSTEM NBS Report 7298, National Bureau of Standards. Boulder, Colorado

(No daté)

$76 \mathrm{p}$

Vapor-liquid equilibria data for the hydrogenmethane system are presented in the form of $K$ factor charts for thirteen isotherms from 90.3 to $188.2^{\circ} \mathrm{K}$ and from 30 to 270 atmospheres pressure. Solid-vapor equilibria data are presented as vapor phase compositions versus temperature and pressure over the range of 57 to $90^{\circ} \mathrm{K}$ and 5 to 15 atmospheres. A bibliography of 390 references on related properties for this system and for the pure components is also presented.

$\# 6500489$ *8.123

Drayer, D. E.; Flynn, T. M.

COMPILATION OF THE PIYYSICAL EQUILIBRIA AND RELATED PROPERTIES OF THE HYDROGEN-CARBON MONOXIDE SYSTEM

NBS Techrical Note 108, National Bureau of St andards, Boulder, Colorado

May 1961

$81 \mathrm{p}$

Available from CFSTI

Literature data have been used to calculate Kfactors for the hydrogen-carbon monoxide system over the range of 68.2 to $122.2^{\circ} \mathrm{K}$ and 10 to 225 atmospheres. K-factors are presented graphically for eight is otherms over this range. Published data on the solid-vapor region are presented separately as composition versus pressure at constant temperature. A bibliography of approximately 450 references is also presented on related properties for this system and for the pure components.

$\# 6500488$

Drayer, D. E.; FIynn, T. M

*8.123

COMPILATION OF THE PHYSICAL EQUILIBRIA AND RELATED PROPERTIES OF THE HYDROGEN-HELIUM SYSTEM

NBS Technical Note 109, Natioral Bureau of Standards, Boulder, Colorado

June 1961

$50 \mathrm{p}$

Available from CFSTI

Published data have been used to calculate Kfactors for the helium-hydrogen system over the range of 17.4 to $21.8^{\circ} \mathrm{K}$ and 2 to 32 atmospheres pressure. K-factors are presented graphically for three is otherms over this range. A bibliography of approximately 290 references is also presented on related properties for this system and for the pure components. 
\# 6500491

Drayer, D. E.; Flynn, T. M.

COMPILATION OF THE PHYSICAL EQUILIBRIA AND RELATED PROPERT1ES OF THE IYYDROGEN-NITROGEN SYSTEM

NBS Technical Note 110 , National Bureau of $S t$ andards, Boulder, Colorado

May 1961

$62 \mathrm{p}$

Available from CFSTI

Published data have been used to calculate factors for the hydrogen-nitrogen system over the liquid-vapor range of 68.2 to $122.2^{\circ} \mathrm{K}$, and 10 to 225 atmospheres. $K$ factors are presented raphically for eleven isotherms within this range. Published data on the solid-gas and solid-liquid regions are presented separately as composition versus pressure at constant temperature. bibliography of 250 references pertaining to the hydrogen-nitrogen system is included.

\section{$\# 6500235,6500236,6500237 \quad * 8.323$}

Dreisback, R. R.

PHYSICAL PROPFRTIES OF CHEMICAL COMPOUNDS, VOLUMI: I, I 1 , AND I11

Advances in Chemistry Series 15, 22, 29, American Chemical Society, Washington, ก. C.

June 1955, Narch 1959,1961

$536 \mathrm{p}, 491 \mathrm{p}, 489 \mathrm{p}$

Data in volume I are listed for 511 organic cyclic compounds and for 17 miscellaneous compounds, in volume II for 476 organic compounds, in volume II for 434 aliphatic compounds and for 22 miscellaneous compounds and elements. Properties studied include purity, freezing point, vapor pressure, density, refractive index, boiling point heat of fusion, heat of evaporation, critical constants, compressibility, viscosity, heat content, surface tension and solubility.

\section{\#6704042}

Dub, M. (Editor)

ORGANOMETALLIC COMPOUNDS, METHODS OF SYNTHESIS PHYSICAL CONSTANTS AND CHEMICAL REACTIONS, VOLUME 1: COMPOUNDS OF THE TRANSITION METALS, SECOND EDITION, COVERING THE LITERATURF FROM 1937 TO 1964 New York: Springer-Verlag New York, Inc. 1966

$856 \mathrm{p}$

Volume I of the series organometallic Compounds covers the derivatives of the transition metals of Groups III through V1II of the Periodic Table. It is a comprehensive, noncritical source of information concerning organometallic compounds containing at least one carbon-metal bond. The information includes methods of preparation, properties, chemical reactions, applications, addition compounds and biological properties. This volume covers the literature from 1937 to 1964 . In addition to the tables of data and information given, a bibliography of over 2300 references and listing of review publications and monographs are included.

\#6 6707010

* $8.323-3.323$

Dub, M.; Weiss, R. W. (Editors)

ORGANOMETALLIC COMPOUNDS, METHODS OF SYNTHESIS PHYSICAL CONSTANTS AND CHEMICAL REACTIONS, VOLUME 1I: COMPOUNDS OF GERMANIUM, TIN AND LEAD IŃCLUDING BIOLOGICAL ACTIVITY AND COMMERCIAL APPLICATION, SECOND EDITION, COVERING THE LITFRATURE FROM 1937 TO 1964

New York: Springer-Verlag New York, Inc.

1967

$727 \mathrm{p}$

Volume 1 I of the series Organometallic Compounds covers organic derivatives of germanium, tin and lead. It is a comprehensive, noncritical source of information concerning organometallic compounds. The scope is limited to compounds containing at least one carbon-metal bond. The information includes methods of preparation, properties, chemical

reactions,

applications addition compounds and biological properties. This volume covers the literature from 1937 to 1964. In addition to the tables of data and information given, a bibliography of over 2700 references and listing of review publications and monographs are included.

\#6500324

* 8.123

Edwards, D. G.

THE VAPOR PRESSURES OF 30 INORGANIC LIOUIDS BETIVEEN ONE ATMOSPHERF: AND THE CR1TICAL POINT

UCRL -7167 , Cali fornia Universi ty

Radiation Laboratory, Livermore, California

June 1963

$148 \mathrm{p}$

Available from CFSTI

Literature values of the vapor pressures of $\mathrm{H}_{2}$, HD, $\mathrm{D}, \mathrm{N}_{2}, \mathrm{O}_{2}, \mathrm{Ne}, \mathrm{Ar}, \mathrm{Kr}, \mathrm{Xe}, \mathrm{CO}, \mathrm{NO}, \mathrm{CH}_{4}, \mathrm{SiF}_{4}{ }^{2}, \mathrm{~N}_{2} \mathrm{O}$, $\mathrm{CO}_{2},{ }^{2} \mathrm{HCl}^{2}, \mathrm{HBr}, \mathrm{HI}, \mathrm{SF}_{6}, \mathrm{H}_{2} \mathrm{~S}, \mathrm{Cl}_{2}, \mathrm{Br}_{2}, \mathrm{NH}_{3}, \mathrm{C}_{2} \mathrm{~N}_{2}$, have been compared wi th the $\mathrm{N}_{2} \mathrm{O}_{4}$ ' Chhoff formula: $\mathrm{CH}_{4}$, and $\mathrm{P}_{1}=-\mathrm{A} / \mathrm{T}+\mathrm{B}$, between the normal boiling point and the critical point, where $A$ and $B$ are determined for each 1iquid. Plots are presented of the deviations computed by the equation: $d=P_{k}$ $\mathrm{P}_{\text {obs }}$, based on accurately determined curves for 17 of these substances. Empirical hypotheses were developed to allow the prediction of the most probable course of the deviation curve in cases where the data appeared to be unreliable. In addition, where precise data were available, the possibility of the existence of fine structure curvature has been demonstrated. The deviation curves have been used to obtain tables of interpolated vapor pressure values which are, in most cases, most accurate by one order of magnitude than any now in the literature, particularly where smoothes regression curves do not discriminate between random experimental errors and systematic deviations of an arbitrarily chosen formula from the real vapor pressure curve. Since the deviation curve is invariably shown to approach the zero ordinate at the critical point with a large negative slope, the correspondence between the critical pressure and temperature is fixed with a high degree of confidence. A particular result of this work is that the critical pressure of NII-3 was shown to be about one atmosphere higher than the presently accepted value. Our values for the critical points of $\mathrm{CH}_{4}, \mathrm{C}_{2} \mathrm{~N}_{2}, \mathrm{HCN}$, and $\mathrm{Br}_{2}$ have also resolved differences' existing between various authorities in the literature.

\#6906009 *3.123-8.123-0.2523

Eisenberg, D.; Kauzmann, W.

THE STRUCTURE AND PROPERTIES OF WATER

New York and oxford: Oxford University Press 1969

$296 \mathrm{p}$

In this book the authors summarize the many experimental observations on water and present theoretical discussions which effectively correlate the data. The topics covered include the water molecule and forces between water molecules; the thermodynamic properties of steam; the structures of the ices; the thermodynamic, electrical, spectroscopic, and transport properties of the ices and of liquid water; hydrogen bonding in ice and water; and models for liquid water. The authors emphasize the relation of the properties of ice and water to their structures, and discuss properties such as infrared and Raman spectra because they reveal a great deal about these structures. The inclusion of some background material in physical chemistry makes the book accessible to the biologist, biochemist, and geologist as well as the chemist and physicist. An addendum listing number of recent articles on the structures and properties of water completes the volume. 
Eldridge, E. A.; Deem, H. W.

REPORT ON PIYSICAL PROPPERTIES OF METALS AND ALLOYS FROM CRYOGENIC TO ELEVATED TEMPERATURES

ASTM Special Technical Publication 296, American Society for Testing and Materials, philadelphia, Pennsylvania

April 1961

$206 \mathrm{p}$

This report is a compilation of the physical properties of the metals aluminum, cobalt, iron, magnesium, molybdenum and nickel, and their alloys. The properties included are thermal conductivity, linear thermal expansion, specific heat, electrical resistivity, density, emissivity, diffusivity and magnetic permability. Data are compiled as a function of temperature, where possible, over the range $-457^{\circ} \mathrm{F}$ to $4500^{\circ} \mathrm{F}$. The data contained in this report came principally from data sheets supplied by companies and organizations who responded to an ASTM-ASME survey, trade bulletins, published technical literature and U.S. Government publications.

$\# 6500375$

Elliott, J. N. THERMODYNAMIC PROPERTIES OF HEAVY WATER

AECL-1673, Atomic Energy of Canada Ltd., Chalk River, Ontario

January 1963

$90 \mathrm{p}$

Tables are presented on the thermodynamic properties of heavy water. Vapor pressure, specific volume, enthalpy, entropy, and internal energy for $D, O$ are tabulated in $B$ ritish and in MKSA units from $390^{\circ} \mathrm{F}$ to $700^{\circ} \mathrm{F}$ in $1^{\circ}$ steps. The viscosity, heat, and conductivity are also tabulated in both systems of units.

$\# 6603001$

*8. 123

Elliott, R. P.

CONSTITUTION OF BINARY ALLOYS, FIRST SUPPLEMENT

New York: McGraw-Hill Book Company

1965

$877 \mathrm{P}$

The present volume contains critical reviews of the literature pertinent to binary equilibrium published through December 1961. The present volume is a supplement to the basic volume Constitution of Binary Alloys by llansen and Anderko. A total of $\frac{100}{8100}$ sstem references were consulted in preparing the volume. Written reviews are included for 1719 binary systems, augmented with 435 figures. For the approximately 7-year period since publication of Constitution of Binary Alloys, reviews have been prepared for $733^{-1}$ sistems for which previously no data were available (compared to 506 for the 22-year period between the two editions of Hansen). Constitution of Binary Alloys and its first supplement now make written reviews available for 2067 binary systems.

\#6906020

*8.221

ENGINEERING SCIENCES DATA. CHEMICAL ENGINEERING SERIES

London: Institute of Chemical Engineers

1969

$300 \mathrm{p}$

The Engineering Sciences Data, Chemical Engineering Series contains graphical and tabular data for the thermal conductivity of liquid aliphatic hydrocarbons, liquid alcohols, and water substance; the specific heat at constant pressure of water substance (ice, water and steam, solid, liquid and gaseous heavy water, and sea water); dynamic viscosity and density of water substances (ice, water and steam, liquid and gaseous heavy water, and sea water); and thermal conductivity of liquid halogenated aliphatic hydrocarbons including commercial refrigerants. Each section contains an index of property entries.

\# 6904013

*8.223

Ernest, G.

Mi chels, H. J. (Trans lator)

CONTRIBUTION TO A UNIVERSAL FORMULA FOR RESIDUAL PART OF THE SPECIFIC HEAT CP OF GASFS PC/T 5, Imperial College, London, England December 4,1968

$46 \mathrm{p}$

In this work the specific heat values $C p$ of four refrigerating fluids (R12, R115, R114, and R113) as well as of propane have been measured in the temperature range between 20 and $90^{\circ} \mathrm{C}$, for pressures between 0,2 and 8 absolute atmospheres; the flow-method has been used and a relative accuracy of approximately $0.1 \%$ was attained. As a result of the high accuracy of these measurements, the residual $\Delta C_{\text {}}$, which for the maximum pressures used is of the orgler of $10 \%$ of the total $C$ value, can be evaluated with a relative accuracy of 1 to $2 \%$. These experimental results, together with the $C_{p}$ data for water, measured by Vukalovitch et al. and the $\mathrm{C}_{\mathrm{p}}$ data for ammonia measured by osborne et al. have been used to obtain empirically a universal expression for the residual part of the specific heat of gases.

\section{\#6902043 $\quad * 8.033$}

Flynn, D. R.; Peavey, B. A., Jr. (Editors)

THERMAL CONDUCTIVITY, PROCEEDINGS OF THE SEVENTII CONFERENCE HELD ÁT THE NATIONAL BUREAU OF STANDARDS, GAITHERSBURG, MARYLAND, NOVEMBER 13-16, 1967

NBS Special Publication 302, National Bureau of Standards, Washington, D. C.

September 1968

80 lp

Available from the Superintendent of Documents, GPO

The Seventh Conference on Thermal Conductivity was held at the National Bureau of Standards on. November $13-16,1967$. This volume contains the texts of the papers presented. Topics covered include surveys of the present state of knowledge regarding the thermal conductivity of different materials, descriptions of different apparatuses for measuring thermal conductivity, new experimental data on the thermal conductivity or diffusivity of a variety of materials, and correlations between experimental results and theoretical predictions.

\section{\#6607035}

$\star 8.143-4.143$

Fomenko, V.S.

Sams onov, G.V. (Editor)

HANDBOOK OF THERMIONIC PROPERTIES, ELECTRONIC WORK FUNCTIONS AND RICHARDSON CONSTANTS OF ELFMENTS AND COMPOUNDS

New York: Plenum Press Data Division

1966

$151 \mathrm{p}$

The experimental and theoretical calculation of the work functions of particular materials (elements and compounds) are done by various methods: thermionic, photoelectronic, field-emission, effusion, contact potential difference, calorimetric and empirical relationships linking the work function to various physical characteristics which are utilized in the theoretical computation of the work function. Richardson constants are also given and references are listed at the end of the book. 
16512020

* 8.133

Forziati, A.F.; Singman, D.

OXYGEN SOURCES FOR FUES CELLS

TR-1128, Harry Diamond Laboratories, Washington,

D.C.

April 25, 1963

$29 \mathrm{p}$

Available from CFSTI

Commercially available gas, liquid, and chemical sources of oxygen for use with fuel cell batteries are compared. Cryogenic sources are shown to be the most efficient on a weight and volume basis. Chemical generators are satisfactory for applications requiring rather large quantities of oxygen gas at infrequent periods. Compressed gas cylinders are convenient when smali quantities of oxygen are desired. A bibliography of selected publications during the past five years is included.

\#6500244

$\approx 8.333$

Francis, A. W.

CRITICAL SOLUTION TEMPERATURES

Advances in Chemistry Series No. 31, American Chemical Society, Washington, D. C.

1961

$250 \mathrm{p}$

This book contains a compilation of critical solution temperatures (CST), a description of the methods used to determine the CST, a guide to the uses of CST data, especially for choosing extraction solvents, and a guide to methods for estimating the CST for untested systems. More than $6000 \mathrm{CST}$ observations are listed. Seventy percent of the systems listed have a hydrocarbon as one component; however, nearly 1100 nonhydrocarbon solvents are listed. In Table I the solvents are listed alphabetically and CST with references are given. Table II gives structure, aniline, and furfural points for high molécular weight hydrocarbons. About 800 aniline points of CST of hydrocarbons with aniline are listed. Table II gives lower critical solution temperatures and upper critical end point or critical temperature, upper layer. Tables IV to VIII present in concise form the data from 5 papers each giving miscibilities of a group of substances. A bibliography of 495 references is included.

\section{$\# 6500517$}

* 8.233

Frost, A. V.

Gerritsen, J. K. (Translator)

VISCOSITY OF VAPORS OF PETROLEUM PRODUCTS

TPRC Translation 6, The rmophysical properties

Research Center, Purdue University, Lafayette,

Indiana

October 29,1959

This work provides empirical formulae and a nomogram for the calculation of the viscosities of vapors of pure hydrocarbons and of petroleum products. The calculated and experimental data are compared in figures and tables, showing viscosity graphs for hydrocarbons and the dependence of viscosity on molecular weight. References are included.

\#6809009

* 8.133

Furukawa, G. T.; Saba, W. G. ; Reilly, M. L.

CRITICAL ANALYSIS OF THE HEAT-CAPACITY DATA OF THE LITERATURE AND EVALUATION OF THERMODYNAMIC PROPERTIES OF COPPER, SILVER, AND GOLD FROM $\cap$ TO $300^{\circ} \mathrm{K}$

NSRDS-NBS-18, Nationa 1 Bureau of Standards,

Washington, D. C.

April 1968

$49 \mathrm{p}$

Available from the Superintendent of Documents, GPO

The literature sources of heat-capacity data on copper, silver, and gold between 0 and $300^{\circ} \mathrm{K}$ have been compiled and the data critically analyzed. Tables of heat capacity $\left(\mathrm{C}_{\mathrm{p}}\right)$, enthalpy $\left(\mathrm{H}-\mathrm{H}_{0}^{\circ}\right)$, entropy $\left(S^{\circ}\right)$, Gibbs energy $\left(\mathrm{G}_{0}-\mathrm{H}_{0}^{\circ}\right)$, enthalpy function $\left(\mathrm{H}-\mathrm{H}^{\circ}\right) / \mathrm{T}$, and Gibbs energy function (G- $\mathrm{H}_{\circ}^{\circ}$ ) $/ \mathrm{T}$ have been obtained from the analyses. The literature values of the heat capacity, the electronic coefficient of heat capacity (gamma) and the $0^{\circ} \mathrm{K}$ limiting Debye characteristic temperature $\left[\theta_{0}(0)\right]$ are compared with the selected values. The sources of the data are tabulated chronologically along with the temperature range of measurements, purity of sample, and the pertinent experimental procedures used. A bibliography of the references is listed. A hrief appraisal of low-temperature calorimetry is given.

$\# 6500396,6500397$

Glushko, V. P. i et al. (F.di tors)

THERMODYNAMIC PROPERTIES OF CHEMICAL SUBSTANCES

VOLUME I. COMPUTATION OF THERMODYNAMIC PROPERTIFS

VOLUME II. TABLES OF TIERMODYNAMIC PROPERTIES SECOND EDITION

Moscow: Academy of Science, U.S.S.R.

1962

$1164 \mathrm{p}, 916 \mathrm{p}$

This two volume handbook contains tables of thermodynamic properties of 335 gases, 44 liquids and 45 solid substances in al1 424 components formed by the following 33 elements and isotopes: $\mathrm{H}, \mathrm{D}, \mathrm{T}, \mathrm{He}, \mathrm{Li}, \mathrm{Be}, \mathrm{B}, \mathrm{C}, \mathrm{N}, \mathrm{O}, \mathrm{F}, \mathrm{Ne}, \mathrm{Na}, \mathrm{Mg}, \mathrm{Al}$, Si, P, S, Ci, Ar, K, Ca, Br, Kr, Rb, Sr, Zr, I, Xe. $\mathrm{Cs}, \mathrm{Ba}$, Ilg, $\mathrm{Pb}$. The thermodynamic properties are calculated for the temperature interval of 293.15 up to 4000 to $20,000^{\circ} \mathrm{K}$. Tables of virial coefficients are giver for 34 gases. Volume 1 of this handbook contains methods of calculation of the rmodynamic properties of individual substances. Critical analysis of literature data up to and including 1916 is given on the constants necessary to calculate tables of thermodynamic properties, such as molecular constants, heat of formation and phase transitions, heat capacity dissociation energy, etc. The reason for the choice of these constants in the handbook is given as we 11 as the estimates in the cases when experimental data are absent. Volume 2 of the handbook contains tables of thermodynamic properties of the individual substances. All the tables have been calculated by the authors of the handhook on the basis of the data chosen in volume 1 .

\#6500228 *8.133

Goldmann, J. B. (Compiler)

PHASE RELATIONSHIPS OF MOLYBDENUM, NIOBIUM AND TUNGSTEN BORIDES, CARBIDES AND SILICIDES: AN ANNOTATED BIBLIOGRAPHY

Special Bibliography SB-63-9, Lockheed Missiles \& Space Company, Sunnyvale, California

May 1963

83p

Available from DDC

This annotated bibliography emphasizes phase relationships and their fabrication as evidenced in the physical properties, refractories, and powder metallurgy of molybdenum, niobium and tungsten compounds which are formed with boron, carbon and silicon. The period 1955-1962 yielded the majority of references included in this bibliography.

\#6500477-6500481 (inclusive) *8.133-4.133 Goldsmith, A. ; Waterman, T.E. ; Hirshhorn, H.J . HANDBOOK 'OF 'THERMOPHYSICAL 'PROPERTIES' OF SOLID MATERIALS, REVISED ENITION,

VOLUME I: ELEMENTS,

VOLUME II: ALLOYS,

VOLUME III: CERAMICS,

VOLUME IV: CERMETS, INTERMETALLICS, POLYMERICS, AND COMPOSITS

VOLUME V:' APPENDIX

New York: The Macmillan Company

1961

I : 752p; II : $1270 \mathrm{p} ;$ I I I :1162p; IV: $798 \mathrm{p} ; \mathrm{V}: 286 \mathrm{p}$

This set of books is an evaluated compilation of all data on the thermophysical properties of solid 
materials published during the period 1940-1957. Materials with melting points above $1000^{\circ} \mathrm{F}$ are included. The following physical properties are included: density, melting point, latent heat of fusion, iatent heat of vaporization, latent heat of sublimation, specific heat, thermal conductivity, thermal diffusivity, emissivity, reflectivity, linear thermal expansion, vapor pressure and electric resistivity. Volume I contains data on the elements; Volume II, alloys; Volume III, ceramics; Volume IV, cermets, intermetallics, polymeric materials, and composite materials. Volume $V$, the Appendix, contains the Materials Index, the Author Index, and an alphabetic list of materials. Wherever possible, a recommended value is given.

\#6500310

* 8.133

Goodwin, R. D. ; et al.

PRESSURE-DENSITY-TEMPERATURE RELATIONS OF FLUID PARAIIYDROGEN FROM 15 TO $100 \mathrm{~K}$ AT PRESSURES TO 350 ATMOSPHERES

Journal of Research of the National Bureau of Standards - A. physics and Chemistry; 6 7A:173-192

Experimental data are presented at closely spaced intervals of temperature and density. The range of experimental densities is from 0.064 to 2.8 times the critical density. In addition, there are presented uniformly interpolated tables in density and temperature and in pressure and temperature.

\#6903054 *8.133

Gosman, A. L.; McCarth, R. D. ; liust, J. G.

THERMODYNAMIC PROPERTIES OF ARGON FROM TIIE TRIPPLE POINT TO $300 \mathrm{~K}$ AT PRESSURES TO 1000 ATMOSPHERES

NSRDS-NBS-27, National Bureau of Standards, Washington, D, C.

March 1969

$146 \mathrm{p}$

Available from the Superintendent of Documents, GPO

Tabular values of density, internal energy, enthalpy and entropy of liquid and gaseous argon are presented for temperatures from 83.8 to $300 \mathrm{~K}$ at pressures of 0.01 to 1000 atmospheres. Diagrams of specific heats, compressibility factor, and entropy are included. The properties presented are calculated from an equation of state which was fitted to experimental p- $p-T$ data from the world literature. Extensive comparisons were made between the equation of state and the experimental data, and deviation plots are presented. The second virial coefficient and Joule-Thomson inversion curve were also calculated and comparisons made with values from other sources. A vapor pressure equation which covers the range from the triple point to the crimical point is also given.

\#6906008

Grigsby, D. L. ; Welles, S.J.

$4.183-8.183$

A BIBLIÓGRAPHY ON SELECT PROPERTIES OF ALUMINUM

Electronic properties Information Center, Hughes Aircraft Company, Culver City, California

April 1969

$59 \mathrm{p}$

This bibliography on pure aluminum and dilute aluminum alloys contains references on the following select properties: macroscopic structure, energy band structure, electrical resistivity, emission properties, magnetic properties, optical properties, thermal properties, Debye temperature and attenuation properties.
$\# 6500653$

* 4.133-3.133-8.133

GROUPS IV, V, AND VI TRANSITION METALS, SELECTED REFERENCES ON PREPARATION, STRUCTURE, PIIYSICAL PROPERTIES, METALLOGRAPIYY, AND TRANSPORT PROPERTIES ORNL-RMIC- 3 (Rev.), Research Materials Information Center, Oak Ridge National Laboratory, Oak Ridge, Tennessee

April 1965

$94 \mathrm{p}$

This bibliography contains lists of selected references on preparation methods, crystal structure, transport properties, and physical and mechanical properties for the Groups IV, V, and VI transition metals. An abstract is included along with each reference entry.

$\# 6902097$

$$
* 4.133-3.133-8.133
$$

GROUPS IV, V, AND VI TRANSITION METALS, SUPPLEMENT NO. 1 , SELLCTED REFERENCES ON PREPARATION STRUCTURE, PHYSICAL PROPERTIES, METALLOGRAPHY, AND TRANSPORT PROPERTIES

ORNL-RMIC-8, Research Materials Information Center, Oak Ridge National Laboratory, Oak Ridge, Tennessee April 1965

$27 \mathrm{p}$

Available from CFSTI

This bibliography is a supplement to ORNL-RMIC-3 (Rev.) of April 1965, and contains lists of selected references on preparation methods, crystal structure, transport properties, and physical and mechanical properties for the Groups IV, V, and VI transition metals.

\section{\#6500465 *8.133-4.133}

Gubareff, G.G.; Janssen, J.E.; Torborg, R.ll.

THERMAL RADIATION PROPERTIES SURVEY, A REVIEN OF THE LITERATURE SECOND EDITION

Minneapolis, Minnesota: Honeywell Regulator Company 1960

$293 \mathrm{p}$

Thermal radiation property values for more than 40 metals and their alloys are given. The properties reported are emissivity, absorptivity, reflectivity, and transmissivity at various surface conditions, temperatures, and wavelengths. In addition, radiation properties of building materials, paints, glasses, papers, cloths, and other miscellaneous solid materials have been compiled, and solar absorptivity and equilibrium temperatures for various materials have been tabulated. These values have been gathered from about 200 references in English, German, French, and Russian and have been carefully analyzed to obtain, in the author's opinion, the most trustworthy data for practical application. Recommended data for technically important materials are also presented graphically. An extensive survey was conducted to compile values for Stefan-Boltzmann's, Planck's, and Wien's constants, and the most reliable values for practical application are listed in a separate table.

\section{\#6809008}

* 8.133

Haar, L.

THERMODYNAMIC PROPERTIES OF MMONIA AS AN IDEAL GAS NSRDS-NBS-19, National Bureau of Standards, Washington, D. C.

August 1968

$10 \mathrm{p}$

Available from the Superintendent of Documents, GPO

Thermodynamic functions for anmonia as an ideal gas at one atmosphere pressure have been evaluated. The contribution of the highly anharmonic out-ofplane vibrational mode, including its large coupling with rotation and its coupling with the other vibrational modes, is considered in detail. Tables of $C_{p}^{\circ} / R,\left(H^{\circ}-E_{0}^{0}\right) / R T,\left(E_{0}^{\circ}-G^{\circ}\right) / R T$, and $S^{\circ} / R$ have been calculated at closely spaced intervals from 50 to $5000^{\circ} \mathrm{K}$ within an overall uncertainty of less than 0.1 percent at $1000^{\circ} \mathrm{K}$. 
\# 6500345

*8. 133

Haar, L.; Friedman, A. S.; Beckett, C. W. IDEAL GÁS THERMODYNAMIC FUNCTIONS AND ISOTOPE EXCHANGE FUNCTIONS FOR THE DIATOMIC IIYDRIDES, DEUTERIDES, AND TRITIDES

NBS Monograph 20, National Bureau of Standards, Washington, D. C.'

May 29, 1961

$271 \mathrm{p}$

Available from the Superintendent of Documents, GPO

This monograph contains a consistent set of tables of thermodynamic properties of a large number of diatomic hydrides, deuterides, and tritides, for the ideal gas state at one atmosphere pressure. In addition to the thermodynamic properties of the molecular gases, the tables also include thermodynamic properties for chemical reactions involving the isotopic exchange of hydrogen. The thermodynamic properties tabulated are the heat capacity, enthalpy, Gibbs free energy, and entropy.

\section{\#6905005}

$* 8.333$

Haas, C. W.; et al.

$1967^{\circ}$ BIBLIOGRAPHY AND SUBSTANCE-PROPERTY INDEX ON PHYSICAL AND THERMODYNANIC PROPERTIES OF ORGANIC SUBSTANCES AND MIXTURES

Thermodynamics Research Certer, Texas A\&्q University, College Station, Texas

September 20,1968

$148 \mathrm{p}$

This report consists of an index by substance and associated physical and/or thermodynamic property (Substance-Property Index) and a bibliography covering the literature on organic substances for the year 1967. As a further aid to users of this index, a "Guide to the Substance-Property Index" based on the standard order of arrangement of the elements and compounds is included in this report. Work in progress throughout the world dealing with physical, thermochemical, and thermodynamic measurements on organic substances and mixtures is summarized in this index by letter-number references, such as A-67, B-42, etc., to the abstracts found in the' IUPAC Bulletin of Thermodynamics and Thermochemistry, No. 11, June 1968. A new feature this year in the bibliography is the incorporation of the title of each article cited.

\section{$\$ 6907009$}

$\star 8.133$

Hala, E.; et al.

VAPOÚR LIQQUID EQUILIBRIUM DATA AT NORMAL PRESSURES Oxford: Pergamon press

1968

$541 \mathrm{p}$

Vapour-Liquid Equilibrium Data at Normal Pressures is a collection of data on a set of selected systems which have been correlated to express the dependence of the activity coefficients or separation functions of the composition of the liquid phase. The quantitative characterization of the systems is thus condensed into the constants of the correlation equations evaluated using the weighted least squares method. For each compound, the tables list the name of the compound, the source of the data quoted, vapour pressure constants, and calculated results, along with the deviations of the calculated from the direct experimental data. This gives information on the quality of the data and on the flexibility of the correlation relations used. Multi-component systems are included in the tables.

\#6807014

*8. 333

Ha11, L. A.

A BIBLIOGRAPHY OF THERMOPHYSICAL PROPERTIES OF METHANE FROM 0 TO $300^{\circ} \mathrm{K}$

NBS Technical Note 367 , National Bureau of Standards, Boulder, Colorado

May 1968

Available from the Superintendent of Documents, GPO
References, together with an abbreviated abstract, are presented for the following mechanical, thermodynamic, and transport properties of methane from 0 to $300^{\circ} \mathrm{K}$ published up to December 1967: density, P-V-T data, compressibility factor, expansivity, compressibility, equation of state, vanor pressure, melting pressure, latent heats, fixed points, specific heat, velocity of sound, Joule-Thomson" coeffjcients, entropy, enthalpy, internal energy, fugacity, Gibbs function, Helmholtz function, thermal conductivity, viscosity, Prandti number, self-diffusion coefficient, surface tension, dielectric constant, refractive index. A total of 660 articles have been indexed. Each article has been reviewed and coded with regard to properties studied, type of article (i.e., experimental, theoretical, etc.), and a method of presentation of data. The temperature and pressure ranges for each property under consideration are also given. An index has been prepared according to property with four subcategories: solid, liquid, gas up to $200^{\circ} \mathrm{K}$, and gas above $200^{\circ} \mathrm{K}$.

$\# 6500483$

$* 8.133$

Ha11, L. A.; llust, J. G.; Gosman, A. L.

BIBLIOGRAPHY OF TUERMOPHYSICAL PROPFRTIES OF ARGON FROM 0 TO $300^{\circ} \mathrm{K}$

NBS Technical Note 217, National Bureau of Standards, Washington, J. C.

July 1964

$102 \mathrm{p}$

Available from the Superintendent of Documents, GPO

A bibliography of 450 references is presented for mechanical, thermodynamic, and transport properties of argon from 0 to $300^{\circ} \mathrm{K}$. Each article has been reviewed and coded with regard to properties studied, type of article (i.e., experimental, theoretical, etc.), and method of presentation of the data. The temperature and pressure ranges for each property under consideration are also given. An index was prepared according to property with 4 sub-categories: solid, liquid, gas up to $200^{\circ} \mathrm{K}$, and gas above $200^{\circ} \mathrm{K}$.

\#6907038 $* 7.033-8.033$

Hame $r, W . J$.

RESUME OF VALUES OF THE FARADAY

Journal of Research of the National Bureau of July-August T968

A resume is given of the determinations of the value of the Faraday. Values obtained by silver deposition, iodide oxidation, oxalate oxidation, the omegatron, and silver dissolution are reviewed. All values are converted to the unified Cli international scale of atomic weights using the international atomic weights of 1967. Values of the Faraday are given in terms of both the NBS (legal) and absolute units of electrical measure. In the latter the new value for the acceleration due to gravity is used in computing the absolute value of electric current. On this basis and using the atomic weight of silver determined by Shields, Craig, and Dibeler, and converting to the $C^{12}$ scale, the value of the Faraday is $96,486.9 \pm 1.6$ absolute coulombs per gram-equivalent which differs by only 1 part per million from the value recommended by the National Academy of SciencesNational Research Council. If the atomic weight of silver recommended in 1967 by the International Atomic Weight Comission is used, the Faraday on the new gravity value is $96,486.5 \pm 1.6$ absolute coulombs per gram-equivalent which differs by 5 parts per million from that recommended by the National Acaderny of Sciences-National Research Council. No change in the value of the Faraday adopted by the NAS-NRC Committee is recommended. 
\#6903025

Hamer, W. J.

STANDÁRD CELLS, THEIR CONSTRUCTION, MAINTENANCE, AND CIIARACTERISTICS

NBS Monograph 84, National Bureau of Standards, Washington, D. C.

January 15,1965

$42 \mathrm{p}$

Available from the Superintendent of Documents, GPO

This Monograph contains information on the construction, maintenance, and characteristics of standard celis. The effects of temperature, pressure, electric current, light, shock, and vibration on standard cells are discussed. A history of the realization and maintenance of the unit of electromotive force is also included. A record of international comparisons of the unit of electromotive force is presented as well as information on the constancy of the National Reference Group of Standard Cells.

\section{\#6907037}

Hame r, W. J.

THEORET ICAL MEAN ACTIVITY COEFFICIENTS OF STRONG ELECTROLYTES IN AQUEOUS SOLUTIONS FROM 0 TO $100^{\circ} \mathrm{C}$ NSRDS-NBS-24, National Bureau of Standards, Washington, D. C.

December 1968

$271 p$

Available from the Superintendent of Documents, GPO

In determining the activity coefficients of electrolytes in aqueous solutions from the freezing point to the boiling point of the solvent, various equations have been used in the treatment of the data. This paper gives values for activity coefficients of electrolytes of various valence types from 0 to $100^{\circ} \mathrm{C}$, and for ionic strengths from zero to 0.1 molal or 0.1 molar, as calculated by seven different equations based on the theory of interionic attraction. These equations are those of Debye and Huckel, Guntelberg, Davies, Scatchard, and Bjerrum, and what may be termed an extended Guntelberg equation and an extended Scatchard equation.

\section{\#6608003}

Hanley, H. J. M.

THE VISCOSITY AND THERMAL CONDUCTIVITY COEFFICIENTS OF DILUTE ARGON BETWEEN 100 AND $2000^{\circ} \mathrm{K}$

NBS Technical Note 333 , National Bureau of Standards, Washington, D. C.

March 1966

Available from the Superintendent of Documents, GPO

The variation of the force constants of the Lennard-Jones, Exp: 6 , and Kihara potential functions was investigated by comparing the Chapman-Enskog kinetic theory expression for the viscosity coefficient with the experimental viscosity of dilute argon. It was found that this variation was more pronounced than expected. It was necessary to rationalize the choice of the force constants of each function before using the function to compare theory with experimental data. of the three, the Kihara was found to give the best correlation, and tables of the viscosity and thermal conductivity coefficients of dilute argon between 100 and $2000^{\circ} \mathrm{K}$ were computed from this potential and the Chapman-Enskog equations.

\#6905033 *8.133

Hanley, H. J. M.: Childs, G. E.

DISCREPANCIES BETWEEN VISCOSITY DATA FOR SIMPLE

GASES

Science, $159: 1114-1117$

March 8, 1968

It has been known for some time that Kestin and his co-workers have reported dilute gas viscosity coefficients which differ from the usually accepted values. Recent work from the Los Alamos Scientific Laboratory supplements Kestin's results. It is shown that there is no evidence for not accepting these different data. The whole subject of dilute gas viscosity measurements above room temperature should be reexamined both from the experimenter's and correlator's viewpoints. There is evidence that published tables may be incorrect by as much as 10 percent above $600^{\circ} \mathrm{K}$.

\#6707003

*8.133

Hanley, H. J. M.; Childs, G. E

THE VISCOSITY AND THERMAL CONDUCTIVITY COEFFICIENTS OF DILUTE NEON, KRYPTON, AND XENON

NBS Technical Note 352, National Bureau of Standards, Washington, D. C.

March 1967

$24 \mathrm{p}$

Available from the Superintendent of Documents, GPO

The coefficients of viscosity and thermal conductivity for dilute neon, krypton, and xenon were examined by a method already proved success ful for dilute argon, oxygen, and nitrogen. This method selects a suitable potential function, and its parameters, which is then used to correlate theory with experimental data, given the kinetic theory expressions for the transport coefficients. The method has recently been expanded and generalized and the results of this general study are applied to this note. The potential functions examined were members of the m-6, Kihara, Exp: 6 and Morse families. It was found that the Kihara was most suitable for neon, and the $\mathrm{m}-6$, with $\mathrm{m}=$ 17 and $m=24$, was most suitable for krypton and xenon, respectively. Viscosity and thermal conductivities were calculated from these functions and tables are given between 100 and $1000^{\circ} \mathrm{K}$.

\#6905027 *8.133-3.133

Hanley, H. J. M. ; Klein, M.

ON THE SELECTION OF THE INTERMOLECULAR POTENTIAL FUNCTION: APPLICATION OF STATISTICAL MECHANICAL THEORY TO EXPERIMENT

NBS Technical Note 360 , National Bureau of Standards, Boulder, Colorado

November 20,1967

$88 \mathrm{p}$

Available from the Superintendent of Documents, GPO

A method has been developed to evaluate quantitatively the relationship between model intermolecular potential functions and macroscopic experimental properties. Specifically, the following function families have been studied: $m-6$, Kihara, exp:6 and Morse; the properties studied are: viscosity coefficient, diffusion coefficient, second virial and Joule-Thoms on coefficients. The principal conclusions from this work are: 1 . a temperature range exists (around room temperature for most substances) in which data are completely insensitive to the potential function; 2 . the function families studied are essentially equivalent; 3 . more than one function is required to represent data over a range of about $1000^{\circ} \mathrm{K} ; 4$. the experimental error which can be tolerated if experimental data are to be used to select a potential function has been estimated. The conclusions are experimentally verified for both transport and equilibrium properties.

$\# 6500249$

Hansen, . $_{\text {. }}$ Anderko, $K$.

*8.133

CONSTITUTION OF BINARY ALLOYS, SECOND EDITION New York: McGraw-Hill Book Company, Inc. 1958

$1305 \mathrm{p}$

This monograph deals with the composition and crystal structure of 1,334 binary alloy systems, compiled from a critical review of the literature. The composition data are presented in the form of phase diagrams for those systems for which such diagrams had been published. Other systems have information about mutual solubility and melting points compiled. The crystal structure data includes symmetry class and lattice parameters. An index to systems is provided. 
6607009

* 8.133

Harned, H. S.: Owen, B. B.

THE PHYSICAL CHEMISTRY OF ELECTROLYTIC SOLUTIONS, THIRD EDITION

New York: Reinhold Publishing Corporation

1958

$803 p$

Basic theory of electrolytic solutions is presented in the first five chapters of this book. Chapters 2,3 and 4 present interionic attraction theory, properties of ionic atmosphere, the thermodynamic properties of aqueous solutions and the theory of irreversible processes in electrolytic solutions. Numerical compilations of physical constants, characteristic slopes and mathematical functions are presented in chapter 5. The experimental methods discussed in chapters 6 through 10 are irreversible processes in solutions of strong electrolytes; conductance, transference numoers, viscosity, diffusion; coulomb forces and ion association, weak electrolytes, frequency and field effects; thermochenical quantities, including partial molal volumes, coefficients of expansion and compressibility; the calculation of activity and osmotic coefficients from freezing points, boiling points and vapor pressures; and the thermodynamics of galvanic cells. No apparatus or experimental techniques are discussed in detail. In the last third of the hook, the emphas is is upon the properties of electrolytes rather than on the methods by which they were obtained. Chapters 11, 12 and 13 deal with solutions of hydrochloric acid, 1-1 electrolytes and polyvalent electrolytes, respectively. Mixtures of strong electrolytes in water form the subject of chapter I4. Ionization constants of weak electrolytes, their temperature coefficients and the ionization of weak electrolytes in salt solutions are considered in chapter 15. Extensive tables of quantities derived from the best available data are given in the appendix. Author, subject and chemical indexes are included.

\section{$\# 6905011 \quad * 8.333$}

Harrison, R. H.; Moore, R. T.; Douslin, D. R.

THERMODYNAMIC PROPERT IES OF COMPRESSED GASES, FINAL TECHNICAL SUMMLARY REPORT, 12 APRIL 1965' TO 14 DECEMBER 1966

Bartlesville Petroleum Research Center, U. S. Bureau of Mines, Bartlesville, Oklahoma

December 1966

$56 \mathrm{p}$

Available from DDC

The thermodynamic properties, $H-H^{\circ}, S-S^{\circ}, G-G^{\circ}$,

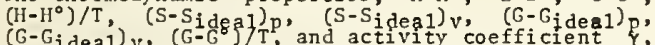
are presented for methane, tetrafluoromethane, and the three mixtures, 75,50 , and 25 mole percent methane, from 0 to $350^{\circ} \mathrm{C}$ and 3 to 400 atmospheres.

\#6704015

$\star 8.233$

Hildegrand, J. H.; Scott, R. L.

THE SOLUBILITY OF NONELECTROLYTES, THIRD EDITION

New York: Dover Publications, Inc.

1964

$488 \mathrm{p}$

This third edition of The Solubility of Nonelectrolytes discusses data and thermodynamic properties for ideal, nonidea1, gas-liquid, liquidliquid, solid-liquid, solid, metallic, and high polymer solutions. Also included are thermochemical properties of mixing (entropy, heat, free energy), intermolecular forces, liquid state, polarity, hydroger bonding, solvation and association, three or more component systems, surface phenomena, and gaseous mixtures. Vapor pressure of binary liquid solutions and chemical kinetics of solutions are presented. The book includes author, subject and formula indexes.
86911032 * 8.133

Hilsenrath, J.; Klein, M.

TABLE OF THERMODYNAMIC PROPERTIES AND CHEMICAL COMPOSITION OF NITROGEN IN CHEMICAL EQUILIBRIUM INCLUDING SECOND VIRIAL CORRECTIONS FROM $1600^{\circ} \mathrm{K}$ TO $15,000 \mathrm{~K}$

AEDC-TR-66-65, Arnold Engineering Development Center, Air Force Systems Command, Arnold Air Force Station, Ternessee

April 1966

$307 \mathrm{p}$

Avaílable from DDC

Tables for the thermodynanic properties for nitrogen are presented which take into account the effect of dissociation and ionization and the limiting-1aw Debye-llucke1 and second virial corrections, upon the the rmodynamic properties and the equilibrium compositions. Values are tabulated from $1600^{\circ} \mathrm{K}$ in steps of $100^{\circ} \mathrm{K}$ to $15,000^{\circ} \mathrm{K}$ at close spacings in the logarithm of the density $\left[\log _{10} \mathrm{p} / \mathrm{D}\right.$. $=-7.0(0.2) 2.2$ ] for the compressibility factor $\mathrm{z}$ $\mathrm{PV} / \mathrm{RT}$; the dimensionless functions for: internal energy, E/RT; eлthalpy, H/RT; entropy, S/R; $\log _{10} \mathrm{P}^{\prime}(\mathrm{atm})$ and $Z^{*}=\Sigma C_{i}^{\prime}$ and the mole fractions of the ${ }^{10}$ species in chemical equilibrium. The underlying equations and the input data are discussed briefly. The effects of the real gas corrections on the equilibrium properties are illustrated graphically. The equilibrium composition is given for selected temperatures over the tabulated density range. The wide range of temperatures and densities over which the thermodyлаліс properties have been tabulated make the tables useful in a variety of engineering design and test programs, and in scientific research and development.

\$ 6500371 $* 8.133$

Hilsenrath, J.; Klein, M.

TABLES OF THERMODYNAMIC PROPERTIES OF AIR IN CHEMICAL EQUILIBRIUM INCLUDING SECOND VIRIAL CORRECTIONS FROM $1500^{\circ} \mathrm{K}$ TO $15,000^{\circ} \mathrm{K}$

AEDC-TR-65-58 Arnold Enoineering Development Center, Amold Air Force Station, Tennessee March 1965

$333 p$

Available from DDC

Tables for the thermodynamic properties for air are presented which take into account the effect of dissociation and ionization and the limiting-law Debye-lluckel and second virial corrections upon the thermodynamic properties and the equilibrium compositions. values are from $1500^{\circ} \mathrm{K}$ in steps of $100^{\circ} \mathrm{K}$ to $15,000^{\circ} \mathrm{K}$ at close spacings in the logarithm of the density $\left[\log _{10} p / 0_{0}=-7 .(0.2) 2.2\right]$ for the compressibility factor $Z^{\circ}=\mathrm{PV} / \mathrm{RT}$; the dimensionless functions for: internal energy, E/RT; enthalpy, H/RT; entropy, S/RX ; $10 \mathrm{~g}, \mathrm{P}(\mathrm{atm})$ and $z^{*}=\Sigma C_{i}$. The underlying equations. 10 and the input data are discussed briefly. The effects of the real gas corrections on the equilibrium properties are illustrated graphically. The equilibriun composition is given for selected temperatures over the tabulated density range. The wide range of temperatures and densities over which the thermodynamic properties have been tabulated make the tables useful in a variety of engineering design and test programs, and in scientific research and development.

\#6500373 *8.133

Hilsenrath, J.; K1ein, $M$.

TABLES OF THERMODYNAMIC PROPERTIES OF NITROGEN IN CHEMICAL EQUILIBRIUM INCLUDING SECOND VIRIAL CORRECT IONS FROM $2000^{\circ} \mathrm{K}$ TO $15,000^{\circ} \mathrm{K}$

AEDC-TDR-63-162 Arnold Engíneering Development Center, Air Force Systems Command

March 1964

$162 \mathrm{p}$

Available from DDC

Tables for the thermodynamic properties for nitrogen are presented which take into account the effect of dissociation and ionization and the 
limiting-law Debye-Huckel and second virial corrections, upon the thermodynamic properties and the equilibrium compositions. Values are tabulated from $2000^{\circ} \mathrm{K}$ in steps of $100^{\circ} \mathrm{K}$ to $15,000^{\circ} \mathrm{K}$ at close spacings in the logarithr of the density [ $\rho / P$. $=-7.0(0.2) 2.4$ ] for the compressibility factor $z=$ PV/RT; the dimensionless functions for: internal energy, E/RT; enthalpy, H/RT; entropy, S/R; $\log _{10} P(\mathrm{~atm})$ and $z^{*}=\Sigma C_{i}$. The underlying equations and the input data are discussed briefly. The effects of the real gas corrections on the equilibrium properties are illustrated graphically. The equilibrium composition is given for selected temperatures over the tabulated density range. The wide range of temperatures and densities over which the thermodynamic properties have heen tabulated make the tables useful in a variety of engineering design and test programs, and in scientific research and development.

\#6905007 *8. 133

Hi lsenrath, J.; Messina, C. G.; Evans, W. H. TABLES OF IDEAL GAS THERMODYNAMIC FUNCTIONS FOR 73 ATOMS AND TIIEIR FIRST AND SECOND IONS TO $10,000^{\circ} \mathrm{K}$ Technical Documentary Report No. AFWL TDR-64-44, Air Force Weapons Lahoratory, Kirtland Air Force Base, New Mexico

August 1964

$441 \mathrm{p}$

Available from DDC

Tables are presented of five thermodynamic properties, $\left(G^{\circ}-E_{0}^{\circ}\right) / R T,\left(H^{\circ}-E_{0}^{\circ}\right) / R T, S^{\circ} / R, C_{p}^{\circ} / R$, and $\left(H^{\circ}-E_{0}^{\circ}\right) / R$, for the electron and for 73 elenents, II through La and $H f$ through Ac (with the exception of At and $\mathrm{Fr}$ ) and, in most cases, for their first and second ions. The tables are given at $100^{\circ}$ intervals starting at $100^{\circ} \mathrm{K}$ for the atoms and at $100^{\circ}$ or $1000^{\circ}$ for the ions and extending to $10,000^{\circ} \mathrm{K}$. They were computed for the atoms and atomic ions considered as ideal monatomic gases hy direct summation over the available energy levels. The summation over energy levels was made from the ground state up to the principal quantum number 5. Where the quantum numer, $n$, for the ground state exceeds 5 , summations were made only over levels having the same $\mathrm{n}$ as the ground state. The sensitivity of the thermal functions to the inclusion of higher energy states in the partition function is discussed briefly, illustrated graphically for the specific heats, and tabulated for the Gibbs free energy function.

$\# 6500343$ *8. 133

Hilsenrath, J.; Ziegler, G. G.

TABLES OF EINSTEIN FUNCTIONS

NBS Monograph 49, National Bureau of Standards, Washington, D. C.

July 1962

$258 \mathrm{p}$

Available from the Superintendent of Documents, GPO

Tables are presented for the contribution of a harmonic oscillator to the free energy function, enthalpy function, entropy and heat capacity of gases. Dimensioniess values of the Planck-Einstein functions are given as a function of $x=h c_{v} / K T$ for $x=0.0010(.0001) 0.1500(.001) 4.000(.01) 10.00(.2) 16.0$. A second $t a b l e$ which gives the contributions in $\mathrm{cal} / \mathrm{mole}{ }^{\circ} \mathrm{K}$ directly as a function of frequency $v$, and temperature $T$, was computed using the values 1.43880 for the second radiation constant hc/k, and 1.98717 for the universal gas constant R. The arguments for the latter table are spaced at 10 wave number intervals from $100 \mathrm{~cm}^{-1}$ to $4000 \mathrm{~cm}^{-1}$.
\#6500346

* 8.133

Hilsenrath, J.; et al.

TABLES OF THERMAL PROPERTIES OF GASES COMPRISING TABLES OF TIIERMODYNAMIC AND TRANSPORT PROPERTIES OF AIR, ARGON, CARBON DIOXIDE, CARBON MONOXIDF IIYDROGEN, NITROGEN, OXYGEN, AND STEAM

NBS Circular 564, National Bureau of Standards, Washington, D. C.

November 1955

$488 \mathrm{p}$

Available from Superintendent of Documents, GPO

Tables are given at close temperature intervals for the thermodynamic and transport properties of air, argon, $\mathrm{CO}, \mathrm{CO}, \mathrm{H}_{2}, \mathrm{~N}_{2}, \mathrm{O}_{2}$ and steam. The thermodynamic properties, compressibility factor, density, entropy, enthalpy, specific heat, specific heat ratio and sound velocity, are tabulated for the real gases at pressures up to 100 atmospheres and to temperatures of $600^{\circ} \mathrm{K}$ for hydrogen, $1500^{\circ} \mathrm{K}$ for carbon dioxide, $850^{\circ} \mathrm{K}$ for steam, and $3000^{\circ} \mathrm{K}$ for the remainder. The ideal gas thermodynamic functions are tabulated uniformly to $5000^{\circ} \mathrm{K}$. Also tabulated are the vapor pressures and transport properties: thermal conductivity, viscosity, and Prandt1 number. These we re fitted either semitheoretically or empirically to the experimental values and are tahulated over the range of the available experimental data. Comparisons of the tabulated values with the existing experimental data are shown in deviation plots which exhibit the range and distribution of the experimental data as well as their agreenent with the tabulated values.

\#6907043, 3 volumes *3.133

Ili lsenrath, J., et al.

THERMODYNAMIC AND SHOCK WAVE PROPERTIES OF ARGON AND NITROGEN IN CHEMICAL EQUILIBRIUM WITII VIRIAL AND IONIC CORRECTIONS,

VOLUTIE I: THIEORY AND EXPLAINATIONS

VOLUME II: TABLES FOR ARGON

VOLURIE III: TABLES FOR NITROGEN

Technical Report No. AFWL-TR-68-60, Volumes I, II \& II, Air Force Weapons Laboratory, Kirtland Air Force Base, New Mexico

May 1969

$360 \mathrm{p}$

Available from DDC

Tables for the thermodynamic properties for argon and nitrogen are presented which take into account the effect of dissociation and ionization and the limiting-law Debye-Huckel and second virial corrections, upon the the rmodynamic properties and the equilibriun compositions. Values are tabulated from $1500^{\circ} \mathrm{K}$ in steps of $500^{\circ} \mathrm{K}$ to $20,000^{\circ} \mathrm{K}$ at close spacings in the logarithr of the density $[\log \rho / \rho$ 。

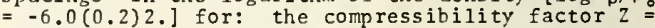
$\mathrm{PV} / \mathrm{RT}$; the dimensionless functions for: internal energy, E/RT; enthalpy, II/RT, entropy, $S / R$; specific heat at constant pressure $C / R$ and constant volume $C_{V} / R$; the velocity of souhd in the

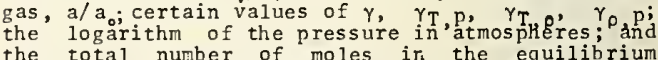
mixture. The concentrations of the molecules. atoms and ions are given in tabular form in mole fractions but the concentration of electrons is also given in particles per cubic centimeter. The properties behind a normal shock are given for a temperature of $300^{\circ} \mathrm{K}$ as an initial condition ahead of the shock. The shock tables include the velocity in kilometers per second and the pressure and density ratios across the shock. The equations underlying the calculations are given in detail and the input data is discussed briefly. The wide range of temperatures and densities over which the thermodynamic and shock properties have been tabulated make the tables useful in a variety of engineering, design and test programs; and in scientific research and development. 
\# 6909008

Ho, C. Y.

*8.233

A CONTINUING SYSTEMATIC PROGRAM ON TABLFS OF

THERMOPHYSICAL PROPERTIES OF MATERIALS

Technical Report AFIL-TR-69-79, Wright Air

Development Center, Naterials Laboratory, Wright-

Patterson Air Force Base, Ohio

April 1969

$101 \mathrm{p}$

Available from CFSTI, AD 688969

This technical report covers work in a continuing systematic program on the thermophysical properties of materials involving the literature search, collection, codification, organization, data extraction, compilation, evaluation, correlation, analys is and synthesis, the preparation of "intermediate tables" presenting the total available experimental information, and the final preparation of internally consistent tables of "best data" referred to as "Tahles of Recommended Most Probable Values." The work reported on consists of data tables projects on the thermal conductivity, specific heat, thermal radiative properties (emittance, reflectance, absorptance, transmittance), thermal diffusivity, and thermal expansion of elements, alloys, compounds, mixtures and systems, and the thermal conductivity, specific heat and viscosity of liquids and gases. property data are presented in hoth tabular and graphical forms with accompanying tables giving specifications and characterizations of the test specimens. The resulting data tables are disseminated at large through the 13-volume TPRC Series on Thermophysical properties of Matter, disseminated thorugh the Thermophysical properties Research Literature Retrieval Guide. This report does not contain the completed thousands of data sheets, but does reproduce in the Appendix, the Table of Contents and the Grouping of Materials and List of Figures and Tables for each of the first seven volumes (which contain over 8000 pages) of the TPRC Series to show the scope of their coverage.

\# 6809011

*8.233-4.233

Ho, C.Y.; Powell, R.W.; Li ley, P.E.

THERIAL CONDUCTIVITY OF SELECTED MATERIALS, PART 2 NSRDS-NBS-16, National Bureau of Standards, Washington, D. C.

February 1968

$146 \mathrm{p}$

Available from the Superintendent of Documents, GPO

The work presented in this report comprises the critical evaluation, analysis, synthesis of the available thermal conductivity data, and the generation of recommended values for twelve metalic elements, mainly for the solid state, for a range of graphites and for three fluids in the gaseous state. These are cadmium, chromium, lead, magnesium, molybdenum, nickel, niobium, tantalum, tin, titanium, zinc, zirconium, Acheson graphite, ATJ graphite, pyrolytic graphite, $875 \mathrm{~S}$ graphite, $890 \mathrm{~S}$ graphite acetone, ammonia and methane. For each of the materials recommended values are given over a wide range of temperature.

$\# 6903060$

*8.133

Ho, J. C.; O'Neal, H. R.; Phillips, N. E.

LOW TEMPERATURE HFAT CAPÁCITIES OF' CONSTANTAN AND MARGANIN

The Review of Scientific Instruments, $34: 782-783$

The heat capacities of Constantan and manganin have heen measured from 0.15 and $0.2^{\circ} \mathrm{K}$, respectively, to $4.2^{\circ} \mathrm{K}$. Both alloys have large hyperfine heat capacities at temperatures of a few tenths of a degree and data are presented which can be used in making corrections to calorimetric measurements in which one of these materials is used as a heater.
$\$ 6511004$

* 8.143

Holley, C. (Compiler and Editor)

TUERMODYNAMIC AND TRANSPORT PROPERTIES OF URANIUM

DIOXIDE AND RELATED PIIASES

IAEA Technical Reports Series 39 (Report of a pane 1 held in Vienna, March 1964), International Atomic Energy Agency, Vienna

1965

$105 \mathrm{p}$

This report deals with the physical, thermodynamic, and transport properties of uranium dioxide and related phases of the uranium-oxygen system. It includes data and a summary of research work done in the field through 1964. The following topics are included: crystal structure, heat capacity, free energy, enthalpy and entropy measurements, vaporization processes, surface and oxidation properties, thermal conductivity, electrical properties, optical properties, and practical implications of thermodynamic and transport properties.

\#6608029

* 8.333

llorsley, L. H.

A2EOTROPIC DATA

Advances in Chemistry Series 6, Anerican Chemical Society, Washington, D. C.

June 1952

$333 \mathrm{p}$

Information is given in tabular form on 14,501 binary systems of azeotropes and nonazeotropes. The boiling points of the pure constituents and of the azeotropes, and the compounds of the azeotropes when available, are given. The boiling points of the azeotropes range from less than $-100^{\circ} \mathrm{C}$ to greater than $2300^{\circ} \mathrm{C}$. Similar data are given for 407 ternary systems. Included are a formula index, a 437 reference bibliography, and three articles, "Vapor-Liquid Equilibrium Diagrams of AlcoholKetone Azeotropes as a Function of Pressure" "Graphical Method for predicting Effect of Pressure on Azeotropic Sys tems," and "Graphical Method for predicting Azeotropism and Effect of pressure on Azeotropic Constants."

\#6500245 * 8.333

Horsely, L. H.; Tamplin, W. S.

A2EOTROPIC DATÁ - II

Advances in Chemistry Series 35, American Chemical Society, Washington, D. C.

1962

$105 \mathrm{p}$

This volume is a supplement to A2EOTROPIC DATA published as Advances in Chemistry Series No.6. American Chemical Society, 1952. It includes revised data on systems in the original table plus new data on azeotropes, nonazeotropes, and vaporliquid equilibria collected since 1952. No at tempt has been made to evaluate the data. Where appreciable differences occur in data from different sources, more than one set of data is recorded. A brief description is included for the calculation of azeotropic data for immiscible systems from vapor pressure data. The tables are arranged in the same manner as the previous volume.

\# 6500303

Hughes, E. E.; Lias, S. G.

VAPOR PRESSURES OF ORGANIC COMPOUNDS IN THE RANGE BELOW ONE MILLINETER OF MERCURY

NBS Technical Note 70 , National Bureau of Standards, Washington, D. C. October 1960

$24 p$

Available from CFSTI, PB 161571

Data for vapor pressures less than one millimeter of mercury for over three hundred organic compounds have been collected. The values are presented in tabular form. No attempt has been made to evaluate the reliability of these data except where several investigators have studied the same compound over 
the same pressure range. A brief discussion of the methods and instruments which have been used to measure low vapor pressures is included.

\#6712007 *8.133-3.133

Hughes, R. L.; Smith, I. C.; Lawless, E. A. Holzmann, R. T. (Fdi tor)

PRODUCTION OF TIHE BORANES AND RELATED RESEARCH

New York, London: Academic Press

1967

$533 \mathrm{p}$

This is a review and analysis of borane chemistry. The topics covered include: precursors to the boranes; formation of the horon-hydrogen bond and diborane; preparation and properties of the boranes, alkylboranes, and carboranes; kinetics and mechanism studies; preparation and properties of pentaborane and decaborane from diborane; separation and purification; analytical methods; horonnitrogen chemistry; acid-base chemistry and appendices on toxicology; infrared spectra, molecular structures, physica

boranes and their derivatives.

\#6511007 *8.133-4.133

Hultgren, R.; Orr, R.L.; Kelley, K.K.

SUPPLEMENT TO SELECTED VALUES OF THERMODYNAMIC PROPERTIES OF METALS AND ALLOYS

California University, College of Engineering and Lawrence Radiation Labóratory, Berkeley, California June, 1970

$500+$ 'loose-leaf pages

This Supplement contains the results of evaluations for 56 metallic elements and 178 binary alloy systems for which thermodynamic data were available. Reevaluation and extension of the selected values will be a continuing process as new data become available. Prior to the publication of a revised edition of the book additional evaluations, as they are completed, will be issued in loose-leaf form at frequent intervals for inclusion with this Supplement. Description of the methods of evaluation and forms of presenting the data may be found in the parent volume of "Selected Values." Results of the evaluations in the form of discussions and analyses of the data and tables of selected values are given. Some of the properties covered include freezing point, vapor pressure, transition temperature, phase diagrams, entropy, heat capacity, free energy function, heat content; heat of transition, sublimation, fusion and vaporization; entropy of transition, fusion and vaporization; free energy of sublimation and vaporization; free energy of formation; free energy - excess integral, relative partial molar and relative partial molar excess; heat of formation relative partial molar; entropy of formation excess integral, relative partial molar and relative partial molar excess; heat capacity relative partial molar.

\section{\#6500386 *8.233}

Hus t, J. G. ; Gosman, A. L.

FUNCTIONS FOR THE CALCULATION, OF ENTROPY, ENTHALPY AND INTERNAL ENERGY FOR REAL FLUIDS USING EQUATIONS OF STATE AND SPECIFIC HEATS

Preprint of paper in Advances in Cryogenic Engineering, Volume 9 , p roceedings of the 1963 Conference, National Büreau of Standards, Cryogenic Data Center, Boulder, Colorado

1963

Available from the Cryogenic Data Center

General integral equations for the determination of entropy, enthalpy, and internal energy are derived. In the first part of this paper the integration path is taken to be on the real gas surface thus yielding one set of equations. In the latter part of this paper the integration path is defined such that ideal gas specific heats and reference properties are introduced. The latter set of integral equations are applied to a particular equation of state resulting in algebraic expressions for the entropy, enthalpy, and internal energy.

\#6500403 *3.233

Hust, J. G.; Stewart, R. B.

THERMODYNAMIC PROPERTY VALUES FOR GASEOUS AND LIQUID CARBON MONOXIDE FROM 70 TO $300^{\circ} \mathrm{K}$ WITH PRESSURES TO 300 ATMOSPHERES

NBS Technical Note 202, National Bureau of Standards, Boulder, Colorado

November 1963

$19 \mathrm{p}$

Available from the Superintendent of Documents, GPO

The internal energy, entropy, enthalpy, and density of carbon monoxide are tabulated as functions of pressure and temperature from 70 to $300^{\circ} \mathrm{K}$ and 0.1 to 300 atmospheres. A compressibility factorpressure chart and a temperature-entropy chart are also included. The $P-p-T$ values have been calculated using the Su principle of corresponding states with nitrogen as a model. Extensive comparisons are included, illustrating the deviations of the calculated values from the experimental data and from other correlated data. Equations representing the $P-p-T$ surface and the vapor pressure are given.

\#6500482

*8.133

Ilust, J. C.; et al.

BIBLIOGRAPHY OF THE THERMOPHYSICAL PROPERTIES OF OXYGEN AT LOW TEMPERATURES

NBS Technical Note 137, National Bureau of St andards, Washington, D. C.

February 1962

$8 \mathrm{p} \quad 709$

Available from the CFSTI, PB 161638

This bibliography of the mechanical, thermodynamic and transport properties of oxygen below $0^{\circ} \mathrm{C}$ presents 325 references and is the result of a thorough search of the world's scientific and engineering literature. In addition to searching abstracting journals and bibliographies, the authors reviewed each docunent for property data and for additional references. Listed for each reference are the properties and the corresponding temperature and pressure range, together with additional pertinent information such as the type of data (i.e. whether the data are derived from experimental measurements, theoretical considerations or as a compilation from other sources), the form and amount of data, etc. An index according to property, sub-indexed for temperature an pressure ranges, and an author index are included.

\#6500463

*8.143-4.143

Janssen, J.; Luck, J.; Torborg, R.

REFLECTANCE OF ANODIZFD TITANIUMA AND BERYLLIUN

Aeronautical Systems Division, Wright-Patterson Air Force Base, ohio

(No date)

$3 p$

Selection of materials having the desired thermal radiation properties is of primary importance for space applications, since heat transfer to and from an object in space occurs only by radiation. The effect of anodizing variables on the reflectance of anodized titanium and beryllium was studied over the range of 0.4 to 22 microns in a vacuum of $10^{-5}$ $\mathrm{mm} \mathrm{IIg}$ with specimen temperatures of 100 to $1300 \mathrm{~F}$. of the anodizing variables studied, the various anodizing solutions generally had the greatest effect on the reflectance. A number of surfaces investigated had high alpha/epsilon ratios, indicating usefulness as potential solar collector surfaces. 
\#500464

$* 8.143-4.143$

Janssen, J.; Luck, J.; Torborg, R. TIHERMAL REFLECTANCE OF ANODIZED MTALS FOR
SPACECRAFT

Aeronautical Systems Division, Wright-Patterson Air Force Base, Óhio

(No date)

$3 \mathrm{p}$

Selection of materials having proper thermal radiation properties permits the engineer to achieve passive temperature control in spacecraft. The effect of anodizing variables on the reflectance of anodized aluninum and magnesium was studied over the range of 0.4 to 22 microns in a vacuum of $10^{-5} \mathrm{~mm}$ of $\mathrm{lig}$ with specimen temperatures of 100 to $825 \mathrm{~F}$. In general, the anodizing process had a greater effect on the reflectance than did alloying elements in the metal. The pressure of water in some coatings, which was driven off at elevated temperatures in the vacuu, gave lower reflectance in the infrared.

\#6711011

* 3.143

Janz, G. J

MOLTEN SALTS IIANDBOOK

New York and London: Academic Press

167

$588 \mathrm{p}$

The data in the handbook was taken from results published in the literature; for the most part, numerical values are those in the original source material. The following categories of data are included: 1. physical properties: atomic and ionic radi, melting and boiling points, density viscosity, vapor pressure, surface tension, refractive index, critical data; 2 . thermodynamic properties: liquid-liquid immiscibility, metalmolten salt systers, netal oxide-moiten salt systems, silver salts-molten salt systems, phaserule studies, solubility, thermal data, cryoscopy, expansivity, compressibility, and high pressure studies; 3. electrochemical properties: reference electrodes and emf series, electrical conductance, polarography, electromigration, dielectric and thermoelectric properties; 4. spectroscopy and structure: vihrational spectroscopy, visible and U.V. spectroscopy, NMIR and FPR spectroscopy, X-ray and neutron diffraction, and fused salt electrolytes; 5. practical features: melt preparation and purification, containment and corrosion, chromatographic analysis, electrolys is chemical processes, fuel cells, hatteries an nuclear technology.

$\# 6711009$

$\star 8.343$

Јалz, G. J.

THERMODYNATIC PROPERTIES OF ORGANIC COMPOUNDS, ESTIMATION METHODS, PRINCIPLES AND PRACTICE, REVISED EDITION

New York, London: Acadenic Press

167

$261 \mathrm{p}$

An updating in practical methods for computing the rmodynamic properties and a compilation of data and correlations for thermodynamic calculations are presented. Part I of the text deals with the calculation of free energy changes and their use as a criterion of thermodynamic properties from molecular parameters, spectroscopic data, and statistical thermodynamics; and estimation methods. Part II presents 78 tables of data and correlations of the many contributors in the field of estimation of thermodynamic properties. These tables have been collected to make application of the estimation methods as convenient as possible. The tables are reproduced as compiled in the original work of each investigator.
甘ै 6811001

Janz, G. J.; et al.

MOLTEN SALTS: VOLUNE 1 , ELECTRICAL CONDUCTANCE, DENSITY AND VISCOSITY DATA

NSRDS-NBS-15, National Bureau of Standards, Washington, D. C.

October 1968

$19 \mathrm{p}$

Available from the Superintendent of Documents, GPO

Data on the electrical conductance, density and viscosity of single-salt melts we re compiled from a comprehensive search of the literature up to December 1966 and a critical assessment made of the compiled data. Recommended values were determined and are presented as functions of temperature in the form of equations and tables. The results for some 174 compounds as single-salt melts are reported; no attempt was made in the present effort to erbrace the results for molten salt mixtures. Data are presented for fluorides, chlorides, bromides, iodides, carbonates, nitrites, nitrates, oxides, sulfides, sulfates and a miscellaneous group.

\#6909063

*8. 143

Janz, G. J.; et al.

MOLTEN SALTS: VOLUME 2. SECTION 1. ELECTROCHFMISTRY OF MOLTEN SALTS: GIBBS FREE ENERGIES AND EXCESS ENERGIES FROI EQUILIBRIUY-TYPE CELLS. SECTION 2. SURFACE TENSION DATA

NSRDS-NBS-28, National Bureau of Standards

August 1969

$116 \mathrm{p}$

Available from Superintendent of Documents, GPO

This book consists of two sections as follows:

Section 1. The critical evaluation of excess free energies of binary molten salt mixtures with a common ion from equilibrium-type electrochemical cells is described in this report. For this purpose calculations using the original emf data were systematically undertaken to establish comparisons of free energy values of various workers that would be significant. The reversibility of electrodes is investigated by comparing the electronotive force of cells with a single molten salt as liquid electrolyte with thernochemical data.

Section 2. Data on the surface tensions of single salt melts have been systenatically collected and evaluated. Results are given for 106 inorganic compounds over a range of temperatures where available.

\#5 511008

$* 8.243$

Jencks, W. P.

pka VALUES

Brandeis University, Waltham, Massachusetts

(No date)

$31 \mathrm{p}$

This is an unevaluated compilation of the pKa values for inorganic acids, phosphates, carboxylic acids, phenols, alcohols and oxygen acids, amino acids, peptides, nitrogen compounds, thiols, carbon acids, and indicators. References are given for each value from a total of 96 sources.

\#6500470,6500472

* 8.143

600474

Johnson, V. J. (Editor)

COMPENDIUM OF THE PROPERTIES OF MATERIALS AT LOW TEMPERATURE (PIIASE 1) PART I. PROPERTIES OF FLUIDS, PART II. PROPERTIES OF SOLIDS, PART III. BIBLIOGRAPHY OF REFERENCES (CROSS-INDEXED)

WADD Technical Report No. 60-56, Parts I, II and

II , Wright Air Development Division, Wright-

Patterson Air Force Base, Ohio

October 1960

I $-400 p$, I I $-300 p$, I II - 159p

Available from CFSTI and DDC, Part I AD 249644,

Part II - AD 249746, Part III - AD 249777 
This first phase of the Compendium covers ten properties of ten fluids (Part I), three properties of solids (Part II), and an extensive bibliography of references including a property and Material Index (Part III). In Part I, density, expansivity, thermal conductivity, specific heat and enthalpy, transition heats, phase equilibria, dielectric constants, adsorption, surface tension and viscosity for the solid, liquid and gas phases of helium, hydrogen, neon, nitrogen, oxygen, air, carbon monoxide, fluorine, argon and methane are given wherever adequate data could be collected. In Part I I thermal expansion, thermal conductivity, and specific heat and enthalpy are given for a number of solids of interest in cryogenic engineering. Data sheets, primarily in graphic form, are presented from "best values" of data collected. The source of the material used, other references and tables of selected values with appropriate comments are furnished with each data sheet to document the data presented. Conversion tables and other helpful information are also included.

\section{\#6500389 *8.143 \\ Keenan, J. H.; Keyes, F. G. \\ THERMODYNAMIC PROPERTIES OF STEAN INCLUDIIT DATA FOR TIIE LIQUID AND SOLID PHASES, FIRST EDITION \\ New York: John Wiley \& Sons, Inc. \\ 163 \\ $89 \mathrm{p}$}

The present tables are computed from entirely new formulations of the properties of water. Table gives absolute pressure, specific volume, enthalpy and entropy for saturation temperatures. Table gives specific volume, enthalpy, entropy and internal energy for saturation pressures. In Table 3 specific volume, enthalpy and entropy are given for superheated vapor. Within the limitations of the size of the volume, tables are included givin the viscosity, the heat conductivity, compressed liquid properties, data on ice and its vapor, isentropic expansion exponents, and certain thermometric calihration data, while at the same time retaining the temperature-scale conversion table, logarithm tables, and conversion factor tables of the 1930 volume. Certain charts of the specific heat hitherto not available are included and show the extraordinarily rapid changes and the large values of the quantity corresponding to volumes at and near the critical state. The range of temperature $\left(1600^{\circ} \mathrm{F}\right)$ and of pressure (5500 psi) of the tables is also greater than the 1930 tables. The inclusion of data for the liquid phase is an innovation made possible by the recent exact measurements of heat quantities and specific volumes. The sources of all the data employed are briefly discussed with references to the devices of formulation employed and the analytical expressions found to be servicable.

\#6911007 *8.143

Keenan, J. II.; et a1.

STEAM TABLES, THERMODYNAMIC PROPERTIES OF WATER INCLUDING VAPOR, LIQUID, AND SOLID PIIASES (ENGLISII UNITS)

New York: John Wiley \& Sons, Inc.

166

$174 \mathrm{p}$

The present Steam Tables are based on the tables of Keenan and Keyes (1936). Included are tables providing measured values of the specific volume of the liquid and of the vapor, enthalpy, heat capacity, P-V-T relation, Gibbs free energy. Also values for the compressed liquid region are included. These tables are in cood accord with the International Skeleton $\mathrm{T}$ ables $(1963)$. The present $t a b l e s$ extend the range of the Keenan and Keyes tables to $2400^{\circ} \mathrm{F}$ and to $15,000 \mathrm{psi}$. A table of the properties of the compressed and metastable liquid is provided, along with a separate and detailerl table for states near the critical point. Transport properties, i.e. viscosity and thermal conductivity, are not included.
\#6512003

Kees om, W. II.

Cook, G. A. (Editor)

HELIUM. ARGON, HELIUM AND TIIE RARE GASES

New York: Elsevier Puhlishing Company

$1942 ; 1961$

pp. 104-108 \& 147; 190-193\&247

These articles were taken from a textbook on helium and other rare gases. Data are presented for the viscosity of helium gas as a function of temperature between $4^{\circ}$ and $300^{\circ} \mathrm{K}$.

\#6500359

$* 8.243$

Kelley, K. K.

CONTRIBUTIONS TO THE DATA ON THEORETICAL METALLURGY

XI I I. HIGII-T EMPERATURE IIEAT-CONTENT, HFAT-CAPACITY, AND ENTROPY DATA FOR THE ELEMENTS' AND INORGANIC COMPOUNDS

Bulletin 584, Bureau of Mines, Washington, D. C.

1960

$232 p$

Available from the Superintendent of Documents, GPO

This publication, as its subtitle indicates, contains high-temperature heat-content, heatcapacity, and entropy data for the elements and inorganic compounds. Some other properties covered include vapor pressure, and heat of transition, fusion, vaporization and sublination. The available experimental and calculated values were compiled and intercompared, and a selection of the "best" values was made. The heat content data are given in tabular form for use by those who make the rmodynamic computations by means of tables, and in algebraic form for use by those who prefer equations.

\section{\#6500357 \\ $* 8.243$}

Kelley, K, K.

CONTRIBUTIONS TO TIIE DATA ON TIIEORETICAL METALLURGY XV - A REPRINT OF BULLETINS $383,384,393$, AND 406 Bulletin 601 , Bureau of Mines, Washington, D. C. 1962

$525 \mathrm{p}$

Available from the Superintendent of Documents, GPO

This work is part of the program of study of the Pacific Experiment Station of the United States Bureau of Mines and is a natural consequence of two earlier publications which dealt with the entropies at $298.1^{\circ} \mathrm{K}$ and the specific heats at high temperatures of inorganic substances. These earlier papers contain the data necessary for writing the thermodynamic equations for the heat and free-energy changes accompanying polymorphic transformations and fusions for all inorganic substances whose heat capacities have been measured in the ranges of temperature where such changes of state occur. It is the purpose of this present work (1) to apply the previously compiled entropy values and specific-heat equations to vaporpressure and other pertinent data in obtaining heat and free energy of vaporization equations for inorganic substances valid up to the normal boiling or sublination points ( $760 \mathrm{~mm}$ pressure), (2) to supplement wherever possible the data on heats and entropies of transformations and fusions, (3) to supplement the data on entropies of gases, and (4) to supply tables of vapor pressures for inorganic substances at various temperatures up to and including the boiling or sublimation point. This work also contains a bibliography of vapor-pressure data, complete so far as possible up to April 1934. 
\#6500356

$* 8.243$

Kelley, K. K.; King, E. G.

CONTRI BUTIONS TO THE DATA ON THEORET I CAL PIETALLURGY XIV. ENTROPIES OF TIIE FLE?ENTS AND INORGANIC COMPOUNDS

Bulletin 592, Bureau of Yines, Washington, D. C.

1961

$149 \mathrm{p}$

Available from the Superintendent of Documents, GPO

The first Federal Bureau of lines compilation of entropy and low-temperature heat-capacity data was Bulletin 350, which appeared in 1932. Revisions and elaborations were issued as Bulletin 394 (1936), Bulletin 434 (1941), and Bulletin 477 (1950). The present hulletin covers data available through Septenber 1959 and is a further elaboration and revision, made desirable hy the fact that nearly 1300 entropy values for inorganic substances at $298.15^{\circ} \mathrm{K}$ now are known, as compared with 800 in the 1950 publication, which covered data available through october ig43. The present bulletin contains the currently pertinent explanatory matter that appeared in its predecessors. It has the same dual purpose of assembling the available entropy values at $298.15^{\circ} \mathrm{K}\left(25.00^{\circ} \mathrm{C}\right)$ for the elements and inorganic compounds and giving enough explanation of methods of measuring and calculating entropies to make the results comprehensible. It should be emphasized that this hulletin supersedes those mentioned above.

\#6500399

$* 8.143-4.143$

Kols ky, H. G.

THE TIIERYODYNAMIC PROPERTIES OF 54 ELIMENTS CONSIDERED AS IDEAL MONATOMIC GASES

LA-2110, Los Alanos Scientific Laboratory, Los Alamos, New Mexico

March 15, 1957

$138 p$

Available from CFSTI

Tables of the thermodynamic properties of fiftyfour elements have been calculated considering each as an ideal monatomic gas. The energy levels as determined by the National Bureau of Standards Spectros copy Section were prepared on IBM cards for one of the LASL IBM 704 computers. The partition functions were calculated for each element and the thermodynamic properties were evaluated from them for 150 temperatures between $10^{\circ} \mathrm{K}$ and $8000^{\circ} \mathrm{K}$. A short table of the harmonic oscillator functions was also done in the same format as the other tables for use in evaluating the vibrational contributions to the the rmodynamic properties. $\Lambda$ brief discussion of the theory and calculational details is given.

$\$ 6602014$ *8. 343

Kreglewski, A.; Zwolinski, B. J.

NEW METIIODS OF CALCULATION OF SOME PIIYSICAL PROPERTIES OF LIOUIDS BASED ON THF ANTOINE EQUATION, II. n-ALKANES AND n-ALKYL COMPOUNDS

Chemical Thermodynanic properties Center, Texas A\&M University, College Station, Texas

August 15, 1965

$11 \mathrm{p}$

Empirical and semi-empirical formulae are derived, enabling one to calculate the normal boiling temperatures and the constants $A, B$, and $C^{\prime}$ of the Antoine vapor pressure equation $\log \mathrm{p}=\mathrm{A}$. $B /\left(C^{\prime}+T\right)$, and the heat of vaporization of n-alkyl compounds $\left(\mathrm{Y}-\left(\mathrm{CII}_{2}\right) \mathrm{m}^{-\mathrm{H}}\right)$. The specific feature of the correlations is that all values converge to those of n-paraffinic hydrocarbons when the number of carbon atons approaches infinity. Sets of internally consistent constants can be readily computed by means of these correlations.
$\# 6902067$

*8.143

Kropschot, R. H.; Birminghan, B. W.; Mann, D. B. (Editors)

TECHNOLOGY OF LIQUID HELIUM

NBS Monograph 1Il, National Bureau of Standards, Washington, D. C.

October 1968

$371 \mathrm{p}$

Available from the Superintendent of Documents, GPO

This treatise is a source document containing information on helium resources, production, conservation, thermodynamic properties, liquefaction and refrigeration techniques, transportation and storage of liquid and safety requirements. It also contains a discussion of uses for liquid and cold gas in cryoelectronics, superconductivity, buhble chambers, cryopumping anc missile and space systens. The book hrings together articles by noted authorities in cryogenic technology in which they discuss their specialized field in great depth.

$\# 6500363 \quad$ *8.143

Kub as chewski, O.; Evans, [.. L.

METALLURGICAL TIERMOCIIEMISTRY, THIRD EDITION

New York: Pergamon Press

1958

$440 \mathrm{p}$

This textbook deals with theoretical and experimental thermodynamics and thernochemistry and contains tables of thermochemical constants for a large number of elenents and compounds of metallurgical importance. Theoretical topics considered include the mass action law, thermodynamic functions and solutions. Experimental methods discussed include calorimetric methods, electromotive force methods, and equilibria with a gaseous phase and a condensed phase. The estimation of the rmochemical data and examples of thermochemical treatment of metallurgical problens are also discussed. The thermochemical data have been compiled from published experimental results of over a century. The authors have, wherever possible, listed the most accurate values and assigned a range of uncertainty to each value. The data has heen included for heats of formation, standard entropies, heats of transformation, fusion and evaporation, heat capacities, vapor pressure, standard free energies of reaction, and crystal structure type.

\#6500364 $* 8.143$

Kubas chewski, 0.; Catteral1, J. A, THERMOCIIEMICAL DATA OF ALLOÝS New York: Pergamon press

1956

$200 \mathrm{p}$

Thermochemical data are given for intermetallic systems and for the transition metals as carbides, phosphides, nitrides, sulphides and oxides. Some of the properties covered include partial free energy, partial heat of solution, partial entropy in solution, integral free energy, integral heat of solution, integral entropy of solution, latent heat of fusion, entropy of fusion, latent heat of transformation, and volume change on formation. References and an index of alloy systems are given at the end to simplify searching.

\# 6500526

$* 0.1151-3.151-5.151-8.151$

LANDOLT-BORNSTEIN, NUNERICAL. DATA AND FUNCTIONAL RELATIONSIIIPS IN PIYYSICS, CHEMISTRY, ASTRONOPY, GEOPIIYSICS AND TECHNOLOGY, SIXTH EDITION,

VOLUME I: ATOMIC AND MOLECULAR PIIYSICS,

PART 2: MOLECULES I (NUCLEAR STRUCTIJRE)

Eucken, A.: Hellwege, K.-H. (Editors)

Berlin, New York: Springer-Verlag

1951

$571 \mathrm{p}$

In German 
This section of the Landolt-Bornstein tables contains atomic physical data of molecules which describes the structure and dynamics of their nuclear framework. The following properties have been tabulated: atomic distances and structures, valence energies of chemical bonds, dissociation energies of chemical bonds, and molecular vibrations and rotations.

\#6602004

$* 0.1151-8.151$

LANDOLT-BORNSTEIN, NUMERICAL DATA AND FUNCTIONAL RELATIONSIIIPS IN PIIYSICS, CIEMISTRY, ASTRONOMY, GEOPHYSICS AND TECHNOLOGY, SIXTII EDITION, VOLUNE II: PROPERTIES OF' MATTER IN ITS AGGREGATED STATES,

PART 2a: VAPOR-LIQUID EQUILIBRIUM AND OSMOTIC PHIENOMENA

Schaefer, K.: Lax, E. (Editors)

Berlin, New York: Springer-Verlag

1960

$974 p$

In German

This section contains 22 mechanical-thermal constants for the equilibrium of heterogeneous systems. Vapor pressure under various conditions, literature references, density, fusion and allotropic changes, temperature changes of crystalline liquids, and other data are given for one component systems. The same type of data as well as heterogeneous equilibrium, freezing point lowering, Lewis-Randall functions, boiling point elevation, osmotic pressure diagrams and references are listed for 222 multi-component systems.

\section{\#6500530 \\ *0.1151-4.151-8.151}

LANDOLT-BORNSTEIN, NUMERICAL DATA AND FUNCTIONAL RELATIONSIIIPS IN PHYSICS, CIIEYISTRY, ASTRONOMY, GEOPIIYSICS AND TECIINOLOGY, SIXTII EDITION,

VOLUME II: PROPERTIES OF MATTER IN ITS AGGREGATED STATES,

PART 2b: EQUILIBRIA EXCEPT FUSION EQUILIBRIA, EQUILIBRIA OF SOLUTIONS I

Schaefer, K.; Lax, E. (Edi tors)

Berlin, New York: Springer-Verlag

1962

$982 \mathrm{p}$

In German

This section of the Landolt-Bornstein tahles contains information on solution equilibria of gaseous substances in liquids, solid and liquid metals and alloys, as well as the solubilities of solid and liquid suhstances in liquid solution media. Except for the section "gases in metals," only solution equilibria in the temperature region of about $-200^{\circ} \mathrm{C}$ to $300^{\circ} \mathrm{C}$ are presented. The data were taken from literature published through 1.962. Solution equilihria are presented for gases with water, aqueous solutions, non-aqueous inorganic liquids, liquids and mixtures of organic and inorganic liquids. A1so, solution equilibria of hydrogen, oxygen and nitrogen in pure metals are presented. Solution equilibria are presented for inorganic materials in water and other inorganic liquids, organic liquids, organic materials in water, and solutions with organic solvents.

\#6510028

${ }^{*} 0.1151-8.151$

LANDOLT-BORNSTEIN, NUMERICAL DATA AND FUNCTIONAL RELATIONSHIPS IN PIIYSICS, CHEMISTRY, ASTRONOM, GFOPIIYSICS AND TECINOLOGY, SIXTH EDITION, VOLUME II: PROPERTIES OF'MATTER IN ITS AGGREGATED STATES,

PART 2C: EQQUILIBRIA EXCEPT FUSION EQUILIBRIA, EQUILIBRIA OF SOLUTIONS II

Sch aefer, K,; Lax, E. (Editors)

Berlin, New York: Springer-Verlag

1964

$731 p$

In German

This section of the Landolt-Bornstein tables includes data on solution equilibria of multicomponent systems for solutions of solid and liquid organic materials in organic liquids and equilibria in systems with several non-miscible phases.

$\# 6500531$

$\star_{0} 0.1151-4.151-6.151-8.151$

LANDOLT-BORNSTEIN, NIMERICAL DATA AND FUNCTIONAL

RELATIONSIIIPS IN PIIYSICS, CHFMISTRY, ASTRONOMT, GEOPHYSICS AND TECINOLOGY, SIXTII EDITION,

VOLUTE II: PROPERTIES OF MATTER IN ITS AGGREGATED STATES,

PART 3: FUSION EQUILIBRIA AND INTERFACIAL

PIIENONIENA

Schaefer, K.; Lax, E. (Edi tors)

Berlin, New York: Springer-Verlag

1956

535 P

In German

This section of the Landolt-Bornstein tables contains data on fusion equilibria and interfacial tensions. The section includes data from literature published through September 1955. Where several values for one measurement were found, the average was presented. Data on fusion equilibria are presented for binary and ternary systems of inorganic compounds, silicate systems, organic systems, and organic-inorganic systems. Interfacial tension data are presented for pure liquids towards their vapors or air, solutions towards air, two non-miscible systems, and interfacial films on water. Data are also presented on absorption from the gas phase and the liquid phase, and on paper chronatography.

\#6500532

$* 0.1151-8.151$

LANDOLT-BORNSTEIN, NUMERICAL DATA AND FUNCTIONAL RELATIONSIIIPS IN PIIYSICS, CIEMISTRY, ASTRONONY GEOPIIYSICS AND TECHNOLOGY, SIXTII EDITION, VOLUME I I: PROPERTIES OF' MATTER IN ITS AGGREGATED STATES,

PART 4: CALORIC QUANTITIES OF STATE

Schaefer, K.; Lax, E. (Edi tors)

Berlin, New York: Springer-Verlag

1961

$863 \mathrm{p}$

In German

This section of the Landolt-Bornstein tables contains data on the the rmodynamic state functions of alloys, compounds and elements, as well as reaction enthalpies for the formation of compounds from the elements and heats of reaction for several inportant reactions, such as acid-base neutralization and moistening of solid surfaces with liquids. The numerical values are given in joules, except that certain tables for the theoretical calculations of functions present values in joules and calories. Because of the large number of substances and systems listed, numerical data could be given only for a selection of compounds; preference was given to substances which had heen accurately investigated or which could claim a greater interest on the part of the user. The following quantities are tabulated: experimental and theoretical hasic quantities of thermochemistry, standard value of molar heats, entropies, formation enthalpies and free formation enthalpies; values of thermodynamic properties as a function of temperature for selected materials; heat capacity as a function of temperature; tables for calculating thermodynamic functions in the presence of internal molecular vibrations (including Planck-Einstein and Debye functions): Joule-Thoms on effect; magnetocaloric effect of lowest temperatures on paramagnetic salts: thermodynamic data of metal solutions; adsorption heats and heats of moistening; and heats of neutralization. 
\# 6909060

$* 0.1151-5.151-8.151$

LANDOLT-BORISTEIN, NIMERICAL DATA AND FUNCT IONAI RELATIONSIIIPS IN PIIYSICS, CHFMISTRY, ASTRONOIY, GEOPHYSICS AND TECINOLOGY, SIXTII FNITION,

VOLUME II: PROPERTIES OF'MATTER IN ITS AGGREGATED STATES,

PART 5b: TRANSPORT PIIENOMENA II, KINETIC HOMOGENOUS GAS EQUILIBRIA

Schaefer, K. (Editor)

Berlin, New York: Springer-Verlag

1968

$397 \mathrm{p}$

In German

Part $5 \mathrm{~b}$ of Volume II contains some kinetic properties of the elements and selected compounds. The first series of tables lists diffusion constants for elements and ions in metals, salts, and alloys, both liquid and solid. A short table of values for diffusion constants of some gases in selected metals is included. The second series of tables deals with thermal conductivity data for gases, liquids and solids. The constants are reported as a function of temperature, pressure and composition. The next series of tables deals with thermal diffusion constants for binary and ternary gas mixtures. Constants are also reported in liquid mixtures and solutions (Ludwig-Soret Effect). The next section lists a series of kinetic rate constants for reaction in gas phase and includes: dissociation, atom-radical and ionmolecule reactions. Another series of tables lists rate constants and equilibrium constants for reactions in solid and mixed phase. Several addenda to the main sections are appended.

\#6500533

*0.1151-4.151-8.151

LANDOLT-BORNSTEIN, NUMERICAL DATA AND FINCTIONAI RLLATIONSIIIPS IN PIIYSICS, CIIEMISTRY, ASTRONORY, GEOPIYYSICS, AND TECINOLOGY, SIXTH EDITION,

VOLUNE II: PROPERTIES OF MATTER IN ITS AGGREGATED STATES,

PART 6: ELECTRICAL PROPIERTIFS I

Ile llwege, K.-11.; Ilellwege, A.M. (Editors)

Serlin, New York: Springer-Verlag

1959

$1018 \mathrm{p}$

In German

This section of the J,andolt-Bornstein tables contains data on the electrical properties of matter (except electrochenical systems) published through February 1959. The properties tabulated include the conductivities of pure metals and certain alloys at $0{ }^{\circ} \mathrm{C}$ and their temperature variations, resistance ratios, effect of magnetic fields on resistance, super-conducting metals and alloys and their transition temperature, magnetic field effects on super conductivity, galvanomagnetic and thermomagnetic effects, ionic conductivity in crystals, transfer numbers in solids. Also tabulated are the constants of homogeneous semiconductors: photoelectric conduction; piezoelectric equations; and the elastic, piezoelectric and dielectric constants of piezoelectric crystals; dielectric properties of inorganic and organic crystals; crystalline fluids and pure fluids; dielectric properties at high frequencies; temperature and pressure dependence of dielectric constants; and the dielectric properties of gases. Thermionic emission and work functions, thermopotentials, Peltier and Thomson heats, and photoemission data are also listed.
\#6500534

* $0.1151-8.151$

LANDOLT-BORNSTEIN, NU:MERICAL DATA AND FUNCTIONAI, RELATIONSHIPS IN PIYSICS, CIIEMISTRY, ASTRONON, GEOPIYSICS AND TECHNOLOGY, SIXTII EDITION,

VOLUME II: PROPERTIES OF'MTTER IN ITS AGGREGATED STATES,

PART 7: ELECTRICAL PROPIRTIES II (ELECTROCHEMICAL SYSTEMS

Hellwege, K.-ll.; Hellwege, A.:. (Editors)

Berlin, New York: Springer-Verlag

1960

$959 \mathrm{p}$

In German

This section of the Landolt-Bornstein tables contains data on the electrical properties of electrochenical systems. The data were taken from the literature published through October 1960 . The data compiled include the electrical conductivity of molten salts, pure liquid, and aqueous electrolyte and field solutions; frequency and field strength dependence of conductivity of aqueous solutions; conductivity of ions in aqueous solutions; electrical conductivity of non-aqueous solutions; electrophoretic potentials and electrokinetic potentials, standard electrode potentials, dissociation constants of inorganic and organic compounds and acid-base indicators.

$\# 6500535$

*0.1151-3.151-4.151-3.151

LANDOLT-BORNSTEIN, NUMERICAL DATA AND FUNCTIONAL RELATIONSHIPS IN PHYSICS, CIIEMISTRY, ASTRONONY, GEOPIIYSICS AND TECINOLOGY, SIXTIY EDITION,

VOLUME II: PROPERTIFS OF'MATTFR IN ITS AGGREGATED STATES,

PART 8: OPTICAL CONSTANTS

llellwege, K.-H.; llellwege, A.M. (Editors)

Berlin, New York: Springer-Verlag

1962

y01p

In German

This section of the Landolt-Bornstein tables contains tables and figures for the optical properties of substances. Data are compiled from literature published through June 1962 . Data are compiled for: the optical constants and reflection of metals, alloys, and thin metal layers; refractive indices of inorganic and organic materials as a function of wavelengths; optical constants of selected solid materials in broad wavelength regions; rotation of the polarization plane in crystals; double refraction; piezooptical and electrooptical constants of piezoelectric crystals; light refraction by glasses and transparent plastic; light permeability, double refraction, Faraday effect and Kerr effect on glasses; refractive index of crystalline fluids; refractive index and optical rotatory power of pure liquids and solutions; Cotton-Mouton effect of liquids; double refraction due to current in liquids; Kerr constants of liquids; refractive indixes of gaseous elements and compounds; refraction and dispersion of air and of hydrogen compounds; Cotton-Mouton effect of gases and Kerr constants for gases.

$\# 6500538$

$* 0.1151-4.151-7.151-3.151$

LANDOLT-BORNSTEIN, NUNERICAL DATA AND FUNCTIONAL RELATIONSIIIPS IN' PIIYSICS, CIIEMISTRY, "ASTRONORY, GEOPHYSICS AND TECHNOLOGY, SIXTH EDITION,

VOLUME IV: TECHNOLOGY

PART 1: MATERIAL VALUES AND MECHANICAL PROPERTIES OF NON-METALS

Schmidt, E. (Editor)

Berlin, New York: Springer-Verlag

$881 p$

In German

This section of the Landolt-Bornstein tables contains material values and mechanical behavior of non-metallic substances, and their technical applications. Data are compiled from literature published through autumn 1964. The following general information is included: measuring 
systems, units and conversion tables, atomic weights, reduction to standard states, densities of water and mercury. Physical and mechanical properties of non-metallic solids are given for naturaI and synthetic building materiaIs, organic natural materials (wood, paper, ceIlulose, wood pulp), fibrous materíals, ceramics, gíasses, synthetic materials, and natural synthetic materials. Also presented are data on friction and rolling resistance of non-metaliic and mctalic substances, viscosity, flow through tubes, circuitous flow of bodies during two dimensional flow and rough flow, flotation, speed of sound, sound radiation and absorption, the human ear, sound spectra, vibrational numers, spatial and building acoustics, sound recording and biological effects of ultrasonics.

$\# 6500539$

*0.II I I - 3. I5 1-4. I 51-7.151-8.151 LANDOLT-BORNSTEIN, NUNERICAL DATA AND FUNCTIONAL RELATIONSHIPS IN PIIYSICS, CHEMISTRY, ASTRONOMY, GEOPIIYSICS AND TECINOLOGY, SIXTII EDITIÓN,

VOLUNE IV: TECINOLOGY,

PART 2a: ILATERIAL VALIJES AND PROPERTIES OF INDUSTRIAL METALS, BASIC PRINCIPLES, TESTINC PROCFSSES, INDUSTRIAL STEFLS

Borchers, II.; Schmidt, E. (Editors)

Berlin, New York: Springer-Verlag

1963

$888 \mathrm{p}$

In German

This section of the Landolt-Bornstein tables includes data on metaliographic principles and concepts, testing properties, and the properties of iron and its alloys. Data are given on diagrams of state, strength testing and destruction free materials. The physical properties of iron tabulated are lattice constants, density, thermaI expansion, diffusion, atomic heats, specific heats, enthalpies, entropies and free enthalpies, phase diagrams and transition points, thermaI conductivity, electrical properties, magnetic properties, optical constants and excitation crosssections. Composition, treatment, strength values and special chemical properties of pure iron, unalloyed steels, highly alloyed steels, speciaI steels, and cast iron are presented.

H 6804008

*0.115I-4.I51-8.151

LANDOLT - BORNSTEIN, MUMERICAI DATA AND FUNCTIONAL RELATIONSIIIPS IN PIYSICS, CIIEMISTRY, ASTRONOMY, GFOPIIYSICS AND TECHNOLOGY, SIXTH EDITIÓN, VOLUME IV: TECHNOLOGY

PART 4a: IIEAT TECIINOLOGY, HEAT TECINOLOGY ENGINEERING MEASURING METHODS, THERMONYNAMIC PROPERTIES OF HOMOGENOUS MATERIALS

Hausen, II. (Editor)

Berlin, New York: Springer-VerIag

1967

$944 \mathrm{p}$

In German

This section of LandoIt-Bornstein is a compilation of data dealing with the technology of thermal measurements and the thermodynamic properties of gases, vapors, liquids, and solid materials. Data are included for such areas as the basis of temperature measurement; the temperature measurement with contact thermometers; opticaI temperature measurement (Pyrometry); hygrometry; thermodynamic properties of homogeneous materiaIs, gases, water and steam, refrigerating agents; thermal expansion of soIids and liquids; and the specific thermal properties of technically important materials.
\#680200 I

$* 0.1151-4.15 \mathrm{I}-8 . \mathrm{I} 51$

LANDOLT-BORNSTFIN, NUMERICAL DATA AND FUNCTIONAL RELATIONSHIPS IN SCIENCE AND TECHNOLOGY, NEW SERIES,

GROUP II. ATOMIC AND MOLECIJLAR PIYYSICS, VOLUME 5: NOLECULAR ACOUSTICS

Schaaffs, $W$.

Hellwege, K.-II. ; Ilellwege, A.M. (Ldi tors)

Berlin, New York: Springer-VerIag

1967

$286 \mathrm{p}$

In German and English

This volume lists data on acoustics based essentialiy on the molecular properties of matter, and values of sound velocity, absorption coefficients, and dispersion data. The measurenents were made at frequencies ranging from $10 \mathrm{Kc}$ to $200 \mathrm{Mc}$. Preference was generally given in compiling data to measurements for which statements on density, chenical purity, and the temperature coefficient were added, Often, however, several statements can be found for a measured value. Generally, the limit of accuracy is $I \%$ to $2 \%$ Data are given on sound velocity in gases and liquids; for pure gases and liquids as a function of temperature, pressure, and structure; also for mixtures of gases, mixtures of liquids, and solution. Data are compiled on sound absorption and dispersion in gases; sound absorption in homogeneous liquids, in solution, and on sound propagation in polymers, also on sound propagation in isotropic or quasi-isotropic solids (metals, inorganic non-metaIs, organic solids). There are special sections on sound propagation in Iiquid helium and shock wave velocity.

H6608030

$* 0.1$ I $51-4.151$

LANDOLT-BORNSTEIN, NUMERICAL DATA AND FUNCTIONAI, RELATIONSHIPS IN SCIENCE AND TECHNOLOGY, NEW SERIES,

GROUP III: CRYSTAL AND SOLID STATE PIIYSICS,

VOLUSIE 1: ELASTIC, PIEZOELECTRIC, PIFZOOPTIC AND ELECTROOPTIC CONSTANTS OF CRYSTALS

Bechmann, R.; llearmon, R.F.S.

llellwege, K.-II.; Ilellwege, A.M. (Editors)

BerIin, New York: Springer-Verlag

1966

$160 \mathrm{p}$

In German and English

This volume of the new Landolt-Bornstein series gives the elastic, piezoelectric, piezooptic, and electrooptic constants of crystals. The data included were taken from data published in the literature through 1965. For materials where one or two sets of measurements of a given constant were found, they are quoted as published; for more than two sets of measurements, the mean and coefficient of variation are given. For nonpiezoelectric constants, the elastic stiffnes and compliance constants, together with their temperature and pressure variations, are given. For piezoelectric constants, the elastic, piezoelectric, dielectric, piezooptic, and electrooptic constants, plus the electromechanical coupling factors are given. The temperature coefficients of the elastic stiffness, compliance, piezoelectric stress and strain constants are also given.

\# 7001036

*0, II $51-4.151-8.15$ I

LANDOLT - BORNSTEIN, NUMERICAL DATA AND FUNCTIONAL RELAT IONSIIIPS IN SCIENCE AND TECINOLOGY, NEW SERIES

GROUP III: CRYSTAL AND SOLID STATE PIIYSICS, VOLUME 2: FLASTIC, PIEZOELECTRIC, PIEŹOOPTIC, ELECTROOPTIC CONSTANTS, AND NONLINEAR DIELECTRIC SUSCEPTIBILITIES OF CRYSTALS

Bechmann, R.; Hearmon, R.F.S.; Kurtz, S.K.

Hellwege, K.-II.; Hellwege, A.N. (Editors)

Berlin, New York: Springer-Verlag

I 969

$242 p$

In German and English 
Volume III/2 of the New Series of Landolt-Bornstein continues Volume III/ only three years after publication of the latter. A1l the tables contained in III/l are brought up to date by supplements in III/2. In addition, the elastic and electrooptic constants of higher order and the nonlinear optical susceptibilities have now heen included. Readers should note that the Index of Substances at the end of III/2 refers to hoth volumes.

\#6911030

$* 8.153$

Landshoff, R. K, M. ; Nagee, J. L. (Editors)

THER'LAL RADIATION PIIENONENA VOLUHE 1 RADIATIVE PROPERTIES OF $A I R$

New York: IFI/Plenur

1969

$664 p$

The editors of this book have prepared an introduction for the tables presented in DASA 19173. A few more figures have heen added and a nurber of errors have been corrected. This volume presents $t a b l e s$ of the equilibrium radiative properties of air and its constituents for a wide range of temperatures and densities. The work is divided into five parts: part $A$ is a summar section designed to give an overview of the field. Some of the data are presented in semi-schematic form and should not be used for quantitative work. Part $B$ gives basic data and references used in the preparation of the subsequent parts. Finally, parts C, D, and $E$ contain the absorption coefficients in the ranges $1000^{\circ} \mathrm{K}$ to $24,000^{\circ} \mathrm{K}$ (Part C), $1 \mathrm{eV}$ to $20 \mathrm{eV}$ (Part D), and above $2 n \mathrm{eV}$ (Part IE).

\#6607005

$* 8.153$

Latiner, W. M.

THE OXIDATION STATES OF THE ELIMENTS AITD THEIR POTENTIALS IN AQUEOUS SOLUTIONS SECOND EDITION Englewood C1iffs, New Jersey: Prentice-Ila11, Inc. 1964

$392 \mathrm{p}$

For each element the heats of formation, free energies of formation and entropies have been tabulated for all important compounds. Many of the free energies are based upon estimated entropy values and a discussion has been given in the Appendix of the methods by which accurate estimations of entropy values may he made. Potential diagrams are given for all the elements which have more than one oxidation state. A discussion of the use of these diagrams in the interpretation of the chemistry of an element is given in Chapter 1. Several hundred new potential values and equilibrium constants also have been added. The treatment of reaction rates and reaction mechanisms has heen extended. The chemistry of uranium, neptunium, plutonium and americium has been greatly anplified and the actinide elements are treated in a new chapter. For the great majority of substances, the U. $S$. Bureau of Standards values for reaction heats have been employed.

\section{\#6902001 *8.153}

Leland, T. W.; Rowlinson, J. S.; Sather, G. A.

THE STÁTISTICAL THERMODYNAMICS OF MULTICOMPONENT SYSTEMS OF MOLECULES OF DIFFERENT SIZES

pc/D17. International Union of pure and Applied Chemistry, Thermodynamic Tables project Centre, London, England

October 12,1967

$35 p$

The central problem in the theory of mixtures is the calculation of the free energy of mixing of molecules of different sizes. An explicit calculation of this free energy is made for a mixture in which all internolecular potentials are of the form $u_{\alpha \alpha}(r)=\sigma_{\alpha q^{r}} n$, where $\sigma$ is a distance characteristic $g q^{2}$ the interactigf of two molecules of species $\alpha$, and where $\sigma_{\alpha \beta}=1 / 2\left(\sigma_{\alpha \alpha^{+}} \sigma_{\beta \beta}\right)$

when $\alpha \neq \beta$. This result follows from a solution of Percus-Yevick integral equation for the pair distribution function of a mixture of lard spheres. The form of the free energy provides a criterion by which existing theories can be judged, and it is shown that an approximation of the type originally suggested by van der Waals is superior to approximations based on the concept of random mixing. Molecules that differ only in size mix with a small and negative excess free energy. The recomended approximation is confirmed by comparison with experiment.

$\# 6500238 * 8.153$

Levin, E. M.; McMurdie, II. F.; Ha11, F. P.

Reser, M. K. (Editor)

PHASE' DIAGRAMS FOR CERAMISTS

Columbus, Ohio: The American Ceramic Society

1956

$286 p$

Phase Diagrams for Ceramists includes 811 phase diagrams. This is a completely revised edition of articles which appeared in the Journal of the American. Ceramic Society. These articles on compilations of phase diagrams date hack more than
30 years. Systems covered include metal oxides, systers containing non-metal oxides, systems containing halides, sulfides, etc, and watercontaining systens. The first 30 pages are devoted to the general discussion of phase diagrams, the phase rule, glossary of terms, interpretations and an annotated bibliography. At the end appears an author and system index to simplify searching.

\#6500239

$* 8.153$

Levin, E. M.; McNurdie, Il. F.

Reser, M. K. (Editor)

PHASE DIAGRAMS FOR CERAMISTS, PART

Columbus, Ohio: The American Ceramic Society 1959

$153 p$

This book brings together in convenient form 462 phase diagrams of ceramic significance that have been developed since the publication of phase Diagrams for Ceramists in 1956. The dia grams included in this volume are an addition to those in the 1956 book, supplementing and not duplicating their data. A combined index for both volumes is included in Part II.

$\# 6500240$

*8. 153

Levin, E. M.; Robbins, C. R.; Mclurdie, If. F.

Reser, II. K. (Editor)

PIIASE DIAGRAMS FOR CERAMISTS

Columbus, Ohio: The American Ceramic Society

1964

$601 p$

This compilation of phase diagrams is a new and revised edition containing over 2000 diagrams. The first 30 pages of introductory material under "General Discussion of Phase Diagrams" remains unchanged except for an addition to experimental methods and a revision of the bibliography. Systens covered include metal-oxygen systems, metal oxides, systems with oxysen-containing radicals (carbonates, perchlorates, hydroxides, nitrates, nitrites, nitrites-nitrates nitrates-carhonates, sulfates, sulfates with metal oxides, sulfates with other oxygen-containing radicals), systems containing halides only, systems containing halides with other substances, systems containing cyanides, sulfides, etc. and systems containing water. Three tables have been added as appendixes. One is a compilation, made at the National Bureau of Standards, of published melting points of metallic oxides. The other two tables are Relative Atomic Weights 1961 (based on ${ }^{12} \mathrm{C}=12$ ) and Molecular Weights of the Oxides. At the end appears an author and system index to simplify searching. 
Lewis, G. N.; Randal1, M.

Pitzer, K. S.; Brewer, L. (Revisers of Second Edition

THERMODYNAMICS SECOND EDITION

New York: Mcfraw-Hill Book Company

1961

$723 \mathrm{p}$

This text reviews the laws and uses of the rmodynamics and includes tables and graphs of thermodynamic properties, constants, and applications. The tables include properties of normal fluids and gaseous solutions, entropy change in translation of an ideal gas to liquid, data for aqueous electrolyte solutions, Debye functions for the thermodynamic properties of solids, tabular summary of thermodynamic formulas thermodynamic properties, electromagnetic work, symbols, and physical constants and numerical factors.

\#6500398

Lick, W. J.; Emons, H. W.

THERIODYNAMIC PROPERTIES OF HELIUM TO $50,000^{\circ} \mathrm{K}$

Cambridge, Massachusetts: Harvard University Press 1962

$122 \mathrm{p}$

The thermodynamic properties of helium have been calculated at temperatures from $8,000^{\circ} \mathrm{K}$ up to $50,000^{\circ} \mathrm{K}$ and at pressures from $10^{-4}$ to 1000 atmospheres. In this range of temperatures and pressures, helium will consist mainly of a mixture of neutral atoms, singly and doubly ionized atoms, and electrons. Two Mollier enthalpy-entropy diagrams are included.

H 6500505

* 8.253

Li ley, P. E.

COLLISION INTEGRALS FOR TIE LFNNARD-JONES 6-12 POTENTIAI,

TPRC Report 15, Thermophysical Properties Research Center, Purdue University, Lafayette, Indiana February 1963

$15 \mathrm{p}$

937

Values are presented for collision integrals occurring in expressions for the transport properties of non-polar gases assuming the LennardJones 6-12 intermolecular potential function. Tables of $\Omega 11$ and $\Omega 12$ are given for $0.100(0.001)$ $1.200(0.005) 2.00(0.01) 5.00(0.05) 10(1) 20=\mathrm{T} / \theta$ where $\theta=\mathrm{e} / \mathrm{k}$. An estimate is made of the accuracy of the tabulation.

\#6500506 $* 8.253$

Liley, P. E.

SURVEY OF RECENT WORK ON THE VISCOSITY, THERMLL CONDUCTIVITY AND DIFFUSION OF GASES' AND GAS MIXTURES

Thermodynamic and Transport Properties of Gases, Liquids and SoIids, pp.40-6 New York: American Society of Mechanical Engineers No Date

$29 \mathrm{p}$

Recent theoretical and experimental studies of transport properties of gases and gas mixtures are reviewed. Emphasis is given to data reported for high temperatures while no data for temperatures below the ice point are reviewed. Summary tables are given to enable easy access to references for the pressure and/or temperature dependence for a particular property and gas. Suggestions are appended for work urgently required and a bibliographic 1 isting of 550 references given.
\# 6500241,6704013

$\star 8.253$

Linke, $\mathrm{W}$. F.

SOLUBILITIES INORGANIC AND MFTAL-ORGANIC COMPOUNDS A CONPILATION OF SOLUBILITY DATA FROM TIIF PERIODICAL LITERATURE VOLUME I: A-Ir, VOLUME I I : K$z$, FOURTH EDITION

Princeton, New Jersey: D. Van Nostrand Company, Inc., Volume I

Washington, D. C.: American Chemical Socicty, Volume I I

1958,1965

$1487 \mathrm{p}, 1914 \mathrm{p}$

Volume I, completed in 1957, includes material abstracted through 1956 and covers the elements and their compounds, alphabetically, from Argon (A) to Iridium (Ir.). The various types of solubility data are arranged in the following order: solubility in water; in aqueous acids; in aqueous bases; in aqueous salts having a cation in common with the solute; in aqueous salts having no common ion; in aqueous solutions of organic compounds; in anhydrous organic solvents; in anhydrous inorganic solvents; in high temperature mixtures (salt melts). The second volume of Solubilities completes the fourth revision of tables of Inorganic and Metal-organic Compounds. It includes the solubilities of compounds from $K$ through $z$. For each compound the solubility data are arranged in the order: solubility in water, solubility in acids, bases, salts, aqueous solvents, anhydrous solvents, and fused salts. Volume II contains a conplete subject Index and Author Index to al1 compounds listed in both Volumes I and II.

\#6500229 * 8.153

Lucks, C. F.; Deem, IH. W.

THERMAL PROPERTIES'OF THIRTEEN METALS

ASTM Special Technical Publication No. 227, American Society for Testing and Materials. Philadelphia, Pennsylvania

1958

$29 p$

Thermal conductivity, linear-thermal-expansion, specific-heat, density, and thermal-diffusivity values are reported over the temperature ranges shown for the following metals: $2024-\mathrm{T} 4$ and $7075-$ TS aluminum from -250 to $800^{\circ} \mathrm{F}$ chromium from 75 to $3000^{\circ} \mathrm{F}$ (thermal conductivity and diffusivity 400 to $1800^{\circ} \mathrm{F}$ ), copper from 75 to $1900^{\circ} \mathrm{F}$ (density from 75 to $3000^{\circ} \mathrm{F}$ ), Inconel and Inconel " $\mathrm{X}$ " from -250 to $1800^{\circ} \mathrm{F}$ AN-M-29 magnesium from -250 to $600^{\circ} \mathrm{F}$ molybdenum from 75 to $3000^{\circ} \mathrm{F}$ (thermal conductivity and diffusivity from 400 to $1600^{\circ} \mathrm{F}$ ), " $\mathrm{K} "$ monel from -250 to $1800^{\circ} \mathrm{F}$. SAE 1010 stee 1 from -250 to $1650^{\circ} \mathrm{F}$ stainless steels types 301,316 , and 347 from -250 to $1800^{\circ} \mathrm{F}$.

\#6500411

$$
\text { * } 8.253
$$

McBride, B. J.: et al

TIIERMODYNAMIC PROPERTIES TO $6000^{\circ} \mathrm{K}$ FOR 210 SUBSTANCFS INVOLVING TIIE FIRST 18 ELEMIENTS

NASA SP-3001 Lewis Research Center, Cleveland, Ohio 1963

$323 \mathrm{p}$

Availab le from CFSTI

Consistent $t$ ables of thermodynamic properties at temperatures from 0 to $6000^{\circ} \mathrm{K}$ were compiled for 210 gaseous and condensed species involving the elements with atomic numbers of 1 to 18 .. The tables give the following the rmodynamic functions for the standard state: heat capacity at constant pressure $C \beta$, sensible enthalpy $11_{T}^{\circ}-H_{0}^{\circ}$, entropy $S_{T}^{\circ}$, sensible free energy ( $\left.F_{T}^{\circ}-I_{0}^{\circ}\right), T$ and $I I_{T}^{\circ}$, the sum of sensible enthalpy and chemlcal energy $T$, $0^{\circ} \mathrm{K}$, as we 11 as values of the enthalpy changes and the logarithms of the equilibrium constants. The latter two functions are given for the reactions of formation of the substances from a set of assigned reference elements $\left[\left(\Delta H_{T}^{\circ}\right)\right.$ and $\left.\log _{10} K_{f}\right]$ and from these elements in thefretomic gaseods state $\left(\Delta \mathbf{H}_{T}^{\circ}\right.$ and $\left.\log _{3} K\right)$. The functions for most of the gases were generated from molecular data, whereas the functions of most of the condensed species are 
based on selected experimental data that were smoothed and made self-consistent.

$\$ 6500401$
McCarty, P. D.; Stewart, R. B.

EQUATION OF STATE FOR CÁLCULATI::G THE TIERMODY:IAIIC PROPERTIES OF HELIUY $\Lambda T$ LOW TFIPERATURES

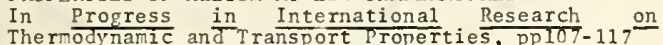
The rmodynamic and Transport properties, pplo7-117

Engineers

1962

$10 \mathrm{p}$

A new equation of state for heliun gas with six adjustable constants is presented. This relation is adequate for the representation of the P-V-T data and for the calculation of the entropy and enthalpy for a range of temperatures from 20 to $300 \%$, with pressures to 100 atm. A comparison of calculated volumes with the original data indicates an average arithmetic deviation of 0.07 percent and maximum deviations of 0.5 percent. $\Lambda$ comparison of calculated pressures with original data indicates about the same average and maximum deviations. $\Lambda$ temperature-entropy chart and a compressibility factor chart have been prepared from values calculated by this equation of state. Values of density, enthalpy and entropy, are tabulated with temperature and pressure as independent arguments.

\#6910021 * 8.153

McCarty, R. D.; Stewart, R. B.

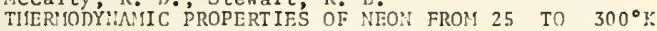
BETHEEN 0.1 AND 200 ATMOSPIIERES

In Advances in Thermophysical properties at Extreme Temperatures and Pressures, pp 84-97, Serge Bratch (Editor)

New Yorl:: The American Society of Mechanical Engineers

1965

$14 \mathrm{p}$

Available from CFSTI

Selected $\mathrm{P}-\mathrm{p}-\mathrm{T}$ data for liquid and gaseous neon have been fitted to an analytical function. The selected data are the available experimental data, supplemented in regions of pressure and temperature where the experimental data are not available hy values calculated from the theory of corresponding states and a generalized equation of state. Deviation plots are given which indicate the accuracy with which the analytical function represents the experimental data. The uncertainty of the tabulated properties is also estinated. The equation of state and the zero pressure specific heat were used to calculate tables of lensity, entropy, and enthalpy for selected temperatures and pressures. These properties are also illistrated in temperature-entropy diagrans.

\section{\#6500311}

*8.153

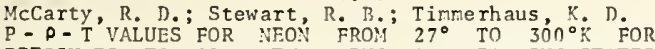
PRESSURES TO 200 ATM IISING CORRESPONDING STATES TIIEORY

Advances in Cryogenic Engineering, 8:135-145 1963

Limited experimental pressure-density-temperature data for neon were compared with the pressuredensity-temperature surfaces of neon hy the law of corresponding states. Thus a hasis is provided for the extrapolation of pressure-density-temperature data to lower temperatures and higher pressures.
\# 6500381 * 8.253

NcCu1lough, J. P. ; Messerly, J. F.

TIE CHEMICAI. THERIODYNAIIC PROPERTIF.S DI ITDROCARBONS AVD RELATYE SIIBSTANCFS USE OF $n$ HEPTANE AS A REIERENCE SIIBSTANCE FOR LOU:TE:TPERATURI: CNLORI:'́ETRY

Bulletin 596, Bureau of Mines, Bartlesville Petroleum Research Center, Bartlesvilie, Oklahoma 1961

Available from the Superintendent of Documents, GPO

This bulletin presents the results obtained for $n$ heptane by the petroleun thermodynamics laboratory of the Bartlesville, Oklahoma, facility. Detailed results of five investigations are given; selected values of the low-temperature thermal properties are tabulated; and comparisons with results that were obtained in other lahoratories are made.

\section{\#6500408}

Mann, D. B.

THERMODYMAIIC PROPERTIES OF IIELIUI! FROII 3 TO $300^{\circ} \mathrm{z}$ BETUEEN 0.5 AND 100 ATHOSPHERES

NBS Technical Note 154, National Bureau of Standards, Boulder, Colorado

January 1962

$95 p$

Available from the Superintendent of Docunents, GPO

The specific volume, enthalpy, entropy, and internal energy values of heliun are presented in tabular form as functions of pressure and temperature. Data are tabulated in one degree Kelvin increments for forty isobars between 0.5 atmospheres and 100 atmospheres. A comparison with previously published data is made where applicable. An expression is presented which represents the pressure-density-temperature surface based on previously published data. The tahulation is presented in the dimensional units of the metric system, but is also available in the dimensional units of the British system in Supplement A of the publication.

\#6500410

* 8.253

Mann, D. B.

TIIERMODYNAMIC PROPERTIES OF TIELIUM FROM 6 TO $540^{\circ} \mathrm{K}$ BETWEE: 10 ANI) 1500 PSIA

NBS Technical Note 154A, National Bureau of Standards, Boulder, Colorado

January 1962

$89 \mathrm{p}$

Available from the Superintendent of Documents, GPO

The specific volume, enthalpy, entropy, and internal energy values of helium are presented in tabular form as functions of pressure and temperature. Data are tabulated in two degree Rankin increments for thirty-six isohars between 10 psia and 1500 psia. A comparison with previously published data is made where applicable. An expression is presented which represents the pressure-density-temperature surface based on previously published data. The tabulation is presented in the dimensional units of the British system, hut is also available in the dimensional units of the metric system.

\# $6706021,6805006,6703017,6903040 \quad$ *7.211-8.211 MATERIALS PROPERTIES DATÁ BOOK

Report 2275, Aerojet-General Corporation, Azuza, California

July 31,1968

$713 \mathrm{p}$

The Materials Properties Data Book provides noncritical reference data of materials properties for use by persons involved with the analysis, design, fabrication or test responsibilities of the NERVA Project. The Data Book includes physical, mechanical, and chemical properties of interest for many materials of significance to the NERVA Progran and, in addition, data on the effects of irradiation on materials. Information is also 
provided concerning material processing, with special emphasis on specifications. Material suppliers are given. The Data Book has been organized for the most effective use of the data by the design engineer. Volune I contains light metal alloys (aluminum, titanium, etc.) data, Volume I-A contains nickel-base alloys, refractory metals and alloys, and other nonferrous metals and alloys (cobalt, copper, etc.) data, Volume 2 contains ferrous alloys data, Volune' 3 contains nuclear materials (fuels, neutron controls, beryllium, etc.), graphites, refractory ceramics, a dhesives and piastics data. The section for each individual material contains descriptive hasic data sheets with pertinent data relative to properties, characteristics and other related information, together with curve sheets for graphic presentation of the parameters.

$\# 6803003$

\section{$* 8.153$}

Meyer, C. A.; et al.

THERIODYNAIIC AND TRANSPORT PROPERTIES OF STEAM CONPRISING TABLES AND CIIARTS FOR STEAM AND MATER

New York: Anerican Society of Mechanical Engineers 1967

$328 \mathrm{p}$

The volume includes nine tables and thirteen charts on stean and water. The data we re calculated from the equations and adopted by the International Formulation Comittee and published in its 1967 "IFC Formulation for Industrial Use." These equations, in turn, were derived from the "International Skeleton Tables of 1963," adopted by sixteen nenber nations of the Sixth International Conference on Properties of Steam. The following thermodynamic properties are listed: 1. saturation pressure and specific volune, entropy and enthalpy for saturated water as we11 as changes upon vaporization from $705^{\circ}$ to $32^{\circ} \mathrm{F}$.; 2 . saturation pressure and specific volume, entropy and enthalpy for steam and water. Specific internal energies from 3200 to $0.1 \mathrm{psia;} 3$. specific volume, enthalpy, and entropy of superheated steam and compressed water for temperatures from $1500^{\circ}$ to $32^{\circ} \mathrm{F}$ in 10 -degree intervals, and pressures of 0.12 to $15,500 \mathrm{psia}$ and also in the critical region $\left(800^{\circ}\right.$ to $650^{\circ} \mathrm{F}$ and 2800 to $3980 \mathrm{psia)}$ at $2^{\circ} \mathrm{F}$ and 20 psia steps; 4. enthalpy as a function of pressure from 0.12 to 15,500 psia, and of the entropy in steps of $0.01 \mathrm{Btu} / 1 \mathrm{bm}{ }^{\circ} \mathrm{F} ; 5$. entropy values for sane pressure range and for enthalpy in steps of $10 \mathrm{Btu} / 1 \mathrm{hm} ; 6$. values of specific volume, enthalpy and entropy of supersaturated steam at pressures of 0.12 to 1500 psia; 7 . specific heat at constant pressure for superheated vapor and compressed liquid. Also presented are viscosity of steam and water from $32^{\circ}$ to $1500^{\circ} \mathrm{F}$ and 1 to 12,000 psia and thermal conductivity and Prandt 1 numbers of steam and water from $32^{\circ}$ to $1500^{\circ} \mathrm{F}$ and pressures from 1 to 7500 psia.

\section{\#6906029 *5.153-8.153}

Mickle, E. A.; Clauson, W. W.; Szego, G. C. FREE RADICALS' AS HIGI ENERGY PROPELLANTS

General Electric Company, Flight propulsion Laboratory, Cincinnati, Ohio

1956

$1062 \mathrm{p}$

This compilation gives data concerning the use of free radicals as high energy propellants as well as discussions on rocket propulsion metastable states, and assumptions and metlods of calculation. Tabulations and figures are included for the $\mathrm{H}_{1} \mathrm{II}_{\mathbf{2}}$; $\mathrm{F}, \mathrm{F}_{2}, \mathrm{H}_{2} ; \mathrm{N}, \mathrm{N}_{2}$, He; $\mathrm{N}, \mathrm{N}_{2}, \mathrm{H}_{2} ; \mathrm{CII}_{4}, \mathrm{CII}, \mathrm{H}$; and ionic systems. Included in the the rmodynamic properties given for each system are: speed of sound in a gaseous mixture in which chemical equilibrium is maintained at all times, speed of sound in a gaseous mixture in which the chemical composition remains fixed, Specific heat capacity at constant pressure for a gaseous mixture in chemical equilibrium, mole ratio of free radical to stable "parent" species in reactant mixture, mole ratio of diluent to stable parent species in reactant mixture, molal enthalpy, equilibrium constants, mass of a particle, molecular weight, specific or total entropy, specific volume, and alpha and beta erissions. Nozzle performance parameters and equilibriun composition in mole fractions are also listed. These data are listed for a hroad range of temperatures and pressures for each systern.

\# 6706013

Milek, J. T.; Welles, S. J

BORON

DS-151, Jiectronic Properties Information Center, Hughes Aircraft Company, Culver City, California 1967

$251 p$

A state-of-the-art survey and a data sheet compilatiof on elenental boron are presented. The thorough literature search uncovered 394 references which were evaluated for information and data on the (1) reduction preparation methods, (2) zonerefining and melting techniques, (3) crystal structures, (4) electrical properties, (5) optical properties, (6) thermal and thernodynamic properties, (7) physical and chenical properties, (8) metailurgy and mechanical properties, (9) applications, and (10) availability and suppliers. Major emphasis was the characterization of horor's semiconductive and acoustic properties with respect to potential seniconductor and delay-line devices. Detailed information on the various methor's of preparing pure horon and the resulting crystal structures plus properties are sumarized in tables.

\#6905016

Milek, J. T.; Welles, S. J 4.153-8.153

CHEMICAL COMPOSITION AND ELJCTRICAL RESISTIVITY of ALUMINUM ALLOYS

DS-161, Electronic Properties Information Center, Hughes Aircraft Company, Culver City, California April 1969

$27 p$

A11 comercially available aluminun alloys are identified hy means of their standard designations. A11 available chemical composition and electrical resistivity data for these alloys are presented in tahular forn for each alloy class. Electrical resistivity data for pure aluminun are reviewed.

\#6910018 *8.353

Moore, R. T.; Harrison, R. T.; Dous 1 in, D. R.

METHANE: BIBLIOGRAPIIY OF TIIE TIIFRMODYNAMIC AND TRANSPORT PROPERTIES ABOVE $300 \mathrm{~K}$

Interin Report AII/B6, IUPAC, Thermodynamic Tables Project, Aliphatic llydrocarbons Working Panel, Bartlesville Petroleum Research Center, U. S. Bureau of Mines, Bartlesville, Oklahoma

1969

$133 \mathrm{p}$

Available from the Bureau of Mines

This bibliography of the properties of methane above $300 \mathrm{~K}$ is sinilar to and complenents the one prepared hy the U. S. Department of Commerce, National Bureau of Standards (Technical Note 367 ) on the thernophysical properties of methane at temperatures helow $300 \mathrm{~K}$.

\#6902008

Muetterties, F. L.

$* 4,153-8,153-3,153$

New York: John Wiley \& Sons, Inc.

1967

$699 \mathrm{p}$

This is a text on the properties of boron and boron compounds containing ten chapters, each written by experts in the different phases of boron chemistry. The chapters discuss the element boron, compounds of high boron content, borates, boric acid, . boron hydrides and halides, boron-nitrogen, carbon, 
phosphorus corpounds, and sulfur and selenium compounds of horon. Each chapter contains a large listing of references as well as tables of the chemical properties of boron compounds.

\# 6706030

* $4.153-3.153-8.153$

Muir, A. Il., Jr.; Ando, K. J.; Coogan, I1. M.

MOSSBAUER EFFECT DATA INDEX 1958-1965

New York: John Wiley \& Sons, Inc.

1966

$351 \mathrm{p}$

The Mossbauer Effect Data Index is an organized index to experimental research results relating to the Mosshauer Effect published through 1965. The index groups experiments first by nuclear transition (daughter isotope and gamna-ray energy), then by source material or host, and then by absorber (or scatter) material or host. Each experiment is described and sumarized on an $80-$ column IB:i card. The data listed for each experiment are: Mossbauer isotope and gamna-ray transition energy, host naterial in which the source atoms are imbedded, source temperature, absorber, absorber terperature, line width, dip of the spectrum (percentage change in counting rate from on-resonance to off-resonance), shift of the absorption spectrum, quadrupole splitting energy (for transitions between spin $3 / 2$ and $1 / 2$ levels), shape of the absorption spectrus and a core for the bibliography reference. A summary sheet is presented for each isotope presenting simplified decay schene gama-ray and $x$-ray energies, halfIife and total internal conversion coefficient of the Mossbauer transition, natural isotopic abundance, and ragnetic and quadrupole moments.

There is an index to references by topic and author.

\# 6500320

*8.153

Mullins, J. C.; Kirk, B. S.; Zieglex, \%. T.

CALCULATION OF TIIE VAPOP PRESSURF AND IIEATS OF VAPORIZATION AND SUDLIMATION OF LIOUIDS AND SOLIDS, ESPECIALLY BELOI ONE ATY IOSDIERT. V. CARBON IONOXIDE AND CARBON DIOXIDE.

Technical Report No. 2, Georgia Institute of Technology, Engincering Experimental Station, Atlanta, Georgia

August 1963

$81 \mathrm{p}$

The vapor pressure and heats of vaporization and sublination of carbon monoxide have been computed at one degree intervals from the normal boiling point $\left(31.633^{\circ} \mathrm{K}\right)$ to $14^{\circ} \mathrm{K}$. The vapor pressure and heats of sublimation of carhon dioxide have been computed at one degree intervals from the normal sublination temperature $\left(194.694^{\circ} \mathrm{K}\right)$ to $40^{\circ} \mathrm{K}$. The agreerent obtained between the computed and experimental vapor pressure data for carbon monoxide in the liquid and solid region down to the solid transition point $\left(61.544^{\circ} \mathrm{K}\right)$ suggests that the computed values may well represent the experimental data within the accuracy of the temperature scales used by the various investigators. The computed $v$ alues of the vapor pressures (Table XIX) are therefore recommended for use as smoothed values, especially below the solid transition temperature where the 1 inited experimental data are scattered and discordant. For carbon dioxide the computed values of the vapor pressures agree well with the best experimental data which have been obtained on the thermodynamic scale. The experimental data measured on the International Temperature Scale appeared to deviate in a manner which is consistant with the available known deviations of the

International Temperature scale from the thermodynamic scale. The values of the vapor pressure of carbon dioxide computed at one degree intervals (Table $\mathrm{XX}$ ) are therefore recomended as a consistent set of values on the thermodynamic temperature scale.
\#6500321 $\star 3.153$

Mullins, J. C.; Ziegler, W. T.

TIIE SYSTFM HIELIUNI-ARGON FRO: $65^{\circ}$ TO $140^{\circ} \mathrm{K}$ UP TO PRESSURES OF 120 ATMOSPIIERES, CORRELATION OF AVAILABLE PHASE EQUILIBRIU: DATA

Technical Report No. 3, Georgia Institute of Technology, Engineering Experiment Station, At lanta, Georgia

January 10,1965

$64 \mathrm{p}$

The available phase equilibrium data for the helium-argon systen have been examined critically and a correlation of the gas and condensed phase compositions are presented from $65^{\circ}$ to $140^{\circ} \mathrm{K}$ at pressures up to 120 atmospheres. No data are available for the solubility of helium in solic argon, but the locus of the three-phase line suggests that the condensed solid phase is very nearly pure argon. The results of the correlations are prescnted in tabular form at $5^{\circ} \mathrm{K}$ and 5 atm intervals with the compositions of the equilibrium gas and liquid phases expressed in terms of mole fractions. In addition, $(K=y / x)$ factors are given for both argon and helium as a function of temperature and pressure. The estimated maximum uncertainty of the gas phase composition in terms of the argon concentration is believed to vary as follows: \pm 4 percent from $65^{\circ}$ to $110^{\circ}$ to $125^{\circ} \mathrm{K}$ and \pm 10 percent at $140^{\circ} \mathrm{K}$. The estimated maximum uncertainty of the liquid phase composition expressed in terms of heliun concentration is believed to be \pm 2 percent up to $110^{\circ} \mathrm{K}$ and \pm 5 percent from $110^{\circ}$ to $140^{\circ} \mathrm{K}$.

\#6500388 *8.153

Mullins, J. C.; Ziegler, W. T. Kirk, B. S.

TIIERMODYNAIIC PROPERTIES OF 'OXYGEN' FROM $20^{\circ}$ TO $100^{\circ} \mathrm{K}$

Technical Report No: 2, Georgia Institute of Technology, Engineering Experiment Station, Atlanta, Georgia

March 1962

$101 \mathrm{p}$

The thermodynamic properties of oxygen have been calculated at two degree intervals from 30 to $100^{\circ} \mathrm{K}$ using published experimental data. The calculations cover the pressure range from approxinately $1900 \mathrm{~mm} \mathrm{Hg}$ at $100^{\circ} \mathrm{K}$ to approximately $10^{-7} \mathrm{~mm} \mathrm{Hg}$ at $30^{\circ} \mathrm{K}$. They include both the saturated and superheated regions. The properties calculated were the vapor pressure, heats of vaporization and sublination, quality, volume, entha1py and entropy. The enthalpy and entropy of solid oxygen at $0^{\circ} \mathrm{K}$ were taken to be zero. Calculations of vapor pressure and heat of sublimation were extended to $20^{\circ} \mathrm{K}$. The tabulated data were used to construct a temperature entropy chart for the range 30 to $100^{\circ} \mathrm{K}$, showing lines of constant pressure, constant volume, constant entha1py, and constant quality.

\section{\#6500387 *8.153}

Mullins, J. C.: Ziegler, W. T.; Kirk, B. S.

TIIERMODYNAMIC PROPERTIES OF PARAHYDRÓGEN FROM $1^{\circ}$ TO $22^{\circ} \mathrm{K}$

Technical Report No. 1, Georgia Institute of Technology, Engineering Experiment Station,

Atlanta, Georgia
November 1,1961

$68 \mathrm{p}$

The thermodynamic properties of parahydrogen have been calculated at one degree intervals from 1 to $22^{\circ} \mathrm{K}$ using existing thermal and equation of state data. The properties calculated include the vapor pressure, heats of vaporization and sublimation, enthalpy and entropy. The enthalpy and entropy of solid parahydrogen at $0^{\circ} \mathrm{K}$ were taken to be zero. The entha1py and entropy of the vapor have been computed from the saturation pressure to $10^{-6}$ man Hg. The tabulated results were used to construct a temperature-entropy diagram with lines of constant pressure, constant volume, constant enthalpy and constant quality over the temperature range 4 to 
$22^{\circ} \mathrm{K}$. In the selection of the experimental data used, heavy reliance has been placed on the work of Woolley, Scott and Brickwedde, and lloge and Arnold of the National Bureau of Standards. A more critical analysis of all existing data might result in slight changes in the computed results. These slight changes are unlikely to affect the use of the computed results for engineering calculations.

\section{\#6809016 *3.153-3.153}

Nels on, R.D., Jr.; Lide, D.R., Jr.; Maryott, A. A. SELECTED VALUES OF ELECTRIC DIPOLE MOMENTS FOR MOLECULES IN TIIE GAS PIIASE

NSRDS-NBS-10, National Bureau of Standards, Washington, D. C.

Septenber i, 1967

$49 \mathrm{p}$

Available fron the Superintendent of Documents, GPO

This table revises, brings up to date, and extends the coverage on numerical values of dipole monents which was included in NBS Circular 537 , Tables of Dielectric Constants and Electric Dipole Monents of

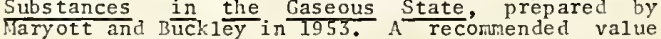
with an estinate of accuracy is presented for more than five hundred organic and inorganic compounds. Extensive coments are given on the definition of dipole moment and principal methods of dipole monent measurement, as well as an exposition of the criteria enployed in selecting the tabulated data.

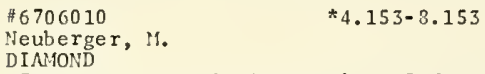

DS-153, Electronic Properties Infornation Center, Hughes Aircraft Company, Culver City, California 1967

$146 \mathrm{p}$

Tables of electrical properties of diamond are presented as follows: conductivity, resistivity, dielectric constant, llall coefficient mobility and thermoelectric power. Emission data are shown as well as a wide variety of irradiation effects. Energy data given are energy bands, energy gap, energy levels, effective mass tables, lifetines and phonon energies. Optical properties included are absorption, reflection, refractive index, magnetoelcctric properties, Debye temperature and thermal conductivity. A summary of crystal structure, phase transitions, and diamond types is included.

\section{\#6706041}

$* 4.153-8.153$

Neuberger, $M$

LEAD OXIDE

DS-155, Electronic Properties Information Center, Ilughes Aircraft Company, Culver City, California May 1967

$71 \mathrm{p}$

These data sheets present a compilation of a wide range of electronic properties for lead oxide. Electrical properties include conductivity, resistivity and dielectric constant. $A$ wide variety of photoclectronic phenomena are shown. Energy data include energy bands, energy gap and energy levels, as well as effective mass tables, phonon energy dispersion and work functions. The optical properties include absorption, reflection and refractive index. Data on several physical phenomena, such as thermal conductivity, Debye temperature, magnetic susceptibility and Richardson's Constant are presented. Thermoelectric data is given. Each property is compiled over the widest possible range of parameters including bulk and film form, from references obtained in a thorough literature search. A summary of crystal structure and phase transitions has been included.
A 7001042

$* 3.153-5.153-3.153$

Niles, F. E.

FORMATION OF IONS IN IONIZEN AIR

BRL-R-1458, Ballistic Research

Aberdeen Proving Grounds, "'aryland

November 1969

$54 \mathrm{p}$

Available from DDC

Schematic representations are given for hoth the formation of positive ions and the formation of negative ions in ionized air. The formation of positive ions can be divided into three regions. Region I contains the ionization of neutral constituents and two-body reactions in dry air. Region II contains reactions involving ionic species which are formed by three-body reactions. Region II I contains reactions requiring water vapor and shows the formation of the hydrated hychroniun ions. The formation of negative ions proceeds predominantly through $\mathrm{O}_{2}{ }^{-}$. The clustering of neutral molecules to $\mathrm{O}^{-}$opens additional channels for the formation of $\mathrm{NO}^{-}$, an ion which is not attacked by atonic oxygen. Rate constants are given, including the experimental values on which they are based, for the reactions in the schenatic representations.

\section{\#6809021 $\quad * 8.163$ \\ Parker, $V$. BB.
TIIERMAL} ELECTROLYTES

NSRDS-NBS-2, National Bureau of Standards, Washington, D. C.

Apri 1 1, 1965

$66 \mathrm{p}$

Available from the Superintendent of Docunents, GPO

The available specific heat, heat of dilution, and heat of solution data for aqueous solutions of uniunivalent electrolytes have been critically reviewed and tables of selected "best" values at $25^{\circ} \mathrm{C}$ prepared. In addition, the neutralization data have been critically reviewed in order to obtain the "hest" value, $13,345 \mathrm{cal} / \mathrm{mole}$, for the heat of ionization of water at $25^{\circ} \mathrm{C}$. The electrolytes reviewed include the hydroxides, halides and oxyhalides, nitrates and nitrites, formates and acetates, cyanides, cyanates and thiocyanates, and permanganates of hydrogen, ammonium and the methyl amonium derivatives, silver, and the alkali metals.

\# 6500169

Parsons, R.

HANDBOOK OF ELECTROCIIEMICAL CONSTANTS

London: Butterworths

1959

$113 \mathrm{p}$

This handbook has values for fundamental constants, properties of ions, salts and solvents, and numerical values of some functions used in the theory of electrolytes. Some of the properties covered include activity coefficients, thermodynamic properties of IICi and NaOI, themal properties of electrolytes, equilibrium constants in solution; molal volumes of electrolytes and their temperature and pressure dependence; properties of molten salts; equilibrium properties of electrodes; viscosity and diffusion in electrolytes; conductivity of electrolytes; kinetic properties of electrodes; and surface tension, dielectric constant and refractivity of aqueous solutions.

\# 6907016

Pomerance, II.

BIBLIOGRAPIIY OF SECOND AND TIIIRD ORDFR ELASTIC CONSTANTS

ORIL-RMIC-9, Research Materials Information Center, Oak Ridge National Laboratory, Oak Ridge, Tennessee December 1968 $27 \mathrm{p}$

Available from CFSTI 
This bibliography of second and third order elastic constants of monocrystalline materials was compilert fron the files of the Research Materials Information Center and from PhysicsAhstracts. The literature since 1964 has heen surveyed. The references are arranged first hy the year of publication, next by the first author's initial, last hy a serial numher. The tables do not show numerical values. The first table is the second order elastic constants, the second tahle is the third order constants; in each the substances are arranged alphabetically by chemical symhol. In the first table, the sccond column shows the crystalline form where it is not cubic. The thir colum shows the temperature range of the measurements, the pressure range or other paraneters. Where the full set of constants is reported, the letters $c$ and s do not appear, but incomplete sets of derivatives are noted. The fourth colunn shows the format, whether the data are graphical or tabular or hy algebraic formula. The last column is the reference number.

\#6706018

$* 8.163$

Pourbai, M. J. M.

ATLAS OF ELECTROCIIEMICAL EQUILIBRIA IN AQUEOUS SOLUTIONS

Oxford, New York: Pergamon Press

1966

$644 p$

The Atlas presents data characterizing the equilibriun conditions of chemical reactions involving water, hydrogen, and 90 of the elements, in the form of potentia1-pll diagrams. By means of the diagrams, it is possihle to predict, on a thermodynamic hasis, for a given element, the equilibrium states of all the possible reactions between this element, its ions, and its solit and gaseous compounds in the presence of water.

\#6500419

Powe 11, R. L.; Blanpied, W. A.

TIIERMUL CONDUCTIVITY OF ITTAAIS AND ALLOYS AT LOI TEMPERATURES, $\Lambda$ REVIEN OF TIIE LITERATURE

NBS Circular 556, National Bureau of Standards, Washington, D. C.

September 1, 1954

$68 p$

Available from the Superintendent of Documents, GPO

An extensive compilation is given of the measured values of thermal conductivity for metals and alloys from room temperature down to approximately $0^{\circ} \mathrm{K}$ The more extensive and inportant data are plotted in $48 \mathrm{graphs}$. The tahles and graphs for the metalic elements and alloys are essentially complete for literature reference from 1900 to early 1254. For comparison, several graphs and tables are given for some representative dielectrics.

\section{\#6500494 *8.163-4.163}

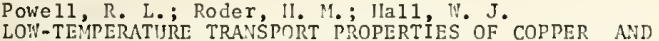
ITS DILUTE ALLOYS: PURE COPPER, ANNEALED AND COLDDRAINN

Physical Review 115:314-323

Ju1y 15, 1959

Experimental results between $4^{\circ}$ and $300^{\circ} \mathrm{K}$ are given for (1), the thermal conductivity, electrical resistivity, and thermoelectric force and power of two high-purity coppers, one annealed and one colddrawn $260 ;$ and (2), the electrical resistivity of a series of sever samples of high-purity copper colddrawn hetween $0 \%$ and $20 \%$ elongation. The total electronic thernal resistivities each consist of three terms: the intrinsic resistivity, $W_{i}$; the imperfection resistivity, $w_{0} ;$ and a deviation term, Wio indicating the departure from strict additivity of $W_{j}$ and $W$. The intrinsic thermal resistivity and intrinsic electrical resistivity vary as T2.8 and $T^{4} .5$ respectively, contrary to the predictions of the usual transport theory using
Bloch approximations and assurations. The resistivity of pure copper is 1.545 ohm cm at $0^{\circ} \mathrm{C}$. The increase in inperfection electrical resistivity is approximately linear with increase in cold-drawn elongation. "lowever, the acder resistivity is not independent of temperature ("latthiessen's rule), but about twice as great at the ice point as it is at $4^{\circ} \mathrm{K}$. The change in thermoelectric power with drawing is positive at the lower temperatures, but negative ahove $38^{\circ} \mathrm{K}$. The Lorenz number does not approach the sominerfeld value at the lowest temperatures, hut flattens out to a value considerably smaller. A discussion of each of the various effects is provided.

\#6809018

* $8.163-4.163$

Powe11, R. W. ; !!o, C. Y.; Liley, P. E.

TIERML CONDUĆTIVITY OF ŚELECTED MATERIALS

iNSRDS-NBS-3, National Bureau of Standards, Washington, D. C.

ioverber 25,1966

$168 \mathrm{p}$

Available from the Superintendent of Documents, GPO

This compilation consists of the critical evaluation and analysis of the available thermal conductivity data on eleven metals and nine nonmetals mainly for the solid state, on seven fluids for both the liquid and gaseous states and two for the liquid state only. The materials studied were selected primarily for their potential applicability as reference standards or because their technical importance. The temperature range for which values are given often exceeds. that for which these values are known with a high degree of certainty. The metals included are aluminum (solic and liquid state), copper, gold, iron (Armco and pure), manganin, mercury, platinum, platinum alloyed with 40 percent rhodium, silver and tungsten. The nonmetallic solids treated include aluminum oxide, heryllium oxide, Corning code 7740 glass, diarond, magnesium oxide, Pyroceran brand code 9606 glass, quartz, thorium dioxide, and titanium dioxide. Data in the literature for the following substances in both $l i q u i d$ and gaseous state were examined and evaluated in this compilation: argon, carbon tetrachloride, diphenyl, helium, nitrogen, m-terpheny 1, p-terpheny 1 , toluene, and water. Graphs, tables, and references are given for each substance.

\#6508035

$* 3.263$

Praunsnitz, J. M

SOLUBILITY OF SOLIDS IN DEIISE GASES

NBS Technical Note 316 , National Bureau of

Standards, Boulder, Colorado

July 1965

450

Available from the Superintencent of Documents, GPO

The thermodynamics of solid-dense gas equilibria is discussed, and two techniques are described for calculating the solubility of a solid component in a gas at high pressure. The first one is based on the recent empirical equation of state of Red1icl which, in turn, is derived from Pitzer's generalized tables of fluid-phase volumetric properties. The second one is hased on the Ilildebrand-Scatchard theory of solutions. Both methods give good semi-quantitative results hut cannot accurately predict solubilities from purecomponent data alone.

\#6500352 $* 3.163$

Predvoditelev, A. S. ; et al.

CIIARTS OF THERMODYNAMIC FUNCTIONS OF AIR FOR TEMPERATURES OF 1000 TO $12,000^{\circ} \mathrm{K}$ AND PRESSURES OF 0.001 TO 1000 ATMOSPIIERES

Glen Ridge, New Jersey: Associated Technical Services, Inc.

1962

$54 \mathrm{p}$

Translated from Russian 
This publication contains graphs of the themodynamic functions of air plotted as a family of curves as a function of temperature and pressure, pressure heing taken as the parameter. Curves for the density of air are not given, and information on this property must he obtained from the equation of state. In addition, this publication describes an approximate method for computing a normal shock and presents universal curves showing the pressure and enthalpy ratios for the shock as a function of the Mach number. At the end of the book charts of "enthalpy vs. entropy" are given for an analysis of isentropic flow.

\#6500351

$* 8.163$

Predvoditelev, A. S. ; et al.

TABLES OF TIIERMODYNAMIC FUNCTIONS OF AIR FOR TEMPLRATURES OF 12,000 TO $20,000^{\circ} \mathrm{K}$ AND PRESSURES OF 0.001 TO 1000 ATMOSPIIERES

Glen Ridge, New Jersey: Associated Technical Services, Inc.

1962

$229 \mathrm{p}$

Translated from Russian

Tables of the thermodynamic functions of air have been compiled for temperatures of 12,000 to $20,000^{\circ} \mathrm{K}$ and pressures of 0.001 to 1000 atmospheres. The tables consist of three parts. In the first part the following properties are given for air: specific enthalpy, specific internal energy, specific entropy, rolecular weight, specific heat capacity at constant pressure, specific heat capacity at constant volume, gamma $=\mathrm{C}_{\mathrm{p}} / \mathrm{C}_{\mathrm{v}}$ and the velocity of sound. In the second part the mole fractions of the air components $\mathrm{N}, \mathrm{O}, \mathrm{Ar}, \mathrm{N}^{+}, \mathrm{O}^{+}, \mathrm{Ar}^{+}, \mathrm{N}^{++}, \mathrm{O}^{++}, \mathrm{Ar}^{++}$, $e$, as well as the density of air, $\rho$ (g/c c) and the quantity $q^{2}=\Sigma z_{i}^{2} X_{i}$, are given at pressures of 0.001 to 0.8 atmospheres. At pressures of 1 to 1000 atmospheres the mole fractions of the air components $\mathrm{N}, \mathrm{O}, \mathrm{Ar}, \mathrm{N}^{+}, \mathrm{O}^{+}, \mathrm{Ar}^{+}, \mathrm{N}_{2}^{+}, \mathrm{O}_{2}^{+}$. NO, e, as well as $\rho$ and $q{ }^{2}$ are given. $A 11^{2}$ quantities in the first two parts of the tables are given at $200^{\circ} \mathrm{K}$ intervals, and 43 values of the pressure are listed for each temperaturee. The thermodynamic functions entropy, internal energy and specific heat at constant volume are given for the air components $\mathrm{N}, \mathrm{O}, \mathrm{Ar}, \mathrm{N}^{+}, \mathrm{O}^{+}$and $\mathrm{Ar}^{+}$in the third part of the tables.

\#6500350 *3.163

Predvoditelev, A. S.: et al.

TABLES OF THERMODYNAMIC FUNCTIONS OF AIR FOR TEMPERATURES OF 6000 TO $12,000^{\circ} \mathrm{K}$ AND PRESSURES OF 0.001 TO 1000 ATMOSPIIERES

Gien Ridge, New Jersey: Associated Technical Services, Inc.

1962

$301 \mathrm{p}$

Trans lated from Russian

Tables of the thermodynamic functions of air have been compiled for temperatures of 6000 to $12,000^{\circ} \mathrm{K}$ and for pressures of 0.001 to 1000 atmospheres. Twenty-two quantities are given for each temperature and pressure: specific internal energy, specific enthalpy, specific entropy, specific heat capacity at constant volume, specific

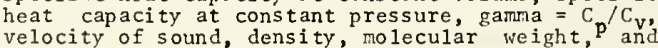
13 mole fractions of the air components $N, 0, A r$, $\mathrm{N}_{2}, \mathrm{O}_{2}, \mathrm{NO}, \mathrm{N}^{+}, \mathrm{O}^{+}, \mathrm{Ar}^{+}, \mathrm{N}_{2}^{+}, \mathrm{O}_{2}^{+}, \mathrm{NO}^{+}$and $\mathrm{e}$. The tables consist of two parts? 'the first part contains a11 the quantities except density and the mole fractions. The thermodynamic functions are given at every $100^{\circ} \mathrm{K}$ interval and for each temperature, there are 97 values of the pressure.

\#6905030

*8, 163

Preston, G. T.; Chapman, T. W.; Prausnitz, J. M. TRANSPORT PROPERTIES OF' CRYOGENIC LIQUIDS' AND TIIEIR MIXTURES

$\frac{\text { Cryogenics, }}{\text { October } 19: 274-279}$
This paper presents correlations for three transport properties: viscosity, thernal conductivity and diffusivity for cryogenic liquids and their mixtures. A method for applying the correlations to the prediction of mixture properties is proposed. The hasis for the correlations is provides hy the theory of corresponding states. Attention is restricted to simple liquids along the liquid-vapor saturation curve from the triple point temperature to temperatures close to critical. The quantum fluids helium and hydrogen have not been included.

\#7001021

Rabinovich, V.A. (Fiditor)

Mos cona, A. (Trans 1ator)

TIIERMOPIYYSICAL PROPERTIES OF GASES AND LIQUIDS NO.

IPST Cat. No. 5554, Israel program for Scientific Translations, Jerusalen, Israel

170

$24 p$

Available from C.FSTI, TT 69-55091

This collection is the first of a series of systematic puhlications initiated hy the GSSSD the Russian equivalent of the I.S. NSRDS - designe? to supply reliable information on the thermophysical properties of suhstances and naterials and aimed nainly at the practical application of the reference data. This collection includes papers dealing with the theoretical calculation and experimental determination of the thermophysical characteristics of substances and materials. It also contains some papers devoted to methods of calculation and the derivation of equations of state for pure substances and gas mixtures. Unlike existing publications, the collection presents the reference data in a form convenient for use: their accuracy is evaluated, and the limits are prescrihed within which the recomended values may vary. The reliability of the data obtained by theoretical calculation methods is checked against well-tested experimental values or confirmed by the accuracy of the calculation method.

\#6905026

Radebaugh,

THERMODYNAMIC RROPIRTIES OF He ${ }^{9}-\mathrm{He}^{*}$ SOLUTIONS WITH APPLICAT IONS TO THE $\mathrm{He}^{3}-\mathrm{He}^{4}$ DILUTION REFRIGERATOR

NBS Technical Note 36?, Nationa1 Bureau of Standards, Washington, D. C.

December 29,1967

$15 \mathrm{p}$

Available from the Supterintendent of Documents, GPO

The thermodynamic properties of liquid $\mathrm{He}^{3}-\mathrm{Ile}^{4}$ solutions between 0 and $1.5^{\circ} \mathrm{K}$ are calculated by using the weakly interacting Fermi-Dirac gas model for $\mathrm{He}^{9}$ in $\mathrm{He}^{4}$. Certain experimental data below about $0.4^{\circ} \mathrm{K}$ are used to evaluate some of the parameters in the model. The properties of both $\mathrm{He}^{3}$ in $\mathrm{He}^{4}$ and the total solution are calculated for concentrations of Ile up to 30 percent. A11 experimental data agree very well with the calculated results, although little data exist below $0.4^{\circ} \mathrm{K}$. The calculated properties are used to analyze the behavior of the $\mathrm{He}^{3}-\mathrm{He}^{4}$ dilution refrigerator in both the continuous and singlecycle processes. The maximun heat absorption below ahout $0.04^{\circ} \mathrm{K}$ is found to he $82 \mathrm{~T}^{2}$ joules per mole of Ile ${ }^{3}$ circulated. The effect of an imperfect heat exchanger and $\mathrm{He}^{*}$ circulation on the refrigeration capacity is discussed. An analys is of two new types of single-cycle processes is also given.

$\# 6609012$ * $8.173-4.153$

Rand, M. II. ; et al. PLUTONIUY: 'PIYSICO-CHEMICAL PROPERTIES OF ITS CONPOUNDS AND ALLOYS

Atomic Energy Review, 4:1-112

June 1966 
This issue covers plutonium and its compounds and includes a critical evaluation of the data on thermodynamic properties, densities, crystallographic structures, compounds with non-metals, intermetallic phases, phase diagrams, equilibrium diagrams and diffusion rates in the condensed states. This assessment includes all the data published up to the end of 1964 .

$\# 6510034$

$* 8.273-3.273$

Reedy, T. L. (Editor)

ICRPG, WORKING GROUP ON THERMOCHEMISTRY PROCEEDINGS OF THIRD MEETING MARCH 17-18, 1965 , VOLUME 1

CPIA Publication No. 82, Johns llopkins Applied Physics Laboratory, Chemical Propulsion Information Agency, Silver Spring, Maryland

July 1965

$172 p$

Available from DDC

This volume contains the unclassified presentations given at the Third Meeting of the Working froup on Thermochemistry, and is concerned with data of interest for rocket motor performance calculations. The 22 papers include reviews of the status of thermochemical data for the heavy element compounds and ionized species. Recent experimental progress is reported in the areas of matrix spectroscopy mass spectroscopy, calorimetry, and high temperature vapor pressure measurements of light element compounds.

$\# 6809001$

$* 8.273-3.273-0.2573$

Reid, R. C.: Sherwood, T. K.

THE PROPERTIES OF GASES AND LIOIIIDS THEIR ESTIMATION AND CORRELATION, SECOND EDITION

New York: McGraw-Hi Il Book Company, Ine.

1966

$646 \mathrm{p}$

This hook presents a critical review of the various estimation procedures for a limited number of properties of gases and liquids. These include critical properties, $\mathrm{P}-\mathrm{V}-\mathrm{T}$ and thermodynamic properties, vapor pressures, 1 atent heats, heats of formation, free energies of formation, heat capacities, surface tensions, viscosities, thermal conductivities, diffusion coefficients, and equilibrium properties of mixtures. Comparisons of experimental and estimated values are shown in the form of tables for indication of the degree of reliability of the methods discussed. The book is designed to be used as a text for molecular physics or a reference source for engineers. A set of Appendixes summarizes material referred to in the chapters and containsa large volume of tabulated data as well as references to the literature.

\#6902061

* 8.173

Renon, N.; Eckert, C. A.; Prausnitz, J. M.

MOLECULAR THERMODYNAMICS OF SIMPLE LIQUIDS, PURE COMPONENTS

IEEC Fundamentals, $6: 52-58$

February 1967

Thermodynamic properties of simple liquids are calculated from an analytical partition function based on a modification of Prigogine's cell theory and on a three-parameter theorem of corresponding states. The partition function gives an excellent representation of the configurational properties of 15 liquids ranging in molecular complexity from argon to neopentane. Three characteristic molecular parameters are sufficient to calculate the configurational energy and entropy, volume, coefficient of expansion, and compressibility. These parameters are a molecular size, a pairpotential energy, and a term closely related to noncentral intermolecular forces. This last parameter is a nearly linear function of Pitzer's acentric factor. The main application of this statistical thermodynamic treatment follows from its straightforward extension to liquid mixtures.
\#6908020

$\star 2.2773-4.173-8.173-7.173$

Rimshaw, S. J.: Ketchen, E. E.

CESIUM- 137 DATA SHEETS

ORNL-4186, Oak Ridge National Laboratory, Oak Ridge, Tennessee

December 1967

27 .

Available fron CFSTI

Data on CS -137 are tahulated. Properties of the fuel forms CsCl, $\mathrm{Cs}_{2} \mathrm{SO}_{4}$ and cesium borosilicate glass are given. Twenty-seven references are included.

\# 6908021

*2.2773-4.173-8.173-7.173

Rimshaw, S. J.; Ketchen, E. E.

CURIUM DATA SHEETS

ORNL-4187, Oak Ridge National Lahoratory,

Ridge, Tennessee

Decemher 1967

$52 \mathrm{p}$

Available from CFSTI

Data on the fuel forms of $\mathrm{Cm}-244$ are presented. Properties of Cm-244 metal, $\mathrm{Cm}_{3} \mathrm{O}, \mathrm{Cm}_{2} \mathrm{O}_{2} \mathrm{~S}, \mathrm{CmF}$, $\mathrm{Cm}=242, \quad{ }^{242} \mathrm{Cm}_{2} \mathrm{O}$, cermet are tabulated. Forty-nine references are given.

\#6908022

*2.2773-4.173-8.173-7.173

Rimshaw, S. J.; Ketchen, E. E.

STRONT IUM-90 DÁTA SHEETS

ORNL-4183, Oak Ridge National Laboratory, Oak

Rirge, Tennessee

December 1967

$45 p$

Available from CFSTI

Data are given on composition, specific power, radiation, critical mass, container compatability, thermophysical properties, mechanical properties, chemical properties, biological tolerance, and shielding for strontium-90 metal, titanate $\left(\mathrm{SrTiO}_{3}\right)$, oxide $\left(\mathrm{SrO}_{3}\right)$, fluoride $\left(\mathrm{S}_{\mathrm{rF}}\right)$, and orthotitanate $\left(\mathrm{Sr}_{2} \mathrm{TiO}_{4}\right)$. The data are indexed to 53 references which are also included.

\#6905025

$* 4.173-8.173$

Roberts, B. W.

SUPERCONDUCTIVE MATERIALS AND SOME OF THEIR PROPERTIES

NBS Technical Note 482, National Bureau of Standards, Washington, D. C.

May 1969

$129 \mathrm{p}$

Available from the Superintendent of Documents, GPO

This is a noncritical compilation of data on superconductive materials that has been extracted from a portion of the literature published up to early 1968. The properties concerned are composition, critical temperature, critical magnetic field, crystallographic data, and lowest temperature tested for superconductivity. The compilation also includes a bibliography, general reference review articles and a special tabulation of high magnetic field superconductors. This compilation supersedes NBS Technical Note 408 having the same title.

\section{\#6607006 *8.173}

Robins on, R. A. : Stokes, R. H.

ELECTROLYTE SOLIJTIONS, THE MEASIIREMENT AND INTERPRETATION OF CONDUCTANCE, CHEMICAL POTENTIAL AND DIFFUSION IN SOLUTIONS OF SIMPLE ELECTROLYTES, SECOND EDITION (REVISED)

London: Butterworths

1965

$571 \mathrm{p}$

This book is restricted chiefly to properties that are fundamental to electrolytes: conductance, chemical potential and diffusion. Conductance constitutes the main characteristic whereby electrolytes are distinguished from other 
solutions. Of the thermodynamic properties, the Gibbs energy is the most useful for the treatment of equilibrium conditions and emphas is has been placed on quantities such as activities and ionization constants, which are related in a simple way to the Gibbs energy. Considerable space is given to the treatment of diffusion in electrolytic solutions. The text deals primarily with aqueous solutions although some recent data have heen introduced for non-aqueous solutions whenever possible. Extensive appendices and $t a b l e s$ are given, including functions and constants useful in computations, as we 11 as compilations of accurate experimental data, especialiv for concentrated solutions. A reasonah $1 \mathrm{y}$ ful1 arcount is also given of the experimental methods wherohv +hese data wore obtained, in order to illustrate the arcurorv and the limitations of modern technique.

\section{\#6500341} *3. 273

Roder, H. M, : Goodwin, R. D.

PROVISIONAL THERMODYNAMIC FUNCTIONS FOR PARAHYDROGEN

NBS Technical Note 130, National Bureau of Standards, Boulder, Colorado"

December 1961

139 p

Available from CFSTI, $\mathrm{Pb} 161631$

PVT data obtained at this 1aboratory were used to compute tabular values of internal energy, enthalpy, and entropy of para-hydrogen. These properties, together with specific volume, are oresented here as functions of temperature and pressure. The data encompased the temperature range 20 to $100^{\circ} \mathrm{K}$ at pressures un to 340 atmospheres. Earlier data were used to extend the ables to $300^{\circ} \mathrm{K}$. Above $100^{\circ} \mathrm{K}$ the upper limit of pressure is 100 atmospheres. The information is also presented in the form of thermodynamic charts. In Supplement $A$ to this report the thermodynamic tables and charts are presented in units of: psia, degrees Rankine, BTU, pounds, and cubic feet.

\section{\#6510032}

* 3.273

Roder, H. M.; Weber, L. A.; Goodwin, R. D. THERMÓDYNAMIC AND RELATED PROPERTIFS OF PARAHYDROGEN FROM THE TRIPLE POINT TO $100^{\circ} \mathrm{K}$ AT PRESSURES TO 340 ATMOSPIIERES

NBS Monograph 94, National Bureau of Standards, Boulder, Colorado

Augus $t 1965$

$109 \mathrm{p}$

Available from the Superintendent of Documents, GPO

Experimental programs on parahydrogen at this 1 aboratory have provided pressure-densitytemperature relations and heat capacities at temperatures from 15 to $100^{\circ} \mathrm{K}$ and at pressures from 2 to $350 \mathrm{~atm}$. The two types of data have been correlated to yield a consistent set of functions. The properties tabulated for selected isobars and isochores are temperature, volume of pressure, the isotherm derivative $(\delta \mathrm{P} / \delta \rho) \mathrm{T}$ the isochore derivative $(\delta \mathrm{P} / \delta \mathrm{T})_{\rho}$ internal energy, enthalpy, entropy, the specific heats at constant volume and at constant pressure, and the velocity of sound. Also presented are the derived Joule-Thomson inversion curve and some comparisons with normal hydrogen near $100^{\circ} \mathrm{K}$.

\section{$\# 6500312$}

$* 8.173$

Roder, H. M.; et al.

ORTHOBARIC DENSITIES OF PARAHYDROGEN, DERIVED HEATS OF VAPORIZATION, AND CRITICAL CONSTANTS

Cryogenics, $3: 16-22$

March 1963

C1osely spaced experimental data are presented and used for defining a constant set of values for orthobaric density, heat of vaporization, and critical constants of parahydrogen. Discussed are units and constants, experimental and derived data, and the various considerations affecting the solution of the critical temperature. Such constants are to aid the calculation of thermodynamic functions in the single phase region near the critical point.

\section{$\# 6500420 * 8.173$}

Rogers, W. M.; Powe 11, R. L.

TABLES OF TRANSPORT INTEGRALS

NBS Circular 595, National Bureau of Standards, Boulder, Colorado

Ju1y 1958

$46 \mathrm{p}$

Available from the Superintendent of Documents, GPO

The transport integrals, $J_{n}(x)$, defined by

$$
J_{n}(x)=\int \frac{e^{z} z^{n} d z}{\left(e^{z}-1\right)^{2}}
$$

are utilized often in the development of theories describing the thermal conductivity, electrical conductivity, thermal electromotive force, specific heat, and other similar transport properties of solids. The tables include values to six significant figures of the integral for the index integer $n$ ranging from 2 through 17 , and for the limit of integration $x$ ranging from 0.1 by 0.1 intervals, to the 1 imiting upper value, that ranges from 25 for $n=2$ to 40 for $n=17$. The three series utilized to represent the integral in different ranges are derived. The limiting values in the upper range and the asymptotic series expansions in the lower range are also given. Auxiliary tables include the values of the Riemann Zeta numhers and Bernoulli numbers utilized in the calculations.

$\# 6500390$

Rossini, F. D.; et al.

$$
\text { *8.273 }
$$

SELECTEN VALUES OF

PROPERTIES

NBS Circular 500, National Bureau of Standards, Washington, D. C.

February 1, 1952

$1263 \mathrm{p}$

Available from the Superintendent of Documents, GPO

The $t a b l e s$ of chemical thermodynamic properties are divided into two series. Series I gives the heat of formation, free energy of formation, entropy, heat capacity, and the logarithm of the equilibrium constant of formation al1 at $25^{\circ} \mathrm{C}$. The heat of formation at $0^{\circ} \mathrm{K}$, molecular formula, description of substance, crystalline form, isomeric name, solvent if in solution, physical state, and a list of specific references are also given for each compound entry. Series II tabulates the heat, temperature, and entropy of transition, fusion, and vaporization (including values of pressure) as we 11 as the molecular formula, description, name of processes studied, initial and final states, and a list of specific references for each compound.

\section{\#6500379}

Rossini, F. D.; et al.

SELECTED VALUES OF PROPERTIES OF IYYDROCARBONS

NBS Circular 461, National Bureau of Standards, Washington, $D$. C.

November 1947

$496 p$

This book includes, in bound form, all the tables of properties of hydrocarbons (and certain closely related compounds) which have been issued as of May 31, 1947, by the American Petroleum Institute Research Project 44. Some explanatory remarks, and tables of fundamental constants, conversion factors, and molecular weights, are also included. 
$\# 6706040$

$\star 8.173-4.173$

Roth, R. S

PHASE EQUILIBRIA STUDIES ON MIXED SYSTEMS OF RARF

EARTH AND OTHER OXIDES

From Progress in the Science and Technology of the Rare Earth 1 , pages 67.202

New York: Përgamon Press

1964

$36 \mathrm{p}$

This report consists of a review of work done on phase equilibria of mixed systems of rare earth and other oxides. "Rare earth" here includes the elements yttrium and scandium as well as the lanthanide series; only trivalent oxides have heen considered. The results are presented in the form of phase diagrams in temperature and percentage compositon and as tables of the lattice constants of oxide crystals.

\#6906014

* 8.253

Rowlins on, J. S.

LIQUIDS AND LIQUID MIXTURES, SECOND EDITION

New York: Plenum Press

1969

$371 p$

The theory of liquids and liquid mixtures is discussed and thermodynamic data for liquid mixtures and pure liquids are given. An account of the equilibrium properties of pure liquids, a development of reliable methods for the calculation of the thermodynamic properties of a liquid in equilibrium with its vapor, the equilibrium properties of liquid mixtures, and an interpretation of the experimental material in terms of the intermolecular forces are presented. Accurate measurements of the properties of pure liquids including water and mixtures of such liquids as argon, nitrogen, oxygen and the lower alkanes are also included. Information is included for mixtures of complex liquids, the critical state, high pressure mixtures, intermolecular forces and the statistical thermodynamics of fluids and mixtures. Indexes and references are given.

$\# 6603017$

Samsonov, G. V.

PLENUM PRESS HANDBOOKS OF HIGH TEMPERATIRFE MATERIALS NO. 2. PROPFRTIES I.NDFX

New York: Plenum Press

1964

$430 \mathrm{p}$

Authorized translation from the Russian

This book contains data on the physical, technical, mechanical, chemical, and refractory properties of refractory compounds which are currently most widely used in technical developments and which offer the most promise of further application in solving the problens of modern engineering. Chapters I-V of the reference book give information of a general character on refractory compounds, data on their crystal structure, specific gravity, thermochemical, thermal, electrical and magnetic, ontical, mechanical. chemical and refractory properties. In Chanters VI-VII, the author has mainly attempted to provide some ides of the resistance of refractory compounds to the action of different chemical reagents and molten media. and to oxidation. Chapter VIII gives concise tables of information on current and prospective fields of application of refractory compounds in different branches of industry. The data are organized according to the properties of more than 600 different materials such as borides, carbides, nitrides, silicides, etc. (oxides are excluded). In each chapter, the following sequence in order of classes of compounds has been adopted: metal-like borides, carbides, nitrides, silicides, phosphides, sulfides, and non-metaliic compounds. The reference book gives the most reliable data and indicates the literature sources in which duplicate values have been obtained by different investigators. Included are 1337 references.
$\# 6500460$

$\star 8.173-4.173$

Sanford, R. L.; Cooter, I. L.

BASIC MAGNFTIC QUANTITIES AND TIIE MEASURFNENT OF THE MAGNETIC PROPERTIES OF MATERIALS

NBS Monograph 47, National Bureau of Standards, Washington, D. C.

May 1962

$36 \mathrm{p}$

Available from the Superintendent of Documents, GPO

This paper gives general information regarding the two basic quantities, magnetic induction, $B$, and magnetizing force, II, and also the magnetic constant $T$ (often designated by the symbols $\mu_{\nu}$ and $\left.\mu_{0}\right)$. Information is also given regarding the magnetic properties of various materials, and methods and apparatus commonly used in the Magnetic Measurements Section for measuring these properties by means of reversed direct current or alternating currents of low frequency. Magnetic measurements peculiar to high frequencies are not discussed. In view of the gradual adoption of the rationalized mksa system of units, this system is included as well as the classical egs electromagnetic system.

\#6610006,6610007

$\star 8.173$

Schick, il. L.

THERMODYNAMICS OF CERTAIN RFFRACTORY COMPOUNDS

VOL UME 1. DISCUSSION OF THEORETICAL STUDIES, SECT IONS I - VI.

VOLUME 2. THERMODYNAMIC TABLES, RIBLIOGRAPHY AND PROPERTY FILE, SECTIONS VII, VIII AND IX.

New York: Academic Press

1966

$630 \mathrm{p}, 775 \mathrm{p}$

This work is a study of the thermodynamics of the borides, carbides, nitrides, and oxides of 31 elements in the temperature range from $0^{\circ}$ to $6000^{\circ} \mathrm{K}$. The elements are group IIA (beryllium, magnesium, calcium, and stronntium), group IIIB (scandium, yttrium, and lanthanum), group IVA (silicon), group IVB (titanium, zirconium, and hafnium), group VB (vanadium, niohium, and tantalum), group VIB (chromium, molybdenum, and tungsten), group VIIB (manganese, technetium, and rhenium), group VIII (rhodium, osmium, iridium, and platinum), and actinides (uranium and thorium). More than 160 thermodynamic tables, together with comprehensive discussions, have been prepared. The work has been summarized in two volumes. Volume 1 , presents a summary of the techniques used to analyze thermodynamic data and gives the data analyses for refractories considered. Volume 2 , is a compilation of thermodynamic tables generated on this project. A bibliography and subject index are also included.

$\# 6500508$

Schleef, D. J .

REVIEW ÓF MODERN THEORY OF THE THERMAL CONDUCTIVITY OF METALS

In The rmodynamic and Transport properties of Gases, Liquids, and Solids Engineers

(no date)

$12 \mathrm{p}$

Conduction of heat in metals occurs through the behavior of the electrons and the crystal lattice under the influence of a temperature gradient. In pure metals, the electronic conductivity is dominant, because of the mobility of free electrons, and the absence of impurities to scatter then. The theory of conduction of heat in an electron gas has been developed on the basis of a relatively simplified model, considering quasi-free electrons in a periodic potential field due to the lattice, and introducing the scattering effect resulting from thermal excitation of the lattice. The results of the theory and its subsequent refinements are in good agreement with experimental data, particularly for the monovalent metals. The theory of thermal conduction in a crystal lattice, treated as a phonon gas, shows that thermal 
resistance is largely governed by phonon interaction (anharmonicity of lattice vibrations), particularly at higher temperatures. The theory is well borne out by experimental data on dielectric crystals. By postulating a characteristic temperature dependence for each of the component thermal resistances corresponding to individual scattering effects, using the elements of the theory for each scattering mechanism, the total resistance curve may be composed in a semiempirical manner, in order to provide a rational basis for the experimental results.

\#6909001

* 8.173

Schmidt, E. (Editor)

PROPERTIES OF WATER AND STEAM IN SI-UNITS, KJ,BAR; $0-800^{\circ} \mathrm{C} ; \quad 1-1000$ BAR; (INCLUDING A MOLLIER H,SDIAGRAM AND A T, S-DIAGRAM)

New York: Springer-Verlag New York Inc., Title No. 1495

1969

$205 \mathrm{p}$

These tables of the properties of water and steam were calculated in their entirety by using a set of equations accepted by the members of the Sixth International Conference on the Properties of Steam under the title "The 1967 IFC Formulation for Industrial Use". Deviations from the figures computed became necessary for smoothing only in small areas of isobaric specific heat capacity. For the most part, the tables on transport properties were also based on internationally accepted sets of equations. The quantity symbols and unit symbols used are those found in international standards. These Tables are mainly intended for use in industry. They are edited in concurrence with the major engineering institutions in a large number of countries and represent a valuable contribution towards international cooperation. These tables take the place of a new edition of the former Version B (published in 1963) of the "VDI-Wasserdamptafeln".

\section{\#6500314}

* 8.173

Schneider, S. J.

COMPILATION OF TIIE MELTING POINTS OF THE MFTAL OXIDES

NBS Monograph 68, National Bureau of Standards, Washington, D. C.

October 1963

$31 \mathrm{p}$

Available from the Superintendent of Documents, GPO

A compilation has been made of the melting points of 70 metal oxides published prior to January 1963. Both the original melting point and the equivalent value based on the International Practical Temperature Scale of 1948 are presented. Included in the survey is information on pertinent experimental details such as the method of temperature measurement, purity, furnace type, and environmental conditions.

\section{\#6500380 $\quad * 8.273$}

Scott, D. W.; McCullough, J.P.

CHEMI CAL THERMODYNAMIC PROPERTIES OF HYDROCARBONS AND RELATED SUBSTANCES, PROPERTIES OF 100 LINEAR ALKANE THIOLS, SULFIDES, AND SYMMETRICAL DISULFIDES IN THE IDEAL GAS STATE FROM $0^{\circ}$ TO $1000^{\circ} \mathrm{K}$

Bulletin 595, Bureau of Mines, Bartlesville, Oklahoma

1961

$68 \mathrm{p}$

Available from the Superintendent of Documents, GPO

Fifty-six tables are presented which record thermodynamic data. The properties covered are the free energy function, heat content function, heat content (enthalpy), entropy, heat capacity, heat of formation, free energy of formation and $10 \mathrm{~g}$ of the equilibrium constant of formation for the above compounds at selected temperatures between $0^{\circ}$ and $1000^{\circ} \mathrm{K}$.
Shaffer, P.T, B.

PLENIM PRESS HANDBOOKS OF

MATERIALS NO. 1 MATERIALS INDEX

HI GH-TFMPERATURE

New York: plenum Press

1964

$760 \mathrm{p}$

Data on the properties of more than 520 different materials, such as carbides, borides, nitrides, sulfides, phosphides, silicides, oxides, as well as mixed oxides and mixed carbides are presented. The data are taken from 698 references, with the large majority from the U.S. Government report literature. The data concern general. $c^{2} e m i c a l$. electrical, mechanical, nuclear, nntical. structural, and thermal properties. The referenres are listed by number as they appear in the tables. as well as alphabetically by name of author. Th extensive table of contents on pages vii to $x x$ makes it easy to find the various materials in the tables.

$\# 6500353$

* 8.173

Shatp, W. E.

THERMODYNAMIC FINCTIONS FOR CARBON DTOXIDE IN THF. RANGE 40 TO $1000^{\circ} \mathrm{C}$ AND 1 TO 1400 BARS

UCRL-7168, California University,

Radiation Laboratory, Livermore. California

December 1962

$52 \mathrm{p}$

Available from CFSTI

Extensive measurements on the pressure-volumetemperature relations of carbon dioxide were carried out sometime ago by Kennedy (1954). Since then, selected values of the thermodynamic functions of carbon dioxide have been published by Price (1955) and by Majundar and Roy (1956). Even though these tables cover the data quite well. it still seemed desirable to make available the more extensive tables which could be calculated from the experimental data. The results of these calculations are iliustrated. Tables are given for the temperature range $40 \cdot 1000^{\circ} \mathrm{C}$ and the pressure range of 1 to 1400 bars which cover the properties: specific volume, entropy, enthalpy, Gibbs free energy, internal energy and Helmholtz free energy.

\#6907018

$\star 8.173$

Shunk, F. A.; Gowdy, B.; Johnson, V. E.

CONSTITUTION OF ALLÓYS ANNUAL REPORT NO. 1, JUNE 1967 TO MAY 31, 1968

I ITRI-B6082-1, IIT Research Institute, Technology Center, Chicago, I1linois

May 31, 1968

$177 \mathrm{p}$

A phased consolidation and updating of Hansen'ss Constitution of Binary Alloys and its two supplements are being perfo rmed. In the first phase, reviews are under consideration for those binary alloy systems wherein at least one member of the pair is one of the elements $\mathrm{Ac}, \mathrm{Ag}, \mathrm{Al}, \mathrm{Am}, \mathrm{Ar}$, As, or Au. These reviews cover the literature which was encompassed by various abstracting services through December 1966. Reviews for 121 systems are presented with this report.

$\# 6905024$

*8.173

Shunk, F. A.

CONSTITUTION OF BINARY ALLOYS, SFCOND SUPPLEMENT

New York: McGraw-llill Book Company

1969

$720 \mathrm{p}$

This book is the second Supplement to the publication Constitution of Binary Alloys by Hansen in the Materials Science and Engineering Series. This supplement is concerned primarily with data which became available during the three-year period 1962-1964 and is intended to be comprehensive for this period. Approximately 8,700 system references were consulted in the preparation of the reviews for the 1,702 binary systems which form the text. 
There are 219 figures. No data were previously available for 313 of these systems.

$\# 6510027$

$* 8.273$

Sillen, L. G.; Marteli, A. E. (Compilers)

STABILITY CONŚTANTS OF MFTAL-ION COMPLEXES,

SECTION I: INORGANIC LIGANDS,

SECTION II: ORGANIC LIGANDS SÉCOND EDITION

Special Publication No. 17, The Chemical Society, London, England

1964

$754 \mathrm{p}$

Each table summarizes the data up to 1960 for the association of one particular 1 igand with all the metallic ions which have heen studied in conjunction with it, as far as the compilers located them. Some data published in 1961-1963 have also been included. Methods of measurement, composition, and temperature of the media to which the data refer, appropriate stability constants for the various complexes formed and references to the original papers are given for each Iigand-metal pair. Acid dissociation constants of the ligands are recorded by including the hydrogen ion among the metals as one of the cations with wich the ligands associate. Redox equilibria are represented by including the electron as a ligand, and hydrolysis of the metallic ions is described hy regarding the hydroxyl ion as one of the ligands. The tables are divided into two sections, the first dealing with inorganic ligands and the second with organic ligands.

$\# 6500217$

$* 8.373-4.373$

Simonds, II. R.; Church, J. M.

A CONCISE GUIDE TO PLAŚTICS, SECOND EDITION

New York: Reinhold Publishing Corporation

1963

$404 \mathrm{p}$

This second edition brings up to date the text of the first edition of $\Lambda$ Concise Guide to Plastics and encompases a much Iarger fierd due $\frac{\text { to the }}{\text { to }}$ expansion of the plastics industry itself. An objective of this book was to answer the questions asked at the 1961 Plastics Exhibit. The subjects discussed include basic chemistry of plastics, manufacturing techniques, stereospecific catalysts, ablative action, borderline materials, forms of plastics, resin manufacture, compounding, processing, applications, production and prices, selection factors, and the business of plastics: One chapter is devoted to plastic manufacturers statements and contains information about the company's materials, trade names and sales for fifty-two producing firms. over fifty pages of tables of the United States Plastics Trade Names are included, as well as numerous graphs and tables of data concerning plastic properties.

\#6500001

Sindt, C. F.: Mann, D. B.

TEMPERATURE- - MNTROPY

TRIPLE-POINT REGION

NBS Technical Note 343, National Bureau of Standards, Washington, D. C.

June 22 , 1966

$6 \mathrm{p}$

Available from the Superintendent of Documents, GPO

The three-phase region at and near the triple point of parahydrogen is presented on the graphical coordinates of temperature and entropy. I sobars from $10 \mathrm{~mm} \mathrm{lg}$ to 340 atmospheres, temperatures from $11^{\circ}$ to $23^{\circ} \mathrm{K}$ and specific volumes covering the range of from $10.5 \mathrm{cc} / \mathrm{gm}$ to $15,000 \mathrm{cc} / \mathrm{gm}$ are included. The energy base of enthalpy and entropy are consistent with previous data published by this laboratory.
$\# 6512021$

*8.173

Singman, D. ; Forziati, A. F.

HYDROGEN SOURCES FOR FUEL CELLS

TR-1168, Harry Diamond Laboratories, Washington, D.C. November 1, 1963

$29 p$

\section{Available from CFSTI}

Commercially available gas, liquid and chemical sources of hydrogen for use with fuel-cell batteries are compared. Cryogenic storage is shown to be the most efficient on both weight and volume bases. Chemical generators are suitable for applications requiring moderate quantities of hydrogen gas at infrequent periods. Compressed-gas cylinders are convenient when small quantities of hydrogen are desired. A bibliography of selected publications during the past five years is included.

\#6510019,6510020 *8.173

Skinner, H. A.; Waddington, G.; Schaefer, K. (Editors)

BULLET IN OF CHEMICAL THERMODYNAMICS NO. 2 , NO. 4

Ann Arbor, Michigan: Iniversity of Michigan Press March 10, 1959; March 20, 1961

$105 \mathrm{p}, 15 \mathrm{lp}$

These annual volumes contain abstracts and bibliographies of papers published in the fields of thermodynamics and thermochemistry, arranged by country and laboratory. Fach abstract contains a short description of the quantities measured or calculated and the method used. Topics of the abstracts include heats of combustion, reaction and formation, thermodynamic properties of pure substances and systems, vaporization studies, and other non-calorimetric thermodynamic properties. An author index and suhstance-property index are included. Each volume covers material from the year previous to the publication of the volume, Volume No. 2 was published in March 1959 and No. 4 in March 1961.

\#6510021, $6510023 \quad * 8.173$

Skinner, H. A. (Editor)

BULLETIN OF THERMODYNAMICS AND THERMOCHEMISTRY NO. 6 , NO. 7

Ann Arbor, Michigan: University of Michigan Press

April 1963, April 1964

$243 \mathrm{p}, 321 \mathrm{p}$

These annual volumes contain abstracts and bibliographies of papers n'blished in the fields of the rmodynamics and thermochenistry, arranged by country and Iaboratory. Ea-h abstract contains a short description of the quantities measured or calculated and the method used. Topics of the abstracts include heats of combustion, reaction and formation, thermodynamic properties of pure substances and systems, vaporization studies, and other non-calorimetric thermodynamic nroperties. An author index and substance-property index are included. Each volume covers material from the year previous to the publication of the volume, Volume No, 6 was published in April 1963 and No.; in April 1964.

\#6602013 *3.373-8.373

Somayajulu, G. R.: 2wolinski, B, J.

ADDITIVITY OF THE POTENTIAL BARRIERS ABOUT DOUBLE BONDS IN HALOGENATED ETIYYLENES

Chemical The rmodynamic Properties Center, Texas AGM University, College Station, Texas September i, 1965

$9 \mathrm{p}$

Potential barriers about double bonds have been found to be additive of the contributions from pairs of bonds separated hy $\mathrm{C}=\mathrm{C}$ bond in substituted ethylenes. On the basis of this work, the torsional frequency of ethylene has heen found to be $825 \mathrm{~cm}^{-1}$. New torsional assignments have been suggested for some molecules. 
Somayajulu, G. R.; Zwolinski, B. J.

A GENERALIZED BOND ENERGY SCIEME INCLUDING BARRIERS TO FREE ROTATION AND CORRECTIONS FOR ROTATIONAI. ISOMERISM

Chemical Thermodynamic Properties Center, Texas Aक्ष: University, College Station, Texas

1965

$21 \mathrm{p}$

A generalized procedure for predicting the energies of the paraffinic hydrocarbons is deduced on the assumption of (i) interactions between pairs of bonds attached to a carbon atom, (ii) interactions between trios of bonds attached to a carbon atom and (iii) interactions between pairs of bonds separated by a $\mathrm{C}-\mathrm{C}$ bond. The derived equation justifies Tatevskii's empirical approach and is shown to be not very different from Allen's equation without the steric terms. Under defined conditions this equation reduces exactly to Allen's equation. Barriers to free rotation were considered in deriving the new equation. This new equation is further modified by including corrections for rotational isomerism in place of trigonal interactions. The new relation is better founded in principle, correctly estimates enthalpies of atomization for normal and isomeric alkanes including those molecules without rotational isomers, and furthemore provides more refined steric terms due to higher order interactions.

\#6603012,6603013,6603014 *8.373-5.373-3.373 Somayajulu, G. R.; Zwolinski, B. J.

RELATIONSIIIP BETWEEN BOND ENERGY AND BOND DISSOCIATION ENERGY,

II. ENTIIALPIES OF' FORMATION OF THE SUBSTITUTED METHANES, SUBSTITUTED MFTHYL RADICALS AND SUBST ITUTED METHANES, III. MAGNITUDES Ó THE BOND AND PAIR BOND CONTRIBUTIONS TO THE ENERGIES OF THE MOLECULES,

IV. ENTHALPIES OF FORMATION OF SURSTITUTED ETHYLENES

Chemical Thermodynamic Properties Center, Texas AfM University, College Station, Texas

August 1965, August 1965 , September 1965

$12 \mathrm{p}, 15 \mathrm{p}, 8 \mathrm{p}$

In Part II, the enthalpies of formation of the substituted methanes have been calculated on the basis of Zahn's model. A method has been developed for the calculation of the dissociation energy of the $R^{\prime}-R^{\prime \prime}$ bond in a substituted ethane. Based on the dissociation energy of the $R^{\prime}-R^{\prime \prime}$ bond, the enthalpies of formation of the substituted methyl radicals and the substituted ethanes have been calculated. The enthalpies of formation of the substituted methyl radicals can also be calculated on the basis of Zahn's model. In Part III methods have been developed for the calculation of the magnitudes of the bond and pair bond contributions based on relationships between bond energy and bond dissociation energy. The pair bond contributions for the pair of bonds $\mathrm{C}<\frac{\gamma}{1}$ in $\mathrm{CX}_{4}, \mathrm{CX}_{3}$, and $\mathrm{CX}_{2}$ have been determined. Also determined were the enthalpies of formation of $\mathrm{CX}$, and $\mathrm{CS}$ radicals, $\mathrm{X}$ being $\mathrm{H}, \mathrm{F}, \mathrm{C} 1, \mathrm{Br}, \mathrm{I}$ or ${ }^{2} \mathrm{CH}$. In $\mathrm{Part}$ IV enthalpies of formation of the $\mathrm{CX}_{2}$ type radicals obtained in Part III have been used to obtain the enthalpies of formation of the CXY type radicals. Based on a relationship between hond energy and bond dissociation erergy, the dissociation energies of the $R^{\prime}-R^{\prime \prime}$ bonds in substituted ethylenes have been calculated. Using the bond dissociation energies and the enthalpies of formation of the substituted methylene radicals, enthalpies of formation of a few substituted ethylenes have been calculated. The calculated values have heen found to be in good agreement with the observed values.
$\# 7001024$

$\star 8.173$

Stims on, H. F.

SOME PRECISE MEASUREMENTS OF THE VAPOR PRESSURE OF WATER IN THE RANGE FROM 25 TO $100^{\circ} \mathrm{C}$

Journal of . Research of the National $\frac{\text { Bureau of }}{\text { Shysics }}$ october 1969

The vapor pressure of water was measured at seven temperatures in the range from 25 to $100^{\circ} \mathrm{C}$ using the boiler and precision manometer with which standard resistance thermoneters were calibrated at the NBS in the $1940^{\prime} \mathrm{s}$. A table gives measure values of pressure, adjusted to even degrees, and indicates estimates of the standard deviations of pressure and of the corresponding temperatures. Except for the measurement at $25^{\circ} \mathrm{C}$, the values of pressure were consistent within one part in 50,000 . The temperature measurements had an estimate standard deviation of 0.00040 . degree.

$\# 6907013$

*8.172-4.172

THERMAL EXPANSION BIBLIOGRAPHY

Research Materials Information Center, 0ak Ridge Nationa1 Laboratory, 0ak Ridge, Tennessee

February 1969

$57 \mathrm{p}$

This is a bibliography of thermal expansion information sources based on papers, reports, and abstracts received by the RMIC. It also contains materials index. Over 350 references are given covering material through February 1969.

\#6500361

$\star 8.373$

Somayajulu, G. R.; Kudchadker, A. P.; Zwolinski, B.

THERMODYNAMICS

Chemical Thermodynamic Properties Center, Texas A\&M University, College Station, Texas

December 1964

$77 p$

This report constitutes a review of thermochemistry and thermodynamics of pure substances for the period December 1, 1963 through December 1, 1964 . The currently recommended values for fundamental constants in chemistry and physics are discussed. Unpublished values of standard enthalpies of formation of certain "key" compounds are summarized. The various mathematical theories of isomers are reviewed and values are quoted for aliphatic alcohols ( $\mathrm{C} 1$ to $\mathrm{C} 20$ ). A critical analysis is presented of various correlation procedures for prediction of enthalpies of formation and bond energies for organic compounds. It is shown that the Laidler, Allen, and Zahn correlations for enthalpies of formation are mathematically equivalent. Various procedures for calculating bond energies are reviewed. Six procedures for correlating normal boiling points for homologous series were compared using the normal alkanes as a basis. The Li-Rossini and the Kreglewski-Zwolinski procedures are the most satisfactory. Parameters were found for predicting the vapor pressures and boiling points of all high molecular weight normal alkanes in the range $C 20$ to C100. New procedures for predicting boiling points and dipole moments were developed.

$\# 6500242,6500243 \quad * 8.273$

Stephen, H.; Stephen, T. (Editors)

SOLUBILITIES OF INORGANIC AND ORGANIC COMPOUNDS, VOLUME I BINARY SYSTEMS, PART 1 AND PART 2

New York: The Macmillan Company 1963

$1933 \mathrm{p}$

Volume 1, parts 1 and 2, comprises the solubilities of elements, inorganic compounds, metallo-organic and organic compounds in binary svstems. There are 2 separate comprehensive indexes of formula and names of compounds presented as in Chemical Abstracts. The compounds are named according to IUPAC. Tables 1-1694 deal with Binary Systems in 
which water is one of the components. Tahles 1695 et seq. are devoted to binary systems consisting of components other than water. In two tables (ppl646-1667) details are given of equilibriun conditions in solid phases fnr svstems with aqueous and non-aqueous solvents. The formulae of the different solid phases in equilibrium with the saturated solution for each compound listed are given and in addition tn the transition temperatures, the solubility of the substance being investigated is shown.

H 6602003 $* 8.173$

Stern, K. H. ; Weise, E. L.

HIGH TEMPERATURE PRÓPERTIES AND DECOMPOSITION OF INORGANIC SALTS PART 1 SULFATES

NSRDS-NBS-7, National Bureau of Standards, Washington, D. C,

1966

$176 \mathrm{p}$

Available from the Superintendent of Documents, GPO

The literature dealing with the high-temperature behavior of inorganic sulfates has been critically reviewed. Free energy functions of reactants and products of the decomposition reactions were calculated and have been tabulated from $298^{\circ} \mathrm{K}$ up to as high a temperature as possible. Free energy functions, equilibrium constants of reactions, and partial pressures of gaseous components were tabulated. Auxiliary data on phase transitions, densities, and kinetics of chemical decomposition have also bcen included.

$\# 6912009$ $* 8.173$

Stern, K. H. ; Weise, F. L,

HIGII TEMPERATURE PRÓPERTIES AND DECOMPOSITION OF INORGANIC SALTS PART 2. CARBONATES

NSRDS-NBS-30, National Bureau of Standards, Washington, D. C.

November 1969

$32 \mathrm{p}$

Available from the Superintendent of Documents, GPO

The literature dealing with the high-temperature behavior of inorganic carbonates has heen critically reviewed. Free energy functions, of reactants and products of the decomposition reactions were calculated and have been tabulated from $298 \mathrm{~K}$ up to as high a temperature as possible. Free energy functions and equilibrium constants of reactions were tabulated. Auxiliary data on phase transitions, densities, and kinetics of thermal decomposition have also been included. The literature of the endothermic decomposition kinetics of solids, as it applies to carbonates, has been reviewed.

\#6500475

*8.143

Stewart, R. B.; Johnson, V. J. (Editors)

COMPENDIUM OF THE PROPERTIES OF MATERIALS AT LON

TEMPERATURE (PIIASE II)

WADD Technical Report 60-56, Part IV, Wright Air Development Center, Wright-Patterson Air Force Base, Ohio

December 1961

$500 \mathrm{p}$

Avai 1 ab 1e from DDC, AD 272-769

Phase II of the Compendium includes data sheets on compressibility factor, velocity of sound and entropy of fluids, vapor-liquid equilibrium concentration of binary mixtures of fluids, and electrical resistivity and thermal conductivity integrals of metallic solids. Data sheets are included for each of these properties for the following materials: compressibility factor (helium, hydrogen, neon, nitrogen, air, methane): entropy (a T-S diagran for neon); velocity of sound (in liquids: helium, hydrogen, nitrogen, oxygen, argon, methane; in gases: helium, hydrogen, neon, nitrogen, oxygen, air, carbon monoxide, argon, methane); liquid-vapor equilibrium concentrations (helium in hydrogen, nitrogen, methane; hydrogen in nitrogen, carbon monoxide, methane; nitrogen in oxygen, carbon monoxide, argon, methane) electrical resistivity (53 of the pure metallic elements); and thermal conductivity integrals (44 pure metallic substances, 36 non-ferrous alloys, ferrous alloys and 4 glasses and plastics). In general, the data sheets present the data primarily in graphiral form, and in addition include tables of selected values, references to the sources of the data and other references. Appropriate comments of interest to the user are also given.

\#6500323 * 3.173

Stewart, R. B.; Timnerhaus, K. D.

TIIERMODYNAMIC PROPERTIES OF CRYOGENIC FLUIDS

R-295, Preprint of Paper No. A-3 presented at 1963 Cryogenic Engineering Conference, University of Colorado, Boulder, Colorado, August 19-21, 1963, National Bureau of Standar's, Boulder, Colorado August 1963

$31 p$

Available from the Cryogenic Data Center, National Bureau of Standards, Boulder, Colorado

Cryogenic Engineering Laboratory, National Bureau of Standards has undertaken a re-evaluation of the pertinent experimental data to provide tabulations of thermodynamic properties for advanced cryogenic systems design. Tables and graphs of properties, as well as analytic functions for compute programing, are now available for several of the cryogenic fluids. These tabulations are intended to provide the engineer with comprehensive and consistent sets of data which are hopefully the best representation of the existing experimental data. These compilations have also served to define the linitations of the existing data and to define the needs for new measurements. This paper summarizes the experimental data for the several cryogenic fluids and outlines some of the procedures utilized in providing the various sets of the rmodynamic property values.

\#6500406

Strobridge, T. R.

* 8.273

THERMODYNAMIC PROPERTIES OF NITROGEN

$300^{\circ} \mathrm{K}$ BETIVEEN 0.1 AND 200 ATMOSPHERES

NBS Technical Note 129, National Bureau of Standards, Boulder, Colorado

January 1962

85 p

Available from CFSTI, PB 161-630

The internal energy, enthalpy, entropy, and specific volume of molecular nitrogen are derived and tabulated as functions of temperature and pressure. In addition to a mathematical model for the pressure-volume-temperature surface, accurate functions are given for the representation of the vapor pressure, density of saturated liquid, specific heat of saturated liquid, and the specific heat at zero pressure. Tahular values in British units over the same pressure and temperature range are available as Supplement A of this Technical Note.

\#6500405

* 8.273

Strobridge, T. R.

THERMODYNAMIC PROPERTIES OF NITROGEN FROM 114 TO $540^{\circ} \mathrm{R}$ BETWEEN 1.0 AND 3000 PSIA, SUPPLEMENT A (BRITISH UNITS)

NBS Technical Note 129A, National Bureau of Standards, Boulder, Colorado

February 1963

$85 \mathrm{p}$

Available from the Superintendent of Documents, GPO

The internal energy, entha1py, entropy, and specific volume of molecular nitrogen are derived and tabulated as functions of temperature and pressure. In addition to a mathematical model for the pressure-volume-temperature surface, accurate functions are given for the representation of the vapor pressure, density of saturated liquid, specific heat of saturated liquid, and the specific heat at zero pressure. Tabular values in metric 
units over the same pressure and temperature range are also available in Technical Note 129.

\#6510042-6510045

*8.273

Stu11, D. R.

JANAF' INTERIM THERMOCHEMICAL TABLES VOLUMFS $1,2,3$ AND 4

Midland, Michigan: Dow Chemical Company

August 1968

1800 looseleaf pages

The JANAF (Joint Arny-Navy-Air Force) Thermochemical Data tables contain data on the thermodynamic properties of materials of interest in rocket propellant and related field calculations. The properties tabulated are: specific heat entropy, Gibbs energy and enthalpy relative to $298.15^{\circ} \mathrm{K}$, enthalpy of formation, and free energy of formation as function of temperature. References to reports from which the data were taken are given for each compound. The compounds are arranged alphabetically by chemical symbol. Volume I $(6510045)$ covers A1 thru B; Volume II $(6510043)$ Be thru C1NO: Volume III $(6510044)$ covers C1Na thru $\mathrm{Hg}_{2} \mathrm{I}_{2}$; Volume IV (6510043) covers I thru $\mathrm{Zr}$ and the electron gas. In each case the formulas themselves are indexed by arranging the elements making them up in alphabetical order of chemical symhols.

\#6510030

*8. 223

Stu11, D. R.

JANAF' THERMOCHEMI CAL TABLES

Midland, Michigan: Dow Chenical Company, Therma1 Research Laboratory

August 1965

1000 looseleaf pages

Available from CFSTI, PB 168370

Thermodynamic properties of the light elements and their compounds are given for the solid, liquid and gaseous state. Some of the properties covered include heat capacity, standard entropy, heat of formation, free energy of formation, free energy function, heat content, log of equilibrilm constant, heat of fusion, heat of sublimation and vaporization, melting point, boiling point. Some references are given.

\section{\#6910028}

$* 8.273$

Stu11, D. R.

JANAF THERMOCHEMICAL TABLES, FIRST, SECOND AND THIRD ADDENDA

Thermochemical Research Laboratory, Dow Chemical Company, Midland, Michigan

August $1966,1967,1968$

$215 \mathrm{p}, 100 \mathrm{p}, 100 \mathrm{p}$

Available from CFSTI, PB 168370-1, PB 168370-2, PB $168370-3$

These three addenda to the JANAF Thermochemical Tables represent the additions and revisions made by the Dow Thermal Research Laboratory during the period June 1965 to June 1968. The addenda represent a changeover to the $\mathrm{C}^{12}$ atomic weight scale and to the physical constants recommended by the National Academy of Science-National Research Council and the National Bureau of Standards. A new element, copper, and some of its compounds have been added to the collection.

\#6810019

*8. 173

Stul1, D. R.; Sinke, G. C. (Compilers)

THERMODYNAMIC PROPERTIES OF THE ELEMENTS

Washington, D. C.: American Chemical Society, Advances in Chemistry Series 18

1956

$233 \mathrm{p}$

Tabulated values of the heat capacity, heat content, entropy, and free energy function of the solid, liquid and gaseous states of the first 92 elements are given for the temperature range $298^{\circ}$ to $3000^{\circ} \mathrm{K}$. Auxiliary data include temperatures and heats of transition, melting and vaporization, and vapor pressures. Literature sources are listed. The puhlished values have been analyzed and are supplemented by estimates when experimental data are lacking.

\#6911010

*8. 173

Stu11, D. R.; Westrum, E. F., Jr.; Sinke, G. C.

THE CHEMICAL TIIERMODYNAMICS OF ORGANIC COMPOUNDS

New York: John Wiley \& Sons, Inc.

1969

$885 \mathrm{p}$

A comprehensive and systematic treatment of organic chemical thermodynamics is provided from a search of the 1iterature up to January 1, 1966. Tabulations have been limited to the temperature range from 298.15 to $1000^{\circ} \mathrm{K}$. The heat capacity, entropy, enthalpy and Gibbs energy of formation for 741 pure organic compounds from 298 to $1000^{\circ} \mathrm{K}$ have been tahulated for the ideal gas state. The entropy, enthalpy, and Gibbs energy of formation are presented as fully as possible for approximately 4400 organic compounds in the ideal gaseous and condensed states at $298^{\circ} \mathrm{K}$. Some regularities existing between hydrocarbons and other classes of organic compounds are pointed out. A review of relevant elements of thermodynamics and a discussion of calculational methods are included in Section I. Brief descriptions of calorimetric measurements are provided and the primary sources of information are tahulated in sections II and III. Chapter 6 introduces the methods used to estimate thermodynamic properties of compounds that have not yet been measured experimentally.

\#6511005

TABLES OF THERMODYNAMIC

* $8.172-2.772$

THERMODYNAMICS OF NUCLEAR MATERIÁLS

Vienna, Austria: International Atomic Fnergy Agency, STI/DOC/10/38

December 1964

$95 \mathrm{p}$

Available from UN Headquarters

A compilation is presented of data on the thermodynamic properties of materials of interest for nuclear technology. The data were released in the Symposium on the Thermodynamics of Nuclear Materials, which was held in May 1962. The properties covered include: heats of formation, free energies of formation, entropies of formation, and standard entropies at $298^{\circ}$ and at other temperatures; enthalpy increments, free energy functions, entropy increments; enthalpies, free energies, entropies of transitions; heats, free energies, entropies of reaction; heat capacities; vapor pressures; thermodynamic properties of mixtures; melting points, densities; surface tensions; and lattice parameters.

\#6511006 *8.172-2.772

THERMODYNAMICS OF NUCLEAR MATERIAL.S, PROCEEDINGS OF THE SYMPOSIUM ON THERMODYNAMICS OF NUCLEAR MATERIALS HELD BY THE INTEPNATIONAL ATOMIC ENERGY AGENCY IN VIENNA, 21-25 Nay 1962

Vienna, Austria:International Atomic Energy Agency, $\mathrm{STI} / \mathrm{PUB} / 58$

September 1962

$822 p$

Available from IAEC

The International Atomic Energy Agency initiated a project to assist in disseminating and assessing data on important nuclear materials. Thus, the symposium was organized in an attempt to present and appraise the results of the work on thermodynamics of nuclear material; to assess their reliability and further to discuss the application of the results and principles of thermodynamics to the solution of current problems in nuclear technology. Particular emphasis was placed on the thermodynamics of actinides and vaporization processes. 
$46500231-6500234$

$\star 8.273$

Timmermans, J.

PHYSICO-CHEMICAL CONSTANTS OF BINARY SYSTEMS IN CONCENTRATED SOLUTIONS,

VOLUME I : TWO ORGANIC COMPOUNDS (WITHOUT HYDROXYL DERIVAT IVES),

VOLUNE II : TWO ORGANIC COMPOUNDS (AT LEAST ONE A HYDROXYL DERIVATIVE)

VOLUME III: SYSTEMS WITT METALLIC COMPOUNDS

VOLUME IV: SYSTEMS WITH INORGANIC + ORGANIC OR INORGANIC COMPOUNDS IEXCEPTING

METALLIC

DERIVAT IVES)

New York: Interscience Puhlishers, Inc.

$1959-1960$

$1269 \mathrm{p}, 1271 \mathrm{p}, 1322 \mathrm{p}, 1332 \mathrm{p}$

The data compiled refer only to hinary systems of concentrated solutions which include systems between 10 and 90 percent hy weight, except those where there is only one measurement hetween 10 and 20 percent. All types of substances are considered as components with the exception of metallic alloys. The data are classified by systems, and are arranged by volume as follows: volume I, both components are organic compounds, excepting the hydroxyl derivatives; Volume II, both components are organic compounds of which at least one is a hydroxyl derivative; Volume III, at least one of the components is a metallic compound; Volume IV, all other systems. Heterogeneous equilibria properties discussed are critical constants, saturated vapor pressure for the triphase equilibrium, vapor pressure curves, boiling curve and azeotropes, composition of liquid and vapor coexisting phases, densities of coexisting phases and rectilinear diameter, composition of the two liquid phases and eventually of the saturated vapor, critical solution point, freezing and melting curves, eutectic and transition points, and equilibria of the condensed phases under high pressure. Properties of the gas, liquid and mixed crystal phases discussed are densities, coefficients of expansion and of compressihility, viscosity and surface tension, refractive index and optical dispersion, dielectric constant, electrical conductivity, optical rotatory power, magnetic rotation, magnetic susceptibility. Thermal constants discussed are specific heat, heat of solution and mixing, heat of vaporization and fusion and thermal conductivity. All numerical data have been taken as given in the original papers. A bihliography and compound and author indexes are included at the end of the fourth volume.

$\# 6500230.6510016$

Timmermans, $\mathrm{J}$.

PIIYSI CO-CHEMI CAL

$* 8.373$

COMPOUNDS, VOLUME I AND VOLUME II

Amsterdam, London, New York: Elsevier Publishing Company

1950,1965

$700 \mathrm{p}, 490 \mathrm{p}$

These volumes contain compilations of critically selected data on the physico-chemical constants of organic compounds, compiled from reports published in the literature. The original volume covers data published through 1950; the second volume covers data published through 1964. Some of the properties covered are boiling point, saturated vapor pressure, critical constants, density, critical solution point, freezing pnint, constants of state with their temperature coefficients, specific weight, compressibility, viscosity, surface tension, refractive index, dielectric constant, specific rotatory power and magnetic susceptibility, heat constants such as specific heat, latent heat of transformation, heat of combustion. The compounds are arranged in the order: saturated and unsaturated acyclic hydrocarhons, aromatic hydrocarbons and cycloparaffins; halogenated derivatives in same order, oxygenated derivatives (alcohols and ethers, aldehydes and ketones, acids and esters), the nitrogen compounds (amines, nitriles, nitroderivatives, amides, etc.), mixed compounds (oxyhalogenated,

nitro-halogenated compounds, etc.), and sulfur and phosphorus derivatives, etc. Bibliographical references and a formula index are included.

\#6500520-6500523

Touloukian, Y, S

DATA BOOK, VOLUME 1 : METALLIC ELEMENTS AND THEIR ALLOYS (SOLID, LIQUID AND GASTOIIS STATES), VOLUMF 2: NONMETALLIC ELEMENTS, COMPOUNDS AND MIXTURES (IN LIOUID AND GASEOUS STATES AT NORMAL TEMPERATURF AND PRIESSURE), VOLUME 3: NONMETALLIC ELEMENTS COMPOUNDS AND MIXTURISS (IN SOLID STATE AT NORNAL TEMPERATURE AND PRESSURE)

Purdue University, The rmoplysical properties Research Center, Lafayette, Indiana

1964

$3000 \mathrm{p}$

One of the major aspects of the Thermophysical Properties Research Center's activities consists of the preparation of data tables. These tables are a compilation of original experimental data on various thermodynamic properties of materials published in government research reports, industrial publications and the world's scientific and technical literature. The primary properties reported are: thermal conductivity; viscosity; the thermal radiative properties emittance, reflectance, absorbance and transmittance; thermal diffusivity; and specific heat. The overall organization of the data tables consists of three volumes: Volume 1 , metallic elements and their alloys (solid, liquid and gaseous states); Volume 2 , nonmetallic elements, compounds and mixtures (Iiquid and gaseous states at NTP); Volume 3 , nonmetallic elements, compounds and mixtures (solid state at NTP). Each substance is assigned a serial code number, and all tables and figures for that substance carry numbers identical with the substance numher. The information is presented in the form of a graph, specification table and data table for each substance. Reference to the original paper is given by number in the specification table. The bihliographic citation of each reference numher is furnished in a numerically ordered list of references at the end of each chapter. Updated and new data sheets are released in June and December of each year.

\#6500497-6500502 *8.273

Touloukian, Y. S. (Editor)

RETRIEVAL GUIDE TO THERMOPHYSICAL PROPERTIES RESEARCH LITERATURE, VOLUME I: BOOKS 1, 2 AND 3 , VOLUME II: BOOKS 1,2 AND 3

New York: McGraw Hill Book Company, Inc. 1960,1963

$1245 \mathrm{p}, 169 \mathrm{p}, 372 \mathrm{p}, 436 \mathrm{p}, 193 \mathrm{p}, 398 \mathrm{p}$

More than 1210 journals, reports, and books have been searched for references to 20,000 articles containing information on seven specified thermophysical properties of more than 14,240 substances. The properties are thermal conductivity, specific heat, viscosity, emissivity, diffusion coefficient, thermal diffusivity and Prandtl numher. Book 1 of each volume primarily constitutes TPRC's Classified Directory of Substances to approximately 14,240 substances and materials for which thermophysical properties information is reported in the volumes, with an indication of the properties of each substance for which references are cited. Book 2 for each volume is the direct output of the digital computer and constitutes the search and retrieval element of the code entries to the sources of information for each property and substance, with an indication of the physical state, subject, language, year of publication of the reference and TPRC serial number. Book 3 for each volume contains the complete hibliographic information for each of the 10,000 references included in each volume, as well as an author index to the contributing authors. Abstracting journals have been searched through 1961 . 
Touloukian, Y. S.; et al.

SYSTEMS AND PROCEDURES DEVIELOPED FOR THE SEARCI, CODING, AND ?ECLIANIZED PROCESSING OF BIBLIOGRAPIIC INFORMATION ON THERNOPIIYSICAL PROPERTIES

In Thermodynamic and Transport Properties of Gases, Liquids and SoIids, pp?8-9l

New York: The Ámerican Society of Mechanical

Engineers

No Date

$13 p$

Methods used by the Thermophysical Properties Research Center for handing collected bibliographical information on themophysical properties are described. Details are given regarding the method of coding abstracts for reproduction on IBM cards. Special consideration is given to the coding of substances and the system of substance classification and notation used for this purpose. Procedures are described which are used to store the punched card information on magnetic tape. Systems for retrieval of this stored information are delineated including a description of the organization of the retireved bibliography and of the machine techniques involved.

\#6704044-6704052 * 8.273

Touloukian, Y. S. (Editor)

THERMOPHYSICAL PROPERTIES OF HIGII TEMPERATURE SOLID MATERIALS,

VOLUME 1: ELEMENTS

VOLUME 2: NONFERROUS ALLOYS,

PART I: NOFERROUS BINARY ALLOYS

PART II : NONFERROUS MULTIPLF, ALLOYS, VOLUME 3: FERROUS ALLOYS

VOLUME 4: OXIDES AND THFIR SOLUTIONS AND MIXTURFS, PART I: SIMPLE OXYGEN COMPOUNDS AND THEIR MIXTURES,

PART II: SOLUTIONS AND TIEIR MIXTIIRFS OF SIMPLE OXYGEN COMPOUNDS, INCLUDING GLASSES AND CERAMIC GLASSES,

VOLUME 5: NÓNOXIDES AND THEIR SOLUTIONS AND MIXTURES, INCLUDING MISCELLANEOUS CERAMIC MATERIALS

VOLUME 6: INTERMETALLICS, CERMETS, POLYMFRS AND COMPOSITE SYSTEMS

PART I: INTERMETALLICS

PART II : CERMETS, POLLYMERS AND COMPOSITE MATERIALS

New York: The Macmillan Company

1967

$1320 \mathrm{p}, 758 \mathrm{p}, 1030 \mathrm{p}, 625 \mathrm{p}, 1005 \mathrm{p}, 1054 \mathrm{p}, 1210 \mathrm{p}$, $758 \mathrm{p}, 961 \mathrm{p}$

A listing of the properties of materials is presented. The properties listed are density, melting point, heat of fusion, heat of vaporization, heat of sublimation, electrical resistivity, specific heat at constant pressure, thermal conductivity, thermal diffusivity, thermal linear expansion, 'radiative properties" (absorptance, emittance, reflectance and transmittance), and vapor pressure as a function $n f$ temperature. An explanatory section is included $w^{*}$ th each volume. The titles of the volumes indicate the type of materials included in each volume.

\#6712001-6712003 * 8.273

Touloukian, Y. S. (Editor)

THERMOPIIYSICAL PROPLRTIES RESFARCH LITERATIJRF RETRIEVAL GUIDE, BOOKS 1, 2 AND 3, SECOND EDITION

New York: plenum press

1967

$819 p, 625 p, 1315 p$

A11 research 1iterature from 1920 to 1964 has been searched for the thermophysical properties of given substances. The specific properties considered are themal conductivity, specific heat at constant pressure, viscosity, thermal radiative properties, diffusion coefficient, permeability, thermal diffusivity and Prandti number. Book 1 contains a listing of substance, the properties compiled for each substance and the classification number for each substance. The Search parameter Index, Book 2, is arranged by property and by substance classification number. For each substance, one or more compilation serial numbers are listed together with information on the state of the material. subject of the publication, language, temperature range considered and year of pubilication of the reference. Book 3 contains the Master Bibliography and an Author Index.

\#6511003

* $8,182-2,782$

URANIUM-CARBON AND PLUTONIUM-CARBON SYSTEMS, A THERMOCHEMICAL ASSESSMENT, A REPORT FROM THE PANEL ON TIIERMODYNAMIC PROPERTIES OF THE URANIUM-CARBON AND PLUTONIUM-CARBON SYSTEMS HELD IN VIENNA, 3-12 OCTOBER 1962

Vienna, Austria:International Atomic Energy Agency, ST I/DOC/10/14

February 1963

$48 \mathrm{p}$

Available from International Atomic Energy Agency

Thermodynamic data reported by various 1aboratories on $\mathrm{U}-\mathrm{C}$ and Pu-C systems are reported. Efforts were made to resolve various discrepancies. Sections are included on preparation of uranium carbides, phase diagrams, heat capacities and thermal properties of the actinide carbides, heat of formation of the uranium carbides, vaporization studies, high-temperature equilibrium measurements, and the $\mathrm{Pu}$ - C system.

\#6500170 *8.183-4.183

Van Kranendonk, J.; Van Vleck, J. H.

SPIN WAVES

Reviews of Modern physics $30: 1-23$

This article reviews the spin waves method of calculating the magnetization for a crystal composed of regularly spaced atoms at low temperatures, where magnetization differs on $1 y$ slightly from that at absolute zero. This article assembles in one place and in a unified fashion many of the results which are rather scattered in the literature, and introduces a simplified version of the quantum-mechanical theory. The approach is based on approximating the magnetic spin system by a system of harmonic oscillators. The treatment is based on the lleisenberg, or localized-spin model for a magnetic solid, which is analogous to the Heitler-London model of chemical bonds. The magnetism is regarded as coming entirely from electronic spins regularly spaced in the crystal.

\# 6902022

* 8.283

Van Thie1, M.; Kusubov, A. S.; Mitche11, A. C. (Editors)

COMPENDIUM OF SIIOCK IVAVE DATA, VOIIME I, SECTION A1. SECTION A-2, VOLIME II, SECTION B, C, D, INDEX UCRL-50108, Volumes I \& II, California University, Livermore, Cali fornia

1967

$900 \mathrm{p}$

Thermodynamic data in condensed media obtained by shock wave techniques are listed. Both dynamic variables and volumetric quantities associated with the shock wave are given. The information tabulated is derived from the shock compression of a sample of known dersity. The measurable quantities tabulated are pressure, volume or density, free surface and particle velocity behind the shock front, as well as shock velocity. The properties of the sample hefore shock compression are listed if available. The volumes are divided into the following sections: Section A-1, elements; Section A-2, inorganic compounds, including intermetallic compounds; Section B, hydrocarbons, organic compounds containing only $\vec{C}$ and II: Section C, other orgaric compounds; and Section D, alloys, solutions and mixtures. A materials index is included as well as a source index for each section. 
\#7001022

$* 8.183$

Vasserman, A. A.; Rabinovich, V. A.

Barouch, A. (Trans I ator)

TIIERMOPIYYSICAL PROPERTIES OF LIQUID AIR AND ITS COMPONENTS

IPST Cat. No. 5555, Israel Program for Scientific Translations, Jerusalem, Is rae 1

1970

243

Availahle from CFSTI, TT 69-55092

The themophysical properties of liquid nitrogen, oxygen, argon and air are examined in this monograph. This book supplements previous studies by the authors on the thermophysical properties of these substances in the gascous state. The book surveys and analyzes the best-known equations of state for liquids. An eauation of state is derived for liquids on the basis of the principles of statistical physics, and is valid for many temperatures and densities. A method is developed for setting up this equation; it is used to describe analytically the thermal properties of the four most important cryogenic liquids. The experimental thermal data for liquid oxygen, argon and air are extrapolated to pressures of 500 bar. As a result, equations of state valid for pressures of practical importance have heen established, and the thermal and caloric properties of these substances have been calculated for parameters which have not been studied experimentally. The values obtained for the density were also used in tabulating the viscosity and conductivity of the four liquids with the aid of the limited experimental material on transfer coefficients. It was thus possible to obtain all the data on the thermodynanic and transport properties of 1 iquid air for the pressures most important for practical applications, extending to the saturation and freezing curves.

\#6500484 * 8.183

Vines, R. G.; Keyes, F. G.

THE TIERMAL CONDUCTIVITY OF. NITROGEN AND ARGON

Technical Report MIT-34-P, Virginia University, Department of Aerospace Engineering, Charlottes: ville, Virginia

July 1963

$22 \mathrm{p}$

Available from DDC, AD 412325

In recent years, a series of measurements of the thermal conductivity were completed for steam over a range of temperature and pressure. Argon and nitrogen were both used as a control check during the course of the measurements of the thermal conductivity of steam. These gases, particularly nitrogen, have been investigated extensively and additional measurements were made for nitrogen and argon using the steam cell. The new data is given together with a correlation of much of the available data for both gases, as a function of temperature and pressure.

\#6807005,6807006

$* 8.183$

Voskresenskaya, N. K, ; et al.

SPRAVOCIINIK PÓ PLAVKKOSTI SISTEMI2 BF.2VONNYKH NEORGANI CHESKIKH SOLEI,

TOM I : DVOINYE SISTE!YY

TOM II: SISTEMY TROINYF, TROINYE VZAIMNYE I BOLEE SLOZHNYE

(HANDBOOK OF FUSED SYSTEMS IN ANITYROUS INORGANIC SALTS

VOLUME I. BINARY SYSTEMS

VOLUME II. TERNARY SYSTEMS, RECIPRICAL TERNARY AND MORE COMPLICATED SYSTEMS)

Moscow, Leningrad: Academy of Sciences, USSR

1961

$585 p, 843 p$

In Russian

This handbook of fused salt systems is hased on 800 sources from authors in Russian, German, French, English, Italian and Japanese. The systems are arranged alphabetically according to the elements involved. Information discussed in these volumes include methods of distillation, thermal analys is, visual observations of first appearing crystals, Xray and optics of quenched phases, chemical analysis of various solid and liquid nhases, the nature of solids and the heat of solution. The first volume deals only with binary sustems and simple salts with a common ion. Systems with double salts as one of the components are given as a part of the corresponding hinary system made un of simple salts. The second volume includes salts without a common ion, ternary systens with a common ion, quarternary, and pentamerous svstems. The compilation shows methods used and gives data or thermal analys is of visual observations. When numerical data is not available or incomplete for construction of phase diagrams, graphs are given for the simplest systems in order to find the desired quantitative value (such as position of liquid-ice) if necessary. Conversions in solic phase are explained when given.

\#6903036

$* 8.283$

Vukalovich, M. P.; Altunin, V. V.

Gaunt, D. S. (Fditor of Translation)

THERMOPIYYSICAL PROPERTIES OF CARBON DIOXIDE

London: Collet's (Publishers) Ltd.

1968

$463 \mathrm{p}$

Discussions and compilations of the thermophysical properties of carbon dioxide covering a wide rango of temperatures and pressures are presented. The material included is based on work carried out by the Department of Theoretical Heat Engineering of the Moscow Power Institute between 1958 and 1964. A thorough list of the relevant literature is given to classify the experimental and theoretical work published previously. The properties listed and discussed include density, phase equilibrium, enthalpy, specific heats, equation of state, thermodynamic functions in the ideal gaseous state, viscosity, self-diffusion and thermal conductivity coefficients, Prandtl number at atmospheric pressure, pressure-dependence of viscosity and thermal conductivity, and mass transfer coefficients of carbon dioxide.

\#6510017,6510018 *8.183

Waddington, f.:; Coops, J.; Skinner, H. A, (Compilers)

THERMOCIEMICAL BULLETIN NO, 2 , NO, 3

International Union of Pure \& Applied Chemistry, Sub-Commission on Fixperimental Thermochemistry

March 1956, March 1957

$27 \mathrm{p}, 43 \mathrm{p}$

These issues of the Thermochemical Bulletin contain abstracts from ten nations including 60 literature sources of data and information on thermochemical properties of chemical elements and compounds. The compilations were made by the sub-Commission on Experimental Thermochemistry of the International Union of Pure \& Applied Chemistry and contains some original data, unpublished as of 1957. A majority of the abstracts in the issues deal with heats of reaction (formation, neutralization, solution, combustion, oxidation, etc.).

$\# 6811004,6905014$ * 8.183

Wagman, D. D.; et al.

SELECTED VALUES OF CHEMICAL

PROPERTIES TABLE FOP TUE ELEMENTS IN THE STANDARD ORDER OF ARRANGFMENT TABLES FOR ELFMENTS 35 TIIROUGH 53 IN TIYE STANDARI ORDER OF ARRANGEMENT

NBS Technical Note $270-3,270-4$, National Bureau of Standards, Washington, D. C.

January 1968 , May 1969

$264 \mathrm{p}, 155 \mathrm{p}$

Available from Superintendent of Documents, GPO

The tables contain values, where known, of the enthalpy and Gibbs energy of formation, enthalpy entropy, and heat capacity at $298.15^{\circ} \mathrm{K}\left(25^{\circ} \mathrm{C}\right)$ and the enthalpy of formation at $0^{\circ} \mathrm{K}$, for all inorganic 
substances and organic molecules containing not more than two carbon atoms. In some instances such as metal-organic compounds, data are given for substances in which each organic radical contains one or two carbon atoms. No values are given in these tables for metal alloys or other solid solutions, fused salts, or for suhstances of undefined chemical composition. The physical state of each substance is indicated in the column headed "State" as crystalline solid (c), liquid (liq), glassy or amorphous (amorp), or gaseous (g). Solutions in water are listed as aqueous (aq). For non-aqueous systems the physical state is that normal for the indicated solvent at $298.15^{\circ} \mathrm{K}$.

\section{\#6906035,6906036 \\ *8.183}

Weatherford, W. D., Jr.; Tyler, J. C. ; Ku, P. M.

PROPERTIES OF INORGANIC WORKING FUUIDS AND COOLANTS

FOR SPACE APPLICATIONS PROPERTIES OF INORGANIC ENERGY CONVERSION AND HEAT-TRANSFER FLUIDS FOR SPACE APPLICATIONS

WADC Technical Reports 59-598 and 61-96, Aeronautical Systems Division, Wright Patterson Air Force Base, Ohio

December 1959 , November 1961

$550 \mathrm{p}, 600 \mathrm{p}$

These reports (WADC TR 59-598 and its revision WADC TR 61-96) are intended to serve as a properties handhook for various inorganic fluids which may have potential value as coolants or working fluids for space applications. The fluids are presented as three distinct classes: liquid metals, liquid nonmetals, and gases. The liquid metals include mercury, cesium, rubidium, potassium, NaK(78), sodium, lithium, bismuth, and lead. The liquid nonmetals included sulfur, phosphorus, lithium hydride, and aluminum bromide. The gases include krypton, argon, helium, hydrogen and aluminum chloride, Data are presented, where available, for temperatures ranging from less than $600^{\circ} \mathrm{F}$ to $4500^{\circ} \mathrm{F}$ for lead, and for pressures ranging from less than one atmosphere to greater than twenty atmospheres. The enumerated properties include density, viscosity, thermal conductivity, specific heat, latent heats of fusion and vaporization, enthalpy, entropy, vapor pressure, critical properties, diffusion coefficients, surface tension, thermai neutron absorption and scattering cross sections, equilibrium species composition, melting points, ionization potential, dielectric constant, electrical resistivity, magnetic susceptibility, corrosion characteristics, and materials compatibility. The characteristics of the various fluids are discussed, and the recommended values for the fluid properties are presented in either tabular or graphical form, or both, with detailed documentation as to bas is and source. Background material, including thermodynamic-cycle, heattransfer, compatibility, and working fluid considerations is discussed. A summary of current research activities in this field is presented.

\section{$\# 6500313$ \\ $\star 8.183$ \\ Weber, L.A.;et a1. \\ VAPOR 'PRESSURE OF $20^{\circ} \mathrm{K}$ EQUILIBRIUM HYDROGEN \\ Cryogenics, $21: 236-238$}

The present work is part of a program for the determination of the volumetric, thermodynamic, and transfer properties of $20^{\circ} \mathrm{K}$ equilibrium hydrogen, hereafter referred to simply as para-hydrogen. The purpose of this report is to present this comparison of these vapor pressure determinations with those of Hoge and Arnold and a smooth representation of this data, useful for computation of densities and thermodynamic functions.
\#6510025,6907045

$6706045,6910004,6908059$

$* 8.183$

West rum, E. F. (Editor)

TIERMOCHFMISTRY NO 8 , NO. 9 , NO. 10 , NO. 11 , NO. 12

Ann Arbor, Michigan: University of Michigan Press May 1965, May 1966, March 1967, June 1968, June 1969

$301 \mathrm{p}, 257 \mathrm{p}, 317 \mathrm{p}, 464 \mathrm{p}, 567 \mathrm{p}$

These annual volumes contain abstracts and bibliographies of papers puhlished in the fields of thermodynamics and thermochemistry, arranged by country and 1aboratory. Each abstract contains a short description of the quantities measured or calculated and the method used. Topics of the abstracts include heats of combustion, reaction, and formation; thermodynamic properties of pure substances and systems; vaporization studies; and ther non-calorimetric thermodynamic properties. An author index and substance-property index are included. Each volume covers material from the year previous to the publication of the volume: Volume No. 8 was published in 1965 , No. 9 in 1966 , No. 10 in 1967 , No. 11 in 1963 , and No. 12 in 1960 .

\#6500382

* 8.283

Wicks, C. E.; Block, F. E.

THERMÓDYNAMIC. PROPERTIES OF 65 ELEMENTS

OXIDES, HALIDES, CARBIDES, AND NITRIDES

Bulletin 605, Bureau of Mines, Albany, Oregon

1963

$146 \mathrm{p}$

Available from the Superintendent of Documents, GPO

This Bureau of Nines hulletin was prepared to compile in readily usable form the heat content, heat of formation, and free-energy-of-formation data for 65 common elements and their respective oxides, halides, carbides and nitrides. The necessary theoretical basis of chemical thermodynamics is discussed in part 1 of this bulletin. It is assumed that the user will be at least acquainted with the fundamentals of chemical the rmodynamics. Accordingly, all derivations of the various functions and their interrelations have been eliminated. This part contains the essential formulas necessary for applying fundamental data to process prohlems, with the accompanying examples included mainly to illustrate their applications. A survey of literature published through 195 ? permitted assemhly of all accepted measured and estimated thermodynamic values. These values are included in part 2 of this bulletin. To satisfy individual needs, these data have been presented in 3 separate forms: (1) Tables of heat content, heat of formation, and free energy of formation at various temperatures as well as values for phase changes; (2) Equations relating the variation of the thermodynamic functions with temperature; and (3) Graphical plots of the variation of free energy of formation with temperature.

\section{$\# 6500504$}

* 8.283

Wienecke, R.

Weinreich, M. I. (Translator)

EXPERIMENTAL AND THEORETICAL DETERMINATION OF TIIE THERMAL CONDUCTIVITY OF TIIE PLASNA IN A HIGIICURRENT CARBON ARC

TPRC Translation 9, Purdue University, Thermophys cal properties Research Center, Lafayette, Indiana September 1960

$24 p$

The total thermal conductivity for the plasma of a high-current carbon arc is determined from the observation of the cooling trend in the arc column after a sudden circuit break of the arc current as a function of the temperature within a range from $4000^{\circ}$ to $10,000^{\circ} \mathrm{K}$, after which the total thermal conductivity is compared with the theoretically calculated values. The qualitative agreement between experimental and theoretical ahsolute values is satisfactory, whereas the two curves (kappa (T) show a displacement relative to each other, in such a way that the curve found 
experimentally is shifted toward temperatures which are lower than those of the theoretical curve. It is presumed that the cause of this phenomenon is to be sought in the existence of relaxation processes during recombination of atoms into molecules.

\#60704035, 6611010

7001040,7001041

Willardson, R. K. Beer, A. C. (Editors)

SEMICONDUCTORS AND SEMIMETALS,

VOLUME 1: PHYSICS OF III-V COMPOUNDS

VOLUME 2: PHYSICS OF III-V COMPOUNDS;

VOLUME 3: OPTICAL PROPERTIES OF III-V COMPOUNDS;

VOLUME 4: PHYSICS OF III $-V$ COMPOUNDS

New York: Academic Press

$1966-1968$

$500 \mathrm{p}, 432 \mathrm{p}, 581 \mathrm{p}, 524 \mathrm{p}$

This set of four volumes deals with the semiconductor, semimetal and optical properties of the III-V compounds. Contributions from ten to fifteen scientists are included in each volume. The books are designed for reference work as well as texts on the graduate level. Volume 1 reviews key properties of the III-V compounds, with special emphasis on band structure, magnetic field phenomena, and plasma effects. In volume 2 , the emphasis is on physical properties, thermal phenomena, magnetic resonances, and photoelectric effects, as well as radiative recombination and stimulated emission. Volume 3 is concerned with optical properties, including lattice effects, intrinsic absorption, free carrier phenomena and photoelectronic effects. Volume 4 includes thermodynamic properties, phase diagrams, diffusion, hardness, and phenomena in solid solutions as well as the effects of strong electric fields, hydrostatic pressure, nuclear irradiation and nonuniformity of impurity distribution on the electrical and other properties of III-V compounds.

$\# 6500248$

$\star 8.383-3.383$

Winchell, A. N.

THE OPTICAL PROPERTIES OF ORGANIC COMPOUNDS, SECOND EDITION, ENLARGED AND COMPLETELY REVISED

New York: Academic Press

1954

$515 \mathrm{p}$

This second edition of The Optical properties of Organic Compounds describesmore than twenty-five hundred substances and nearly two thousand are placed on one or both of the two diagrams which are enclosed with the book for the determination of compounds. All organic compounds whose optical properties were described prior to October 1, 1952 are included.

\#6500218

Wood, L.

PHYSICAL CHEMISTRY OF SYNTHETIC RUBBERS

Chapter 10 from Synthetic Rubber, edited by G. S. Whitty, pp. 316-372

New York: John Wiley \& Sons

1954

$57 \mathrm{p}$

This chapter discusses the value of the principles of physical chemistry in the development of synthetic rubbers and their application particularly in the American synthetic-rubber industry in the period between 1940 and 1954. It presents information about the structure and properties of rubbers and some possibilities of altering the structure to produce new rubbers with desired properties. Much attention is given to the Government-produced butadiene-styrene copolymer, the GR-I (Butyl rubber), GR-S, Neoprene, and other rubbers which were produced on a large scale during that period. A large amount of data concerning the physiochemical properties of these rubbers is tabulated as well as a list of 407 references to work that has been done in this area.
$\# 6500215$

$\star 8.383$

Wood, L. A.

SYNTHETIC RUBBERS: A REVIEW OF THEIR COMPOSITIONS, PROPERTIES, AND USES

NBS Circular 427, National Bureau of Standards, Washington, D.C.

June 25,1940

$31 \mathrm{p}$

Available from the Superintendent of Documents, GPO

Twenty-nine commercial varieties of synthetic rubber are classified as.(1) chloroprene polymers, (2) butadiene polymers (including co-polymers), (3) organic polysulfides, (4) isobutene polymers, (5) plasticized vinyl chloride polymers, and (6) dimethylbutadiene polymers. Familiar examples of each of these types are, respectively, (1) Neoprene, (2) the German Buna rubbers, (3) Thiokol, (4) Vistanex, (5) Koroscal, and" (6) "methyl rubber". Since the last type is chiefly of historical interest, it is treated only briefly. For each of the first five types a brief discussion is presented concerning the raw materials used, the chenical reactions during manufacture, and the general conditions of polymerization or condensation. There is also some discussion of compounding and vulcanization, and of the properties and uses of the product. Published data on the different types are summarized in comparative tables of values of density, refractive index, identity period along the fiber axis, swelling in liquids, tensile strength, and permeability. The reported values of dielectric constant, power factor, and resistivity are also given. In Germany and Russia synthetic rubber is used to displace natural rubber in all its applications; in other countries, especially the United States, only those applications where natural rubber is notably deficient are of present importance. Synthetic rubbers show outstanding superiorities to natural rubber in resistance to the effects of liquids, especially petroleum products, and in resistance to deterioration by light, heat, ozone, and oxygen. These superiorities offset the higher cost of the synthetic materials, which is in most cases at least three or four times that of natural rubber. A bibliography of over 200 references covers the literature of the decade 1930-40.

$\# 6500219$

$\star 8.383-4.383$

Wood, L. A.

VALUES OF THE PHYSICAL CONSTANTS OF RUBBER

In "Rubber Technology Conference, London, May 23 25, 1938, First proceedings," pp. 933-953

Cambridge, England: W. Heffer and Sons, Ltd.

1938

$21 \mathrm{p}$

A critical survey has been made of published values for sixteen of the principal physical constants of rubber in the fields of mechanics, heat, optics and electricity, namely: dimensions of unit cell, density, expansivity, thermal conductivity, specific heat, heat of fusion of crystalline rubber, heat of combustion, volume compressibility, poisson's ratio, velocity of sound, refractive index and dispersion, stress-optical coefficient, dielectric constant, power factor, and conductivity. The value for each constant which seems to be the most reliable has been indicated, and, when necessary, corrected to the standard conditions of normal atmospheric pressure and temperature of $25^{\circ} \mathrm{C}$. Temperature coefficients are given when possible. The values are given for rubber in four different forms: the purified hydrocarbon, commercial raw rubber, soft vulcanised rubber containing $2 \%$ combined sulfur, and hard rubber containing $32 \%$ combined sulfur. Relationships between expansivity and change of density with temperature, expansivity and change of refractive index with temperature, and refractive index and dielectric constant are discussed. 
\#6603019

$\star 8.183-4.183$

Wood, W. D.; Deem, H. W.; Lucks, C. F.

PLENUM PRESS HANDBOOKS OF HIGH-TEMPERATURE MATERIALS NO. 3 . THERMAL RADIATIVE PROPERTIES New York: Plenum Press

1964

$474 \mathrm{p}$

This report is a compilation of data on thermal radiative properties. It also includes a brief discussion of the basic fundamentals of thermal radiation and of the methods of measuring these properties. Much of the information has previously been distributed in DMIC memoranda; however, it is consolidated in this report for the benefit of those with a broad interest in radiant heat transfer. Thermal radiative data are included for the following materials: titanium and its alloys; stainless steels; iron-, nickel-, and cobalt-base superalloys; the refractory metals (chromium, columbium, molybdenum, tantalum, and tungsten) and their alloys; coated materials for elevatedtemperature service; and ceramics and graphite. Forty-one references are included.

\#6907022

$* 3.193-8.193$

Working Group on Infrared Backgrounds

INFRARED TARGET AND BACKGROUND

EASUREMENTS CONCEPTS, UNITS ANI TEC

IRIA Report 2389-64-T, Michigan University, Institute of Science and Technology, Ann Arbor, Michigan

January 1962

$43 \mathrm{p}$

Available from DDC

This report discusses concepts, units, and techniques for making and describing measurements of radiation from targets and backgrounds. Graphs, figures, and references are included.

\#6902006

* 8.293

Yatsimirskii, K. B.; Vasil'ev, V. P.

INSTABILITY CONSTANTS OF COMPLEX COMPOUNDS

New York: Consultants Bureau

1960

$214 \mathrm{p}$

Translated from Russian

This book gives the instability constants of 1381 complex compounds prefaced with an introductory section of a general theoretical nature examining methods of calculating instability constants from experimental data, the effect of external conditions on the stability of complexes, and the main factors determining the stability of complex compounds in aqueous solutions. This compilation includes original and abstract literature through 1954 and some work published in 1955-56.

\#6500308
Younglove, B. A.; Diller, D. E. E

THE SPECIFIC HEAT ÁT CONSTANT VOLUME OF PARAHYDROGEN AT TEMPERATURES FROM 15 TO $90^{\circ} \mathrm{K}$ AND PRESSURES TO 340 ATMOSPHERES

Cryogenics, $2: 1-5$

December 1962

The computation of thermodynamic properties, relative to that of ideal gas, of compressed fluid by means of the equation of state data requires the data to be extremely accurate. The uncertainties that are introduced through the derivatives of the equation of state would be minimized by supplementing the calculations with the constant volume specific heat data of the compressed fluid. Specific heats for both ideal gas and compressed liquid states, together with PVT data will permit computations around closed paths to check the overall consistency of the data. For these applications the current program on hydrogen properties includes calorimetric specific heats of parahydrogen at constant volume. There are reported in this paper measurements of $C_{v}$ of the compressed fluid from well below to above the critical temperature.

\#6500307 *8.193

Young love, B. A.; Diller, D. E.

THE SPECIFIC HEAT OF SATURATED LIQUID PARAHYDROGEN FROM 15 TO $32^{\circ} \mathrm{K}$

Cryogenics, $2: 1-5$

September 1962

The specific heat of saturated liquid parahydrogen $\mathrm{C}_{\sigma}$ has been measured as part of a program for determining the thermodynamic properties of parahydrogen at high densities and low temperatures: Thermodynamic properties of the compressed liquid have been computed using $C_{0}$ as the baseline. The specific heat of argon has been measured for comparison with that of Flubacher, Leadbetter, and Morrison. Also the $C_{\sigma}$ of parahydrogen has been measured by others, which provides an additional comparison. The results obtained in this work serve to evaluate the accuracy that may be expected in future measurements of compressed fluids with this apparatus.

$\# 6500316 \quad * 8.393$

Ziegler, W. T.; Mullins, J. C.; Kirk, B. S

CALCULATION OF THE VAPOR PRESSURE AND HEATS OF VAPORIZATION AND SUBLIMATION OF LIQUIDS AND SOLIDS, ESPECIALLY BELOW ONE ATMOSPHERE, 1. ETHYLENE

Technical Report No. 1, project A-460, Engineering Experimental Station, Georgia Institute of Technology, Atlanta, Géorgia

June 2, 1962

$47 \mathrm{p}$

A number of thermodynamic relations are presented for computing, or assessing the probable accuracy of, the vapor pressure and heat of vaporization and sublimation of liquids and solids as a function of temperature. The relations are exact and take into account gas imperfection in the vapor phase. The solution of the equations, in general, requires iterative procedures which can be readily handled on an automatic digital computer. Applications of certain of the relations to the computation of the vapor pressure and heat of vaporization and sublimation of ethylene from its normal boiling point $\left(169.40^{\circ} \mathrm{K}\right)$ to $20^{\circ} \mathrm{K}$ are presented. The computed results are given at 2 degree intervals from 168 to $100^{\circ} \mathrm{K}$ and 5 degree intervals from 100 to $20^{\circ} \mathrm{K}$. The calculated results are thermodynamically consistent with the available experimental data and represent a thermodynamically acceptable extrapolation of these data to $20^{\circ} \mathrm{K}$.

$\# 6500317$

*8.193

Ziegler, W. T.; Mullins, J. C.; Kirk, B. S. CALCULATION OF THE VAPOR PRESSURE AND HEATS OF VAPORIZATION AND SUBL IMATION OF LIQUIDS AND SOLIDS, ESPECIALLY BELOW ONE ATMOSPHERE, 2. ARGON

Technical Report 2, Georgia Institute of Technology, Engineering Experimental Station, Atlanta, Géorgia

June 15,1962

$43 \mathrm{p}$

The vapor pressure and heats of vaporization and sublimation of argon have been calculated from $88^{\circ}$ to $20^{\circ} \mathrm{K}$ using thermodynamic relations. The calculations made use of published thermal data for saturated liquid and solid argon and second virial coefficient data for the gas. Comparison of the computed vapor pressures with available experimental vapor pressure data showed that the experimental vapor pressure data were not thermodynamically consistent with the published thermal and second virial coefficient data. However, it was found that by decreasing the assigned value of the heat of vaporization at the normal boiling point by about one percent, the computed vapor pressures agreed quite well with the experimental measurements of Flubacher et a1. which appear to be the most accurate data. The 
effects of small adjustments of the other thermal and the second virial coefficient data on the computed vapor pressure were also examined. It was found that no reasonable adjustment of these data alone could bring the computed and experimental vapor pressure data into agreement. The adjusted heat of vaporization at the normal boiling point, together with our choice of the best experimental values for the other necessary thermal and second virial coefficient data, have been used to compute the vapor pressure and heats of vaporization and sublimation of argon at one degree intervals from $88^{\circ}$ to $50^{\circ} \mathrm{K}$ and two degree intervals from $50^{\circ}$ to $20^{\circ} \mathrm{K}$.

\# 6500315

* 8.393

Ziegler, W. T.; Mullins, J. C.; Kirk, B. S.

CALCULATION OF' THE VAPOR PRESSURE AND HEATS OF VAPORIZATION AND SUBLIMATION OF LIQUIDS AND SOLIDS, ESPECIALLY BELOW ONE ATMOSPHERE, 111. METHANE

Technical Report No. 3, Project A-460, Engineering Experimental Station, Georgia Institute of Technology, Atlanta, Georgia August 31, 1962

$60 \mathrm{p}$

The vapor pressure and heats of vaporization and sublimation of methane have been calculated at 2 degree intervals from the normal boiling point to the triple point and at 5 degree intervals from the triple point to $20.4^{\circ} \mathrm{K}$ by a thermodynamic method. Two sets of calculations were made. One calculation, given in Table Vil, made use of a selected set of "best" experimental values for the necessary physical and thermal data. The other calculation, given in Table VIII, made use of the same input data except for slight adjustments in the heat of vaporization and temperature of the normal boiling point. The calculated vapor pressures have been compared with the available experimental data. It was found that the computed vapor pressures agreed quite well with the smoothed experimental data of Brickwedde and Scott down to about $70^{\circ} \mathrm{K}$. Below $70^{\circ} \mathrm{K}$ the agreement, while progressively poorer, still appears to be within the uncertainty of the experimental measurements. There appears to be little basis on which to choose between the computed values given in Tables VI1 and VIII. Uncertainties in the "best" values of thermal data used, as well as uncertainty in the temperature scale corrections, no doubt still exist. However, since the computed vapor pressures given in Table VilI agree slightly better with the extensive and careful measurements of Brickwedde and Scott, the vapor pressures and heats of vaporization and sublimation given in Table V1II are recommended for use. A table of vapor pressures interpolated to one degree intervals is included for convenience. The thermodynamic functions $\left(\mathrm{H}^{\circ}-\mathrm{H}_{0}^{\circ}\right) / \mathrm{T}, \mathrm{S}^{\circ} / \mathrm{R}$ and $-\left(\mathrm{F}^{\circ}-\mathrm{H}^{\circ}\right) / \mathrm{T}$ for methane in the ideal gas state at one atmosphere have been computed at two degree intervals from $90^{\circ}$ to $200^{\circ} \mathrm{K}$, assuming a rigid rotator, harmonic oscillator model, using a summation procedure for the rotational partition function which takes into account nuclear spin. These functions have been used in the calculation of the vapor pressure and heats of vaporization and sublimation mentioned above, assuming that no interconversion of the nuclear spin species occurs (i.e., the room temperature equilibrium state is "frozen in"). The vapor pressure and heats of vaporization and sublimation have also been computed assuming the classical statistical mechanical form of the rotational partition function. The rotational contribution to the ideal gas thermodynamic functions of methane $\left(\mathrm{E}^{\circ}-\mathrm{H}_{\circ}^{\circ}\right) \mathrm{rot} / \mathrm{R}$ and $\left(\mathrm{C}^{\circ}\right.$ rot $) / \mathrm{R}$ for the individual nuclear spin species $A, E$, and $F$ and the "frozen" high temperature equilibrium mixture (A: $E: F=5: 2: 9$ ) have been computed down to $50^{\circ} \mathrm{K}$.
4500318

*8.193

Ziegler, W. T.; Mullins, J. C.

CALCULATION OF THE VAPOR PRESSURE AND HEATS OF VAPORIZATION AND SUBLIMATION OF LIQUIDS AND SOLIDS, ESPECIALLY BELOW ONE ATMOSPHERE, 4. NITROGEN AND FLUOR1NE

Technical Report 1, Georgia Institute of Technology, Engineering Experimental Station, Atlanta, Georgia

April 1963

$59 \mathrm{p}$

The vapor pressure and heats of vaporization and sublimation of nitrogen and fluorine have been computed at one degree intervals from the normal boiling point to $10^{\circ} \mathrm{K}$ and $14^{\circ} \mathrm{K}$, respectively, using available thermodynamic data. The computed results have been compared with the published experimental data. The excellent agreement obtained between the computed and experimental vapor pressure data for nitrogen in the liquid and solid range suggest that the computed values may we 11 represent the experimental data within the accuracy of the temperature scales used by the several investigators. The computed values are therfore recommended for use as smoothed values, especially in the solid range below $50^{\circ} \mathrm{K}$ where the available experimental data are scattered and discordant. For liquid fluorine the agreement between the computed values and the experimental data of Hu, White and Johnston is good except near the triple point. The limited measurements of other investigators, which appear to be less accurate than those of $\mathrm{Hu}$ et al, show significant disagreement with the computed values. For solid fluorine the computed values differ considerably from the single published set of limited experimental data. In view of the limited and scattered nature of the experimental data for fluorine, it is recommended that the computed vapor pressure values be used as a smoothed representation of the vapor pressure of fluorine, especially in the solid range.

$\# 6500319$

*8.193

Ziegler, W. T.; Yarbrough, D. W.; Mullins, J. C. CALCULATION OF THE VAPOR PRESSURE AND HEATS OF VAPORIZATION AND SUBLIMATION OF LIQUIDS AND SOLIDS BELOW ONE ATMOSPHERE PRESSURE, 6. KRYPTON

Technical Report 1 , Georgia Institute of Technology, Engineering Experimental Station, Atlanta, Georgia

July 1964

$44 \mathrm{p}$

The "best" available experimental thermal and second virial coefficient data, together with suitable thermodynamic relations have been used to compute the vapor pressure and heats of vaporization and sublimation of krypton at one degree intervals from $119.786^{\circ} \mathrm{K}$ (normal boiling point) to $12^{\circ} \mathrm{K}$. The effect on the computed results of varying separately the heat of vaporization at the normal boiling point and the second virial coefficient of krypton was examined. In order to obtain reasonably good agreement between the experimental and computer vapor pressures it - was found necessary to use a heat of vaporization at the normal boiling point of $21: 4$ cal/gm mole, a value approximately 0.35 per cent smaller than the best experimental value. Th input data for the calculations were used to compute the heat of sublimation of krypton at $0^{\circ} \mathrm{K}$ and the calorimetric entropy of krypton in the ideal gas state at $119.786^{\circ} \mathrm{K}$. The computed vapor pressures are believed to represent the vapor pressure of krypton as a function of temperature below $119.786^{\circ} \mathrm{K}$ very nearly within the uncertainty of the experimental data. They are especially recommended for use below $75^{\circ} \mathrm{K}$ where experimental data are scanty or lacking. 
$\$ 6500322$

2iegler, W. T.; et al.

CALCULATION OF THE VAPOR PRESSURE AND HEATS OF VAPORI2ATION AND SUBLIMATION OF LIQUIDS AND SOLIDS BELOW ONE ATMOSPHERE PRESSURE, VII. ETHANE

Technical Report No. 2, Project A-764, Engineering Experimental Station, Georgia Institute of Technology, Atlanta, Georgia

December 3i, 1964

$57 \mathrm{p}$

Thermodynamic relations have been used to compute the vapor pressure and heats of vaporization and sublimation of ethane at one degree intervals from $184.52^{\circ} \mathrm{K}$ (the normal boiling point) to $20^{\circ} \mathrm{K}$. The results are presented in Table VIII, These calculations made use of the "best" available experimental data for the normal boiling point and triple point temperatures, the heat of vaporization at the normal boiling point and heat of fusion at the triple point, heat capacity and density of the saturated condensed phases, and a representation of the second virial coefficient as a function of temperature based on the Kihara core model. The necessary thermodynamic properties of the ideal gas phase were computed from statistical mechanical relations using an internal rotation barrier of $2875 \mathrm{cal} / \mathrm{gm}$ mole. Comparison of the computed vapor pressures with the available experimental data strongly suggest that only the vapor pressure data of Loomis and Walters and the smoothed data of the American Petroleum Institute Research Project 44 (which are based at least in part on the data of Loomis and Walters) are reasonably consistent with the thermodynamic data used to compute the vapor pressures. By variation of the internal rotation barrier height and the theoretical representation of the second virial coefficient computed vapor pressures winch agree somewhat better with the data of Loomis and Walters than those given in Table VIII were obtained. However, in view of the uncertainty in the temperature scale of Loomis and Walters and the thermal input data (including the second virial coefficient) it is believed that the vapor pressures and heats of vaporization and sublimation given in Table VIII represent these properties of ethane as satisfactorily as they are known at this time. No comparison of the computed heats of vaporization and sublimation with experimental values was possible, since no measured values of these quantities appear to have been made. The thermodynamic functions $\left(\mathrm{H}^{\circ}-\mathrm{H}_{\circ}^{\circ}\right), \mathrm{S}^{\circ}$ and $\mathrm{C}_{\mathrm{p}}^{\circ}$ of ethane in the ideal gas state at one atmosphere pressure have been computed at $10^{\circ} \mathrm{K}$ intervals from 20 to $300^{\circ} \mathrm{K}$ using an internal rotation barrier of 2875 cal/gm mole. They are given in Table XI.

\section{$\# 6606026$}

* 8.193

Ziegler, W. T.; Mullins, J. C.; Berquist, A. R.

CALCULATION OF' THE VAPOR PRESSURE AND HEATS OF VAPORI2ATION AND SUBLIMATION OF LIQUIDS AND SOLIDS BELOW 1 ATMOSPHERE PRESSURE, VIII XENON

Technical Report 3, Georgia Institute of Technology, Engineering Experiment Station, Atlanta, Georgia

April 1966

$43 p$

The "best" available experimental thermal data, together with suitable the rmodynamic relations, have been used to compute the vapor pressure and heats of vaporization and sublimation of xenon at one degree intervals from 165.02 (the normal boiling point $)$ to $20^{\circ} \mathrm{K}\left(\mathrm{p}=1.05 \times 10^{-34} \mathrm{~mm} \mathrm{Hg}\right)$. The computed vapor pressures have been compared with the available experimental vapor pressure data. The computed triple point pressure, $612.45 \mathrm{~mm} \mathrm{Hg}$, is in excellent agreement with the best experimental value $(612.5 \mathrm{~mm} \mathrm{Hg})$. It is believed that the computed values represent the experimental data within the present limits of uncertainty of the measurements and the temperature scales used by the different investigators. The computed values are especially recommended for use in the temperature range below the triple point $\left(161.37^{\circ} \mathrm{K}\right)$ where experimental data are scanty or lacking. The computed values are compared with the independently computed values or Grutter and Schorrock. The heat of sublimation of xenon at $0^{\circ} \mathrm{K}$ has been computed to be $3798 \mathrm{cal} / \mathrm{gm}$ mole. The calorimetric entropy of the ideal gas has been re-computed to be 37.708 cal/gm mole ${ }^{\circ} \mathrm{K}$.

\#6905041

$$
\text { *3. 393-8. } 393
$$

Zwolinski, B. J.: Wilhoit, R. C.

COMPREHENSIVE INDEX OF API 44-TRC SELECTED DATA ON THERMODYNAMICS AND SPECTROSCOPY

publication 100, Chemical Thermodynamic Properties Research Center, Texas A\&M University, College Station, Texas

1968

$507 \mathrm{p}$

The API-TRC Comprehensive Index is a key to the contents of publications presenting critically evaluated numerical property values and selected spectral data that are prepared, compiled, published and distributed by the Thermodynamics Research Center of Texas AGM University. This index contains in codified form the contents of twelve distinct loose leaf data sheet compilations of physiocochemical, thermodynamic and spectroscopic data. Included are a compound name index and a formula index.

$\# 6500362$

*8.293

Zwolinski, B. J.

OPERAT IONAL AND TECHNICAL RULES AND PROCEDURES FOR THE PREPARATION OF THE CONT INUING CRIT ICAL TABLES OF THE API RESEARCH PROJECT 44 AND THE MCA RESEARCH PROJECT CONVERSION AND USE OF TEMPERATURE SCALES Operations Memo 19, Texas AGM University, Chemical Thermodynamics Properties Center, College Station, Texas

June 1965

$18 \mathrm{p}$

A history and detailed description of temperature scales is given and procedures for the conversion of temperature to scales used by API and MCA tables.

\#6601019-6601023

$* 8.393$

Zwolinski, B. J.

SELECTED VALUES OF HYDROCARBONS AND RELATED COMPOUNDS, VOLUMES 1-5

Chemical Thermodynamics properties Center, Texas AGM University, College Station, Texas

October 1964

$2500 \mathrm{p}$

New and revised values of physical and thermodynamic properties of hydrocarbons and related sulfur and nitrogen compounds of primary interest to the petroleum industry are presented in tabular form. Some of the properties include density; vapor pressure; boiling point and freezing point; refractive index; transition temperature specific dispersion; viscosity, surface tension, compressibility factor; critical temperature, pressure and volume; density; heat content function; free energy function; standard entropy and enthalpy; heat capacity; heat of reaction, heat of formation, heat of combustion; free energy of formation; log of equilibrium constant of formation; heat of vaporization, fusion, transition; entropy of vaporization, fusion, transition; and free energy of vaporization. For each table of numerical values, specific references by author are cited. A periodically revised cumulative General List of References is included.

$\$ 6500325,6500326$

$* 8.393$

Zwolinski, B. J.

TRC TABLES SELECTED VALUES OF PROPERTIES

OF CHEMICAL COMPOUNDS, VOLLMES I AND II

Chemical Thermodynamic Properties Center, Texas A\&M University, College Station, Texas

December 1964

$600 \mathrm{p}$ 
New and revised values of physical and thermodynamic properties for classes of compounds of interest to the chemical industry which are hydrocarbons and nonhydrocarbons including halogen, oxygen, and some heterogeneous nitrogen compounds and also many simple inorganic compounds. Some of the properties include density; vapor pressure; boiling point and freezing point; temperature of transition; surface tension; viscosity; refractive index; cryoscopic constant; critical temperature, pressure and volume; density; and compressibility factor; heat content function; free energy function; entropy; enthalpy, heat capacity; log of equilibrium constant of formation; heat of formation; free energy of formation; heat capacity of vaporization, of fusion and transition; entropy of vaporization fusion and transition; and heat of vaporization, fusion and transition. For each table of numerical values, specific references by author are cited. A periodically revised cumulative General List of References is included. 



\section{PERIODICALS}

JOURNAL OF RESEARCH reports National Bureau of Standards research and development in physics, mathematics, chemistry, and engineering. Comprehensive scientific papers give complete details of the work, including laboratory data, experimental procedures, and theoretical and mathematical analyses. Illustrated with photographs, drawings, and charts.

Published in three sections, available separately:

\section{- Physics and Chemistry}

Papers of interest primarily to scientists working in these fields. This section covers a broad range of physical and chemical research, with major emphasis on standards of physical measurement, fundamental constants, and properties of matter. Issued six times a year. Annual subscription: Domestic, $\$ 9.50$; foreign, $\$ 11.75 \%$.

\section{Mathematical Sciences}

Studies and compilations designed mainly for the mathematician and theoretical physicist. Topics in mathematical statistics, theory of experiment design, numerical analysis, theoretical physics and chemistry, logical design and programming of computers and computer systems. Short numerical tables. Issued quarterly. Annual subscription: Domestic, $\$ 5.00$; foreign, $\$ 6.25 \%$.

\section{Engineering and Instrumentation}

Reporting results of interest chiefly to the engineer and the applied scientist. This section includes many of the new developments in instrumentation resulting from the Bureau's work in physical measurement, data processing, and development of test methods. It will also cover some of the work in acoustics, applied mechanics, building research, and cryogenic engineering. Issued quarterly. Annual subscription: Domestic, $\$ 5.00$; foreign, $\$ 6.25 *$.

\section{TECHNICAL NEWS BULLETIN}

The best single source of information concerning the Bureau's research, developmental, cooperative and publication activities, this monthly publication is designed for the industry-oriented individual whose daily work involves intimate contact with science and technology-for engineers, chemists, physicists, research managers, product-development managers, and company executives. Annual subscription: Domestic, $\$ 3.00$; foreign, $\$ 4.00 *$.

* Difference in price is due to extra cost of foreign mailing.

\section{NONPERIODICALS}

Applied Mathematics Series. Mathematical tables, manuals, and studies.

Building Science Series. Research results, test methods, and performance criteria of building materials, components, systems, and structures.

Handbooks. Recommended codes of engineering and industrial practice (including safety codes) developed in cooperation with interested industries, professional organizations, and regulatory bodies.

Special Publications. Proceedings of NBS conferences, bibliographies, annual reports, wall charts, pamphlets, etc.

Monographs. Major contributions to the technical literature on various subjects related to the Bureau's scientific and technical activities.

National Standard Reference Data Series. NSRDS provides quantitative data on the physical and chemical properties of materials, compiled from the world's literature and critically evaluated.

Product Standards. Provide requirements for sizes, types, quality and methods for testing various industrial products. These standards are developed cooperatively with interested Government and industry groups and provide the basis for common understanding of product characteristics for both buyers and sellers. Their use is voluntary.

Technical Notes. This series consists of communications and reports (covering both other agency and NBS-sponsored work) of limited or transitory interest.

Federal Information Processing Standards Publications. This series is the official publication within the Federal Government for information on standards adopted and promulgated under the Public Law 89-306, and Bureau of the Budget Circular A-86 entitled, Standardization of Data Elements and Codes in Data Systems.

Order NBS publications from:

Superintendent of Documents

Government Printing Office

Washington, D.C. 20402 
U.S. DEPARTMENT OF COMMERCE

WASHINGTON, D.C. 20230

OFFICIAL BUSINESS

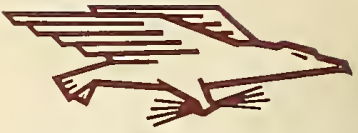

POSTAGE AND FEES PAID

U.S. DEPARTMENT OF COMMERCE

Penalty far Private Use, $\$ 300$ 